\title{
Die Gradpartikeln im Deutschen
}

Untersuchungen zu ihrer Syntax, Semantik und Pragmatik

\section{INAUGURAL-DISSERTATION}

zur Erlangung des

Doktorgrades der Philosophie

der Ludwig-Maximilians-Universität zu München

Fachbereich 14

"Sprach- und Literaturwissenschaft II«

vorgelegt von

HANS ALTMANN

Muinchen

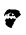

$416 \quad 040392 \quad 800 \quad 16$

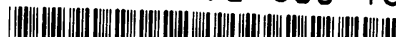

Tuibingen: Max Niemeyer Verlag 
Referent: Prof. Dr. Hans Fromm

Korreferent: Prof. Dr. Theo Vennemann

Tag der mündlichen Prüfung: 28.2.1975

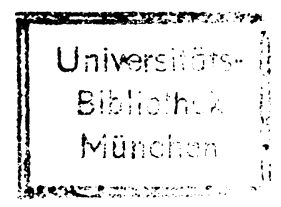

Die Arbeit erscheint gleichzeitig als Bd. 33 der Reihe Linguistische Arbeiten im Max Niemeyer Verlag Tübingen 
INHALTSVERZEICHNIS

1. EINLEITUNG

1.1. Zur Terminologie und zum Untersuchungsgegenstand

2. THEORETISCHE GRUNDLAGEN

2.1. Zum Verhältnis von Theorie und Empirie bei der Beschreibung von Gradpartikeln ................................. 4

2.1.1. [Grammatiken des Deutschen] 4

2.1.2. [Wörterbücher des Deutschen] 5

2.1.3. [Nevere Gradpartikel-Literatur] 5

2.1.4. [Vorschlag für eine ausgeglichene Gestaltung des
Verhältnisses von Theorie und Empirie]

2.1.5. [Beschreibungsgegenstand; Materialbasis] 9

2.1.6. [Materialauswertung] 12

2.1.7. [Strukturierung der Gradpartikel-Beschreibung] 12

2.2. Zur syntaktischen Beschreibung der Gradpartikeln im Deutschen ..... 13

2.2.1. [Syntax und Kategorisierung] 13

2.2.2. [Zur Kategorisierung der Gradpartikeln in der Forschung] 15

2.2.3. [Probleme der Kategorisierung von Gradpartikeln im Deutschen] 16

2.2.4. Syntaktische Analyse- und Beschreibungsverfahren 18

2.2.4.1. [Kommtationstest; Klassenbildung] (18)

2.2.4.2. [Permutationstest; Stellungsgesetze] (19)

2.2.4.3. [Transformationstests: Satztypen, Negationsmöglichkeiten] (22)

2.2.5. [Syntax-Themen im Rahmen der Beschreibung von Gradpartikeln in einer generativen Transformationsgrammatik]

2.2.5.1. [Spaltsatz ('clefting')] (22)

2.2.5.2. [Topikalisierung] (24)

2.2.5.3. [Extraposition] (25)

2.2.5.4. [Linksversetzung ('left dislocation')] (25)

2.2.5.5. [Aktiv - Passiv] (25)

2.2.6. Exkurs zur Diskussion um die Darstellung von Gradpartikeln in einer generativen Transformationsgrammatik

2.3. Probleme des Skopus von Gradpartikeln .................. 28

2.3.1. [Skizzierung der Fragestellung] 
2.3.2. [Arbeitshypothesen für die Skopusbeschreibung] 29

2.3.3. [Zur Gliederung des Analysenteils nach Skopustypen] 33

2.4. Zur Topic-Comment-Gliederung bei Gradpartikel-Sätzen.

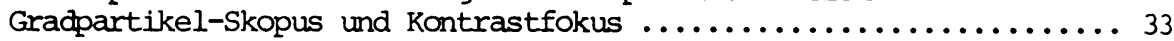

2.4.1. [Bedeutung dieses Themas für die Gradpartikel-Beschreibung] 33

2.4.2. [Topic, Fokus, Fragesatztest] 33

2.4.3. [Topic und Fokus in der generativen Transformationsgrammatik]

2.4.4. [Normal- und Kontrastierungsfälle] 38

2.4.5. [Gradpartikel-Sätze und Kontrastierung] 41

2.4.6. [Beziehungen zwischen Gradpartikel-Skopus und Kontrastfokus. Skopustypen. Subtypen durch Kontrastierung]

2.5. Probleme der semantischen Beschreibung von Gradpartikeln

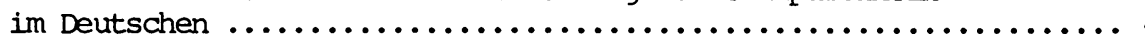

2.5.1. Zu allgemeinen Problemen im Zusammenhang mit der Beschreibung des Gradpartikel-Semantik

2.5.2. [Aufgaben der Gradpartikel-Semantik; Gradpartikel-Semantik in der Forschung]

2.5.3. Assertion

2.5.4. Präsupposition

2.5.4.1. [Gradpartikel-Semantik und Präsuppositionsforschung] (55)

2.5.4.2. [Frege, Russell und die Präsuppositionsthematik]

2.5.4.3. [Grundlinien der Präsuppositionsthematik in der Logik; Wahrheitswert, Negation] (58)

2.5.4.4. [Grundlinien der linguistischen Präsuppositionsdiskussion] (60)

2.5.4.5. [Logisch-semantische und pragmatische Präsuppositionen ] (63)

2.5.5. Konversationelle Implikatur

2.5.5.1. [Orientierung] (66)

2.5.5.2. [Konversationsmaximen von Grice] (66)

2.5.5.3. [Konversationelle Implikaturen bei Grice] (67)

2.5.5.4. [Konversationelle Implikatur bei L. Horn] (68)

2.5.6. Verfahren zur Analyse von Semantik und Pragmatik der Gradpartikeln

2.5.6.1. Der Negationstest (71)

2.5.6.2. Ermittlung von Bedeutungselementen durch geeignete Kontexte (78)

2.5.6.3. Der Suspensionstest (80)

2.5.6.4. Der Konjunktionstest mit und (82)

2.5.6.5. Der monologische Widerspruchstest (aber - Test) (84)

2.5.6.6. Dialogische Analyse von Gradpartikelsätzen (84) 
3. ANALYSENTEIL I: UNTERSUCHUNG DER GRADPARTIKEIN NACH SKOPUSTYPEN

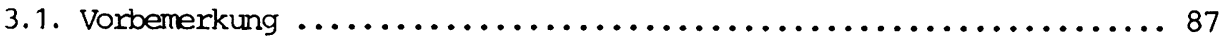

3.1.1. Liste der untersuchten Gradpartikeln 87

3.1.2. Analyseprogramm 87

3.2. Skopustyp: Nominalphrasen $\ldots \ldots \ldots \ldots \ldots \ldots \ldots \ldots \ldots \ldots \ldots \ldots \ldots$

3.2.1. Skopus: Einfache Nominalphrasen 89

3.2.1.1. nur - Gruppe $\quad 89$

Syntax (89); Skopus (96); Semantik (99)
auch - Gruppe

3.2.1.2. auch - Gruppe
Syntax (107); Skopus (112); Semantik (113)

3.2.1.3. Sogar - Gruppe
Syntax (118); Skopus (120); Semantik (122)

117

3.2.2. Exkurs: Skopustyp komplexe Nominalphrase 130

3.2.2.1. nur - Gruppe (130)

3.2.2.2. auch - Gruppe (134)

3.2.2.3. sogar - Gruppe (135)

3.2.3. Exkurs: Skopustyp Nominalphrase mit kontrastiv betontem Attribut

3.2.3.1. nur - Gruppe (135)

3.2.3.2. auch - Gruppe (138)

3.2.3.3. sogar - Gruppe (140)

3.2.4. Skopustyp: Präpositionalobjekte

141

3.2.4.1. nur - Gruppe (141)

3.2.4.2. auch - Gruppe (145)

3.2.4.3. sogar - Gruppe (146)

3.2.5. Skopustyp: P'ronominalisierte Nominalphrasen

146

3.2.5.1. nur - Gruppe (146)

3.2.5.2. auch - Gruppe (149)

3.2.5.3. sogar - Gruppe (150)

3.2.6. Skopustyp: Gliedsätze

3.2.6.1. nur - Gruppe (151)

3.2.6.2. auch - Gruppe (157)

3.2.6.3. sogar - Gruppe (159)

3.2.7. Exkurs: Skopustypen mit kontrastiv betonten Konjunktionen 160

3.3. Skopustypen im Bereich des Prädikats $\ldots \ldots \ldots \ldots \ldots \ldots \ldots \ldots \ldots . \ldots \ldots$

3.3.1. Skopustyp: Verbum 164

3.3.1.1. nur - Gruppe 164

Syntax (164); Skopus (168); Semantik (169)

3.3.1.2. auch - Gruppe
Syntax (175); Skopus (176); Semantik (176)

3.3.1.3. sogar - Gruppe
Syntax (181); Skopus (181); Semantik (181)

175

181

3.3.2. Exkurs: Skopustyp mit kontrastiv betonten Elementen des Auxiliar-Bereiches 
3.3.3. Skopustyp: Prädikatives Adjektiv und Prädikatsnomen

3.3.3.1. nur - Gruppe

Syntax (187); Semantik (190)

3.3.3.2. auch - Gruppe

3.3.3.3. sogar - Gruppe

3. 4. Skopustyp: Verbalphrase ........................ 202

3.4.1./2./3. nur -, auch -, sogar - Gruppe

Skopus (202); Syntax (204); Semantik (206)

3.5. Skopustypen mit kontrastiv betonten Quantoren

208

3.5.1. Skopustypen mit kontrastiv betonten Zahlwörtern

3.5.1.1. nur - Gruppe

Syntax (208); Skopus (210); Semantik (211)

3.5.1.2. auch - Gruppe

Syntax (216); Semantik (217)

3.5.1.3. sogar - Gruppe

Syntax (220); Skopus (221); Semantik (221)

3.5.2. Skopustypen mit den kontrastiv betonten Quantoren viel wenig; viele - wenige - einige - alle

3.5.2.1./2./3. nur -, auch-, sogar - Gruppe

Syntax (223); Semantik (224)

3.6. Skopustyp: Adverbien .......................... 227

3.6.1. Skopustyp: Adverbiale Präpositionalphrase

3.6.1.1. nur - Gruppe (227)

Syntax (227); Skopus (228); Semantik (229)

3.6.1.2. auch - Gruppe (230)

3.6.1.3. sogar - Gruppe (231)

3.6.2. Exkurs: Skopustypen mit kontrastiv betonten Präpositionen

3.6.2.1./2./3. nur -, auch -, sogar - Gruppe (231)

3.6.3. Skopustyp: Adjektivadverbien und reine Adverbien

3.6.3.1. nur - Gruppe (233)

Syntax (233); Skopus (234); Semantik (234)

3.6.3.2. auch - Gruppe (239)

3.6.3.3. sogar - Gruppe (240)

3.6.4. Skopustyp: Satzadverbien

3.6.4.1./2./3. nur -, auch -, sogar - Gruppe (241)

3.6.5. Skopustyp: Limitierende Adverbien

3.6.5.1./2./3. nar -, auch -, sogar - Gruppe (245)

Syntax (245); Semantik (246)

3.7. Skopustyp: Satz

3.7.1. sogar - Gruppe (249)

3.7.2. auch - Gruppe (252)

3.7.3. nur - Gruppe (254) 
4. ANALYSENTEIL II: EINZELTHEMEN

4. 1. Besondere Verwendungsweisen von Gradpartikeln .............. 256

4.1.1. Betonte Gradpartikeln

4.1.1.1. nur - Gruppe (256)

4.1.1.2. auch - Gruppe (258)

4.1.1.3. sogar - Gruppe (262)

4.1.2. "Bindestrich-Zusammensetzungen" mit Gradpartikeln

4.1.2.1. nur - Gruppe (262)

4.1.2.2. auch - Gruppe (263)

4.1.2.3. sogar - Gruppe (263)

4.1.3. Das Auftreten von mehreren Gradpartikeln in einem einzigen Satz

4.1.4. Kombinationen von Modalpartikeln mit Gradpartikeln 269

4.1.5. Feste Verbindungen von Gradpartikeln mit anderen Wörtern

4.1.5.1. nur - Gruppe (271)

4.1.5.2. auch - Gruppe (273)

4.1.5.3. sogar - Gruppe (275)

4. 2. Nicht-Gradpartikel-Funktionen der hier behandelten Partikeln ... 275

4.2.1. Konjunktionale Verwendung

4.2.1.1. nur - Gruppe (275)

4.2.1.2. auch - Gruppe (278)

4.2.1.3. sogar - Gruppe (279)

4.2.2. Modalpartikel-Funktion der untersuchten Elemente

4.2.2.1. nur - Gruppe und Modalpartikel-Funktion (280)

4.2.2.2. auch - Gruppe und Modalpartikel-Funktion (283)

4. 3. Negationsmöglichkeiten bei Gradpartikeln .

4.3.1. nur - Gruppe (288)

4.3.2. auch - Gruppe (291)

4.3.3. sogar - Gruppe (293)

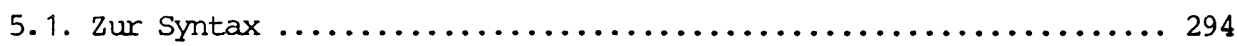

5.1.1. Zur Frage der syntaktisihen Klassenbildung 294

5.1.2. Austauschbarkeit 294

5.1.2.1. [nur - Gruppe] (295)

5.1.2.2. [auch - Gruppe] (295)

5.1.2.3. [sogar - Gruppe] (296)

5.1.2.4. Untergruppen nach distributionellen

Eigenschaften (296)

5.1.3. Stellungsgesetze 
ABKÜRZUNGSVERZEICHNIS

$\begin{array}{llll}\text { GP, GPn } & \text { Gradpartikel (n) } & \text { ASS, ASSen } & \text { Assertion(en) } \\ \text { MP, MPn } & \text { Modalpartikeln(n) } & \text { konv. IMPL } & \text { konversationelle Implikatur } \\ \text { NEG, NEGen } & \text { Negation(en) } & \text { PSP, PSPen } & \text { Präsupposition(en) } \\ \text { NP, NPn } & \text { Nominalphrase (n) } & & \\ \text { PP, PPn } & \text { Präpositionalphrase (n) } & \text { skal. } & \text { skalierend } \\ \text { VP, VPn } & \text { Verbalphrase (n) } & \text { quant. } & \text { quantifizierend } \\ \text { V } & \text { Verb } & & \\ \text { IMP } & \text { Inperativsatz } & & \\ \text { XU } & \text { Fragesatz } & & \end{array}$

BENUTZUNGSHINWEISE

1. Auf ein Register wurde verzichtet, da der Analysenteil mit diesem Verfahren nicht sinnvoll erschlossen werden kann. Um die Orientierung zu erleichtem wurde das Inhaltsverzeichnis ungewöhnlich kleinteilig angelegt. Zieht man das Arbeitsprogramm für den Analysenteil S. 87/88 hinzu, dann entsteht ein so engmaschiger Raster, daß auch für Detailfragen die entsprechende Information aufgefunden werden kann.

2. Für die Wiedergabe von Alternativformulierungen bei Tests wurden unterschiedliche Markierungen gewählt, die sich häufig gegenseitig stützen: Schrägstrich [/], Einrückung, Querstrich [-] und runde (Fakultativ-) Klammem [( )]. Auf diese Weise konnte die störende Wiederholung von Sätzen oder Satzteilen reduziert werden. Das Verfahren müßte trotzdem so unmittelbar plausibel sein, daß der Leser unschwer das jeweils Gemeinte rekonstruieren kann. 
1.1. Zur Terminologie und zum Untersuchungsgegenstand

Die vorliegende Untersuchung beschäftigt sich mit den drei Wörtern nur, auch, sogar und ihren jeweiligen Synonymen bzw. Teilsynonymen, sowie mit ihren negativen Entsprechungen. Für die Gesamtheit dieser Wörter werde ich im folgenden den Terminus "Gradpartikeln" gebrauchen, und zwar nur insoweit, als sie in einer Verwendung vorliegen, auf die die Kennzeichnung "Quantifizierung mittels logischer Operatoren "zutrifft (Bartsch 1972: 192). Dieser Ausdruck meint, daß die Elemente der bezeichneten Wortgruppe mit einer (im allgemeinen kontrastiv betonten) Konstituente semantisch verknüpft werden und diese Konstituente in eine quantifizierende Beziehung zu typgleichen Konstituenten setzen. ${ }^{2}$ Diese Skopuskonstituente ist in den folgenden drei Sätzen die Subjekts-NP:

(1) Von dem Sondertrupp bleibt kein Gefolgsmann am Leben. Nur A g u i $r$ $r e$ übersteht die Fährnisse im Fieber.

(2) Diese Würde kann auch ein $M$ ö $r d$ e $r$ nicht verwirken.

(3) Längst hatte er die Grenzen überschritten, die sogar $N$ a $t$ i o n a I sozialisten dem deutschen Machtstaat zogen.

Es wird eine der Aufgaben der Untersuchung sein, zu zeigen, daß eine vielleicht noch wichtigere Funktion dieser Partikeln ${ }^{3}$ darin besteht, bestimmte Skalen zu

1 In Clément/Thümmel. (1975) werden diese wörter als "Rangierpartikeln" bezeichnet. Da sich dieser Terminus nur auf syntaktische Merkmale bezieht, wodurch auch Partikeln mit anderer semantischer Funktion einbezogen werden, folge ich diesem terminologischen Gebrauch nicht.

2 Eine Konkretisierung der Bezeichnung "typgleiche Konstituente" kann erst in den semantischen Einzelanalysen gegeben werden.

: Auf den Terminus "Partikeln" näher einzugehen und meinen Gebrauch davon aus seiner Geschichte innerhalb der sprachwissenschaftichen Forschung zu rechtfertigen muß ich mir leider versagen. Mir bleibt nur der Hinweis darauf, daß ich mich bei der Abgrenzung wie Bezeichnung der Teilgruppen stark an den Usus innerhalb der russischen Sprachwissenschaft halte, wie er in Admoni (1960), Arndt (1960), Kriwonossow (1963. 1965 a und b. 1966), sowie im Anschluß daran bei Helbig (1968. 1970) und Schubiger (1965) zum Ausdruck kommt. Zur Geschichte des Terminus "Partikeln" in der Indogermanistik 
induzieren und der jeweils mit ihnen semantisch verbundenen Konstituente (dem "Skopus") einen bestimmten Platz oder "Grad" in dieser Skala zuzuweisen: gerade auf diese Leistung, das Plazieren eines Wertes in einer Skala mit Angabe der "Richtung" der Skala, bezieht sich melne Bezeichnung "Gradpartikeln".

Die oben genannten drei Gruppen können jedoch keinesfalls als lückenlose Liste der Gradpartikeln, weder in syntaktischer noch in semantischer Hinsicht, betrachtet werden. Syntaktisch gehört zum Beispiel die Partikel-Gruppe gerade, eben, genau, ausgerechnet in einer Verwendung wie

(4) Daß ausgerechnet/gerade die $T$ ü b ing e $r$ ohne Chancen bleiben sollten, erklärten die betroffenen Assistenten mit der politischen Abstinenz des Tübinger Mittelbaues.

trotz zahlreicher Idiosynkrasien voll und ganz zu den Gradpartikeln, in semantischer Hinsicht stellt sie aber einen Ubergangstyp dar. ${ }^{4}$ Hinzu kommen die zahlreichen Partikeln und auch nicht-Partikel-Konstruktionen, die ähnliche semantische Funktionen wie die Gradpartikeln erfüllen, z.B. höchstens, mindestens, ungefähr, die pure/reine/bloße schikane. ${ }^{5}$ Doch ging es mir um die syntaktischen und semantischen Eigenschaften der Kerngruppe, und nur am Rande um die hochinteressante Erscheinung des "Ausfransens" einer syntaktischen und semantischen Kategorie.

Dort, wo keine andere Möglichkeit der Bezugnahme besteht, werde ich mich unter Vernachlässigung der oben bezeichneten Einschränkung auf eine bestimmte Funktion in Emangelung einer funktionsneutralen Bezeichnung mit dem Ausdruck "Gradpartikeln" auf die Wortgruppe an sich beziehen. - Diese teminologische Neuprägung wurde notwendig, weil in der einschlägigen Jiteratur kein passender Gruppenbegriff auftritt. Das resultiert in der deutschen grammatischen Literatur aus der fehlenden Einsicht in die gemeinsamen Eigenschaften, also in den Gruppencharakter selbst; bei der relevanten englischsprachigen Literatur zu den entsprechenden Wörtern des Englischen (wie in den sich daran anschlieBenden deutschen Veröffentlichungen) überwiegt das Interesse an der semantischen Analyse einzelner Elemente. - Im Zusammenhang mit der Diskussion um präsuppositionsähnliche Erscheinungen in natürlichen Sprachen ist aber die Be-

vergleiche E.G. Hartman (1966) in seiner Einleitung. Festzuhalten ist noch, daß in der angelsächsischen ebensowenig wie in der französischen oder deutschen Sprachwissenschaft "Partikeln" die Rolle eines Terminus, also einer Wortartbezeichnung spielt; hier wird diese Bezeichnung überwiegend vortheoretisch gebraucht.

4 In der ursprünglichen Fassung der Untersuchung war die Gruppe mitbehandelt worden. Die Ergebnisse, soweit sie über das hier Mitgeteilte hinausgehen, werde ich in einer gesonderten Abhandlung veröffentlichen.

5 Persönliche Mitteilung Marga Reis. 
zeichnung "Partikelsemantik" zu einem gern gebrauchten Verständigungssignal für einen bestimmten Forschungsbereich geworden, ohne daß damit nur die oben genannten Wörter gemeint wären. Die unscharfe Abgrenzung hängt sicherlich auch damit zusammen, daß unter dieser Fragestellung die Wortklassenproblematik natürlich keine entscheidende Rolle mehr spielt.

Da ich im Laufe der Untersuchung auch intensiver auf andere als die oben genannten Funktionen der Gradpartikeln eingehen werde, muß ich fuir den weiteren Bereich der Partikeln eine teminologische Regelung anbieten. Sie berïcksichtigt so weit als möglich die jeweilige Tradition der einzelnen Termini und lehnt sich darüberhinaus eng an den derzeitigen Sprachgebrauch an: "Partikeln" verwende ich für den Gesamtbereich der nicht flektierten Wörter. Für semantisch oder syntaktisch motivierte Teilgruppen verwende ich eine Verbindung aus "Partikel" und einem die jeweilige Funktion kennzeichnenden Bestimmungswort, also: "Negationspartikeln", "Modalpartikeln", 6"Konjunktionspartikeln", "Interjektionspartikeln", 7 "Vergleichspartikeln", 8 "Steigerungspartikeln", 9 "Gradpartikeln".

Eine Verwechslung der Gradpartikeln mit den Steigerungspartikeln ("Graduierung") scheint mir aufgrund dieser teminologischen Regelung nicht sehr wahrscheinlich. - Die Etablierung der Gradpartikeln als sowohl syntaktische wie auch semantische Kategorie betrachte ich als eine meiner wichtigsten Aufgaben.

"Modalpartikeln" nach dem terminologischen Usus bei Arndt (1960), Admoni (1960), Kriwonossow (1963. 1965 a und b. 1966): also für Partikeln wie ja, doch, denn, aber usw. Bei Weydt (1969) findet sich die Bezeichnung "Abtönungspartikeln", allerdings trennt der Verfasser nicht sauber genug zwischen der Abtönungsfunktion dieser Wörter und ihren weiteren Funktionen. Etwa zu umschreiben mit den in der Duden-Grammatik (1966: 343 ff.) aufgelisteten Interjektionen, soweit sie unflektiert und einigermaßen stabile sprachliche Zeichen sind, z.B. ah, ach, oweh, hoppla, nanu, aha.

8 So auch in der Duden-Grammatik (1966: 233) genannt: als, denn, so ... wie.

9 Gemeint sind damit Partikeln wie noch, weit, etwas, sehr, höchst, überaus, ungemein, besonders, immer usw., soweit sie zusammen mit attributiven oder prädikativen Adjektiven bzw. Adjektivadverbien auftreten. 
Dieser Abschnitt dient der Vorbereitung der Analyse der Gesetzmäßigkeiten von Gradpartikeln im Deutschen: also der Schilderung der Forschungslage, der Darlegung der wichtigsten Fragestellungen im Zusammenhang mit der Untersuchung von Gradpartikeln, der argumentativen Absicherung der eignen Forschungsposition und der Einführung in die angewandten untersuchungsmethoden. Demgemäß sind die Hauptstationen, die sich in der Kapitelgliederung wiederfinden: das Verhältnis von Theorie und Empirie bei der Untersuchung der Gradpartikeln; Probleme der Syntax von Gradpartikeln; die Beziehungen zwischen Kontrastfokus und dem Skopus von Gradpartikeln; und schließlich die Probleme der Gradpartikelsemantik, die weit in die linguistische Pragmatik hineinreichen.

2.1. Zum Verhältnis von Theorie und Empirie bei der Beschreibung von Gradpartikeln

Man tut der bisherigen Gradpartikel-Forschung wohl nicht Unrecht, wenn man bei ihr einen Mangel an Ausgewogenheit im Verhältnis Theorie - Empirie global konstatiert. ${ }^{1}$ Bei dieser notwendigerweise pauschalierenden Feststellung müssen natürlich individuelle Schwächen oder auch Vorzüge unberücksichtigt bleiben. Drei erstaunlich homogene Untergruppen hinsichtlich des jeweiligen Verhältnisses zum Analysematerial wie der Auffassung von Regelbildung kann man erkennen:

2.1.1. Da ist zum einen die große Gruppe der Grammatiken des Deutschen, in denen die Behandlung der Gradpartikeln naturgemäß sehr knapp ausfallen muß. ${ }^{2}$ Bei

1 Von diesem Vorwurf muß man Clëment/Thümmel (1975) ausnehmen. Ihre Art, sich an Belegen zu orientieren, ähnelt sehr stark meinem eigenen Vorgehen.

2 Vergleiche etwa H. Paul (1916-1920); O. Behaghel (1923-1932); H. Glinz (1961); W. Admoni (1960); Duden-Grammatik (1966; 1973); J. Erben (1964); W. Schmidt (1964); H. Brinkmann (1962). Detaillierte Seitenangaben zur Behandlung von Gradpartikeln in diesen Werken finden sich im Literaturverzeichnis. - Der Aufsatz von Helbig (1970) kann zu dieser Gruppe hinzugerechnet werden. 
aller Verschiedenheit dieser Untermehmungen bleibt doch ihr gemeinsames Kennzeichen die schmale Materialbasis, die zusammen mit dem Fehlen selbständiger Untersuchungen, auf die man sich beziehen könnte, die Beobachtungen und die daraus folgenden Feststellungen unsystematisch, lückenhaft, ja fehlerhaft macht. Das Scheitern des Wortartenkonzeptes bei einer morphologischen "Negativklasse", zusammen mit der Polyfunktionalität der meisten Partikeln läßt nur die verstreute Verzeichnung von zahlreichen Idiosynkrasien $\mathrm{zu}^{3}$ Das bloße, weitgehend vortheoretische Erfassen der möglichen Konstruktionstypen hätte eine ungeheuer große Materialsamlung erforderlich gemacht, die zudem die kritiklose Vermischung der unterschiedlichsten Herkünfte und Verwendungsweisen und damit auch diachroner und synchroner Tatbestände hätte vermeiden müssen.

2.1.2. Nur vordergründig davon völlig verschieden ist die Gruppe der wörterbücher des Deutschen. "Sie enthalten viel, ja unüberschaubar viel sprachliches Material, und zwar aus den unterschiedlichsten quellen, was deren Abfassungszeit und Abfassungszweck betrifft, aber auch von den Verfassem ad hoc gebildet. Fast immer wird diese Verschiedenartigkeit bei der Darstellung bzw. Regelbildung, soweit eine solche überhaupt stattfindet und nicht nur einzelne Gebrauchsweisen verzeichnet werden, ignoriert. Die Auswertung des iberreich gebotenen Materials, das in jedem Fall Berücksichtigung verdient, weist zudem zahlreiche Mängel auf. Es handelt sich fast immer um reine Wortmonographien, 5 gekennzeichnet von einem fast krampfhaften Festhalten an der Einheit des Wortes, die meist nur mit unausgesprochen diachronen Argumenten aufrecht erhalten werden kann, und vom Fehlen der Querverweise auf Synonyme,

Antonyme sowie weitere Elemente der gleichen syntaktischen Kategorie. Daraus resultiert eine sehr hinderliche Zersplitterung der Darstellung, die einhergeht mit einer unzulänglichen Semantiktheorie; deren Mittel erschöpfen sich meist in weitgehend beliebigen Paraphrasen.

2.1.3. Die Arbeiten von R. Bartsch und P. Blumenthal ${ }^{6}$ sowie die angelsächsische Forschung zu only, even und $a l_{s o} /$ too $^{7}$ zeigen ein genau umgekehrtes Er-

3 Ein extremes Beispiel hierfür ist Erben (1964). Vergleiche die Angaben im Literaturverzeichnis! Siehe die Zusammenstellung im Literaturverzeichnis mit Angabe der ausgewerteten Artikel!

5 Abgesehen von Systematisierungsversuchen in Klappenbach/Steinitz (1961 ff).

6 R. Bartsch (1972); P. Blumenthal (1974).

7 B. S.-Y. Kuroda (1965. 1969); S. Fischer (1968); N. Chomsky (1969);

B. Fraser (1971); A. Akmajian (1970a); R.S. Jackendoff (1969 b. 1972);

S. R. Anderson (1972); Ch. J. Fillmore (1965; 1971); L. Horn (1969. 1972);

G. Lakoff $(1970 ; 1971)$; S. F. Schmerling (1972). 
scheinungsbild. Ihr gemeinsames Merkmal ist die äußerst schmale empirische Basis, meist nur bestehend aus wenigen und zumeist selbstgebildeten Sätzen. Diese Tatsache wird erst erwähnenswert durch die Folgen: jede Untersuchung befaßt sich praktisch nur mit den Regularitäten von Idiokompetenzen. Unvereinbare Akzeptabilitätsurteile sind dadurch die Regel und behindern fortwährend die Diskussion. Da die eigene Phantasie meist nicht weit genug trägt, sind Lücken in der Beschreibung unvermeldlich, und fehlerhafte Verallgemeinerungen werden erleichtert. Ferner wird die Bedeutung des Kontextes in aller Regel unterschätzt. In dieser Praxis jedenfalls zeigt sich ein deutlich gestörtes Verhältnis von Theorie und Empirie, sichtbar in der dogmatischen Verkürzung des Beschreibungsgegenstandes, die eine empirische Uberprüfung der Regelbildung fast unmöglich macht. Gestützt auf nur geringes Sprachmaterial zu einem stark eingeschränkten Untersuchungsbereich werden weitreichende theoretische Folgerungen gezogen, und in vielen Fällen wird zudem die Beweislast auf andere verlagert.

2.1.4. Eine Untersuchung, die um einen merklichen Fortschritt auf dem Gebiet der Gradpartikel-Forschung bemiht ist, muß einerseits möglichst alle in der Literatur zum Thema bereits geleisteten Arbeiten aufnehmen, also die in der angelsächsischen Literatur sowie im Gefolge davon bei Bartsch (1972) und Blumenthal (1974) erarbeiteten theoretischen Fragestellungen ebenso wie die zahlreichen Einzelbeobachtungen zur Syntax und Semantik der Gradpartikeln im Deutschen in den einschlägigen Grammatiken, aber auch das in den Würterbüchern des Deutschen bereits gesammelte Sprachmaterial auswerten, soweit es für eine Beschreibung des gegenwärtigen Deutschen relevante Informationen liefern kann. Allein der Versuch, alle diese verstreuten Einzelleistungen zu einer Synthese zusammenzuführen, wäre schon die Anstrengung wert. Darüberhinaus sind aber auch die einleitend bereits knapp charakterisierten Mängel dieser Arbeiten zu vermeiden. Dies kann sich zuerst erweisen in der praktikablen und nützlichen Abgrenzung des Untersuchungsgegenstandes, in unserem Fall also des Sprachsystems oder auch der Sprachsysteme, denen diese Wortgruppe angehört. Hierbei kann die überzogene Idealisierung des Chansky'schen Kompetenzbegriffes als uberwunden gelten. ${ }^{8}$ Einmal ist dieses theoretische Konstrukt der empirischen iberprüfung nicht umittelbar zugänglich, andererseits schließt es eine nicht unbedeutende Menge von Sprechdaten als irrelevant von vormherein von der Beschreibung aus. - Aber auch der in letzter Zeit, infolge der Problematik des Chomsky'schen

8 Dazu nur die folgende Auswahl: N. Chomsky (1965; 1969); S. Kanngießer (1972 a und b); W. Abraham (1970); Althaus/Henne (1971); J. Habermas (1972). 
Kompetenzbegriffes und aus tberdruß an den endlosen Diskussionen um die Akzeptabilität von Belegsätzen, von vielen Forschern gewählte Ausweg, nur noch eine Idiokompetenz, melst die eigene, zu beschreiben ("in my dialect") scheint mir nicht sinnvoll gangbar. Denn gerade die Kompetenz elnes Sprachwissenschaftlers diirfte kaum homogen sein, sondern eher ein Kompetenzgemisch aus Fachund Sondersprachen, Dialekt, Hochsprache, Literatursprache, dtachronen Systemen der jüngsten Vergangenheit darstellen. Zudem bestehen meist noch deutliche Differenzen zwischen aktivem und passivem Sprachbesitz. Erfahrungsgemäß überschätzt man leicht die Fähigkeit, einzelne Sprachäußerungen unterschiedlichen Teilkompetenzen zuzuordnen. Die immer wieder angefühtten Vorzüge dieses Verfahrens, nämlich beliebige Verfügbarkeit des Sprachmaterials und Fehlerfreiheit, existieren tatsächlich nicht: eigene Äußerungen miissen ebenso einer genauen Untersuchung ihrer Fehlerfreiheit, ihrer Zugehörigkeit zu einem bestimmten Sprachsystem unterzogen werden wie fremdes Sprachmaterial. Wirde hierzu wieder nur die untersuchte Idiokampetenz herangezogen, so käme es zu einem Aufaddieren der Fehler. Die beliebige Verfügbarkeit ist vollends ein Phantom: kaum jemand ist in der Lage, die relevanten Beispiele für alle denkbaren Konstellationen eines untersuchungsbereiches zu produzieren, ganz zu schweigen von dem gravierenden Mangel, daß alle diese sprachlichen Äußerungen kontextfrei, eben als linguistische Beispiele produziert werden: dadurch werden sie weitgehend wertlos für alle Erscheinungen, in denen der Kontext eine entscheidende Rolle spielt, wie in Kontrastkonstruktionen, bei Akzentfragen, bei TopicCorment-Untersuchungen; alle diese Themen spielen aber bei der untersuchung der Gradpartikeln eine entscheidende Rolle. - Schließlich bleibt bei diesem unter bestimmten Voraussetzungen durchaus berechtigten Verfahren die Tatsache, daß sich Sprecher untereinander verständigen können, daß sie sich also auf ein gemeinsames Regelsystem beziehen, völl1g unberücksichtigt.

In gleicher Weise mß man sich allerdings vor dem gegentelligen Extrem hüten: dem undifferenzierten Einbringen von sprachlichem Materlal aus unterschiedlichsten Epochen, Räumen, Verwendungsweisen, Textsorten usw. in die sprachwissenschaftiiche Analyse. In diesem Zusarmenhang spielt das Gesetz der grammatischen Toleranz eine nicht unbedeutende Rolle: es ermöglicht einerseits die Verständigung bei tellidentischen Kompetenzsystemen, andererseits erschwert es aber die Trennung dieser Systeme, also die Zuweisung bestimter Äußerungen zu bestimmten Sprachsystemen, ungemein. Trotzdem darf man sich der schwierigen Aufgabe nicht entziehen, wenn die Daten nicht ins Unendliche wachsen und damit die Regelfindung unerträglich erschweren sollen. Die Fähigkeit der Zuweisung von Äußerungen zu Kampetenzsystemen gehört trotz der 
dabei häufigen Fehler zu den relevanten Sprachdaten bzw. Fähigkeiten des kompetenten Sprechers, die in eine Sprachbeschreibung notwendig eingehen müssen.

Daraus folgt, daß jede Sprachbeschreibung, auch die von Teilbereichen einer Grammatik wie Syntax und Semantik von Gradpartikeln, das Kompetenzsystem, das sie zum Gegenstand der Beschreibung macht, nennen muß: in diesem Falle die iberregionale deutsche Standardsprache ${ }^{9}$ der Gegenwart, bezogen auf einen differenzierten Kompetenzbegriff. Das heißt:

a. Die deutsche Standardsprache ist in sich selbst inhornogen, in rascher Entwicklung begriffen. Sie weist Bereiche auf, in denen es nur konkurrierende regionale Varianten gibt; Bereiche mit Regelunsicherheit (etwa bei Stellungsgesetzen von Gradpartikeln); Homonymenangst (etwa bei den verschiedenen Funktionen von allein und sel,bst.). Die Vielzahl der vorhandenen Daten zwingt hier zu einer vorsichtigen Idealisierung, die aber nicht in unverantwortlichen Reduktionismus fïhren darf.

b. Schließlich kennzeichnen sie zahlreiche nahtlose Übergänge zu angrenzenden Kompetenzsystemen: zu veralteten Sprachformen (wie bei der Nachstellung von nur; oder bei einzig), zu Regionalsprachen, zu Dialekten usw. Ideal wäre natürlich die Einbeziehung all dieser angrenzenden Systeme in die Beschreibung, doch verbietet der begrenzte Raum (und die begrenzte Arbeitskraft) mehr als gelegentliche Bemerkungen hierzu.

Nach dieser globalen äußeren Abgrenzung des Beschreibungsgegenstandes und der Nennung einiger allgemeiner Eigenschaften des Sprachsystems "Deutsche Standardsprache der Gegenwart", die in unterschiedlichem Grade auf die meisten natürlichen Sprachen zutreffen, ist der Beschreibungsgegenstand noch nach seinen Binnenstrukturen zu kennzeichnen, also nach den wichtigsten Fähigkeiten, die nótwendig mit der Beherrschung des Regelsystems einer Sprache verbunden sind. Speziell bei der Beschreibung von Gradpartikeln sind folgende Fähigkeiten des kompetenten Sprechers zu berücksichtigen:

a. Die Fähigkeit zur Bildung von Paraphrasen. Hier bieten die lexikalischen Paraphrasen nicht allzu viele Schwierigkeiten:

(1) Nur/lediglich/bloß/ausschließlich $H$ a ns lügt.

Sehr viel schwieriger ist jedoch folgende Paraphrasenform zu beurteilen:

(2) Nur $H$ a n s lügt.

Hans lügt, und niemand, der nicht Hans ist, lügt.

9 Auf diesen Begriff einzugehen muß ich mir leidex versagen. Es bleibt nur (für eine erste Information) der Verweis auf das LGL (Althaus/Henne/Wiegand 1974: III.21, 271-275). 
Denn diese Paraphrase gibt zwar die Bedeutung des num-Satzes zutreffend wieder, "gewichtet" aber die einzelnen Bedeutungsteile anders (bzw. falsch). b. Die Fähigkeit zum Erkennen und Auflösen von Ambiguitäten. Sie treten bei Gradpartikeln in bestimten Positionen und Satzarten zwischen verschiedenen Funktionen auf, etwa im folgenden Satz zwischen Gradpartikel-Funktion und Modalpartikel-Funktion:

(3) G e h nur!

c. Die Fähigkeit zur Rekonstruktion von Annahmen des Sprechers einer Äußerung. So kann ein Hörer aus der Äußerung

(4) Sogar $G$ e $1 d$ verachtet er.

erschließen, daß für den Sprecher Geld auf der Skala des Erstrebenswerten weit oben steht, bzw. daß er annimmt, daß für den Hörer oder allgemein für eine bestimmte Bezugsgruppe Geld diese Rolle erfüllt.

d. Die Fähigkeit zur Bestimmung von Feststellungen, die aus bestimmten Äußerungen folgen. So "folgt" (in einer noch gänzlich prätheoretischen Verwendung dieses Wortes) aus der Äußerung von Satz (4), daß derjenige, auf welchen diese Äußerung zutreffen soll, auch noch andere (weniger wichtige) Dinge verachtet, bzw. daß der Sprecher dieser Äußerung das glaubt.

2.1.5. Daß direkte Regelkenntnis qua Kompetenz nicht Grundlage der grarmatischen Beschreibung sein kann, ist' wohl allgemein akzeptierte Praxis. Aber auch gegen ein geschlossenes Corpus von Äußerungen, ${ }^{10}$ dem in jedem Fall die Repräsentativität fehlen mu und das auch immer defekte Äußerungen enthält, wie gegen eine undifferenzierte Materialsammlung als Beschreibungsgegenstand bzw. Analysebasis wurden bereits Gründe angeführt. Alle Ansätze zeigen, daß die Kompetenz des Forschers in jedem Fall bei der Materialerhebung leitend sein muß, so wenig sie zur Materialproduktion taugt und so problematisch sie bei der Materialbewertung ist; sie kann nämlich die corpusbildung wesentlich erleichtem. Als Materialquellen aber können zunächst die bereits vorhandenen corpora in Wörterbüchem und Grammatiken, soweit sie verwertbares Material enthalten, dienen. Den Kern des Analysematerials bilden Äußerungen aus vergleichbaren Quellen, die in möglichst großer Menge gesammelt werden: diese große Zahl sichert zu einem gewissen Teil bereits die Repräsentativität. - So dienten mir als quellen für Gradpartikel-Sätze (oder besser:-Texte) wissenschaftliche Wörter-

10 Siehe hierzu J. Miller (1973) und G. Schank (1973). 
bücher des Deutschen, ${ }^{11}$ deutsche Tageszeitungen, politische Magazine, wissenschaftliche Zeitschriften (vorwiegend linguistische), wissenschaftliche Monographien verschiedener Fachbereiche, Unterhaltungsliteratur der Gegenwart, Mitschnitte von Rundfunk- und Fernsehsendungen (insbesondere Parlamentsdebatten), mündliche Äußerungen in Gesprächen, an denen ich teilnahm. Dabei versuchte ich, wo immer möglich, außer dem eigentlichen Belegsatz noch mindestens einen Vorgänger- und einen Folgesatz zu erfassen. Daß dies bei den Wörterbüchem meist nicht möglich war, minderte deren Quellenwert beträchtlich. ${ }^{12}$ Soweit es sich um münlliche Quellen handelte, wurden die beobachteten Akzente miterfaßt. ${ }^{13}$

Daran schließt sich vernünftigerweise eine erste Phase der Auswertung. Dazu gehört als erstes das Sortieren des Materials nach oberflächensyntaktischen Kriterien: dies gewährleistet zunächst ein relativ theorieneutrales Vorgehen. Im Falle der Gradpartikel-Belege erfolgte die Sortierung nach Skopustypen, ${ }^{14}$ zunächst basierend auf der Plazierung des Kontrastakzentes. Dabei ergab sich, neben vereinzelten Schwierigkeiten bei der Identifizierung des wahrscheinlichsten von mehreren möglichen Skopi, eine Restgrmppe, die mit diesem Kriterlum nicht zu erfassen war. Aus ihr ließen sich die konjunktionalen Verwendungen und die Verwendungen als Modalpartikel relativ sicher aussortieren, ebenso Temporaladverbien, Adjektiva und Adjektivadverbien (bloß, ausschließlich), sowie Numeralia (einzig). Danach verblieb meist ein nicht ganz klärbarer Rest.

Schließlich erfolgt noch die Zuordnung korrekter Belege zu verschiedenen Kompetenzsystemen (soziale, regionale, veraltete Varianten, Neuerungen, stilistisch markierte Varianten) und die Bewertung des Sprachmaterials. Dabei ist der Begriff der Akzeptabilität ${ }^{15}$ leitend: um die Akzeptabilität einer bestimmten Äußerung bezüglich eines bestimmten Sprachsystems einigemaßen sicher festzustellen, genügt meist nicht die eigene Sprachkompetenz. Eine gewisse Wahrscheinlichkeit erhält man erst durch Ruickfragen bei kompetenten Sprechem, de-

11 Verzeichnis mit Angabe der jeweils ausgewerteten Artikel S. 322.

12 Der verbale Kontext muß in der Untersuchung meist wegfallen. Er wird teilweise ersetzt durch normierte Kontrastsätze.

13 In allen Fällen wurden die Quellenangaben auf den Belegen verzeichnet. In der Arbeit wird auf diese Information jedoch verzichtet. Abgesehen von Aufwand und Fehlermöglichkeiten würde man nämlich dadurch nur überflüssige Information bieten, da daraus noch nicht auf die zuordnung zu einem bestimmten Sprachsystem oder auf die Korrektheit bezüglich eines Sprachsystems geschlossen werden kann. In jedem Fall ist eine Überprüfung durch kompetente Sprecher des Deutschen notwendig.

14 Zum Begriff 'Skopus' siehe Abschnitt 2.3. S. 28-33.

$15 \mathrm{Zu}$ diesen Begriffen vergleiche die Ausführungen bei N. Chomsky (1957; 1969: $13 \mathrm{ff}$. und 1965; 1969: $22 \mathrm{ff}$. und 188-194); D. Bolinger (1968: 34-40); A. Steube (1968); N. J. Spencer (1973); Quirk/Svartvik (1966). 
ren Kompetenz aber wiederum nicht zweifelsfrei konstatiert werden kann: man gerät also in die Nähe eines wohl unvermeidlichen Zirkels. - Weiterhin beobachtet man sehr häufig Unsicherheit der befragten Personen in ihren Akzeptabilitätsurteilen. Da es sich bei "Akzeptabilität" um einen vorwissenschaftlichen Terminus, gewissemaßen um ein Prädikat natürlicher Sprachen handelt, hat man, wie allgemein, mit einem gewissen Vagheitsbereich ${ }^{16}$ zu rechnen. Allerdings reduziert sich diese Unsicherheit beträchtlich, wenn man situierte Äußerumgen bei diesen Befragungen verwendet. Dies zeigt, daß die Befragungssituation häufig wichtige Informationen ausfiltert. - In den empirischen Teilen der folgenden Untersuchung kommen nur Akzeptabilitätsurteile vor. Ich beschränke mich darauf, voll akzeptable Sätze ungekennzeichnet zu lassen, sicher inakzeptable mit : zu markieren, und bei Fällen, die kein klares Urteil zulassen, ein ? davorzusetzen. Alle darüber hinausgehenden Informationen, etwa bezüglich der Ursache der Inakzeptabilität, werden ausformuliert.

Alle diese Arbeiten setzen Hypothesenbildung voraus und erfordern parallele Theoriebildung. Sie konkretisiert sich z.B. in der Ausarbeitung eines Schemas der denkbaren Kombinationen, in denen ein bestimmtes Element erscheinen kann. So wurde für die Gradpartikeln ein Feinraster erarbeitet, der innerhalb der einzelnen Skopustypen zu folgender Einteilung führte: verschiedene Stellungstypen der Gradpartikeln relativ zu ihrem Skopus; semantische Subtypen der Skopustypen (meist mit unterschiedlichen semantischen Funktionen der Gradpartikeln verknuipft); verschiledene Satzarten (Funktionswechsel!); negierte und unnegierte Sätze, Negationstypen; Kontrastierungstypen; Fälle von Thematisierung einzelner Bedeutungselemente (z.B. Präsuppositionsproteste).

Schließlich muissen kontrollierte Testsituationen geschaffen werden, die über bestimmte Eigenschaften des Untersuchungsgegenstandes Auskunft geben können. Aufgrund dieser vorläufigen Ordnungsschemata ist es wiederum möglich, Lïcken im Belegmaterial aufzuspüren und durch gezielte Belegsammlung aufzufüllen. - In vielen Fällen gelang dies bei den Gradpartikeln nicht: entweder weil idiosynkratische Lücken vorlagen, oder weil gewisse Konstellationen generell nicht möglich waren, oder weil es sich um äußerst selten gebrauchte Konstruktionen handelte. In jedem Fall wurde versucht, die Liicken durch die Abänderung vorliegender Beispiele aufzufüllen. Gerade für Tests werden derartige Manipulationen unvermeidlich bleiben, doch steigt mit ihnen erfahrungsgemäß auch die Rate der Unsicherheit bei Akzeptabilitätsurteilen. - Das Cor-

16 Diese Überlegungen wurden angeregt von U. Blau (1973/74), 'insbesondere durch den Abschnitt 1.2. "Unbestimmte Sätze" (20-57). 
pus bleibt somit in jeder Phase der Arbeit prinzipiell offen, entspricht also dem Prinzip der ständigen Modellrevision, wobei die Möglichkeit immer neuer Daten von vomherein einkalkuliert werden muß.

2.1.6. Das eben skizzierte Verfahren der Materialerhebung bringt fast automatisch ein breites Fundament für solide Aussagen über Regularitäten, sofern man der Gefahr widersteht, in den Materialmengen zu versinken. In gleicher Weise muß man sich vor der nur scheinbaren Objektivität und Materialnähe operationalistischer Verfahren hüten: denn der Operationalismus verkennt die Natur sprachlicher Regeln. Andererseits liefern aber auch deduktive Systeme kaum mehr als formal korrekte Ergebnisse. - Die Regelaussagen setzen demach umfassendes Sprachmaterial wie auch Kenntnis der theoretischen Fragestellungen voraus. Die Auswertung selbst muß sich sorgsam zwischen Operationalismus und Deduktivismus halten, d.h., die theoretischen Voraussetzungen müssen expliziert und die wichtigsten Grundkonzepte so weit als möglich operationalisiert werden, so daß, darauf aufbauend, die Regularitäten quasi experimentell aufgedeckt werden können, ohne daß man vergißt, daß die Basis derartiger "Tests" Regelbeherrschung wie Hypothesenbildung sind. Dabei genügt es nach meiner Ansicht, die bestätigt wird durch zahlreiche und prominente Beispiele, ${ }^{17}$ nicht, nur die Ergebnisse der Tests mitzuteilen. Erst die Mitteilung des Verfahrens selbst, also der Tests und ihrer Bewertung wie Auswertung, sichert die Kontrollierbarkeit der Ergebnisse und damit ihre Verwertbarkeit, so miihevoll das im einzelnen auch für den Autor wie den Leser sein mag. Dadurch allein wird schon die Begrenzung der Reichweite theoretischer Schlußfolgerungen gesichert und den Lesem das Material zu differenziertem Argumentieren an die Hand gegeben, so daß durch immer neue Erprobung der mitgeteilten Regeln an immer neuem Material die Richtigkeit uberprüft bzw. eine Modellrevision erzwungen werden kän.

2.1.7. Für den Bereich der Gradpartikeln ergeben sich aus den "Irrwegen" der bisherigen Forschung wie aus den im Vorangehenden formulierten Prinzipien der Regelgewinnung einige grundsätzliche Entscheidungen: Es erscheint inadäquat, nur eine Anzahl von Wortmonographien aneinanderzureihen, etwa in der Form,

17 Siehe etwa die Rezension zu R. Bartsch, Adverbialsemantik (1972) durch R. Steinitz und E. Lang (1975), insbesondere "2. Heuristik" (Seite 14): "Als Fazit bleibt, daß Bartschs Testbatterien, sobald der Leser anfängt, sie durchzuprüfen, das Vertrauen in Bartschs empirische Bais sehr ins Wanken bringen, ganz zu schweigen von der leserunfreundlichen Art ihrer Anlage." 
daß man an der Einheit des Wortes festhält und in den unterschiedlichen syntaktischen Funktionen nur unterschiedliche Ausprägungen derselben "Grundsemantik", bzw. positionsbedingte Varianten derselben Einheit sieht. Gewiß ist die Einheitlichkeit des Wortkörpers ein nicht gering zu veranschlagender Faktor. Aber er verleitet auch bisweilen zum unbemerkten Einbau von verkappten diachronen Argumenten. Für mich steht also eine bestimmte syntaktische und semantische Funktion im Vordergrund der Untersuchung, und erst in zweiter Linie die Kategorie, die diese Funktion ausfüllen kann. Andererseits wäre es ebenso unklug, nur diese eine Funktion zu untersuchen, da ihre Merkmale keineswegs von vornherein so klar sind, daß eine zweifelsfreie Trennung der Elemente dieser Kategorie von anderen Funktionen (z.B. Konjunktion oder Modalpartikel) möglich wäre, zumal unter bestimmten Voraussetzungen Funktionswechsel oder Funktionsambiguität auftreten kann. - Es gilt also, das "Muster" der Überlagerung von Wortgruppe und Kategorien bzw. Funktionen herauszuarbeiten. Die Aufgabe der Trennung so nahe beieinander liegender Funktionen erzwingt allein schon die Berücksichtigung nicht nur der syntaktischen Beschreibungsebene (auf der diese Funktionen in Grenzbereichen nicht auseinanderzuhalten sind). Die Semantik bietet dariber hinaus forschungsgeschichtlich derzeit die interessantesten Probleme, kann aber auf dem Wege iber die Syntax zahlreiche Hinweise aufnehmen. Die Tatsache, daß Kontrastäußerungen unabdingbar sind zur Eruierung der Gradpartikel-Semantik und diese wiederm die Berücksichtigung des Kontrastakzentes notwendig machen, zeigt, daß man mit dieser Vermehrung der Beschreibungsebenen eine regelrechte Kettenreaktion auslöst. Sie beweist nur, daß sinnvollerweise das Geflecht der Bedingungen und Bedingtheiten durch die Beschreibung nicht willkürlich zerschnitten werden soll. Tut man es nicht, so gewinnt man von der einen Beschreibungsebene viele Argumente für Entscheidungen auf anderen Ebenen.

\subsection{Zur syntaktischen Beschreibung der Gradpartikeln im Deutschen.}

2.2.1. Syntax umfaßt die Beschreibung von Einheiten (besser: Klassen von Einheiten = Wörtern, Morphemen, Lexemen) und deren mögliche Kombinationen in Sätzen natürlicher Sprachen. Der Beschreibungsbereich der Syntax ist also die Oberflächensyntax. So selbständig diese Beschreibungsebene ist, so gibt sie doch zahlreiche Hinweise auf die semantische und pragmatische Struktur einer Äußerung bzw. der Sprache, der sie angehört, wenn auch keine eins-zu-eins Zuordnung zwischen den syntaktischen und semantischen Strukturen eines Tex- 
tes bestehen. ${ }^{18}$ Diese Hinweise auf Gesetzmäßigkeiten in anderen Beschreibungsebenen machen die Untersuchung der Gradpartikel-Syntax so interessant.

Wenn man obige Umschreibung von 'Syntax' akzeptiert, dann mïnden alle Arten syntaktischer Regelfindung in Kategorisierung. Klassifikationen ${ }^{19}$ stellen ein notwendiges Mittel zur Vereinfachung von Beschreibungen dar: es geht dabei um die Zusammenfassung von Elementen mit gleichem Verhalten, mit gleichen Eigenschaften in einer Menge oder Klasse, die intensional durch diese Eigenschaften, ausdrückbar durch Regeln, extensional durch ihre Mitglieder oder Elemente definiert ist. Derartige Klassifikationen werden unumgänglich bei formalen Beschreibungen, denn diese können sich immer nur auf Klassensymbole und darauf anwendbare Regeln beziehen.

Viele herkömmliche Wortarten ${ }^{20}$ sind in erster Linie morphologische Klassen, Flexionsklassen, insbesondere die Hauptwortarten Nomen, Adjektiv und Verbum. Da diese Klassen jeweils über die mit ihnen verbundenen Kategorien = Formkategorien weitgehend in der gleichen Menge von Strukturstellen oder syntaktischen Funktionen auftreten, werden diese Strukturstellen meist nach ihnen benannt.

Für die syntaktische Kategorisierung empfiehlt sich prinzipiell folgender Ansatz: man legt zunächst anhand eigener linguistischer Intuitionen und anhand der Grammatiktradition probeweise bestimmte Strukturstellen innerhalb von möglichen Sätzen einer bestimmten Sprache fest und ordnet ihnen bestimmte Eigenschaften zu, etwa ihre möglichen umgebungen (die in anderen syntaktischen Kategorien zu definieren sind), ihre möglichen Positionen innerhalb verschiedener Satzmuster, und nicht zuletzt mu die Menge der in ihnen möglichen Elemente angegeben werden. Umgekehrt definiert natürlich auch die Menge der dort möglichen Elemente eine bestimmte Strukturstelle. Trotzdem ist eine säuberliche Trennung zwischen der syntaktischen Funktion (= eine bestimmte Strukturstelle in Sätzen einer Sprache) und der syntaktischen Kategorie (= die Menge der in einer bestimmten syntaktischen Funktion möglichen Elemente) notwendig. Wortarten hingegen sind definiert durch einen bestimmten Komplex von syntaktischen Rollen (Plural!). Eine Einteilung nach Wortarten bietet sehr viele Unklarheiten durch die zahlreichen Idiosynkrasien auf diesem Gebiet. Insbesondere im Bereich der Partikeln weist beinahe jedes einzelne Element eine typische Kom-

18 Wegen syntaktischer Ambiguitäten und wegen der aus den Bereichen Akzent, Intonation, lexematische Bedeutung, enzyklopädisches Wissen hinzukommenden Informationen; dies macht die explizite zuordnung von bestimmten Äußerungen zu logisch-semantischen Explizitfassungen so schwierig.

19 Vergleiche dazu A. Juilland und H.H. Lieb (1968).

20 Siehe dazu W. P. Schmid (1970) und L. Lipka (1971). 
bination von für es möglichen syntaktischen Funktionen auf.

Es scheint ferner geraten, sorgsam zwischen Kategorien der Oberfläche als syntaktischen Kategorien und Kategorien der semantischen Repräsentation als semantischen Kategorien zu trennen. ${ }^{21}$ Daß einfache Zuordnungsregeln zwischen diesen beiden möglich sind, muß nach dem bisherigen Stand der Diskussion füglich bezweifelt werden.

Bei der negativ definierten Formklasse der Partikeln besteht von vormherein wenig Anlaß, syntaktische Strukturstelle und morphologische Klasse zu identifizieren. Dazu kommt noch die Erscheinung, daß diejenigen Elemente, die in einer bestimmten Funktion gemeinsam auftreten, nur in den seltensten Fällen auch in weiteren möglichen Funktionen gemeinsam auftreten. So können keineswegs alle Gradpartikeln auch Modalpartikel-Funktion oder Konjunktionspartikel-Funktion erfüllen. Dadurch ist es besonders wichtig, bestimmte Strukturstellen oder Funktionsweisen zu identifizieren und die Menge der jeweils in ihnen möglichen Elemente zu ermitteln. Folgende Themen missen also behandelt werden: die Strukturstelle und ihre Eigenschaften bzw. distributionellen Merkmale; die einzelnen Morpheme, hier die Gradpartikeln, und ihre sämtlichen distributionellen Merkmale; idiosynkratische syntaktische Eigenschaften einzelner Elemente, die zu Lücken in der Distribution (bezüglich einer bestimmten syntaktischen Funktion) führen. So kann etwa selbst nur in jenen Positionen, in denen es nicht als Reflexivum auftreten kann, zweifelsfrei die Gradpartikel-Funktion erfüllen. Und schließlich können auch noch semantische Eigenarten von Elementen zu Liicken und Störungen in der Distribution führen.

\subsubsection{Bei Beachtung all dieser Bereiche ergibt sich bei den Gradpartikeln} meist ein sehr kompliziertes Bild. Das führt bei den oben genannten Gramatiken des Deutschen, trotz oder vielleicht gerade wegen der vordergrünig klassifikatorischen Absicht, dazu, daß man nirgends über die verstreute Verzeichnung der zahlreichen Idiosynkrasien hinauskommt. Hier muß der Versuch, Wortarten mit einem weitgehend einheitlichen Muster syntaktischer Verwendungsweisen oder mit einem wie auch immer zu bestimmenden gemeinsamen Bedeutungskern zu etablieren, scheitem. Die in einigen Grammatiken angebotenen strukturalistischen Untersuchungsmethoden zur Emittlung der jeweiligen Distribution werden wegen der Vielfalt der möglichen Satzmuster, der Zuordnung zu bestimmten Konstituenten und der Positionen gerade bei den Gradpartikeln nicht durchgehalten: ${ }^{22}$

21 Zur Reduktion der grammatischen Kategorien innerhalb der "Generativen Semantik" siehe R. I. Binnick (1972: 23-27).

22 Vgl. etwa H. Glinz (1961: 220-223 und 231). 
nur auf diesem Wege wäre aber eine begründbare Trennung der verschiedenen Funktionen möglich. Insbesondere die Frage der Zuordnung der Gradpartikeln zu bestimmten Konstituenten wird nicht klar in ihren formalen (Stellung, Akzent) und semantischen (Vorgang der semantischen Überlagerung: Operator - Skopus) Aspekten behandelt. Bezeichnend hierfür ist die Beschreibung dieses Zuordnungsverhältnisses als "Attribut" in der Duden-Grammatik (1966) oder als "appositiv" bei Erben (1967). - All diese Faktoren haben zu der verbreiteten Meinung geführt, keine zwei Elemente zeigten bei den Partikeln dieselben syntaktischen Eigenschaften, es handele sich sozusagen um reinen Rohstoff der Sprache; die Funktionen würden von Fall zu Fall neu definiert. ${ }^{23}$ Es wird Aufgabe der folgenden Untersuchung sein, diesen Eindruck zu beseitigen.

Die Frage der syntaktischen Kategorienbildung wird auch in der englischsprachigen transformationellen Literatur zu diesem Bereich nicht angegangen. Kennzeichnend hierfür ist, daß in den vorwiegend an den syntaktischen Gesetzmäßigkeiten dieser Partikelgruppe orientierten Arbeiten, ${ }^{24}$ in denen even, also, too, only meist in gleicher Weise wie NEG, QU, IMP USW. (also als Satzoperatoren) behandelt werden, bei Strukturbäumen nur jeweils die Elemente selbst und nicht irgendein Kategoriaisymbol als 'Name' der betreffenden Knoten auftreten. ${ }^{25}$ Mit der Verlagerung des Interesses auf die semantische Analyse verstärkt sich diese Tendenz noch. 26

2.2.3. Die geschilderte Forschungssituation läßt es als eine zentrale Aufgabe erscheinen, nach der bereits erfolgten Etablierung der Gradpartikeln als seman-

23 So z.B. H. Glinz (1961: 221). Aber auch M. Immler (1974b: 24) meint: "Es gibt keine ein für allemal feststehende, begrenzte, im absoluten Sinne endliche Zahl von syntaktischen Kategorien (Wortklassen, Distributionsklassen); dies wird schon nahegelegt durch die vielen fehlgeschlagenen versuche, eine endgültige Aufstellung der Wortklassen einer Sprache zu geben ...; es läßt sich leicht erhärten, indem man zeigt, daß im Bereich der sogenannten Partikeln (nur, nicht, noch, schon, erst, ja, nie, gleich etc.) wahrscheinlich keine zwei genau die gleichen distributionellen Eigenschaften haben."

24 Dies gilt für S. - Y. Kuroda (1965. 1969), S. Fischer (1968), B. Fraser (1971), S. R. Anderson (1972).

25 Auch hier muß man Clément/Thümmel (1975) wieder ausdrücklich ausnehmen. Sie betonen den Gruppencharakter der genannten Elemente und führen dafür das Kategorialsymbol RP (=Rangierpartikeln) ein. Ihre nur syntaktisch definierte Klasse RP umfaßt mehr Elemente. Die Subkategorisierung in $R_{1}$ bis ${ }^{R}{ }_{6}$, beruhend auf (möglicherweise nicht erkannten) Skopuseigenschaften, haite ich nicht für sinnvoll. Der Ausschluß von betontem auch (Seite 130) beruht auf einem Irrtum. Hier zeigt sich, daß die Vernachlässigung der Semantik die syntaktische Analyse nicht erleichtert, sondern erschwert.

26 Siehe etwa Fillmore (1965), Lakoff (1970), Horn (1969 und 1972), Blumenthal (1974)! 
tische Klasse auch deren Konstituierung als syntaktische Kategorie zu versuchen. Dabei kann man sich nur auf die eigentlichen syntaktischen Kriterien stützen, also gegenseitige Austauschbarkeit und weitgehend gleiche Stellungsgesetze sowie gleiches Verhalten bei syntaktischen umformungen. Dabei darf man sich weder von den zahlreichen Distributionslücken noch von den Idiosynkrasien einzelner Elemente täuschen lassen: in den meisten derartigen Fällen lassen sich einleuchtende Erklärungen finden, seien sie nun formal-syntaktisch oder semantisch. Für das Deutsche ist es unumgänglich, stets auch die übrigen Funktionen der Gradpartikeln, soweit sie mit der Gradpartikel-Funktion gewisse Eigenschaften teilen und aufgrund dieser Tatsache schwer von ihr zu trennen sind, in die untersuchung mit einzubeziehen. 27 Die wichtigsten sind konjunktionale und Modalpartikel-Funktion. Als konjunktionale Funktion der untersuchten Partikeln bezeichne ich nur jene semantischen Funktionen, die sich nicht als "Quantifizierung mittels logischer Operatoren", bzw. in einer weiteren Differenzierung mit den typischen quantifizierenden, skalierenden und polaren Interpretationsweisen erfassen lassen. Als syntaktisches Kriterium wähle ich die Beschränkung auf zwei Positionen im Satz: die Satzanfangsstellung sowie die Position unmittelbar nach dern finiten Verb. Die Ersetzbarkeit durch zweifelsfrei als Konjunktionen zu klassifizierende Elemente ist leider nicht hinreichend für die $\mathrm{Zu}-$ weisung zur konjunktionalen Funktion, gibt aber immerhin wichtige Hinweise. ${ }^{28}$

Unter Modalpartikel-Funktion verstehe ich ebenfalls eine Verwendungsweise, deren semantische Eigenschaften nicht mit den oben genannten Interpretationsweisen zu erfassen ist. Über die genauen semantischen Funktionen dieser Verwendungsweise gibt es in der hierfür relevanten Literatur keine einheitliche Meinung. ${ }^{29}$ In neueren Arbeiten werden sie meist als Indikatoren für die illokutionäre Rolle bestimmter Äußerungen angesehen. - Die syntaktischen Funktionen und Eigenschaften sind ebenso schwer zu beschreiben. Mit Sicherheit läßt sich nur sagen, daß die Modalpartikeln nie in Konstituentensätzen auftreten, außer in zitierter Rede, und daß sie bei Matrixsatzwortstellung immer einen Platz nach dem finiten Verbum einnehmen. Bestimmte Konstituenten können zwischen finitem Verbum und Modalpartikel stehen, ihre Eigenschaften sind aber nur schwer aus-

27 Das tun bis zu einem gewissen Grad auch Clément/Thümmel (1975), wenn auch die übergänge bzw. Ambiguitäten nicht genau genug herausgearbeitet werden. Unverständlich bleibt die kommentarlose Ausschließung der konjunktionalen Funktion.

28 Siehe hierzu Abschnitt 4.2.1. S. 275-279. Zur Problematik der Trennung von konjunktionaler Funktion und Gradpartikel-Funktion mit Satz-Skopus siehe Abschnitt 3.7. S. 248-254.

29 Genauere Informationen sowie Literaturhinweise bei Abschnitt 4.2.2. S. 280-287. 
zumachen.

\subsubsection{Syntaktische Analyse- und Beschreibungsverfahren.}

In den folgenden Abschnitten sollen die syntaktischen Analyseverfahren, wie sie heute üblicherweise angewendet werden, für die Zwecke der GradpartikelBeschreibung abgewandelt und kurz zusammengefaßt werden. Derartige Verfahren können natürlich nicht im strengen Sinn als absolut induktiv und objektiv gelten, da ihnen in jedem Falle gewisse elementare Annahmen über die Natur der Sprache bzw. über mögliche Beschreibungsarten zugrundeliegen. Sie setzen darüberhinaus linguistische Intuition und Wohlverhalten von Informanten und überprüfem voraus und werden folglich verwendet als Mittel, um die linguistische Intuition zu stützen und zu kontrollieren und bestimmte Gesetzmäßigkeiten (informal) darstellbar zu machen.

2.2.4.1. Bei der Bildung syntaktischer Kategorien geht es um die Zuordnung bestimter sprachlicher Einheiten zu einer sich durch gemeinsame syntaktische Eigenschaften auszeichnenden Menge (Klasse) von Einheiten (Elementen). Zu diesen Eigenschaften gehört ein hoher Grad gegenseitiger Austauschbarkeit: die einzelnen Elemente sind im Idealfall gleich bezüglich ihrer Fähigkeit, einen bestimmten Platz im Satzmuster einzunehmen. Deshalb ist die Prüfung der gegenseitigen Austauschbarkeit das wichtigste Mittel zur Erarbeitung der Menge der Elemente, die an bestimmten syntaktischen konstruktionsstellen möglich sind. In der Anwendung auf die Gradpartikeln ergibt sich eine gewisse Schwierigkeit aus der großen Zahl der möglichen syntaktischen Umgebungen, in denen die Gradpartikeln auftreten können. ${ }^{30}$ Der größeren übersichtlichkeit halber wurden deshalb die einzelnen Stellungstypen, definiert nach dem jeweiligen Skopus der Gradpartikeln bzw. nach der kontrastiv betonten Konstituente innerhalb des Gradpartikel-Skopus in der syntaktischen Oberflächenstruktur, getrennt untersucht. Komplizierend wirkt, daß semantische Merkmale einzelner Gradpartikeln ihre Zuordnung zu bestimmten Skopuswörtern in zunächst recht schwer zu durchschauender Weise behindem; femer syntaktische Idiosynkrasien, etwa, daß sich in bestimmten Positionen andere Verwendungsweisen des gleichen Elementes gegenüber der Funktion als Gradpartikel durchsetzen, siehe etwa:

(1) Er fährt selbst mit dem Auto.

30 Wenn auch das Grundmuster der Zuordnung zu bestimmten Konstituenten der Oberflächenstruktur weitgehend konstant bleibt. 
(Dieses Beispiel kann übrigens durch kontrastiven Akzent auf Auto nicht völlig disambiguiert werden).

Bei den Austauschproben werden die Akzentverhältnisse konstant gehal.ten. Das ist relativ einfach und sicher, wenn ein Kontrastakzent vorliegt, also auch ein gut abgrenzbarer Skopus (dies kann gegebenenfalls durch entsprechende Vorgänger- und Folgesätze verdeutlicht werden). Wo das nicht der Fall ist (wie beim Skopus Verbalphrase bzw. Satz) ergeben sich wegen der zusätzlichen Stellungsproblematik fließende Übergänge zu anderen Verwendungsweisen (insbesondere zu den Modalpartikeln). - Zunächst werden nur die Gradpartikeln der jeweiligen semantischen Gruppe gegeneinander ausgetauscht. Erkennbare semantische Differenzen können hier übrigens, wo möglich und nötig, durch passend gewählte Folgesätze verdeutlicht werden. Die übrigen Gradpartikeln einzusetzen arübrigt sich weitgehend, da ja ohnehin eigene Abschnitte für sämtliche Gradpartikel-Gruppen zum jeweiligen Skopustyp vorhanden sind. Schließlich werden, unter strenger Wahrung der Akzentverhältnisse, weitere Elemente eingesetzt. Echte Ersetzungsmöglichkeiten liefern eigentlich nur einige Adverbgruppen sowie Negationspartikeln, insbesondere die Kontrastnegation. Über deren mögliche Unterscheidung von der Gradpartikeln wird bei den einzelnen Abschnitten berichtet.

Als extreme Form des Austauschtests kann man die Entfernung des betreffenden Elementes (Reduzieren) bzw. seine Ersetzung durch zwei oder mehr Elemente (Erweitern) betrachten. Bei diesen Manipulationen geht es darum, optionale und obligatorische Konstituenten zu ermitteln. Diejenigen Elemente, welche die Funktion 'Gradpartikel' erfüllen, sind, wie man leicht feststellen kann, frei hinzufügbar und wegstreichbar, ohne daß sich dabei syntaktisch inakzeptable Sätze ergeben. Die Auswirkungen auf die Semantik sind zwar beträchtlich, führen aber ebenfalls nicht zu Inakzeptabilität. - Hingegen sind die Elemente dieser Gruppe, soweit sie konjunktional und in Anfangsstellung vorliegen, nicht tilgbar, wenn Inversion der Wortstellung vorliegt. 31

2.2.4.2. Das Herausarbeiten der Stellungsgesetze der Gradpartikeln im Deutschen ist aufgrund der Vielgestaltigkeit der möglichen Kombinationen und der Bedeutung der Gradpartikel-Stellung für die Skopusabgrenzung und damit für eine ganze Reihe theoretischer Probleme von besonderem Interesse.

31 Also nicht bei konjunktionalem allein; da diese konjunktionspartikel keine Inversions-Wortstellung bedingt. Dies zeigt, daB damit kein notwendig mit der Konjunktionsfunktion verbundenes Trennungskriterium gewonnen wurde. 
In den Grammatiken wie in den meisten Wörterbüchem zum Deutschen finden sich kaum Regel-Angaben zu Stellungsgesetzen von Gradpartikeln von größerem Allgemeinheitsgrad. Dies liegt nicht zuletzt daran, daß die Basis aller Stellungsgesetze, nämlich die Zuordnung der Gradpartikeln zu bestimmten Konstituenten, ihrem Skopus, nicht klar in ihren formalen (Stellung, Akzent) und semantischen (Verhältnis Operator - Skopus) Aspekten erkannt wird.

Nicht sehr viel günstiger ist in diesem Bereich die Lage in der englischsprachigen Forschung. Als d a s syntaktische Merkmal der Zuordnung von englischen Gradpartikeln zu ihren Skopuskonstituenten werden zwar von Fraser (1971 und Anderson (1972) die Stellungsgesetze eingehender behandelt, doch bleiben wegen einer Reihe von Mängeln tragfähigere Aussagen aus: das Analysematerial ist zu schmal, die Zahl der untersuchten Skopustypen ist zu gering, und das enge Zusammenspiel zwischen Stellung, Akzent, Kontrastsätzen und Vorwissen zur Abgrenzung des Gradpartikel-Skopus wird nicht erkannt. Auf der anderen Seite räumt Susan Schmerling (1972) dem Kontrastakzent eine überragende Stellung bei der Skopusmarkierung ein, die Stellung der Gradpartikel wertet sie demgegenuiber als zweitrangig, wodurch sie zu ganz extremen, höchstens münlich möglichen Stellungstypen kormt. - Zudem darf man nicht vernachlässigen, daß zwischen den Stellungsgesetzen englischer und deutscher Gradpartikeln doch beträchtliche Unterschiede bestehen, etwa beim Skopustyp Verb oder Verbalphrase, wo das Englische Voranstellung, das Deutsche Nachstellung der Graduartikel (in bezug auf das finite Verb) aufweist: zugleich ein schöner Beleg für die unterschiedlichen Wortstellungstypen, die beide Sprachen verkörpern.

Mein Untersuchungsziel ma es also sein, die Stellungsvarianten möglichst vollständig zu erfassen und nach Möglichkeit eine Reduktion der fast unzähligen Stellungsmöglichkeiten auf wenige Stellungstypen zu erarbeiten. Die ist deswegen von so großer Bedeutung, weil von einer syntaktischen Kategorie nur dann die Rede sein kann, wenn auf ihre Elemente weitgehend dieselben Stellungsgesetze anwendbar sind. Die Basis, die Grundkonstellation für alle Untersuchungen von Stellungsregeln ist die Zuordnung zwischen Gradpartikel und Skopus. Diese Zuordnung kann prinzipiell variiert werden, wobei sich gleichzeitig die semantische Interpretation ändert.

(2) Nur Ha ns lügt (niemand sonst).

(3) Hans $I \ddot{u} g t$ nur (er tut sonst nichts; er tut nichts Schlimmeres).

Diese Grundkonstellation kann in mehreren Ebenen variiert werden:

a. Die Gradpartikel nimmt eine bestimmte Position relativ zu ihrer Skopuskonstituente ein (z.B.: sie steht unmittelbar davor). Diese feste Einheit wird 
dann im Satz umgestellt. Es fällt auf, daß es zwar bevorzugte Plätze gibt, etwa am Satzanfang oder am Satzschluß, daß aber die Stellungsfreiheit sehr gro ist. Semantische Änderumgen treten dabei nach meinen Erfahrumgen nicht auf. b. Die Gradpartikel wird im Satz umgestellt. Dabei wechselt in der Regel der Skopus.

c. Will man aber dabei den Skopus konstant halten, so sind nur noch bestimmte Gradpartikel-Positionen möglich oder es müssen neue Zuordnungsmerkmale wie der Kontrastakzent als Hilfsmittel eingesetzt werden. - Da ohnehin alle möglichen Skopustypen der Reihe nach vorgestellt werden, wäre ein Durchprobieren aller Stellungsvarianten einer Gradpartikel in einem bestimmten Satz sinnlos. Also wird die Verbindung mit der jeweiligen Skopuskonstituente aufrechterhalten, und zwar durch einen Kontrastakzent auf der Skopuskonstituente, in Zweifelsfällen durch einen kontrastiven Vorgänger- oder Folgesatz. Schwierigkeiten erwarten uns dort, wo Mehrfachbetonungen möglich sind, ohne daß der Skopus wechseln wïrde, also bei mehrgliedrigem Skopus:

(4) In Wirklichkeit war nur ein Hauptschalter in der zentralen Umspannstation Rhodesiens ausgefallen.

Der Skopus umfaßt ein Hauptschalter bzw. ein Hauptschalter in der zentralen Umspannstation Rhodesiens. Praktisch jedes Glied davon ist kontrastiv betonbar. - Aber auch in jenen Fällen, wo die Skopuskonstituente nicht durch elnen Kontrastakzent markiert ist, muß man mit Problemen rechnen, also beim Skopus Verbalphrase bzw. Satz:

(5) Wehner hat die freie Marktwirtschaft gelobt, und Schmidt hat sogar ein Loblied auf das Stabilitätsprogramm gesungen.

Aus diesen Versuchen schälen sich 6 Positionstypen heraus:

i. GP unmittelbar vor der Skopuskonstituente, die aus einem Wort besteht:

(6) Er hat nur $H$ a n $S$ angelogen.

Bei Teilkonstituenten besteht meist die Möglichkeit, daß die GP unmittelbar vor der betreffenden Konstituente, die den Kontrastakzent trägt, oder vor der Gesamtkonstituente steht:

(7) Nur eine $q u a n t i f i z i$ e $r$ e $n d$ e Interpretation erklärt das.

(8) Eine nur $q u a n t i f i z i e r e n d e$ Interpretation erklärt nichts.

ii. GP unmittelbar nach der Skopuskonstituente:

(9) Er nur wird uns verraten.

iii. GP in der Skopuskonstituente, wo diese mehrgliedrig ist: 
iv. GP in Distanzstellung vor oder nach der Skopuskonstituente. Sie tritt in der mündlichen Sprache relativ häufig auf, in der schriftlichen Form bleibt. jedoch meist ein gewisser Grad von Inakzeptabilität:

(11) Ein $L$ ü $f \in h$ e $n$ war es nur, das ihn bewegte.

v. Die drei möglichen Positionen der GP relativ zu einer topikalisierten Skopuskonstituente: unmittelbar vor und nach der Skopuskoristituente, sowie in Distanzstellung danach, wobei die GP auch betont werden maß:

(12) Nur $k$ ü $s$ s e $n$ wollte er sie.

(13) In die $s t$ a $d t$ nur wollte er fahren.

(14) $K$ üs $s$ e $n$ wollte er sie nur.

(15) $K \ddot{u} s s$ e wollte er sie a $u_{c h}$.

vi. In jedem Falle wird untersucht, ob eine Stellungsvariante möglich ist, in der die GP kontrastiv betont wird. Es wird versucht, die daraus folgenden semantischen Verschiebungen zu registrieren:

$$
\text { Hans und nur Hans lügt. }
$$

Zu beachten ist ferner, daß bei umstellungen trotz konstant gehaltener Akzentverteilung und Akzentstärke bisweilen Funktionswechsel oder auch Funktionsambiguitäten auftreten, etwa das iberwechseln zur Funktion MP:

$$
\begin{gathered}
\text { Ge h nur! (im Sinne von: } F \text { ahr nie mit dem Auto! } \\
\text { bzw. als Aufforderung zum Gehen) }
\end{gathered}
$$

Derartige Fälle zu sammeln und zu systematisieren ist eine wichtige Beschreibungsaufgabe. - Natirlich muß man auch darauf achten, ob ein bestimter Stellungstyp etwa für gesprochene oder geschriebene Sprache, für bestinmte Gruppensprachen typisch ist, oder ob diachrone Entwicklungen sichtbar werden. Die Umformungstests des folgenden Abschnitts werden zu den Stellungsgesetzen noch reiche Informationen bieten, insbesondere die Untersuchung der Satztypen.

2.2.4.3. Zu den eben behandelten Grundoperationen treten dann noch zahlreiche subtile Einzeltests, meist als Umformmgstests oder Transformationstests bezeichnet, hinzu, die gerade in der angelsächsischen Literatur zu den Gradpartikeln zu hoher Reife entwickelt wurden. Die meisten von ihhen beschränken sich nicht auf syntaktische Tatbestände, sondern venitteln auch wichtige semantische Einsichten. Hier werden nur diejenigen behandelt, die nicht auf eine spezifische Fragestellung der generativen Transfomationsgrammatik zuge- 
schnitten sind.

Die Umformung von Gradpartikel-Sätzen in die verschiedenen Satztypen dient der Kontrolle und dem Vergleich der Stellungsgesetze und der Beobachtung der Bedingungen, unter welchen eine andere syntaktische Funktion bei Elementen der Kategorie 'Gradpartikeln' auftritt. Letzteres zielt auf die Tatsache, daß die Modalpartikeln häufig nur bei ganz bestimmten Satztypen auftreten können; so etwa nur in der Wortfrage und im Imperativ:

(18) $G$ e h nur!

(19) Was $h$ a $s t$ du nur?

Der Reihe nach werden folgende Satztypen untersucht: Aussagesatz; die Fragesatztypen Wortfrage, Satzfrage, rhetorische Frage, Vergewisserungsfrage; dann der Imperativ, der besonders oft Funktionswechsel zur Modalpartikel aufweist; Ausruf (formal meist schwer von Imperativ bzw. metorischer Frage zu trennen, aber wichtig für die Beobachtung von Funktionswechseln zu Modalpartikeln); und schließlich die Konstituentensatzwortstellung. Diese umformung ist besonders wichtig, wenn der Skopus im Bereich einer Verbalphrase mit einer analytischen Verbform liegt: da diese in der Hauptsatzwortstellung in diskontinuierlicher Form vorliegt, sind hier Fragen der Skopusabgrenzung besonders schwierig.

(20) Er hat sogar seinen $H$ u $t$ vergessen.

(21) ... weil er sogar seinen $H u t$ vergessen hat.

Das Thema "syntaktische Möglichkeiten der Negierung eines Satzes mit Gradpartikeln" ist eng verknüpft mit Fragen der funktionalen Satzperspektive (Skopus der Negation = Fokus des jeweiligen Satzes), mit Fragen der Präsuppositionen eines Gradpartikel-Satzes (Konstanz der Präsupposition unter interner = starker Negation) und mit dem Problem der Kontrast-/Korrektursätze. - Folgende Negationsmöglichkeiten werden unter fomalen Aspekten erprobt:

i. Die mögliche "Inkorporation" der Negation in die jeweilige GP: nicht nur. ii. Wenn NominalF' asen im Skopus der Gradpartikel stehen: die Möglichkeit, die Negation zwischen Gradpartikel und Skopus-Nominalphrase zu plazieren und ev. mit dem indefiniten Artikel zu verbinden: nur nicht $H$ a $n s$, nur kein $W$ a s $s$ e . In allen diesen Fällen setzt sich der Skopus der lokalen Negation (Kontrastnegation) gegen den der Gradpartikel durch: die GP wird in konjunktionale Funktion oder in GP-Funktion mit Satzskopus abgedrängt. iii. Die möglichen Positionen der Satznegation (starken Negation):

(22) Nur Hans hat nicht gelogen. 
2.2.5. Zum Schluß seien noch einige vorwiegend syntaktische Themen angefuhrt, die in der englischsprachigen transformationellen Iiteratur eine nicht unbedeutende Rolle in dem Streit um interpretative oder generative Semantik bzw. um die Bedeutung der Oberflächenstruktur für die semantische Interpretation von Sätzen gespielt haben.

Als mögliches Argument gegen die Relevanz von oberflächenstrukturellen Eigenschaften bestimmter Äußerungen für die Zuordnung von Satzoperatoren wie even zu bestinmten Korstituenten als ihrem Skopus fuihrte B. Fraser (1971) die Erscheinung der "geschützten Umgebung" ('protected environment') an: dies bedeutet, daß der GP-Skopus durch Transformationen nicht verändert wird, z. B. auch nicht durch das clefting.

2.2.5.1. Als Test hat die Spaltsatzkonstruktion eine doppelte Funktion. Er soll die Unverletzlichkeit des GP-Skopus zeigen:

Nur ein Hauptschalter im U mspannwerk ist ausgefallen.

Es ist nur ein $H$ a upt $s$ chal te $r$ / Ein $H$ a upts $c h a l$ -

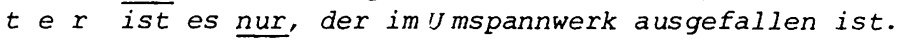

Das Ergebnis ist zwar akzeptabel, aber bedeutungsverschieden vam Ausgangssatz. Damit besitzen wir prinzipiell eine Möglichkeit, die Grenzen des Skopus zu finden. - Eine weitere Aufgabe besteht darin, die Vereinbarkeit der Funktion der Spaltsatzform mit der Semantik verschiedener Gradpartikeln zu prüfen. So wird sowohl von Fraser (1971: 173f.) als auch von Horn (1969: 106) behauptet, daß das engl. even nicht mit der Spaltsatzbildung vereinbar sei, da die Spaltsatzkonstruktion die Einzigkeit "impliziere", während even Nichteinzigkeit präsupponiere. Leider ist aber der Spaltsatz nur ein stumpfes Analyseinstrument: ábgesehen davon, daß dieser Satztyp generell leicht inakzeptabel wirkt, ist er auch keineswegs auf alle vorkormenden GP-Skopustypen anwendbar, insbesondere nicht bei komplexen Nominalphrasen, wo die größten Abgrenzungsprobleme auftreten.

2.2.5.2. Die gleichen Gesetzmäßigkeiten (auch hinsichtlich der Einschränkumgen) gelten im Grunde für das Topikalisieren der Skopuskonstituente von Gradpartikeln, ein Thema, das bislang in der Gradpartikel-Literatur noch nicht behandelt wurde, das aber wegen der engen Beziehungen zwischen Syntax und Semantik und wegen der Probleme bei der formalen Beschreibung dieses Wortstellungstyps 32

32 Eine eingehende Behandlung der Thematik habe ich in einem Vortrag auf dem $\mathrm{X}$. Linguistischen Kolloquium 1975 in Tübingen "Gradpartikeln und Topikalisierung" versucht. Siehe Altmann(1976). 
einiges Interesse wert ist. Da diese Konstruktion als sechster Stellungstyp bereits eingeordnet wurde, werden entsprechende Fragen jeweils dort abgehandelt.

2.2.5.3. Fraser (1971) erwähnt als weiteren Umordnungstyp noch die Extraposition. Versteht man darunter die Rechtsverlagerung von Subjekts-, Objektsund Relativsätzen, so treten bei Beibehaltung der Anordnung Gradpartikel Konstituentensatz keinerlei Besonderheiten auf. Für das Herausbewegen einer Konstituente aus dem Skopus-Konstituentensatz gilt wieder das Gleiche wie beim Clefting. Beweiskraft liegt darin nach meiner Ansicht nicht, da die gleichen Gesetzmäßigkeiten für die entsprechenden Sätze ohne Gradpartikeln gelten.

\subsubsection{Auch die Zusammenhänge zwischen Gradpartikeln und Left Dislocation} ihrer Skopuskonstituente blieben bislang unbehandelt, obwohl diese Konstruktion in der heutigen mündlichen Ungangssprache das häufigste Mittel der Hervorhebung einzelner Konstituenten sein dürfte. Die Besonderheit ist hierbei, daß die Gradpartikel, wenn ibberhaupt, nur dem pronominalen Platzhalter zugeordnet werden darf.

Da in bezug auf die Skopusabgrenzung alle diese Konstruktionen gleichwertig sind, beschränke ich mich auf die Behandlung von Spaltsätzen, zumal dort auch die entscheidenden semantischen Fragen auftreten.

2.2.5.5. Die formalen Beziehungen zwischen Aktiv- und Passivformen von Sätzen haben in der englischsprachigen Literatur zu den Gradpartikeln eine entscheidende Rolle in der Diskussion um die zuordnung eines bestimmten Skopus zu einer Gradpartikel in der Oberflächenstruktur oder bereits in der Tiefenstruktur gespielt. Die Argumentation verläuft dabei in folgender Weise:

a. Die Phrasenstrukturregeln einer Transformationsgrammatik erzeugen nur Aktivsätze. Passivsätze werden durch eine nicht bedeutungsverändernde Transformation davon abgeleitet: es handelt sich also um eine rein fomale Beziehumg zwischen zwei Sätzen, welche die Wahrheitswertbedingungen des betreffenden Satzes nicht verändert. Die Tatsache, daß die Passivierung in Sprachen mit fester Wortstellung e i $n$ wenn auch nicht $d$ a s Topikalisierungsmittel ist, berührt zwar die Gebrauchsregeln des betreffenden Satzes, nicht aber seine semantische Repräsentation. - Nur wenn es gelänge, nachzuweisen, daß dadurch auch die Wahrheitsbedingungen eines Satzes verändert werden (können), wären unterschiedliche Formalisierungen notwendig, d.h. die gesamte folgende Argumentation wäre zum Einsturz gebracht. 
b. Skopus einer Gradpartikel können immer nur Konstituenten sein, die vom gleichen Knoten dominiert werden. Das würde nun zwar die Darstellung in einem Aspekte-Modell oder im revidierten Standardmodell sehr erleichtern, doch fehlen dafür noch alle empirischen Argumente.

c. In der Oberflächenstruktur liegen jeweils nur aufeinanderfolgende Konstituenten im Skopus von Gradpartikeln.

d. Kann man nun nachweisen, daß in der Oberflächenstruktur Konstituenten im Skopus von Gradpartikeln liegen, die in der Tiefenstruktur nicht vom selben Knoten unmittelbar dominiert werden, so hat man nach diesem Ansatz "bewiesen", daß die Zuordnung der Gradpartikeln nur in der Oberflächenstruktur erfolgen kann. - Dieses Thema wird eigentlich nur dann relevant, wenn in einem Belegsatz im Passiv die Verbalphrase insgesamt im Skopus liegt (sie darf dabei nicht nur aus der analytischen Verbalform bestehen!), bzw. wenn man einen Belegsatz im Aktiv mit der gesamten Verbalphrase im Skopus einer Gradpartikel auf seine Umformungsmöglichkeiten ins Passiv untersucht.

\subsubsection{Exkurs zur Diskussion um die Darstellung von Gradpartikeln in einer generativen Transformationsgrammatik.}

Mit der Frage des Skopus von Gradpartikeln in Passivsätzen ist generell die Frage der adäquaten Behandlung der Gradpartikeln in einer generativen Transformationsgrammatik angesprochen. Der Gang der Diskussion in der angelsächsischen Literatur, der alle Phasen des Streits um das Aussehen einer generativen Transformationsgrammatik umfaßt, soll hier nur sehr kurz geschildert werden.

Für die "Syntactic Structures"-Fassung der Transformationsgrammatik gibt es keinen Lösungsversuch für das Gradpartikel-Problem. Prinzipiell wären in ihr zwei Lösungen denkbar: die Erzeugung der Gradpartikeln in allen Positionen, in denen sie in der Oberflächenstruktur von Sätzen auftreten können, durch Phrasenstrukturregeln, die dadurch aber sehr komplex werden würden. Andererseits bestünde die Möglichkeit, die Gradpartikeln durch Einsetzungstransformationen unmittelbar an den Stellen zu plazieren, an denen sie in der Oberflächenstruktur auftreten. Dies würde zwar eine Vereinfachung des Regelapparates ermöglichen, aber es handelte sich um eine bedeutungsverändernde Transformation, die nach der Durchsetzung der Katz/Fodor-Hypothese, daß alle Transformationen bedeutungserhaltend $z u$ formulieren seien, nicht mehr geduldet werden konnte. Eine erste Lösung dieses Dilemmas versuchte S. -Y. Kuroda (1969: $337 \mathrm{ff}$.$) . Ausgehend von einer empirisch nur sehr schwach abgesicherten Hypo-$ these, nämlich daß Gradpartikeln grundsätzlich nur ein Mal je Satz auftreten können, und der Ähnlichkeit'dieses Verhaltens mit dem von Negationen, schlug er vor, ein Platzhaltersymbol für diese Gruppe von Elementen durch Phrasenstrukturregeln, unmittelbar dominiert vom Satzsymbol, zi erzeugen und es dann jeweils einer skopusfähigen Konstituente durch eine (allerdings auch nicht völlig bedeutungserhaltende) sogenannte zuordnungstransformation ('attachment transformation') zuzuweisen. Die Skopuskonstituente soll mit der Gradpartikel zusammen wieder eine Konstituente vom gleichen Typ bilden. - Gegen die Darstellung des Skopus-Operator-Verhältnisses durch eine Dominanzrelation sprechen nun allerdings einige syntaktische wie semantische Eigenschaften: so z.B. 
die Tatsache, daß sich die Gradpartikeln jeweils nux an den Nahtstellen zwischen den Hauptkonstituenten eines Satzes aufhalten dürfen (von Nominalphrasen mit kontrastiv betonten Adjektivattributen abgesehen), daß sie keineswegs mit ihrer skopuskonstituente permutiert werden müssen, und schließlich, daß die Dominanzrelation für den semantischen Vorgang der Uberlagerung der Bedeutung einer Konstituente durch ein anderes Element, also für die operator Skopus - Relation keine adäquate Darstellung ist. Interessant, insbesondere für eine semantische Interpretation der Gradpartikeln, ist jedoch Kurodas (1969: 347 f.) Hinweis, daß ein Gradpartikel-Satz durch eine Konjunktion von Sätzen, die sich nur hinsichtlich des jeweiligen Skopuselementes unterscheiden, erklärt werden kann. Diese Paraphrase berücksichtigt allerdings noch nicht den unterschiedlichen Status dieser Sätze (also Assertion bzw. Präsupposition des jeweiligen Gradpartikel-Satzes zu sein). Ungelöst ist bei diesem Vorgehen die Frage der Zuteilung des kontrastiven Akzentes. - Hierfür bietet der Ansatz von Susan Fischer (1968) eine Lösung, die gleichzeitig näher an das Ideal der Bedeutungserhaltung durch die zuordnungstransformation herankommt. Eine beliebige Konstituente soll in der Tiefenstruktur mit dem Merkmal [+Prominent] gekennzeichnet werden: sie ist dann diejenige Konstituente, der in der oberflächenstruktur durch die phonologischen Interpretationsregeln der Hauptakzent zugewiesen wird. Gleichzeitig kann nur dieser Konstituente durch eine zuordnungstransformation eine Gradpartikel zugewiesen werden. Danach können dann optionale Stellungsregeln angewendet werden. Ungelöst bleibt aber die Frage der Regelfolge. - Chomsky (1969; 1971: 199-206) und Jackendoff (1972: 237-242) behaupten demgegenüber, daß die Frage der Akzentzuweisung nicht in der Tiefenstruktur zu lösen sei; vielmehr glauben sie Beispiele beibringen zu können, die beweisen sollen, daß Akzentzuteilung und davon abhängige Skopuseingrenzung für logische Operatoren durch Merkmale der Oberflächenstruktur gesteuert werden: als Beweis führen sie die Akzentzuteilung bei transformationell abgeleiteten Konstituenten (z.B. bei Passivsätzen) an. Dieser Ansatz führt jedoch zu sehr künstlichen Lösungen, da Merkmale der Oberflächenstruktur von größter Bedeutung für die semantische Interpretation eines Satzes werden: es muß also eine Möglichkeit gefunden werden, die Informationen der Oberflächenstruktur der bislang allein über der Tiefenstruktur operierenden interpretativen semantischen Komponente zuzuführen. - Diese Lösungen beruhen auf einer Reihe von falschen Annahmen: nämlich daß kontrastiv betonte Konstituente und GradpartikelSkopus jeweils identisch seien, und daß der Skopus von Gradpartikeln jeweils einen vollständigen Teilbaum der angesetzten Tiefenstruktur umfassen müsse. Genau an diesem Punkt der Diskussion setzen auch B. Fraser (1971) und S.R. Anderson (1972) an. Fraser geht von der Annahme aus, daß Kontrastakzent und Skopus logischer Partikeln bereits in der Tiefenstruktur festgelegt werden müssen, und prüft die Argumente, die ev. gegen eine solche Lösung sprechen könnten, z.B. die von Chomsky angeführten Sätze, die angeblich auf transformationell abgeleitete Skopi schließen lassen. Er ist der Meinung, daß alle derartigen Beispiele nicht voll akzeptabel seien und deshalb keine sicheren Beweise darstellten. Hingegen kann er eine ganze Reihe von Fällen aufzeigen, in denen eine tiefenstrukturelle Lösung gewisse Vorteile böte: das gilt vor allem für das verbot, aus einem Konstituentensatz, dex den Skopus von even bildet, nach der Regel der Negations-Transportation einzelne Elemente in den Matrixsatz zu bewegen; für das bereits erwähnte Verbot, Telle des Skopus von even in einen Spaltsatz zu verlagern oder zu extraponieren. Ferner macht er plausibel, daß Teile von Idiomen nicht den Skopus von even bilden können. All dies deutet nach Fraser darauf hin, daß even schon in der Tiefenstruktur einen vor allen transformationellen Veränderungen geschützten Skopus zugewiesen erhält. - Genau die gegenteilige Position vertritt S. R. Anderson (1972). An neuen Argumenten führt er dabei an, daß die durch eine tiefenstrukturelle Lösung notwendigen Umstellungstransformationen Verstöße gegen den ComplexNP-Constraint und den Sentential-Subject-Constraint ermöglichen. 
2.3. Probleme des Skopus von Gradpartikeln.

2.3.1. In den Bemerkungen zur Syntax der Gradpartikeln im Deutschen wurde bereits mehrfach, insbesondere bei den Stellungsregeln (S. 19), bei der Thematik "geschützte Umgebung" (S. 24) sowie bei der Skizzierung der Darstellungsproblematik in einer generativen Transfomationsgrammatik (S. 26) darauf hingewiesen, daß die Zuordnung Gradpartikel - Gradpartikel-Skopus die Grundkonstellation für alle syntaktischen und semantischen Beschreibungsansätze darstellt. Diese Zuordnung wurde in ihren Eigenschaften von der deutschen Grammatikforschung, wohl wegen ihrer Logikferne, bis vor kurzem nicht erkannt. ${ }^{34}$ Hingegen ist die operator-Eigenschaft der Gradpartikeln in der generativ orientierten Forschung sehr früh in ihrer auch methodisch so wichtigen Brückenfunktion, die das Thema auch in dieser Untersuchung haben soll, erkannt worden. ${ }^{35}$ Hierbei hat die vorwiegend an der semantischen Analyse interessierte Gruppe 36 in der Regel die Zuordnung als gegeben hypostasiert und sich nicht weiter um die formalen (oberflächenstrukturellen) Aspekte dieser Relation gekümmert. Da sie meist nur Sätze mit einfachem Skopus untersuchte, erwuchsen ihr aus dieser Vereinfachung auch keine Schwierigkeiten. - Andererseits hat die stärker syntaktisch orientierte Forschung, neben der gleichen Beschränkung auf einfache Skopustypen, ${ }^{37}$ die Situation künstlich, d.h. unempirisch. vereinfacht, indem sie entweder die Stellung der Gradpartikel allein, ${ }^{38}$ oder den kontrastiven Akzent auf der Skopuskonstituente ${ }^{39}$ bzw. eine Kombination aus Kontrastakzent und Gradpartikel-Stellung als hinreichend für die formale Kennzeichnung der Zuordnung ansahen. Die Ausklammerung der pragmatischen Aspekte der Kontrastierung wie der Frage nach den möglichen Skopustypen ermöglichte

34 Dies zeigt die Kennzeichnung als "Attribut" in der Duden-Grammatik (1966) und als "appositiv" bei Erben (1967). - Einen gewissen Neuansatz bringen clément/Thümmel (1975). Sie sprechen im Zusammenhang mit ihrer Gruppe Rangierpartikeln von "Bezugsbereich" (Siehe "Einleitung" S. 11 und passim). Doch wird dieser Terminus, da syntaktisch nicht explizierbar, nur ganz vage umschrieben. Mehrere Beispiele im Verlaufe der Untersuchung lassen jedoch erkennen, daß die zwar eng verknüpften, aber doch unterscheidbaren Erscheinungen von Skopus und Kontrastfokus vermischt, ja verwechselt werden.

35 Siehe Kuroda (1965 und 1969).

36 Also Fillmore (1965; 1971), Horn (1969 und 1972), Bartsch (1972) und Blumenthal (1974).

37 AuBer der Frage des Satzskopus bei Fraser (1971) und Schmerling (1972).

38 So bei Fraser (1971) und Anderson (1972).

39 So etwa Chomsky $(1969 ;$ 1971), Akrnajian (1970a), Jackendoff (1969b una 1972). 
diese Haltung.

2.3.2. Ich gehe im Gegenzug, um die empirischen Arbeiten nicht unzulässig einzuschränken, von der weitestmöglichen Hypothese aus, nämlich daß die Oberflächenstruktur einer nicht defekten Äußerung zusammen mit Hinweisen aus Kound Kontext hinreichend Anhaltspunkte für eine richtige Dekodierung dieser Äußerung durch einen beliebigen kompetenten Sprecher/Hörer aufweist und aufweisen muß. Damit ist der pragmatische Aspekt, der sich im Verlauf der empirischen Arbeiten zu Kontrastäußerungen allgemein wie zu Gradpartikel-Sätzen im besonderen als sehr wichtig erwiesen hat, in die Untersuchung einbezogen. Um die bekanntermaßen daraus folgenden methodischen Schwierigkeiten gering zu halten, ist man gezwungen, entweder alle Faktoren, welche mit situationellen Gegebenheiten zusammenhängen, rigoros auszuschalten, oder von der Hypothese auszugehen, daß grundsätzlich alle Merkmale des situationellen Kontextes versprachlicht und auf diese Weise in eine linguistische Untersuchung einbezogen werden können. Der obige Grundsatz der Dekodierbarkeit zwingt ferner dazu, alle Formen elliptischen oder indirekten Sprechens entweder als rekonstruierbar zu betrachten, oder von der linguistischen Untersuchung auszuschließen - beides sehr weitgehende Idealisierungen. Wenn man also nur ein hinreichend genaues Verfahren anwendet, dann muß für jede semantische Erscheinung auch eine beschreibbare formale (aberflächenstrukturelle) Entsprechung aufzufinden sein. Als Testgebiet für diese Behauptung bietet sich die Erscheinung des Skopus von Gradpartikeln an.

Die Gradpartikeln kann man nun, aus dem Blickwinkel einer der Prädikatenlogik angenäherten Darstellung gesehen, der Gruppe der Operatoren zurechnen; mit ihnen verbindet sich das "Gefiuhl, daß sie semantisch andere Elemente überlagern" (P. A. M. Seuren 1969:118). Diese semantisch von den Operatoren überlagerten Konstituenten werden als 'Skopus' des operators bezeichnet. Natürlich wird jeweils der gesamte Satz von den semantischen Auswirkungen eines Operators betroffen. - Feststellbar ist bei den Operatoren femer eine sehr vielgestaltige syntaktiscine Zuordnung zu diesen Skopus-Konstituenten, ohne daß sie aber in irgendeiner Weise Teil dieser Konstituenten werden würden. Die semantische Relation ist in den formalen Systemen der Logik (z.B. durch Klammerung) gut darstellbar, ein Problem bildet jedoch die Zuordnung dieser Explizitfassungen zu den oberflächensyntaktischen Merkmalen der Verbindung zwischen Gradpartikeln und bestimmten Konstituenten der Oberflächenstruktur, welche ihrem 
Skopus in einer formalen Darstellung entsprechen. ${ }^{40}$ Anhaltspunkte sind hierbei: a. Die Stellung der Gradpartikel. Schon durch eine flüchtige Überprüfung konnten wir in Abschnitt 2.2.4. S.21 feststellen, daß Gradpartikeln vor und in sehr begrenztem umfang auch $\mathrm{n}$ a $\mathrm{ch}$ ihren vermuteten Skopuskonstituenten auftreten können (zur weiteren Komplizierung der Situation in gewissen Fällen auch in Distanzstellung, doch will ich dies zunächst einmal vernachlässigen).

(1) Nur e $r$ kann uns helfen.

(2) Er nur kann uns helfen.

(3) E r kann uns nur helfen.

Die Zuordnumg ist dadurch nicht mehr eindeutig, etwa in dem Sinne, daß man sagen könnte, jeweils die erste auf die Gradpartikel folgende Konstitixente in der Oberflächenstruktur entspricht ihrem Skopus, es sei denn, es besteht kein Unterschied zwischen den beiden Stellungsvarianten vor und nach einer bestimmten Konstituente bezüglich ihrer Funktion, ihres Skopusbereiches: dann wären die Grenzen des Skopus durch eine einfache Umstellung der Gradpartikel zu ermitteln. Eine weitere Möglichkeit wäre, daß sich nie mehr als eine Konstituente der Oberflächenstruktur im Skopus befindet, also weder: zusammengesetzte Konstituenten, Teile aus verschiedenen Konstituenten, Teile einer einzigen Konstituente (Wortteile, grammatische Kategorien). - Da diese Annahmen als zu weitgehend betrachtet werden mïssen und auch durch Beobachtungen rasch widerlegt werden können, scheidet die Stellung als Mittel der Skopusabgrenzung weitgehend aus. Dagegen spricht auch, daß die Gruppe der Gradpartikein noch in einer Reihe anderer syntaktischer Funktionen vorkommt, deren mögliche Positionen innerhalb von Sätzen sich durchaus mit den möglichen Positionen von Gradpartikel-Funktionen decken können, ohne daß aber dabei ein Skopus auftreten wïrde.

b. Der Satzakzent. Man kann von der Hypothese ausgehen, daß der Skopus von Gradpartikeln gleichzeitig Fokus des jeweiligen Satzes sein muß, eine These, die auch von Chomsky (1969; 1971: 207), Akmajian (1970a:203 ff.) und Jackendoff (1972: 249) vertreten wird und die im Zusammenhang mit der kommunikativen Funktion der Gradpartikeln, insbesondere im Bezug auf ihr Auftreten in kontrastierenden Satzpaaren, noch genauer untersucht werden muß. Fermer kann man die von Akmajian (1970a) und Sgall (1973: $162 \mathrm{ff.}$ ) vertretene und auch unmittelbar einleuchtende Ansicht heranziehen, daß in jeder einfachen Äußerung jeweils

40 Einfachheitshalber verwende ich zukünftig auch für diesen oberflächensyntaktischen Sachverhalt nur 'Skopus'. 
nur ein Fokus vorliegen kann. Daraus würde übrigens auch folgen, daß in jedem Satz jeweils nur eine Gradpartikel auftreten kann. ${ }^{41}$ Da der Fokus eines Satzes jeweils durch den Satzakzent markiert ist, wobei es bestimmte phonologische Regeln für die Zuweisung des Akzents zu einer bestimmten Silbe in einem mehrgliedrigen Fokus gibt, müssen in bezug auf die Lage bei den Gradpartikeln zwei Fälle unterschieden werden:

i. Das Akzentmuster deckt sich mit dem "pattern" für den normalen Satzakzent, wenn die Verbalphrase oder ein ganzer Satz den Skopus der Gradpartikeln bildet. ii. In allen anderen Fällen handelt es sich um einen Kontrastakzent, dessen Natur allerdings in der Forschung kontrovers ist. Jedenfalls lassen sich in allen diesen Fällen Kontrastsätze bilden, bzw. sie sind zu ergänzen, häufig sind sie auch im Diskurs verbalisiert.

Bei diesem Verfahren ergeben sich allerdings auch bestimmte Schwierigkeiten: mit dem Satzakzent wird immer nur eine bestimmte Silbe markiert, bzw. diejenige terminale Konstituente, in der sie enthalten ist. Wenn aber der Skopus von Gradpartikeln, was nicht von vornherein auszuschließen ist, da leicht beobachtbar und in der Literatur zu diesem Thema nicht prinzipiell umstritten, auch nicht-terminale Konstituenten umfassen kann, vielleicht sogar die ganze Verbalphrase oder den Satz, dann bleibt nach wie vor das Problem der Abgrenzung. Daß der Skopus im Hinblick auf die Position des Akzentes tatsächlich ambig sein kann, wird im Analyseteil an passender Stelle noch demonstriert.

Um das Ergebnis zusammenzufassen: weder die Stellung der Gradpartikel noch die Lage des Kontrastakzentes reichen für sich allein in allen Fällen zur zweifelsfreien Markierung und Abgrenzung des Gradpartikel-Skopus. Aber auch zusammen erfüllen sie nicht diese Funktion, und zwar dann nicht, wenn die durch Kontrastakzent markierte Silbe Betonungsträger für sprachliche Einheiten unterschiedlicher Größe sein kann, ein Fall, der bei vielgliedrigen Verbalphrasen nicht gerade selten ist. Klarheit bringt erst die Einbeziehung von Kound Kontext. Wenn dies bislang in der Gradpartikel-Forschumg übersehen wurde, so deswegen, weil die eingeschliffenen Dekodierungsmechanismen Skopusambiguitäten bei normaler Kommunikation ausschließen. Methodisch läßt sich dies durch Tests mit kontrastierenden Vorgänger- und Folgesätzen, die mit dem kontrastierten Satz bis auf den Skopus identisch sind, erreichen:

(4) Niemand lügt. Nur $H$ a ns lügt.

41 Behauptung von Kuroda (1965) bis Fraser (1971) und Horn (1969 und 1972). Gegenbeispiel von Anderson (1972). Siehe hierzu auch Abschnitt 4.1.3. S. $264-269$. 
(5) Nur $H$ a n s lügt, und niemand sonst (lügt).

Dadurch ist ohne weiteres ersichtlich, daß in allen Fällen, in denen die Konstituente mit dem Kontrastakzent mehr als eine Silbe umfaßt, der Skopus ganz unterschiedliche Ausdehnung aufweisen kann. Erst Ko- und Kontext können eine Disambiguierung bewirken. Im Analyseteil wird dies an vielen Beispielen durchgespielt. - Aber trotz der engen Verbindung zwischen Gradpartikel-Skopus und Kontrastfokus darf man beide nicht einfach gleichsetzen. So muß man vor allen anderen Aufgaben darauf achten, ob es Fälle gibt, in denen die beiden Einheiten entweder unterschiedliche Ausdehnung haben oder gar völlig verschiedene Elemente umfassen. - Folgende Fragen sind also im Zusammenhang mit der Skopusproblematik bei der Untersuchung des Belegmaterials zu klären:

a. Welche einfachen Konstituenten künnen den Skopus der verschiedenen semantischen Gruppen der Gradpartikeln bilden? Weiøen sie bestimmte Merkmale, wie Akzent-/Fokusfähigkeit auf, und läßt sich für die Gesamtheit dieser Erscheinungen eine plausible Erklärung finden?

b. Können Teile von Konstituenten einen Gradpartikelskopus bilden, und, wenn ja, welche? Auch grammatische/morphologische Kategorien?

c. Welche zusammengesetzten Konstituenten können als Gradpartikel-Skopus bestimt werden? Eventuell auch Verbalphrase und Satz?

d. Wie gestaltet sich jeweils das Zusammenwirken der drei Hauptmerkmale der Skopusidentifizierung: Stellung der Gradpartikeln, Positionen des Akzents und Kontrastsatz?

e. Wie verhält sich der Skopus von Gradpartikeln zum Skopus anderer Operatoren, also etwa der Negationen?

Zu diesen Fragen liefern die im Abschnitt "Syntax" beschriebenen Tests zahlreiche Informationen. So kann mit Hilfe des Austauschtests die für jede einzelne GP typische Kombination von möglichen Skopustypen ermittelt werden. Die dabei auftretenden "Liicken" in der Verteilung, wie etwa bei selbst im Bereich der VP, bediurfen der Erklärung; im Falle von selbst besteht sie darin, daß sich hier die Funktion als Reflexivpronomen durchsetzt. Bei der Emittlung der Stellungstypen (Umstelltest) ist automatisch die Korrelation zwischen Gradpartikel-Position und Skopusgrenzen zu prüfen. Zu dieser Frage sollen aber auch die Tests im Zusammenhang mit der Hypothese von der "geschützten umgebung" Entscheidungshilfen liefern, also insbesondere das Clefting.

Ein weiteres Untersuchungsmittel im Zusammenhang mit dem Thema "Skopus" ist die Ermittlung der möglichen Akzent-Positionen bei festliegender Gradpartikel-Position und die durch Verlagerungen des Kontrastakzentes ausgelösten semantischen Änderungen, die Riickschliusse auf die Skopusgrenzen zulassen. - 
Hinzu kammen die schon mehrfach erwähnten Kontrastsätze mit nomierten Kontrastierungsformeln. Sie geben Auskunft über die Skopusgrenzen sowie über mögliche semantische Interpretationen bestimmter Gradpartikel-Sätze. Außerdem können sie in kritischen Fällen als eine Art normierter Kontext helfen, die Testbedingungen stabil zu halten, insbesondere Kontrastakzent und die jeweilige Interpretation. - Nicht vergessen darf man, im Zusammenhang mit der Prüfung der Negationsmöglichkeiten auf das Zusammenspiel von Negationsskopus und Gradpartikel-Skopus zu achten.

2.3.3. Um die Ergebnisse der Untersuchungen zur Festlegung der Skopusgrenzen nicht in unzulässiger Weise zu präjudizieren und zugleich weitgehende Theorieneutralität wenigstens im Bereich der Materialerfassung zu gewährleisten, wird im Analyseteil nicht nur nach den wenigen Skopustypen unterteilt, die sich durch die Analysen herausgeschält haben: S, NP, VP, V, PP, ADV. Nicht zuletzt, um die Gesetzmäßigkeiten der Kontrastierung mitzuerfassen, wird die kontrastive Binnenstruktur der Skopuskonstituenten in der Typisierung berücksichtigt.

\subsection{Zur Topic - Comment - Gliederung bei Gradpartikel-Sätzen Gradpartikel-Skopus und Kontrastfokus}

\subsubsection{Im Rahmen des Abschnitts "2.3. Skopus" war bereits mehrfach von den} Beziehungen zwischen Gradpartikel-Skopus und Kontrastfokus die Rede. Da diese Problematik nicht nur von der Seite des Gradpartikel-Skopus her angegangen werden kann, muß auch die Thematik Fokus, Kontrastfokus und Kontrastierung für sich und im Zusammenhang mit Gradpartikeln betrachtet werden.

Das Thema wirde eigentlich erfordern, auf die Topic-Comment-Artikulation sowie Darstellungsprobleme derartiger Erscheinungen grundsätzlich einzugehen, denn die Diskussion auf diesem Gebiet leidet nach wie vor unter einem großen Wirrwarr der Terminologie und der jeweils berücksichtigten Faktoren. Das würde aber einerseits den vorhandenen Raum ungebührlich beanspruchen und andererseits doch nur wenig zur Klärung des, jedenfalls in der Forschungstradition, relativ peripheren Kontrastthemas beitragen. Deshalb gehe ich vor der Behandlung des für die Analyse der Gradpartikeln Nötigen nur ganz kurz auf die Grundorientierungen und auf die Teminologie ein.

2.4.2. Die Topic-Comment-Artikulation (TCA) oder Thema-Rhema-Gliederung des Satzes ist ein ureigenstes Thema der Prager Linguistenschule, und das bis in 
die Gegenwart, wie die Veröffentlichung von Sgall e.a. (1973) zeigt. An seinem teminologischen Usus, seinen Definitionen orientiere ich mich zunächst, denn obwohl die TCA inzwischen ein Dauerthema für Formalisierungsversuche im Rahmen von Modifikationen der Montague-Grammatik und bei Kategorialgrammatiken geworden ist, so hat diese emsige Beschäftigung keine wirklich neuen Perspektiven bezüglich der Grundkonzepte und der darauf aufbauenden Teminologie gebracht: nach wie vor geht man von Topic und Focus aus und definiert beide operational mit Hilfe des Fragetests.

Das Topic (auch 'Thema', 'Satzgegenstand', 'psychologisches Subjekt') ist durch folgende Eigenschaften ausgezeichnet: es mß beim Fragesatztest in der Frageformulierung erscheinen; es umfaßt die kontextuell gebundenen bzw. nicht zum 'Vordergrumd' gehörigen Elemente eines Satzes; die Nominalphrasen in seinem Bereich sind vorwiegend definit und generisch; der ibergang zum Focus/ Comment ist fließend, also eine Frage der Abstufung; und es kann, in bestimmten Grenzen, frei vom Sprecher gewählt werden. Chomsky (1969) meint jedoch, daß in einem wohlgeformten Diskurs aufeinanderfolgende Sätze dieselben Präsuppositionen (sein Ausdruck für 'Topic') aufweisen muissen.

Die Termini 'Focus', 'Comment' und 'psychologisches Objekt' (auch 'Satzaussage') bilden zu den oben genannten die jeweiligen Komplementärbegriffe. Sgall (1973: 25) schreibt dem damit Gerneinten die Funktion zu, die im Hörergedächtnis verankerten Informationen zu modifizieren, während Halliday (1967: 204), etwas vorsichtiger formulierend, als Focus denjenigen Teil der Äußerung bezeichnet, von dem der Sprecher wïnscht, daß er als informativ interpretiert wird. Eine formale Definition versucht als einziger Chomsky (1969; 1971: 205 f.). Focus ist in seinem Verständnis "a phrase containing the intonation center ...; the focus must be composed of full lexical items - more generally, items that make a contribution to the meaning of a sentence that is in some sense independent of anything outside the focus." Die Schwäche dieser Definition ist, daß sie keine A b g r e n z u n g des Focus emöglicht. Diese ist bestenfalls aufgrund des Tests der natuirlichen Antwort möglich, obwohl dieser bei mehreren Foci in einem Satz bzw. bei einem mehrgliedrigen Focus sehr unscharf werden kann. Die zweite, nur vordergründig formale Kennzeichnung, erweist sich bei näherem Hinsehen als nicht praktikabel, obwohl sicherlich jeder zu wissen glaubt, was damit gemeint sein könnte. Odo Leys (1973) geht allerdings in die falsche Richtung, wenn er als Erweiterung vorschlägt, daß auch Elemente oder Merkmale komplexer Symbole unter umständen focusmäßig bei der Interpretation der Oberflächenstruktur in den Vordergrund treten könnten. Denn es ging ja Chomsky gerade darum, eine Abgrenzung des gewöhnlichen Focus gegenüber dem 
Kontrast- oder Korrekturfocus zu finden.

Die den Terminus 'Focus' definierenden Eigenschaften sind also folgende: im Fragetest merkiert der Skopus der Frage den Bereich des Focus in der "natürlichen Antwort"; er unfaßt vorwiegend kontextuell ungebundene Elemente, doch kann auch ein kontextuell gebundenes Element zum Focus gehören (ihn aber nicht allein bilden); demgemäß sind die Nominalphrasen in seinem Bereich vorwiegend indefinit. Während Sgall behauptet, daß für den Sprecher immer ein bestimmter Bereich vorhanden ist, aus dem er den Focus relativ frei wählen kann, nimmt Chomsky an, daß der Focus jeweils vom Intonationszentrum des Satzes determiniert wird: dadurch gäbe es in jedem Satz jeweils nur eine Phrase, dle als Focus interpretiert werden kann. Ferner ist Sgall (1973: 164) im Gegensatz zu Chomsky der Ansicht, daß ein Focus nicht notwendigerweise mit einer vollständigen Phrase (ob nun in der Oberflächenstruktur oder Tiefenstruktur) zur sammenfallen muß. Dagegen versucht Jackendoff (1972: 237) festzulegen, daß nur die Domänen der phonologischen Zyklen, in welchen die Silbe mit dem Hauptakzent [l stress] zugeteilt erhält, als mögliche Foci in Frage kommen. Alles andere rechnet er unter Korrektur- und Kontrasterscheinungen.

Dies scheint mir zu beweisen, daß ein großer Teil der Divergenzen auf eine unterschiedliche Grenzziehung zwischen normalen Äußerungen (mit ungestörter Topic-Focus-Gliederung) und Kontrast- bzw. Korrekturäußerungen zurückzuführen ist. Dabei ist beiden Parteien zu widersprechen: Kontrastierung ist in erster Linie keine formale, sondem eine funktionale Angelegenheit, demgemäß auch nicht immer aufgrund formaler Kriterien zweifelsfrei als solche bestimmbar. So deutet zwar ein Satzakzent, der nicht dem (durch "Aufaddieren" der Phrasenakzente entstandenen) Normalakzent entspricht, auf Kontrastierung hin; aber Kontrastierung kann auch bei normaler Position des Satzakzentes vorliegen.

In den knappen Beschreibungen von Topic und Focus/Comment wurde bereits mehrfach der Fragetest erwähnt. Er entspricht völlig dem von Chomsky (1969; 1971: 199 ff.) entwickelten Test der natürlichen Antwort und ist das einzige einigermaßen praktikable operationale Verfahren auf diesem Felde. Zu dem jeweils zu untersuchenden Satz wird eine Wortfrage gebildet, zu welcher dieser Satz eine mögliche Antwort darstellt. Der Frageskopus markiert dabei relativ genau den Focus im zugehörigen deklarativen Satz, also in der natürlichen Antwort darauf. Allerdings wird dadurch die Beweispflicht nur um eine Stufe verschoben, nämlich zur Abgrenzung des Frageskopus. Außerdem weist dieser Test noch weitere Mängel auf: er ist sehr unscharf im Bereich des fließenden Übergangs vom Topic zum Comment, und er bereitet große Schwierigkeiten, wenn in einem Satz mehrere Foci vorliegen, bzw. wenn der Focus mehrgliedrig ist. 
Dieser Fall hat auch eine gewisse Bedeutung für die Untersuchung von Gradpartikeln. Sgall (1973: 56) ist der Ansicht, daß in einem einzigen Satz auch mehrere positionen ungebunden sein können, so daß also der Focus mehrgliedrig ist bzw. daß ein Satz mehrere Foci enthält. Dies verdeutlicht er durch folgenden Fragesatz:

(1) Auf welche Art und Weise, wohin und wem hat Karl was gesandt?

Nun kann nicht geleugnet werden, daß Sätze dieser Art tatsächlich vorkommen, ja es lassen sich sogar Sätze konstruieren, in denen alle denkbaren Position $\in$. erfragt werden. Doch sollte man auch nicht übersehen, daß in diesen Sätzen gewöhnlich nur völlige Uninformiertheit signalisiert wird, bzw. eine Fehleinschätzung der Kommunikationsbedingungen durch den Kommunikationspartner verdeutlicht wird. Als Normalfall sollte gelten, daß nur eine Position erfragt wird, und daß für die Erfragung mehrerer Positionen auch entsprechend viele Fragen gebildet werden. Falls man diese Regel auch auf Gradpartikel-Sätze anwenden kann, bedeutet das, daß zwar grundsätzlich mehrere focusbindende Gradpartikeln mit disjunktem Skopus je Satz möglich sind (soweit dadurch nicht semantische Kombinationsgesetze verletzt werden), daß aber eine Häufung von ungebundenen Elementen die Analyse, das Verständnis erschwert.

2.4.3. Nachdem anfänglich in der generativen Transformationsgrammtik unterschiedliche Topic-Comment-Gliederungen desselben Satzes als reine Stellungsvarianten behandelt worden waren, die durch nicht bedeutungsverändernde Transformationen aus einer Tiefenstruktur-Wortfolge abgeleitet werden, gestand Chomsky mit dem Aufsatz von 1969 die semantische Relevanz dieser Erscheinungen zu. Aber wie bei der eng verwandten Frage des Skopus von Gradpartikeln leugnet er die Möglichkeit, den Focus einer Äußerung schon in der Tiefenstruktur zu markieren. Vielmehr sei der Akzent, der nach seiner generativen Phonologie 42 des Englischen eine ausschließlich oberflächenstrukturelle Erscheinung ist, das eigentlich steuernde Element: Focus und damit auch Skopus würden erst durch die Oberflächenstruktur bestimmt. Eine ganz ähnliche Position vertritt Jackendoff (1972: 232 ff.). Damit sind aber äußerst künstliche Lösungen, wie etwa die von Jackendoff vorgeschlagene Indizierung von einzelnen Konstituenten, notwendig, um die Formulierung von Antitransformationen zu ungehen. - Eine quasi tiefenstrukturelle Lobsung, wie sie in ganz informellex Weise Akmajian (1970a) vorschlug, wurde von Chomsky und Jackendoff (1972: 233-236) abgelehnt. Akmajian hatte an Spaltsätze (cleft-sentences) erinnemde Tiefenstrukturen

42 Chomsky/Halle (1968), siehe vor allem zu "Stress Placement" S. 68-162. 
vorgeschlagen, wobei der übergeordnete Satz den Focus, der Relativsatz die "Präsupposition" darstellten sollte:

(2) Nur Ha ns lügt. - Es ist nur Hans, der lügt.
(x lügt) $(x=$ nur Hans $)$

Über die logische Form der Verbindung zwischen beiden Sätzen konnte Akmajian keinerlei Angaben machen. Jackendoff's Einwände stützten sich darauf, daß eine ganze Anzahl von möglichen Focus-Konstituenten keine akzeptable Spaltsatzformulierung erlauben würden.

Einzelne Hinweise in den vorausgehenden Abschnitten ließen bereits erkennen, daß sowohl von Sgall als auch von Chamsky der Gradpartikel-Skopus als ein normaler Satzfocus eingeordnet wird. Die einzige Besonderheit wird darin gesehen, daß eine bestimmte Partikel-Klasse automatisch den Satzfocus an sich zieht, bzw. als Focus-Markierung fungiert. Dles liegt nicht zuletzt daran, daß Kontrastäußerungen einerseits nicht rigoros von Nomaläußerungen unterschieden werden, und daß andererseits, daraus folgend, die Eigenschaften von Kontrast- und Korrekturäußerungen nicht hinreichend genau erfaßt werden.

Wie S. Schmerling (1972) in ihrem Artikel zur Kontrastbetonung zutreffend feststellt, wurde das Thema Intonation und Satzakzent in den ersten beiden Phasen der generativen Transfomationsgrammatik (und nicht nur dort) ungebïhrlich vernachlässigt bzw. inadäquat behandelt. So gingen Chomsky und Halle (1968: 69-172) bei ihrer Beschreibung des englischen Satzakzentes davon aus, daß er in einer Art "Aufaddieren" der Wortakzente zu Phrasenakzenten, sowie der Phrasenakzente zum Satzakzent beschrieben werden könnte. Dieses Verfahren ist sicherlich für die unmarkierten Fälle möglich, fast überall aber, wo Kontrastierung vorliegt, erzeugen diese Regeln eindeutig fehlerhafte Akzentmuster. So gesteht auch Chomsky in einer Anmerkung von "Deep structure, surface structure and semantic interpretation" ${ }^{43} \mathrm{zu}$, daß für alle Fälle expressiver oder kontrastiver Betonung eine solche Iösung nicht in Frage kormt. Da sich aus einer solchen Betonung, wie er meint, deutliche semantische Unterschiede gegenïber der normalen Akzentverteilung ergeben, scheint ihm eine Markierung mit entsprechenden Merkmalen schon bei der Satzerzeugung (um die Bedeutungsneutralität des Ableitungsprozesses zu gewährleisten) notwendig. Als Basis für die Formulierung geeigneter Akzentregeln nimmt Chamsky an, daß es sich nur um eine tberlagerung der normalen Akzentkontour handle. Für diese Annahme spricht aber nach den bisherigen Untersuchungen nichts.

Sgall (1973: 32-38, Abschnitt 2.4.) geht nur sehr knapp auf das Thema ein. 43 Siehe N. Chomsky (1969; 1971: 199, Anm. b). 
Er unterscheidet zunächst eine Normalform (basic layer), in der CD-Skala (= communicative dynamism) und CI-Skala (= communicative importance) übereinstimmen, und eine Spezialform (second instance), bei der diese beiden Skalen divergieren. Da die beiden Skalen aber sehr unklar definiert sind, können wir damit nicht sehr viel anfangen. - Immerhin hat Sgall (1973) wie auch Schmerling (1972) den Zusammenhang von Satzakzent und Skopusmarkierung von Gradpartikeln gesehen. Unter Kontrastfocus versteht er aber die Hervorhebung eines einzelnen Elementes, das mit dem vorausgehenden Text, genauer mit einem Element darin, kontrastiert. Ferner ist er der Ansicht, daß die Topic-FocusStruktur des kontrastierten Satzes im Kontrastsatz erhalten bleibt. In dieser Fragen möchte ich mich jedoch dem von Sgall kritisierten Firbas (1973) anschließen, der meint, daß man nur das Kontrastelement als nicht kontextuell gebunden betrachten könne.

2.4.4. Um eine leichtere Verständigung über die gemeinten Sachverhalte herbeizufïhren, will ich im folgenden die mir möglich erscheinenden Normal- und Kontrastierungs-Fälle aufzählen:

a. Unmarkierte Sätze: normale Verteilung der kontextuellen Gebundenheit, der kormunikativen Bedeutung der Einzelelemente und ihrer jeweiligen Rollen im Satz. Normaler Satzakzent und damit keine erkennbaren Beschränkungen für Vorgänger-und Folgesätze.

(3) Anja ärgerte einen $S$ t u $d$ e $n t$ e $n$.

Aus Gründen der leichteren Unterscheidbarkeit möchte ich teilidentische Sätze bei den Vorgänger-Sätzen ausschließen. - Die Kommunikationsabsicht des Sprechers, soll eine Mitteilung sein, er mu also annehmen, daß die betreffende Äußerung informativ ist für den Hörer, im Sinne der Griœe'schen Konversationspostulate. Insofem liegt auch, allerdings nur im weitesten Sinne, eine Art Kontrast vor: von den an einer bestimmten Diskursstelle möglichen Äußerungen wird eine ausgewählt, die durch den Kontrast zu den an dieser Stelle ebenfalls möglichen Äußerungen informativ wird.

Derselbe Satz kann auch mit einem "emphatischen" Akzent gesprochen werden, einer Verstärkung des gewöhnlichen Satzakzentes. Ein dafür typischer Diskurs könnte etwa folgendermaßen aussehen:

(4) A: Du, stell dir vor, Anja ärgerte einen $S$ t u d e $n$ t $e n$ ! B: Was, einen $S t u d e n t e n$ ? Ist die noch $z u \quad r$ e $t$ e $n$ ?!

Bestimmte Vorgänger und Folgesätze sind jedoch nicht vorgeschrieben. Ublicherweise wird es sich um die Mitteilung einer wichtigen, ev. ganz uner- 
warteten Tatsache, also mit hohem Informationswert, handeln. Diese Sprecherannahme kann natürlich zurückgewiesen werden:

(5) C: Wieso, was ist daran Schlimmes? / Na und?

Utbrigens zeigt die Antwort nicht selten elnen Focuswechsel an:

(6) D: Die Anja? Hätt' ich nie von ihr gedacht!

Natürlich kann dieses Satzpaar nicht als wohlgeformtes Diskurspaar im üblichen Sinn gelten. - Wie der Fragesatztest zeigt, ist der Focus auch in diesem Falle keineswegs auf eine einzige terminale Konstituente beschränkt:

(7) A: Wen hat Anja geärgert?

B: Einen Studenten.

A: Was hat Anja getan?

B: Einen Studenten geärgert.

Als Mittel der Hervorhebung fungiert auch die Topikalisierung der Focuskonstituente, meist mit emphatischem Akzent verbunden, sowie die Spaltsatzbildung:

(8) Einen $S t u d$ e $n$ te $n$ ärgerte Anja!

(9) Es war ein $S t u d$ e $n t$, den Anja ärgerte!

Derartige Sätze können auch als Korrektursätze verwendet werden, doch setzt dies einen Vorgängersatz voraus, der bis auf die Focuskonstituente (vielleicht sogar nur die betonte Konstituente) identisch ist.

b. Kontrast-/Korrektursätze: Die unterschiedlichen Typen werden jewells durch Vorgängersätze verdeutlicht, außerdem empfiehlt sich die Darstellung der Tilgungsmöglichkeiten:

i. Phonetische Korrektur:

(10) A: Anja hat einen $S c h$ t u p e $n t$ e $n$ geärgert.

B: Nein, das heißt: Anja hat einen $s t u d$ e $n t$ e $n$ geärgert. / Nein, das heißt: $s$ t u d e n $t$ e n.

ii. Informationskorrektur:

(11) A: Anja hat einen ordina $x i$ us geärgert.

B: Oh nein, sie hat nicht einen/keinen ordin a $r i$ us geärgert, (sondern) sie hat einen $S t u d e n t e n$ geärgert./ Oh nein, keinen Ordinarius, sondern einen $S t$ u $d$ e $n t e n$. / Aber nein, einen $S t u d$ e $n$ te $n$.

Die Kontrastnegation dürfte für diesen Fall ziemlich typisch sein. Ubrigens kann hier natürlich auch Topikalisierung auftreten:

$$
\begin{aligned}
& \text { B: Einen } O r d i n a r i u s \text { hat sie } n i c h t \text { geärgert, aber } \\
& \text { einen } S t u d e n t e n \text {. }
\end{aligned}
$$

In allen diesen Fällen ist man nicht auf die Korrektur der Focuskonstituente festgelegt: 
(13) C: Aber nein, $H$ a $n s$ hat einen Ordinarius geärgert./ Aber nein, $H$ a $n s$ war das. / Aber nein, $H$ a $n s$.

D: Aber nein, einen Ordinarius hat sie u $m$ a $r m t$. / Aber nein, u $m$ a $r m t$ hat sie ihn.

Die Korrektur kann auch Teilkonstituenten betreffen, und dabei muß es sich keineswegs um eine phonetische Korrektur handeln:

(15) A: Anja hat einen $S t$ u d e $n t$ e $n$ geärgert.

B: Aber nein, eine $S t u d$ ent $i n /$ Studen $t i n$.

(16) C: Sie hat ihn nicht geärgert, aber sie wi $r d$ ihn ärgern.

Daneben scheint auch, mit gewissen Einschränkungen, die Korrektur mehrer Konstituenten möglich zu sein:

(17) A: Anja hat einen $s t u d$ e $n t$ e $n$ geärgert.

B: Aber nein, Anja hat nicht einen $s t u d$ e $n t$ e $n g$ e ä $r g$ e $r t$, sondern einen $O r d i n a r i$ us umarmt. / Erstens war es nicht ein $s t$ u $d$ e $n t$, sondern ein $O r d i n a r i u s$, und zweitens hat sie den nicht $g e \ddot{a} r g e r t$, sondern u $m$ a $r m t$.

Alle diese Formulierungen wirken reichlich gekünstelt. Es scheint so, als mißßte zumindest ein gewisser inhaltlicher Zusammenhang oder doch ein formaler Gleichlauf zwischen korrigierter und Korrekturphrase vorhanden sein.
C: Anja hat nicht einen $S t u d$ e $n t e n g$ e är $g$ e $r t$, sondern einen $k \circ 1$ l e gen $d e n u n z i$ e $r t$.

Alle Elemente des kontrastierten Satzes können nur in einem Radio-Eriwan-Witz korrigieri werden:

(19) D: Erstens war das nicht Anja, sondern $H$ a $n s$, und geärgert hat ex nicht einen $s t u d$ en $t$ e $n$, sondern einen ordinari us, und $g$ e är gert hat er ihn eigentlich a uch nicht, sondern $u m a r m t$.

Sgall vermischt in seinem Ansatz Emphase- und Kontrastsätze. Das erscheint nicht tunlich, da man deutliche formale und funktionale Unterschiede ausmachen kann. - Korrektursätze können, soweit ich sehe, nur in den seltensten Fällen auch als Nommalfom-Sätze verwendet werden, und zwar dann, wenn sie keine Tilgungen aufweisen, wenn der Normalfocus des betreffenden Satzes kontrastiert wird, und wenn der Kontext beseitigt wird.

Eine Bemerkung mu man wohl noch zum aminösen Kontrastakzent machen: die kontrastierte Silbe trägt jeweils den Satzakzent. 'Es ist sehr schwierig, zu entscheiden, ob es sich hierbei um einen bezüglich seiner phonetischen Qualitäten besonderen Akzent handelt, der etwa durch Druckstärke oder Tonhöhendifferenz bzw. Tonhöhenverlauf vom "normalen" Satzakzent unterscheidbar wäre. 
Diese Eigenschaften würde er aber mit dem normalen Emphaseakzent teilen. Viel wichtiger sind für die Identifizierung der Kontrastfunktion elner bestimmten Äußerung bzw. einer bestimmten Konstituente andere Merkmale, wie Kontext, Divergenz zwischen normaler Akzentposition und kontrastiver Akzentposition, Tilgungen usw.

2.4.5. Die weitaus meisten Gebrauchsweisen von Sätzen mit Gradpartikeln gehören dem kontrastiven Typ an: wie bei diesem besteht weitgehende Freihelt in der Wahl der kontrastierenden Konstituente (dem Skopus), und sowohl Tilgungen als auch Sätze mit Kontrastnegation kommen vor. Daneben gibt es allerdings auch Beispiele ohne diese expliziten Kontrastmerkmale; in diesen Fällen kann man aber von einer impliziten Kontrastierung sprechen. Die betreffende Äußerung kontrastiert mit einer Äußerung, die an dieser Stelle eigentlich erwartet wurde. Wie diese allerdings im jeweiligen Fall auszusehen hätte, ist kaum entscheidbar, ohne daß man alle Situationsfaktoren zur Beurteilung hinzuzieht. Doch kann man aus dieser Eigenschaft kein Argument dafür machen, daß man den einzelnen Gradpartikeln eine Art Erwartungspräsupposition zuordnet. - Daß es sich beim Gradpartikel-Skopus nicht um einen nomalen Satzfocus handeln kann, zeigt auch die Tatsache, daß der Fragetest nicht sinnvoll auf GradpartikelSätze angewendet werden kann, und daß dies in gleicher Weise von zweifelsfreien Kontrastäußerungen gilt

(20) A: Wen hat Anja geärgert?

B: “Anja hat nicht einen $S t$ u de $n t$ e $n$ geärgert. Anja hat einen ordinari us geärgert.

Dies dürfte eher die Antwort auf folgende Frage sein:

(20a) A: Hat Anja einen Studenten geärgert?

Dabei handelt es sich aber um eine für die Focusemittlung ungeeignete Satzfrage. - Dieses Scheitem rührt vermutlich von der kamunikativen Funktion des Kontrastes her: er wird nicht als Antwort verwendet, sondern als Gegenbehauptung. Gleiches gilt im Prinzip von Sätzen mit Gradpartikeln. Als Frage läßt sich nur die Informationserwartung formulieren:

(21) A: Haben alle Assistenten die ordinarien geärgert?

$B$ : Nein, nur $A n j$ a hat die Ordinarien geärgert.

Eine Folge wie:

(22) A: Wer hat die Ordinarien geärgert?.

B: Nur $A \cap j$ a hat die Ordinarien geärgert.

scheint nicht ganz akzeptabel. Hingegen gibt es in diesem Spezialfall noch 
eine besonders trickreiche Variante:

(23) A: Wer alles hat die Ordinarien geärgert?

B: Nur $A$ n j a.

Hierbei dient nur zur Zurückweisung der Nichteinzigkeitspräsupposition des Fragesatzes.

Die Explizitformulierung des Kontrastsatzes mit Kontrastnegation (Skopus der Kontrastnegation!) ist hinreichend für die Festlegung des Kontrastfocus: durch diese Bezeichnung glaube ich ihn auch hinreichend vom Nomnalfocus unterschieden. - Der Kem des Problems besteht darin, daß ein Satz mehr oder weniger vollständig kopiert wird, und daß ein oder mehrere Elemente durch ein semantisch irgendwie äquivalentes, aber kontrastierbares Element ersetzt werden. Diese Problemstellung ist mit einer reinen Satzgrammatik nicht zu bewältigen.

2.4.6. Eine zentrale Aufgabe der Beschreibung von Gradpartikeln ist also, wie hoffentlich die vorausgehenden Darlegungen gezeigt haben, die Untersuchung der Beziehungen zwischen Gradpartikel-Skopus und der Erscheinung der Kontrastierung. Es steht fest, daß der Gradpartikel-Skopus in jedem Falle den (oder doch einen) Satzakzent erhält, daß also die Gradpartikel focusbindend ist. Im Hinblick aber auf diejenigen Fälle, in denen dieser Skopus-Focus auch nichtkontrastiv interpretiert werden könnte, also bei den Skopustypen $S$ und VP sowie in bestimmten Fällen auch V,kann nicht von vorneherein gesagt werden, ob es sich beim Gradpartikel-Skopus in jedem Falle um einen Kontrastfocus handeln muß, zumal die Fälle von impliziter Kontrastierung bei Gradpartikel-Sätzen eher die Regel bilden. Dies bedingt, daß die Eigenschaften der Kontrastierung laufend erforscht werden. - Ferner kann aus der Tatsache, daß (Kontrast-)Focus und Gradpartikel-Skopus fest miteinander verbunden sind, noch nicht auf Gleichheit, z.B. bei der Abgrenzung, geschlossen werden. Man muß vielmehr zunächst von der Hypothese ausgehen, daß beide Erscheinungen relativ selbständige Phänomene sind, damit die möglichen Fragestellungen nicht ohne hinreichende empirische Absicherungen (unzulässig) eingeschränkt werden.

Hilfsmittel bei der Erforschung dieser Regularitäten kann, wie bereits gezeigt, nur in sehr begrenztem Maße der Fragesatztest sein. Dennoch wird er immer wieder eingesetzt, da auch sein Scheitern wie die gegebenenfalls nötigen Modifikationen wichtige Einsichten vermitteln, vor allem auf dem Gebiet der Gradpartikel-Semantik. Ein weit wirksameres Mittel sind hingegen Kontrastäußerungen. Sie wurden intuitiv und unsystematisch schon immer bei der Beschreibung der Gradpartikel-Semantik eingesetzt, beruht ja doch auch die Me- 
thode des "factoring" darauf. Ansätze zur theoretischen Elnbeziehung finden sich bei Kuroda (1969: 333 ff. und 346) und bel Hom (1969; 1972: 222 f.). Diesen "Test", der eng verwandt ist mit weiteren von mir eingesetzten semantischen Analyseverfahren, verwende ich systematisch in den Formen des der Gradparti-

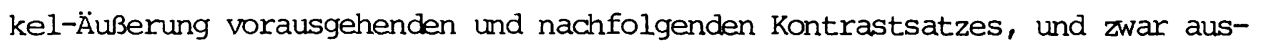
schließlich in monologischer Form (also ohne die Annahme eines Sprecherwechsels). Dadurch sind die formalen wie inhaltlichen Anforderungen strenger, die Testbedingungen werden eher kontrolllerbar. Auf diese Weise können einzelne Bedeutungsaspekte von Gradpartikel-Sätzen expliziert werden. In den für die einzelnen Gradpartikeln sowie (mit Einschränkungen) die verschiedenen Interpretationsarten typischen Kontrastierungsformeln, die meist selbst wieder Gradpartikeln enthalten, wird das Zusammenspiel der Funktionen von Gradpartikeln deutlich.

Geht man so vor, so kann man sich bei der Gliederung des Analysenteils nicht mehr auf die wenigen Skopustypen beschränken. Mit der Frage nach den möglichen Skopustypen ist ja auch nach den Bedingungen von Kontrastierbarkeit gefragt. Da sich zeigte, daß die vielgestaltigen Möglichkeiten der Kontrastierung eine kleingliedrige Binnendifferenzierung innerhalb der Hauptskopustypen bewirken und da sich zudem eine an oberflächenstrukturellen Gesichtspunkten orientierte Skopusgliederung nicht ganz mit einer Gliederung nach semantischen Skopustypen zur Deckung bringen läßt, war ein Kompromiß zwischen diesen drei Gliederungsprinzipien nötig. Er sieht folgendermaßen aus:

1. Das Gerüst bilden die (semantischen) Hauptskopustypen, nämlich: Nominalphrasen (hier vertreten durch die Subjekts-NP), Prädikate, Verbalphrasen, Adverbien, Sätze. - Die diesen hinzugerechneten Skopustypen Quantoren und Idiome müßten eigentlich auf die übrigen Typen aufgeteilt werden, weisen aber so typische und selbständige syntaktische wie semantische Eigenschaften auf, daß ich diese Einordnung für gerechtfertigt halte.

2. Thnen werden formale (syntaktische) Subtypen zugeordnet.

Im Bereich der Nominalphrasen: Subjekts-, Objekts-NP, Präpositionalobjekte sowie Pronamina und Gliedsätze.

Im Bereich des Prädikats: Verbum, prädikatives Adjektiv, Prädikatsnomen.

Im Bereich der Quantoren: Kardinalzahlen, Ordinalzahlen, viel - wenig, viele wenige - alze - manche.

Im Bereich der Adverbien: adverbiale Präpositionalphrasen, Adjektıv-Adverb, reine Adverbien. Satzadverbien und limitierende Adverbien bilden demgegenüber relativ selbständige Subtypen. Hier einzuordnen wären auch die Adverbialsätze, doch hat sich gezeigt, daß sie dem Skopus-Subtyp Gliedsatz 
(zum Haupttyp NP) ähnlicher sind als den übrigen Adverbien. So wurden sie auch dort zugeordnet.

3. Nun sind noch die überwiegend dem Kontrast-Thema gewidmeten Rand-Skopustypen zu nennen:

Im Bereich der Nominalphrasen: Kontrastierung eines Wortteils, Kontrastierung des Artikels, kontrastiv betonte Präposition bei Präpositionalobjekten, kontrastiv betonte Konjunktion bei Gliedsätzen, kontrastiv betontes Adjektivattribut.

Im Bereich des Prädikats: kontrastiv betonte trennbare Verbvorsilbe, Kontrastierung von Auxiliar-Elementen bzw. -Merkmalen.

Im Bereich der Adverbien: kontrastiv betonte Konjunktionen von Adverbialsätzen, kontrastiv betonte Präposition in adverbialen Präpositionalphraser.

Hier verfahre ich nun teilweise inkonsequent, aber doch ökonomisch. So fasse ich die verschiedenen kontrastiv betonten Präpositionstypen zu einem selbständigen Abschnitt zusammen; gleiches gilt für die verschiedenen Konjunktionstypen. Ein echtes Problem bietet aber der Subtyp kontrastiv betontes Adjektivattribut. Dieses gehört je nach der Stellung der Gradpartikel zum (semantischen) Skopustyp (komplexe) Nominalphrase oder aber zum Skopustyp Prädikat, genauer prädikatives Adjektiv.

In der Uberschau resultiert daraus ein Durchgehen aller einfachen und zusammengesetzten Konstituententypen (mit Ausnahme der Negation). Ich hoffe, daß sich dies als akzeptabel und sinnvoll erweist. ${ }^{44}$

44 Zur Kennzeichnung des Kontrastakzentes verwende ich in den Belegsätzen, wie wohl allgemein üblich, die sperrung. Dort, wo der Kontrastakzent mit dem normalen Satzakzent zusammenfällt, oder wo eine komplexe Skopuskonstituente mit komplizierterer Akzentkontur vorliegt, unterbleibt diese Auszeichnung meist; natürlich auch dann, wenn mehrere mögliche Positionen des Kontrastakzentes untersucht werden sollen. 
2.5. Probleme der semantischen Beschreibung von Gradpartikeln im Deutschen.

2.5.1. Zu allgemeinen Problemen im Zusammenhang mit der Beschreibung der Gradpartikel - Semantik.

So interessant die bisher behandelten Beschreibungsebenen fuir die Analyse der Gradpartikeln sind, das Hauptaugenmerk gilt doch mit der raschen Entwicklung der letzten Jahre der Semantik: hier strömen die Ergebnisse aus den übrigen Bereichen zusammen, Syntax und besonders die eng verknüpften Erscheinungen von Skopus und Kontrastierung liefern entscheidende Hinweise für die semantische Analyse der Gradpartikeln, eine Tatsache, die bislang in der GradpartikelForschung weitgehend ungenützt blieb.

Die Beschreibung der Gradpartikeln setzt einen relativ weiten Bedeutungsbegriff voraus, z.B. so, wie er in dem Versuch von L. Wittgenstein zum Ausdruck kormt, 'Bedeutung' und 'Gebrauch' zu identifizieren:"Man kann für eine g $r \circ \beta$ e klasse von Fällen der Benützung des Wortes 'Bedeutung' - wenn auch nicht für a 1 l e Fälle seiner Benützung - dieses Wort so erklären: Die Bedeutung eines Wortes ist sein Gebrauch in der Sprache". (Wittgenstein 1967: 35, $\S 43)$. Dieser Ansatz bedarf einer gewissen Präzisierung: Es kann nicht um eine individuelle Verwendung eines bestimmten Wortes in einer ganz bestimmten Situation gehen, sondern nur um die allgemeinen Regeln des Gebrauchs, soweit sie sich nicht auf die formalen Eigenschaften von sprachlichen Einheiten beziehen, sondern auf ihre kormunikative Funktion. Der Vorteil eines solchen Ansatzes wäre, daß er auch die nicht wahrheitswert-relevanten Eigenschaften des Gebrauchs von sprachlichen Einheiten in die Untersuchung der Bedeutung einzubeziehen erlaubte: etwa reine Angemessenheitsbedingungen, Bedingungen des Gliuckens sprachlicher Handlungen usw.

Im Hinblick auf Bedeutung als Gebrauchsregel/wurde behauptet, daß natürliche Sprachen nicht als Zeichensysteme mit eindeutig festgelegten Bedeutungen verstanden werden könnten. ${ }^{45}$ Die Verwendungsregeln würden sich zum Teil erst in der Kommunikation konstituieren und die Bedeutungen wirden je nach Kontext und Situation variieren. Diese Annahme scheinen gerade die Gradpartikeln zu bestätigen, und zwar auch dann, wenn man die nicht-Gradpartikel-Funktionen dieser Elemente sorgfältig ausschließt: während Teile ihrer Bedeutung relativ stabil sind, wechseln andere Teile, wie es scheint, regellos, oder in

45 So etwa B. Henn (1973: 41); ebenso W. L. Schmidt (1967). 
Abhängigkeit vom Ko(n) text, wobei die steuemden Kotextmerkmale nur schwer auszumachen sind. Mit Sicherheit handelt es sich hierbei aber nicht um je nach Sprecher variierende Bedeutungsregeln. Die Erfahrung prinzipieller Verstehbarkeit trotz mannigfaltiger Mißverständnisse kann nicht nur auf einer zufälligen Identität der Realitätserfahrungen beruhen, sondem ist zurückzuführen auf die Giltigkeit semantischer Regeln mit hinreichender Stabilität und Homogenität. Daß daneben auch im Bereich der Semantik das Toleranzprinzip wirksam ist, wodurch gewisse Regelunterschiede weitgehend belanglos bleiben, widerspricht dem nicht grundsätzlich.

2.5.2. Die semantische Analyse der deutschen Gradpartikeln hat mehrere recht gut trennbare Teilaufgaben: so hat sie die syntaktisch, wie sich zeigen wird, recht homogene Gruppe daraufhin zu untersuchen, ob sie auch semantisch als Gruppe gelten kann. Innerhalb der deutschen Grammatiktradition wurde dies ebenso vernachlässigt wie der syntaktische Gruppencharakter. - In der amerikanischen transformationellen Literatur wurde der semantische Gruppencharakter nie geleugnet, stillschweigend wohl sogar meist vorausgesetzt, aber nirgends Gegenstand einer intensiveren Untersuchung. Als semantische Gruppe ausdrücklich konstituiert wurden die Hauptvertreter erst durch R. Bartsch (1972: 192 ff.). Dagegen wurde die zweite Aufgabe, die Erarbeitung der Binnengliederung, vorwiegend von der deutschen Wörterbuchtradition angegangen, wenn auch mit einem unzulänglichen Synonymie- und Homonymiebegriff. So wurde zwar die Grobgliederung in drei Hauptgruppen erreicht, aber nicht die teilweise Überlagerung von auch- und sogar-Gruppe gesehen. Ebensowenig gelang die Nachzeichnung der Feinstruktur innerhalb der einzelnen Gruppen oder gar die Unterscheidung mehrerer Interpretationstypen. Zwar wurden Idiosynkrasien registriert, aber die regelhafte semantische Erklärung hierfür stand noch nicht zur Verfügung: beachtete man doch einen weg nicht, der in der Logik seit langem begangen wird und den die englischsprachige Gradpartikel-Forschung ibemomen oder aber "wiederentdeckt" hat: das Zerlegen ('factoring') eines Gradpartikel-Satzes in mehrere Teilbehauptungen. So lautet etwa die in der Logik seit langem übliche und durch Fillmore (1965) in die Linguistik ibernommene Paraphrase für nur:

(1) Nur Hans lügt.

Hans lügt, und niemand, der nicht Hans ist, lügt.

Zunächst einmal kann man das als eine der üblichen Paraphrasen werden, die zwar eine wichtige Fähigkeit des kompetenten Sprechers spiegeln, für deren Uberprüfung es aber keine objektiven Kriterien gibt. ${ }^{45}$ Daruiberhinaus mu man 46 Zum Paraphrasenproblem siehe R. Nolan (1970). 
darauf hinweisen, daß auch die Zurückführung beliebiger Äußerungen auf standardisierte Paraphrasen noch keine vollständige semantische Beschreibung darstellt. Tatsächlich handelt es sich nur um ev. auch formalisierte Explizitfassungen, jedoch ohne echte semantische Interpretationsregeln bzw. ohne Angaben der Bedingungen des Glückens. Man kann auch nicht übersehen, daß im gewöhnlichen Diskurs Paraphrasen, die benützt werden zur Beseitigung von Mißverständnissen, zu definitorischen Zwecken, zur Bewältigung von Kompetenzunterschieden, nicht eigentlich dazu dienen, die Bedeutung eines Ausdruckes darzulegen, sondem durch Angabe von Synonymen, durch das Eingrenzen eines Ausdrucks auf eine einzige standardisierte Bedeutung, durch die Angabe einfacherer Ausdrücke zusätzliche Hilfen zur semantischen Interpretation zu geben. Jede Paraphrase mit sprachlichen Mitteln muß zirkulär bleiben. Eine echte semantische Interpretation kann man nur durch die Anwendung eines Ausdruckes auf eine bestimmte Situation geben. Nur für die Art dieser formgerechten Anwendung kann man Bedingungen, Regeln aufstellen. - Das muß dann kein Einwand sein, wenn man derartige Bedeutungselemente nur zur Beschreibung von fast grammatikalisierten Bedeutungskategorien verwendet, um also gewisse interne Strukturen des Lexikons zu explizieren, aber deswegen keineswegs behauptet, man hätte bereits eine vollständige Semantik geschaffen. - Unausweichlich und unwiderlegbar scheint hingegen der Einwand, daß zwischen dem Ausgangswort und seiner Dekomposition keine echte Äquivalenzrelation, sondern immer nur eine unidirektionale Implikation herrsche. ${ }^{47}$ Das heißt, daß durch die Paraphrasierung die Wahrheitsbedingungen eines Satzes verändert werden, die Semantik sich also ändert. Dies ist bei den Gradpartikeln auf eine eigenartige Weise zugespitzt: während etwa bei nur-Sätzen beide Teilbehauptungen zutreffen müssen, wenn der nur-Satz sinnvoll anwendbar bzw. war.r oder falsch sein soll, ist dies bei den so ähnlichen auch- und sogar-Sätzen durchaus nicht immer der Fall: hätte ein bestimmtes Element den Wahrheitswert falsch, sc hätte das für den unzerlegten Gradpartikel-Satz kaum Folgen, der Wahrheitswert wäre nach wie vor $w$, für die nomierte Paraphrase im Sinne des 'factoring' 'hingegen $f$.

Die Ähnlichkeit dieser Lösung mit Russell's Formalisierungsvorschlag für defirite Deskriptionen, also für das Problem der Existenz-(Referenz-)Präsuppositionen in natürlichen Sprachen ist augenfällig. Hier wie dort kann man mit D. Franck (1973: 19) kritisieren, daß eine derartige Explikation die grundlegenden Unterschiede im Status der einzelnen Bedeutungselemente unzulässig ni-

47 So auch Bartsch/Vennemann (1972: 21f.). 
velliert: also die Art des Gegebenseins im Sinne von Assertion und Präsupposition. - Dieser Unterschiede hat sich die englischsprachige GradpartikelForschung mit Nachdruck angenommen. In seinem für die Gradpartikel-Semantik richtungweisenden Aufsatz von 1965 wies Fillmore (1971: 537) auf einige Schwächen bzw. Lïcken der interpretativen Semantik nach Katz/Fodor hin. Er zeigte darin insbesondere, daß es Sätze gibt, die nicht aus sich selbst heraus mit Hilfe der üblichen semantischen Regeln interpretiert werden können. Als Lösung schlug er vor, daß, aufbauend auf die grammatische Struktur einer Äußerung X, "entailment rules" diesem X eine Menge von Sätzen Y zuordnen, die mit Hi]fe der üblichen semantischen Regeln interpretiert werden können. Die Gesamtheit ihrer Interpretationen könnte dann als die semantische Interpretation von $x$ gelten. Unter dem Begriff "entailment" faßt Fillmore hier allerdings noch die unterschiedlichsten Folgerungserscheinungen zusammen. - Dieser Vorschlag bedeutet im Falle von even, daß die einzige Möglichkeit, seine semantische Funktion anzugeben, im "Zerlegen" des Partikelsatzes besteht:

She even reads Sanskrit.

- She reads Sanskrit.

- One would not expect that she does read Sanskrit.

Zwischen dem analysierten Satz und den Zerlegungen bestehe eine semantische Folgebeziehung, deren formale Natur allerdings von Fillmore nicht näher erläutert wird. Die von ihm erstmals formulierte "Erwartungspräsupposition" wird imer wieder von anderen Autoren, sogar von L. R. Horn (1969; 1972: 234), aufgenommen, als nicht präzisierbar aber nirgends näher behandelt. Ich hoffe zeigen zu können, daß diese "Erwartungspräsupposition" einerseits die unterschiedlichsten Ausprägungen erhalten kann, andererseits zur Erklärung verschiedener Erscheinungen gar nicht nötig ist, sondern die eigentliche Funktion der Gradpartikeln nur verdeckt. Sie läßt sich darüberhinaus auch nicht als konstantes Bedeutungselement durch operationale Verfahren erfassen.

Der klassische Aufsatz für die präsuppositionelle Analyse von only, even und too ist jedoch der von L. Horn (1969). Die späteren Äußerungen zu diesem Thema beziehen sich alle, explizit oder implizit, auf diese Analysevorschläge, deren schwerwiegendster Mangel in dem Ignorieren des Zusammenspiels von Syntax, Kontrastierung, Skopus und Semantik liegt. Eine Anzahl von semantischen Eigenschaften von Gradpartikel-Sätzen, vor allem die skalierende Interpretation bei even sowie die Suspendierbarkeit von einzelnen Bedeutungselementen ${ }^{48}$ untersucht Horn in seiner Dissertation von 1972. Hier geht er auch auf konversatio-

48 L. Horn (1972: 22-34); siehe auch G. Lakoff (1970; 1971: 41-45 und 56-59). 
nellen Implikaturen, und zwar im Zusammenhang mit Quantoren, ein. Alle diese Konzepte für den unterschiedlichen Status von Teilbehauptungen bei Gradpartikel-Sätzen werden im nächsten Abschnitt noch diskutiert. - Dem Wortlaut nach weitgehend mit denen von Horn (1969 und 1972) identische Bedeutungsanalysen von even geben auch Fraser (1971: 153) und S. R. Anderson (1972: 903 f.). Doch verwenden sie für die Nichteinzigkeits-Präsumposition die Bezeichnung 'Implikation' ("implication") und beziehen sich hiermit auf Austin (1962; 1972: $64,68,186$, 187). Für diese Relation gibt es auch die von Hungerland (1960) stammende Bezeichnung 'contextual implication'. Sie bezieht sich darauf, daß man mit dem Aussprechen einer Behauptung in der Regel zu verstehen gibt, daß man selber glaubt, daß diese Behauptung zutrifft, z.B.

(3) John is sick and I believe that John is sick. ${ }^{49}$

Es handelt sich also eher um eine Annahme über den guten Glauben, der jeder sprachlichen Kommunikation zugrundeliegt, also um die Bedingung, sprachliche Äußerungen nicht mißbräuchlich zu verwenden. Folgerichtig wurde sie von Searle (1968; 1970: 60) unter die'preparatory conditions' eingereiht, allgemeine Voraussetzungen für das Glücken von Kommunikationsakten, Bedingungen, die erfüllt sein missen, damit eine Äußerung als normal gelten kann. Aus diesem Grund scheint es nicht sehr sinnvoll, sie bei den logischen Folgerungen ganz bestimmter Äußerungen einzureihen. Andererseits hat die direkte Verneinung dieser "Voraussetzung" gewisse Ähnlichkeiten mit der Verneinung einer logischen Implikation:

(4) Hansens Kinder haben Glatzen, aber ich glaube es nicht. (Austin 1962; 1972: 68)

Hingegen weist die mißbräuchliche Verwendung etwas andere Merkmale auf: wer A sagt, obgleich B falsch ist, muß als unehrlich gelten. - Die Analyse eines sogar-Satzes würde bei der Verwendung dieser Kontextimplikation folgendemaßen lauten:

Sogar $H$ a $n s$ ist müde.

impliziert: Andere Menschen sind müde.

Oder genauer: Ein Sprecher, der den Satz Sogar $H$ a ns ist müde. äußert, muß an die Wahrheit von Andere Menschen sind müde glauben. Dies entspricht genau der Konstellation bei Karttunens Definition des 'semantic entailment'. Dazu muß gesagt werden, daß es sich um eine mögliche, aber nicht sehr erhellende Ausdehnung des Austin'schen Gebrauchs von "imply" handelt. Abgesehen davon ist die Beschränkung auf eine quantifizierende Interpretation bei sogar-Sätzen nicht möglich, wie ich im Analyseteil noch zeigen werdei.

49 Das Beispiel stammt von Fillmore (1965; 1971: 540). 
Die semantische Analyse deutscher Gradpartikeln hat also, aufbauend auf die syntaktische Vergleichbarkeit dieser Partikeln und auf die zuverlässige Ermittlung der jeweiligen Skopusgrenzen, folgende Fragen zu klären:

a. Bilden diese Gradpartikeln auch in semantischer Hinsicht eine Gruppe? Diese Frage kann als beantwortet gelten, wenn sie eine in ihren Strukturen gemeinsame semantische Beschreibung zulassen.

b. Welche semantischen Untergruppen lassen sich bilden? Diese Frage kann dann als gelöst betrachtet werden, wenn die Synonymie bzw. Teilsynonymie zwischen den einzelnen Elementen expliziert ist.

c. Gibt es bestimmte Interpretationstypen quer durch alle Gruppen von Gradpartikeln, und wodurch sind sie gekennzeichnet?

d. Wie läßt sich die Semantik der einzelnen Elemente explizieren? Als Basis bietet sich natürlich das 'factoring' an. Glückt die semantische Einzelbeschreibung mit diesem Verfahren, so besitzt man gleichzeitig die Mittel zur Beantwortung der ersten drei Fragen.

e. Welche Eigenschaften haben die durch das factoring erzielten Bedeutungselemente? Zur Klärung dieser Frage wird ein umfänglicher Apparat von semantischen Tests eingesetzt, der im Anschluß an die Diskussion der theoretischen Basis dargestellt wird.

f. Ist aufgrund der festgestellten semantischen Eigenschaften dieser Teilbehauptungen eine Statuszuweisung möglich?

Lediglich die Beantwortung von Frage f. ist auch abhängig von definitorischen Entscheidungen bzw. Zweckmäßigkeitserwägungen. Sollte sich aufgrund neuer Ergebnisse zeigen, daß die Summe gewisser Eigenschaften nicht hinreicht, um eine der Teilbehauptungen als Präsupposition zu klassifizieren, so berührt das keineswegs den Kern der Arbeit. Auf jeden Fall kann man feststellen, daß die einzelnen Teilbehauptungen unterschiedlichen Status haben mïssen, da sie unterschiedliche statusrelevante Eigenschaften aufweisen.

Ich bin mir durchaus im Klaren dariber, daß die Beantwortung aller sechs Fragen immer noch keine komplette Semantik der deutschen Gradpartikeln darstellt: dies wäre erst gegeben in einer Darstellung im Rahmen einer (an formallogischen Systemen orientierten) dtsambiguierten Beschreibungssprache, der semantische Interpretationsregeln zugeordnet sind, also z.B. im Sinne der Montague-Granmatik oder verwandter kalküle. - Ansätze hierfür, die ausnahmslos auf dem 'factoring' basieren, liegen bereits vor (z.B. Bartsch 1972, 192 ff.). Ihr gemeinsamer Mangel ist die Beschränkung auf eine quantifizierende Interpretation. Diese Untersuchung soll zeigen, inwieweit jene Vorschläge mit den empirischen Daten übereinstimmen. 
Die in Frage kommenden Statustypen, nämlich Assertion, Präsupposition und konversationelle Implikatur, bedürfen genauerer Darlegungen. Es geht hierbei um die Entwicklung der theoretischen Konzepte sowie um die Sammlung der jeweiligen Eigenschaften, die zumeist unmittelbar aus den Definitionen abgeleitet werden künnen. Dabei muß ich mich trotz der Komplexität der Fragestellungen wie der Forschungslage im Hinblick auf den begrenzten Raum und die begrenzte Thematik dieser Untersuchung auf das in diesem Zusammenhang zum Verständnis der Fragestellungen wie der Analyseansätze unbedingt Notwendige beschränken.

\subsubsection{Assertion}

Als Assertion wird in den gängigen Analysen der Bedeutungskem_ganz allgemein gesprochen) einer Äußerung verstanden, also ein Bedeutungsteil, der im Vordergrund der mit einer Äußerung vemittelten Information steht und in der Regel nicht durch Schliusse aus dem Wortlaut abgeleitet wird. Insofem bildet dieser Begriff die Basis für die Definition der übrigen Folgerungsrelationen. In diesem Zusammenhang muß auch auf den eng verwandten Begriff 'Proposition' eingegangen werden. Er wurde in der klassischen Logik zunächst nicht deutlich von dem der 'Assertion' unterschieden, da ja nur assertorische Äußerungen untersucht wurden. In einer ganz ungefähren ersten Annäherung kann man Proposition als das, was eine Rolle bei der Zuteilung eines Wahrheitswertes spielt, bezeichnen. Sie besteht, auch formal, aus einem Prädikat und seinen Argumenten, deren Zahl sich nach der Menge der Leerstellen dieses Prädikats richtet. Diese Formel ist allerdings auch identisch mit den traditionellen Formeln für Assertionen ('statements'). Mit der Sprechakttheorie wurde die Assertion zu nur einem unter vielen möglichen Sprechhandlungstypen. Dadurch wird aber eine rigorose Trennung zwischen dem Indikator der illokutionären Rolle und der Proposition notwendig und auch möglich. Bei Searle (1968) wird dieser Indikator der illokutionären Rolle als Frädikat über der jeweiligen Proposition dargestellt. Die Proposition ist in dieser Darstellung derjenige Teil der Sprechaktformel, der in allen möglichen sprechakttypen konstant bleiben kann. Wenn also zwei illokutionäre Akte die gleiche Referenz und die gleiche Prädikation enthalten, so wird stets die gleiche Proposition ausgedrückt. Dieser propositionale Akt ist nach Searle auch bei Außerungen in natürlichen Sprachen streng von illokutionären Akten zu trennen, da er nicht selbständig vorkormt und andererseits ein illokutionärer Akt nicht notwendig einen propositionalen Akt beinhaltet. In konkreten Sprechakten ist jedoch diese theoretisch so einleuchtende Trennung keineswegs problemlos zu bewerkstelligen, und zwar aus 
zwei Gründen. Einmal handelt es sich bei gewöhnlichen Äußerungen nicht nur um explizite performative Formeln und eingebettete daß-Satz-Komplemente (ev. verkürzt zu Infinitiven). In der Regel fehlt sogar, wie D. Wunderlich (1972: $15 \mathrm{ff.}$ ) und Ehrich/Saile (1972: 255-287) überzeugend dargestellt haben, diese performative Fonmel, abgesehen von ritualisierten oder institutionalisierten Sprechhandlungen. Die Festlegung der illokutionären Kraft einer bestimmten Äußerung erfolgt vielmehr meist durch den Kontext, und zwar den sprachlichen wie den situationellen, durch Intonation, Akzent und Emphase, sowie durch Partikeln, insbesondere Modalpartikeln, die sehr viel zur Disamblguierung bezüglich der illokutionären Rolle beitragen. Es ist ganz bezeichnend, daß gerade sie lange Zeit als bedeutungs- wenn nicht funktionslos galten. - Die Proposition ist also nur dann einigemaßen klar abgrenzbar, wenn der zu analysierende Satz in der Konstituentensatzform vorliegt. Sie ist identisch mit der semantischen Informationsmenge eines Satzes, und zwar unabhängig von dessen Form. Die Proposition ist also ein semantisches Objekt im Gegensatz zum Satz, der ein syntaktisches Objekt darstellt, und auch im Gegensatz zur konkreten Äußerung, die ein pragmatisches Objekt bildet. Sie kann als eine Anweisung auf eine mögliche Welt gelten, deren Funktion erst durch einen 1llokutionären Indikator festgelegt wird.

Ein weiteres Problem bei der Festlegung der Proposition einer natürlichen Äußerung stellen jene Satzgebilde dar, bei denen der Wortlaut nicht weitgehend identisch mit der Proposition ist, wie dies etwa bei nur-Sätzen im Gegensatz zu auch- und sogar-Sätzen der Fall ist. Deshalb wählt man besser eine umfassendere Definition, die auch derartige Fälle mit einschließt. Dafür bietet sich folgende Regelung an: Äußerungen in natürlichen Sprachen sind komplexe Gebilde im Sinne der Logik. Sie enthalten zum einen Hinweise auf die Funktion dieser Äußerung (ihre illokutionäre Rolle). Zum anderen bedingen sie bestimmte Voraussetzungen, die erfüllt sein missen, wenn die Außerung sinnvoll sein soll, und zwar unabhängig davon, ob nun diese Äußerung in positiver oder in negierter Form vorliegt. (z.B. Präsuppositionen). Und schließlich sind aus der Äußerung eine Reihe von Folgerungen abzuleiten, deren gemeinsames Merkmal ist, daß sie negationssensitiv sind. Sie lassen sich wiederum in verschiedene Untertypen unterteilen, darunter auch die "eigentliche" Proposition: sie ist dadurch ausgezeichnet, daß sie im Vordergrund der Behauptung steht. Deshalb nenne ich sie im Zusammenhang dieser Untersuchung weiterhin Assertion, da diese Analysen ohnehin nur auf assertive Außerungen angewendet werden. Damit erreiche ich eine einfache Dreiteilung: Behauptetes (Assertion) gegen Vorausgesetzes (Präsupposition) und gegen Gefolgertes (z.B. konversationelle Implikatur). Gerade we- 
gen dieser Vereinfachung muß man nachdrücklich darauf hinweisen, daß elne assertorische Äußerung nur eine unter zahlrelchen möglichen Sprechakttypen darstellt; sie besteht in der Behauptung des Inhaltes elner Proposition. Dieser Sprechakttyp kann (in einer expliziten Illokutionsformel) durch zahlreiche Verben ausgefiuhrt werden, z.B. behaupten, feststellen, versicherm, für wahr erklären usw., andererseits dient deren Semantik meist zur Beschreibung der Eigenschaften des Sprechakttypes.

Behauptung und Widerspruch, verteilt auf Sprecher und Hörer wie auch beschränkt auf eine Person, stellen eine sehr häufige Sprechhandlungssequenz dar. Sie kann zur Analyse der Semantik bestimmter Äußerungen verwendet werden, da z.B. von einem globalen Widerspruch ("Nein.") in der Regel eine Präsupposition nicht betroffen wird, hingegen mit Sicherheit die Assertion (genauer: die behauptete Proposition). Logische Folgerungen ('entailment') verhalten sich zwar unter Negation ebenso wie die Assertion, d.h. sie kehren sich in ihrem Wahrheitswert um, aber bei der globalen Zurückweisung werden sie nicht thematisiert. Damit besitzen wir ein dialogisches Instrument zur Trennung der Bedeutungskomponenten. Die gleichen Gesetzmäßigkeiten gelten bei Selbstkorrekturen. Die Schwierigkeit liegt. in jedem Fall bei der Explikation einer globalen Zurückweisung. - Ebenso kann ein Sprecher nicht gleichzeltig einen bestimmten Sachverhalt behaupten und verneinen, hier gilt allerdings das Gleiche für alle mit einer Äußerung "implizierten" Propositionen. Damit ist diese Konstellation ungeeignet zur Trennung der verschiedenen Bedeutungskomponenten, aber man kann mit ihr immerhin feststellen, ob eine bestimmte Proposition notwendiger Bestandteil der Interpretation einer bestimmten Äußerung ist oder nicht. Eine weitere Schwierigkeit der Bestimming von Bedeutungskomponenten liegt darin, daß sich mit einer oberflächenstrukturell einfachen Außerung mehr als eine Assertion verbinden kann, ${ }^{50}$ etwa durch die Auflösung eines kormlexen Prädikates. Für die Zweckmäßigkeit solcher Iösungen gilt es allerdings linguistische Grïnde zu finden.

Die Zuweisung einzelner Konstituenten zu Präsupposition oder Assertion, wie sie auch Chomsky's 'focus-presupposition'-Terminologie ${ }^{51}$ nahelegt, 1st nicht

50 Darauf weist u. a. auch Marga Reis (1974: 75) hin.

51 Darauf scheint sich D. Franck (1973: 21) zu beziehen, wenn sie 'Assertion' als denjenigen Teil der Satzbedeutung definiert, der im betonten Bereich des explizit Behaupteten steht; dieser folge logisch aus der Gesamtaussage. Allerdings sei die Grenze zwischen behauptetem und präsupponiertem Gehalt nur bei ganz einfachen Sätzen identisch mit der Grenze zwischen prädikativem und referentiellem Satzteil. 
möglich, und zwar auch dann, wenn man zuzugeben bereit ist, daß die TopicFocus-Gliederung des Satzes in bestimten Fällen die Wahrheitsbedingungen eines Satzes beeinflussen kann, also assertionsrelevant ist: zumindest dann, wenn man an dem Kriterium der Umkehrung des Wahrheitswertes unter intemer Negation festhält. Diese Negation betrifft jeweils nur den Focus einer Äußerung, womit sich bei einer Veränderung des Focus eine andere Assertion ergäbe:

(6) Deutsch spricht man in ös terreich.52 In Österreich spricht man $D e u t s c h$.

Aber in jedem Fall handelt es sich um vollständige Propositionen und nicht um einzelne Konstituenten.

Eine gewisse Sicherheit bei der Identifikation der Assertion bieten allein die oben bereits erwähnten monologischen und dialogischen Äußerungsfolgen, z.B. a. Die globale Verneinung einer Äußerung:

(7) A: Nur $H$ a n s ging weg. B: Nein. Auch K.a $r l$ ging weg.

Die Explikation des Nein fiuhrt mit einiger Sicherheit zur Assertion. Die Antwort (7a):

(7a) C: Nein. Hans ging nicht weg.

ist nur als expliziter (und nicht formgerechter) Präsuppositionsprotest zu werten. Das Nein genügt in diesem Falle nicht zur Verdeutlichung der Kommunikationsabsicht.

b. Die Ablehnung der Assertion durch den Sprecher selbst ist nicht möglich:

(8) A: "

c. Sçiließlich ist auch die Suspension der Assertion nicht möglich: ${ }^{53}$

(9) A: "Nur Hans ging weg, wenn es tatsächlich niemand sonst tât. / $\because \overline{\text { Aber }}$ möglicherweise hat es jemand anderer getan.

Diese Ansätze werden im Abschnitt 2.5.6. S.70 zu Tests systematisiert sowie deren Anwendung auf Gradpartikel-Sätze eingehend erläutert.

52 Siehe Sgall e.a. (1973: 132).

53 Siehe hierzu auch L. R. Horn (1972: 21). 


\subsubsection{Präsupposition}

2.5.4.1. Schon mehrmals wurde im Vorhergehenden der Terminus 'Präsupposition' erwähnt, für welchen Sachverhalt übrigens Strawson (1950: 330) und in seinem Gefolge auch andere englische Forscher 54 das Verbum "to imply" ("mitbehaupten" im Gegensatz zu "saying outright") verwendet. Selt L. Hor (1969) ist nicht nur die semantische Analyse von Gradpartikeln eng mit der PräsuppositionsProblematik verknüpft, die Gradpartikeln spielen auch umgekehrt eine nicht unbedeutende Rolle bei der Diskussion um die Integration dieses Konzeptes der formalen Logik in die linguistische Semantik. Eine verwertbare Darlegung würde das Eingehen auf zahlreiche Forschungsansätze bedingen. Ich befinde mich aber in der glücklichen Lage, mich in dieser Sache auf eine Untersuchung von Marga Reis (1975) beziehen zu können. Dort wird anhand zahlreicher Beispiele zu den unterschiedlichsten Präsuppositionstypen, die bislang in der Forschung behandelt wurden, der Nachweis zu führen versucht, daß es sprachliche Präsuppositionsgaranten im Sinne von logisch-semantischen Präsuppositionen (die also in allen denkbaren Kontexten konstante präsuppositionale Eigenschaften aufweisen) nicht gibt, daß vielmehr die präsuppositionalen Eigenschaften natürlichsprachlicher Ausdrücke wechseln, wenn auch zumeist durchaus regelmäßig, abhängig von Eigenschaften des Ko- wie Kontextes, und damit beschreibbar. Die u.a. auch von R. Hausser (1975) vertretene Ansicht, es gäbe diese Präsuppositions-Garanten ${ }^{55}$, und nur die bei ihnen auftretenden Folgerungs-Erscheinungen könnten, da auf sie allein die strenge logische Präsuppositions-Definition zutreffe, sinnvoll Präsuppositionen genannt werden, ist einerseits unnötig als Forderung für natürliche Sprachen, die ja bekanntlich vielfach ambig bzw. sensitiv für den pragmatischen Kontext sind, andererseits unumgänglich für (per definitionem) disambiguierte logisch-semantische Beschreibungssprachen. In bezug auf natürliche Sprachen wäre diese restriktive Definition m.E. unempirisch oder doch zumindest hinderlich für die Untersuchung präsuppositionaler Erscheinungen in Einzelsprachen.

Ich verstehe diese Untersuchung als einen Beitrag in der Diskussion um die geschilderten Forschungspositionen. Gradpartikeln bilden in dieser Hinsicht ein exzellentes Experimentierfeld, das beinahe alle denkbaren Konstellationen exemplifizieren kann.

\footnotetext{
54 Z.B. Max Black (1958; 1973: 63-66).

55 Wenn auch mit der Einschränkung der sogenannten präsuppositionstilgenden Kontexte, wozu intensionale Verben zu rechnen sind.
} 
2.5.4.2. Die Präsuppositions-Diskussion in der Logik befaßt sich selt Frege (1892; 1966) fast ausschließlich mit Existenz- bzw. Referenz-Präsuppositionen, die mit definiten Deskriptionen verbunden sind. Das Zutreffen der ExistenzPräsupposition ist nach Frege die Bedingung dafür, daß die Gesamtbehauptung, in der der präsupponierende Ausdruck enthalten ist, "Bedeutung" hat, also einen Wahrheitswert besitzt. Frege le1tet daraus die Forderung $a b$, daß in einer Begriffsschrift, wie er sich ausdrückt, jeder Eigenname eine Referenz, elne Bedeutung haben müsse: daß es sich also bei einem Logikkalkül urn eine in präsuppositionaler Hinsicht disambiguierte Sprache handeln müsse. Dies könnte jedoch nur über die explizite Behauptung der Existenz wie der Einzigkeit und die anschließende Interpretation (= Wahrheitswertzuteilung) für diese Behauptungen geschehen, würde also der von Russell vorgeschlagenen Iösung sehr nahe kommen. Sätze, die einen Eigennamen oder eine Kennzeichnung ohne Bedeutung (= ohne Referenten) enthalten, hält Frege für weder wahr noch falsch, er würde ihnen vielmehr jede Bedeutung (= Wahrheitswert) absprechen. Dies kann sicherlich nicht im Sinne eines dritten Wahrheitswertes 'unentschieden' oder 'unentscheidbar' gedeutet werden, eher im Sinne von 'nicht formgerecht'.

Russell's klassischen Beitrag zur Präsuppositions-Thematik erwähne ich hier nur deshalb, weil sein früher Versuch einer formalen Lösung des PräsuppositionsProblems frappierende Ähnlichkeiten mit dem 'factoring' bei Gradpartikeln aufweist. - Die Präsuppositions-Problematik glaubt Russell (1905; 1969. Femer 1957; 1969) dadurch lösen zu können, daß er bezeichnende Ausdrücke in Subjektsfunktion bei der Formalisierung in mehrere Propositionsfunktionen auflöst, die als Subjekt jeweils die Variable $\mathrm{x}$ enthalten, und zwar:

a. die Existenzbehauptung:

(10a) Es gibt etwas, das König von F rankreich ist.

b. die Einzigkeitsbehauptung:

(10b) Es gibt nur ein etwas, das König von F rankreich ist.

Diese einem Subjektsausdruck entsprechenden Teilbehauptungen werden untereinander und mit der ursprünglichen Behauptung durch die logische Konjunktion verbunden. Die Folge davon ist, daß die Gesamtaussage falsch wird, wenn ein Teilglied, z.B. die Existenzbehauptung, den Wahrheitswert 'falsch' erhält. Damit wird die Einführung eines dritten Wahrheitswertes umgangen. Dies ist eine

56 Bei dem Terminus 'Präsupposition' dürfte es sich übrigens um die engl. Übersetzung des bei Frege noch eher "umgangssprachlich" benutzten "Voraussetzung" handeln. Ein Rückgriff auf diesen deutschen Ausdruck ist nicht mehr sinnvoll, nachdem sich 'Präsupposition' bereits durchgesetzt hat. 
technisch elegante Lösung, doch bewirkt sie bei natürlichen Sprachen einige Erklärungslücken. Wie D. Franck (1973: 19) feststellt, sind Existenz- und Einzigkeitsbehauptung zusammen noch keine adäquate Explikation der Sprechhandlung des Referierens, höchstens die notwendige Voraussetzung für das Gelingen dieser Sprechhandlung. Dies betrifft insbesondere einen pragmatischen Unterschied, der der spezifischen Erleichterung der nommalsprachlichen Kommunikation dient: Existenz und Einzigkeit gelten bei der Handlung des Referierens mit allgemein akzeptierten sprachlichen Mitteln als geprüft. Dadurch fällt die Notwendigkeit, sich hierüber erst noch zu einigen, in der Regel weg. - Damit hängt ein weiterer Einwand aus pragmatischer Sicht zusammen: die einzelnen Teilbehauptungen haben einen unterschiedlichen Status, eine verschiedene Funktion; dieser Unterschied wird durch die formale Gleichbehandlung, betont durch die logische Konjunktion, verwischt.

Und schließlich der entscheidende Einwand: scheitem bei der Russell'schen Formalisierung Existenz- oder Einzigkeitsbehauptung, so erhält die gesamte Behauptung den Wahrheitswert 'falsch'. Scheitert hingegen die Existenz- bzw. Referenz-Präsupposition in einer Behauptung einer natürlichen Sprache, so wird sie nicht als falsch, sondern als nicht wahrheitswert-fähig, als 'bedeutungslos' bewertet. Damit ist aber der Russell'sche Kalkül nicht mehr zur Beschreibung natürlicher Sprachen geeignet. - Soweit bei der semantischen Analyse von Gradpartikeln das 'factoring' in gleicher Weise angewendet wird, d.h. wenn gleichwertige Teilbehauptungen durch die logische Konjunktion verbunden werden, gelten dafür die gleichen Einwände. Hinzu kommt, daß in gewissen Fällen, nämlich bei auch und sogar unter bestimmten Voraussetzungen, das Zutreffen der als Präsupposition bezeichneten Teilbehauptung nicht zwingende Voraussetzung für Wahrheit oder Falschheit, der Äußerung ist; der Gradpartikel-Satz wird in diesen Fällen beim Nichtzutreffen der Präsupposition lediglich eigenartig. In der 'factoring'-Explikation hingegen würde er den Wahrheitswert 'falsch' erhalten. Schon aus diesem Grund kann eine derartige Beschreibung nicht befriedigen.

2.5.4.3. 'Präsupposition' wie oben dargestellt ist also zunächst ein Thema der klassischen Logik, die nur Äußerungen assertorischen Charakters untersucht. Das Kemproblem ist dabei die Zuteilung eines von zwei möglichen Wahrheitswerten an Behauptungen. Die Referenz von definiten Kennzeichnungen in Subjektsposition bzw. -funktion bildet hierbei ein entscheidendes Problem: $w$ und $f$ prädizieren nämlich nur das Zutreffen eines bestimmten Prädikats auf den jeweiligen Subjektstem; dessen Gultigkeit, d.h. die Tatsache, daß es tat- 
sächlich etwas gibt, worauf mit diesem Ausdruck verwiesen werden kann, ist damit stillschweigende Voraussetzung für die Ertellung eines Wahrheitswertes.

Die Frage des Wahrheitswertes von Behauptungen ist also von Anfang an die Kemfrage der Präsuppositionsproblematik: wie missen elementare Aussagen intern beschaffen sein, damit ihnen ein Wahrheitswert zuerkannt werden kann?

Mit der Definition der Wahrheitswerte ist die Frage der Negation verknüpft, definieren sie sich doch meist gegenseitig. Damit ist dann auch von dieser Seite der Anschluß zur Präsuppositions-Definition gegeben, daß die (starke!) Negation einer Behauptung, die den Wahrheitswert von Assertion wie logischen Folgerungen ins Gegenteil verkehrt, nicht die Präsuppositionen dieser Behauptung berïhrt. Es ist zu klären, inwieweit diese Eigenschaft essentiell mit der Präsuppositionsproblematik verbunden ist, und ob - und wenn ja, welche - Erweiterungen für jene Fälle möglich sind, bei denen dieses Negationskriterium nicht oder nur schwer anzuwenden ist, etwa bei nichtassertorischen Äußerungen sowie bei Sätzen mit Modalpartikeln oder Gradpartikeln. Man sollte dabei allerdings nicht ibbersehen, worauf u.a. auch Cohen (1973: 30) hinweist, daß bei einer Präsuppositions-Definition allein anhand von Wahrheitswerten bestimmter Propositionen die eigentliche semantische Verbindung zwischen einer Assertion und ihren Präsuppositionen vernachlässigt wird, so daß man behaupten könnte, jeder beliebige Satz präsupponiere alle Tautologien. 57

Nach Strawson (1970: 74) haben nur Äußerungen, nicht Sätze oder Satzformeln einen Wahrheitswert: "... it is not the invariant type-sentences themselves that are naturally said to be true or false, but rather the systematically varying things that people say, the propositions, they express, when they utter those sentences on different occasions." - Als Basis für die Zuteilung eines bestimmten Wahrheitswertes an eine Äußerung dient üblicherweise Tarski's Konvention für den Wahrheitsbegriff: " $\mathrm{X}$ ist wahr genau dann, wenn $\mathrm{p}$ " (Tarski 1944; 1972: 59). Demnach mu eine Wahrheitstheorie entwickelt werden, die aus einer Menge von Axiomen besteht: aus ihnen folgt zu jedem Satz eine Aussage, die dem Satz die Bedingungen zuordnet, unter denen er wahr ist, und zwar immer in Begriffen der jeweils analysierten Sprache. 'wahr' und 'falsch' sind metasprachliche Prädikate für Sätze/Assertionen der Objektsprache. - Es kormt also jeweils darauf an, für eine offene Satzformel die möglichen Variablenbewertungen festzulegen, also diejenigen Element-n-Tupel, welche eine bestimmte Satzformel "erfüllen" können: dieser Begriff der "Erfüllung" ist der Kembegriff der Wahrheitstheorie von Tarski. Mit ihm werden die Wahrheitswerte als Desig-

57 Eine Möglichkeit zur Vermeidung dieses Problems bietet Hausser (1975: 8). 
nate von Sätzen zu mengentheoretischen Entitäten. Auf diesen Begriff der Erfüllung baut eine weitere interne Definition der Wahrheitswerte auf, sie ist nicht unabhängig denkbar: eine wahre Behauptung erhält, wenn verneint, den Wert 'falsch'; eine falsche Behauptung erhält durch ihre Negierung den wert 'wahr'. Dies scheint nur die zwei klassischen Wahrheitswerte zuzulassen, doch bleiben einige Fragen zunächst offen: was geschieht, wenn ein Subjektsterm keinen Referenten besitzt? Oder wenn Unsicherheit besteht, ob ein bestimmtes Prädikat auf einen bestimmten Subjektsterm zutrifft? Und schließlich muß noch festgelegt werden, was als die Verneinung eines Satzes zu gelten hat.

Die Frage nach der Form der Vemeinung ist für die klassische Logik unerheblich: geht man von nur zwei Wahrheitswerten aus, so erhält man sowohl bei der starken wie bei der schwachen Vermeinung jeweils den entgegengesetzten Wahrheitswert. Doch entspricht dies nicht den erkennbaren Unterschieden im Gebrauch der verschiedenen Negationsarten in natürlichen Sprachen, außerdem wird auf diese Weise eine adäquate Behandlung der Präsuppositionsproblematik unmöglich. Den Ansatz zur Lösung der Frage bietet Schnelle (1973: 186 f.) mit seinem Hinweis, daß Tarski's Wahrheitsdefinition nicht in direkter Weise widerspruchsfrei auf natürliche Gemeinsprachen übertragbar sei, da diese Vagheiten und Mehrdeutigkeiten aufweisen. Demgegenuiber vertritt U. Blau (1973/74: Kap. 1.2.) mit überzeugenden Argumenten den Standpunkt, daß zumindest zwei Vagheitsgründe, nämlich die beiden oben zuerst genannten, in keiner Sprache, also auch nicht in einer Standardsprachform, vermeidbar seien. Daher komme es, daß Sätze zwar nur auf eine Weise wahr, aber auf sehr verschiedene Weise nicht wahr sein könnten. Den dieser Beobachtung zugrundeliegenden Sprachgebrauch verdeutlicht Horn (1972: 9), der eine ganz ähnliche Argumentation verfolgt, mit folgendem Diagramm:

\begin{tabular}{|c|c|c|}
\hline wahr & \multicolumn{2}{|c|}{ nicht wahr } \\
\hline w & $\mathrm{u}$ & $\mathrm{f}$ \\
\hline nicht falsch & falsch \\
\hline
\end{tabular}

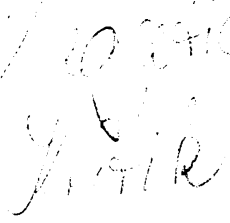

Mit den drei Wahrheitswerten $w$, f und u lassen sich nun auch die Unterschiede zwischen starker und schwacher Negation darstellen (Blau 1973/74:72):

\begin{tabular}{c|c|c}
$p$ & $-p$ & $7 p$ \\
\hline$w$ & $f$ & $f$ \\
$f$ & $w$ & $w$ \\
$u$ & $u$ & $w$
\end{tabular}


Während die starke, "präsupponierende" Negation die Unbestimmtheitsgründe unberührt läßt, führt die schwache, externe Negation bei Anwendung auf unbestimmte Sätze zum Wahrheitswert 'wahr'. Das zweiwertige Wahrheitswertsystem ist also nur mit dieser Negation zu retten.

Damit befinden wir uns bereits in einer günstigen Ausgangsposition für die Beschreibung natürlicher Sprachen. Es bedarf jedoch noch, um die verschiedenen Formen der Präsupposition und Vagheit erfassen zu können, einer Ausdehnung nach zwei Richtungen: eine Wortsemantik mu in die Beschreibung eirbezogen werden, und wir müssen ein Bewertungskriterium für nichtassertorische Sätze finden. Denn für Sprechhandlungen wie Aufforderung, Befehl, Frage usw. sind 'wahr' und 'falsch' wie 'unbestimmt' keine adäquaten Bewertungskriterien. Sie wären vielleicht gegeben mit der Ausformulierung der "Bedingungen des Glückens" von Sprechhandlungen, unter die sicherlich auch assertorische Äußerungen subsumiert werden könnten. Es mïßten also jeweils die Regeln oder Bedingungen spezifiziert werden, die bei der Benützung bestimmter Sprechhandlungen oder syntaktischer Konstruktionen oder Lexeme $z u$ beachten bzw. zu erfüllen sind, damit pragmatisch, syntaktisch und semantisch wohlgeformte Äußerungen entstehen. - Also eine Erweiterung des Tarski-Kriteriums der 'Erfülllung'. Auch Präsuppositionen wären dann als Bedingungen für das 'Glücken' bestimmter Sprechhandlungen zu betrachten. Zwei Nachteile hat aber diese Ausdehnung: Einmal muß man mit mehreren TYpen von Anwendungsbedingungen rechnen, muß also von neuem mit der Aufgliederung beginnen. Zum anderen würde das Verifikationsverfahren für die Wohlgeformtheit von Äußerungen bezüglich einer Anwendungssituation in die Nähe der Beurteilung der Akzeptabilität rücken, wäre also noch sehr viel vager als das zugegebenemaßen schwer zu handhabende Negationskriterium.

2.5.4.4. Im Gegensatz zu der Mehrzahl der Logiker beschäftigen sich Linguisten nicht nur mit dem Sprechhandlungstyp 'Behaupten', sondern mit allen nur denkbaren Formen sprachlichen Handelns; sie widmen sich ferner nicht nur definiten Kennzeichnumgen und den mit ihnen verbundenen Existenz-Präsuppositionen, sondem zahlreichen weiteren präsuppositionsähnlichen Erscheinungen, die keineswegs so konstante Eigenschaften aufweisen. Damit wird eine Adaption der PräsuppositionsDefinition an linguistische Bedirfnisse notwendig, wie sie z. B. Ch. Fillmore (1971b: 380), J. Bellert (1971; 1973: 221-239), L. Karttunen (1972: 247), Urs Egli (1971:75), D. Wunderlich (1973: 472) und H. Ebert (1973: 423) versuchten. In der Auseinandersetzung mit diesen linguistischen Präsuppositionsdefinitionen lassen sich einige wichtige Grundlinien entwickeln: 
1. Zur Präsuppositionsrelation: Bei den genannten Forschern ist die Präsuppositions-Relation kein logischer Junktor und damit auch keine diesen Junktor definierende Wahrheitsfunktion mehr, ebensowenig eine Relation zwischen logischen Formen von Sätzen/Propositionen/Sprechakten; eher eine intuitive Folgeoder Bedingungsbeziehung zwischen wahren Aussagen, bzw. genereller formuliert, zwischen angemessenen Äußerungen. Bei Ebert (1973) wird sie als Relation iberhaupt nicht mehr erwähnt.

2. Zum Vor und Nachbereich dieser Relation: was im Vor- und Nachbereich der Präsuppositionsrelation stehen soll, ist aus den linguistischen Definitionen nur annähernd zu erschließen; im Vorbereich (= präsupponierende Elnheit) bei Irena Bellert eine nicht weiter spezifizierte "Äußerung", bei Karttunen wohl eine sprechaktunabhängige Proposition P; bei Egli bleibt unklar, ob er mit s eine Proposition oder deren Behauptung meint; bei Wunderlich ist es die Äußerung eines Satzes s. In der Definition von Ebert schließlich ist der jeweilige Sprecher Träger von Präsuppositionen. Letzteres ist nun sicherlich eine plausible (wenn nicht triviale) Erklärung. Abgesehen davon, daß eine Handlungstheorie ohne Zweifel nützlich und notwendig für die Erklärung und Beschreibung sprachlicher Handlungen ist, sollte man bei der rein linguistischen Analyse sprachlicher Gesetzmäßigkeiten nicht vorschnell kapitulieren. Wenn Voraussetzungen in "die Formulierung einer Mitteilung eingehen", so kommt es darauf an, die sprachlichen Indikatoren dieser Voraussetzungen herauszufinden und zu beschreiben. Es scheint mir nötig, auch im Bezug auf präsupponierende sprachliche Einheiten, daran festzuhalten, daß regelgeleitetes sprachliches Handeln und nur solches ist Gegenstand linguistischer Beschreibung - qua Regel unabhängig von einzelnen Sprechern und Hörern existiert und beschrieben werden kann. - Ich möchte demgegenuiber daran festhalten, daß man fuir den Vorbereich der Präsuppositionsrelation ausgeht von formallogischen Usus, nur Behauptungen als wahrheitswertfähige Gebilde zuzulassen. Dies muß natürlich für linguistische Zwecke erweitert werden auf Äußerungen (also beliebige Sprechakttypen), die denselben propositionalen Kern aufweisen.

Als Nachbereich, d.h. als Präsupponiertes (oft auch nur abgekürzt 'Präsupposition' genannt) treten bei Irena Bellert als wahr geltende Konsequenzen auf, also wohl Behauptungen; bei Karttunen wird über die Natur des Nachbereiches $Q$ nur gesagt, daß er vom Sprecher geglaubt werden maß (also wohl auch eine Behauptung); um Behauptungen muß es sich auch bei U. Egli handeln, da man wohl nur sie bejahen und verneinen kann. Wunderlich nennt sie Voraussetzungen des Sprechers, die aber der Hörer nach grammatischen Regeln aus der Äußerung des Sprechers rekonstruieren können muß, und die der Sprecher in Behauptungssätzen 
explizieren können muß (was zumindest eine ungliuckliche Formulierung ist). Bei Ebert schließlich ist nur mehr recht vage von Urteilen des Sprechers die Rede. Wiederum scheint es mir geraten, ja unumgänglich, sich an der formallogischen Analyse zu orientieren: danach miissen, Strawsons Axiom vorausgesetzt, auch im Nachbereich wahrheitswertfähige Gebilde, also Behauptungen stehen. Daran ändert sich auch nichts, wenn im Vorbereich beliebige Sprechakte mit identischem propositionalem Kem stehen, da wir ja wohl die Sprechaktneutralität der Präsuppositionsrelation beibehalten wollen. Diese Regelung ist zugegebenermaßen nicht ganz problemlos; denn in der Oberflächenstruktur von Sätzen ist gewöhnlich nur die Assertion explizit vorhanden. ${ }^{58}$ Auch bei der überführung in eine Explizitfassung und bei deren anschließender Formalisierung wird nur die Assertion in ihrer Struktur sichtbar gemacht. Das Präsupponierte ist zumeist nur erschließbar, implizit vorhanden. Wenn aber das Präsupponierte, wenigstens der Struktur nach, als Behauptung zu repräsentieren ist, ergibt sich in praktisch allen Fällen das Problem, dafür eine verbindliche (begründbare) Formulierung zu finden; dies ist insbesondere bei recht vagen "Erwartungs-Voraussetzungen" wie. etwa bei sogar ein fast aussichtsloses Unterfangen. - Natülich hat Ebert recht (und sie trifft sich darin mit D. Franck 1973: 19), wenn sie darauf verweist, daß eine explizite Behauptung keine angemessene Darstellung der spezifischen Eigenschaften und Funktionen von Präsupponiertem ist. Andererseits scheint es bis jetzt keinerlei verwertbare Hinweise darauf zu geben, daß nicht alles, was als Präsupponiertes auftritt, auch assertiert werden kann. Kategoriale oder konzeptuelle Unterschiede zwischen Assertion und Präsupponiertem sind also nicht zu erwarten.

2.5.4.5. Damit sind aber zunächst nur Formalia abgeklärt. Der Kern der Diskussion, nämlich die Qualität der Präsuppositionsrelation, wurde nur gestreift: nämlich, gibt es sprachliche Indikatoren für diese Präsuppositionen, die unter allen denkbaren Umständen stets dieselben Präsuppositionen auslösen, und sind diese Präsuppositionen (per definitionem) stets und unter allen Umständen Bedingungen für Wahrheit oder Falschheit der sie auslösenden Äußerungen? Bejaht man alle diese Fragen, so hat man einen strengen logisch-semantischen Präsuppositionsbegriff formuliert. - In dieser Fonm wird aber die Definition sicherlich von keinem Sprachwissenschaftler mehr für die Untersuchung natürlicher

58 Ein Gegenbeispiel stellen Sätze mit nur in Gradpartikel-Funktion dar. Sie enthalten eine explizite Präsuppositions-Formulierung, die Assertion muß erschlossen werden. 
Sprachen aufrechterhalten, allein schon wegen der intensionalen Kontexte, die eine Relativierung der sprachlichen Gesetzmäßigkeiten erzwingen. Aber auch im Blick auf die übrigen Gesetzmäßigkeiten bei natürlichen Sprachen ist die Annahme stabiler Präsuppositions-Garanten zwar nicht unmöglich, aber doch unwahrscheinlich; man denke etwa an die Polysemie von Lexemen, an syntaktische Ambiguitäten, an die kontextuell variierenden Bedeutungen von Lexemen und Konstruktlonen. Die Definition wäre außerdem viel zu eng etwa für "PräsuppositionsGaranten", die wie die Gradpartikeln ihre Präsuppositionen erst im Zusammenwirken mit anderen sprachlichen Einheiten (ihren Skopuskonstituenten) konkretisieren können. - Bleibt als letzte Barriere die Festlegung, daß die Gültigkeit des Präsupponierten Voraussetzung für die Zuteilung eines Wahrheitswertes an die auslösende Äußerung ist. Wie weit diese Forderung (abgesehen von der Modifikation für nichtassertorische Äußerungen) variiert werden kann und muß, miissen erst weitere Untersuchungen ergeben.

Für eine logische Kalkülsprache hingegen bleibt die Definition, vielleicht mit geringfügigen Modifikationen, sinnvoll. Hier ist es tatsächlich notwendig, daß Präsuppositions-Garanten eindeutig markiert sind, und daß ihre präsuppositionalen Eigenschaften ganz stabil sind, auch mit Bezug auf die generell als präsuppositionstilgend beschriebenen Kontexte.

So sind die oben dargestellten Modifikationen der Präsuppositions-Definition durch Linguisten unnötig für die Beschreibung in formalen Sprachen, aber auch unnötig für die Untersuchung natürlicher Sprachen. Es geht ja nicht darum, die Definitionen so auszudehnen, bis alle Zufälligkeiten, alle denkbaren pragmatischen Faktoren, alle Idiosynkrasien mit erfaßt sind. Vielmehr kommt es darauf an, herauszufinden, unter welchen regulären Bedingungen welche sprachlichen Erscheinungen welche Präsuppositionen auslösen, und gegebenenfalls die Abweichungen durch zusätzliche GesetzmäBigkeiten zu erklären.

Ich ziehe also einen scharfen Trennungsstrich zwischen der formalen Expl1kation präsuppositionaler Erscheinungen in Logikkalkuilen (und zwar auch, soweit sie für die Beschreibung natürlicher Sprachen konzipiert sind) und der für die Zwecke der Analyse präsuppositionaler Erscheinungen in natürlichen Sprachen notwendigen Präsuppositionsdefinition. Während im ersten Fall die strenge Fassung erforderlich ist mit den Faktoren: eindeutige Zuordnung zwischen Präsuppositionsgaranten und Präsupponiertem, stabile Präsuppositions-Formulierung, Voraussetzung für die Zuteilung eines Wahrheitswertes und damit zusammenhängend Gultigkeit des Negationskriteriums, um die formale Beschreibbarkeit zu sichern muß für präsuppositionale Erscheinungen in natürlichen Sprachen, die in mannigfaltiger Hinsicht ambig sind, mit einer kamplizierteren Sachlage gerechnet 
werden: keine stabile Verbindung sprachlicher Elemente mit präsuppositionalen Erscheinungen, Abhängigkeit vom verbalen Kontext, fehlende Anwendbarkeit des Wahrheitskriteriums sowie des Negationskriteriums bzw. Zwang zur Modifikation dieser Kriterien, wechselnde "Füllung" der von einem Element ausgelösten Präsuppositionen. Trotz dieser Modifikationen, die ja nicht den Kern des Präsuppositionskonzeptes antasten, ist der übergang von den logisch-semantischen zu den traditionell als "pragmatisch" bezeichneten Präsuppositionen nicht leichter geworden. Als unterscheidendes Merkmal gilt gewöhnlich die Tatsache, daß die "Füllung" der von einem Element ausgelösten Präsupposition wechseln kann. Hierfür wären gerade die Gradpartikeln ein geeignetes Demonstrationsobjekt: wechselt doch die Präsuppositionsformulierung sowohl mit dem jeweiligen Skopus als auch mit dem Interpretationstyp. Letzteres läßt möglicherweise nur den (nicht sehr befriedigenden) Ausweg der Polysemierung, es sei denn, man betrachtet eine Interpretationsart nur als durch pragmatische Faktoren verursachte "engere" Auslegung. Ersteres hingegen ist kein echtes Gegenargument: zur Präsupposition gehört bei den Gradpartikeln nur die lexematisch nicht gefüllte Quantifizierungsformel, etwa in der Art von Bartsch (1972:195). Dies ist nicht grundsätzlich verschieden von der Situation bei Referenz-Präsuppositionen.

Schwieriger ist die Sachlage bei den von U. Blau (1973/74:51f.) behandelten Beispiele, die man auf Anhieb für Fälle von logischen Präsuppositionen halten wïrde, deren Interpretationen aber durchaus von den pragmatischen Gegebenheiten abhängen kann: so würde man für a freut sich, $b$ zu sehen als Präsupposition ansetzen: $a$ = Lebewesen; für a ist schwanger als Präsupposition ansetzen: $a$ = weiblich; für Jakob ist von München nach Frankfurt geflogen als Präsupposition ansetzen: Jakob = Mensch. In allen drei Fällen kann man jedoch bei Veränderung der pragmatischen Bedingungen ("sehender" Computer, der "Gefühle" ausdrücken kann; künstlicher Uterus; nur Vögel können fliegen) auch andere Präsuppositionen ansetzen. - Diese Argumentation kann aber in zweifacher Weise durchkreuzt werden: entweder hat man es mit unterschiedlichen Sprachsystemen (und damit auch Verwendungssituationen) zu tun, die nicht in dieser Weise verglichen werden diirfen, oder aber man hat es mit nur erdachten welten zu tun, also den bekannten Fall des präsuppositionstilgenden Kontextes vor sich. Ein gewisses, linguistisch irrelevantes Variieren mit Ko- und Kontext ist aber wohl ebenso einzuschätzen wie das Variieren assertierter Bedeutung im Zusammenhang mit "Weltwissen".

Bleibt noch die von K. Ebert (1973: $423 \mathrm{ff.}$ ) angesetzte Unterscheidung von Logischen Präsuppositionen als "notwendigen Voraussetzungen für das Verstehen 
und die Beurteilung eines Sprechaktes", und pragmatischen Präsuppositionen als "Annahmen des Sprechers in bezug auf das Wissen des Hörers, die in der sprachlichen Formulierung ihren Niederschlag findet". Letztere werden wieder aufgespalten in starke pragmatische Präsuppositionen als "echte Voraussetzungen" für den Hörer ("Annahme der Bekanntheit und Identifizierbarkeit von Referenten") und schwache pragmatische Präsuppositionen als "Annahme der Bekanntheit bestimmter Sachverhalte, die z.T. aus dem Text ableitbar, zum Teil für das Verständnis nicht wichtig sind". Das Prinzip "Voraussetzung für das Verstehen elnes Sprechaktes" ist nicht so weit von dem Kriterium 'Bedingung für einen Wahrheitswert' (= Designat eines Satzes) entfernt, wie das vielleicht scheinen mag. Zwelerlei ist dazu festzustellen: einmal beruhen die Unterschlede in dieser Hinsicht bei den von K. Ebert angefiuhrten Beispielen (darunter ein auch-Satz) wohl in erster Linie auf der Kontextlosigkeit bzw. auf dem Charakter als isoliertes linguistisches Beispielsmaterial. Im Dialog ist nach meinen Beobachtungen der Grad der Beunruhigung des Hörers bei nicht verifizierbaren Gradpartikel-Präsuppositionen nicht geringer als bei nicht verifizierbaren Referenz-Präsuppositionen usw. Zum zweiten wird keinerlei Operationalisierung für diese Bestimmung angegeben, eine Tatsache, die aber auch fuir den Wahrheitswert gilt (jedenfalls bei der Analyse konkreter Äußerungen bezüglich einer Situation). Ich habe auf der Suche nach einem operationalen Kriterium einen Typ von dialogischem Text ausgemacht, der sich vielleicht dazu eignet. Er besteht in folgender Sequenz:

A: Gradpartikel-Satz.

B: Zurückweisung der vermutlichen Präsupposition verbunden mit Zustimmung zur wahrscheinlichen Assertion.

Oder: Zustimmung zur Präsupposition verbunden mit Zurückweisung der Assertion.

Gerade der erste Fall mïßte qua Präsuppositions-Definition klare Inakzeptabilität verursachen, also eine echte Testsituation zulassen. Die Urteile kann man nun tatsächlich als sehr sicher bezeichnen, wozu wohl auch die Sequenzierung ihr Teil leistet. Das Beunruhigende daran ist, daß die Ergebnisse selbst bei denselben Gradpartikeln variieren. Die intuitiv erkennbare Regelmäßigkeit des Variierens konnte ich nicht explizieren. Der Leser muß daher zu den einzelnen Analysen verwiesen werden. - In jedem Falle kann man vorläufig festhalten, daß eine derartige Unterteilung von Präsuppositionen kaum sinnvoll ist, solange nicht die empirische Relevanz erwiesen ist. Dieser Nachweis aber ist erst möglich, wenn man ibber entsprechende operationale Hilfsmittel verfügt. 


\subsubsection{Konversationelle Implikatur}

2.5.5.1. Ein weiterer Folgerungstyp, der bei der semantischen Analyse von Gradpartikeln, genauer bei einer der dabei auftretenden Interpretationsarten, eine nicht unbedeutende Rolle spielt, ist die konversationelle Implikatur ('conversational implicature'). Sie wird erklärt durch die von Griœ erarbeiteten "Konversationsmaximen", die ijber diesen Spezialfall hinaus mehrfach bei der Beschreibung und Erklärung von Eigenschaften von Gradpartikel-Sätzen gute Dienste leisten. Deshalb zunächst eine knappe Zusammenfassung dieser Maximen und erst im Anschluß daran die Darlegung von zwei Typen von Implikaturen.

2.5.5.2. Griœe wollte mit der Formulierung von Konversationsmaximen ${ }^{59}$ nach eigener Deutung die Enge der Searle'schen Bedingungen: keine Behauptung ohne Behauptbarkeit; keine Bemerkung, ohne daß etwas bemerkenswert ist - iberwinden und erklären, durch welche Regeln ein effektiver Informationsaustausch sowie die Beeinflussung und Lenkung der Handlung anderer gesichert werden. Diese Regeln betreffen nicht nur den Gebrauch einiger sprachlicher Einheiten wie der performativen Verben. Sie sind dariber hinaus geeignet, die spezifischen Unterschiede zwischen einer formalen Logik und einer Logik der natürlichen Sprachen sichtbar zu machen. Dies betrifft insbesondere die Diskussion um die sogenannten Trugschlüsse ('fallacies'). 60

1. Das Kooperationsprinzip: Es besagt, daß jeder Teilnehmer an einem Gespräch seine Diskussionsbeiträge so gestalten muß, wie man das vernünftigerweise von ihm erwarten kann. Auf die Erfiullung dieses allgemeinsten Prinzips bauen alle ibrigen Maximen sowie alle Schlußfolgerungen (die konversationellen Implikaturen) auf. Insofem kann man dieses Prinzip den Maximen nur überordnen, aber nicht beiordnen.

2. Die Maxime der Quantität. Nach Griœ besteht sie aus zwei Teilen: ein Beitrag muß so informativ wie gefordert sein; er soll nicht informativer als gefordert sein. - Der zweite Teil ist nicht nur, wie Griœe meint, weniger wichtig, sondem überhaupt überflüssig, da seine Aussage in der ersten Teilregel bereits enthalten ist, wenn man sie als "genau so informativ wie gefordert" versteht. - Auf diese Maxime bezieht sich der für uns interessante Typ der

59 Grice (1968: Lecture II \& III). Siehe zu diesem Thema auch: Gordon \& Lakoff (1971); Robin Lakoff (1972 und 1973); L. Horn (1972 und 1973); Don Larkin \& M.H. O'Malley (1973); J. Murphy (1975); D. Wunderlich (1972: 54-58).

60 Siehe hierzu Geis \& Zwicky (1971). 
konversationellen Implikatur: sie besagt etwa für Skalen, daß man jeweils den höchsten möglichen Wert, wobei man auf die "Richtung" der Skala zu achten hat, angeben muß. Diese Grenzmarkierung kann allerdings bei Unsicherheit über den noch möglichen Grad mit bestimmten Formulierungen suspendiert ('cancel') werden. 61

3. Die Maxime der Qualität. Sie besteht aus der Supemaxime: Der Beitrag zu einem Gespräch soll wahr sein - und zwei untergeordneten Teilmaximen: man soll nichts sagen, was man für falsch hält; man soll nichts sagen, wofür man nicht hinreichende Evidenz geltend machen kann. - Diese Maxime entspricht weitgehend der Searle'schen Aufrichtigkeitsbedingung.

4. Die Maxime der Beziehung ('relation'). Sie lautet bündig: sei relevant in deinen Beiträgen. - Auch diese Maxime ist die Basis für einen bestimmten Typ von Implikaturen: sie emöglicht bei Mitteilungen ïber längst bekannte Tatsachen den Schluß, daß die Mitteilungsabsicht notwendig eine andere sein muß als der wörtliche, kognitive Inhalt der Äußerung, sle muß also erst erscilossen werden: sicherlich ein unlösbares Problem für eine reine Sprechakttheorie. Andererseits kann man mit Hinweis auf diese Maxime die Angriffe von Larkin und O'Malley (1973) zurüdkweisen. Sie führen gegen die Konversationspostulate, die sie für zu stark informationstheoretisch orientiert erklären, ins Feld, daß sie die in der Alltagssprache häufigen, oberflächlich deklarativen, tatsächlich aber nicht-informativen Äußerungen nicht erfassen könnten. Gerade die Maxime der Relation erklärt sie aber: da diese Äußerungen für jeden erkennbar nichtinformativ sind, ist der Schluß auf andere Funktionen, die von den beiden Verfassern selbst reichlich aufgezählt werden (Vorwurf, Aufmerksammachen, Drohung, Ausdruck von Mitgefühl und Solidarität usw.) für jeden Hörer zwingend.

5. Die Maxime der Art und Weise. Sie kann eine ganze Reihe von Teilmaximen umfassen: vermeide Verworrenheit des Ausdrucks; vermeide Ambiguitäten; sei knapp im Ausdruck; sei präzise etc. Auch nach Auffassung von Grice handelt es sich hierbei um sehr viel schwächere Konventionen, denen auch ästhetische, soziale und moralische Maximen beigefügt werden könnten. Von ihnen ist deshalb auch keine sehr große Reichweite zu erwarten.

2.5.5.3. Interessant für die Analyse sprachlicher Handlungen sind die verschiedenen (zum Teil schon kurz angesprochenen) Möglichkeiten, die oben genannten Maximen zu verletzen: Der Sprecher kann sich z.B. weigern, zu kooperieren; damit ist eine Verständigung von vornherein unmöglich. - Der Sprecher kann in

61 Vergleiche dazu den Abschnitt über den Suspensionstest 2.5.6.3. S. 80ff. 
einen Normenkonflikt geraten, etwa zwischen der Maxime der Qualität und derjenigen der Relevanz. Dem Hörer kann dies durch die eindeutige Verletzung der jeweils für weniger relevant gehaltenen Maxime signalisiert werden. - Daneben gibt es natürlich auch den Fall der bewußten Irreführung: sie besteht in der stillschweigenden Verletzung von Konversationspostulaten und wird erst durch die Erwartung der Befolgung aller Konversationsmaximen möglich.

Theoretisch interessanter ist es, wenn ein Sprecher durch die bewußte und erkennbare Verletzung einer Maxime dem Hörer etwas nahelegen will, den eigentlichen Fall der konversationellen Implikatur, die zu den nichtkonventionellen Implikaturen zählt. Sie beruht auf der vom Hörer zu lösenden Fragestellung: wie kann die Tatsache, daß der Gesprächspartner das gesagt hat, was er gesagt hat, vereinbart werden mit der Annahme, daß er sich kooperationsbereit, also bereit zur Einhaltung der Konversationsmaximen, gezeigt hat? - Als Beispiel führt Grice (1968: II, Seite 4) an: A und B unterhalten sich über C, einen Bankangestellten. Auf die Frage von A, wie es C gehe, antwortet B:

(11) B: Oh, quite well, I think; he likes his colleagues, and he hasn't been to prison yet.

Das, was B mit dieser Äußerung meinte ('imply', 'suggest') ist verschieden von dem, was er tatsächlich sagte: er wollte hiemit darauf hinweisen, daß C potentiell unehrenhaft ist. - Diese konversationelle Implikatur muß bestimmte Bedingungen erfüllen (Griœ 1968: II, Seite 23 f.):

a. Es muß mindestens das Kooperationsprinzip beachtet werden.

b. Man muß die konventionelle Kraft der betreffenden Äußerung kennen, um die konversationelle Implikatur, die nicht Teil der Bedeutung des betreffenden Ausdrucks ist, erkennen zu können.

c. Man muß iber die nötigen Hintergrundsinformationen verfügen.

d. Nicht die betreffende Äußerung bewirkt die konversationelle Implikatur, sondern die jeweilige Art der Äußerung.

Grice glaubt ibrigens, daß prinzipiell alle konversationellen Implikaturen widerrufbar sind, und zwar durch die Hinzufügung der Formel: I do not mean to imply that p. (Grice 1968: III, Seite 5).

2.5.5.4. Deutlich davon verschieden ist die Konzeption der konversationellen Implikatur bei L. R. Hom (1972: 40-52), dessen Vorschlägen ich bei meiner Analyse der deutschen Gradpartikeln in dieser Hinsicht im Prinzip folgen werde. Er stützt sich bei seiner Definition auf die Maxime der Quantität, also: die Information muß so informativ wie gefordert sein; die Information soll nicht 
informativer als gefordert sein. Das bedeutet, daß die Feststellung Das ist mein Freund Hans, wenn es sich um eine Sprecherin handelt, konversationell impliziert, daß die Person 'Hans' weder ihr Liebhaber noch ihr Ehemann ist. Im anderen Falle liegt eine Irreführung des Hörers vor.

Horn untersucht diese Relation überwiegend bei Behauptungen, die Quantoren enthalten, z. B.

(12) A: Hat Hans (mindestens) drei Kinder?

B: Ja, er hat (tatsächlich) vier.

Diese Antwort ist durchaus akzeptabel. Hingegen ist sie auf die Frage

$$
\text { A: Hat Hans (genau) drei Kinder? }
$$

nicht akzeptabel. Beides kann unschwer mit Hilfe der oben angeführten konversationellen Maximen erklärt werden. - Grundsätzlich verhält es sich so, daß bei Zahlen assertiert wird, daß es sich um nicht weniger als angegeben handelt ("upper bound", wie Hom (1972: 43) dies bezeichnet), und daß gleichzeitig konversationell impliziert wird, daß es sich um nicht mehr als angegeben handelt ("lower bound"). Diese Gesetzmäßigkeit kann jedoch z.B. durch die Hinzufügung von nur ins Gegenteil verkehrt werden.

(14) Hans hat nur $v i$ e $r$ Kinder.

Damit wird behauptet, daß er nicht mehr als vier kinder hat, und konversationell impliziert, daß er nicht weniger kinder hat. ${ }^{62}$ Dies läßt sich übertragen auf Zahlenangaben, die sozusagen ein nur fordem, aber nicht explizit enthalten, wie die folgende Äußerung (Horn 1972: 43):

Maria kann von 150 D-Mark im Monat leben.

Es läßt sich ferner übertragen auf skalare Prädikate wie hübsch - schön, warm - heiß, kühl - kalt, gut - hervorragend usw. ${ }^{63}$ - Das Interessanteste an dieser Relation ist, daß sie eine Reihe von Operationalisierungen (Tests) zuläßt ; ${ }^{64}$ man kann z.B. die konversationelle Implikatur einer Äußerung anschließend ausdrücklich behaupten:

62 Die Meinung Horn's zu dieser Thematik ist nicht völlig klar. Eine Skalenumkehr durch nur scheint er aber auszuschließen; siehe Horn (1972: 49). Die Überprüfung könnte durch den Suspensionstest erfolgen:

Hans hat nur 3 Kinder, "wenn nicht 4. / wenn nicht (sogar nur) 2.

$63 \mathrm{Vgl}$. Horn (1972: 47f.). Nicht für alle hier von Horn aufgeführten skalaren Prädikate gibt es deutsche Entsprechungen mit denselben Eigenschaften. Außerdem spielt der Kontext (insbesondere die Gradpartikeln) bei der "Definition" von Skalen eine entscheidende Rolle.

64 Siehe dazu Horn (1972: 49). 
(16) Hans hat vier Kinder, (aber) nicht fünf.

Man kann der konversationellen Implikatur widersprechen:

(17) Hans hat nicht nur $v i$ e $r$ Kinder, sondern $f \ddot{u} n f$.

(18) Hans hat vier Kinder, in der Tat, er hat fünf Kinder.

Und man kann schließlich die konversationelle Implikatur suspendieren:

(19) Hans hat vier Kinder, wenn nicht fünf.

(20) Hans hat vier oder sogar $f$ ü $n f$ Kinder.

(21) Hans hat vier Kinder, und möglicherweise hat er sogar $f \ddot{u} n f$.

Diese Tests lassen sich leider auf Sätze mit Gradpartikeln teilweise nur mit großen Schwierigkeiten anwenden. Doch hoffe ich mit dem Suspensionstest sowohl ein Erkennungsmittel für die konversationelle Implikatur als auch, daraus folgend, für einen bestimmten Interpretationstyp von Gradpartikel-Sätzen in der Hand $z u$ haben.

In der Literatur haben auch noch die sogenannten 'suggerierten Schlußfolgerungen' ("invited inferences") eine gewisse Rolle gespielt. ${ }^{65} \mathrm{Da}$ ich, im Gegensatz zu Geis/Zwicky (1971), der Ansicht bin, daß die Maxime der Relation für ihre Erklärung ausreicht, bilden sie nur einen Untertyp der konversationellen Implikaturen und brauchen nicht eigens behandelt zu werden. - Als Beweis für ihre Zugehörigkeit $z u$ den konversationellen Implikaturen mag auch gewertet werden, daß sie suspendierbar sind. Dies hat Karttunen (1971a: 566-569) nachgewiesen, während er gleichzeitig, in Auseinandersetzung mit Lakoff (1970), feststellte, daß Präsưpositionen nicht suspendiert werden können; damit aber besäße man ein Trennungskriterium zumindest für diese beider Bedeutungselemente.

2.5.6. Verfahren zur Analyse von Semantik und Pragmatik der Gradpartikeln

Die gesamte folgende Testbatterie wurde erstellt, um Ersatzmöglichkeiten für den einerseits unsicheren, andererseits bei allen nichtassertorischen Äußerungen nicht anwendbaren Negationstest zu finden. Dabei ergab es sich, daß mit einer Reihe von Verfahren auch nicht-präsuppositionelle Erscheinungen untersucht werden können. Der Zweck liegt also in einer doppelten Ausdehnung der untersuchungsbasis: in der Einbeziehung auch anderer Bedeutungselemente in die

65 Siehe zu diesem Thema M. L. Geis / A. M. Zwicky (1971); L. Karttunen (1972: 271); A. Grosu (1973). 
Untersuchung, und in dem Versuch, diese Bedeutungselemente mit möglichst vielen sprachlichen Erscheinungen in Beziehung zu setzen. Allerdings maß man die Erwartung, daß man dadurch zu einer zweifelsfreien Formulierung der einzelnen Bedeutungselemente und zu ihrer sicheren Klassifizlerung kommen könnte, von Anfang an stark einschränken: dazu sind insbesondere bei den Gradpartikeln die zahlreichen, nicht immer sicher isolierbaren und erklärbaren intervenierenden Gesetzmäßigkeiten zu kompliziert.

Die folgenden Beschreibungen der einzelnen Tests haben die Aufgabe, die bisherige Anwendung in der sprachwissenschaftlichen Forschung kritisch zu erfassen, die theoretische Basis der darauf aufbauenden Aussagen zu klären, das Testverfahren detailliert zu schildern und die Wirkung auf die einzelnen Bedeutungselemente je nach ihrem Status an einigen Beispielen zu demonstrieren. Nafürlich wird die Diskussion um diese "Tests" im Verlauf der Einzelanalysen fortgesetzt; sind sie doch selbst in hohem Maße kontrovers und in ihrem jeweiligen Aussagewert umstritten.

\section{2:5.6.1. Der Negationstest}

Dieses zunächst einzige Präsuppositions-Kriterium war von Anfang der Diskussion an eine unselige Hypothek. Schon Frege (1892; 1966: 54 f.) erwähnt die Konstanz der Referenz-Präsupposition unter Negation als bestimmendes Merkmal, allerdings in einer so unklaren Formulierung, daß man nur aus den analysierten Sätzen erschließen kann, daß er vermutlich die starke (Interne) Negation meinte. Für den genannten Präsuppositions-Typ bringt der Russell'sche Beschreibungsvorschlag im Hinblick auf das Negationskriterium zunächst einmal deutliche Vorteile: die Zerlegung einer komplexen Information, wie sie ja wohl häufig in einem Satz einer natürlichen Sprache vorliegt, in Teilinfonmationen enöglicht eine Festlegung der zugelassenen Negations-Skopi. Jede einzelne dieser Teilinformationen kann zum Skopus einer Negation werden, in diesem Fall also die Existenzbehauptung, die Einzigkeitsbehauptung und die eigentliche Prädikation. Den bereits in 2.5.4.2. S. 57 vorgebrachten Einwänden gegen ein solches Verfahren, basierend auf Divergenzen gegenibber den Verhältnissen in natürlichen Sprachen, kann man unter der neuen Themenstellung ganz ähnlich gelagerte Gegenargumente hinzufügen. Den drei logisch möglichen Negationsskopi entspricht in der präsupponierenden natürlichsprachlichen Äußerung nichts: Existenz- und Einzigkeitspräsupposition können nicht direkt negiert werden (dialogische Präsuppositionsproteste sind hiervon natürlich ausgenammen). Ganz zu schweigen davon, daß die Negation einer Existenzbehauptung keineswegs das 
Scheitem der mit einer definiten Kennzeichnung verbundenen Präsupposition in jedem Falle erklären kann; man denke nur einerseits an die Fälle, in denen die Referenz scheitert, weil dem Hörer der Äußerung das Bezeichnete nicht bekannt, vielleicht nur unter dieser Kennzeichnung nicht bekannt ist, und andererseits an die Möglichkeit, daß jemand nicht an die Existenz der mit bestimmten Kennzeichnungen belegten Objekte glaubt, dennoch aber diese Ausdrücke referierend gebraucht und ihren referentiellen Gebrauch versteht. Als stabile Gebrauchsbedingung für bestimmte Ausdrücke ist die Referenz-Präsupposition per definitionem nicht negierbar.

Alle diese Teilaspekte münden in die Fragen nach den Entsprechungen der 10 gischen Negationsformen in natürlichen Sprachen; nach dem Skopus der verschiedenen Negationsformen in natürlichen Sprachen; und nach den jeweiligen Interrelationen zwischen den verschiedenen Präsuppositions-Arten und den Negationsarten und ihrem jeweiligen Skopus.

Die Erklärung, daß bei Subjektstemen nur deswegen der Negationstest weitgehend ohne Komplikationen angewendet werden könne, weil sich diese im Normalfall der Negation nicht in deren Skopus befinden (z.B. bei D. Franck 1973: 30), ist nicht sehr hilfreich. Denn dann wäre eine Erklärung nötig, warum andere Präsuppositions-Garanten, die nach diesem Sprachgebrauch zweifelsfrei im Skopus von starken Negationen liegen können, dennoch unter diesen Bedingungen ihre Präsuppositionen beibehalten. - Zur Lösung der hier aufgeworfenen Fragen ist zweierlei nötig: eine knappe Zusammenfassung der Behandlung der Negation in der formalen Logik und ein ebenso knapper Uberblick ibber die linguistische Negationsforschung.

Als Junktor oder monadischer Satzoperator hat die logische Negation definition'sgemäß die Funktion, den Wahrheitswert eines Ausdrucks umzukehren. Dies gilt allerdings nur für ein zweiwertiges System. In einem solchen System existiert auch kein Unterschied zwischen interner und externer Negation. Der Skopusunterschied zwischen den beiden Negationsformen kommt erst in einem dreiwertigen System zur Geltung, ${ }^{66}$ und zwar in folgender Weise:

1. Die interne, starke Negation (= natüliche Negation) entspricht in natülichen Sprachen folgendem Beispiel :

(23) Der König von F rankreich ist nicht glatzköpfig.

formalisiert als: $\sim B(k)$ oder $\neg B(k)$. Diese Negation ist konträr, d.h. S und $7^{S}$ können beide nicht-wahr, aber nicht beide wahr sein. Die Referenz-Präsuppo-

66 Darauf bin ich schon im Abschnitt 2.5.4.3. S.59 kurz eingegangen. Vgl. auch Horn (1972: 5-12) und U. Blau (1973/74: 72). 
sition bleibt von diesem Negationstyp unberihrt. Trifft sle nicht zu, so ist der Satz, ob nun positiv oder negativ in seiner Form, als $u=$ unentscheidbar $\mathrm{zu}$ werten.

2. Die externe, schwache Negation entspricht etwa folgender Formulierung in natuirlichen Sprachen:

(23) Es trifft nicht zu/ist nicht wahr, daß der König von F rankreich glatzköpfig ist.

formalisiert als: $\overline{\mathrm{B}(\mathrm{k})}$ oder $-\mathrm{B}(\mathrm{k})$. Diese Negation ist kontradiktorisch, also immer bivalent: da sie nach logischen Konventionen auch die Präsupposition betrifft, ist sie die Vorbedingung für die Aufrechterhaltung eines zweiwertigen Systems. - Äquivalente zur Kontrastnegation bzw. lokalen Negation gibt es innerhalb der formalen Logik nicht. Sie scheint auch eine bloß pragmatische Variante der starken Negation zu sein, die gegenüber dieser keine Unterschiede in der Wahrheitswertzuteilung verursacht (es sei denn durch das $\mathrm{Zu}$ sammenwirken mit anderen operatoren).

Beim Versuch, die Regeln für die logischen Negationsformen mit bestimmten Erscheinungen in natuirlichen Sprachen in Bezlehung zu setzen, muissen zwei Themenbereiche in Betracht gezogen werden: die Möglichkeit einer konstanten $\mathrm{Zu}$ ordnung zwischen den Negationstypen und -regeln in Logikkalkülen und in natürlichen Sprachen, vorausgesetzt, daß diese beiden Bereiche überhaupt aufeinander bezogen werden können; und als entscheidendes Teilproblem die Frage des Skopus von Negationen. Da in der Logik jeweils ein atomarer Satz als Skopus der Negation gilt, muß dessen Pendant in natürlichen Sprachen gefunden werden: ein schwieriges Unterfangen, da wohl die meisten Sätze natürlicher Sprachen als komplexe Sätze im Sinne der Logik zu gelten haben.

Das Problem des Skopus von Negationen wurde in der Sprachiwissenschaft in Ansätzen schon relativ früh erfaßt, sichtbar in der Unterscheidung Satznegation - Satzgliednegation. Aber für die "traditionelle" einschließlich der strukturalistischen Sprachwissenschaft blieb die Beschreibung der Gesetze der verschiedenen Negationsformen notwendig aufgrund der theoretischen Voraussetzung (Beschränkung auf die Oberflächenstruktur) und der Beschreibungsziele und -methoden (Klassifizierung, Darstellung in reinen Konstituenzsystemen) eine unlösbare Aufgabe. Der unvereinbare Widerspruch zwischen erkennbarer gemeinsamer Funktion und etwa den unterschiedlichen Wortartzugehörigkeiten, Stellungsgesetzen etc. der einzelnen Negationsfonmen konnte nichts anderes als Verwirrung bewirken. - Das Skopusproblem wurde aber auch in den ersten Phasen der generativen Transformationsgrammatik mit unzureichenden Mitteln angegangen; die Lösungsvorschläge entsprechen denen bei den Gradpartikeln. Der Versuch zur 
Syntaktisierung von primär semantischen Fragestellungen, sichtbar im Versuch der transformationellen Einfiuhrung und Zuordnung des Negationselementes in der Syntactic-Structures-Fassung; Erzeugung des Negationselementes in der Basis und transformationelle Zuoranung zu bestimmten Konstituenten; ${ }^{67}$ und schlle $\beta-$ lich Verknüpfung der Skopusabgrenzung mit relnen Oberflächenstruktur-Merkmalen: ${ }^{68}$ alle diese Schritte haben Parallelen in der Gradpartikel-Forschung. Auch die generative Semantik, obwohl sie die entscheidenden Lösungsansätze bereits schuf, lehnte zunächst die Einbeziehung der Topic-Focus-Satzgliederung, die die notwendige Voraussetzung für die adäquate Beschrelbung des Negationsskopus ist, ab. Schließlich wiesen aber Bach (1968), Zemb (1972: Kap. V) und Heidolph (1970) auf die Bedeutung von Betonung bzw. Focusabgrenzung für die Begrenzung des Negationsskopus hin, die der folgende Satz exemplifizieren kann:

(24) The professor didn't sign a petition.

Damit war auch die Forderung verbunden, die wichtigsten Krtterien der funktionalen Satzperspektive in einer Semantischen Repràsentation darzustellen. Für die nichtkontrastive Verwendung der starken Negation kann man nach Sgall (1973: 81-97) zwei Hauptgruppen unterscheiden:

a. Das Verb ist nicht kontextuell gebunden; der Negationsskopus relcht dann von der Gebundenheitsgrenze (BJ) bis zum Ende der Semantischen Satzrepräsentation. Negiert wird in diesem Falle die Relation zwischen den gebundenen Elementen und dem Satzfocus.

b. Das Verb ist kontextuell gebunden; wenn die Negation nach diesem gebundenen Verb zu stehen karmt, dann reicht ihr Skopus von der Gebundenheitsgrenze (BJ) bis zum Ende der Semantischen Satzrepräsentation. Der Funktion nach handelt es sich um eine Negation der Prädikation.

Ähnliche Ansätze gibt es auch in anderen Grammatikmodellen, die die Elnbezlehung der Topic-Comment-Gliederung in die formale Beschreibung versuchen. Als generelle Regel läßt sich dazu formulieren: die Vemeinung einer assertor1schen Äußerung bedeutet in natürlichen Sprachen die Negation des Focus-Bereiches; oder genauer: die Negation vermeint, daß die Prädtkations-Relation zw1schen Topic und Focus gültig ist, wobel der Focus den Negationsskopus bildet.

Noch einmal zur linguistischen Seite der externen oder sctiwachen Negation: normalerweise wird in der Literatur dazu behauptet, daß der Skopus dieser Negation die gesamte Proposition unfasse, und daß damit auch die Präsuppositionen betroffen seien. Dies halte ich fiur etne unbegrindete Ubertragung logi-

67 Beispiele hierfür sind: Klima E. S. (1964) und G. St1ckel (1970).

68 Wie in Chomsky (1969) und R. S. Jackendoff (1972: 254 ff.) 
scher Gesetze auf natürliche Sprachen. Auch Horn (1972: 5-12) welst darauf hin, daß diese Form der Negation in natürlichen Sprachen zumindest ambig sei, was die Löschung der Referenz-Präsupposition anbetrifft. Ich möchte noch einen Schritt weiter gehen. Betrachtet man die folgende Äußerung:

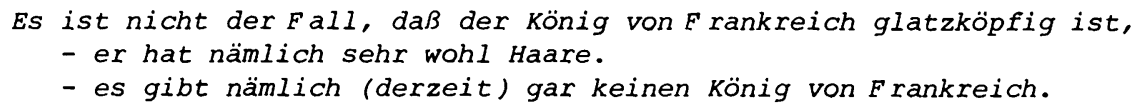

so wird man die erste Fortsetzung als durchaus möglich und passend bewerten missen, die zweite hingegen läßt den Einleitungssatz wie eine bewußte Irreführung des Hörers/Lesers erscheinen. Vielleicht liefert hierfür die Griœ'sche Maxime "be relevant" eine Erklärung: den ersten Satz zu äußern hat nur dann einen Sinn, wenn zumindest denkbar wäre, daß er zutrifft; dies ist jedoch nur dann der Fall, wenn es einen (möglicherweise glatzköpfigen) König von Frankreich gibt.

Die geschilderten Tatsachen beachtend muß man bei der Verwendung des $\mathrm{Ne}-$ gationstestes zur Analyse der präsuppositionalen Eigenschaften bestimmter Äußerungen folgendes berücksichtigen:

- er darf nur bei assertorischen Äußerungen angewendet werden.

- er darf nur bei Äußerungen mit normaler Topic-Focus-Struktur verwendet werden, also nicht bei kontrastiven Äußerungen.

- um Unsicherheiten zu vermeiden, sollte man nur die starke Negation ver-wenden.Der Skopus der Negation ist hierbei das kontextuell ungebundene Satzsegment, also der Satzfocus. Im Zweifelsfall kann man dies durch den Fragetest/Test der angemessenen Antwort überprüfen.

- Kontrast- und Korrekturnegation (lokale Negation) sollte man wegen der dort auftretenden semantischen Eigenheiten und der möglichen Kollisionen des Negations-Skopus mit dem Skopus anderer operatoren vermeiden.

- die Negationen sollte man auf eine einzige für die gesamte Außßung beschränken. Iexikalisch inkorporierte Negationen sowie die Folgen von Negationstransportation, falls es derartiges iberhaupt gibt, ${ }^{69}$ können allerdings vor unlösbare Probleme stellen.

- in jedem Falle sollte man eine Riickführung auf das dem Negationstest zugrundeliegende Prinzip versuchen, nämlich daß die Präsupposition eines Satzes Voraussetzung für die Zuteilung eines Wahrheitswertes ist. Diese Bedingungen zeigen schon, daß eine unbeschwerte vortheoretische Hand-

69 Siehe zu dieser Frage Bartsch (1973). 
habung des Negationskriteriums ausgeschlossen 1st. Bezleht man sie auf die präsuppositionale Analyse von Gradpartikel-Sätzen, so ergibt sich folgendes Bild: a. Die Beschränkung auf assertorische Äußerungen ist einschneidend, stört aber die Analyse nur in Grenzfällen. Alternativen scheint es dann nicht zu geben. b. Normale Topic-Focus-Struktur liegt bei Gradpartikel-Sätzen höchstens bei den Skopustypen Verbalphrase und Satz sowie bei konjunktionalem Gebrauch vor. c. Damit ist auch die Bedingung, nur die starke Negation zu verwenden, nicht mehr zweifelsfrei zu erfüllen.

d. Über den TYP der Kontrastnegation in durch Gradpartikeln bereits kontrastiven Sätzen kann man erst nach eingehenden empirischen Untersuchungen verläßliche Aussagen machen.

Zusammengefaßt: die präsuppositions-neutrale Negation von Gradpartikel-Sätzen steht keineswegs von Anfang an fest. Andererseits ist aber die Lage wiederum nicht so aussichtslos, wie es zunächst scheinen mag. So sind die meisten oben genannten Beschränkungen mit geeigneten Maßnahmen durchaus überwindbar: nämlich durch die Abwandlung, vielleicht auch Ausdehnung auf die Verneinung im Dialog:

A: Nur $H$ a $n s$ war in Hamburg.

B: Nein. (Nicht nur $H$ a $n s$ war in Hamburg, sondern) auch Karl und Detlev.

(27) A: Hans, Karl und Detlev waren in Hamburg.

B: Nein, nur $H$ a $n s$ war in Hamburg.

(28) A: War nur $H$ a $n s$ in Hamburg?

B: Nein, auch Karl und Detlev (waren in Hamburg).

Allerdings kann man nicht generell davon ausgehen, daß eine verneinende Antwort im normalen Gebrauch nach der Intention des Sprechers jeweils nur die Assertion des betreffenden Satzes betrifft. Oft (und dies wird vor allem bei nur noch genauer zu diskutieren sein) wird von Sprechern des Deutschen formal kein Unterschied gemacht zwischen Assertionsverneinung und Präsuppositionsprotest. Nur ein Beispiel: A und B erwarten, daß mehrere Personen nach Hamburg fahren.

(29) A: Stell dir vor, nur $H$ a $n s$ ist nach Hamburg gefahren.

B antwortet lakonisch Nein. ind beharrt bei Riickfrage unerschütterlich darauf, damit hätte er gemeint und könne man natürlich auch nur meinen, Hans wäre auch nicht nach Hamburg gefahren, es wäre also iberhaupt niemand gefahren. - In dieser Situation bleiben nur 3 Auswege: a) das Verhalten von B auf elnen Kampetenzdefekt zurickzufiuhren, den Dialog damit als defekt zu bewerten; b) darin eine begründete (und begrindbare) Abweichung zu sehen; c) den Dialog als ak- 
zeptabel zu bewerten und damit die Verpflichtung zu ibernehmen, ihn als regulär zu beschreiben.

Handelt es sich bei der dialogischen Vemeinung um eine Assertions-Verne1nung, dann läßt sich die präsuppositionsneutrale Negation finden, indem man die dieser Antwort Nein entsprechende negative Formulierung des Gradpart1kelSatzes bildet, in unserem Falle also:

(30) Nicht nur $H$ ans war in Hamburg (sondern auch Karl und Detlev). Daneben lohnt es sich, wegen der Nähe der Gradpartikeln zur lokalen Negation (Kontrastnegation), schon im Rahmen der syntaktischen Analyse die denkbaren Negationsformen in Gradpartikel-Sätzen zu sammeln und auf diese Weise Material für die Beswhreibung der Beziehungen zwischen Operatoren und deren jeweiliyem Skopus zu gewinnen. Von besonderem Interesse sind cabei jene Fonnen, die eine feste Verbindung zwischen Negation und Gradpartikeln bringen, wie richt nur, nicht einmal usw. Die einzelnen Schritte des Untersuchungsprogramms sehen also folgendermaßen aus:

a. Wie lautet die direkte Negation (so möchte ich die Entsprechung der Anthort Nein nennen) für Sätze mit Gradpartikeln?

b. Hat man diese zweifelsfrei festgelegt, so kann man damit klären, welche Bedeutungsaspekte bei Negation in der Bedeutung der betreffenden Äußerung konstant geblieben ist: dies dirften Präsuppositionen sein.

c. Wo dieser Ablauf nicht möglich ist oder nicht zu klaren Ergebnissen führt, kann man eine versuchsweise Umkehrung der Argumentation anbieten: man setzt eine bestimmte Bedeutungskomponente als Präsupposition an und versucht eine Negation zu finden, die diese Komponente umberührt läßt. Auf diese Weise hat man eine bestimmte Evidenz für die Art der anzuwendenden Negation erhalten.

obwohl es sich bei dem Kriterium der Negationsstabilität der Präsupposition (abgeleitet von der Eigenschaft, Voraussetzung für die Zuteilung eines Wahrheitswertes an die präsupponierende Außerung zu sein) um das wichtigste Merkmal handelt, sollte man sich generell bei Klassifizlerungen (und um solche handelt es sich bei der Statuszuweisung an Bedeutungselemente) nicht nur von einem einzigen Kriterium leiten lassen, und schon gar nicht bei den Gradpartikeln. Vielmehr sollte ein Bündel von regelhaften Erscheinungen, die möglichst einfach aus der Grunddefinition etwa von 'Präsupposition' abgeleitet werden können, zur Identifizierung herangezogen werden. Genau so sind die nun folgenden "Tests" konzipiert. 
2.5.6.2. Ermittlung von Bedeutungselementen durch geeignete Kontexte

Bevor die Statuszuweisung an einzelne Bedeutungselemente angegangen werden kann, müssen diese selbst erst feststehen. Für diesen Zweck besonders geelgnet sind im Falle von Gradpartikel-Sätzen spezifische Kontexte. Unter 'Kontext' will ich im folgenden jeweils einen Vorgänger- oder Folgesatz zu einem Gradpartikel-Satz verstehen. Derlei Sätze wurden, zur Verdeutlichung des jeweils Gemeinten, in beinahe allen Äußerungen zum Gradpartikel-Problem in unsystematischer Weise verwendet. Ich versuche deshalb, die vorhandenen Möglichkeiten und ihren jeweiligen Einsatz bei der Analyse von Gradpartikelsätzen möglichst vollständig zu erfassen und in das Analyseprogramm einzubauen.

1. Die eigentlichen Kontrastsätze. Sie sind immer dialogisch zu denken, und zwar in folgenden Formen:

a. A äußert einen Gradpartikel-Satz, B stellt eine Gegenbehauptung auf.

(31) A: Nur $H$ a $n s$ ist nach Hamburg gefahren.

B: Nein, auch Karl und Fritz (sind nach Hamburg gefahren).

b. A äußert irgendeinen Satz, und B stellt dazu mit Hilfe eines GradpartikelSatzes eine Gegenbehauptung auf:

(32) A: Hans, Kari und Fritz sind nach Hamburg gefahren. B: Nein, nur $H$ a $n s$ (ist nach Hamburg gefahren).

2. Vorgänger- und Folgesätze, in denen einzelne Bedeutungsaspekte von Gradpartikelsätzen bestätigt werden. Hier ist sowohl eine dialogische wie eine monologische Form denkbar. Zunächst die beiden dialogischen Formen:

a. A äußert einen Gradpartikel-Satz und B stimmt ihm mit einem nomalen Satz zu:

(33) A: Nur $H$ a $n s$ ist nach Hamburg gefahren.

$\mathrm{B}: \overline{\mathrm{Ja}}$, kein anderer hat sich dazu aufschwingen können.

b. A äußert einen normalen Satz und B führt diesen in zustimmender Weise durch einen Gradpartikel-Satz fort:

(34) A: So gut wie niemand ist nach Hamburg gefahren.

$\mathrm{B}$ : Ja, nur $H$ a $n \mathrm{~s}$ (ist nach Hamburg gefahren).

Und jetzt die beiden monologischen Formen:

c. A äußert einen Gradpartikel-Satz und expliziert ein bestimmtes Bedeutungselement dieses Satzes durch einen Folgesatz:

A: Nur $H$ a $n s$ ist nach Hamburg gefahren, niemand sonst (ist nach Hamburg gefahren).

d. A äußert einen normalen Satz und erläutert ihn mit einem Gradpartikel-Satz: 
Ein Kontrastsatz in monologischer Form ist nicht möglich, da dies zu unauflösbaren Widersprüchen und damit zur Inakzeptabilität führen würde.

Diese Formen von Kontexten zeigen gewisse Ähnlichkelten mit anderen Testarten: alle dialogischen Formen sind verwandt mit der dialogischen Bedeutungsanalyse (siehe 2.5.6.6. S. $84 \mathrm{ff.}$ ), doch können hier nicht, wie bel dieser dialogischen Bedeutungsanalyse, alle im Gradpartikelsatz enthaltenen Bedeutungselemente thematisiert werden. Die monologischen Formen wiederum sind verwandt mit dem Konjunktionstest (siehe 2.5.6.4. S. 82 ff.).Sie bieten aber mehr Mbglichkeiten als der Konjunktionstest, da ihre Form nicht so starr festgelegt ist. - Doch möchte ich, um die Konstanz oder doch Vergleichbarkeit der Testbedingungen zu wahren, eine Formforderung stellen: in den jeweiligen Vorgänger und Folgesätzen soll nur immer der Skopus der Gradpartikeln durch typgleiche Konstituenten ersetzt werden dïrfen. Die Explikation von 'typgleich' ist eine der wichtigsten Aufgaben dieses Tests.

Aus seiner sehr wenig spezifischen Anlage heraus bietet der Test einerseits zwar wenig präzise Informationen, dafür hilft er aber bei der Beantwortung sehr vieler und sehr unterschiedlicher Fragen. Seine Kernaufgabe ist die Isolierung der einzelnen Bedeutungselemente von Gradpartikèl-Sätzen; allerdings erfahren wir hier kaum etwas über den Status dieser Bedeutungselemente. Mit dieser Analyse decken wir gleichzeitig Grundlegendes über die Eigenarten der drei Interpretationsmöglichkeiten (quantifizierend, skalierend, polar) und die für sie jeweils kennzeichnenden, je nach Gradpartikel-Gruppe verschiedenen Kontrastierungsformeln auf. Letztere geben auch Aufschluß über die jeweiligen Skopusgrenzen und ermöglichen damit sowie auf dem Wege des fortlaufenden Austausches eine Spezifizierung von 'typgleiche Skopuskonstituente', abhängig von Skopustyp und Interpretationsart. Unschätzbares leistet dieser Test aber für die semantische Analyse von einzelnen Graopartikeln innerhalb einer semantischen Gruppe, d.h. für ihre semantische Unterscheidung.

In keinem Fall wird das oben ausgebreitete Analysenprogramm vollständig durchgeführt. Meist beschränke ich mich, wegen der besseren Kontrollierbarkeit, auf monologische Vorgänger- und Folgesätze. - Eine weitere Funktion überschreitet die Grenzen dieses Tests: in allen Fällen, wo es auf die Konstanz aller Faktoren in einem Gradpartikel-Satz ankonmt, wird dies durch Vorgänger- und Folgesätze abgesichert. Sie bieten in den meisten Fällen ausreichenden Schutz gegen unkontrollierte Akzentveränderungen und damit Bedeutungsänderungen. 


\subsubsection{Der Suspensionstest}

Er wird im Zusammenhang mit Gradpartikel-Sätzen erstmals von G. Lakoff (1970; 1971: 41 ff.) angewendet, aber erst von L. Horn (1972: Ch. 1: scalarity and suspension, 3-69) systematisch behandelt und eingesetzt. Zu beachten ist ferner, daß Karttumen (1971a: 567-569) in Auseinandersetzung mit Lakoff (1970) auf Probleme bei der Suspension von Präsuppositionen hingewiesen hat.

Man muß sich diesen Test monologisch, also ohne Sprecherwechsel, denken, und voraussetzen, daß sich der Sprecher bezüglich bestimmter Bedeutungskomponenten nicht sicher ist und dies durch Relativierung, Aufhebung der Komponenten zum Ausdruck bringen will.

Das Vorgehen gestaltet sich so, daß an den zu analysierenden GradpartikelSatz eine Suspensionsformel angehängt wird, die irgendein konjunktivisches Element sowie sogar, nicht einmal, überhaupt oder ein semantisch verwandtes Element enthalten muß. Folgende Suspensionsformeln sind also möglich:

- wenn überhaupt...

- und/aber möglicherweise ... sogar/nicht einmal ...

- und/aber es ist möglich, daß ... sogar/nicht einmal ...

Um einigermaßen die Vergleichbarkeit der Analysen zu gewährleisten, beschränke ich mich in der Regel auf die Formel und möglicherweise ... sogar/nicht einmal...

Die Anwendung auf alle auftretenden Bedeutungselemente von GradpartikelSätzen, also nicht nur auf diejenigen, denen der Status einer Präsupposition zuerkannt wird, soll die Schlußfolgerungen, die nicht nur die jewelligen Interpretationen von Gradpartikel-Sätzen, sondern auch Aussagen über die Art des Tests selber betreffen, absichern helfen durch die größere Streuung des untersuchten Materials.

Daß die Akzeptabilitätsurteile bei der Suspension von Bedeutungselementen mit gleichem Status, aber von verschiedenen Gradpartikeln, durchaus unterschiedlich ausfallen können, zeigen die folgenden Beispiele, die eine gewisse Orientierung über das Verfahren ermöglichen sollen.

1. Suspension von Assertionen:

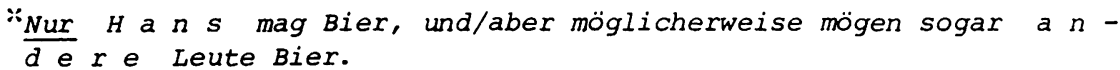

"Auch $H$ a n $s$ mag Bier, und möglicherweise mag nicht einmal Han s Bier.

(40) 'Sogar Hans mag Bier, und möglicherweise mag nicht einmal

Die Ergebnisse sind, wie zu erwarten, eindeutig inakzeptabel. 
2. Suspension von Präsuppositionen:

(41) Nur $H$ a $n s$ mag Bier, und möglicherweise nicht einmal e $r$.

(42) "Sogar $H$ a n $s$ mag Bier, und möglicherweise nicht einmal sonst jemand/wenn überhaupt sonst jemand.

(43) "Auch $H$ a ns mag Bier, wenn überhaupt sonst jemand.

Die Akzeptabilität des nur-Satzes zeigt bereits die Sonderstellung dieser Gradpartikel, gleichzeitig begründen die auch- und sogar-Beispiele Zweifel daran, daß die Suspendierbarkeit primär etwas mit dem Präsuppositions-Status von Bedeutungselementen zu tun haben könnte.

3. Suspension von konversationellen Implikaturen:

Hier muß man vorausschicken, daß Bedeutungselemente mit diesem Status nur bei Gradpartikel-Sätzen mit skalierender Interpretation auftreten.

(44) Er besitzt nur $f$ ü n $f$ Mark, und möglicherweise sogar nur $v i$ e $r$.

(45) Er verdient jetzt sogar $t$ a u s e n d Mark, und möglicherweise sogar elfhundert.

Die Akzeptabilität dieser Beispiele scheint zu bestätigen, daß Karttunen (1971a) recht hatte, als er konversationelle Implikaturen bzw. suggerierte Schlußfolgerungen als das eigentliche operationsgeblet von Suspensionsformeln bezeichnete. Daß dies nur zum Teil stimmt und daß wir damit noch kein Kriterium für die sichere Trennung von Präsuppositionen und konversationellen Implikaturen besitzen, soll der Analysenteil zeigen. Zahlreiche Einzeltests beweisen, daß es keine feste Verbindung zwischen dem Status eines Bedeutungselementes und seiner Suspendierbarkeit gibt (jedenfalls bei Gradpartikel-Sätzen), sondern daß diese Relation von einer Gradpartikel-Gruppe zur anderen wechselt, innerhalb der einzelnen Gradpartikel-Gruppen aber ziemlich stabil ist. Es zeigt sich femer, daß Suspendierbarkeit eng mit skalierender Interpretation sowie der jeweiligen Skalenrichtung zusammenhängt. Dies geht so weit, daß die Suspendierungsformel bei Gradpartikel-Sätzen, die normalerweise quantifizierend interpretiert werden, skalierende Interpretation erzwingt, sowie die für die Suspension notwendige Skalenrichtung induziert. Daß alle diese Eigenschaften mit dem sogar/nicht einmal der Suspensionsformel zu tun haben, liegt auf der Hand. 
2.5.6.4. Der Konjunktionstest mit und:

Er beruht auf dem Prinzip, mit dem zu analysierenden Satz bestimte Elemente seiner eigenen Bedeutung zu verbinden. Man möchte nun annehmen, daß es für derlei Kombinationen generelle Regeln der Verbincbarkelt gibt, die sich an dem jeweiligen Status des Bedeutungselementes orientieren: ${ }^{70}$ so, daß eine Assertion zum Zwecke der nachdrücklichen Behauptung verdoppelt werden darf; daß die explizite Präsuppositions-Formulierung dem zugehörigen Satz zwar vorausgehen, aber nicht folgen darf (weil sie ja in dem betreffenden Satz ohnehin als gültig vorausgesetzt wird) usw. Diese Regeln werden jedoch in mannigfacher Weise bei Gradpartikel-Sätzen durchbrochen bzw. modifiziert. Diese Eigenarten zu erfassen und zu erklären ist Aufgabe des Analysenteils. - Ich bescinränke mich nicht, wie Horn (1972), darauf, die explizite Formulierung auf den Analysesatz folgen zu lassen, sondern ich untersuche auch die umgekehrte Anordnung, da sie sich bisweilen (insbesondere bei den Präsuppositionen) signifikant anders verhält. - Bei der Exemplifizierung des Testablaufs ist darauf zu achten, daß die aufgeführten Beispiele mit nur keineswegs typisch für alle Gradpartikeln bzw. für alle Skopustypen sind. Der Analysesatz lautet:

(46) Nur Ha n s lügt.

1. Assertion:

a. Assertionsformulierung - und - Gradpartikel-Satz.

(47) Niemand (außer Hans) lügt, und nur $H$ a $n s$ lügt.

(48) Karl, Egon ... lügen nicht, und nur $H$ a $n s$ lügt.

b. Gradpartikel-Satz - und - Assertionsfomulierung.

(49) Nur $H$ a n s lügt, und niemand sonst (lügt).

(50) Nur $H$ a $n s$ lügt, und nicht Karl, Egon ...

2. Präsupposition:

a. Explizite Präsuppositionsformulierung - und - Gradpartikel-Satz.

(51) Hans (lügt) und $\underline{n u r}$ Hans lügt.

b. Gradpartikel-Satz - und - explizite Präsuppositionsformulierung.

(52) $\because$ Nur $H$ a n $s$ lügt, und hans lügt/ und er lügt.

3. konversationelle Implikatur: für sie mu ein geeigneterer Analysesatz verwendet werden:

70 Solche Regeln versuchte L. Horn (1972: 76-81) zu formulieren. 
a. Explizite Fonmulierung der konversationellen Implikatur - und - GP-Satz:

"Hans besitzt nicht weniger als fünfzig Mark, und Hans hat nur $f \ddot{u} n f z i g$ Mark.

b. GP - Satz - und - explizite Formulierung der konversationellen Implikatur.

(55) ?Hans besitzt nur $f$ ün $f z i g$ Mark, und Hans/er besitzt nicht weniger als fün $\overline{f z i g}$ Mark.

Einige Störfaktoren müssen genau beobachtet werden: die Voranstellung der Präsuppositionsformulierung bedingt eine kontrastive Betonung auf nur. Es bleibt zu prüfen, ob es Äquivalente bei den anderen Gradpartikeln gibt, und wie diese Anordnung erklärt werden kann. - In vielen Fällen könnte Akzeptabililtät durch den Austausch von und gegen aber erreicht werden: dieser Austausch würde nichts an den wahrheitswertfunktionalen Eigenschaften des jeweiligen Satzes ändern, wohl aber an seinen Gebrauchsbedingungen. Dies zeigt, wie stark die Semantik bzw. die Gebrauchsregeln dieser belden Konjunktionen in die Analysenbedingungen eingehen. Das erforderte eigentlich ein genaueres Eingehen auf sie, zumal es bislang keine überzeugenden Darstellungen des Bereiches gibt; doch wïrde diese Aufgabenstellung den Rahmen der Untersuchumg weit überschreiten. Die Akzeptabilität ließe sich außerdem in vielen Fällen durch Tilgungen bzw. Pronominalisierungen verbessem. Es ist aber auch hier unklar, wie weit dies die Testbedingungen verändert. In beiden Fällen muß ich mich daher auf die bloße Erwähnung der jeweiligen Möglichkeiten beschränken.

Hingewiesen sei auch noch auf die Ähnlichkeit dieser Testanordnung mit dem formal weniger stark festgelegten Test mit Vorgänger und Folgesätzen in monologischer Form. - Wie dieser liefert er viele Informationen zur semantischen Analyse der einzelnen Gradpartikeln bzw. Gradpartikel-Gruppen; zur Frage der Unterscheidung der einzelnen Interpretationsarten (quantifizierende, skalierende, polare Interpretation); zur Klärung des Status der einzelnen Bedeutungselemente. Es kann vermutet werden, daß die einzelnen Typen von Bedeutungselementen jeweils eine typische Kambination von Reaktionen zeigen. Und schließlich könnte die Sachlage bei den konversationellen Implikaturen einiges zur Klärung der Skalenrichtung beitragen. 
2.5.6.5. Der monologische Widerspruchstest (aber-Test)

Er beruht auf dem Prinzip, daß ein Sprecher nicht den aus einem eben geäußerten Satz ableitbaren Bedeutungselementen (ob nun Assertion oder Präsupposition oder konversationelle Implikatur) explizit widersprechen darf. In jedem Falle entstehen dadurch unauflösbare Widersprüche. Lediglich bei einigen Arten des 'Entailments' (das bei Gradpartikeln nicht auftritt) ist man versucht, den betreffenden Satz dann als eine Art Rätsel zu interpretieren.

Er wird im Prinzip ganz so wie der Konjunktionstest mit und durchgefuihrt, d.h. ein Analysesatz wird vermittels der Konjunktion aber mit der expliziten Verneinung seiner Assertion, Präsupposition oder konversationellen Implikatur verbunden, und zwar sind wieder beide Reihenfolgen denkbar. Die Beispiele aus dem vorhergehenden Abschnitt müssen also nur geringfügig umgeformt werden:

$$
\begin{aligned}
& \ddot{N}_{\text {Nur }} H \text { a n s lügt, aber irgendjemand sonst lügt. } \\
& \because \underline{\text { Nur }} \text { Hans lügt, aber Hans lügt nicht. }
\end{aligned}
$$

Da der Test im Normalfall nur inakzeptable Ergebnisse liefert, hat er nur sehr geringen Erkenntniswert. Aus diesem Grunde wird er nur in Ausnahmefällen vollständig durchgeführt. Zu hüchster Vorsicht ist allerdings Anlaß gegeben, wenn der Test akzeptable Ergebnisse liefert. Dann ist genau zu prüfen, ab die jeweilige explizite Formulierung eines Bedeutungselementes tatsächlich zutrifft. Zur Problematik der Semantik von aber gilt das im vorhergehenden Abschnitt zur Konjunktion und Gesagte sinngemäß.

\subsubsection{Dialogische Analyse von Gradpartikel-Sätzen.}

Dieser Test ${ }^{71}$ beruht auf der Fiktion, daß der Hörer eines Gradpartikel-Satzes einzelnen Bedeutungselementen dieses Satzes zustimt, sie zurückweist, erfragt. oder seiner bisherigen Unkenntnis Ausdruck gibt. Natiurlich kann eine Antwort auch aus der Kombination verschiedener Reaktionen auf unterschiedliche Bedeutungselemente bestehen. Seine Aussagekraft besteht darin, daß signifikante Unterschiede zwischen den verschiedenen Typen von Bedeutungselementen hinsichtlich der Möglichkeit, ihnen direkt zuzustimmen oder sie abzulehnen, bestehen; leider gibt es jedoch keinerlei Möglichkeit, z.B. die zurückweisung der Assertion, der Präsupposition oder der konversationellen Implikatur jeweils anhand dafür vorgeschriebener spezifischer Formeln oder Verfahren zu identifizieren.

$71 \mathrm{Zu}$ diesem Test erhielt ich wertvolle Anregungen von K. H. Ebert (1973: 431) und von D. Zaefferer (1974) sowie in Diskussionen mit D. Zaefferer. 
Man kann nur etwa folgende Grundregeln aufstellen:

1. Widerspruch gegen die Assertion: allein hier ist ein globaler Widerspruch mit nein (bei positiven Sätzen) bzw. doch (bei negativen Sätzen) möglich, also Anfügung der expliziten Formulierung der verneinten Assertion.

(58) A: Nur $H$ a n s lügt.

B: Nein. ( = Nicht nur $H$ a n $s$ lügt, sondern auch Egon und Karl.)

Die Verneinung kann natürlich auch durch eine Gegenbehauptung ausgedrilckt werden:

(59) B: Aber Karl und Egon lügen doch a $u c h$.

Übrigens wird die Assertionsverneinung gern mit der Präsuppositionszustimmung verbunden:

(60) B: Hans lügt zwar, das stimmt schon, aber Karl und Egon lügen doch a u c h.

2. Widerspruch gegen die Präsupposition: eine globale Zurückweisung mit Hilfe von nein scheint hier in den meisten Fällen nicht hinreichend, zumindest ist das Ergebnis ambig. In der Regel kann man wohl mit ausgesprochenen Protestfomeln rechnen, bei denen in jedem Fall die zurückgewiesene Präsupposition explizit genannt, ev. durch eine Gegenbehauptung gekontert wird. Für die konversationelle Implikatur gilt dasselbe:

(61) A: Nur $H$ a n s lügt.

B: $\overrightarrow{A b e r}$ Hans lügt doch gar nicht! /Nein.

(62) A: Nur $f \ddot{u} n f z i g$ Mark sind in der Kasse.

B: Aber wie kommst du denn auf einen solchen Unsinn? Da sind doch nur $v i$ e r z i g Mark drin.

Ob bestimmte Kombinationen, wie Assertionszustimmung (globale und explizite!) mit Präsuppositions-Protest,möglich sind, bedarf einer genauen Überprufung.

3. Zustimmung zur Assertion: wiederum ist allein hier eine globale Zustimmung mit ja (bei positiven Sätzen) bzw. nein (bei negativen Sätzen) möglich, die Explikation der Assertion wird meist durch wirklich oder tatsächlich verstärkt.

(63) A: Nur $H$ a n s lügt.

$\mathrm{B}: \overline{\mathrm{Ja}}$ (es lügt wirklich sonst niemand).

Die globale Assertionszustimmung mit ja kann nicht mit der Zurückweisung der Präsupposition verknüpft werden:

B: 'Ja, und/aber Hans lügt nicht.

Dies liegt daran, daß die globale Zustimmung mit ja auch die Zustimming zu allen mit dem Satz verknüpften Bedeutungselementen bedeutet. 
4. Zustirmung zur Präsupposition und konversationellen Implikatur: sie kann nur durch explizite Nennung der Präsupposition bzw. konversationellen Implikatur erfolgen, oft kombiniert mit der Zurickweisung der Assertion.

(65) A: Nur $H$ a n s lügt.

B: Na ja, der Hans lügt zwar, das stimmt schon, aber die anderen lügen doch genauso.

Eine weitere Möglichkeit ist der Ausdruck von Unbekanntheit durch den Hörer eines Gradpartikel-Satzes. Sie ist fiur die Assertion nicht sehr wahrscheinlich, da sie ja als Information über Neues - in der Regel - gedacht ist. Für die Präsupposition ist diese Reaktion hingegen von großer Bedeutung. Etwas ganz Ahnliches scheint beim Focuswechsel durch den Hörer einer Äußerung vorzuliegen, wenn er also nicht die Focusinformation in seiner Antwort thematislert, sondern Teile des Topic, des gebundenen Segmentes also.

Dieser Test soll Informationen liefern zu folgenden Punkten: er soll helfen bel der Trennung der verschiedenen Bedeutungselemente und der Zuweisung des jeweiligen Status. - Er soll - via globale Vermeinung - helfen, die richtige, d.h. die präsuppositionsneutrale Negation von Gradpartikel-Sätzen zu finden. - Er soll Aufschluß geben dariber, ob die Präsuppositionen von Gradpartikel-Sätzen, der Definition von 'Präsupposition' entsprechend, Voraussetzung für die Verwendbarkeit bzw. für die Zutellung eines Wahrheitswertes sind. Dazu dienen insbesondere die oben genannten Kombinationen. - Und er soll es emöglichen, auf diese Weise Unterschiede zwischen den Gractpartikel-Gruppen in Bezug auf die genannten Gesetzmäßigkeiten herauszufinden.

Dieser Test ist also in ganz besonderer Welse mit der Definition der jeweiligen Bedeutungselemente und mit der Angabe ihrer Eigenschaften verbunden. Wegen der Probleme bei der Anwendung sollte man jedoch nicht restlos klare Entscheidungen von ihm erwarten.

2.5.6.7. Der Umfang der Testbatterle darf nicht zu der Annahme verleiten, es handle sich um überflüssigen Aufwand. Da kaum einer der Tests elndeutige Ergebnisse liefert, kann in den meisten Fällen erst die Komblnation der verschiedenen Testergebnisse zu einer einigermaßen tragfähigen Entscheidung führen und unkontrollierbare Einflüsse ausschalten. Auch die Entsprechungen zwischen den verschiedenen Tests erfilllen durch den vielfach dabei stattfindenden Aspektwechsel wichtige Funktionen. 
3. ANALYSENTEIL I: UNTERSUCHUNG DER GRADPARTIKELN NACH SKOPUSTYPEN

\subsection{Vorbemerkung}

3.1.1. Die drei Gradpartikel-Gruppen umfassen folgende hauptsächlich untersuchte Mitglieder:

1. nur-Gruppe: nur, bloß, lediglich, allein, einzig, einzig und allein, ausschließlich.

2. auch-Gruppe: auch; dazu die syntaktisch stark beschränkten Elemente gleichfalls, ebenfalls, ebenso, fermer, daruberhinaus, außerdem etc.

3. sogar-Gruppe: sogar, selbst, nicht einmal.

\subsubsection{Analyseprogramm}

Um leichtere Orientierung innerhalb der einzelnen Kapitel zu gewährleisten, wird hier das Untersuchungsprogramm stichwortartig zusanmengefaßt. Dabei muissen natürlich die z.B. die Syntax übergrelfenden Untersuchungspunkte der Syntax allein zugeordnet werden.

\section{Belegsätze}

syntax-Arbe1tsprogramm

Klassenb1ldung/Austauschbarke1t:

- Elemente elner GP-Gruppe untereinander.

- ibrige Gradpartikeln.

- weitere Ersetzungsmöglichkeiten.

Stellungsgesetze:

Typ 1: GP unmittelbar vor dem Skopus.

Typ 2: GP unnittelbar nach dem Skopus.

Typ 3: GP in der Skopuskonstituente.

Typ 4: GP in Distanz vor und nach dem Skopus.

Typ 5: Skopus topikalisiert, (IP in Distanzstellung danach.

TYP 6: Kontrastiv betonte GP. 


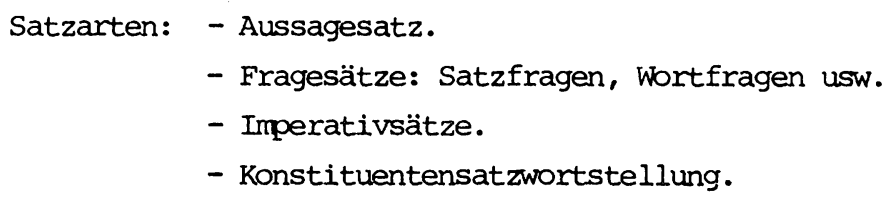

Syntaktische umformungen:

- Spaltsatzbildung.

- transformationell abgeleiteter Skopus (z.B. Passiv)

Negationsmöglichkeiten:

TYp 1: NEG unmittelbar vor der Gradpartikel.

Typ 2: NEG zwischen GP und Skopuskonstituente.

TYp 3: NEG in Distanzstellung nach dem Skopus.

TYp 4: Lokale/kontrastive NEG.

$\mathrm{s} \mathrm{k} \circ \mathrm{p} \mathrm{u} \mathrm{s}-\mathrm{F} \circ \mathrm{k} \mathrm{u} \mathrm{s}$

Skopustypen (syntaktisch; semantische Subtypen).

Skopusgrenzen:

- Relation zwischen Stellungsmöglichkeiten der GPn und den Skopusgrenzen.

- Relation zwischen Akzent-Positionen und Skopusgrenzen.

- Abgrenzung durch kontrastive Vorgänger- und Folgesätze.

Semantische Skopusmerkmale (mit Hilfe der Kontextmethode).

Verhalten des GP-Skopus zum Skopus anderer operatoren.

Test der natürlichen Antwort/Fragetest.

Semantik-A r b e itsprogram m

Bisher vorgeschlagene semantische Analysen von GPn.

Negationstest, Wahrheitswert-Kriterium.

Test der passenden Kontexte (monologisch, dialogisch; Vorgängersätze, Folgesätze; Zustimmung, Widerspruch).

Suspensionstest.

Konjunktionstest mit und.

Monologischer Widerspruchstest mit aber.

Dialogische Analyse

(Widerspruch, Zustimmung, Erfragen; Mißverständnisse; Kombinationen) .

z u s a m m $\mathrm{n}$ a s u n g der Analyseergebnisse. 


\subsection{Skopustyp: Nominalphrasen}

\subsubsection{Skopus: Einfache Nominalphrasen}

Es empfiehlt sich, bei diesem Skopustyp das gesamte Analyse- und Beschreibungsprogramm möglichst lückenlos abrollen zu lassen, und zwar einmal deswegen, weil in der vorliegenden Literatur zu den GPn meist Belspiele dieses Typs verarbeitet wurden und daher auch die meisten Aussagen zum Fragenkomplex auf diese Konstellation abgestellt und beschränkt sind. Zum anderen deswegen, well hier auf ökonomische Weise alle relevanten Fragen angeschnitten oder doch vorstrukturiert werden können, so daß bei den übrigen Skopustypen die Aussagen weitgehend auf divergente Erscheinungen beschränkt werden können. - In der Regel werden nur Subjekts-NPn analysiert. Nur dann, wenn bei NPn in anderen Funktionen abweichende Gesetzmäßigkeiten oder Besonderheiten auftreten, wird das gesondert erwähnt.

\subsubsection{1. nur-Gruppe}

1. Subjekts-NPn:

(1) Von dem Sondertrupp ... bleibt kein Gefolgsmann am Leben. Nur A $g$ u $i \quad r \quad r e \quad \ldots$ übersteht die Fährnisse im Fieber.

(2) In Wahrheit war nur ein $H$ a u $p$ t schalter durchgebrannt.

(3) Ihn schreckte allein der $U$ m z u g.

(4) Allein der $G$ e d’̀ $n k$ e entsetzte uns.

2. NPn als Genitiv-, Dativ-, Akkusativobjekt:

(5) Eine Reformklinik, in der es nur L u x us zimmer geben soll...

(6) Sein Besuch in der Nürnberger Klinik gelte nur einer $R \circ$ u t $i n e-$ untersuchung.

(7) Er ging dabei allein seiner $F$ ä $h$ i g keiten verlustig.

Klassenbildung/Austauschbarkeit

Für nur, allein, einzig, bloß, lediglich, ausschließlich, einzig und allein sind zumindest für die ersten drei Sätze keine Beschränkungen der Einsetzbarkeit erkennbar, abgesehen von der Tatsache, daß einzig in der Verwendung als GP antiquiert wirkt, und daß allein am Satzanfang offensichtlich eher zu anderen Verwendungsweisen tendiert: etwa zu konjunktionaler Verwendung, die für (4) näher zu liegen schelnt, oder zu einer möglicherweise nur semantisch abweichenden Funktion, die mit der Bedeutung von schon in dieser Position ${ }^{1}$

1 Siehe auch H. Glinz (1961: 222). 
identisch sein dïrfte. Beides zusammen könnte elne Erklärung liefern für dle offenkundige Meidung dieser Varianten durch Sprecher und für die Bevorzugung des eindeutigen einzig und allein in diesen Positionen. Hinzuwelsen 1st vielleicht auch noch auf die in den Austauschtests sichtbar werdenden semantischen Differenzen zwischen nur und bloß sowie lediglich einerseits und allein, einzig, einzig und allein, ausschließlich andererseits. - Mit den ubblichen 1diosynkratischen Beschränkungen sind auch die ubrigen Gradpartikeln der anderen Gruppen einsetzbar. - Weitere Ersetzungsmöglichkeiten bieten eine Reihe anderer Adverbien wie erst, schon, allerdings mit deutlich verschledenen semantischen Funktionen. Bei Objekts-NPn sind diese Elemente nur in threr Funktion als Temporaladverbien möglich:

(7a) Er ging dabei erst/schon seiner Fähigkeiten verlustig. In der Position nach dem finiten Verb sind praktisch alle Adverbien einsetzbar, ebenso auf allen Positionen die Kontrastnegation nicht.

Stellungsgesetze

Typ 1: GP unmittelbar vor der Skopuskonstituente. Alle Belegsätze gehören diesem Typ an. Gewisse Beschränkungen sind, wie oben bereits erwähnt, nur bezüglich allein am Satzanfang erkennbar (durch das Uberwiegen anderer Funktionen). Typ 2: GP unmittelbar nach der Skopuskonstituente.

(1a) A guirre allein/?einzig/"bloß/"lediglich/”ausschließlich/”einzig und allein/" nur übersteht die $F$ ährnisse im F ieber.

Man beachte, daß allein in dieser Position auch betont u.d.h. als Modaladverb vorkommen kann. Ansonsten aber scheint diese Position gegeniber der Satzanfangstellung von allein bevorzugt zu werden. Die übrigen GPn der Gruppe können zwar in dieser Position beobachtet werden, doch können keine einheitlichen Akzeptabilitätsurteile erreicht werden. Da Beispiele für diese Nachstellung insbesondere in älteren literarischen Werken "hoher" Stillage zu finden sind, liegt der Schluß nahe, daß hier entweder diachrone Erscheinungen vorliegen, oder daß dieser Stellungstyp als der normalen Stillage unangemessen empfunden wird. Andere Ergebnisse sind auch für Satzschlußstellung und indefintte NPn nicht zu registrieren:

(3a) Ihn schreckte ein $U$ m z u g allein/?einzig/"bloß/" schließlich/"einzig und allein/?nur.

Auch die scheinbar eindeutigen Belspiele

(8) Dieses $B \cup C h$ nur interessiert mich.

(9) Ein $B$ e is pi e 1 nur: schon in der Kindheit...

erweisen sich bei näherem Hinsehen als recht prekär. So sind für (8) folgen- 
de Kontrastsätze möglich:

- nicht $j$ e n e s. Hierbei mïßte aber wohl im vorausgehenden Satz dieses betont werden, die nomale Betonung, wie mir scheinen w11l; dann handelt es sich aber um einen anderen Skopustyp.

- nicht dieses $B i l d$. Ich könnte mir aber keine Situation vorstellen,

in der dies nicht eine beinahe metasprachliche Korrekturäußerung wäre.

- nichts Wichtigeres/Bedeutenderes.

Bei Satz (9) ist zwar der Kontrastakzent eindeutig auf Beispiel festgelegt. Die möglichen Kontrastsätze verwirren jedoch das Bild:

- nicht viele. Die nomale Version dïrfte hierfür allerdings nur $e i n$ Beispiel lauten.

- kein Beleg. Das wïrde eine skalare Interpretation im Sinne von nicht mehr als ein Beispiel wie auch eine bloße Unterscheidung zweier gleichwertiger Varianten zulassen.

- nichts sonst. Nur dieser Folgesatz würde eine quantifizierende Interpretation kennzeichnen. Sie scheint aber der üblichen Verwendung dieses Satzes kaum angemessen. 2

Eine klarere Lage ergibt sich in dieser Hinsicht bei den Objekts-NPn. Nachstellung der GP ist bei ihnen dann möglich, wenn der Objektskopus den Satzschluß bildet: Dadurch sind Skopusambiguitäten von vornherein ausgeschlossen.

(10) Er gedachte dabei der $r \circ t$ e $n$ nur/bloß/allein..

Typ 3 (GP in der Skopuskonstituente) ist hter nicht sinnvoll zu verwirklichen, da die GP nicht zwischen Artikel und Nomen stehen kann.

Typ 4: Distanzstellung der GP vor oder nach der Skopuskonstituente. Im Gegensatz zu der von B. Fraser (1971) und Susan Schmerling (1972) für das Englische festgestellten Möglichkeit dér Distanzstellung (allerdings nur an anderen Skopustypen) besteht im Deutschen offenbar nur eine theoretische Möglichkeit, die mit iberzogenem Akzent vielleicht gerade noch gerettet werden kann:

(lb) Aguirre übersteht ( nur $\because a l l e i n / . .$.$) die Fährnisse ("nur/$ "allein/ ...) im Fieber ("

Im Allgemeinen scheint aber die Bindung an die unmittelbar folgende Konstituente zu überwiegen. Daneben gibt es einen TYp, bei dem Nachstellung der GP in Distanz voll akzeptabel ist. Er trifft sich mit Typ 5: Position der GP relativ zu einer topikalisierten Skopuskonstituente.

2 Unberücksichtigt bleibt, ob nicht ein Beispiel in diesem Fall - nach Ergänzung von ich gebe - als Akkusativ zu deuten ist. 
Topikalisierung ${ }^{3}$ im eigentlichen Sinn (als Umstellung einer Konst1tuente von weiter "rechts" im Satz in die Spitzenposition) ist natürlich beim Subjekt nicht möglich, jedoch im Sinne einer herausgehobenen Position für die Fokuskonstituente von GPn durchaus typgleich mit anderen Skopuskonstituenten.

(11) Der $M$ ens $c h$ interessiert mich nur/?allein/bloß/lediglich/einzig/ einzig und allein/ausschließlich, nicht das Vermögen.

Ein $L \ddot{u} f t c h$ e $n$ war es nur/allein/..., was uns erschreckte.

Man könnte nun beim Vergleich mit (lb) auf den Gedanken verfallen, daß die GP nur dann in Distanzstellung am Satzschluß akzeptabel ist, wenn sich zwischen finitem Verb bzw. Kopula und Satzschluß nur Pronomina oder Platzhalter finden. Hier jedoch der Gegenbeweis:

(1la) Der Mens $c h$ interessierte Hans in diesen Fährnissen nur.

Auch die Position der GP nach Hans ist möglich, was vielleicht auf den quasiparenthetischen Charakter von in diesen Fährnissen zurückgeführt werden kann. Syntaktische Gründe für den Akzeptabilitätsunterschied zwischen (lb) und (1la) scheiden aus. Für eine semantische Erklärung via Zwang zur skalierenden Interpretation in diesem Konstruktionstyp fehlen aber noch die Voraussetzungen.

Es bleiben noch die Möglichkeiten für eine Distanzstellung der GPn vor der Skopuskonstituente zu prüfen:

(3b) “Nur/"allein/?einzig/bloß/:lediglich/ausschließlich/?einzig und allein Das Ergebnis täuscht jedoch: nur und $b$ loß liegen in der konjunktionalen Verwendung vor, wie die Ersetzbarkeit durch hingegen, jedoch usw. zelgt. Allein wïrde in konjunktionaler Verwendung eine andere Wortstellung (allein, ihn schreckte...) erfordem. einzig scheint nur im Sinne von einzigartig, ausschließlich nur im Sinne von fortwährend möglich.

(3c) Ihn (" "nur/"allein/"einzig/...); schreckte (nur/allein/einzig/bloß) ... , - in diesen Tagen der $U$ m zu $g$.

Das Ergebnis ist bei Zweitstellung der GP eindeutig negativ, das positive Ergebnis für die Mittelstellung täuscht jedoch wiederum: nur und bloß können wiederum in konjunktionaler Verwendung vorliegen (Ersetzbarkeit durch hingegen, jedoch usw.), allein, einzig, einzig una allein sowie ausschließlich könnten in der Bedeutung von als einziges/einzigen vorliegen. Es bleibt jedoch eine gewisse Möglichkeit, daß es sich auch um echte GPn mit dem Skopus umzug han-

3 Das Thema "GPn und Topikalisierung" habe ich in einem Vortrag auf dem $x$. Linguistischen Kolloquium in Tübingen eingehend behandelt: Altmann (1976). 
deln könnte, dies mag von der quasiparenthetischen Funktion von in diesen Tagen, das dadurch kaum als Skopuskonstituente in Frage kommt, herrühren. TYp 6: kontrastiv betonte GP. Sie tritt nur bei Verdoppelung der Skopuskonstituente auf:

(2a) In Wahrheit war ein Hauptschalter und nur/? a l le in / e in zig/bloB/? Iediglich/ausschließlich/ einzigund al l e in ein Hauptschalter durchgebrannt.

In diesem Fall ist die Skopuskonstituente unbetonbar. Dies scheint gewisse Konsequenzen für den Test 'Konjunktion PSP - präsupponierender Satz' zu haben.

Es zeigte sich also, daß die Stellungsgesetze iberaus komplex sind. Uneingeschränkt akzeptabel ist nur der TYp l (GP unmittelbar vor der Skopuskonstituente), weitgehend akzeptabel Typ 5 (Skopuskonstituente topikalisiert, GP am Satzschluß), unklar bleibt die Akzeptabilität von Typ 2 (GP unmittelbar nach der Skopuskonstituente), der ergänzende Typ 4 (GP in Distanzstellung) sowie der TYP 3 (GP in der Skopuskonstituente) scheiden völlig aus.

Stellung der Einheit GP + Skopus im Satz:

Offensichtlich ist der Komplex GP + Skopus ohne Folgen für Semantik oder.pragmatische Gebrauchsregeln des Gesamtsatzes frei verschiebbar. Zwischen den einzelnen Positionen bestehen lediglich stilistische Unterschiede: Satzanfang und Satzschluß kann man als bevorzugte Positionen für kontrastiv betonte Konstituenten bezeichnen, handelt es sich doch um die Nachdruckstellen des Satzes. Ob dies allerdings soweit geht, daß die Nachstellung der Partikeln am Satzanfang erleichtert und am Satzschluß erschwert wird, läßt sich nicht mit Sicherheit sagen. - Die GP besitzt gegenüber der Skopuskonstituente eine gewisse Unabhängigkeit. Doch kann dies allein nicht als hinreichender Beweis gegen die Behauptung gewertet werden, daß die GP im Sinne der syntaktischen Konstituenz direkt von der Skopuskategorie dominiert wird (siehe etwa die diskontinuierlichen Konstituenten). Die Vorstellung von einer bloßen Erweiterung eines Satzgliedes durch eine GP scheint weniger syntaktisch als semantisch abwegig. In allen Fällen kann die GP nur die Stellen zwischen den Satzgliedern, also zwischen den größeren Konstituenten, einnehmen, in ihre Skopuskonstituente integriert werden wie andere Erweiterungen (z.B. Adjektivattribute, Zahlwörter) kann sie nicht (mit gewissen Ausnahmen beim Skopustyp NP mit kontrastiv betontem Adjektivattribut).

Syntaktische umformungen:

Zunächst $z u$ den verschiedenen Satzarten. Für die Aussagesatzform stehen die Sätze (1) bis (7). Die Satzfrage belegt (3d): 
(3d) Erschreckte ihn nur/allein/ $\ldots$ der $U$ m z ug ?
Erschreckte ihn $\frac{\text { der }}{U m z}$ ug nur/allein ... ?

Die Variante mit den nachgestellten GPn bewegt sich aus den oben genannten Grïnden an der Grenze der Akzeptabilität.

(3e) Nur/allein/... der $U$ m z ug erschreckte ihn? Wortfrage:

(3f) Wen schreckte nur der $J m z$ u g ?

(2b) Nur was war durchgebrannt?

Während (3f) mit gewissen Einschränkungen durchaus als Frage akzeptabel ist, ist die Erfragbarkeit der Skopuskonstituente selbst nur als Echofrage denkbar (siehe dazu auch die Ausfiuhrungen über den Fragesatztest S. 98!). Imperativ: Er ist natürlich in diesem Zusammenhang nur in jenen Fällen möglich, in denen eine Subjekts-NP auftritt. Da diese aber im Imperativ redundant ist, dürfte eine kontrastive Betonung nur in Ausnahmefällen möglich sein:

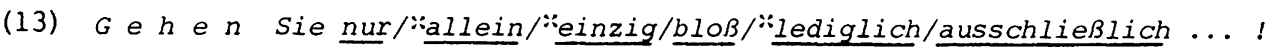
In diesem Normalfall des Imperativs ist die Subjekts-NP Sie nicht Skopus der GP. Vielmehr befindet sich in allen akzeptablen Fällen das Verbum im Skopus, wie der Folgesatz ... schwimmen Sie nicht! verdeutlicht. Bei nur und bloß ist darüber hinaus noch die Verwendung als MP möglich, verdeutlicht durch die Folgesätze: ... ich erlaube es germe. und ... ich lege keinen Wert auf ihre Gegenwart. Die Grïnde für die Inakzeptabilität von allein, einzig usw. werden im Abschnitt iber den Skopus Verb noch behandelt.

(13a) ?Gehen nur/"allein/"einzig/bloß/lediglich/ausschließlich sie! Geh doch nur/bloß/lediglich/ausschließlich Du!

Wodurch die gewisse Inakzeptabilität der beiden Formen bedingt ist, weiß ich nicht zu sagen. In der Regel wird aber wohl in der für diese Außerung allein denkbaren Situation die Formulierung Nur $S i$ e solzen/können gehen! gewählt.

Es zeigt sich also, daß bei allen diesen umformungen eine Distanzstellung der GP ausgeschlossen ist. Dariber hinaus konnte man feststellen, daß beim Imperativ Funktionswechsel zur MP auftreten.

Spaltsatzbildung: Auf sie wurde oben bereits kurz verwiesen, und es zeigte sich, daß die Ergebnisse vollkommen akzeptabel sind, da zum einen die Funktionen der nur-Gruppe und des Spaltsatzes zueinander passen, und da in diesem Fall nicht Teile der Skopuskonstituente abgespalten werden können.

Di.e Umwandlung ins Passiv wird nur der Vollständigkeit halber erwähnt:

(1c) Nur von Aguirre wurden diefährnisse bewältigt. 
Da hierbei keine Konstituenten geteilt werden usw., treten auch keinerlei Schwierigkeiten auf.

Möglichkeiten der Negierung:

Typ 1: "Inkorporierung" der Negation in die Gradpartikel:

(2c) In Wahrheit war nicht nur/allein/ einzig/?einzig und allein/ lediglich/ bloß/?ausschließlich ein $H$ a u $p$ t schalter durchgebrannt, sondern auch/sogar ...

Diese Form der Negation ist völlig regulär. Die inakzeptablen Versionen können nur als idiomatische Beschränkungen gedeutet werden.

TYP 2: NEG zwischen GP und Skopuskonstituente:

(2d) In Wahrheit war nur/bloß/ lediglich/ allein/ ausschließlich/ einzig nicht ein/kein $\mathrm{Ha}$ up t schalter durchgebrannt, sondern ...

Die Verteilung der Akzeptabilitätsurteile legt den Schluß nahe, daß sich in diesen Fällen der Negationsskopus gegen den GP-Skopus durchsetzt; es liegt damit die konjunktionale Verwendung von nur und bloß vor, allein ist nur deshalb inakzeptabel, weil es in konjunktionaler Verwendung Spitzenstellung (bei normaler Wortfolge) fordert. Auch die häufiger auftretende Version

(2e) Alles mögliche war durchgebrannt, nur nicht ein/kein $H$ a u $p t$ schalter.

läßt nur die Deutung von nur als Konjunktion zu. Unterschiede zwischen den beiden Negationsformen konnte ich nicht feststellen.

Typ 3: die Form der starken Negation/Satznegation:

(3g) Nur der $U$ m z ug schreckte ihn nicht.

Diese Form ist chne alle Beschränkungen möglich. Zusammen mit nicht nur ist sle die Diskussionsbasis für den Negationtest. - Die Kombination dieses Negationstyps mit abjekt-Skopus fiuhrt jedoch zu Schwlerigkeiten durch die Stellung des abjektskopus unmittelbar nach dem finiten Verb:

(5a) Eine Peformklinik, in der es nur L u, $x$ us zimmer nicht geben soll...

(6a) ?Sein Besuch in der Nürnberger Klinik gelte (nicht) nur einer $R O u$ $t$ i $n$ e untersuchung (nicht).

Aber auch im Nomalfall entsteht hierbei eine Ambiguität zwischen Satznegation und lokaler Negation, wenn die NEG-Partikel unmittelbar vor dem Objekt-Skopus zu stehen kommt.

Typ 4: Formen der lokalen Negation/Kontrastnegation:

(3h) ?Nicht i hn schreckte nur der $J m z u g$, sondern $d$ e $n$ da. Der Satzrest ist in allen Fällen dieser Art nur als quasi zitiert denkbar, in 
der Regel fällt also der Kontrastakzent auf Umzug weg, oder es ist ein Kontrastsatz notwendig, der sowohl ihn als auch Umzug in einer Parallelkonstruktion kontrastiert: ... sondem den da schreckte nur die $H$ e $i \mathrm{~m}$ reise.Derartige Konstruktionen bewegen sich aber offensichtlich am Rande der Akzeptabilität. - Erstaunlich ist an diesem Negationstyp noch, daß eine Umstellung der kontrastierten Konstituente ohne Veränderung der Semantik nicht möglich ist:

(3i) Nur der 'J $m z$ ug schreckte nicht i.h $n$...

Dieser Satz ist nur bei konjunktionaler Interpretation von nur voll akzeptabel. Die Problematik von mehreren kontrastiv betonten Konstituenten ist in dieser Version verschärft.

Skopus und Fokus

Wie oben im Austauschtest bereits festgestellt wurde, können alle Glieder der Gruppe den Skopus Subjekts-NP (wie natürlich auch Objekts-NP) besitzen (jedenfalls in der Position unmittelbar vor der Skopus-Konstituente). Die Ausfälle bei anderen Stellungstypen bleiben in diesem Zusammenhang ohne Bedeutung. Es konnte auch nicht festgestellt werden, daß die Skopuskonstituenten bestimmte syntaktische oder semantische Merkmale (wie Definitheit, Zählbarkeit usw.) aufweisen muißten. - Zu klären bleibt nun die Frage, anhand welcher Merkmale die genauen Skopusgrenzen festgelegt werden können. Als erstes bietet sich die Position der GP relativ zur kontrastiv betonten Konstituente an. ${ }^{4}$ Die linke Grenze der Skopuskonstituente wird in der Stellung der GP unmittelbar vor der Skopuskonstituente/betonten Konstituente klar markiert, jedenfalls bei diesem Skopustyp. Da die Stellung der GP unmittelbar danach lückenhaft oder doch recht ungewöhnlich ist, können wir aus der Stellung der GP keine sicheren Kriterien für die rechte Skopusgrenze gewinnen. Gänzlich unsicher ist die Skopusabgrenzung bei den - allerdings seltenen - Fällen von Distanzstellung der GPn.

Umgekehrt können wir auch nicht aus der Position der GP auf einen bestimmten Kontrastakzent schließen. Dies mag zwar für die (primäre) mündliche Sprache irrelevant sein, gibt aber m.E. doch wichtige Hinweise auf die steuernden Faktoren im Zusammenhang mit der Skopusfrage. In der folgenden Satzreihe wird die Position der GP wie alle übrigen Merkmale des Ausgangssatzes konstant gehalten, lediglich der Kontrastakzent wechselt: dabei wird (aus Vereinfachungsgründen) der Variationsbereich auf die Konstituente rechts von der GP beschränkt.

(2f) In Wahrheit war nur e in Hauptschalter durchgebrannt, - und nicht $f \ddot{u} \bar{n} f$, wie zunächst berichtet. 
(2f)

In Wahrheit war nur ein $H$ a $u p t$ schalter durchgebrannt,

- und nicht ein $N$ e $b$ e $n$ schalter.

- und nicht ein $R$ e 1 a $i s$.

- und nicht eine $B \circ m b e$ explodi e $r t$.

In Wahrheit war nur ein Haupt $s c h$ a $l t$ e $r$ durchgebrannt,

- und nicht eine Haupt $s i c h$ e $r$ un $g$.

In Wahrheit war nur ein Hauptschal $t$ e $r$ durchgebrannt,

- und nicht eine Hauptschal t ung.

Die erste Variante kann man ausschalten, da es sich dabei um den Skopustyp kontrastiv betontes Numerale handelt (siehe Abschnitt 3.5.1.). Der letzte Typ ist eine recht unwahrscheinliche variante. In der Regel wird hier Haupt $s$ c $h$ a $l$ ter und Haupt $s c h$ a $l$ tung betont. Dadurch können allerdings die einleitenden Sätze in der vorletzten und letzten Version nicht mehr unterschieden werden; doch ist dies gleichgültig, da in solchen Fällen ohnehin ein Korrektursatz vorausgehen oder nachfolgen muß: dies gilt für alle Fälle, in denen nicht eine ganze Konstituente kontrastiert wird, also meist bei der Kontrastierung von Wortteilen. Bleibt also nur die zweite Version (abgesehen von $N$ e $b$ e $n$ schalter). Hier liegt nur eine Verstärkung des normalen wortakzentes vor. Beide verbleibenden Interpretationen des Vordersatzes können ohne expliziten Kontrastsatz auftreten, sind also in diesem Falle äußerlich nicht zu unterscheiden. In einer Äußerungssituation muß aufgrund des $\mathrm{Ko}$ bzw. Kontextes entschieden werden, ob es sich um einen Skopus NP oder Subjekts-NP + infinites Verb handelt: - und nicht sonst irgend etwas (z.B. ein Relais, eine Birne, eine Leitung usw.), was schlimmer wäre.

- und nicht sonst irgendetwas passiert (z. B. ein Krieg ausgebrochen, die Sonne verlöscht), was schlimmer wäre.

In beiden Fällen ist durch die angegebene Wirkung (plötzlicher Stromausfall in ganz Rhodesien) ein ziemlich scharf abgrenzbarer Bereich von möglichen Ursachen, die nach ihrer Bedeutung für die dortige Bevölkerung in schlimmere und weniger schliume eingeteilt werden können, bezelchnet. Aus pragmatischen Gründen nicht gut mögliche Kontrastsätze wären also:

- und nicht die Frau des Kraftwerkleiters.

- und nicht eine Rose verblüht. 5

Die Testserie bestätigt die These, daß die Abgrenzung des Skopus von GPn in den meisten Fällen nicht aufgrund oberflächensyntaktischer Merkmale, sondem nur aufgrund der Merkmale des Ko-/Kontextes möglich ist. Wenn ich hier trotzdem von festen Skopustypen ausgehen, so geschieht dies aus darstellungs- und

5 Man beachte allerdings die stilistische wirkung derartiger pragmatisch abweichender Texte! 
forschungsökonomischen Gründen: ich hypostasiere immer Kontexte, die nur den betreffenden Skopustyp zulassen (bzw. ich gebe sie selber bei).

Zu diesem Abschnitt ist noch ein kurzer Hinweis nötig: Ein Skopus SubjektsNP + infinites Verb wäre, die bisherige Forschung zu den GPn vorausgesetzt, völlig undenkbar, da danach als Skopus nur eine vollständige Konstituente (in der Tiefen- bzw. Oberflächenstruktur) in Frage kommt. Eine mögliche Erklärung wäre, daß sich in diesem Fall der 'Kernsatz' im Skopus befindet, und daß in Wirklichkeit einem übergeordneten Satz angehört: In Wirklichkeit ist es nur so, daß ein Hauptschalter durchgebrannt ist. Diese Analyse hat manches für sich, da in Sätzen mit Dativ und Akkusativobjekten nichts Vergleichbares zu beobachten ist. - Das verläßlichste methodische Hilfsmittel zur Festlegung des GPSkopus bleiben die Kontrastsätze, wie sie oben demonstriert wurden, und wie sie im Laufe der semantischen Analyse noch häufig angewendet werden.

Die in der Literatur mehrfach behauptete Identität von Satzfokus und GPSkopus ließe sich nur in der kombinierten Verwendung von Kontrastsätzen und dem Test der 'natürlichen Antwort' mit einiger Zuverlässigkeit beweisen. Bei Sätzen mit GPn ist jedoch letzterer mit vermutlich uniberwindbaren Schwierigkeiten verbunden. Auf die Frage:

(1d) Wer übersteht die F ährnisse im F ieber?

ist die 'natüliche Antwort': A guirre (übersteht die Fährnisse im Fieber), jedoch nicht: Nur A guirre übersteht die Fährmisse im Fieber. Dies ist eigentlich nur die 'natürliche Antwort' auf die Frage: Wer alles übersteht/welche Leute überstehen die Fährmisse im Fieber? ${ }^{6}$ Dieser Satz enthält eine 'Nichteinzigkeitspräsupposition'. Sie wird explizit durch das nur zurückgewiesen. Es handelt sich dabei un eine recht ausgefallene Verwendungsweise sowohl des Frage-Antwort-Typs als auch von nur, und ich zweifle, ob damit der Kern des Tests der natiurlichen Antwort noch getroffen ist: gerät doch eine PSP in den Fokus der Äußerung. Diese Fragestellung verschärft sich noch bei den ïbrigen GP-Gruppen. - Bei der Identifizierung des Skopus allein aufgrund der Stellung der GP entstehen bei Objekts-NPn noch größere Schwierigkeiten als bei Subjekts-NPn: läßt man den kontrastiven Akzent zunächst unberücksichtigt, so muß keineswegs automatisch die auf die GP folgende ObjektsNP im Skopus stehen. Folgt nämlich die GP auf das finite Verb, so kann ebensogut dieses im Skopus stehen; das hat allerdings seine kontrastive Betonung zur Folge. Auch die gesamte VP kann in diesem Fall den Skopus bilden: in bei- 
den Fällen scheinen allerdings Kontrastsätze unabdingbar.

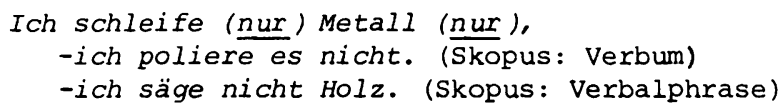

Um derartige Ambiguitäten auszuschließen, werden (zumindest bel schriftlichen Äußerumgen) meist andere Formulierungen gewählt.

$$
\begin{aligned}
& \text { (14a) ... weil ich Metall nur } s c h l \text { e } i f e \text {, nicht poliere. } \\
& \text { (14b) } . . \text { weil ich nur } M \text { e } t a l \text { l schleife, aber nicht Holz säge. }
\end{aligned}
$$

\section{Semantik}

In der Logik und Sprachphilosophie wurde nur + Skopus bislang als a und nicht nicht-a analysiert. Erst Horn (1969) schlug vor, die Giltigkeit von a als präsupponiert und nur die Ablehnung von nicht-a als assertiert zu betrachten. 7 Dieser Analyse ist, soweit ich sehe, bisiang nicht widersprochen worden. Ich werde sie im folgenden und an gleicher Stelle bei den übrigen Skopustypen auf ihre linguistische Relevanz überprïfen und sie gegebenenfalls erweitem oder differenzieren. Für die oben gegebenen Belegsätze sind nach Horn (1969) folgende semantische Analysen anzusetzen:

(1) Nur A g u i r $r$ e übersteht die Fährnisse im Fieber.

PSP: Aguirre übersteht die Fährnisse im Fieber (und)

ASS: Niemand, der nicht Aguirre ist, übersteht die Fährnisse im Fieber.

(2) In Wahrheit war bloß ein $H$ a u $p$ t schalter durchgebrannt.

PSP: In Wahrheit war ein Hauptschalter durchgebrannt (und)

ASS: Nichts, was nicht ein Hauptschalter ist, war durchgebrannt.

Diese Analyse kann nicht voll befriedigen: es fehlt der Bedeutungsaspekt, daß nichts Wichtigeres durchgebrannt/nichts Schlimmeres passiert ist.

Ihn schreckte allein der $J m z u g$.

PSP: Ihn schreckte der'J mzug (und)

ASS: Ihn schreckte nichts sonst.

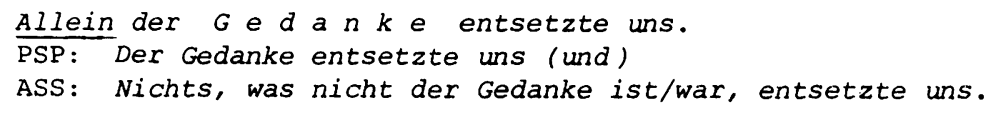

Auch diese Analyse ist aus analogen Grinden umbefriedigend. Doch mag dies an einer ev. abweichenden Funktion von allein liegen.

Negationstest: Es gilt nun, diese Analyse durch den wichtigsten PSPs-Test abzusichern. Um dies aber erreichen zu können, muß erst einmal der 'richtige' Negationstyp herausgefunden werden. Zu diesem Zweck kehre ich vorläufig die

7 Die semantische Analyse in Fillmore (1965) kann ebenfalls in dieser Weise verstanden werden. 
Argumentationsrichtung um und suche eine Negationsform, die eines der oben genannten Bedeutungselemente nicht in seiner Bedeutung umkehrt.

(2g) Nicht nur ein $H$ a u p t schalter war durchgebrannt.

Dieser Satz kann folgendermaßen paraphrasiert werden: Ein Hauptschalter war durchgebrannt, und auch noch anderes war durchgebrannt.

(2h) In Wahrheit war nur nicht ein $H$ a u $p$ t schalter durchgebrannt.

Die Paraphrase hierzu lautet: Nicht ein $H$ a u $p$ t schalter war durchgebrannt, sonderm irgendetwas anderes (war durchgebrannt).

(2i) Nur ein $H$ a up $t$ schalter war in wirklichkeit nicht durchgebrannt.

Die Paraphrase dazu lautet: Ein Hauptschalter war nicht durchgebrannt, (und) alles andere war durchgebrannt. - Der Vergleich mit der Analyse des entsprechenden positiven Satzes zeigt, daß nur die Negation vor der GP, also (2g), ein unverändertes und ein negiertes Bedeutungselement bewirkt. Hingegen kehrt die normale Satznegation die beiden Bedeutungselemente um. - Als nächstes soll nun versucht werden, die oben angeführten Bedeutungselemente zu suspendieren. Suspension der 'Präsupposition' :

(2j) In Wahrheit war nur ein $H$ a u $p$ t schalter durchgebrannt, und möglicherweise nicht einmal dieser/falls überhaupt der.

(3j) "Ihn schreckte allein der $U$ m zug, und möglicherweise nicht einmal dieser.

Dies verweist bereits auf gravierende Unterschiede in der Semantik von nur und arlein.

(3k) "Ihn schreckte nicht allein der $U m z u g$, und möglicherweise nicht einmal dieser.

(4a) ?Allein der $G$ e d a $n k e$ schreckte uns, und möglicherweise nicht einmal dieser.

Hieran kann man unschwer sehen, daß entweder die semantische Analyse unzutreffend ist, oder daß ein anderer Gebrauch von allein vorliegt. Suspension der Assertion:

(31) ?Ihn schreckte allein der $U m z u g$, und möglicherweise auch noch der Berufswechsel.

Dieser Satz klingt seltsam, ohne daß man ihn direkt inakzeptabel nennen könnte. - Bei geringfügiger Änderung des Satzes kann auch noch ein anderer Bedeutungsaspekt suspendiert werden, dessen Charakter vorerst unklar ist: 
(2k) In Wahrheit war nur/bloß/lediglich ein $M i t$ t $e$ l schalter durchgebrannt, "und möglicherweise sogar ein Hauptschalter./und möglicherweise sogar nur ein Nebenschalter.

Man könnte glauben, daß es sich um eine Suspension der Assertion handeln milßte. Dann müßte man aber annehmen, daß diese ASS 'gerichtet' wäre, da sonst das negative erste Ergebnis unverständlich wäre. Das scheint jedoch nicht plausibel. Dazu noch ein weiteres Beispiel mit einer Subjekts-NP, die in eine Skala von Beamtenpositionen eingeordnet werden kann:

(15) Gestern ist nur/bloß/lediglich/“einzig/ “allein/ ausschließlich ein

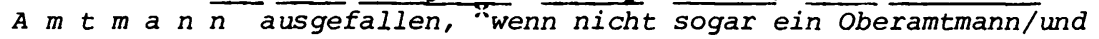
möglicherweise sogar nur ein Oberinspektor.

Dieser Test zeigt folgendes:

a. Zur Markierung einer (unerwartet niedrigen) Stufe auf einer Skala sind allein, einzig und ausschließlich ungeeignet, da sie nur eine quantifizierende Funktion erfüllen können. Hingegen können nur, bloß und lediglich sowohl quantifizierende als auch skalierende Funktion erfüllen. Ungeklärt ist einstwellen, wodurch jeweils die Wahl der einen oder anderen Funktion verursacht wird. b. Es ist unklar, ob die ASS in einer solchen Skala bei nur ausschlieBlich "stärkere" Werte betrifft, oder nur "schwächere", oder alle Werte außer dem bezeichneten.

c. Man kann nicht verkennen, daß die Suspension der Grenze in Richtung auf einen "höheren" Wert in einem Satz mit nur einen Widerspruch produziert: er ist U.U. akzeptabel, da die Suspensionsformel durch ihre unscharfe Formulierung auch die Interpretation zuläßt, daß der Sprecher sich selbst korrigiert (und nicht nur seiner Unsicherheit über den höchstmöglichen Wert Ausdruck gibt) . d. Man kann weiterhin nicht leugnen, daß die erste Suspensionsformel nur für den gleichen Satz ohne nur gilt:

(15a) Gestern ist ein Amtmann ausgefallen, und möglicherweise sogar ein Oberamtmann.

Diese Suspensionsformel kann aber durch die Hinzufügung von nur für nur - Sätze akzeptabel gemacht werden:

(15b) Gestern ist nur ein A $m t$ mann ausgefallen, und möglicherweise sogar nur ein Oberinspektor.

Dies würde heißen, daß eine bestimmte Berufsskala mit einer vorherrschenden Richtung vorliegt, und daß diese von nur + Skopus in der umgekehrten Richtung durchlaufen wird. - Eine semantische Analyse des skalierenden nur mïßte also so lauten:

- präsupponiert wird die Gültigkeit des genannten Wertes. 
- assertiert wird die Unguiltigkeit eines oder mehrerer "objektiv" höherer Werte.

- konversationell impliziert (nach der Grice'schen Maxime der Quantität) wird die Ungültigkeit eines "objektiv" niedrigeren Wertes.

Konjunktionstest: zunächst ein Beispiel für das quantifizierende allein.

(3m) Ihn schreckte allein der $U m z u g$,

- "und ihn schreckte der Umzug. (Wiederholung der PSP)

- und ihn schreckte nichts sonst. (Wiederholung der ASS)

Die Umkehrung des Tests ergibt folgendes Bild:

(3n) Ihn schreckte der'J mzug und ihn schreckte allein der $U m z$ u $g$. (PSP des zweiten Satzes vorneweg behauptet)

Ihn schreckte nichts sonst (als der Umzug) und ihn schreckte allein der $U m z u g$. (ASS vorneweg behauptet)

Während der erste Test die erwarteten Ergebnisse bringt, bedarf der zweite eines kurzen Kammentars: mit der angedeuteten kontrastiven Betonung auf Umzug sind für mich beide Sätze inakzeptabel (der zweite zugegebenermaßen weniger). Durch die Verlagerung des kontrastiven Akzentes auf die GP (allein ist hierfür weniger geeignet, da offenbar bei Betonung die Interpretation als Modaladverb überwiegt) wird der erste Satz voll akzeptabel, beim zweiten verhindert die Schwierigkeit, eine gesicherte ASS-Formulierung zu finden, ein eindeutiges Akzeptabilitätsurteil. - Nun noch ein skalierendes Beispiel:

(15c) Gestern ist nur ein $A m t m$ a $n n$ ausgefallen,

- "und ein Amtmann ist ausgefallen. (Wiederholung der PSP)

- ? und niemand sonst ist ausgefallen. (Wiederholung der ASS?)

- und (es ist) kein Oberamtmann (ausgefallen). (Wiederholung der ASS)

- "und (es ist) kein oberinspektor (ausgefallen). (Wiederholung der konv. IMPL)

(15d) - Gestern ist ein Amtmann ausgefallen, (PSP)

- ?Gestern ist niemand sonst ausgefallen, (ASS?)

- Gestern ist kein Oberamtmann ausgefallen, (ASS)

- "Gestern ist kein Oberinspektor ausgefallen, (konv. IMPL) und gestern ist nur ein $A m t m$ a $n n$ ausgefallen.

Wieder gilt, daß im zweiten Fall die GP nur anstelle von Amtmann kontrastiv zu betonen ist. - Der Test zeigt, daß auch in diesem Fall die quantifizierende Interpretation nicht völlig auszuschließen ist: dadurch würde allerdings die konv. IMPL zu einem Teil der ASS werden, womit die Vorwegnahme wie die Wiederholung akzeptabel werden mïßten: das ist auch in der Tat der Fall. Beides sei auch noch kurz an den beiden Hauptnegationsformen von Sätzen mit nur demonstriert: 
(30) Ihn schreckte nicht allein der $U$ m $z$ ug,

- "und ihn schreckte der Umzug. (Wiederholung der PSP)

- und/sondern ihn schreckten noch andere Dinge. (ASS)

(3p) Ihn schreckte der U mzug und ihn schreckte nicht a 1 le in der der U mzug. (PSP)

Ihn schreckten noch andere Dinge, und ihn schreckte nicht allein der $U m z u g$. (ASS)

(15e) Nur ein $A m t m a n n$ ist gestern nicht ausgefallen, - "und ein Amtmann ist gestern nicht ausgefallen. (PSP)

- und/aber alle anderen sind ausgefallen. (quant. ASS)

(15f) Ein Amtmann ist gestern nicht ausgefallen und nur ein Amtmann. (PSP)

Alle anderen Dienstgrade sind gestern ausgefallen, und nur ein $A m t$ mann ist gestern nicht ausgefallen. (quant. ASS)

Bei dieser Form der Negation setzt sich die quantifizierende gegenüber der skalierenden Interpretation durch. Deshalb noch kurz die oben angewandte Negation:

(15g) Gestern ist nicht nur ein $A m t m$ a $n n$ ausgefallen,

- “und ein Amtmann ist ausgefallen. (PSP)

- ?und noch andere Beamte sind ausgefallen. (quant. ASS)

- ?und höhere Dienstränge sind ausgefallen. (skal. ASS)

- ?und tiefere Dienstränge sind ausgefallen. (konv. IMPL)

(15h) - Gestern ist ein Amtmann ausgefallen, (PSP)

- Gestern sind noch andere Beamte ausgefallen, (quant. ASS)

- Gestern ist ein Oberamtmann ausgefallen, (skal. ASS)

- *Gestern ist ein Oberinspektor ausgefallen, und nicht nur ein $A m t m a n n$ (ist ausgefallen).

Auch die nicht-direkte Negation eines nur - Satzes läßt also das 'factoring' in Bedeutungskomponenten unterschiedlicher qualität zu.

Der monologische Widerspruchstest sei nur der Vollständigkeit halber aufgeführt:

(1e) Lediglich Aguirre übersteht die Fährnisse im Fieber, - "aber Aguirre übersteht die F ährnisse nicht. (NEG dex PSP)

- “aber auch andere Leute überstehen die Fährnisse. (NEG der ASS)

(21) In Wahrheit war nicht nur ein $H$ a u $p t$ schalter durchgebrannt, - “aber es war kein Hauptschalter durchgebrannt. (NEG der PSP)

- "aber es war nichts anderes ausgefallen. (NEG der ASS)

Bessere Ergebnisse bringt dieser Test auf anderen Gebieten:

a. Bei der Trennung der verschiedenen Funktionen von allein.

(4b) Allein der $G$ e $d$ a $n k$ e schreckte uns,

- ※aber der Gedanke schreckte uns nicht. (NEG der PSP)

- () aber uns schreckte auch anderes. (NEG der ASS)

Im Falle der Interpretation im Sinne von schon ist der zweite aber - Satz akzeptabel.

b. Bei der Herauslösung konversationeller Implikaturen: 
(15i) Gestern ist nur ein $A m t m$ a $n n$ ausgefallen,

- *aber ein Amtmann ist nicht ausgefallen. (NEG der PSP)

- :aber auch ein Oberamtmann ist ausgefallen. (NEG der ASS)

- ?aber auch ein Oberinspektor ist ausgefallen. (NEG der konv. IMPL)

Die Verbindung eines Satzes mit der Negation seiner konv. IMPL erhält eher den Charakter eines Rätsels als eines kruden Widerspruchs.

Wesentlich mehr Einsichten in die Semantik und Pragmatik der GPn erbringen die dialogischen Analysen:

(2m) A: In Wirklichkeit war nur ein $H$ a up $t$ schalter durchgebrannt.

Mögliche ablehnende Reaktionen von B:

(2n) B: Nein. (Auch ein Relais war durchgebrannt).

Nein, viel schlimmer: ein Attentat hat die stromversorgung von Rhodesien unterbrochen.

Diese direkten Negationen können offensichtlich nur die ASS von A's Äußerung betreffen. In den beiden Antworten kommt sowohl die quantifizierende als auch die skalierende Interpretation zum Ausdruck.

(20) B: - Nein, ein Hauptschalter ist ja gar nicht durchgebrannt.

- Du gehst von einer falschen Voraussetzung aus: es ist nämlich gar kein Hauptschalter durchgebrannt.

- Aber es ist doch gar kein Hauptschalter durchgebrannt.

- Nicht einmai das!

Alle diese Entgegnungen betreffen die PSP. Ablehnungen einer PSP sind insofern problematisch, als es zum einen keine verbindliche Form dafür gibt; zum andern muß man irmer wieder feststellen, daß die verlangte explizite Zurückweisung einer PSP (im Gegensatz zur ASS, die allein mit nein zurückgewiesen werden kann) von vielen Sprechem im Falle von nur nicht für notwendig gehalten wird, und zwar, wenn ich richtig beobachtet habe, besonders bei skalierender Interpretation.

(2p) B: - Schlimm genug.

- Ich finde einen durchgebrannten Hauptschalter schlimmer als eine pevolution der F relimo.

- Ich kann deine Erleichterung gar nicht verstehen.

Diese häufig zu beobachtenden Reaktionen lehnen die vom Sprecher A etablierte Bezugsskala, bzw. die Einordnung bestimmter Ereignisse in diese Skala ab.

(2q) B: - Die Sache ist noch viel weniger aufregend. Der, Kraftwerksmechaniker hat mal wieder gepennt.

Diese Reaktion mu man wohl als Widerspruch gegen die konv. IMPI bei skalierender Interpretation werten.

Dieselbe Gruppierung ist für zustimmende Reaktionen möglich. 
B: - Ja, (es ist tatsächlich sonst nichts passiert.) (ASS)

- Ja, es war wirklich nichts Schlimmeres/kein Attentat.

- Ja, ich habe auch gehört, daß es ein Hauptschalter war. (PSP)

- Ja, die Voraussetzung, daß ein Hauptschalter durchgebrannt ist, stimmt, aber...

- Ich finde das auch nicht gar zu tragisch. (Skala selbst)

- So schlimm wie eine Revolution der Frelimo ist das nun wirklich nicht.

- Na, deine Erleichterung kann ich verstehen.

- Ja. Eine weniger aufregende U rsache hatte es allerdings auch nicht. (?) (konv. IMPL)

Dies sei auch noch kurz, reduziert auf ablehnende Antworten, an den wichtigsten zwei Negationsformen dieses Satzes demonstriert:

(2s) A: ... Nicht nur ein $H$ a u $p$ t schalter war durchgebrannt.

B: - Nein, sonst war nichts durchgebrannt. (ASS)

- ?Irrtum, sonst ist nichts durchgebrannt.

- ?Doch!

- Du irrst dich: ein Hauptschalter war gar nicht durchgebrannt. (PSP)

- Ja. Aber ich wußte gar nicht, daß ein Hauptschalter durchgebrannt war. (PSP)

- Was, hat's noch Schlimmeres gegeben?

- Find' ich schon schlimm genugl (Skala selbst)

(2t) A: Nur ein $H$ a u $p$ t schalter war nicht durchgebrannt.

B: - Nein, es ist sonst gar nichts durchgebrannt. (ASS)

- Aber auch der war doch durchgebrannt. (PSP)

- Doch! (Zweifelhaft, ob als PSP-Protest möglich)

- Ich finde das'auch nicht schlimm. (Skala selbst?)

- Aber das ist doch eine Katastrophe. (Skala selbst)

Die letzten beiden Reaktionen, zu denken im Kontext:

(2u) Wir haben erreicht, daß alle Schaltelemente durchbrannten. zeigen, daß auch bei nur...'nicht skalierende Interpretation möglich ist.

Vielleicht kann eine kurze Zusammenfassung der Ergebnisse der semantischen Tests etwas mehr Ubbersicht bringen: für diesen Skopustyp beweisen die Tests in weiten Bereichen die thertragbarkeit der Horn'schen Analyse auf das Deutsche. Probleme ergeben sich nur daraus, daß die angebliche PSP nicht so stark "backgrounded" zu sein scheint, daß sie nicht direkt verneint werden könnte; ferner aus der großen Zahl von möglichen Negationsformen, die die Anwendung des Negationstests erschweren. - Es sei deshalb gestattet, die Situation in einfachen Diagrammen zu verdeutlichen.

1. Quantifizierende Interpretation: sie ist möglich bei nur $x$, nicht nur $x$, nur $x$... nicht. Zur Darstellung werden Venn-Diagramme verwendet. Dabei sind zwei Mengen zu unterscheiden: die Gesamtmenge der durch die Skopuskonstituen- 
te von nur bestimmten typgleichen NPn, und die echte Tellmenge der von der Skopus-NP bestimten NPn: diese muß nicht eine Einemenge sein. Die von der jeweiligen Kombination der Negation mit nur als gültig bezeichneten Tellmengen werden schraffiert, die als ungültig bezeichneten werden weiß belassen.

PSP = die vom nur - Skopus bezeichnete echte Teilmenge.

ASS = die Schnittmenge von Gesamtmenge und präsupponierter Menge.

nur $x$

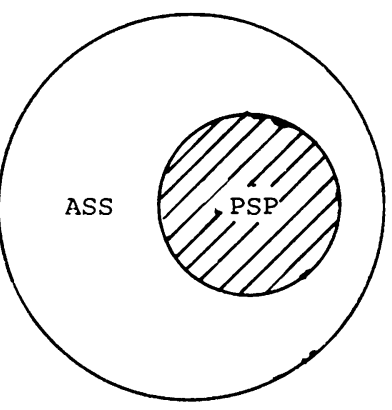

nicht nur $x$

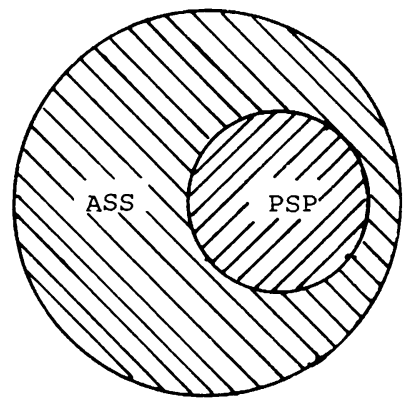

$\operatorname{nur} x \ldots$ nicht

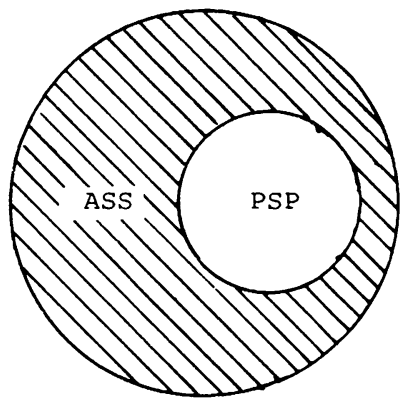

2. Skalierende Interpretation: grundsätzlich kann der Sachverhalt durch die oben verwendeten Venn-Diagramme dargestellt werden. Doch empfiehlt sich eine aussagekräftigere Darstellung. Dabei wird die durch das Zusammenwirken der GP und ihrer Skopuskonstituente definierte Skala durch eine skalierte senkrechte Linie dargestellt. Die Richtung der Skala, jeweils relativ zu der beigegebenen Normalskala, wird durch Pfeilung der Iinie verdeutlicht. Der jeweils durch die Formulierung bezeichnete Basiswert wird durch einen querpfeil identifiziert. Grenzmarkierungen durch irgendwelche Bedeutungselemente sollen kurze senkrechte Pfeile mit Index verdeutlichen, gültige oder ungültige Skalenberelche werden durch indizierte Klammern (mit + oder - respektive) iberdeckt.

normale Skala

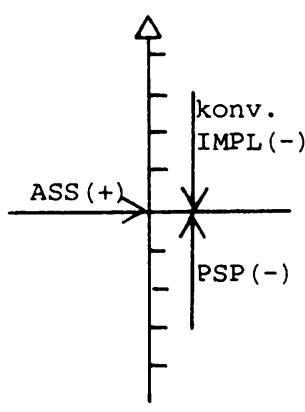

nur-x Skala

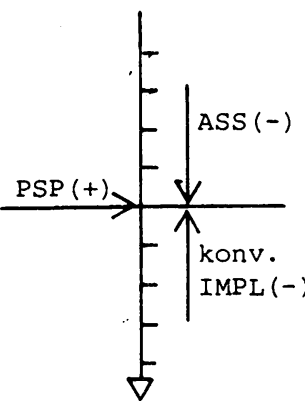

$$
\text { nicht-nup-x Skala }
$$

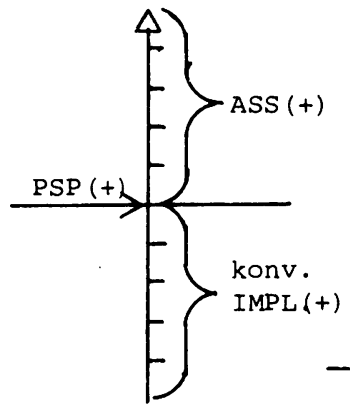

?nur $x \ldots$ nicht Skala 
Es bleibt noch darauf hinzuweisen, daß die Differenzierung zwlschen Skalen, deren Werte sich gegenseitig ausschließen, und Skalen, deren Werte sich nicht gegenseitig ausschließen, wie sie z.B. für den Skopustyp Prädıkat notwendłg ist, bei diesem Skopustyp noch keine Bedeutung hat. Die gegebene Darstellumg kann also noch keinen allgemeinen Aussagewert beanspruchen.

Bei den Elementen der Gruppe sind folgende Spezialisierungen zu registrieren: nur, bloß, lediglich: sie sind sowohl in quantifizlerender wie in skalierender Funktion möglich.

allein, einzig, ausschließlich: sie sind nur in quantifizierender Funktion möglich.

3.2.1.2. auch - Gruppe.

1. Subjekts-Nominalphrase:

(1) Nun beklagt also auch der $s p$ i e ge 1 den armen Steuerzahler.

(2) Auch $z$ we $r g e$ haben klein angefangen.

(3) Dagegen hätte auch ein Chris t demokrat nichts einzuwenden.

(4) Diese würde kann auch ein $M$ ö $r d$ e $r$ nicht verwirken.

(5) Auch Mi cha e I schreibt.

2. Genitiv-, Dativ-, Akkusativ-Objekts-Nominalphrase:

(6) Die Knüppelhiebe, die in westdeutschen Städten prasselten, demolierten auch die $I n n$ e $n$ welt (bei den uniformierten Tätern).

(7) Um den "besonderen Status der Politischen" nicht zu betonen, setzt Ost-Berlin zugleich auch $K r i m i n e l 1$ e auf freien $F$ uß.

(8) Britanniens Monarch Edward VII., nie abgeneigt, Damen zu verführen, die auf seinen priváten Festen tanzten, ließ auch den $B$ ü $r g$ e $n$ ihre $F$ reiheit.

Klassenbildung/Austauschbarkeit

Eine Ersetzung durch Partikeln mit gleicher oder doch sehr ähnlicher Semantik scheint nicht möglich, abgesehen davon, daß man in (4) sogar bzw. selbst einsetzen kann. Von den übrigen Wörtern, die man als gleichbedeutend mit auch betrachtet, können in den oben angebotenen syntaktischen Mustern keine eintreten:

(1a) Nun beklagt also "ebenso/"ebenfalls/"gleichfalls/"ferner ... der $s p i$ e $g$ e $l$ den armen Steuerzahler.

Die Elemente der ïbrigen. GP-Gruppen können frei eingesetzt werden. Mit welteren Austauschmöglichkeiten steht es nicht anders als bei der nur - Gruppe: erst und schon usw. können in allen auftretenden Positionen eingesetzt wer- 
den, vielleicht, leider usw. jedoch nur in der Position hinter dem finiten Verbum.

Stellungsgesetze

TYp 1: GP unmittelbar vor der Skopuskonstituente. Siehe dazu die Sätze (1)-(8). Typ 2: GP unmittelbar nach dem Skopus.

(2a) $\because z$ we $r g$ auch haben klein angefangen.

Eine Überprüfung zeigt, daß die Nachstellung von auch (bei kontrastiv betonter Skopuskonstituente) in keinem Fall möglich 1st. Dies gilt auch für die oben genannten semantisch verwandten Elemente.

Typ 3: GP in der Skopuskonstituente. Wie der folgende Beispielsatz zeigt, fällt auch diese Möglichkeit aus:

(3a) "Dagegen hätte ein auch $C h r i s t$ demokrat nichts einzuwenden. Typ 4: GP in Distanzstellung vor und nach der Skopuskonstituente.

(1b) ("'Auch) nun ("auch) beklagt ("auch) also der $S p i$ e g e 1 den armen ("auch) Steuerzahler.

Auch weitere Tests erhärten nur diesen völlig negativen Befund. Bei der scheinbaren Ausnahme

(3b) Auch/ferner/ebenso/überdies/obendrein hätte dagegen ein Chri s $t$ demokrat nichts einzuwenden.

zeigen die angeführten Austauschelemente wie auch die Unmöglichkeit eines Folgesatzes wie - nicht nur ein Sozialdemokrat, daß es sich hier um die konjunktionale Verwendungsweise von auch handelt.

Typ 5: Skopuskonstituente topikalisiert, GP in Distanzstellung.

(2b) $: z$ w e $r g$ e haben auch klein angefangen.

Bei kontrastiver Betonung des auch kann aber sofort volle Akzeptabllität erreicht werden:

(2c) Zwe $r g$ e haben $\underline{a u c h}$ klein angefangen.

In dieser Position ist auch durch betontes ebenso, ebenfalls, gleichfalls ersetzbar. Die restlichen oben zitierten Elemente, also fermer, überdies, obendrein können nur konjunktional verwendet werden. - Betontes auch und seine Ersetzungsmöglichkeiten können darüberhinaus jeweils unmittelbar hinter der (dann wohl schwächer betonten) Skopus-NP stehen, soweit diese nicht satzeinleitend, sondern nach dem finiten Verb auftritt (Inversion):

(2d) Klein angefangen haben $z$ we $r g e$ a $u c h$. 
Komplikationen entstehen jedoch, wenn der Satz eine Negation enthält:

(4a) Diese Würde kann ein $M$ ö $r d$ e $r \underline{a u c h}$ nicht verwirken.

(3c) Dagegen hätte ein Christdemokrat a $\underline{\mathrm{a} C h}$ nichts einzuwenden.

In allen Fällen befinden sich eher die Konstituenten am Satzanfang im Skopus von auch. Auch für (2d) ist dies bei passendem Kontext möglich, so daß nicht die Negation, sondern die Spitzenstellung einer bestimten Konstituente das entscheidende Kriterium wäre.

Typ 6: Kontrastiv betonte GP. Eine der Möglichkeiten wurde bereits im vorausgehenden Abschnitt behandelt. Die andere ist das formelhafte Hans und a $u c h$ Hans ..., das nicht in gleicher Weise wie die parallele Konstruktion bel nur akzeptabel ist. -

Zusammenfassend kann man zu den Stellungsgesetzen von auch in Relation zur Skopuskonstituente Subjekts-NP sagen, daß offenbar nur zwei mögliche Positionen existieren: 1. GP unmittelbar vor der kontrastiv betonten Skopuskonstituente; 2. GP kontrastiv betont, und zwar a. in Distanzstellung nach der topikalisierten Skopus-NP; b. unmittelbar nach der nicht topikalisierten Skopus-NP. Syntaktische Umformungen

Zunächst zu den Satzarten. Für die Aussagesatzform stehen die Sätze (i)-(6) . Fragesatz:

(2e) Haben auch $z$ we $r g e /$ zwerge a $u c h$ klein angefangen?

(1c) Wann beklagt auch der $s p i$ e ge l / der Spiegel a uch die armen Steverzahler?

(1d) Auch der $S p$ i e g e 1 beklagt den armen Steuerzahler/ der Spiegel beklagt ( $\underline{a \mathrm{u} c h}$ ) den armen Steverzahler ( $\underline{a \mathrm{u} c h}$ ) ?

In keinem Fall kann ein Funktionswechsel beobachtet werden. Interessant ist, daß in jedem Fall auch eine Altemative mit kontrastiv betonter GP existiert. Die betonte GP (die auch hier durch ebenfalls, gleichfalls usw. ersetzt werden kanni steht (wegen der Inversion) rinter ihrer Skopuskonstituente. Bel größerer Distanz, wie bei (ld) ist Skopusambiguität wohl nicht zu vermeiden. In gesprochener Sprache wirken aber Akzent und Kontext meist disambiguierend. Bei dieser GP-Gruppe scheint es auch angezeigt, die Form des Ausrufs kurz zu betrachten:

(9) Du bist aber auch ein Einfaltspinsell

Folgende Akzentverteilungen sind hierbei möglich: betontes auch, Skopus du; Einfaltspinsel betont, damit auch Skopus; ohne registrierbaren kontrastiven Akzent, tberwiegen der "entrüsteten Intonation": dann erfüllt auch die Funktion 
einer $\mathbb{P}$, sichtbar daran, daß kein Kontrastsatz möglich ist: ... nicht nur ein Zappelphilipp. Dieser Funktionswechsel ist nur in der Position nach dem finiten Verb, ev. mit dazwischengeschobenem aber möglich (ev. können auch Pronomina dazwischentreten).

Imperativ:

(10) Bitte, fangen auch $S i$ e / Sie $\underline{a u c h}$ klein an.

Die Ujberprüfung der verschiedenen Satztypen erbringt also keine neuen Stellungsgesetze und nur einen einzigen Fall von Funktionswechsel.

Spaltsatzbildung: sie liefert ein von der nur - Gruppe deutlich verschiedenes Ergebnis:

(4b) “Es sind auch Mö $r$ d e $r$ / "auch Mö $r d$ e $r$ sind es/ "Mörder sind es $a$ u $C h$, die diese würde (nicht) verwirken können.

Dies ist in der Semantik von auch begründet: während nämlich auch die nichtEinzigkeit der Skopuskonstituente präsupponiert, dient die Spaltsatzbildung der Betonung der Einzig(artig)keit der herausgehobenen Konstituente (wie auch der kontrastive Akzent allein).

Negationsmöglichkeiten

TYP 1: NEG + GP + Skopuskonstituente.

(3d) :Dagegen hätte nicht auch ein Chris t demokrat etwas einzuwenden. Diese Parallele zu nicht nur gibt es bei auch nicht. Das nicht auch in Fragesätzen kann dies nicht widerlegen, da es sich bei diesem nicht um eine reine Fragepartikel handelt. Plausible Gründe für diese "Lücke" kann ich nicht nennen. Typ 2: Negation zwischen GP und Skopuskonstituente.

(3e) 'Dagegen hätte auch nicht ein $C h r$ is t demokrat etwas einzuwenden. Dagegen hätte auch kein $C h r i s t$ demokrat etwas einzuwenden.

(11) Das erlaubt dir niemand, weder ein Sozialdemokrat, noch ein F reidemokrat, und auch nicht ein $C h r i s t$ demokrat.

Mir ist nicht völlig klar, ob die zweite Fassung von (3e) und Satz (11) wirklich akzeptabel sind. Auf jeden Fall scheint zwischen auch und der Skopuskonstituente (außer bei Reihungen wie in (11)) nur dann eine Negation möglich zu sein, wenn letztere indefinit ist. Normalerweise werden indefiniter Artikel und Negation dann zu kein vereinigt, es sei denn in der Konstruktion niemand, auch nicht. Die Gesetzmäßigkeiten lassen sich in diesem Falle besser an Objekt-NPn als Skopus zeigen:

(7a) ?Um aber den "besonderen Status der Politischen" nicht zu betonen, setzt ost-Berlin auch nicht Krimine 1 I e auf freien $F u$. 
(7b) Ost-Berlin setzt auch nicht $K r i m i n e l l$ e auf freien $F$ uß, um den besonderen Status der Politischen nicht zu betonen.

(7c) "Auch nicht $K r i m i n e l 1$ e setzt Ost-Berlin auf freien $F$ uß, um den besonderen Status der Politischen nicht $z u$ betonen.

(7d) Auch setzt Ost-Berlin nicht Kriminelle auf freien $F u ß$, sondern ...

Folgende Grundregeln können für die Kombination auch + nicht formuliert werden: 1. Die unmittelbar vor einer NP stehende NEG ist eine lokale oder Kontrastnegation. Sie darf nicht mit dem indefiniten Artikel zu keine vereinigt werden (obwohl man das oft beobachten kann) und verdrängt auch aus seinem Skopus. Dadurch ist auch nur mehr mit Satzskopus möglich, vorausgesetzt daß es sich in einer dafür geeigneten Stellung befindet, wie in (7b) und (7d). Allerdings ist (7c), trotz Erfüllung beider Forderungen inakzeptabel; als Erklärung bleibt nur die Topikalisierung von nicht Kriminelle. Beachte aber:

(7e) Auch Nicht-Kriminelle setzte Ost-Berlin auf freien $F u ß$.

Hierbei liegt auch in GP-Funktion mit einem NP-Skopus (mit inkorporierter Negation) vor.

2. Der Kamplex auch + nicht steht zwischen einem finiten Verbum und einer NP. Die Negation ist dann Satz-NEG und muß als solche in einen folgenden indefiniten Artikel inkorporiert werden. auch ist in diesem Fall nur mit Satzskopus (bzw. konjunktional) interpretierbar: z.B. (7b).

(7f) Ost-Berlin setzt auch keinen Kriminellen auf freien $F u ß$.

(7g) Ost-Berlin setzt auch nicht die Kriminellen auf freien $F$ uß.

Damit wäre auch die Inakzeptabilität von (7a) erklärt.

Typ 3: Starke Negation/Satznegation.

(12) $\underline{\text { Auch }} R$ i e $s$ e $n$ haben nicht groß angefangen.

Zusammen mit (4) zeigt dieser Satz die völlig nomale Situation bel dieser Negationsart.

Typ 4: Lokale/kontrastive Negation.

(le) Nun beklagt also auch der $s p$ i e ge l nicht den armen, $s t$ e u e $r$ zahler, sondern...

Nicht vernachlässigen dürfen wir einen knappen thberblick über die möglichen Negationsformen bei betontem $a u c h$.

(2f) “Kein $z$ we $r g$ hat a $u c h$ klein angefangen/ $n i c h t$ ein $z w$ e $r g$ hat auch klein angefangen.

$\because z$ w e r ge haben nicht a uch klein angefangen.

$\because z$ w e $r g$ e haben a $u c h$ klein nicht angefangen.

$z$ we $r g e$ haben $\underline{a c h}$ nicht klein angefangen. 
Es zeigt sich also auch hier, daß ausschließlich die Satz-NEG im zusammenhang mit der GP auch akzeptabel ist.

Skopus und Fokus

Wie schon für nur und seine Synonyme festgestellt, lassen sich auch für auch keine bestimmten Bedingungen bezüglich der semantischen oder syntaktischen Eigenschaften der Skopuskonstituente ausmachen. - Die Problematik eines Versuchs, den jeweiligen Skopus aufgrund der Stellungsmöglichkelten von auch abzugrenzen, verschärft sich durch die Tatsache, daß zwar die vordere Grenze durch die Position von auch unmittelbar vor der Skopuskonstituente zweifelsfrei gegeben ist, und daß dann der kontrastive Akzent den Skopuskem markiert, daß aber andererseits die "rechte" Grenze wegen des Fehlens der Nachstellung bei unbetontem auch sowie der Möglichkeit zur Kontaktstellung bei nachgestelitem betontem auch nicht festgelegt werden kann. - Die Möglichkeiten der Akzentpositionierung bei betontem Skopus gehen nicht über das bei der nur - Gruppe Gesagte hinaus. - Die einzig sichere Methode der Skopusabgrenzung bieten auch hier wieder Kontrastsätze:

(13) A: Nur $R i$ e s e $n$ fangen wirklich ganz klein an. B: Oh nein, auch $z$ we $r g e$ fangen ganz klein an.

(13a) Auch $z$ we $r g$ e fangen klein an, nicht nur Riesen.

Hierbei müssen die kontrastierenden Sätze bis auf die Skopuskonstituenten identisch sein (Versicherungsformeln usw. Sind davon auszunehmen). Die Möglichkeiten zur Kontrastierung von zwei Skopuskonstituenten sind dabei beschränkt, ohne daß man dafür sichere semantische Kriterien nennen könnte:

(13b) Auch $z$ we $r g$ e fangen klein an, "nicht nur Schreibtische. Hingegen:

(13c) $\underline{\text { Auch }} z$ we $r g$ e fangen klein an, nicht nur multinationale Konzerne. Trotzdem scheinen die Regeln eng genug zu sein, um auch eine nichtkontrastierte Außerung wie etwa den Filmtitel

(2) Auch $z$ we $r g e$ fangen klein an.

richtig zu interpretieren, d.h. die Menge der möglichen Kontrastsätze in etwa abzuschätzen, bzw. die sie definierende Grundstruktur.

Bei abjekt-NPn können auch Vollverben unmittelbar vor der GP auch zu stehen kommen. Dađurch entsteht in Einzelfällen Skopusambiguität:

(14) Er dankte auch dem Entgegenkommenden.

Sowohl das Verbum als auch das Dativobjekt, aber auch die gesamte VP können 
Skopus von auch sein. Die Position von GPn unmittelbar nach dem finiten Verb erweist sich also schon hier als besonders vielschichtig. - Hingegen entstehen keine Skopusambiguitäten, wenn auch nach einer anderen NP zu stehen kommt: das Verbot der Nachstellung von auch bei NP-Skopus verhindert dies.

(15) Er spielte den Ball auch dem L i b e $r \circ \quad z u$.

Semantik

Wiederum stehen frïhere sprachphilosophische und logische Analysen von auch $x$ im Sinne von $x$ und nicht-x der von Horn (1972: 80) vorgeschlagenen präsuppositionellen Analyse gegenüber, die ins Deutsche ïbertragen etwa lauten mu:

(16) $\frac{\text { Auch }}{H}$ a $n$ s ging weg.

ASS: Hans ging weg.

PSP: Wenigstens einer, der nicht Hans ist, ging weg.

Nach dieser nur quantifizierenden Bedeutungsanalyse hätten die Sätze (1) bis

(5) folgende Semantik:

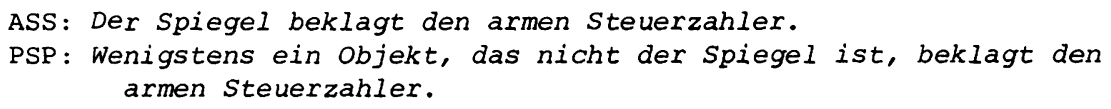

Die Formulierung der PSP ist in dieser Fassung sehr unzulänglich. Es muß allerdings bezweifelt werden, daß eine einheitliche Fassung für alle denkbaren Anwendungssituationen überhaupt möglich ist.

(2) ASS: Zwerge haben klein angefangen.

PSP: Wenigstens ein objekt, das kein zwerg ist, hat klein angefangen.

Das Überraschungsmoment der Aussage, das etwa durch die Einsetzung von sogar gekennzeichnet werden kann, bleibt in dieser Analyse völlig unberücksichtigt.

(3) ASS: Ein Christdemokrat hätte nichts dagegen einzuwenden.

PSP : Wenigstens ein weiterer Mensch, der kein Christdemokrat ist, hätte nichts dagegen einzuwenden.

Der in (3) ganz deutliche skalierende Bedeutungsaspekt kormt in der Analyse überhaupt nicht vor.

(4) ASS: Ein Mörder kann diese Würde nicht verwirken.

PSP: Wenigstens einer, der kein Mörder ist, kann diese würde nicht verwirken.

Bei der Kambination von auch und nicht sind die Mängel einer nur quantifizierenden Interpretation besonders deutlich. Hingegen scheint sie für (5) völlig auszureichen:

(5) ASS: Michael schreibt.

PSP: Wenigstens einer, der nicht Michael ist, schreibt. 
Suspensionstest

Suspension der Präsupposition:

(2g) Auch $Z$ we rge haben klein angefangen, - "und möglicherweise sogar niemand sonst.

- "und möglicherweise sogar nur diese allein.

(4c) Diese würde kann auch ein $M$ ö $r d$ e $r$ nicht verwirken,

- "und möglicherweise sogar niemand sonst.

- "und möglicherweise sogar nur ein Mörder allein.

(5a) Michael schreibt a $\mathrm{uch}$,

- und möglicherweise sogar niemand sonst.

- und möglicherweise sogar nur er allein.

Dies bestätigt die bereits von Horn (1972) erwähnte Unmöglichkeit, die jeweiligen PSPen von engl. also/even zu suspendieren. Als Ursache komt in Frage, daß nur bei skalierenden Interpretationen die Suspension eines Bedeutungsaspektes in Richtung auf einen höheren oder niedrigeren Wert möglich ist: dies würde zudem erklären, warum in der Suspensionsformel sogar oder nicht einmal vorkommen müssen.

Suspension der Assertion:

(1f) Nun beklagt also auch der $s p i$ e ge $l$ den armen Steuerzahler,

- " und möglicherweise nicht einmal der spiegel.

- "falls er ihn überhaupt beklagt. (?)

(5b) Michael schreibt a u ch / e b e n fa 1 is ...,

- "und möglicherweise schreibt nicht einmal er.

- "falls er überhaupt schreibt.

Offenbar entstehen auf diese Weise nur unaufhebbare Widersprüche.

Die Suspension einer konv. IMPL würde voraussetzen, daß wir uns auf eine skalierende Interpretation beschränken: in ihr wird ein bestimmter wert als gültig assertiert, "tiefere" Werte werden als gültig präsupponiert und die Ungültigkeit "höherer" Werte konversationell impliziert (Maxime der Quantität).

(4d) Diese würde kann auch ein $T \circ t s c h \perp$ ä $g$ e $r$ nicht verwirken, und möglicherweise nicht einmal ein Mörder.

(17) Auch ein Amt ma $n$ war ausgefallen, und möglicherweise sogar ein Oberamtmann.

(4e) ?Ein Mörder verwirkt diese Würde, ein Totschläger kann sie a uch verwirken, und möglicherweise sogar ein nieb.

Daraus kann man schließen, daß die Suspensionsformel tatsächlich nur zur Anwendung auf skalierend interpretierbare Sätze geeignet ist: sie kann hierbei nicht dazu dienen, einen bestimmten Wert aufzuheben, sondern nur dazu, die unsicherheit über eine bestimmte Grenzziehung, geschehe sie nun in der ASS, PSP oder konv. IMPL, auszudricken, also eine bestimmte Grenziberschreitung zu er- 
möglichen. Da bei auch nur die konversationelle Implikatur eine Grenze markiert, hingegen die ASS einen Wert und die PSP einen Bereich als gültig bezeichnet, kann nur die konv. IMPL suspendiert werden. - Dies läßt für (3) und

(4) folgende Interpretation zu:

(3) ASS: Christdemokraten haben nichts dagegen einzuwenden.

PSP: Leute, die weniger rigoros/konservativ ... sind als Christdemokraten, haben nichts dagegen einzuwenden.

konv. IMPL: Leute, die rigoroser/konservativer... sind als christdemokraten, haben etwas dagegen einzuwenden. (?)

(4) ASS: Ein Mörder kann diese würde nicht verwirken.

PSP: Leute, die weniger verbrochen haben, können diese würde nicht verwirken.

konv. IMPL: Leute, die schlimmer sind als Mörder, können diese würde verwirken.

Die angebotenen Formulienungen für die konv. IMPL sind deswegen problematisch, weil in solchen GP-Sätzen häufig bereits der höchste Wert der jeweiligen Skala assertiert wird.

Konjunktionstest:

(1g) Nun beklagt also auch der $s$ p i e g e 1 den armen Steuerzahler,

- "und andere Zeitschriften/Personen ... beklagen den armen Steuerzahler. (quant. PSP)

- "und der Spiegel beklagt den armen Steuerzahler. (ASS)

Die umkehrung erbringt für die PSP ein akzeptables Ergebnis, für die ASS ein nichtakzeptables Ergebnis, verläuft also völlig regulär.

Diese Würde kann auch ein $M$ ö $r$ e $r$ nicht verwirken,

- : und weniger schlimme Verbrecher können sie nicht verwirken. (PSP)

- " und diese Würde kann ein Mörder nicht verwirken. (ASS)

- ' und Leute, die schlimmer sind als Mörder, können diese Würde verwirken. (konv. IMPL)

- Leute, die weniger schlimme Verbrecher als Mörder sind, können diese würde nicht verwirken, (PSP)

- 'Diese Würde kann ein Mörder nicht verwirken, (ASS)

- “Leute, die schlimmer sind als Mörder, können diese würde verwirken, (konv. IMPL)

und diese würde kann auch ein $M$ ö $r$ d e $r$ nicht verwirken.

Zugegebenermaßen ist Satz (4) nicht ideal für die Demonstration dieser Gesetzmäßigkeiten. Mörder repräsentiert i.d.R. den höchsten Grad der damit verbundenen Skala, so daß eine konv. IMPL nicht mehr sehr sinnvoll ist. Die weitergehende Frage ist allerdings, ob nicht die Formullerung auch ... nicht in diesen Zusammenhängen in jedem Fall eine "höchste Stufe" anzeigt. 
aber - Test/monologischer Widerspruchstest:

(2h) Auch $z$ we $r g e$ haben klein angefangen, - aber niemand sonst hat klein angefangen. (NEG der PSP)

- "aber zwerge haben nicht klein angefangen. (NEG der ASS)

Und noch ein Beispiel für die negierte Form mit skalierender Interpretation:

(4h) Diese würde kann auch ein $M$ ö $r d$ e $r$ nicht verwirken,

- “aber weniger schlimme Verbrecher können sie verwirken. (NEG der PSP)

- *aber ein Mörder kann sie verwirken. (NEG der ASS)

- “aber schlimmere Verbrecher können sie nicht verwirken. (NEG der konv. IMPL).

Einen geringeren Grad der Inakzeptabilität bei der konv. IMPL kann ich hier allerdings nicht erkennen.

Dialogische Tests:

(2i) A: Auch $z$ we $r g e$ haben klein angefangen.

B: - Nein. (Zwerge haben nicht klein angefangen). (ASS-Verneinung)

- "Nein. Andere haben nicht klein angefangen. (verunglückter PSP-Protest).

- Aber es fängt doch sonst niemand klein an. (PSP-Protest)

- Aber das ist doch klar. (Hinweis auf die "be relevant" - Maxime)

- Ja. Zwerge haben klein angefangen. (ASS-Zust.immung)

- Ich bin zwar auch der Meinung, daß eine Menge Leute klein anfängt, aber bei Zwergen habe ich da so meine Zweifel. (PSP-Zustimmung + ASS-Verneinung)

(4i) A: Diese würde kann auch ein $T \circ t$ schläger nicht verwirken.

B: - Doch. Das kann er durchaus. (ASs-Verneinung)

- Aber viel weniger schlimme Verbrecher können sie doch verwirken. (PSP-Protest)

- Aber ein Mörder kann sie doch auch nicht verwirken. (Zurückweisung der konv. IMPL)

- Nein, das kann er nun wirklich nicht. (ASS-Zustimmung)

- Na ja, für weniger schlimme Verbrecher kann ich das ja akzeptieren. (PSP-zustimmung)

- Aber das heißt doch, daß ein Mörder z.B. diese würde verwirken kann. (Vergewisserungsfrage nach der konv. IMPL)

Die Tests haben die quantifizierende Analyse prinzipiell bestätigt, aber auch die Erweiterung um eine skalierende Version als notwendig erwiesen.

Die folgenden Diagramme sollen wieder eine übersichtliche Zusammenfassung der Ergebnisse bringen. Für sie gelten wieder die in 3.2.1.1. S. 105 formulierten Grundsätze und Erläuterungen.

1. Quantifizierende Interpretation: hier existieren nur die Möglichkeiten auch $x / x \ldots$ a uch, auch $x \ldots$ nicht. Eine präsuppositionsneutrale Negationsfom nicht auch ist zwar denkbar, mir sind aber keine akzeptablen Beispiele bekannt. 
auch $x$

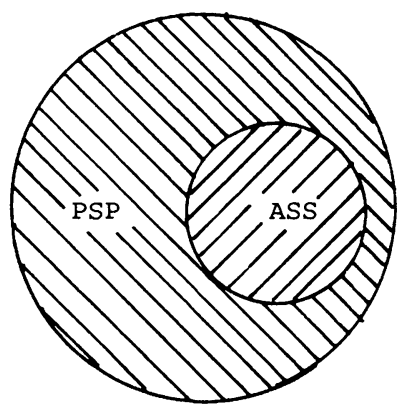

auch $x$... nicht

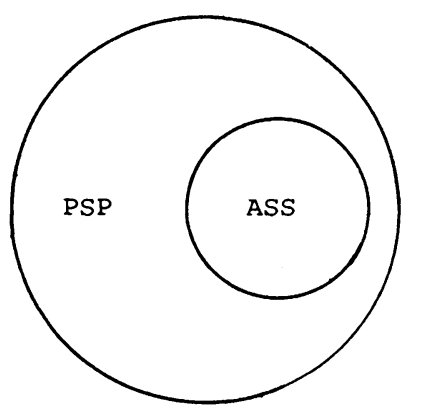

?nicht auch $x$

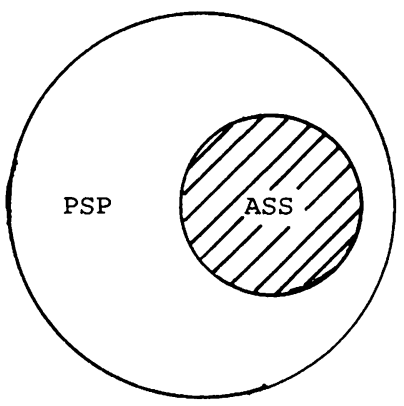

Die Identifizienung der PSP kann also nicht zuverlässig aufgrund ihrer Stabilität unter Negation bewerkstelligt werden, sondem nur aufgrund ihres verhaltens in den übrigen Tests.

2. Skalierende Interpretation: sie ist nur bei auch $x$ und auch $x \ldots$ nicht zugelassen.

auch $x$

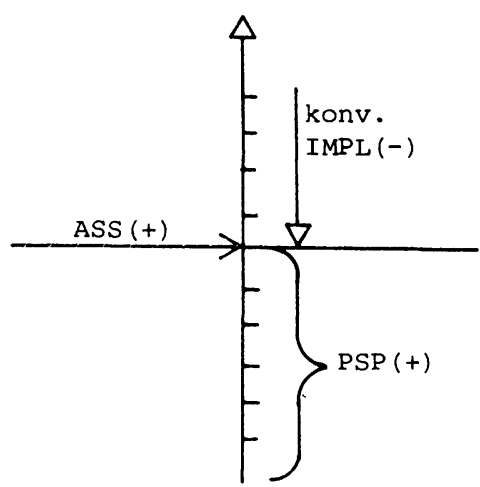

auch $x$... nicht

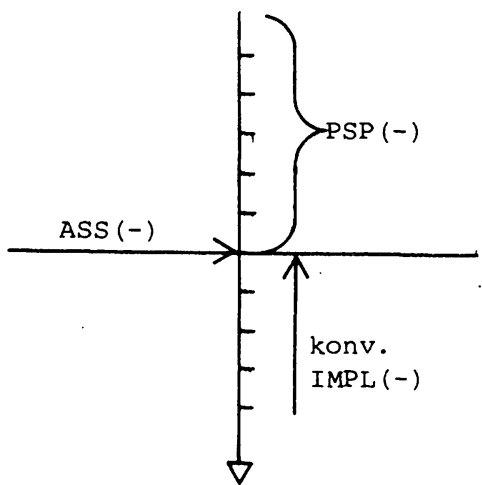

\subsubsection{3. sogar - Gruppe}

1. Subjekts-Nominalphrasen:

(1) Sogar J J han a hat nicht "Auf Wiedersehen" gesagt.

(2) Längst hatte er die Grenzen überschritten, die selbst $N$ a $t$ i $\circ n$ a $1-$ sozialisten dem deutschen Machtstaat zogen.

(3) Nicht einmal $S c h w e i n e$ nehmen bei ständiger Musikberieselung zu. 
2. Genitiv-, Dativ-, Akkusativ-Objekts-Nominalphrasen:

(4) Spaniens berühmte Kupplerin, sie, die sogar $B$ i s $c h$ ö $f$ e zu ihren Kunden machte...

(5) ... daß in allen Kulturstaaten des Westens selbst $V$ e $r$ b $r$ e $h$ e $r n$ volle Einsicht in das sie betreffende Dossier gewährt wird.

(6) Der Plan der Mondlandung war den Sowjets derartig waghalsig erschienen, daß sie seine Verwirklichung nicht einmal den $A m$ e $r$ i $k$ $n$ e $r$ zutrauen mochten.

Klassenbildung/Austauschbarkeit

In den vorhandenen Belegsätzen sind die drei Elemente der sogar - Gruppe uneingeschränkt austauschbar. Schwierigkeiten können nur durch die bei nicht einmal "inkorporierte" Negation auftreten, wenn der Satz elne weitere Negation aufweist (siehe hierzu aber den Abschnitt "Negationsmöglichkeiten" S. 120). Auch die übrigen GPn sind (abgesehen von gewissen Stellungseigenheiten usw.) beliebig einsetzbar. Die weiteren Austauschmöglichkeiten (erst, schon, nicht, Satzadverbien usw.) entsprechen denen bei nur und auch.

Stellungsgesetze:

Typ 1: GP unmittelbar vor dem Skopus; siehe dazu die Sätze (1) bis (6). Typ 2: GP unmittelbar nach dem Skopus. Zunächst scheint es hierfür keinerlei Möglichkeiten zu geben:

(1a) J $\mathrm{O}$ a n na ?sogar/"selbst/"nicht einmal hat "auf Wiedersehen" gesagt.

Beachte aber:

(3a) Eine Gewichtszunahme bei ständiger Musikberieselung verzeichnet man bei Rindern und Masthühnern, bei Menschen und bei $S c h$ we $i$ e n sogar.

(7). - Nicht nur Riesen, $z$ we $r g$ e sogar haben klein angefangen.

Diese Sätze kann man nur mit starken Einschränkungen als akzeptabel bezeichnen. Ich vermute, daß sie nur wegen ihrer oberflächlichen Ähnlichkeit mit Sätzen, in denen die Kontaktstellung zwischen toplkalisierter Skopuskonstituente und betontem nachgestelltem sogar durch Tilgung dazwischenstehender identischer Konstituenten entsteht, siehe Satz (7a):

(7a) Alles mögliche haben wir da, ... ja $z$ we $r g$ e sogar/selbst.

TYP 3 (GP in der Skopuskonstituente) kammt für diesen Skopustyp nicht in Frage. TYp 4: GP in Distanzstellung vor und nach dem Skopus.

(2a) ... die "sogar/"selbst/"nicht einmal dem deutschen Machtstaat $N$ a $t i$ o n a l sozialisten zogen.

(2b) ... die sogar/selbst/nicht einmal glücklicherweise/in der Pegel/im allgemeinen $N$ at $i$ on l sozialisten dem deutschen Staat zogen. 
Distanzstellung vor dem Skopus ist also nur dann in gewissem umfange möglich, wenn zwischen GP und Skopus-NP nur nahezu parenthetische Filgungen, dle in dieser Funktion selbst nicht in den Skopus der GP kommen können, stehen. Typ 5: Skopus topikalisiert, GP in Distanzstellung danach. Hier sind in der sogar - Gruppe dieselben Möglichkeiten vorhanden wie in den anderen Gruppen: die GP folgt auf einem Platz nach dem finiten Verb:

(8) Joh a n a fragte mich sogar/(selbst)/nicht einmal.

(2c) Die $N$ a $t i \circ n$ a 1 sozialisten zogen sogar/(selbst)/nicht einmal dem deutschen Machtstaat Grenzen.

Bei selbst tritt in dieser Position automat1sch Funktionswechsel zum Reflexivum ein. Satz (2c) ist eine typisch mindliche Version. In der schriftlichen Version dürfte (wenn Akzentmarkierung oder vereindeutigender Kontext fehlen) i.d.R. dem deutschen Machtstaat für den Skopus gehalten werden.

TYP 6: Der Fall der kontrastiv betonten GP ist bei dieser Gruppe ausgeschlossen. selbst ist zwar kontrastiv betonbar, wechselt aber damit autamatisch in die Funktion des Reflexivums.

Zusammenfassung der Stellungsgesetze: in dieser Hinsicht hat man es mit einer weitgehend homogenen Gruppe zu tun, deren "Nomalposition" Typ 1 bzw. Typ 5 ist. Alle anderen Versionen sind inakzeptabel oder nur miindlich möglich.

Syntaktische unformungen

Satzarten: Fïr die Form des Aussagesatzes stehen die Belegsätze (1) bis (3). Fragesätze:

(3b) Nehmen bei ständiger Musikberieselung sogar/selbst/nicht einmal $S c h w e i n e$ zu?

(3c) Sogar $S c h w e i n e$ nehmen bei ständiger Musikberieselung zu?

(9) Wann beklagt sogar der $S p i$ e g e 1 den armen Steuerzahler?

Imperativ:

(10) Verabschiede dich sogar $d u / d$ u sogarl

Die sehr geringe Akzeptabilität spiegelt den Gegensatz zwischen der Redundanz der Subjekts-NP im Imperativ und der Funktion des Kontrastfokus.

Konstituentensatzwortstellung: siehe die Sätze (2) und (4) bis (6).

In keinem Fall sind Besonderheiten der Stellungsgesetze oder Funktionswechsel erkennbar.

Spaltsatzbildung: Von Fraser (1971) wird behauptet, daß der Skopus von engl. even nicht gleichzeitig Kern eines Spaltsatzes seln diurfte, und er erklärt dies mit dem Widerspruch zwischen der Funktion des Spaltsatzes, Einzig(artig)- 
keit anzuzeigen, und der Semantik von even, die Nichtelnzigkeit beinhalte. Dies scheint im allgemeinen auch für die sogar - Gruppe zu gelten, doch möchte ich auf eine sonderbare Abweichung aufmerksam machen:

(11) A: Nun, diese unerfreulichen Nachrichten hat dir sicher niemand gesagt, der dich besonders gut leiden mag.

B: Stell dir vor: $H$ a $n s$ war es sogarles war sogar $H$ a $n s$, der mich darauf aufmerksam gemacht hat.

Zwei Lösungsmöglichkeiten sind denkbar: a. es handelt sich um kein echtes Clefting; dafür gibt es aber keinen überzeugenden Anhaltspunkt. b. sogar bewirkt hier keine Nicht-Einzigkeits-PSP. Ein Hinweis darauf ist, daß dieser Satz ohne Clefting kaum akzeptabel ist, die Ursache hierfür kann nur in dem Unterschied zwischen den Skopustypen Subjekts-NP und prädikatives Nomen liegen. Siehe dazu auch Abschnitt 3.3.3.3. S. 198.

Negationsmöglichkeiten:

Typ 1: Inkorporierung in die GP; sie ist ebenso unmöglich wie bei auch.

(1b) "Nicht sogar/selbst J o ha n n a hat "Auf Wiedersehen" gesagt. Diese "Lücke" kennzeichnet die Asymmetrie zwischen der nur - Gruppe einerseits und den auch - und sogar - Gruppen andererseits.

Typ 2: Negation zwischen GP und Skopuskonstituente.

(1c) "Selbst nicht Joh a n n a hat "Auf wiedersehen" gesagt.

(3d) "Selbst kein $S c h$ we $\mathrm{n}$ nimmt bei Musikberieselung $z u$.

(12) "Das erlaubt dir niemand: weder ein Sozialdemokrat, noch ein $F$ reidekrat, sogar nicht ein/kein $C h r i s t$ demokrat.

aber: ... selbst/sogar ein Chris t demokrat nicht.

... nicht einmal ein $C h r i s t$ demokrat.

Noch viel rigider als bei auch gilt hier also die Regel, daß die Negation nicht in die Skopus-NP integriert werden darf.

Typ 3: Starke Negation, Satznegation. Siehe dazu (1). Die Negationsform nicht einmal, wie sie in (3) vorliegt, entspricht dieser völlig: für mich sind keine Differenzen zwischen sogar... nicht und nicht einmal erkennbar.

Typ 4: Die lokale NEG bleibt in diesem Zusammenhang ohne Bedeutung.

Skopus und Fokus

Auch bei sogar usw. sind keine bestimmten Bedingungen hinsichtlich der syntaktischen und semantischen Merkmale der betreffenden Skopuskonstituenten feststellbar. - Bei der Festlegung der Skopusgrenzen begegnet man den gleichen Schwierigkeiten wie bei der auch - Gruppe: die "linke" Grenze ist durch die Normalstellung (TYP 1) gut markiert, für die"rechte" Grenze hingegen finden sich keine klaren Kriterien aus der Stellung von sogar usw. Auch hinsicht- 
lich der möglichen Akzentpositionen gibt es keine neuen Beobachtungen. Das sicherste Mittel zur Skopusabgrenzung sind auch hier Vorgänger und Folgesätze:

(1d) Hans und Eva, die ja immer sehr unhöflich sind, haben sich nicht verabschiedet. Sogar J $\mathrm{J}$ a $n \mathrm{n}$ a hat nicht "Auf Wiedersehen" gesagt.

(le) Hans und Eva waren sehr lieb zu mir. "Sogar $J \circ h$ a $n$ a hat nicht "Auf Wiedersehen" gesagt.

Eine Grundregel über die Art dieser Vorgängersätze ist nicht formal zu fassen. In jedem Fall muß die kontrastierte Konstituente eine Interpretation als "geringere Stufe" im Rahmen einer Skala zulassen. Eine wörtliche Wiederholung (abgesehen von der kontrastierten Konstituente) ist nicht nötig.

(1f) Selbst J $J$ a $n$ a hat nicht "Auf wiedersehen" gesagt, nicht nur $\overline{E v a}$ und Hans, von denen man ja Unhöflichkeit gewöhnt ist.

(3e) Nicht einmal $S c h w e i n$ e nehmen bei ständiger Musikberieselung schneller $z u$, von Menschen ganz zu schweigen.

Der Variationsbereich für Folgesätze scheint wesentlich enger zu sein: da häufig auch Tilgungen vorkommen, ist zumindest semantische wenn schon nicht formale Identität vorausgesetzt.

Beim Test der "natürlichen Antwort" zur Festlegung des Satzfokus treten bei sogar die gleichen Schwierigkeiten auf wie bei nur und auch:

(13) A: Wer begleitet denn Ibykus/wer ist es, der Ibykus begleitet?

B: $K r$ a $n$ i $c h$ e begleiten ihn/es sind $K r$ aniche, die ihn begleiten. -

?Nur $K r$ a $n i c h e$ begleiten $i h n / E s$ sind nur $K r$ a $n i c h e$, die ihn begleiten.

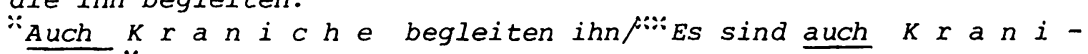
$c \overline{h e} \mu_{K} r a n i c h e$ sind es auch , die $\overline{i h n}$ begleiten.

“Sogar $K r$ a n $i$ che begleiten ihn les sind sogar $K r$ a $n i$ $c \overline{h e}$, die ihn begleiten.

Es sei noch darauf hingewiesen, daß die nur - Antwort durchaus eine akzeptable Interpretation haben kann: die wer - Frage präsupponiert einen Antwortbereich, der mit dem Merkmal [Hum] gekennzeichnet werden kann. Dieser Bereich wird durch die Antwort Kraniche verlassen, insofern könnte nur der Löschung des präsupponierten Fragebereiches dienen, und zwar in Richtung auf elnen "minderen" Bereich (Mensch - Tier). Eine ähnliche Lösung für sogar, z.B.

(13a) Verschiedene F reunde und Tiere begleiteten ihn und sogar $K r$ a $n i$ $c h e$.

halte ich nicht für möglich. Auch eine der bei nur aufgezeigten Möglichkeit der Zurïkweisung einer Nicht-Einzigkeits-ṔSP entsprechende Verwendung kann bei sogar (hier mïßte es die Zunückweisung einer Einzigkeits-PSP sein) nicht nachgewiesen werden. 


\section{Semantik}

Zum engl. even gibt es Ansätze zur Beschrelbung der Semantik in Horn (1969), Fraser (1971), Horn (1972) und bei Anderson (1972), jedoch nur Horn versucht eine Analyse nach ASS und PSP. Bei Fraser ${ }^{8}$ und Anderson werden nur (wohl intuitiv gewonnene) Bedeutungselemente aufgezählt, ohne deren Status vïllig zu klären. - Dabei erscheinen immer drei Grundelemente:

1. Der Satz ohne sogar(ASS).

2. Die Behauptung, daß auf andere (Horn: mindesten 1) "vergleichbare Elemente" die gleiche Aussage zutrifft (bei Horn: PSP).

3. Schließlich der Aspekt der "Unerwartetheit". Horn, der auch dies als PSP bezeichnet, verwendet hierfür die Formulierung "Man hätte nicht erwartet, daß (ASS)" (sinngemäß), Fraser "C. The speaker would not expect or would not expect the hearer to expect (Assertion)". Anderson zerlegt diesen Bedeutungsaspekt in zwei Teilaspekte, die für sein Beispiel

(14) Even JONES hates Millard Fillmore.

folgendemaßen lauten:

$$
\begin{aligned}
& \text { "c. Jones is about the last person one would expect to find hating } \\
& \text { someone like Millard Fillmore ... John is basically charitable. } \\
& \text { d. Because of c) one would expect Jones to like Millard Fillmore, } \\
& \text { thus a)is surprising, and shows that Millard Fillmore must be } \\
& \text { especially repugnant." }
\end{aligned}
$$

Diese Interpretation verrät vor allem Mangel an Phantasie bei der Interpretation von Beispielsätzen; ich könnte mir folgende Möglichkeiten (zumindest beim parallelen deutschen sogar - Satz) denken:

a. Millard Fillmore ist lieb, wohlgeachtet, und Jones ist lieb und wohlgeachtet. Der $\mathrm{H} a ß$ des einen auf die andere ist völlig unverständlich, wie auch der Haß anderer Leute auf Millard Fillmore.

b. Millard Fillmore ist lieb und wohlgeachtet, Jones ist hingegen bös (wie die anderen Hasser). Der Sprecher ist ganz entsetzt iber die Bosheit dieser Welt.

c. Millard Fillmore ist bös und widerlich, und auch Jones ist bös und widerlich; beide mïßten sich eigentlich lieben, aber sogar Jones haßt Millard Fillmore, wie die anständigen Leute auch ...

Diese Reihe ist wohl noch ziemlich lange fortsetzbar, und es sieht nicht so aus, als ob es in dieser Richtung irgendwelche Möglichkeiten zur Generalisierung gäbe. Es muß sich also um einen Holzweg der semantischen Analyse handeln:

8 Fraser (1971:152) und im Anschluß daran Anderson (1972: 904) sprechen statt von einer Präsupposition von einer Implikation (im Sinne Austins). Ihre Gründe hierfür können nicht überzeugen. 
einzelne Aspekte des situationellen Kontextes sind nicht der lingutstischen Analyse zugänglich. Skepsis ist wohl auch gegenüber den Analysen von Horn und Fraser angebracht: insbesondere scheint es keine Regeln für die genaue Formulierung dieser "Präsupposition" zu geben.

Vorläufige semantische Analyse der Sätze (1) bis (3):

Ich halte mich dabei zunächst an die Vorschläge von Fraser (1971) und Horn

(1969) und (1972):

(1) Selbst J h a $n$ a hat nicht "Auf Wiedersehen" gesagt.

ASS: Johanna hat nicht "Auf Wiedersehen" gesagt.

PSP: Mindestens eine weitere Person hat nicht "Auf Wiedersehen" gesagt.

"Erwartungs-PSP": Es ist erstaunlich, daß Johanna nicht "Auf Wiedersehen" sagte/der Sprecher erwartete nicht oder erwartete nicht, daß der Hörer erwarte, daß Johanna nicht "Auf Wiedersehen" sagt.

(2) Längst hatte er die Grenzen überschritten, die sogar $N$ a $t$ i $\circ$ n a 1 sozialisten dem deutschen Machtstaat zogen.

ASS: Die Nationalsozialisten zogen dem deutschen Machtstaat Grenzen.

PSP: Mindestens eine weitere Personengruppe zog dem deutschen Machtstaat Grenzen.

"Erwartungs-PSP": Man erwartet nicht ... daß die Nationalsozialisten dem deutschen Machtstaat Grenzen zogen.

Dieser Satz kann jedoch nach meinem Verständnis nicht mit einer "ErwartungsPSP" verbunden werden, im Gegensatz vielleicht zu:

Sogar die $N$ a $t$ i o n a 1 sozialisten zogen dem deutschen M. Grenzen.

Satz (2) bedeutet ja gerade, daß man selbstverständlich von jedem Menschen erwartet, daß er dem deutschen Machtstaat Grenzen zieht, also auch von den Nationalsozialisten (von denen man allerdings noch am ehesten erwarten könnte, daß sie es nicht tun).

(3) Nicht einmal $S c h$ we ine nehmen bei ständiger Musikberieselung schneller zu.

ASS: Schweine nehmen bei ständiger Musikberieselung nicht schneller zu. PSP: Mindestens eine weitere Gruppe von Lebewesen nimmt bei ständiger Musikberieselung nicht schneller zu.

"Erwartungs-PSP": Es ist unerwartet ... daß Schweine ... nicht schneller zunehmen.

Die Analysen sind im Bereich der PSPen wenig befriedigend. Zwar geben sie die Grundlinien der jeweiligen Bedeutung richtig wieder, aber sogar erhält dadurch einen Sonderstatus gegenüber den übrigen GPn (wenn Ähnliches auch schon für nur behauptet wurde); und die "Erwartungs-PSP" ist in ihrem tatsächlichen Inhalt außerordentlich schwer zu fassen. - Einen Hinweis auf eine, wie ich hoffe bessere bzw. zutreffende, Iösung gibt' die Ersetzbarkeit von sogar durch auch und andererseits von bestimmten Verwendungsweisen von auch durch sogar. 
Auch zeigt in allen diesen Fällen eine skalierende Funktion. Dies helßt, daß auch prinzipiell ambig ist bezüglich quantifizierender oder skalierender Funktion (der jeweilige Ko-/Kontext kann disambiguierend wirken), daß hingegen sogar prinzipiell nur in skalierender Funktion möglich ist. Eine graphlsche Darstellung könnte etwa so aussehen:
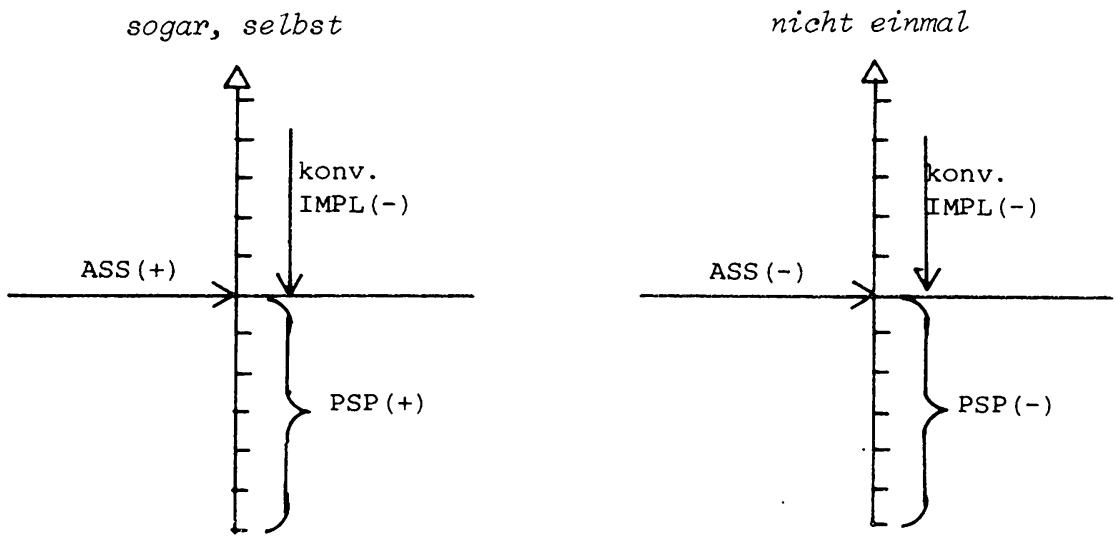

Ein sogar - Satz assertiert also die Giltigkeit eines extremen Wertes auf einer bestimmten Skala, präsupponiert die Gültigkeit für die "tieferen" Werte, und impliziert konversationell, daß kein "höherer Wert" gilt. Umgekehrt assertiert ein nicht einmal - Satz, daß ein bestimmter (hoher? tiefer?) Wert nicht gilt, präsupponiert, daß die(?) tieferen Werte nicht gelten, und impliziert konversationell, daß die höheren Werte auf der Skala gelten. Unklar bleibt hierbei einstweilen, ob die durch sogar und nicht einmal definierten Skalen in einer bestimmten Relation zu "objektiven" Skalen stehen muissen. Folgender Satz spricht jedoch dagegen:

(15) ?Hans kommt sogar mit $f \ddot{u} n f$ hundert Mark im Monat aus, und möglicherweise sogar mit (nur) 400 (wenn er sich sehr einschränkt).

Der Satz wird aber erst durch die Einfügung von nur voll akzeptabel.

Zur Suspendierbarkeit der einzelnen Bedeutungsaspekte Suspension der möglichen PSPen:

Selbst $J \circ h$ a $n$ a hat "Auf Wiedersehen" gesagt,

- "und möglicherweise hat sogar sonst niemand "Auf Wiedersehen" gesagt.

- “und möglicherweise ist sie sogar die einzige, die "Auf Wiedersehen" gesagt hat.

- ?und möglicherweise habe ich das sogar erwartet.

- *und möglicherweise hat sogar jemand, der höflicher ist als sie, "Auf Wiedersehen" gesagt. 
Sogar die $N$ a $z$ i $s$ zogen dem deutschen Machtstaat Grenzen,

- "und möglicherweise hat sogar sonst niemand mehr dem deutschen Machtstaat Grenzen gezogen.

- und möglicherweise ist das nicht einmal erstaunlich.

- "und möglicherweise hat sogar jemand, der weniger machthungrig war als die Nazis, dem deutschen Machtstaat keine Grenzen gezogen.

Nun hat Horn (1972) behauptet, daß die PSP eines Satzes mit even keinesfalls suspendiert werden könne, ohne daß er allerdings Gründe dafür angeben konnte. Dies wird für sogar durch die obigen Ergebnisse bestätigt. Andererseits kann die angebliche Erwartungs-PSP ohne Sciwierigkelten suspendiert werden, ohne daß man allerdings von einer typischen "PSP-Suspension" sprechen könnte.

Schließlich führt die Suspension der aus meiner skallerenden Interpretation folgenden PSP zu inakzeptablen oder doch nicht sehr glücklichen Ergebnissen. Alles zusammengenommen muß das aber nicht gegen meine Interpretation der Sachlage sprechen, vor allem dann nicht, wenn die Suspensionsformel vorwiegend dazu dient, die Unsicherheit des Sprechers iber Grenzziehungen bei skalierenden Interpretationen auszudrücken. Klarheit kann aber vielleicht der Versuch bringen, die aus der skalierenden Interpretation folgende konv. IMPL zu suspendiere Suspension der ASS:

(1h) Selbst J h a n a hat nicht "Auf Wiedersehen" gesagt, - "und möglicherweise hat sogar Johanna "Auf Wiedersehen" gesagt.

Sogar die $N$ a $z$ i $s$ zogen dem deutschen Machtstaat Grenzen, -"und möglicherweise haben sogar sie ihm keine Grenzen gezogen.

Dieses negative Ergebnis entspricht den Erwartungen.

Suspension der konv. IMPL:

(16) Ein Gefreiter, ein Jnteroffizier und sogar ein $L$ e u $t n$ a $n t$ ist an Befehle gebunden, und möglicherweise sogar ein General.

... und sogar der $\ddot{A} r m$ e $l$ kanal ist für Schwimmer bezwingbar, und möglicherweise sogar die Adria an ihrer breitesten Stelle.

Beide Beispiele halte ich für uneingeschränkt akzeptabel. Wäre die PSP dieser Sätze nur quantifizierend, wie bislang vorgeschlagen, so wïrde es sich bei diesen Beispielen nur um die ausdrückliche Behauptung von präsupponierten Tatsachen handeln, (16) und (17) müßten also auf jeden Fall inakzeptabel sein. 9 Daraus kann man nur folgern, daß nicht die Gilltigkeit der Aussage für irgendjemand/irgendetwas (nicht identisch mit dem in der ASS genannten Objekt) präsupponiert wird, sondern für einen ganz bestimmten Bereich, für eine ganz be- 
stimmte Menge, und daß andererseits die Ungulltigkeit für eine ebenfalls klar abgegrenzte Menge zwar nicht behauptet, und auch nicht präsupponiert wird, daß sie aber aus der Aussage gefolgert werden kann, solange nicht ausdrilcklich das Gegenteil festgestellt wurde. - Die Schwierigkeit liegt hierbei darin, daß diese Mengen nur sehr selten einwandfrei aus dem Text erschließbar sind, da die Skalen, an denen sie sich orientieren, in manchen Fällen sehr vielfältig sein können, so etwa für Satz (1):

- Skala der Verwandtschaft (Johanna sehr nahe verwandt).

- Skala der Höflichkeit (Johanna sehr höflich).

- Skala der gegenseitigen Sympathie (Johanna sehr sympathisch).

- Skala der Nächstenliebe (Johanna sehr versöhnlich), etc.

Wenn aber diese Skala nicht aufgrund der Oberflächenstruktur eines GP-Satzes zweifelsfrei festgelegt werden kann, dann kann auch keine intensionale Definition für die präsupponierte Menge gegeben werden. Für eine linguistische Behandlung dieser Frage ist dies jedoch nicht unabdingbar notwendig. Es genügt, daß wir die Struktur der Gebrauchsmöglichkeiten der GP sogar erkennen.

Konjunktionstest:

(1i) Nicht einmal $J \circ h$ a $n$ a hat "Auf Wiedersehen" gesagt,

- ?und mindestens ein weiterer Mensch hat nicht "Auf Wiedersehen" gesagt. (quant. PSP)

- "und alle weniger höflichen/geliebten ... Menschen haben nicht "Auf Wiedersehen" gesagt. (skal. PSP)

- "und Johanna hat nicht "Auf Wiedersehen" gesagt. (ASS)

Eine konv. IMPL ist für diesen Fall nicht sinnvoll, da der Satz überwiegend folgende (trotz des Wortlautes skalierende!) Interpretation hat: Niemand hat "Auf Wiedersehen" gesagt, nicht einmal Johanna. Typisch für sogar - Sätze ist das allerdings nicht.

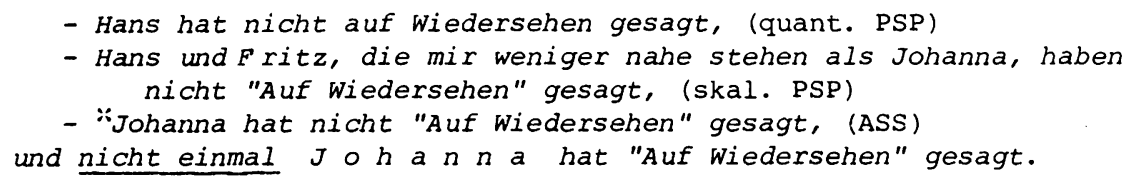

Die verwendeten Formulierungen für die vorgeschlagenen PSPen lassen keine Entscheidung $\mathrm{zu}$, welche die richtige ist. Dies ist jedoch vielleicht möglich mit der folgenden Fassung:

(1k) "Hans, der mir viel näher steht als Johanna, hat nicht "Auf Wiedersehen" gesagt, und nicht einmal $J \circ h$ a $n$ a hat "Auf Wiedersehen" gesagt.

Die PSP-Formulierung in (1k) entspricht einer quantifizierenden PSP; sie be- 
wirkt jedoch klare Inakzeptabilität. Bei skalierender Interpretation ist eine solche PSP ausgeschlossen. - Daß das Hinzufügen der ASS in beiden Anordnungen inakzeptabel ist, bedarf ebenfalls der Erklärung: es könnte daran liegen, daß nicht einmal offensichtlich nicht betont werden darf, daß aber andererseits dies die einzige Möglichkeit für eine Wiederholung der ASS 1st. Die andere Erklärungsmöglichkeit wäre, daß nur hierin eine untypische Ausnahme bildet: nur dort, wo der jeweilige Satz die ASS nicht in einer expliziten Formulierung enthält, darf diese in expliziter Form hinzugefügt werden. - Da die Klärung dieser Fragen sehr wichtig ist, wiederhole ich die Tests mit einem vielleicht eindeutigeren Beispiel:

(16a)

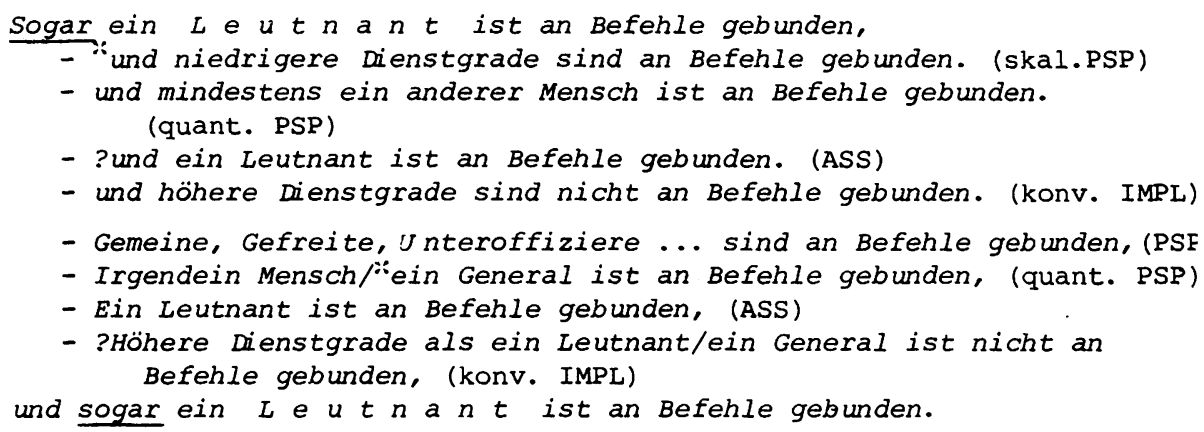

Dies zeigt, daß sich die PSP in der skalierenden Interpretation völlig regulär verhält, während die quantifizierende PSP irreguläres Verhalten zeigt, und zudem je nach Füllung verschiedene Akzeptabilität erhalten kann. Vielleicht ist auch noch der Hinweis darauf, daß u.U. die Kombination

(16c) Ein General ist an Befehle gebunden und sogar ein $L$ e $u$ t n a $n$ ist an Befehle gebunden.

als akzeptabel erscheinen kann (nach Abzug einer auf Sachkenntnis bezogenen Wahrscheinlichkeit): sogar begründet in diesem Falle eine der Dienstgradskala entgegenlaufende Skala der "Befehlsgebundenheit". - Die Wiederholung der ASS erbringt hier - wahrscheinlich aufgrund der Betonbarkeit von sogar - etwas akzeptablere Ergebnisse. - Irregulär ist auch das Verhalten der konv. IMPL: ihre explizite Wiederholung müßte inakzeptabel sein, das Vorwegnehmen akzeptabel. Die abweichenden Ergebnisse könnten dadurch verursacht sein, daß die "Grenzziehung" bei sogar weniger scharf ist, ja daß in ihm geradezu eine Grenzunsicherheit zum Ausdruck kommt.

Monologischer Widerspruchstest:

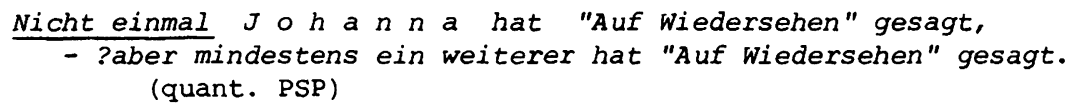


- “aber alle weniger höflichen/geliebten ... Menschen haben "Auf wiedersehen" gesagt. (skal. PSP)

- "aber Johanna hat "Auf Wiedersehen" gesagt. (ASS)

- ?aber höflichere Menschen als sie haben "Auf Wiedersehen" gesagt. (konv. IMPL)

(16d) Sogar ein $L$ e $u$ t $n$ a $n t$ ist an Befehle gebunden,

- ?aber mindestens ein weiterer Dienstgrad ist nicht an Befehle gebunden. (quant. PSP)

- *aber niedrigere Dienstgrade sind nicht an Befehle gebunden. (skal. PSP)

- "aber höhere Dienstgrade sind an Befehle gebunden. (konv. IMPL)

Die Tatsache, daß es für die quantifizierende Interpretation bei der Kombination des sogar - Satzes mit der quant. PSP akzeptable Auslegungen gibt (wenn auch nur solche, die nicht interessieren), oder aber daß ein völlig widersinniges Gebilde entsteht, aber kein logischer Widerspruch, zeigt m. E. eindeutig, daß diese Interpretation an der Funktion von sogar vorbeigeht. Die skalierende Interpretation hingegen produziert klare Widersprüche (von einer möglichen Umkehrung der Skala abgesehen; unter dieser Voraussetzung wären derartige Kombinationen wieder akzeptabel).

Dialogische Analyse

(1m) A: Nicht einmal J $J$ h a n a hat "Auf Wiedersehen" gesagt.

B: - Doch. Sie hat ("Auf Wiedersehen" gesagt). (ASS)

- Aber es hat sich doch gar niemand sonst wortlos verdrückt! (quant. PSP)

- Aber Hans und Karl, die viel weniger nett sind zu dir, haben doch "Auf Wiedersehen" gesagt. (skal. PSP)

- Aber Eva, die du doch viel mehr liebst, hat doch auch nicht "Auf wiedersehen" gesagt. (konv. IMPL)

- Hast du vielleicht etwas anderes von ihr erwartet? ("Erw.-PSP")

- Ja. (Johanna hat nicht "Auf Wiedersehen" gesagt.) (ASS)

- "Jemand anderer hat auch nicht "Auf wiedersehen" gesagt. (quant.PSP)

- Ja, bei Hans und Karl hätte ich mir auch weniger gedacht. (skal.PSP)

- Na ja, wenigstens Eva hat "Auf Wiedersehen" gesagt. (konv. IMPL)

- Ja, das hat mich auch ziemlich überrascht/gekränkt/gewundert. ("Erwartungs-PSP")

(16e) A: Sogar ein $L$ e u $n$ a $n t$ ist an Befehle gebunden.

B: - Nein. Ein Leutnant ist bestimmt nicht mehr an Befehle gebunden. (ASS)

- ?? Ja, natürlich, aber sonst doch niemand mehr. (quant. PSP)

- $V$ on einem Leutnant weiß ich es ja nicht. (ASS) Aber die niedrigeren Pänge sind doch gar nicht an Befehle gebunden. (skal.PSP)

- Aber Generäle sind doch auch an Befehle gebunden! (konv. IMPL)

- Na ja, ein Leutnant ist ja auch kein besonders hoher Dienstgrad. (Skala selbst)

- Ja, so ist das. (Ein Leutnant ist an Befehle gebunden). (ASS)

- ?Ja, das weiß ich. Und ich gebe auch $z u$, daß noch mindestens ein anderer (Dienstgrad) an Befehle gebunden ist. Aber ... (quant. PSP) 
- Ja, weiß ich. Und natürlich sind auch niedrigere Dienstgrade an Befehle gebunden. Aber ... (skal. PSP)

- Na ja, zum Glück sind ja die höheren Dienstgrade nicht an Befehle gebunden. (konv. IMPL)

- Und ein Leutnant ist ja nun wirklich ein hohes Tierl (Skala selbst)

In diesem Test wird klar wie sonst nirgends die skalierende Interpretation bestätigt: vor allem durch die Unmöglichkeit, eine quantifizierende PSP abzulehnen oder zu bestätigen, femer durch die Möglichkeit, die zugrundeliegende Skala selbst zu thematisieren. Bleibt noch die Aufgabe, eine der obigen Globalverneinung entsprechende direkte Negation für Sätze mit sogar/selbst zu finden. Legen wir die skalierende Interpretation zugrunde, so mißste dieser Satz folgende Bedeutungselemente umfassen (für Satz (2)):

ASS: Die Nazis zogen dem deutschen Machtstaat keine Grenzen.

PSP: Weniger radikale politische Gruppen zogen dem deutschen Machtstaat Grenzen.

konv. IMPL: Radikalere politische Gruppen als die Nazis zogen dem deutschen Machtstaat keine Grenzen.

Dafür kann ich keine Oberflächenrealisierung finden. Wirde man die konversationelle Implikatur (zumindest in diesem Falle) für überflüssig befinden, so wäre die Negation

(2g) Nur die $N$ a z i $s$ zogen dem deutschen Machtstaat keine Grenzen. doch wären dann ASS und PSP gegeneinander vertauscht. Eine andere, allerdings nicht ganz akzeptable Lösung wäre:

(2h) Nicht auch die $N$ a $z$ is zogen dem deutschen Machtstaat Grenzen. Die parallele Version

(2i) "Nicht sogar die $N$ a z is zogen dem deutschen Machtstaat Grenzen. ist zwar interpretierbar, wird aber einhellig von allen Befragten abgelehnt. Nicht einmal und das gleichwertige sogar ... nicht hingegen kehren nicht nur die ASS, sondern auch die skal. PSP und natürlich auch die konv. IMPL in ihrem Wert um, können also nicht als direkte Negation gelten, obwohl sie eindeutig die übliche Form der Satznegation/starken Negation darstellen. - Eine Erklärung könnte sich darauf beziehen, daß die übliche Form der Satznegation in allen Fällen von kontrastivem Akzent nicht die Verbindung von Topic und Focus negiert, sondern nur eine Negation innerhalb des Topic darstellt, die PSPneutrale Negation hingegen jeweils den gesamten Satzfocus umfassen muß, also den Skopus der GP. Dies erklärt allerdings immer noch nicht die Asymetrie zwischen nur einerseits und auch und sogar andererseits. Dazu müssen erst 
noch eindeutige Fälle von skalierender Interpretation bel nur betrachtet werden, die jedoch offensichtlich nur beim Skopustyp Prädikat vorkommen.

Ergebnisse der semantischen Tests:

Die Art der Assertion von Sätzen mit sogar/selbst/nicht einmal unterlag von Anfang an keinen Zweifeln. Bei der Formullerung der PSP erwies sich eine skalierende Interpretation als deutlich überlegen gegenuber einer Kambination aus quantifizierender und "Erwartungs-PSP". Das Skopus-Element von sogar bezeichnet dabei den Endpunkt der Gilligkeit (ASS), das Zutreffen des Prädikates auf die "niedrigeren" Elemente der Skala wird präsupponiert, das Nicht-Zutreffen auf die "höheren" Elemente der Skala wird konversationell impliziert (wobei man allerdings nicht übersehen kann, daß sogar nicht selten den obersten Punkt der Skala überhaupt markiert.) Die Richtung der Skala (also "aufwärts" oder "abwärts") ist nicht an sogar oder nicht einmal zweifelsfrei zu erkennen:

(18) Nicht einmal $z$ ig e un e $r$ wollen hier wohnen. Sogar $z i g$ e $n$ e $r$ wollen hier nicht wohnen.

3.2.2. Exkurs: Skopustyp komplexe Nominalphrase.

3.2.2.1. nur-Gruppe.

1. Mit attributivem Adjektiv:

(1) Der CSU-Nachwuchs verlangte... nur eine $g$ e wis $s$ e $R \ddot{u} \subset k$ $k \circ p$ p 1 u $n g$.

(2) Es gibt kaum Zweifel, daß die sogenannte Bezinknappheit bloß ein la us i g e r T $r$ i ck ist.

2. Koordinierte Nominalphrasen:

(3) Eine Reformklinik, in der es statt dessen nur $E$ in - und $z$ w e i bettzimmer gibt...

3. Nominalphrasen mit Genitiv- oder Präpositionalergänzung:

(4) ... Statt dessen nur Ein- und Zweibettzimmer mit Telefon.

(5) Böse reagierten in Osteuropa nur die Häretiker in Albanien.

4. Nominalphrasen mit Apposition:

(6) Ein Kommuniqué, das über den Zweck der Luna-Peise nur $P$ h $r$ a $s$ e $n$ vage und verlogen wie immer - enthielt.

(7) Der CSU-Nachwuchs verlangte lediglich eine Rückkoppelung, unverbindlich wie eine gut katholische Ehe.

5. Nominalphrasen mit Konstituentensatz:

(8) Für nicht angefertigte Arbeiten folgte bloß die wiederholte Nachfrage, ob die Arbeit inzwischen fertiggestellt sei, aber keine Sanktion.

Die notwendigen Ergänzungen konzentrieren sich bei diesem Skopustyp auf die Fragen der Skopusabgrenzung und im Zusammenhang damit auf die Problenatik der Akzentpositionierung und der Bildung passender Kontrastsätze. 
Zu 1: Hier müssen wir (bei Beschränkung auf die Konstituente nach der GP) folgende Akzentmöglichkeiten berücksichtigen:

- Betonung auf definitem oder indefinitem Artikel. Dann handelt es sich aber um den Skopustyp Numerale (3.5.1.) oder Demonstrativpronomen (3.2.5.).

- Betonung des attributiven Adjektivs (in unseren Beispielen aus semantischen Gründen nicht möglich). Siehe hierzu den folgenden Abschnitt $3.2 .3 . !$

- Betonung des Nomens.

- Betonung sowohl des attributiven Adjektivs wie des Nomens.

Welche Elemente jeweils tatsächlich im Skopus der GP liegen, läßt sich nur durch kontrastive Vorgänger-bzw. Folgesätze festlegen:

(9) Sein Verhalten konnte nur tiefe Verwunderung bewirken,

(a) - nicht $v \circ r$ ü b er gehende $V$ erwunderung.

(b) - aber nicht 1 a u $t e$ z $\mathrm{s} t \mathrm{i} m \mathrm{~m}$ u $\mathrm{g}$.

(c)- aber nicht tiefe $N$ i e dergeschlagenhe $i t$.

Wohlwollen vorausgesetzt, ist in (9a) und (9c) die Betonung im Ausgangssatz klar: Adjektiv bzw. Nomen werden kontrastiv betont. Im Fall ( $9 \mathrm{~b}$ ) wären beide Akzentpositionen nicht akzeptabel. Vielmehr müssen hier beide Wörter kontrastiert werden: das ist leichter vorstellbar bei schweigende Entrüstung laute zustimmung. Derartige beinahe idiomatische Kontrastierungsmöglichkeiten sind jedoch nicht allzu häufig. - Nach meinen Beobachtungen sind zwei Betonungsmuster möglich: zwei gleichstarke Auf- und Abschwünge aufeinanderfolgend bzw. Aufschwung über dem ersten kontrastierten Element und Abschwung über dem zweiten. Die Artikulationsstärke dürfte in beiden Fällen gleich sein. In jedem Fall aber handelt es sich um eine seltene und weitgehend schriftsprachliche rhetorische Figur. In mündlicher Rede wird hingegen offenkundig die Kontrastierung von Einzelelementen vorgezogen.

Mit impliziter Kontrastierung kann dieser Skopustyp sehr viel häufiger verwendet werden, wobei allerdings Ambiguitäten (und dementsprechende Mißverständnisse unvermeidbar sind. Die Unterscheidung explizit - implizit kontrastiv hat übrigens deutliche Auswirkungen auf die Semantik des so verwendeten Satzes. Bei implizit kontrastiver Verwendung wird z. B. bei Satz (9) die Unmöglichkeit aller anderen Reaktionen als tiefe Verwunderung assertiert, bei explizit kontrastiver Verwendung hingegen der Bereich der negativen ASS z.B. auf laute $z$ ustimmung eingeschränkt (in der PSP sind beide Versionen gleich). Ferner steht fest, daß bei kontrastiver Betonung des attributiven Adjektivs nur eine restriktive Interpretation möglich ist, bei kontrastiver Betonung des Nomens hingegen nur eine nichtrestriktive. Welche von beiden Erklärungsweisen für die kontrastive Betonung beider Elemente zutrifft, ist mir unklar, doch halte ich die restriktive für wahrscheinlicher.

Zu 2: Bei koordinierten NPn liegt folgendes Akzentmuster nahe: jede NP erhält verstärkte Betonung. Doch ist, wie die folgenden Kontrastsätze zeigen, auch eine andere Lösung denkbar:

(3a) Eine Reformklinik, in der es statt dessen nur Ein- und Zweibettzimmer gibt,

(a) - nicht jedoch Mehrbettzimmer oder gar Säle.

(b) - nicht jedoch Ein- und Dreibettzimmer.

Im Falle a) tragen tatsächlich beide NPn verstärkte Betonung, im zweiten Falle jedoch nur die zweite NP des Ausgangssatzes. - $\mathrm{Zu}$ beachten ist ferner, daß koordinierte NPn mit einer vorangestellten GP bedeutungsverschieden sind von koordinierten NPn mit je einer "eigenen" GP:

(3b) Reformkliniken, in denen es (entweder) nur $E i n$ - und (oder) nur $z$ we $i$ bettzimmer gibt, jedoch nicht Mischformen, d.h. Kliniken, in denen es sowohl Ein- als auch Zweibettzimmer gibt. 
Die Nachstellung der GP ist bei diesem Skopustyp völlig ausgeschlossen.

Zu 3: Nominalphrasen mit Genitiv- bzw. Präpositionalergänzung. Hier häufen sich die Möglichkeiten der Akzentverteilung und Kontrastsatzbildung, wie Satz (4) ganz deutlich zeigt. Die "normale" Interpretation dürfte für diesen Satz mit folgendem Kontrastsatz zu verdeutlichen sein:

(4a) ... nicht jedoch $M$ e hr bettzimmer ohne Telefon.

Das heiBt also, alle Elemente der NP werden kontrastiert. Bei impliziter Kontrastierung werden alle Elemente der komplexen NP zur Abgrenzung des Gültigkeits- bzw. Ungültigkeitsbereiches herangezogen. Die normale Realisierung zeigt Betonungsspitzen auf allen diesen Hauptelementen, doch findet man sehr häufig auch bloße Endbetonung, d.h. nur das letzte Nomen trägt einen Hauptakzent, offenbar gefördert durch den normalen Satzschlußakzent.

Die Nachstellung der GPn ist in diesen Fällen zwar möglich, doch erzwingt sie nach meinem Verständnis eine andere Interpretation:

(4b) ... statt dessen Ein- und zweibettzimmer mit $T$ e 1 e fon nur/ bloß/lediglich ...

Diese stellung bewirkt in aller Regel einen besonders starken Akzent auf dem letzten Element, das dann offenbar allein im Skopus steht: übrigens ist dies die einzige Möglichkeit, um eine derartige Wirkung zu erzielen, da die Formulierung

(4c) ... statt dessen Ein- und Zweibettzimmer nur mit $T$ e 1 e fon. wohl immer ambig ist, da nur hier eher als nachgestellt gegenüber dem Skopus Ein- und Zweibettzimmer empfunden wird, es sei denn, man bindet es durch nachdrückliche Phrasierung an mit Telefon. In der Regel wird aber diese Ambiguität durch die Formulierung

(4d) ... Ein- und zweibettzimmer, und zwar nur/ausschließlich mit $T$ e $l$ e fon.

beseitigt. Dieser Satz ist auch von der Semantik her interessant. Er meint nämlich nicht, wie man zunächst vielleicht vermuten könnte, daß die zimmer außer dem Telefon keine weiteren Ausstattungsdetails aufweisen, sondern daß alle zimmer ein Telefon besitzen. Die gleiche Interpretation besitzt übrigens auch der Satz, in dem sich die ganze komplexe NP im Skopus von nur befindet: hier ist jedoch die semantische Analyse nicht weiter problematisch.

Zu 4: NP mit Apposition. Die Apposition gehört offenbar nicht mehr zum Skopus (oder kann jedenfalls bei der semantischen Analyse dieser GP-Sätze vernachlässigt werden), wie verschiedene Texte beweisen:

(6a) ... nur $P h r a s$ e - vage und verlogen wie immer - enthielt, keine $\overline{e c h}$ ten In $n$ ormationen.

(6b) ?... keine Informationen, genau und richtig.

Da Appositionen nur die Semantik ihres Bezugsnomens wiederholen bzw. verstärken, lassen sie sich nicht selbständig kontrastieren (was nicht unbedingt heißen muß, da $B$ sie auch nicht focusfähig sind).

(6c) ... nur vage und verlogene Phrasen enthielt, keine genauen und richtigen Informationen.

(6d) ... nur $P h r$ a $s$ en, "丷nur vage und $v e r l \circ g$ e $n$ wie immer, enthielt.

(6e) $\because .$. Phrasen, nur $v a g e$ und $v e r l \circ g$ e $n$ wie immer...

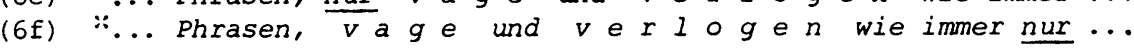

Die elnzige vielleicht mögliche Form der Nachstellung bestätigt diesen Befund: 
(6g) ...Ph $r$ a $s$ e nur, vage und verlogen wie immer, enthielt.

Nichtliterarischer Verwendung dürfte diese Formulierung jedoch schwerlich angemessen sein. - Nichtrestriktive Relativsätze sind natürlich in gleicher Weise zu behandeln.

Zu 5: NP mit Konstituentensatz. Im Skopus liegt hier der gesamte Ausdruck einschließlich des Konstituentensatzes (Konstituentensätze ohne Bezugsnomen bzw. nur mit einem Platzhalter an dessen Stelle im Skopus werden in einem eigenen Abschnitt behandelt), der die Ergänzung des nominalisierten Verbes darstellt. Der Nachweis hierfür nur aufgrund der GP-Positionen ist allerdings relatiy schwierig, da jede Form von Nachstellung der GP inakzeptabel ist:

(8a) $\because .$. folgte die Nachfrage, ob die Arbeit inzwischen fertiggestellt sei, nur, aber keine Sanktion.

Die mangelhafte Akzeptabilität rührt aber wohl kaum daher, daß etwas nicht zum Skopus Gehöriges eingeschlossen wird, sondern sie resultiert aus der Länge des Ausdruckes, die nur durch nachdrückliche Zusammenfassung in der Artikulation kompensiert werden könnte. Die Gegenprobe:

(8b) ... folgte die wiederholte Nachfrage bloß, ob die Arbeit inzwischen fertiggestellt sei, aber keine Sanktion.

bringt ein auf den ersten Blick akzeptables Ergebnis (abgesehen von den allgemeinen Problemen der GP-Nachstellung), doch wird dadurch nur eine Skopusambiguität zwischen Nomen und ob-Satz erzeugt, keineswegs der Skopus auf Nachfrage eingeschränkt. Klarer ist die Umformung:

(8c) ... folgte bloß die wiederholte $F$ rage nach der $F$ ertigsteilung der Arbeit, keine Sanktion ...

(8d) ... weil er bloß nach der F ertigstellung der Arbeit fragte ...

In diesen beiden Fassungen sind alle Elemente der komplexen NP im Skopus der GP enthalten, dafür spricht auch die Kontrastierbarkeit mit der einfachen NP keine Sanktion. Die Entscheidung hinsichtlich des Akzentes ist damit aber nicht sehr viel leichter, am wahrscheinlichsten dürfte aber sowohl für (8c) wie für (8d) Arbeit als Träger des kontrastiven Akzentes sein. Die Unklarheit ist wohl dadurch bedingt, daß bei mehreren Elementen eine Kontrastierung Element für Element nicht sinnvoll bzw. üblich ist, daB daher mit dem Ausgangssatz nicht auch gleichzeitig der Kontrastsatz gegeben bzw. ziemlich genau erschlieBbar ist: damit herrscht die implizit-kontrastive Verwendungsweise vor, worauf ev. auch die Möglichkeit hindeutet, keinen klaren Akzent zu setzen.

Bei den Negationsmöglichkeiten ergibt sich gegenüber dem Skopustyp "einfache Nominalphrase" nur ein unwesentlicher Zusatz, nämlich die Möglichkeit zur lokalen Negation etwa bei Adjektivattributen, jedoch nicht beim nominalen Kern der komplexen NP:

(2a) ?... daß die sogenannte Benzinknappheit nur ein nicht lausiger Trick ist.

Damit kann man aber vermutlich nicht die anderen Möglichkeiten zur Plazierung einer Negation zwischen GP und Skopusnomen erklären:

(3c) ... eine Reformklinik, in der es alle technischen Raffinessen, nur nicht/nur keine $E$ i $n$ - und $Z$ we i bettzimmer gibt.

Die Tatsache, daß nur hier zwar durch bloß, aber nicht durch lediglich, ausschließlich usw. ersetzt werden kann, spricht dafür, daß es sich um die konjunktionale Verwendung von nur handelt. Beachte aber folgenden Satz: 
(1a) Der CSU-Nachwuchs verlangte nur keine/nur nicht eine $R \ddot{u} c k-$ koppelung ...

(1b) Der CSU-Nachwuchs verlangte alles Mögliche, nur verlangte er keine Rückkoppel ung.

(1c) Der CSU-Nachwuchs verlangte alles Mögliche, nur e in es verlangte er nicht: eine Rückkoppelung.

Ich bin mir nicht sicher, ob im letzten Satz wirklich eine konjunktionale Verwendung vorliegt (trotz der Ersetzbarkeit durch aber usw.), bzw. ob es sinnvoll ist, eine scharfe Trennlinie zwischen beiden Verwendungsarten zu ziehen. Zur Entscheidung dieser Frage sind Paraphrasen mit außer bzw. in der Form $x$ und nicht nicht-x ein zu stumpfes Instrument, da sie zu tiefgreifenden syntaktischen Umformungen zwingen:

(1d) Der CSU-Nachwuchs verlangte alles Mögliche, außer einer Rückkoppelung.

(1e) Der CSU-Nachwuchs verlangte keine Rückkoppelung, und er verlangte alles Mögliche.

In beiden Paraphrasen ist das Muster von foregrounding und backgrounding von (1c) zerstört, Unterschiede in wahrheitswertfunktionaler Hinsicht kann ich jedoch nicht feststellen. Letzteres ist auch für konjunktionales nur nicht zu erwarten, ebensowenig wie für aber gegenüber und.

\subsubsection{2. auch - Gruppe}

1. Mit attributivem Adjektiv:

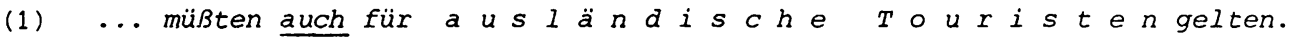

2. Koordinierte Nominalphrasen:

(2) Inzwischen wirbt nicht nur die Henkel-"Seife Fa" mit der "wilden Frische von Limonen", auch das Scheuermittel $A t$ a und das Geschirrspülmittel $P$ r $i$ l verfärben sich plötzlich gelb.

3. Nominalphrasen mit Genitiv- oder Präpositionalergänzung:

(3) Denn die Krise im Selbstverständnis der Ordnungshüter bot auch die Chance einer $N$ e uorientierung.

4. Nominalphrase mit Apposition:

(4) - Wie berichtet hatte die Gemeinde Grünwald auch ein Raumordnungsverfahren, so umständlich wie ja immer, für die stadtnächste Trasse I gefordert.

5. Nominalphrase mit Konstituentensatz:

(5) Auch eine welt, die von den Geißeln des U mweltschmutzes b e f $r$ e i $t$ ist, würde ohne Wachstumsstop unweigerlich untergehen.

6. Zusammengesetzte Nomina:

(6) Erst, seit auch die $P \circ i$ i zei-Bi ide $r$ laufen lernten, sieht der Verkehrsfahnder eine positive Einwirkung auf die Einsichtsfähigkeit der Autofahrer.

Gegenüber den Ausführungen in 3.2.1.2. sowie 3.2.2.1. ergeben sich keine neuen Gesetzmäßigkeiten. Sie sind hier sinngemäß anzuwenden. 


\subsubsection{3. sogar - Gruppe}

1. Mit attributivem Adjektiv:

(1) Die SPD erkennt der verfaßten Studentenschaft sogar "selbst/nicht einmal ein $p \circ 1 i t i s c h$ e $s$ a $n d a t$ zu.

2. Koordinierte Nominalphrasen:

(2) Paul fliegt sogar $S t a r f i g h t e r$ und $A l p h$ a Jets.

(3) Längst hatte er die Grenzen überschritten, die selbst $S A$ und $S S$ dem deutschen Machtstaat zogen.

3. Nominalphrasen mit Genitiv- oder Präpositionalergänzungen:

... ein Wiederaufleben antideutscher Gefühle oder sogar Demonstrationen gegen $G$ ä $s t e$.

... ist so allgemein geworden, daß es außer Poptribünen selbst die Amtszimmer der $P \circ I i t i k$ e $r$ erreichte.

4. Nominalphrasen mit Apposition:

(6) Die massivsten Luftangriffe der Geschichte seien selbst den A $m e-$ $r i k$ a $n r n$, reich wie sie sind, $z u$ teuer geworden.

5. Nominalphrasen mit Konstituentensatz:

(7) Und Sonderemissär Rosen erhielt nicht einmal die Erlaubnis, seine Agenten in der Zelle zu besuchen.

Hinzuweisen bleibt hier nur ergänzend auf die Schwierigkeit, diejenigen Positionen ausfindig $z u$ machen, in denen selbst offenbar nicht oder doch nicht in GP-Funktion auftauchen kann. Als Regel gilt hierbei: weist ein Element außer der Verwendung als GP noch andere Funktionen und Bedeutungen auf, so wird es in allen Positionen, in denen auch diese Funktionen auftreten können, nicht als GP akzeptiert, d. h. die mögliche andere Funktion setzt sich gegen die GP-Funktion durch, oder es kommt zu Funktionsambiguität.

3.2.3. Exkurs: Skopustyp Nominalphrasen mit kontrastiv betontem Attribut.

3.2.3.1. nur - Gruppe

1. Attributive Adjektiva:

(1) ... daß Wachstum und Fortschritt nur für einen $h \circ h$ e $n$ Preis $z u$ haben sind.

(2) Die $F$ achdidaktik spielt nur eine $k l$ e i n e Rolle.

(3) Ressortchef Dohnanyi will nur in un w e n t i chen Details nachgeben.

(4) Uns adelt nur ein e d 1 e $r$ Geist.

(5) Nur $r e i \bar{n}$ e $n$ Herzen duftet der Abendtau.

(6) Die F achdidaktik spielt nur eine $k 1$ e $i n$ e $r$ e / die kle in$s$ t e Rolle.

(7) Uns spricht der Scheinfreund, so wie du, allein bei gut en/ $b$ e s $s$ ren/best e n Tagen zu.

(8) Nur ein 1 e $i d e n s c h$ a $f t l i c h e r$ Jäger wird soviel für ein Revier bezahlen.

(9) Nur ein $s$ t a $r k$ e $r$ Raucher trägt ein erhöhtes Lungenkrebsrisiko. 
2. Attributive Partizipien:

(10) ... weil es für ihn keine Realität, sondern nur die e i gene, $s$ e $l b s$ geschaffene wirklichkeit gibt.

(11) In Mödlareuth gebe es nur. $b e s c h r a ̈ n k t e$ Parkmöglichkeiten für $P K W$ und Busse.

(12) ... das Druckhaus, dem lediglich ein $v$ e $r$ a $l$ t e $t$ e $r$ Maschinenpark verblieben war.

3. Genitiv- und Präpositionalattribute:

(13) Tatsächlich blühte die wirtschaft zunächst in den Ländern nur des $K \circ n t i n$ e $t s$ auf, nicht in denen der dritten welt.

(14) Sind Sie so vorsichtig mit Rücksicht auf die Arbeitslosenzahlen, oder die Preissteigerungen, oder mit Rücksicht nur auf die Gewinnentwicklung der Großunternehmen?

Die Gruppen 1. und 2. mit ihren Untergruppen zeigen in ihrem syntaktischen Verhalten als Skopus von GPn keine signifikanten Unterschiede. Soweit 3. davon abweicht, wird es jeweils vermerkt.

Klassenbildung/Austauschbarkeit

Syntaktische Gründe außer möglichem Funktionswechsel von allein und einzig in der Position nach dem finiten Verb für eine Beschränkung der Austauschbarkeit existieren nicht. Die Inakzeptabilität von allein und einzig in. (2) (die sich auch durch Umstellung usw. nicht beseitigen läßt) kann nur einen semantischen Grund haben, zumal diese beiden GPn z.B. in (7) voll akzeptabel sind: es ist natürlich wieder der Ausschluß der skalierenden Interpretation durch allein und einzig.

Stellungsgesetze

Die GP steht bei diesem Skopustyp normalerweise vor der gesamten NP. In poetischen bzw. rhetorischen Texten findet man auch die Stellung nach der gesamten NP. Darüberhinaus gibt es die stellung zwischen definitem oder auch indefinitem Artikel und dem kontrastiv betonten Adjektivattribut: sie ist spezifisch für diesen Skopustyp.

(15) Nur eine $q$ u a $n t i f i z i$ e $r$ e $n d e$ Interpretation...

(16) Eine nur $q u a n t i f i z i$ e $r$ e n $d e$ Interpretation...

Der Stéllungsunterschied ist mit einem semantischen Unterschied verknüpft, der durch die folgenden Paraphrasen verdeutlicht werden kann:

(15a) Nur eine Interpretation, die $q$ u a $n t i f i z i$ e $r t \ldots$

(16a) Eine Interpretation, die nur $q$ u a $n t i f i z i r t \ldots$

Bei (15a) liegt also der Skopustyp komplexe subjekts-NP vor, verknüpft mit quantifizierender Interpretation. Bei (16a) hingegen handelt es sich um den Skopustyp Verbum, verknüpft mit skalierender Interpretation. Insofern ist dieses Beispiel falsch eingeordnet. Für weitere Informationen verweise ich auf den Abschnitt 3.3. Das Satzpaar ist zudem das vielleicht überzeugendste Beispiel, daß die Erscheinungen GP-Skopus und Kontrastfokus unterschieden werden müssen.

Außer der Stellung der GP am SatzschluB bei Topikalisierung der NP, die das kontrastiv betonte Attribut enthält, kommt keine Distanzstellung vor. Für Genitiv- oder Präpositionalattribute ist die Stellung zwischen Bezugsnomen und Attribut kennzeichnend. In allen übrigen Positionen der GP unterscheidet sich dieser Skopustyp nicht von einer gewöhnlichen Skopus-NP. 
Skopus/Fokus

Alle Versuche, das Attribut allein abzuspalten, schlagen fehl. Hingegen ist die Abspaltung ('Clefting') der gesamten NP (unter Beibehaltung des Kontrastakzentes auf dem Attribut) durchaus möglich:

(4a) "Es ist nur ein edler $\dddot{M}_{E i n}$ e $d 1$ e $r$ ist es nur, der uns adelt Geist.

(4b) Ein e d 1 e $r$ Geist ist es nur, der uns adelt.

Dieses Ergebnis macht es wahrscheinlich, daß nicht nur das Attribut, sondern die gesamte NP jeweils den Skopus der GP bildet.

Zur semantischen Analyse

Ein Blick auf die möglichen Kontrastierungen zeigt bereits die "Vorzüge" dieses Skopustyps :

(1a) ... nur für einen $h \circ h$ e $n$ Preis, nicht für einen niedrigen.

(2a) ... nur eine $k l$ e in e Rolle, keine große/bedeutende.

(4c) ... n nur ein e $d l$ e $r$ Geist, nicht ein gemeiner/hinterhältiger.

Das Kernnomen darf also keinesfalls verändert werden:

(5a) “

Damit ist der Assertionsbereich schon sehr stark eingeschränkt. Darüberhinaus stehen meist nur geringe Wahlmöglichkeiten bei den Attributen zur Verfügung: i.d.R. handelt es sich um polare Paare, die skalierend und quantifizierend interpretiert werden können, nur selten um mehrgliedrige Skalen. Deshalb die Analysesätze (2) für skalierenden und (9) für quantifizierenden Gebrauch.

(2b) Die $F$ achdidaktik spielt nur eine $k l$ e $i \mathrm{n} e$ Rolle.

PSP: Die $F$ achdidaktik spielt eine kleine Rolle.

ASS: Die $F$ achdidaktik spielt keine große Rolle.

(konv. IMPL: Die $F$ achdidaktik spielt nicht überhaupt keine Rolle.)

(9) Nur ein $s t a r k$ e $r$ paucher trägt ein erhöhtes Lungenkrebsrisiko.

PSP: Ein starker ucher trägt ein erhöhtes Lungenkrebsrisiko.

ASS: Jemand, der wenig raucht, trägt kein erhöhtes Lungenkrebsrisiko.

Suspensionstest:

(2c) Die $F$ achdidaktik spielt nur eine $k l$ e i n e Rolle,

- und möglicherweise spielt sie nicht einmal eine kleine Rolle. (PSP)

- und möglicherweise spielt sie sogar überhaupt keine Rolle.

(konv. IMPL)'

- “und möglicherweise spielt sie sogar eine große Rolle. (ASS)

(9a) Nur ein $s t a r k$ e $r$ Raucher trägt ein erhöhtes Lungenkrebsrisiko,

- ?und möglicherweise nicht einmal. ein starker Raucher. (PSP)

- "und möglicherweise sogar jemand, der wenig raucht. (ASS)

Hier zeigt sich, daß nur eine skalierende Interpretation durchaus zuläßt. Mit allein wäre eine Suspension der PSP inakzeptabel gewesen.

Konjunktionstest:

(2d) Die $F$ achdidaktik spielt nur eine $k$ l e i n e Rolle,

- "und sie spielt eine kleine polle. (PSP)

- und die $F$ achdidaktik spielt keine große Rolle. (ASS)

- ?und die $F$ achdidaktik spielt nicht überhaupt keine Rolle.

(konv. IMPL)

(2e) - Die F achdidaktik spielt eine kleine Rolle, (PSP; betontes nur!)

- Die $P$ achdidaktik spielt keine große Rolle, (ASS)

- "Die F achdidaktik spielt nicht überhaupt keine Rolle, (konv. IMPL) und die $F$ achdidaktik spielt nur eine $k l$ e $i n e$ Rolle. 
In beiden Fällen scheint die Anbindung der konv. IMPL mit aber voll akzeptabel (abgesehen von der seltsamen Negationsmöglichkelt).

Dialogische Analyse:

(2f) A: Die F achdidaktik spielt nur eine $k l$ e $i \mathrm{n}$ e Rolle.

B: - Nein, sie spielt eine große Rolle. (ASS)

- Na ja, daß sie eine kleine Rolle spielt, mag ja stimmen, (PSP)

"aber sie spielt doch eine große Rolle. (ASS-Verneinung)

- ?Na ja, daß sie keine große Rolle spielt, mag ja stimmen, (ASSZustimmung) aber sie spielt doch keine kleine Rollel(PSP-Protest)

- Ich find' das gar nicht so schlimm. (Skala selbst)

- Ja, sie spielt wirklich keine große Rolle. (ASS)

- Na ja, die Rolle, welche die Fachdidaktik spielt, ist sicherlich eine kleine und keine große. (PSP + ASS)

Bei diesem ja sehr einfachen, eindeutigen Fall wiegen die gewissen Abweichungen von den Normalergebnissen umso schwerer. Es zeigt sich, daß es sich um ein geordnetes polares Paar in PSP und ASS handelt, wobei nur die untere Stufe mit nur kombiniert werden kann. Eine ad-hoc-Erweiterung zu einer Skala mit mehr Gliedern ist zwar immer möglich, dadurch verändern sich die Testbedingungen. Hingegen setzt sich in allen Fällen, wo dies möglich ist, die polare Interpretation durch. Dies entspricht, wenn ich recht sehe, völlig der Beschreibung der relativen Adjektiva in Bartsch/Vennemann (1973). - Während also NPn mit Adjektivattributen im Skopus von GPn ein von den übrigen NPn erkennbar verschiedenes semantisches Verhalten zeigen, kann das Gieiche nicht bei Genitivund Präpositionalattributen im Skopus gesagt werden. Abgesehen davon, daß sich nur sehr schwer akzeptable Belege hierfür finden lassen, kann man für die wenigen Beispiele keinen semantischen Unterschied zur gesamten NP im Skopus finden.

(13a) In den Ländern nur des $K \circ n t i n$ ent $s=\underline{n u r}$ in den Ländern. des Kontinents.

\subsubsection{2. auch - Gruppe}

1. Attributive Adjektiva:

(1) Die bange $F$ rage, ob weitere Expansion sinnvoll sei, beschlich linke und oft auch durchaus $k \circ n s$ e $v$ a $t i v$ e Politiker.

(2) Die Kriminelien haben sich um das alte waffengesetz nicht gekümmert, und sie werden sich auch um das $n$ e u e nicht kümmern.

(3) ... Puderweiß, wie einst Skifreund Thomas Mann die flockigen Kristalle genannt hat, ohne die auch die $s c h$ ön $s$ e skipiste nicht auskommt.

(4) Da darf auch nicht der $k I$ e i n $t$ e Fehler passieren.

(5) Das erfuhr der Präsident, der ... den Spitznamen "Kugel-Schreiber" hat und neuerdings auch auf ganz $z i v i l$ e $n$ Partys nicht mehr ohne PPK-Pistole anzutreffen ist...

(6) In den Aufnahmefragebogen werden nämlich als "Vorstrafe" auch die rein $p \circ 1$ i $t i s c h$ e $n$ DDR-Urteile eingetragen.

(7) Ich meine nicht die politische bimension. Aber ich würde erwarten, daß jetzt auch $k$ u $l$ t u r e 11 e Konsequenzen gezogen werden.

(8) Auch ein $\overline{l e} i d e n s c h$ a $t$ t $l i c h e r$ Jäger wird nicht so $\overline{v i e l}$ für ein Revier anlegen wollen.

2. Attributive Partizipien:

$F$ reilich, auch ein $g$ e bä $n d i g t$ es wachstum würde wohl kaum das sichern, was... 
(10) Auch eine von den Geißeln des Umweltschmutzes bef $r e i t e$ Welt würde ohne Wachstumsstop unweigerlich untergehen.

3. Genitivattribute:

(11) Er prüft die Anregungen der Laien. Die Vorschläge der Domkapitel und auch der $B$ i $s$ C h o f $s$ konferenzen müssen ...

(12) Für diesen Vorschlag votierten die Rheinischen Kommunalbeamten. Die überwältigende Mehrheit auch der $R$ u h $r g$ e bi e t s-Oberbürgermeister hatte...

Stellungsgesetze:

Typ 1: GP unmittelbar vor der kontrastiv betonten Konstituente. Dieser Stellungstyp ist in diesem Fall (außer bei den Genitivattributen und bei den NPn ohne Artikel) nicht anwendbar. Es gibt also nicht die bei der nur-Gruppe beschriebene GP-Position zwischen Artikel und kontrastiv betontem Adjektivattribut mit ihren semantischen/Skopusfolgen.

Typ 2: GP unmittelbar nach der Skopuskonstituente. Hier gilt ebenso, daB die GP nach der Konstituente stehen kann, die das kontrastiv betonte Attribut enthält. - Auch bei diesem Skopustyp gibt es die Möglichkeit der Kombination von topikalisierter Skopus-NP mit kontrastiv betontem Attribut und betontem auch nahe dem Satzschluß: nur scheint es in denjenigen, hier etwas zahlreicheren Fällen, in denen auch dieselbe Semantik wie sogar hat, gewisse Schwierigkeiten zu geben:

(3a) 2... die s chö ns te skipiste kommt ohne sie a u ch nicht aus. ?... der $k l e i n s t e$ Fehler darf a ${ }^{2} c h$ nicht passieren.

Man kann, glaube ich, nicht übersehen, daß diese Position von auch ausschließlich die Funktion der Hinzufügung, also eine rein quantifizierende Funktion hat. Nun ist aber offenbar auch in Verbindung mit einem Superlativ-Adjektivattribut sowie mit einer Satznegation (bei Normalform des Adjektivs) nur in der Bedeutung von sogar möglich: dies scheint die gewisse Inakzeptabilität von (3a) und (4a) zu verursachen. (Siehe auch Altmann: 1976).

Zur semantischen Analyse:

Hierfür gilt zunächst einmal dasselbe wie bei der nur-Gruppe (3.2.3.1.) verzeichnet: das jeweilige Bezugsnomen definiert sehr genau den präsuppositionellen Bereich, und zwar für nur quantifizierende Interpretation die Menge der mit dem betreffenden Nomen verbindbaren Attribute, für die skalierende Interpretation kommt noch die Skalendefinition durch das Attribut selbst und die Angabe der Richtung der Skalł durch die GP hinzu. - Das interessanteste Thema ist jedoch die Erfassung der formalen wie der semantischen Kriterien für die Zuweisung der quantifizierenden bzw. der skalierenden Interpretation. Die formalen wurden oben bereits genannt; davon gilt die Verbindung mit dem Superlativ offenbar in jedem Fall, ganz sicher jedoch die Kombination auch + Superlativ-Attribut + Satznegation. Die Negation allein ist jedoch kein sicheres zeichen: siehe Satz (2). Ambig scheint (5) zu sein, eindeutig im Sinne der sogar-Interpretation Satz (8). Völlig auszuschließen ist die sogar-Interpretation auch bei den meisten anderen Beispielen nicht.

(8) Auch ein 1 e $i d$ e n schaftlicher Jäger wird nicht soviel für ein Revier anlegen wollen.

ASS: Ein leidenschaftlicher Jäger wird nicht soviel für ein Revier anlegen wollen.

PSP: Andere Jäger (quant.)/weniger begeisterte Jäger (skal.) werden nicht soviel für ein kevier andegen wollen.

konv. IMPL: Ein der Jagd völlig verfallener Mensch wird soviel für ein Revier anlegen wollen. (?) 
Suspensionstest:

(8a) Auch ein 1 e $i d$ e n schaftlicher Jäger wird nicht soviel für ein Pevier anlegen wollen,

- "und möglicherweise werden sogar andere Jäger soviel für ein Revier anlegen wollen. (quant. PSP)

- *und möglicherweise werden sogar weniger begeisterte Jäger soviel für ein Pevier anlegen wollen. (skal. PSP)

- und möglicherweise werden nicht einmal dem Jagen völlig verfallene Menschen soviel für ein Revier anlegen wollen. (konv. IMPL)

Das Ergebnis entspricht völlig dem bei der Interpretation von sogar in vergleichbaren Fällen. Es wird durch den dialogischen Test nachdrücklich bestätigt.

(8b) A: Auch ein 1 e $i d$ e n schaftlicher Jäger wird nicht soviel für ein Revier anlegen wollen.

B: - Doch, das wird er durchaus tun. (ASS)

- ?Aber alle anderen Jäger wollen doch soviel anlegen! (quant. PSP)

- Aber weniger leidenschaftliche Jäger wollen doch soviel anlegen. (skal. PSP)

- Sogar ein der Jagd völlig verfallener Mensch wird das nicht tun. (konv. IMPL)

- Das mag schon sein, daß ein leidenschaftlicher Jäger nicht soviel für ein Revier anlegen will, aber die neureichen AuchJäger werden die Summe schon hinblättern. (ASS-Zustimmung + PSP-Protest!)

Erstaunlicherweise ist die letzte Kombination hier nicht inakzeptabel. Ev. könnte es sich um einen Skalenwechsel handeln, und zwar von der der emotionalen Verbundenheit mit der Jagd zu der "Prestige-Skala", die hier gegensätzliche Richtungen aufweisen (Wechsel der Bezugsmenge).

- Nein, natürlich wird er das nicht tun. (ASS)

- Daß normale Jäger nicht soviel Geld anlegen wollen, nehme ich schon auch an. Aber bei einem leidenschaftlichen Jäger zweifle ich doch sehr. (Zustimmung zur skal. PSP, ASS-Verneinung)

- Du schließt aber doch nicht aus, daß ein der Jagd völlig verfallener Mensch soviel blechen könnte? (konv. IMPL)

- ?Daß andere Jäger (gewehrtragende, grünbehoste, dickleibige) das nicht tun werden, glaube ich eigentlich auch. (quant. PSP)

\subsubsection{3. sogar - Gruppe}

1. Attributive Adjektiva:

(1) Der Bayernkurier sprach sogar von einer $k r i m i n$ e $l$ l e $n$ Mehrheit, die ... für Bonn abfalle.

(2) Aber das Lillehammer $\neg$ Kommando schaltete selbst $k$ le ine Risiken aus.

(3) ... um das Transatlantik-Kabel sogar vor den mo $d$ e $r n s t e n$ Schleppnetzen zu schützen.

(4) In seinen $F$ irmen herrschte Hubmann wie ein absoluter Fürst, selbst die $k I e i n s t e n$ Entscheidungen behielt er sich vor.

(5) Sogar unsere e $i g$ e $n$ e U rteile können wir jetzt nicht mehr vertreten.

(6) Die Tünche des liberalen Rechtsstaats fällt jedesmal dann ab, wenn es um - selbst nach den e $i \mathrm{~g} e n$ e $n$ Verfassungsvoraussetzungen legale Angriffe gegen die herrschende Klasse geht. 
2. Attributive Partizipien:

(7) Freilich, sogar ein derart $g$ e bä $n d i g t$ e $s$ wachstum würde wohl kaum das sichern, was...

3. Genitivattribute:

Dem Miramare-Beach-Hotel in Korfu wird angekreidet, daß dort der tägliche Gebrauch selbst des $L i$ e ges $t$ u h 1 s noch etwas koste.

Die hier geltenden Gesetzmäßigkeiten können weitgehend von den parallelen Abschnitten 3.2.3.1./2. bzw. von den vorausgehenden sogar-Abschnitten übertragen werden. Besonders vermerkt sei nur, daß, wie bei auch, die Position der GP zwischen Artikel und kontrastiv betontem Adjektivattribut nicht möglich ist.

\subsubsection{Skopustyp: Präpositionalobjekte}

\subsubsection{1. nur - Gruppe}

(1) Er pocht nur auf sein $R$ e cht.

(2) Ich denke nur über die $A r b$ e $i t$ nach.

(3) Gunther spricht nur über/von $G \circ$ e $t h e$.

(4) Das hängt allein von $K$ a $r l a b$.

(5) Es ist daher in keiner Weise ausgemacht, daß die sprachlichen Abweichungen sich aus dem aphasischen Symptom allein ergeben. Man denke nur an das Heer von $V$ e $r k$ e $h r s$ polizisten.

Stellungsgesetze:

Typ 5 (Skopuskonstituente topikalisiert, GP in Distanz danach) kann Auskunft geben daribber, ab tatsächlich nur das Präpositionalobjekt im Skopus steht, oder ab die gesamte VP den Skopus bildet:

(la) Auf sein $R$ e $c h t$ pocht er nur.

(6a) An das Heer von $V$ e $r k$ e $r s$ polizisten denke man nur.

Auch durch diese Umstellung wird eine Slopuskonstituente Präpositionalobjekt nicht diskontinuierlich, ebensowenig aber die.VP (abgesehen von analytischen Verbalformen): erst letzteres wäre aber ein starkes Argument gegen einen Skopus Präpositionalabjekt.

Typ 6: Kontrastiv betonte GP. In allen oben aufgeführten Sätzen ist eine starke Akzentuierung der GPn möglich, wenn auch nicht gerade eine kontrastive. Daneben kann der Kontrastakzent auf der Skopuskonstituente fast völlig verschwinden. Man könnte allerdings auch der Ansicht sein, daß es dadurch zu einem Bedeutungs- bzw. Funktionswandel kammt: nur erhielte dann die Bedeutung immer nur, fortwährend, ausschließlich. Ob dies aber gar die einzig mögliche Analy- 
se auch fur die unbetonte GP ist, können erst Kontrastsätze zeigen.

Syntaktische Umformungen: Interessant ist hier nur der Imperativ.

(1b) Poch nur auf dein Recht!

(2a) Denk nur über die Arbeit nach!

(3a) Sprich bloß von Goethe!

Die Akzente wurden absichtlich nicht markiert; es gibt nämlich elne ganze Reihe von Möglichkeiten:

1. Kontrastiver Akzent auf dem Verb: in diesem Fall offenbar eher elne theoretische Möglichkeit, da sehr enge Restriktionen bestehen, nämlich Kontrastlerung des gesamten Prädikates:

(2b) Denk nur über die Arbeit $n a c h, s c h r e i b$ sie nicht/ $s$ p r $\overline{i c h}$ nicht über sie.

(2c) Du sollst nur über die Arbeit $n$ a $c h$ denken, aber sie nicht $s c h r e \overline{i b}$ en.

Eine weitere Möglichkeit ist, daß mit einem Verb im gleichen Satzrahmen kontrastiert wird, d.h. mit dem gleichen Präpositionalobjekt einschließlich Präposition. Aber auch in diesem Fall klingen sowohl Ausgangs- wie Kontrastsatz recht seltsam.

2. Lediglich emphatische Akzente auf Verb und Nomen des Präpositionalobjektes. In diesem Falle sind Kontrastsätze ungrammatisch. Es sind lediglich Fortsetzungen denkbar wie zu (1b): ... du wirst die Leute schon uberzeugen.

zu (2a): ... vielleicht kommst du dann auf deine Fehler.

zu (3a): ... du wirst schon sehen, wie alle einschlafen.

Hierbei handelt es sich um die Verwendungsweise 'MP'. Denkbar wäre bel den gegebenen Merkmalen (Stellung, Akzent) auch eine Verwendung im Sinne einer GP mit dem Skopus Satz. Eine solche Lösung hat aber nur dann einen Sinn, wenn die syntaktischen und semantischen Eigenschaften von GPn dabei erhalten bleiben:

(1c) Tu nichts anderes, poch nur auf dein Pecht!

(1d) Tu nur e in e s : auf dein Pecht pochen.

Eine Interpretation dieser Art kann aber für (1b), (2a) und (3a) ausgeschlossen werden.

3. Kontrastiver Akzent auf dem Nomen, soweit dieses kontrastierbar ist. Damit ist aber die gesamte Präpositionalphrase Skopus der GP (siehe dazu auch den Abschnitt uber kontrastive Vorgänger und Folgesätze!).

4. Kontrastive Akzente oder auch nur emphatische Akzente auf verb und auf dem Nomen der Präpositionalphrase; dies wïrde einen Skopus VP bedeuten. Die Kon- 
trastierung einer ganzen VP scheint aber bei dieser Stellung der GP Innerhalb der VP eher gemieden zu werden, man bevorzugt Ersatzformulierungen mit der Stellung GP - gesamte VP:

(le) Du sollst nur auf dein $R e c h t$ pochen, aber nicht...

(1f) Ich befehle dir, nur auf dein $R$ e $c h t$ zu pochen und nicht Unfug $z u$ machen.

Spaltsatzbildung: die dabei entstehenden sehr eigenartigen Gebilde verweisen nachdrücklich auf die Schwierigkeit, nur einen Teil des Gebildes Verb + Präpositionalphrase als Skopus einer GP zu betrachten:

(2d) ?Es ist nur die $A r b$ e $i t /$ die $A r b$ e $i t$ ist es nur, worüber ich nach denke.

(2e) ${ }_{E s}$ ist nur über die $A r b$ e $i t /$ Uber die $A r b e i t$ ist es nur, worüber $\overline{i c h}$ nachdenke.

(2f) ?Es ist nur über die $A r b$ e $i t$ nachdenken, was ich tue/?tber die die $A r \overline{b e} i t$ nachdenken ist es nur, was ich tue.

Utber die normale geringe Inakzeptabilität von Spaltsätzen im Deutschen hinaus scheinen (2d) und (2e) abweichend. (2f) kann insofern nicht in die Wertung einbezogen werden, als eine VP im Fokus eines Spaltsatzes in jedem Fall höchst prekär ist.

Negationsmöglichkeiten:

Typ 1 (nicht nur) zeigt bereits die Schwierigkeit, einleuchtende Kontrastsätze für diesen Skopustyp zu finden:

(1g) Er pocht nicht nur auf sein $R$ e $c h t$, sondern...

Typ 3, die Satz-NEG, bereitet ebenfalls gewisse Probleme:

(3b) Gunther spricht nur von $G \circ$ e $t h e$ nicht.

(3c) Nur von $G \circ$ e the spricht Gunther nicht.

In beiden Fällen kann eine konjunktionale Interpretation von nur in der Art von (3d) nicht völlig ausgeschlossen werden, doch bedingt sie wohl eine andere Akzentverteilung:

(3d) Nur, Gunther spricht nicht von Goethe/Nur spricht Gunther nicht von Skopus und Fokus

Für die Akzentpositionierung, das Kernthema dieses Abschnitts, ergeben sich gewisse Probleme aus der Tatsache, daß die Abfolge Verb - GP - Präpositionalobjekt generell auch als GP mit dem Skopus V oder VP interpretiert werden kann. Diese Interpretation wird noch durch die enge Bindung zwischen Verb und Prä- 
positionalobjekt gefördert, ${ }^{10}$ ferner durch gewisse fast idiomatische Fügungen mit einem Präpositionalobjekt: hierbei ist das Verb in seinen möglichen Ergänzungen sehr eingeschränkt, ja es weist manchmal sogar nur eine einzlge Möglichkeit zur Ergänzung auf. In diesen Fällen ist schon aus technischen Gründen kein Kontrastsatz möglich:

(lh) Er pocht nur auf sein $R$ e $c h t$, er macht keinen Unfug.

(li) *Er pocht nur auf sein $R e c h t$, nicht an die tür.

Bei (lh) liegt die gesamte VP im Skopus: hierbei ist sowohl implizit wie explizit kontrastive Verwendung möglich, doch dïrfte die implizite Kontrastierung (wie bei allen Skopustypen aus mehreren Elementen) überwiegen, wobei die normale Satzschlußbetonung kaum verstärkt wird. ${ }^{11}$ - In Satz (1i) liegt zwar der kontrastive Akzent eindeutig auf Recht, doch paßt das kontrastierende Nomen nicht. Die hier angeführte Möglichkeit brächte zusammen mit dem Wechsel der semantischen Merkmale bei für einen Wechsel vom Präpositionalobjekt zur adverbialen Präpositonalphrase: das Ergebnis ist unbestreitbar inakzeptabel. Natürlich gibt es auch Beispiele, bei denen eine Kontrastierung möglich ist:

(3e) Gunther spricht nur über Go e $t h$ e, keineswegs über Handke/nie über ein anderes Thema.

Das hängt allein von $K$ a $r 1$ ab, nicht von Helmuth/von niemandem sonst.

Semantik: Auch hier führen die nichtkontrastierbaren Präpositionalobjekte zu Komplikationen. So ist die nomale Analyse für Satz (1):

(1) PSP: Er pocht auf sein pecht.

ASS: Er pocht auf nichts anderes.

nicht sehr sinnvoll, da sie einen Wechsel vom Präpositionalobjekt (in der PSP) zur adverbialen Präpositionalphrase (in der ASS) erzwingt. Überdeutlich zeigt dies der Suspensionstest:

(1j) :Er pocht nur auf sein $R e c h t$, und möglicherweise nicht einmal das/und möglicherweise pocht er nicht einmal darauf.

Hingegen bringt der gleiche Test, angewendet auf die VP als Skopus, wesentlich bessere Ergebnisse:

10 Siehe auch R. Steinitz (1969: 2.9. Adverbial und Präpositionalobjekt,40-46.)

11 Bei explizit kontrastiver Verwendung läßt sich meist zum Satzschluß hin steigende Satzintonation, die eine Fortsetzung erwarten läßt, bzw. tatsächlich einleitet, feststellen. Geht der Kontrastsatz voraus, so liegen i.d.R. emphatische Akzente sowohl auf nur wie auch auf Recht. Leider sind die Aussagen über den Akzent bei einem Skopus VP noch sehr vage und spekulativ, es ist auch sehr wohl möglich, daß sich in ihnen phonetische und semantisch-funktionale Aussagen mischen. 
(1k) Er pocht nur auf sein $R$ e $c h t$, und möglicherweise tut er nicht einmal das/und möglicherweise pocht er nicht einmal auf sein Recht.

Zweifelhaft ist lediglich, ob diese Art von VP irgendwelche skalierende Interpretationen zuläßt, und dies ist ja die Voraussetzung für die Suspension.

\subsubsection{2. auch - Gruppe}

(1) Die Kriminellen werden sich auch um das $w$ a f $f$ e $n$ gesetz nicht scheren.

(2) Warum sollte in einem Staat nicht auch einer so frei sein, diese F reiheit auch für die $\ddot{A} r z t$ e organisation herbeizusehnen?

(3) Auch auf die ohnmächtige öf $\mathrm{f}$ e $t \mathrm{l}$ i chkeit nahm Nixon keine Rücksicht.

(4) Ind wir könnten auch an der Entwicklung der sowjetischen Produktion mitwirken und zu einem Energieverbund kommen.

Austauschbarkeit/Klassenbildung

Der Eindruck, daß bei diesem Skopustyp eine größere Zahl von Austauschmöglichkeiten für das umbetonte auch, z.B. durch ebenso, uberdies, obendrein, weiters usw. besteht, täuscht, jedenfalls hinsichtlich der GP-Funktion: denn nur dann, wenn man auch, wie in dieser Stellung möglich, im konjunktionalen Sinn interpretiert, bestehen diese Möglichkeiten, wie sich durch Umstellung zeigen läßt:

(3a) Auch/"ebenso/"überdies/"obendrein ... auf die ohnmächtige óf f e nt lichkeit nahm Nixon keine Rücksicht.

(3b) Auch/ebenso/überdies/obendrein ... nahm Nixon auf die ohnmächtige óf fent lichkeit keine Rücksicht.

Stellungsgesetze: Wiederum betreffen Zusätze nur die Typen 5 und 6. Es zelgt sich nämlich, daß die betonte Version des auch (sie muß nachgestellt werden!) zum einen immer vor der Satznegation stehen mu, und daß diese Kombination besonders geläufig ist:

(la) Um das $w$ a $f$ e $n$ gesetz werden sich die Kriminellen $\underline{a} u_{c h}$ nicht scheren.

(3c) Auf die ohnmächtige Öffentlichkeit nahm Nixon a

\section{Satzarten}

Fragesätze: während die normale wortfrage

(3d) Warum hat Nixon auch auf die ohnmächtige ö f $f$ e $n$ t lichkeit keine Rücksicht genommen?

noch kaum Ansätze zu einer anderen Interpretation des auch zeigt, wird sie bei Verkürzung des Satzes unausweichlich: 
(3e) Warum auch auf die Öffentlichkeit Rücksicht nehmen! 12

In diesem Satz ließe sich auch eine Betonung auf dem Fragewort und ein enger Anschluß von auch an das Fragewort denken, beides Zeichen für die Verwendung von auch als Fragepartikel bzw. MP. Die gleiche Konstruktion ist ubrigens auch bei nur möglich, allerdings verkürzt auf Fragewort und nur, oder mit nur am Satzschluß, oder nur kombiniert mit immer.

\subsubsection{3. sogar - Gruppe}

(1) ... daß mein Mann sich als Werkstudent durchsetzen konnte und es sogar bis zum $s$ e n a t $s$ r t brachte.

(2) CDU-Barzel hielt seine Erklärung vom letzten $F$ reitag vorher selbst vor den $P$ a $r$ te $i$ f $r$ unden geheim.

(3) Nicht einmal an das $R$ a si e $r$ was $s$ e hat sie gedacht.

Außer dem üblichen Funktionsumschlag von selbst in Positionen, in denen es auch als Reflexivum möglich ist, ist auch alles andere aus den jeweiligen Parallelkapiteln hier anwendbar.

\subsubsection{Skopustyp: Pronominalisierte Nominalphrasen}

3.2.5.1. nur - Gruppe

1. Personalpronomina:

(1) Nur ihr, ih $r$ habt verloren.

(2) Peiß denn von einem Kummer dich los, der $d i c h$ nur angeht.

(3) Einzig und allein $d i / d i r$ einzig und allein gilt meine Sorge.

(4) Einzig ih $r$ wollte er sich anvertrauen.

2. Demonstrativpronomina:

(5) Nur/allein $d e r / d i$ eser/jener/finsol che $r$ kann uns helfen, ...

(6) Nur/allein der/derjenige/jener kann uns helfen,...

3. Artikel: Definite und indefinite Artikel können, gegen Bartsch (1972:192), nicht Skopus von GPn sein. Soweit sich Artikelformen im Skopus befinden, handelt es sich um Demonstrativpronamina bzw. Zahlwörter.

$$
\text { Nurlallein } d \text { e } r / \text { e i } n \text { Mann kann uns helfen... }
$$

12 Zur Einordnung infinitivischer Fragen siehe Gordon/Lakoff (1971). 
4. Reflexivpronomina:

(8) Der Eigensüchtige hilft nur $s i c h$ ( $s$ e 1 b s $t)$.

(9) Er denkt bloß/lediglich/ausschließlich an sich.

5. Possessivpronomina:

(10) Nur s e in wille geschehe.

6. Relativpronomina, Fragepronomina: ${ }^{13}$

(11) " ${ }_{\text {Nur }}$ we $r /$ we l c he $r / d$ e $r$ die Sehnsucht kennt, weiß...

7. Indefinitpronomina: ${ }^{14}$

(12) "Nur/allein je m a nd / i r g e ndwelcher/irgendStellungsgesetze:

Typ 1 (GP unmittelbar vor dem Skopus) ist auch hier die Normalstellung.

Typ 2 (GP unmittelbar nach dem Skopus) ist überall möglich, allerdings mit den bekannten stilistischen Folgen, wie (2) zeigt. Dabei darf die GP die Grenze zwischen zwei größeren Konstituenten (Satzgliedern in der Regel) nicht verlassen. So z.B. darf nur nicht zwischen Demonstrativpronomen und Bezugsnomen stehen (das gilt auch für Possessivpronomina).

(13) "De $r$ nur Mann kann uns helfen.

Grundsätzlich verboten ist offenbar die Nachstellung der GPn bei Reflextva:

(8a) “Der Eigensüchtige hilft $s i c h$ ( $s$ e l b s $t$ ) nur.

Als Begründung kann ich nur die zweifelhafte Betonbarkeit von sich angeben. Die Tatsache hingegen, daß nachgestelltes nur bei Fragepronomina akzeptabel ist, hebt nicht die generelle Feststellung auf, daß Fragepronomina nicht im Skopus von GPn stehen können:

(14) Wa r um nur/"iallein/"einzig/?bloß ... hat er das getan?

Schon die fehlende Austauschbarkeit zeigt, daß es sich nur um die Mp-Funktion handeln kann. Identisch damit ist:

(14a) Wa $r$ u m hat er das nur/bloß getan? / Warum $h$ a $t$ er das nur getan? Syntaktische Umformungen:

Von den verschiedenen Satztypen ist wiederum nur der Imperativ interessant: sobald nur und $b$ LOB in die Position nach dem finiten Verb rücken, werden sie

13 Siehe hierzu Keenan (1971: 268).

14 Siehe auch B. Fraser (1971: 153). 
als MPn interpretiert, es sei denn, die folgenden Konstituenten weisen höhere Bindungskräfte auf, wie es etwa beim Demonstrativpronomen der Fall ist:

(15) Hilf dir nur $s$ e l b s $t$ ! (MP)

(16) Hilf nur di e s e m! (GP)

Spaltsatzbildung: sie weist bei diesem Skopustyp einen erstaunlich hohen Grad an Akzeptabilität auf. Komplikationen können wegen der Elngliedrigkeit des Skopus nicht auftreten.

Skopus und Fokus:

Wie schon bei den Belegsätzen deutlich wurde, treten bei diesen Skopustypen eine ganze Reihe von Beschränkungen hinsichtlich der syntaktischen bzw. semantischen Merkmale der Skopuspronomina auf. Sie gllt es der Reihe nach zu erfassen und zu erklären:

1. Von den Personalpronomina kann es nicht im Skopus von GPn stehen: ${ }^{15}$

(17) “Nur es (das Kind) kann uns helfen.

Als Erklärung bleibt nur die fehlende Betonbarkeit. Mangelnde Kontrastierbarkeit (semantisch) kann es jedenfalls nicht sein, doch ist dies eine plausible Erklärung für den Ausschluß des Platzhalter - es als Skopus von GPn:

(18) 'Nur es regnet/schneit.

(19) 'Ich hoffe nur es.

2. Sämtliche Artikel können nicht den Skopus von GPn bilden, obwohl eine gewisse Kontrastierbarkeit, z.B. zwischen definiten und indefiniten Artikeln vorhanden wäre; diese Form des Kontrastes gleicht jedoch der Kontrastierung von Wortteilen (oder es handelt sich um metasprachlichen Gebrauch).

(20) Ich habe nux e in, nicht de $r$ Idiot gesagt.

In allen anderen Fällen tritt bei starker Betonumg Funktionswechsel ein.

3. Mangelnde Kontrastierbarkeit ist auch der Grund für den Ausschluß der Reflexivpronomina bei reflexiven Verben von der Skopusfähigkeit:

(21) "Sie treffen nur $s i c h$.

(22) :Die F reunde streiten nur sich.

Der Grund hierfür ist sicherlich, daß es sich um obligatorische Elemente handelt. 4. Die gemeinsame Ursache des Ausschlusses all dieser Elemente von der Skopusfähigkeit ist ihre Indefinitheit: auch die Fragepronomina fallen unter diese Kennzeichnung.

15 Vgl. Fraser (1971: 176). 
Akzentprobleme treten nur dort auf, wo ein Nomen hinzutreten kann, also bel Demonstrativpronomina und Possessivpronomina. Betonungsalternativen gibt es aber wohl nur bei den Possessivpronomina, wohl kaum bei Demonstrativpronomina:

(23) “Während das Bettenangebot allein in diesem $J$ a h $r$ um 30 ooo Betten anstieg ...

"Einzig aus diesem $G r$ un $d$ e hatte er Erfolg.

Der Grund hierfür könnte darin liegen, daß das Demonstrativpronomen automatisch den Satzakzent an sich zieht, also grundsätzlich Fokuskonstituente ist (dies betrifft nicht die Fälle von Textreferenz). Dazu paßt auch, daß mehrere Demonstrativpronomina je Satz recht seltsam wirken: also eine Anzahl von Parallelen mit nur, nicht zuletzt auch mit dessen identifizierender Funktion.

\subsubsection{2. auch - Gruppe}

1. Personalpronomina:

(1) Kaum war Barzel zu Ohren gekommen, daß sich sein Gegner vorbereitet, ließ auch e $r$ sich von der Parteizentrale einen Reiseplan ausarbeiten.

(2) Das schließt nicht aus, daß auch wi r sehr daran interessiert sind.

(3) "Auch es (das Kind) kann uns helfen.

(4) 'Auch es regnet/ schneit ...

2. Demonstrativpronomina:

(5) Auch der/dieser/jener/einsolcher kann uns heifen.

(6) Auch $d$ e $r / d$ e $r$ jenige/ $j$ e $n$ e $r$ kann uns helfen, der...

3. Artikel:

(7) Auch de $r /$ i $n$ Mann kann uns helfen...

4. Reflexivpronomina:

(8) Aber er schützt auch sich ( $s$ e l b st).

(9) "Die F reunde streiten auch sich.

5. Possessivpronomina:

(10) Auch nach deinem e i g e n e $n$ Ansatz...

6. Relativpronomina, Fragepronamina:

(11) "Auch we $r /$ we l che $r / d$ e $r$ die Sehnsucht kennt, weiß...

(12) "Ich frage dich: auch w a s hast du getan? 
Nicht zu verwechseln mit folgenden Konstruktionen:

(13) Auch, wer die $s$ ehnsucht kennt, weiß nicht...

(14) I $C h$ frage dich $\underline{a c h}$ : was hast du getan?

7. Indefinitpronomina:

(15) ${ }_{\text {'Auch }}^{\text {gehabt. }} j$ emand/irgend welcherl i $r g$ e $n d$ einer hat Glück Wie schon durch die Auswahl der Belegsätze angedeutet, gelten für auch dieselben Regeln wie für nur (3.2.5.1.) hinsichtlich dieses Skopustyps sinngemäß.

\subsubsection{3. sogar - Gruppe}

1. Personalpronomina:

(1) Man beschuldigte selbst $m$ i $c h$.

(2) Nicht einmal i hr hat man geholfen.

(3) ?Sogar e s (das Kind) kann uns helfen.

(4) "Sogar es regnet/schneit...

2. Demonstrativpronamina:

(5) Sogar $d$ e $r / d i$ ese $r / j$ ene $r /$ eins o l che $r$ kann uns

(6) Selbst $d$ e $r / d$ e $r$ jenige/ je $n$ e $r$ kann uns helfen, der...

3. Artikel können nicht Skopus von sogar, selbst, nicht einmal sein.

4. Reflexivpronomina:

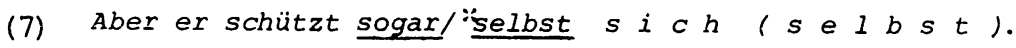

Das Beispiel zeigt sehr deutlich die aufgrund der lautlichen Gleichheit erwartete Inakzeptabilität der GP selbst, wenn ein Reflexivpronamen den Skopus bildet.

(8) "Sie treffen sogar sich/einander.

5. Possessivpronomina:

(9) Sogar/selbst dein/dein e igene $r$ wille geschehe.

6. Relativpronomina, Fragepronomina:

(10) 'Sogar, we r die Sehnsucht kennt, weiß was ich leide.

(11) 'Ich frage dich: sogar w a s hast du getan?

7. Indefinitpronomina können nicht Skopus von sogar usw. seln. - Auch im übrigen gelten die aus den anderen Abschnitten bekannten Gesetzmäßigkeiten. 


\subsubsection{Skopustyp: Gliedsätze}

\subsubsection{1. nur - Gruppe}

1. Subjektsätze:

(1) Mich wundert $\underline{n u r} / \underline{\text { nur }}$ die $T$ a $t$ sache, daß das dem 'Spiegel' entgangen sein sollte.

(2) So kann nur $d$ e $r$ jenige/ $n u \underline{r}$ vier $F$ rauen aus ihrem Lebenskreis herauslösen, wer/der wie Arwed Imiela auf Pealitäten nicht Rücksicht zu nehmen braucht.

(3) Bestraft wurde $\underline{b l o \beta} / \underline{b l o \beta} d$ e $r$ jenige, wer/der eine Waffe mißbrauchte.

(4) $\quad$ Nur/ nur de $r$ jenige, wer/der die Sehnsucht kennt, weiß, was ...

2. Objektsätze:

(5) Er weiß nur /nur $F \circ 1$ gendes/nur e $i n e s$, nämlich/ daß frische Luft und frisches wasser dazugehören.

(6) Es bleibt nur /nur e $i n e s$, nämlich zu hoffen, daß auch die Verantwortlichen für diese Taten einmal ihren Richter finden.

.(7) ... Imiela ... $\frac{N u r}{\text { hur }} d a s$, was man ihm hundertprozentig nachweisen kann, gibt er zu, heißt es in dem Gutachten.

(8) Ich wollte nur ( e i nes, nämlich) sehen, wie ihm das Lächeln vergeht.

(9) Er hatte lediglich gesagt, er beabsichtige nicht, amerikanischer Botschafter in Moskau zu werden.

(10) Wir geben $\underline{n} u r$ (nur $d$ a $s$ ), was wir von euch empfingen.

(11) Ich wollte nur wissen, ob überhaupt jemand jemandem etwas verraten hat, und nicht, wer wem was.

\section{Adverbialsätze:}

(12) Opitz: " $\underline{N u r}$ (nur $d$ a n n ), wenn wir sofort anfangen können."

(13) Hat nicht die alte welt, nur ( $d$ e $s w e g e n$ ) weil sie anders glaubte, die neue wüst gemacht?

(14) Beglückest du die Menschen $\underline{n u r}$ (nur $d$ es $h$ a 1 b), um von der Welt geehrt $z u$ werden?

(15) Ich möchte $\underline{n u r}$ (nur $d \circ r t$ ) schneiden, wo es wieder wächst.

(16) Zur Reise nach Rom ist Küng denn auch lediglich d a $n \mathrm{n}$ bereit, wenn seine Bedingungen erfüllt werden.

Klassenbildung/Austauschbarkeit

Um in dieser Frage zu klaren Verhältnissen zu kommen, muß man die folgenden Konstruktionstypen sauber trennen:

a. nur usw. betont, kein Bezugsnomen zwischen GP und Gliedsatz.

b. nur unbetont, Bezugsnomen zwischen GP und Gliedsatz. 
c. infinite Verbform zwischen GP und Gliedsatz; die GP scheint hter gewöhnlich unbetont zu sein, doch kann ich betonte Formen nicht ganz ausschließen (siehe (6), (8), (9), (11) und (15)).

d. Ev. existiert auch eine Version mit unbetonter GP und ohne Bezugsnomen: es mïßte sich dann um die konjunktionale Funktion von nur handeln.

In den Konstruktionstypen b. und c. sind, wie schon die Belegsätze zeigen, alle Elemente der nur - Gruppe ohne Beschränkungen verwendbar. In der Version a. wirken allein, einzig und ausschließlich nicht völlig akzeptabel, dies mag mit ihrer Betonbarkeit zusammenhängen. - In der hypothetischen Version d. müßten bei konjunktionaler verwendung automatisch allein, einzig, lediglich und ausschließlich ausscheiden. Doch ist das wegen der dann möglichen Skopusambiguität (bei betontem Verb kann auch dieses im Skopus stehen) nicht zweifelsfrei $z u$ entscheiden. - Weitere Ersetzungsmöglichkeiten (abgesehen von den anderen GP-Gruppen) kann ich bei strenger Einhaltung der Akzentmuster nicht erkennen. Stellungsgesetze

Die Grundanordnungen wurden oben bereits aufgezählt. Sie müssen bei den folgenden Stellungstypen jeweils einzeln untersucht werden.

Typ 1: GP unmittelbar vor dem Skopus. Sie ist in allen Beispielen der Konstruktionstypen a. und b. gegeben, abgesehen von Satz (2). Für den Konstruktionstyp ist vorher zu klären, ob die infinite Verbalform jeweils zum Skopus gehören muß, kann oder auch nicht kann.

Typ 2: GP unmittelbar nach der Skopuskonstituente. Die GP kann höchstens die Position nach der Bezugs-NP einnehmen, jedoch nicht die nach dem gesamten Skopus-Gliedsatz. Offenbar ist dessen Länge der einzige Grund hierfür.

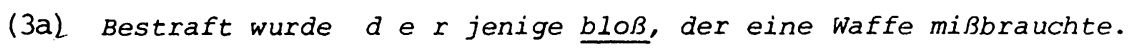

(3b) *Bestraft wurde d e $r$ jenige, der eine waffe mißbrauchte, bloß.

TYP 3: GP in der Skopuskonstituente. Hierfür lassen sich trotz der Mehrgliedrigkeit der Skopuskonstituente keine akzeptablen Beispiele beibringen (aber (3a)!). Typ 4: GP in Distanz vor und nach der Skopuskonstituente. Die Einheit GP + Bezugs-NP ist relativ zum Skopusgliedsatz frei beweglich: dafür ist Satz (2) ein überzeugendes Beispiel. Die betonte GP allein hingegen nach dem Konstruktionstyp a. kann höchstens unittelbar auf das finite verb folgen (ebenfalls Satz (2)), doch wirken derartige Sätze recht seltsam. Für die Distanzstellung nach der gesamten Skopuskonstituente habe ich (abgesehen von TYp 5) keinerlei akzeptable Beispiele gefunden.

Typ 5: Skopuskonstituente topikalisiert. Hierbei missen wieder verschiedene Möglichkeiten berücksichtigt werden: a. die betonte GP allein vor dem Skopus- 
Gliedsatz: siehe Satz (4) in der ersten Version. Bel daß - Sätzen scheint diese Lösung nicht in jedem Fall akzeptabel zu sein. - b. Voll akzeptabel ist es, wenn GP und Bezugs-NP vor dem Skopusgliedsatz am Satzanfang stehen. - c. Für die Stellung der Skopuskonstituente am absoluten Satzanfang gibt es nur eine akzeptable GP-Position, in Verbindung mit Left Dislocation:

(6a) Was wir von euch empfingen, nur $d$ a $s$ geben wir.

Typ 6: Kontrastive Betonung der GP. Bei der Betonung der GP Im Falle des Fehlens eines "Platzhalters" kann man nicht elgentlich von einer kontrastiven Betonung sprechen, eher von einer Ersatzbetonung nach Löschung des Platzhalterelements. Bedeutungs- oder Gebrauchsunterschlede gegeniber den unbetonten Formen mit "Platzhalter" sind nicht festzustellen.

Satzarten: Hier sind nur der Imperativ (wegen des bekannten Funktionswechsels von nur und $b$ LOß zu MPn) und die Konstituentensatzwortstellung erwähnenswert:

(b) ... weil wir nur das (geben), was wir von euch empfingen, (geben). (6c) ... weil wir nu geben, was wir von euch empfingen.

Im Falle (6c) entsteht die auch für (6), (8), (9) und (11) registrierte Skopusambiguität. Sie wird im Abschnitt "Skopus und Fokus" eingehend behandelt. Spaltsatzbildung führt bei diesem vielgliedrigen Skopustyp natürlich zu inakzeptablen Ergebnissen, Extraposition hingegen bleibt ohne Auswirkungen.

Skopus und Fokus

In der Mehrzahl der hier geschilderten Fälle wäre eine Skopusabgrenzung nur aufgrund der Stellung der GP und der Betonungsverhältnisse möglich: kann doch die GP nur unmittelbar davor und im Konstruktionstyp Left Dislocation danach stehen. Auch in jenen Fällen, in denen GP + Platzhalter weiter vom Gliedsatz entfernt sind, kann aus zwei' Gründen kein Abgrenzungsproblem entstehen: bei Tilgung des Platzhalters rückt die GP autamatisch an den Skopus-Gliedsatz heran, außerdem kann nicht zweifelhaft sein, daß Platzhalter und Gliedsatz fest miteinander verbunden sind: der Gliedsatz ist eine semantisch notwendige Ergänzung des Platzhalters. - Wesentlich mehr Mihe machen diejenigen Sätze, in denen zwischen GP und Skopus-Gliedsatz eine infinite Verbalform steht (automatisch bei Konstituentensatzwortstellung (hier finites Verbum) und bei analytischen Verbformen). Die Stellung der GP ummittelbar vor dem Gliedsatz ist in diesen Fällen ausgeschlossen:

$$
\begin{aligned}
& \because E s \text { bleibt zu hoffen } \underline{n u r} \text { (nur } F \circ 1 \text { ge ndes ), ... } \\
& { }_{E r} \text { hatte gesagt lediglich, daß er nicht beabsichtige; ... }
\end{aligned}
$$

Doch gibt es mehrere Versionen, in denen die Vollverbform von der Stellung her 
keineswegs zum Skopus der GP gehört (falls nicht betont!) :

(9b) Er sagte lediglich, daß ...

(6e) Hoffen können wir nur, daß die verantwortlichen ...

andererseits aber auch Versionen, in denen das Verbum zum Skopus gehört:

(6f) Es bleibt nur e i nes, nämlich hoffen, daß...

Einziges Mittel der Disambiguierung bleibt in diesen Fällen ein Kontrastsatz:

(6g) Es bleibt nichts mehr zu tun. Es bleibt nur $z u$ hof $f$ e $n, d a \beta \ldots$

(6h) Es bleibt nicht zu hoffen, daß... Es bleibt nur zu hoffen, daß...

Die angedeuteten Akzentunterschiede kann ich allerdings nicht mit Sicherheit behaupten. Möglicherweise handelt es sich nur um Unterschiede der Phrasierung.In der folgenden Version befindet sich übrigens nur das infinite verb im Skopus:

(13a) Nur wiss e n wollte ich, ob..., nicht...

In einer Reihe von Fällen kann allerdings das Verbum gar nicht zum Skopus gehören: wenn es nämlich nicht kontrastiert werden kann. Überhaupt sind Kontrastsätze unter Einbeziehung des Verbums recht schwierig zu bilden:

(8a) Ich wollte nur sehen, wie ihm das Lächeln vergeht, nichts sonst / nicht hören, wie er zusammenbricht.

Akzentprobleme

In aller Regel fehlt bei diesem Skopustyp das Bezugsnomen, das den Akzent stellvertretend für den Gliedsatz tragen würde. Statt dessen wird oft die GP betont bzw. auf sie trifft die progrediente Intonation, d.h. die übliche Vorbereitung eines Konstituentensatzes. Beide Erscheinungen sind schwer auseinanderzuhalten. In einigen Fällen, insbesondere bei Subjektsätzen, kann man auch beobachten, daß das der GP unmittelbar vorausgehende finite Verbum (auch wenn Pronomina dazwischen stehen) betont wird; die konjunktionale Interpretation von nur und bloß liegt in diesen Fällen allerdings näher (zumal ein Platzhalter diese Art von Betonung verhindert). - Auch die Akzentzuteilung im Konstituentensatz erweist sich als recht kompliziert:

a. Die Konjunktion kann kontrastiv betont werden; das ist nur bei daß ohne Schwierigkeiten möglich. Für diesen Fall überwiegt die polare Interpretation von nur: kontrastiert wird z.B. die Faktizität gegenijber der Zahl. Zu diesem Zweck muß natürlich Faktizität festgestellt werden: ${ }^{16}$

(1a) Nur, $d$ a $\beta$ das dem spiegel entgangen ist, wundert mich, nicht wie oft. 16 Zu diesem Problemkreis vergleiche D. Schnurre (1973). 
(17) Mich interessiert nur, $d$ a $\beta$ dem Spiegel Skandale entgangen sind (sein sollen), nicht jedoch wieviele.

b. Das Hauptverb kann betont werden. In diesem Falle sind z.B. zu den Sätzen

(1) bis (4) sehr unterschiedliche Folgesätze möglich:

zu (1): - der Spiegel ist doch sonst inmer so helle.

zu (2): - sonderm die Kealrtat nacn belieben umdichtet.

zu (3): - nicht hingegen, wer die Waffe legal trug.

zu (4): - nicht hingegen, wer nur von der Sehnsucht redet.

Bei Relativsätzen geht es also darum, aus der Menge, die durch Bezugsnomen und Relativpronomen bezeichnet wird, diejenigen Elemente auszugrenzen, auf die das Prädikat zutrifft: (auf Realitäten) nicht Rücksicht nehmen; (Waffe) mißbrauchen; (Sehnsucht) kennen. Für die daß - Sätze ist wahrscheinlich der Folgesatz von (1) nicht repräsentativ (vermutlich bedingt durch das wundert des Matrixsatzes). Die Folgesätze werden in diesem Falle eher nach dem Schema aber nicht, daß der $x y$ (Prädikat). konstruiert. Hierbei mu wohl das Kontrastprädikat in einer semantischen Relation zum Akzentprädikat stehen. Aber in welcher? Oder: aus der Menge von Prädikaten, die dem Subjekt des Konstituentensatzes zugeordnet werden können, wird eines abgetrennt: dies liefe auf die Einzigkeitsinterpretation von nur hinaus.

c. Die Subjekts-NP des Konstituentensatzes wird betont:

(lb) Mich wundert $\underline{n} u \underline{r}, d a ß d$ a $s$ dem Spiegel entgangen sein sollte, wo er doch für diesen Bereich als besonders informiert gelten kann/ nicht aber, daß ihm so viele andere Dinge entgangen sind.

Die Funktion des nur wäre hierbei, aus der Menge der Subjekts-NPn, auf die ein bestimtes Prädikat zutreffen kann, eine einzige als gültig zu kennzeichnen. d. Schließlich kann auch noch die Objekts-NP des Konstituentensatzes betont werden. Mögliche Folgesätze wären dann:

zu (1): - nicht aber, daß es "B $i l d$ " und "W e $l t$ " entgangen ist.

zu (2): - ??

zu (3): - nicht aber, wer ein we rkz e u g miBbrauchte.

zu (4): - nicht aber, wer irgendetwas anderes kennt.

Die Funktion der GP wäre hier, aus der Menge der Sachverhalte, die zu einer bestimmten Subjekt-Verb-Kombination gehören, denjenigen als allein gültig zu bezeichnen, auf den das objekt zutrifft. - In dieser Weise kann man wohl alle Akzentstellungen durchgehen, die in Konstituentensätzen auftreten können; es wurde hier nur einmal demonstriert, um zu zeigen, daß eine bestimmte GP-Position keineswegs eine bestimte Akzentverteilung zur Folge hat, und daß damit die Bedeutungen auch noch bei festgelegtem GP-Skopus variieren können. - 
Bisher sind jedoch nirgends diejenigen Interpretationen aufgetaucht, die wir intuitiv den Sätzen (3) und (4) zuweisen würden (Relativsätze!). Sie können etwa folgendermaßen umschrieben werden: aus der Menge derjenigen Elemente, auf die der Platzhalter zutrifft, wird diejenige Teilmenge als gültig bezelchnet, auf die der im Konstituentensatz bezeichnete Sachverhalt zutrifft. Dieser Bedeutungsfunktion entspricht der nur implizit kontrastive normale Satzakzent. Er kann natürlich die gleichen Elemente treffen, die auch elnen Kontrastakzent tragen können, und dies trägt sicherlich zur Verwirrung bei.

Semantik

Wie nicht anders zu erwarten erbringt die semantische Analyse bei diesem Skopustyp nichts anderes als das von der Analyse des Skopustyps einfache NP schon Bekannte. Dabei scheint allerdings die quantifizierende Interpretation einen klaren Vorrang zu besitzen, so etwa bei Satz (3):

(3) PSP: Bestraft wurde, wer eine waffe mißbrauchte.

ASS: Bestraft wurde niemand sonst.

Auf dem Umweg über eine Suspension der PSP kann man aber offenbar eine skalierende Interpretation induzieren. Interessant ist der Vergleich mit der NichtGP-Fassung dieses Satzes:

(3c) Bestraft wurde, wer eine Waffe mißbrauchte.

Dies könnte man im Sinne eines einfachen Konditionals auslegen:

(3d) Wenn jemand eine waffe mißbrauchte, so wurde er bestraft.

Nach dem von Geis/Zwicky (1971) erwähnten Prinzip der Vollendung von Konditionalen in natürlichen Sprachen könnte man daraus eine suggerierte Schlußfolgerung ableiten:

(3e) Wenn jemand keine waffe mißbrauchte, so wurde er nicht bestraft.

Dies entspricht nun völlig den Bedeutungselementen des Satzes (3), allerdlngs besitzen diese Bedeutungselemente unterschiedlichen Status: die PSP von (3) wird in (3c) zur ASS, die ASS von (3) wird in (3c) zur suggerierten Schlußfolgerung. Danach ist nur (und möglicherweise jede GP) ein Instrument zur Neugewichtung von Informationsbestandteilen, bzw. ein Element im Mechanismus von "foregrounding" und "backgrounding" bei natürlichen Sprachen. 


\subsubsection{2. auch - Gruppe}

1. Subjektsätze:

(1) Daß Hans vorhin gelogen hat, stimmt nicht, und daß er überhaupt ein schlechter Mensch ist, stimmt $a u c h / e b e n f a 11 s .$. nicht.

(2) Auch die Tatsache, dem Krieg lebend entkommen zu sein, ist keine Schand

2. Objektsätze:

(3) Als er die "letzten Paradiese" der Erde belichtete, erkannte er auch, daß die Menschen an ihren eigenen Abfällen ersticken werden.

(4) Die Erfahrung zeigt, daß es dies nicht ist. Die Erfahrung zeigt auch, daß es nicht automatisch andere gibt, die stellvertretend für uns dafür sorgen, daß...

3. Adverbialsätze verschiedener Typen:

(5) Unsere Kollegen wissen das. Sie wissen auch, wo die Verantwortlichen für jede Art von Preistreiberei sitzen: imU nternehmerlager.

(6) Auch, als er wieder gesund war, veränderte er sein Verhalten nicht.

(7) ... umgezogen - manchmal freiwillig, weil ein Arbeitsplatz in einer anderen Stadt höheren Lohn versprach, oft aber auch/"ebenfalls/“gleichfalls, weil die vertraute Tätigkeit nichts mehr einbrachte.

(8) Auch “ “qleichfalls/ ’ebenfalls, wenn wir ein Taxi nehmen, können wir den $z$ ug nicht mehr erreichen.

(9) Wenn er auch ein Scharlatan oder ein Verrückter wäre, bestünde kein Anlaß, so voreingenommen zu fragen.

Klassenbildung/Austauschbarkeit

In dieser Hinsicht bieten die Sätze (1) bis (9) ein etwas wirres Bild, das sich aber schnell ordnen läßt: in den Stellumgen vor dem Skopus-Konstltuentensatz kann nur auch stehen. Das betonte auch hingegen in Distanzstellung nach dem Skopus kann durch ebenfalls, ebenso usw. ersetzt werden. Danach bleibt nur der Schluß, daß das auch in den Positionen vor dem Skopus-Konstituentensatz nicht betont ist, oder jedenfalls nicht in der gleichen Weise wie das nachgestellte auch.

Stellungsgesetze: hier sind gewisse Abweichungen gegeniber der nur - Gruppe festzustellen. Folgende Stellungstypen kann man erkennen:

a. auch unmittelbar vor dem Skopus-Konstituentensatz wie in (5), (6), (7) und (9). Zwischen GP und Konstituentensatz kann sich ein Platzhalter für die Gliedsätze einschieben. Es ist nicht ganz klar erkennbar, wann ein solcher Platzhalter zwingend vorgeschrieben ist und wann nicht: wahrscheinlich ist er die Regel, wenn auch den Satzanfang bildet. Gegenbeispiele bieten hier aber die Sätze mit Auch, wenn ... - Die Art des Platzhalters richtet sich völlig nach 
der Art des Gliedsatzes (der-, die-, dasjenige bei Relativsätzen; die l'atsache bei daß - Sätzen; darüber bei ob - Sätzen, damals bei Temporalsätzen; deswegen bei Kausalsätzen usw.). Da er automatisiert ist, kann er auch wegbleiben. b. auch unmittelbar nach dem Konstituentensatz, in dem bekannten starren "pattern":

(2a) Dem Krieg entgangen zu sein, auch $d$ a $s$ ist keine Schande.

(4a) Daß es nicht automatisch andere gibt, die stellvertretend für uns sorgen, auch d a $s$ lehrt die Erfahrung.

In mysteriöser Weise scheren jedoch die Adverbialsätze aus:

(6a) ?Als er wieder gesund war, auch $d$ a $n n$ veränderte er...

(8a) ?Wenn wir ein Taxi nehmen, auch d a $n n$ erreichen wir...

Diese Inakzeptabilität kann auch nicht durch das Verhalten dieser Sätze ohne auch erklärt werden:

(6) :Als er wieder gesund war, dann veränderte er sein Verhalten.

(8b) Wenn wir ein Taxi nehmen, dann erreichen wir ...

c. auch in Distanz vor dem Konstituentensatz, insbesondere bei analytischen Verbformen: $\mathrm{Zu}$ den dabei auftretenden Skopusambiguitäten siehe Abschnitt 3.2.7.1. Bei auch scheint in allen diesen Fällen auch die konjunktionale Interpretation möglich zu sein.

d. Betontes auch in Distanz nach dem Skopus: diese Besonderheit von auch ist bei diesem Skopustyp voll entwickelt.

Vollig mysteriös ist der Stellungstyp von Satz (9), der sich auch bei (8) imitieren läßt, bei anderen Adverbialsätzen aber offenbar nicht. Man könnte nun zunächst denken, daß es sich um eine besondere Verbindung zwischen wenn und auch handelt. Andererseits ist aber die "Nommalversion" bedeutungsgleich:

(9a) Auch dann, wenn er ein Scharlatan oder ein ...

(9b) Auch, wenn er ein Scharlatan wäre ...

(9c) Wenn er ein Scharlatan wäre, bestünde $\underline{a u c h}$ kein Anlaß ...

Daß Scharlatan den Skopus von auch bildet, kann wohl dadurch widerlegt werden, daß die dazu passende Version mit nachgestelltem betontem auch kaum akzeptabel bzw. deutlich bedeutungsverschieden ist:

(9d) Wenn er ein Scharlatan $\underline{a u c h}$ wäre, bestünde keine Anlaß ...

Andererseits kommt für die übrigen GP-Gruppen nur diese Interpretation in Frage bei vergleichbarer Stellung, obwohl auch in dieser verwendung eindeutig die skalierende Funktion von sogar aufweist: 
(10) Sogar, wenn er ein Scharlatan wäre, bestünde kein Anlaß ...

(10a) Wenn er sogar ein Scharlatan wäre, bestünde kein Anlaß ...

Es kann sich also nur um eine idiosynkratische Eigenschaft von auch handeln, oder um einen fehlerhaften Satz, wie ich nicht ganz ausschließen will.

Hingewiesen sei an dieser Stelle auch noch auf das von Dagmar Schnurr (1973) beschriebene Zusammenwirken zwischen der Abstraktionsfunktion von uberhaupt und der quantifizierenden Funktion iles nachgestellten betonten auch. Danach mu das erste Glied einer Reihung einen speziellen Fall bezeichnen, das zweite Glied einen allgemeinen: nur hięr darf dann überhaupt stehen.

(1a) Daß Hans vorhin gelogen hat, stimmt nicht, und daß er überhaupt ein schlechter Mensch ist, ist $\underline{a c h}$ nicht wahr.

(1b) :Daß Hans überhaupt vorhin gelogen hat, stimmt nicht, und daß er ein schlechter Mensch ist, ist auch nicht wahr.

3.2.6.3. sogar - Gruppe

1. Subjektsätze:

(1) Mich wundert nicht einmal, daß das dem Spiegel entgangen sein soll.

(2) Sogar/?selbst die Tatsache, dem Krieg entgangen zu sein, ist keine Schande.

2. Objektsätze:

(3) ... kann man mit -deren- Rechtsauffassung sogar sagen, alle Entlassenen sind Kriminelle.

(4) Der Kriegsminister hatte sogar gewagt, Zeitungen mit Klagen zu bedrohen, die über seine Beziehungen zu dem Mädchen berichteten.

(5) Ich möchte sogar sagen, daß Israel ein ausgesprochen deutscher $S$ taat ist

(6) Die hettbewerbshüter prüfen derzeit sogar/ selbst (!), ob sie gegen die ölkonzerne einschreiten müssen.

3. Adverbialsätze unterschiedlicher Typen:

Selbst $d \circ r t$, wo er sonst in Krisenlagen stets seine zuflucht gesucht hatte, in der Außenpolitik, fühlte sich Nixon...

(8) Sogar/selbst/nicht einmal, als ich auf Mallorca war, hat es geregnet.

(9) Auch, wenn diese Vereinigung, wirtschaftlich gesehen, gleichgültig, ja selbst/sogarl nicht einmal, wenn sie schädlich wäre, sie müßte dennoch stattinden.

Die Glieder der sogar - Gruppe zeigen sehr deutlich, daß GPn in der Position unmittelbar vor dem Skopus-Konstituentensatz auf keinen Fall "kontrastiv" betont werden können (gegen den Anschein bei einigen Beispielen mit nur und auch). 


\subsubsection{1./2./3. nur-, auch- und sogar-Gruppe}

Im Rahmen der im vorausgehenden Kapitel behandelten Subjekt-, Objekt-, und Adverbialsätze waren viele unterordnende Konjunktionen Teil des Skopus von GPn. Die entscheidende Frage ist nun, ob die nach GPn kontrastiv betonten Konjunktionen jeweils allein im Skopus dieser GPn liegen, oder doch wieder der ganze jeweilige Konstituentensatz. Trifft letzteres zu, so ergäbe sich nur eine Vermehrung der bereits behandelten Fälle wechselnden Kontrastakzentes im Rahmen von Konstituentensätzen, die im Skopus von GPn liegen. - Hinderlich ist bei der Lösung dieser Frage der große Mangel an Belegsätzen: außer für die Kombination nur we $i \quad l \quad .$. ließen sich bislang keinerlei Beispiele finden. Für die Behauptung von Fraser (1971: 162), daß even vor den Konjunktionen if, after, before, as, though, while, when, where, ev. until, nicht jedoch vor since, although, for, because, however stehen kann (wobei unklar ist, welchen Skopus er mit dieser Beschreibung meint), ließen sich im Deutschen keine vergleichbaren Gesetzmäßigkeiten finden: ob man nun Gliedsatzskopus mit diesen Konjunktionen oder auch Konjunktions-Skopus meint.

Wegen der vergleichsweise ephemeren Bedeutung dieses Kapitels, des außerordentlichen Mangels an Beispielen und der geringen Differenzierung zwischen den verschiedenen GPn und GP-Gruppen werden alle GPn gemeinsam abgehandelt. In der Einteilung der Konjunktionen halte ich mich an die (sicher sehr anfechtbare) semantische Einteilung des Duden (1966), berücksichtige aber die formalen Unterklassen (deren Diskussion ich mir hier leider versagen muß): da Kontrastierbarkeit sowohl ein semantisches wie auch ein formales Phänomen sein kann, hat keine der beiden Einteilungsarten prinzipielle Priorität. Da aber naturgemäß die semantische Kontrastierbarkeit der eigentliche Gegenstand ist, wird sie hier als leitend verwendet.

\section{Kopulative (anreihende) Konjunktionen: und, wie, sowie, außerdem.}

In dieser semantischen Gruppe, die außerdem formal homogen ist (soweit man 'nebenordnend' als "formal" einreihen kann; man beachte, daß die Wortstellungsbedingungen damit $\mathrm{n} i \mathrm{ch} t$ gemeint sind, sonderr "eher" das Kriterium der Gleichrangigkeit/Verschiedenrangigkeit zweier Teilinformationen), finden sich keine akzeptablen Möglichkeiten. Die Gründe hierfür können syntaktischer Natur sein (Konjunktion gehört formal keinem der beiden Sätze an; dies ist dann entscheidend, wenn es sich nur um Kontrastierung der Konjunktion bei Skopus Gliedsatz handelt), aber auch semantischer: es läßt sich ganz einfach keine Kontrastierungsmöglichkeit denken bei dieser Funktion, abgesehen von der Ausschließung aller anderen Möglichkeiten der Verbindung der jeweiligen Sätze. - Den natürlich immer möglichen metasprachlichen Gebrauch will ich hier ausdrücklich ausschließen, um Mißverständnisse zu vermeiden:

Er sagte (stets) nur/"allein/"einzig/ bloß/? lediglich ausschließlich (das Wort) "und", nicht (nie) "oder".

Offenbar besteht in diesem Gebrauch nur die Möglichkeit, die Ausschließlichkeit zu behaupten (Normalverwendung von ausschließlich!), nicht jedoch zu skalieren. Dies wäre aber vielleicht mit einer umstellung zu erreichen:

(2) Er sagte (bei dieser einzigen Äußerung) nur o $d e r$, nicht $u n d$, wir haben also nur eine von beiden Möglichkeiten.

Es wird also eine Skala mit zwei Werten etabliert, der "untere" der beiden Werte "gilt". In diesem Falle wäre ausschließlich aus semantischen Gründen inakzeptabel. 
2. Disjunktive Konjunktionen: oder, entweder - oder, sonst, andernfalls.

Die Verhältnisse entsprechen hier ganz denen bei den kopulativen Konjunktionen. Denkmögliche Kombinationen mit andernfalls sind in diesem Zusammenhang irrelevant: sie betreffen nur Fälle, in denen dieses wort als verkürztes Konditional verwendet werden kann: nur $d$ a $n n$, wenn das Andere der Fall ist... Es scheint mir ohnehin fraglich, ob die konjunktionale verwendung von andernfalls noch üblich ist.

3. Adversative Konjunktionen: aber, allein, doch, nur. - während.

Für die nebenordnenden Konjunktionen gilt dasselbe wie in 1. Aber auch für das unterordnende während ergibt sich nichts anderes:

(4) () Sie setzte sich neben die Alte in den Fond des Wagens, nur/auch/ sogarl... wä hr e $n d$ die jungen Mädchen die Rückplätze einnahmen.

Man macht beim Lesen/Hören dieses Satzes automatisch den Versuch, während temporal zu interpretieren. Doch ist hier wohl nur die adversative Interpretation möglich (siehe auch den nächsten Abschnitt!).

4. Temporale Konjunktionen (nur nebenordnend): als, während, solange...

Hier zeichnen sich gewisse Möglichkeiten ab:

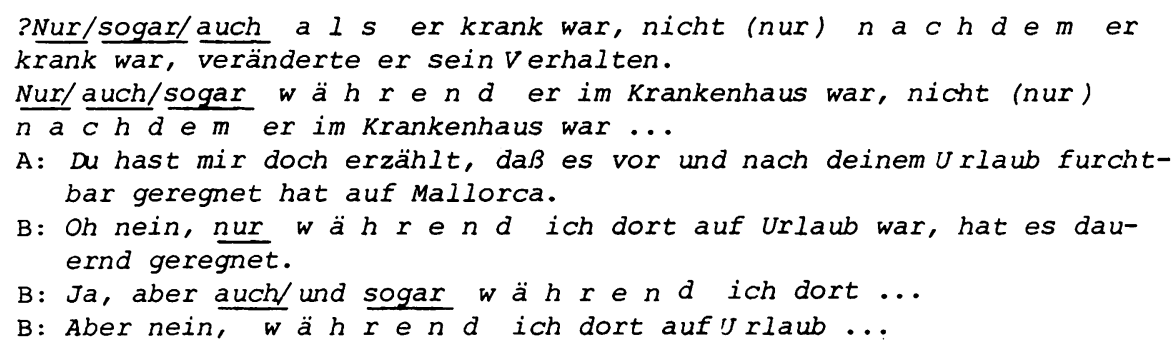

Entscheidend ist also, daß es kontrastierende Formulierungen gibt. Dann sind auch wieder alle Funktionen der GPn möglich: nur in (7) ausschließlich quantifizierend, da bei einer skalierenden Interpretation die "Richtung" der Skala nicht stimmen würde, ebenso auch. Sogar hingegen bringt die "Regenzeiten" in eine Skala der Widerlichkeit.

5. Modale Konjunktionen: wie, indem, als ob ...

(8) Unter Wissenschaftlern besteht nur Einigkeit, $w i$ e man solche $S$ toffe nachweisen soll, nicht jedoch, woz $W$ auf wessen Kosten.

"Einen Maibaum kann man auch transportieren, i $n d$ e $m$ man ihn umlegt. ?Er läuft nur so, als o $\bar{b}$ er ein Dieb wäre, in hirklichkeit ist er ein raffinierter Polizeispitzel.

Offenbar besteht also bei den modalen Konjunktionen eine gewisse Möglichkeit der Kontrastierung, wie (8) und vielleicht auch (10) zeigen. In beiden Fällen ist ein Bezugsnomen oder doch ein Platzhalter notwendig. Dies deutet darauf hin, daB der Skopustyp Adverbialsatz mit kontrastiv betonter Konjunktion vorlieg

6. Kausale Konjunktionen: nebenordnend denn, nämlich; ja und doch sind, gegen Duden, MPn. Unterordnend weil, da, zumal, wo doch.

Akzeptable Beispiele scheint es hier nur bei weil zu geben:

(11) Hat nicht die alte helt nur, $w$ e $i$ l sie anders glaubte, (und nicht $\circ b$ w $\circ h 1$ sie anders glaubte) die neue wüst gemacht?

(12) Sie verlassen die kleinen Orte, obwohl hier jeder jeden kennt, oft aber auch, we $i$ l hier jeder jeden kennt. 
Für sogar usw. konnte ich keinen akzeptablen Belegsatz finden: möglicherweise liegt das an der skalierenden Funktion von sogar, die sich mit der polarität von weil - obwohl nicht verträgt (da sich die beiden Konjunktionen gegenseitig ausschiließen).

7. Konsekutive Konjunktionen: nebenordnend also, folglich, infolgedessen, daher, deshalb, deswegen, darum ... Unterordnend: daß, so daß, als daß.

Auch hier mag es auf den ersten Blick so aussehen, als ob Kombinationen wie nur deshalb, auch deswegen usw. Beispiele für die Akzeptabilität des Skopustyps liefern würden. Aber es handelt sich bei deshalb, deswegen usw. nicht um Konjunktionen, wie der Duden meint (auch wenn sie am Satzanfang stehen), sondern um pronominalisierte Präpositionalphrasen (beachte ihr syntaktisches Verhalten!). - Auch für die unterordnenden von äen konsekutiven Konjunktionen gilt, daß Kombinationen mit GPn nicht akzeptabel sind.

8. Finale Konjunktionen: nebenordnend dazu, darum; unterordnend damit, daß. In allen denkbaren akzeptablen Beispielen handelt es sich nicht um Kombinationen mit Konjunktionen, sondern mit pronominalisierten Präpositionalphrasen. Auch bei den unterordnenden finalen Konjunktionen gibt es keine Kontrastierungsmöglichkeit.

9. Konditionale Konjunktionen: nebenordnend sonst, andernfalls; unterordnend: wenn, falls, sofern, wofern.

Im Falle der unterordnenden konditionalen Konjunktionen scheint es gewisse Kontrastierungsmöglichkeiten zu geben:

(13) Nur, we $n n$ ich Alexander wäre, würde ich mich so verhalten, aber $\overline{i c h}$ bin es nicht.

(14) Ich bin nicht Alexander, und selbst/sogar/auch $w$ e $n$ ich Alexander wäre, würde ich mich nicht so verhalten.

Hypothetische Annahme und Realität werden also jeweils miteinander kontrastiert. Für falls, sofern, wofern ist dieselbe konstruktion wohl nicht völlig auszuschließen, aber doch ungebräuchlich.

10. Konzessive Konjunktionen:

Während auch hier die nebenordnenden Konjunktionen (zwar - aber, jedoch, allein, trotzdem) nicht kontrastierbar sind, ist dies für die unterordnenden konjunktionen dieses Typs (obgleich, obwohl, obschon, obzwar, wenngleich, wenn auch) nicht ganz auszuschließen.

$$
\begin{aligned}
& \text { Nur } t r o t z d e m, \text { nicht } w e i l \text { es schwer ist, macht uns } F \text { ran- } \\
& \text { zösischlernen spaß. }
\end{aligned}
$$

Es handelt sich also um eine Kontrastierung innerhalb der Ebene der Kausalität.

11. Instrumentale Konjunktionen: unterordnend indem, dadurch daß, damit daß.

Die für dadurch daß und damit daß erkennbaren Möglichkeiten sind irrelevant, da es sich dabei jeweils um pronominalisierte Präpositionalphrasen handelt, und da diese zum zweiten nicht wirklich kontrastierbar sind.

12. daß, ob.

Für beide Konjunktionen gibt es akzeptable Beispiele:

(16) Ich weiß nur, $d a \beta$ er jemanden mitbringt, nicht aber $w e n$.

(17) Es interessiert mich nicht, $w e n$ er mitbringt, sondern nur, $0 b$.

offenbar liegt hier skalierendes nur (mit der Unterscheidung allgemeiner spezieller Fall)vor. 
(18) Ich frage dich nicht nur, we $n$ er mitbringt, sondern auch, $0 b$ er jemanden mitbringt.

Satz (18) ist nur dann akzeptabel, wenn es eine rein quantifizierende Interpretation für ihn gibt. Da dies für sogar ausgeschlossen ist, muß Satz (19) inakzeptabel sein:

(19) :Ich fragte dich nicht nur, we $n$ er mitbringt, sondern sogar, o b er jemanden mitbringt.

Zur Syntax

in den wenigen Fällen, in denen dieser Skopus möglich ist, sind keine neuen Fakten hinsichtlich der Klasseneigenschaften der GPn sichtbar geworden. Hingegen sind an mehreren Stellen die semantischen Unterschiede zwischen den einzelnen GP-Gruppen deutLicn nervorgetreten. Für weitergehende Aussagen ist die Materialbasis zu schmal und zu unsicher. - Die wenigen akzeptablen Beispiele sind ausschließlich den Konstituentensätzen zuzurechnen. Demgemäß lassen sich auch keine gravierenden Unterschiede gegenüber den beim Skopustyp "Gliedsätze" registrierten Stellungsgesetzen feststellen. Allerdings scheint im Allgemeinen die Kontaktstellung der GP unmittelbar vor der kontrastiv betonten Konjunktion vorgezogen zu werden.

Skopus und Fokus

Die Stellung bietet einerseits mit der Bevorzugung der Kontaktstellung der GP zur kontrastiv betonten Konjunktion ein starkes Argument für den nur aus der Konjunktion bestehenden Skopus. Andererseits liefert sie aber auch einige Gegenargumente: die Nachstellung der GP unmittelbar nach der Konjunktion ist völlig ausgeschlossen; zwischen GP und Konjunktion kann (bzw. muß) sich noch ein Platzhalter einschieben (vergleiche Satz (8) und (10)); auch die übrigen Stellungsgesetze beim Skopustyp Gliedsätze kommen hier zur Anwendung. - Die Akzentposition auf der Konjunktion wäre für sich genommen eindeutig. Aber leider bietet sie (auch nicht im Zusammenwirken mit der stellung der GP) kein zwingendes Argument gegen die Vermutung, daß auch der gesamte Gliedsatz den Skopus bilden könnte. Ebensowenig führen Kontrastsätze (wie sie oben in jedem Fall gebildet wurden) zu einer klaren Entscheidung, obwohl jeweils nur die Konjunktionen kontrastiert wurden.

Zur Semantik

Die semantische Analyse müßte etwa für Satz (16) lauten:

(16a) PSP: Ich weiß, daß er jemanden mitbringt.

ASS: Ich weiß nichts sonst/ich weiß nicht, wen er mitbringt.

Die ASS a. scheint eher auf einen Skopus Gliedsatz (Objektsatz) mit normaler Betonung dieses Teilsatzes hinzudeuten. Träfe jedoch die ASS b. zu, so würde dies heißen, daß bei dieser Version üblicherweise die ASS explizit angeschlossen wird. - Die üblichen semantischen Tests helfen in diesem Falle nicht weiter. So ist der Suspensionstest nicht anwendbar, da es sich jeweils nur um die Kontrastierung polarer Paare von Konjunktionen handelt. Der Konjunktionstest ist in diesem Falle weitgehend identisch mit dem Test kontrastierender Vorgängerund Folgesätze. - Zusammenfassend läßt sich also nur folgendes feststellen: die Mehrzahl der Argumente spricht für einen Skopus Gliedsatz mit kontrastiv betonter Konjunktion. In jedem Falle ist die Kontrastierbarkeit der Konjunktion unverzichtbare Voraussetzung für diesen Konstruktionstyp. Sie scheint nur innerhalb eines homogenen semantischen Bereiches gegeben zu sein (also nicht lokal - temporal gemischt z.B.), und zwar in der Form polarer Paare. Diese können unter Umständen auch "gerichtet" sein, etwa nach mehr - weniger, vorher - nachher, kürzer - länger usw. 
3.3. Skopustypen aus dem Bereich des Prädikats.

3.3.1. Skopustyp Verbum

3.3.1.1. nur - Gruppe

1. Synthetische Verbformen:

(1) Blinder Eifer $s c h$ a $d$ e $t$ nur.

(2) Ich $d e n k e$ nicht bloß so, sondern ich $s$ a $g$ es auch.

(3) Dies $s$ a $g t$ er nicht allein, dies 1 üg $t$ er meisterlich.

2. Analytische Verbformen einschließlich Passiv:

(4) ImV orfeld dieser Veränderungen werde seit einigen Jahren eine $S$ ymboldiskussion geführt, die sich am Fall Goetz nur $k \circ n k r$ e:t i $s i$ e $r$ habe.

(5) ... von denen man nicht weiß, ob sie nur d a $v \circ n g e l$ a u $f e n$, oder ob sie, wie in Houston, e $r$ m $\overline{r d}$ e $t$ worden sind.

(6) Wir haben die Schlange nur $v e r w u n d e t$, nicht $g e t o ̈ t e t$.

(7) Du hast das bloß ge $\mathrm{t} r$ ä u $m t$ (nicht wirklich erlebt).

(8) Er ist bloß we g ge gange $n$.

(9) Mein Mann wurde unter Rehor in den Krankenpflegebeirat berufen und von mir in dieser $F$ unktion nur $b$ e $s t \ddot{a} t i g t$.

(10) Nur geli e he $n$ war die stunde.

(11) Die tür war bloß ange $l$ e $h n t$.

3. Verb + Modalverb:

(12) ... kühl, auf die seit seinem Erstling "Lebenszeichen" praktizierte Weise, kalkulierte er Bildeffekte, wie man sie nux $t r \ddot{a} u m$ e $n$ kann.

(13) Ich kann nur $b$ e $d$ a $u$ e $r n$, bekundete Aufsichtsratsvorsitzender Max U eber, daß Herr Bartholmeh gegangen ist.

(14) Man kann nur $a b w a r t e n$, was dem Spiegel nach der Abqualifizierung Kohls als nächstes einfallen wird.

(15) Ich kann nur $s$ t a u $\mathrm{e} n$ und b e w u n d e rn.

(16) Wie häufig legasthenische $S$ törungen bei Schülern in anderen Bundesländern auftreten, läßt sich nur $v \in r m u t e n$.

Klassenbildung/Austauschbarkeit

Bei diesem Skopustyp lassen sich keine prinzipiellen syntaktischen Beschränkungen für die einzelnen Elemente dieser GP-Gruppe erkennen. Die auf threr stilistischen Merkmalhaltigkeit beruhenden Beschränkungen für allein und einzig sind auch hier wieder zu beobachten. Der von der jeweiligen Position relativ zum Verb abhängige Funktionswechsel von allein und einzig muß im nächsten Abschnitt noch genauer behandelt werden, und die auffälligen semantischen Beschränkungen für die Verwendbarkeit von lediglich, allein, ausschließlich, 
einzig und ev. auch $b$ loß sind Gegenstand des Semantik-Abschnitts, und dort vor allem des Tests mit Folgesätzen. - Die Austauschbarkeit gegen die übrigen GPn ist prinzipiell gewährleistet, weitere Austauschmöglichkeiten llefern fast alle Adverbialgruppen, allerdings mit vielen semantischen und syntaktischen Restriktionen, insbesondere dann, wenn eine Negation vorhanden ist. Da dies nicht sehr viel zum Verständnis der Syntax der GPn beiträgt, unterlasse ich eine genauere Darstellung dieses Bereiches.

\section{Stellungsgesetze}

Ich möchte, um die Vergleichbarkeit der Ergebnisse zu sichem, zunächst die 6 Stellungstypen durchbehandeln und erst danach andere Einteilungsprinziplen in kurzen Überblicken berïksichtigen.

Typ 1: GP unmittelbar vor dem Skopuselement. Fur synthetische Verbformen, soweit sie in Matrixsätzen stehen, ist diese GP-Position völlig ausgeschlossen.Bei synthetischen Verbformen in Konstituentensätzen und bei allen analytischen Verbformen sowie bei der Verbindung Modalverb + Vollverb bildet dieser Stellungstyp die Normalstellung.

Typ 2: GP unmittelbar nach dem Skopuselement. Für synthetische Verbformen im Matrixsatz ist dies die Normalstellung der GP (siehe (1)). Welche Elemente sich dazwischenschieben können, wird bei Stellungstyp 4 behandelt. - Fir synthetische Verbformen im Konstituentensatz ist diese GP-Anordnung ausgeschlossen. Das gleiche gilt für alle analytischen Verbformen, ob sie nun in Matrix- oder Konstituentensätzen auftreten. Für "poetischen" bzw. "veralteten" Gebrauch und bei topikalisiertem infinitem Verb möchte ich diese GP-Position allerdings nicht vemeinen.

TYP 3: GP innerhalb der Skopuskonstituente. Für synthetische Verbformen kommt diese GP-Position natürlich nicht in Frage (abgesehen von Partikelverben). Bei analytischen Verbformen und in der Kombination Modalverb + Vollverb existiert in der Matrixsatzwortstellung bei absoluten Verben ein solcher Stellungstyp, vorausgesetzt, man rechnet die finiten Verbformen zum Skopus hinzu, wogegen eben dieser Stellungstyp ein gewisser Gegenbeweis ist.

Typ 4a: GP in Distanzstellung vor der Skopuskonstituente. Fir synthetische Verbformen in der Matrixsatzwortstellung kommt diese Position nicht in Frage. - In der Konstituentensatzwortstellung ist sie jedoch nicht völlig unmöglich:

(2a) ... weil ich nicht $\underline{\text { bloß }}$ so an euch $d$ e $n k e$, sondern...

(la) ?... weil blinder Eifer nur dem lieben Charly $s c h$ a $d e t$.

(lb) $\because .$. weil blinder Eifer nur doch $s c h$ a $d e t$.

Nach meinen Beobachtungen werden alle diese Positionen in schriftlichen Äuße- 
rungen gemieden, da sie zu Skopusambiguitäten führen können (VP-, NP-Skopus). Für die analytischen Verbformen und die Kombinationen von Vollverb + Modalverb besteht sowohl in der Matrix- als auch in der Konstituentensatzwortstellung diese Möglichkeit, doch führt sie ebenfalls zu Skopusambiguitäten und wird deshalb meist gemieden, es sei denn, die dazwischenstehenden Konstituenten kommen für den Skopus nicht in Frage:

(13a) Ich kann nur von Herzen $b$ e $d a$ u e $r n$, daß...

(9a) Er wurde von mir nur in dieser Funktion $b$ es $t$ ä $t i g t$.

Typ 4b: GP in Distanzstellung nach der Skopuskonstituente. Für synthetische Verbformen ist dies eine relativ häufige GP-Position. In fast allen Fällen muß dabei die GP die letzte Stelle im Satz einnehmen; welche Konstituenten ihr allerdings folgen können, ist schwer abzugrenzen; jedoch keinesfalls Genitiv-, Dativ- und Akkusativobjekte. - Bei allen analytischen Verbformen kommt hingegen diese GP-Position nicht in Frage.

Typ 5: Skopuskonstituente topikalisiert, GP unmittelbar davor, also am Satzanfang. Für die synthetischen Verbformen scheidet diese Stellung der GP aus. In den wenigen akzeptablen Beispielen liegt konjunktionale Fumktion vor:

(1c) Nur $s c h$ a $d$ e $t$ blinder Eifer.

Für alle analytischen Verbformen hingegen ist dies eine durchaus übliche GPPosition (insbesondere für nachdrückliches Sprechen): siehe etwa Satz (10)! In nicht wenigen Fällen kann es dabei allerdings zu Ambiguitäten zwischen der GP-Funktion und der konjunktionalen Interpretation von nur und bloß komen. Die Möglichkeit der Distanzstellung der GP entfällt für synthetische Verbformen. Für die analytischen Verbformen hingegen ist sie ganz normal. Offenbar muß in allen diesen Fällen die GP am Satzschluß stehen:

A $n g$ e $l$ e hnt war die türe bloß.

TYP 6: Kontrastiv betonte GP. Diese Möglichkeit entfällt bei diesem Skopustyp. Der geschilderte Sachverhalt macht es verständlich, daß in aller Regel die Position der GP unmittelbar vor dem Verbum im Skopus bevorzugt wird: häufen sich doch im anderen Falle, also bei synthetischen Verbformen, die Skopusambiguitäten. Hingegen bietet die Kombination GP + infiniter Verbalteil bzw. synthetische Verbalform im Konstituentensatz den zusätzlichen Vorteil, daß die kontrastiv betonte Skopuskonstituente an den Satzschluß gerät oder aber in die topikalisierte Position. Außerdem kann auf diese Weise den systemwidrigen Verbstellungsgesetzen ausgewichen werden. 
Syntaktische Umformungen

Zunächst wieder zu den Satzarten. Für die Form des Aussagesatzes stehen alle obigen Belegsätze. - Fragesatzformen:

(1d) $S c h$ a $d e t$ blinder Eifer nur?

(7a) Hast du das bloß $g$ e $t r \ddot{a} u m t$ ?

(15a) Kannst du nur s $t$ a $u n$ e $n$ und b e w u n d e rn ?

(le) Wem $s c h$ a $d e t$ blinder Eifer nur?

(2b) Warum hab' ich das bloß $g$ e $t r$ ä $u m t$ ?

(15b) Wie kannst du nur s t a u n $n$ und b e w u n e $n n$ ? Die Sätze (1e), (2b) und (15b) demonstrieren sehr deutlich die Skopus- bzw. Funktions-Ambiguitäten von nur und bloß in dieser Position und in dieser "Aussageweise": vor allem die Funktion als MP.

Imperativ:

(3a) S a g' das nur!

(6a) $V$ e $r$ w un $d$ e die Schlange nur, tö t e sie nicht!

(14a) wa $r$ t e nur a $b$, was dem Spiegel nach der Abqualifizierung Kohls als nächstes einfallen wird.

In allen Imperativformen tritt bei dieser GP-Stellung bei nur und bloß Ambiguität zwischen der GP- und der MP-Funktion auf (in (14a) ev. nur MP-Funktion). Konstituentensatzwortstellung: für analytische Verbformen stehen bereits oben genügend Beispiele zur Verfügung: (4), (5), (12). Hier noch zwei Beispiele für synthetische Verbformen:

(1f) ... weil blinder Eifer nur $s c h$ a $d e t$.

(2c) ... wenn er nicht bloß so $d$ e $n k e$, sondern es auch $s$ a $g e$. Die zitierten Beispiele zeigen sehr deutlich die Vortelle dieser Konstruktion für die klare Skopusabgrenzung.

Spaltsatzbildung: Clefting ist bei finiten Verben generell nicht möglich, aber auch bei infiniten Vollverben sehr ungewöhnlich. Ließe sich die Abspaltung durchfïhren, so hätte man im Falle der analytischen Verbformen ein starkes Argrment für die Abgrenzung des Skopus: für seine Beschränkung auf die infinite Vollverbform. Immerhin ist das Mit-Abspalten des Modalverbs noch inakzeptabler:

(15c) “Es ist nur $s$ t a u $n$ e $n$ können, was man tut.

Aber auch hier beruht die Inakzeptabilität nicht auf nur, sondern auf der Abspaltung der gesamten Verbalform allgemein. Der Test ist also ungeeignet, um daraus Schlußfolgerungen über den Verb-Skopus von GPn zu ziehen.

Negationsmöglichkeiten:

Typ 1: Inkorporierung der Negation.

(1g) Blinder Eifer $s c h a d e t$ nicht nur, er $n \ddot{u} t z t$ manchmal auch. 
Wie üblich ist diese Negationsform bei den GPn allein, einzig, lediglich und ausschließlich nicht gebräuchlich. Sie hat als die PSP-neutrale NEG zu gelten. TYp 2: NEG zwischen GP und Skopuskonstituente. Diese Position ist naturgemäß für synthetische Verbformen in Matrixsatz-Wortstellung nicht möglich. Hingegen in der Konstituentensatz-Wortstellung:

$$
\text { ... weil er das nur nicht s a g.t ... }
$$

Dies ist aber äquivalent mit:

(3c) Er sagt das nur nicht.

In beiden Fällen handelt es sich um die konjunktionale Verwendung von nur, die auch noch bei bloß und allein auftreten kann. Dasselbe gilt für alle analytischen Verbformen:

(1lb) Die tür war bloß nicht ang e le hnt/ bloß war die tür nicht ang $\mathrm{l}$ e $\overline{h \mathrm{n} t}$.

Dies entspricht semantisch nicht der Version:

(11c) Die tür war alles mögliche: bloß e $i n e s$ war sie $n i c h t$ : nämlich a $n$ g $l$ e $h n t$.

Darauf gehe ich jedoch im Abschnitt 4.2.2.1. S. 274 ein.

Typ 3: Satznegation. Da sie jeweils die Prädizlerung betrifft, läßt sich beim Skopus Verbum keine andere starke Negation finden als die im Typ 1.

Typ 4: Die lokale Negation für einzelne Konstituenten, abgesehen von der Skopuskonstituente, ist natürlich ohne Beschränkungen möglich:

(6b) wir haben keine/nicht $S$ ch 1 a $n g$ e n nur $v$ e $r$ w unde $t$, sondern $D r$ a chen e $r$ s ch 1 a g e $\bar{n}$.

Skopus und Fokus

Aufgrund der bisherigen Tests und Untersuchungen kann man keine spezifischen Anforderungen an die syntaktischen oder semantischen Elgenschaften der Skopuskonstituenten erkennen, und zwar gilt dies für die nur - Gruppe insgesamt wie für einzelne ihner Mitglieder. Natürlich können Verben, soweit sie Teile von Idiomen darstellen (also auch bei Funktionsverbgefügen usw.) nicht allein im Skopus der GP stehen (siehe auch Abschnitt 3.8.!). Gewisse Schlüsse auf die Skopusgrenzen aufgrund der Stellung der GP sind nur in den Fällen möglich, in denen Kontaktstellung zwischen GP und kontrastiv betonter Konstituente vorliegt. Dazu sind wir bei synthetischen Verbformen in Konstituentensatzwortstellung und bei analytischen Verbformen in nichttopikalisierten Wortstellungsarten in der glücklichen Lage, die Abfolge GP + Vollverb am Safzschluß zu haben, wamit wir eine sichere Skopusabgrenzung besitzen (vorausgesetzt, daß diskontinuierliche Elemente keinesfalls in den Skopus von GPn eingehen).

Die Positionierung des Akzentes bietet in der Regel bei diesem Skopustyp keine besonderen Probleme: es kamt nur das Verbum selbst oder die trennbare 
Vorsilbe in Frage. Der Fall, daß ein Verb mit trennbarer Vorsilbe nicht auf der Vorsilbe, sondern auf der Stammilbe betont wird, gehört zum Thema "Kontrastierung von Wortteilen".

(17) Er wollte nur auf $s$ e $h e n$, nicht aufs $p r i n g e n$.

Kontrastive Vorgänger- und Folgesätze sind bei diesem Skopustyp ein wirksames Hilfsmittel, um die semantischen Differenzen zwischen den elnzelnen Elementen dieser GP-Gruppe aufzudecken und die damit zusammenhängenden unterschiedlichen Auswirkungen auf den Skopus:

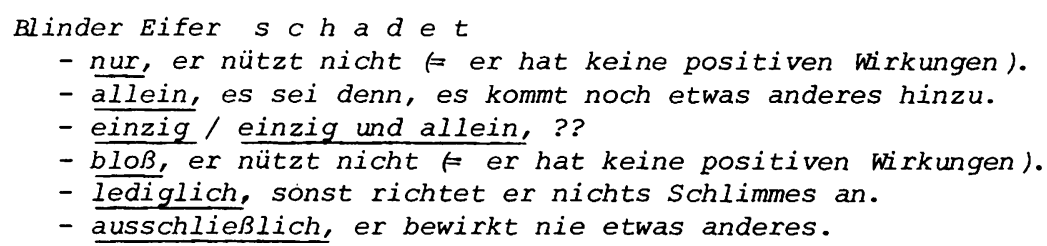

Nur bei nur und $b$ Zoß sind also alle Möglichkeiten der quantifizierenden, skalierenden und polaren Interpretation vorhanden. Bei lediglich scheint ein starkes Element zur "Verharmlosung" vorhanden zu sein (Skalierung), bei ausschließlich überall dort, wo es möglich ist, eine temporale Komponente. Allein, einzig und einzig und allein sind wohl beim Skopus Verbum nicht als GPn möglich, oder doch nicht üblich. Ohne kontrastive Betonung des Verbums würden sie vermutlich in anderer Funktion interpretiert, nämlich als Modaladverbien. -

Der Fragesatztest zur Ermittlung des Satzfokus begegnet hier den gleichen Schwierigkeiten wie bei allen anderen Sätzen mit GPn. Im folgenden Dialog

(li) A: Was tut/bewirkt blinder Eifer?

B: Blinder Eifer $s c h$ a $d$ e $t$ (nur/ausschließlich....).

ist ein nur, ausschließlich usw. in der Antwort eine zusätzliche, überflüssige Information. Lediglich bei der Zurückweisung unzutreffender Nichteinzigkeits-PSPen erfüllen die GPn eine echte Funktion in der Antwort:

(1j) A: Was bewirkt denn blinder Eifer alles?

B: Blinder Eifer $s c h$ a $d$ e $t$ nur (sonst bewirkt er nichts).

Semantische Analyse

Für nur mit dem Skopus Verbum liegt bereits eine Analyse in Bartsch (1972:197) vor: danach ist die Funktion von nur reduzierbar darauf, daß das Skopusprädikat als allein auf die jeweilige Kombination von Argumenten zutreffend bezeichnet wird. Eine Trennung nach ASS und PSP ist, jedenfalls in der fomalen Darstellung, nicht klar erkennbar. Skalierende Informationen werden nicht berücksichtigt. - Hingegen betont Horn (1969: 226f.) bei only mit dem Skopus Prädikat (wobei ich zunächst einmal die terminologischen Unterschiede zwischen 'Prädikat.' und 'Verbum' vernachlässige) die skalare Funktion. Seine Explikation der 
Vernältnisse ist aber einigemaßen wirr:

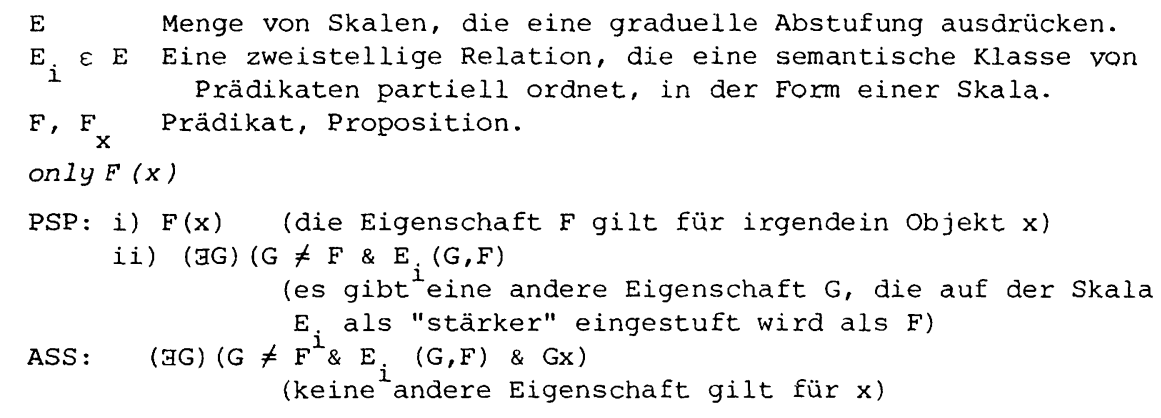

Demonstriert wird dies an den Beispielen:

Muriel only campained for Hubert, she didn't vote for him. John only likes rice (... he doesn't love it).

Unklar bleibt bei dieser Analyse der Charakter der von Horn postulierten Skalen: sind sie in jedem Falle lexikalisiert, wie Horn am Beispiel like - Zove zu demonstrieren versucht, oder gibt es gewisse lexikalisierte Teilordnungen, bei denen die Elementenfolge sowie die Endpunkte usw. nur einigermaßen sicher, andererseits aber auch nicht völlig frei bestimmbar sind? Oder sind die Skalen tatsächlich völlig arbiträr und jederzeit unbegrenzt, ev. gerade durch die verwendung von GPn, neu schaffbar? Weiter müßte man klären, was unter "stärker" tatsächlich zu verstehen ist. - Eine ebenfalls nicht unwesentliche Frage ist, ob es sich, wie die Analyse von Hom nahelegt, tatsächlich um eine zusammengesetzte PSP handelt, also um isolierbare Einzigkeits-PSP und Skalen-PSP. Zu prüfen bliebe ferner, ob die verwendung von nur usw. nicht durch eine Bedingung an die lexikalischen Eigenschaften seiner Skopuskonstituenten zu steuern wäre. Für den Satz (1) mïßte eine Analyse nach Horn folgendermaßen lauten:

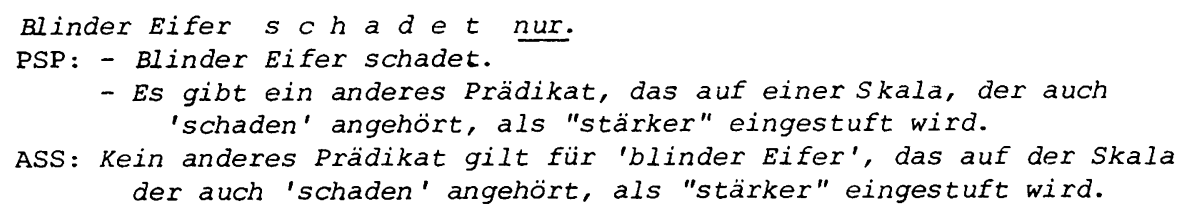

Wir müßten also versuchen, eine - möglicherweise lexikalisierte - Skala von Prädikaten, die "Schädlichkeit" bezeichnen, aufzubauen oder auch nur zu finden: daraus müßte dann folgen, daß kein Prädikat, das auf dieser Skala "stärker" ist als schaden, gilt. Dies ist natürlich eine fragwïrdige Interpretation. Da man schadet in diesem Zusammenhang nur mit nützt kontrastieren kann, müßte man vielleicht eine durchlaufende "Nützlichkeitsskala", die auch Negativwerte aufweist, aufbauen. In lexikalisierter Form sehe ich dafür aber außer den beiden genannten Verben keine weiteren Möglichkeiten. 
Der Bartsch'sche Vorschlag bietet für diesen Fall gewisse Vorteile: er läuft ja darauf hinaus, alle denkbaren Prädikate für blinder Eifer auszuschließen, abgesehen von schaden. Damit ist natürlich auch nützen ausgeschlossen, aber auch eine Menge von Prädikaten, die wir weder ausschließen wollen noch können. So birgt etwa der folgende Satz keineswegs einen Widerspruch:

(11) Blinder Eifer ist ja immer ganz lustig, aber (blinder Eifer) $s c h$ a $d e t$ nur.

Dies zeigt sehr deutlich, daß die ASS eines nur - Satzes mit einem Verb-Skopus immer nur Prädikate einer bestimmten Dimension als ungültig bezeichnet. Diese Dimensionen für einige unserer Belegsätze anhand von kontrastiven Folgesätzen zu verdeutlichen ist die nächste Aufgabe.

(1) ... er nützt nicht/er hat nie positive wirkungen.

(4) ... aber nicht an ihm sich entzündet hat.

(8) ... nicht abgereist/richt krank ...

(9) ... nicht berufen/nicht besonders gefördert ...

(10) ... nicht wirklicher Besitz ...

(11) ... nicht fest verschlossen/nicht eingehängt/nicht sorgfältig gestapelt.

(12) ... nicht wirklich sehen/erleben.

(13) ... nicht billigen/mich nicht darüber freuen ...

(14) ... nicht helfen/ nichts tun ...

(15) ... nicht gleichgültig bleiben/nicht anders reagieren ...

(16) ... nicht sicher sagen/nicht argumentativ absichern....

Bei den meisten Sätzen ist nicht nur eine Kontrastierungsmöglichkeit, sondern eine ganze Reihe davon je nach Kontext zu registrieren, allerdings eine keineswegs beliebige Auswahl, sondern streng auf die Dimensionen ausgerichtet, in die das mit nur gekennzeichnete Verbum eingeordnet werden kann. Eine regelrechte Skala läßt sich nur in sehr wenigen Fällen etablieren:

(5a) ... nur davongelaufen, oder entführt, oder vergewaltigt, oder sogar getötet worden sind.

Das passende Kontrastwort ist also nur selten ohne Konkurrenten. Zudem ist weder bei mehrgliedrigen Skalen noch bei polaren Paaren, wie ich sie vorderhand nennen will, die "Richtung" der Skala von vornherein festgelegt; sie wird erst durch das nur etabliert:

(4a) ... eine symboldiskussion geführt, die sich am Fall Goetz nur e $n t$ $z$ ü $n d e t$, nicht aber $k \circ n k r e t i s i e r t$ hat.

Hier ist auch noch eine günstige Gelegenheit, auf den Bedeutungsunterschied zwischen den einzelnen GPn hinzuweisen. Betrachtet man das Verhalten dieser GPn gegenüber dem folgenden Satz, der eine "natürliche" Skalierung aufweist, so stellt man fest, daß jeweils ein "unteres" Verb im Skopus von nur mit einem 
höherrangigen kontrastiert werden muß:

(18) Ich soll nur $g$ e $h e n$, nicht $t r a b$ e $n$ oder $l$ a $u f e n$ oder $r$ e $n$ e $n$ oder gar $r$ a s e $n$.

Erstaunlicherweise geht es aber auch umgekehrt:

(18a) Ich soll nur $r$ a $s e n$, nicht aber 1 a u $f$ e $n$ oder gar $g$ e $h e n$. Der Bedeutungsunterschied ist klar erkennbar. Ersetzt man nun in beiden Fällen nur durch ausschließlich, so ändert sich die Bedeutung von (18), und zwar in Richtung auf eine temporale oder quantifizierende Interpretation: bel nur können beide Bedeutungsvarianten vorkommen, die wichtigste ist aber die skalierende, die von ausschließlich nicht getragen werden kann. In (18a) hingegen bewirkt der Austausch keinerlei Veränderungen, da hier eine skalierende Interpretation ohnehin nicht möglich ist: hier bleiben nur die Interpretationen immer nur bzw. Keine andere Fortbewegungsart. Lediglich hingegen, für das offenkundig nur eine skalierende Interpretation möglich ist (bei bloß ist das noch unsicherer), ist nur in (18) einwandfrei $z u$ verwenden.

Suspensionstest:

(Im) Blinder Eifer $s c h$ a $d$ e $t$ nur,

- "und möglicherweise tut er sogar noch "Stärkeres". (ASS)

- ?und möglicherweise nützt er sogar. (ASS)

(6c) Wir haben die Schlange nur ve $r$ w unde $t$,

- ?und möglicherweise haben wir die Schlange sogar getötet. (skal. ASS)

(15d) Ich kann nur $s t a$ u $n$ e und b e w unde $r n$,

- "und möglicherweise kann ich sogar noch "s tärkeres" tun. (skal. ASS)

- ?und möglicherweise kann ich auch noch etwas anderes tun. (quant.ASS)

(18b) Ich soll nur $g$ e $h$ e n,

- ? und möglicherweise soll ich sogar traben/ laufen... (skal. ASS)

Die Suspension der ASS muß in jedem Falle zu logischen Widersprüchen fuhren

(auch bei den nur mit ? gekennzeichneten Sätzen). Aber dieser Widerspruch läßt sich doch recht deutlich unterscheiden von schlichtweg unsinnigen Abweichungen: etwa durch eine "verkehrte" Skalenrichtung (wie bei der ersten Version von (1m)), oder durch das Fehlen einer Skala, wie in (15d).

(1n) Blinder Eifer $s c h$ a d e $t$ nur, "und möglicherweise tut er nicht einmal das. (Suspension einer - kaum vorstellbaren - skal. PSP)

(3d) Wir haben die Schlange lediglich $v$ e $r$ w u $d e t$, und möglicherweise nicht einmal das. (Suspension einer skalaren PSP)

(15e) Ich kann ausschließlich $s$ t a u $\mathrm{e} n$ und b e w undern," und möglicherweise nicht einmal das. (Suspension einer "skal. PSP")

(18c) Ich soll nicht nur gehen, "und möglicherweise nicht einmal das. (Die Suspension muß wegen der Negation scheitern)

Der Versuch, die zweite PSP im Sinne Horn's zu suspendieren, fihrt wegen des Wechsels von Objekt- zu Metasprache zu völlig unsinnigen Ausdriicken: 
(10) Blinder Eifer $s c h$ a $d$ e $t$ nur, "und möglicherweise gibt es gar kein stärkeres Prädikat auf dieser Skala.

(6d) Wir haben die Schlange nur $v$ e $r$ w u n de $t$, "und möglicherweise gibt es gar kein stärkeres Prädikat auf dieser Skala.

Eine konv. IMPL tritt in diesen Fällen offensichtlich nicht auf.- Dieser Suspensionstest bestätigt also, daß in vielen Fällen weder mit einer skalierenden Interpretation im Sinne Horn's noch mit einer quantifizierenden Interpretation im Sinne von Bartsch die Bedeutung zu erfassen ist.

Konjunktionstest:

(lp) Blinder Eifer $s c h$ a $d e t$ nur,

- "und blinder Eifer schadet. (PSP)

- und blinder Eifer nützt nicht. (ASS polar)

- ?und blinder Eifer bewirkt nichts Schlimmeres. (ASS skal.)

- ?und blinder Eifer bewirkt sonst nichts. (ASS quant.)

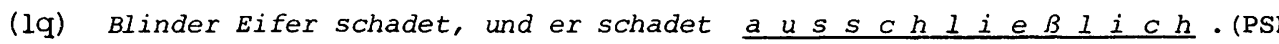

- ?Blinder Eifer nützt nicht, (ASS polar)

- ?Blinder Eifer bewirkt nichts Schlimmeres, (ASS skal.)

- ?Blinder Eifer bewirkt sonst nichts, (ASS quant.) und blinder Eifer $s c h$ a $d$ e $t$ nur.

(6e) wir haben die Schlange nur ve $r$ w u n d e $t$,

- "und wir haben die $\overline{\text { Schlange verwundet. (PSP) }}$

- und wir haben die Schlange nicht getötet. (ASS polar bzw. skal.)

- und es trifft nichts "Stärkeres" als 'verwunden' zu.

- ?und wir haben der Schlange sonst nichts getan. (ASS quant.)

(6f) Wir haben die Schlange verwundet und wir haben sie $\underline{n u \underline{r}}$ verwundet. (P:

- Wir haben die Schlange nicht getötet, (ASS skal. bzw. polar)

- Wir haben die Schlange nichts Schlimmeres als verwundet, (ASS skal

- ? Wir haben der Schlange nichts sonst getan, (ASS quant.) und wir haben die Schlange nur $v e r w$ u $d e t$.

Die polare Interpretation von nur bringt in fast allen Fällen akzeptable Ergebnisse. Andererseits sind aber auch quant. und skal. Interpretation nicht völlig auszuschließen, wenn auch ihre schwerfälligen Formulierungen schwer in das Sche' ma dieser Tests einzupassen sind. Das gute Ergebnis bei allen Anwendungen der polaren ASS mag darauf zurückzuführen sein, daß diese Form der ASS doch ein Nehr an Information gegenüber der tatsächlich in der nur - Formulierung enthaltenen ASS bringt, oder doch zumindest einen disambiguierenden Effekt hinsichtlich der intendierten "Kontrastebene" zeitigt.

Monologischer Widerspruchstest:

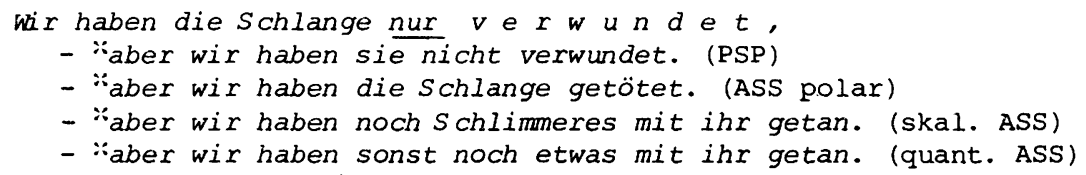

Alle drei ASS-Formulierungen erbringen klare Widersprüche, können also nicht von vornherein ausgeschlossen werden. 
Dialogische Analyse:

(6h)

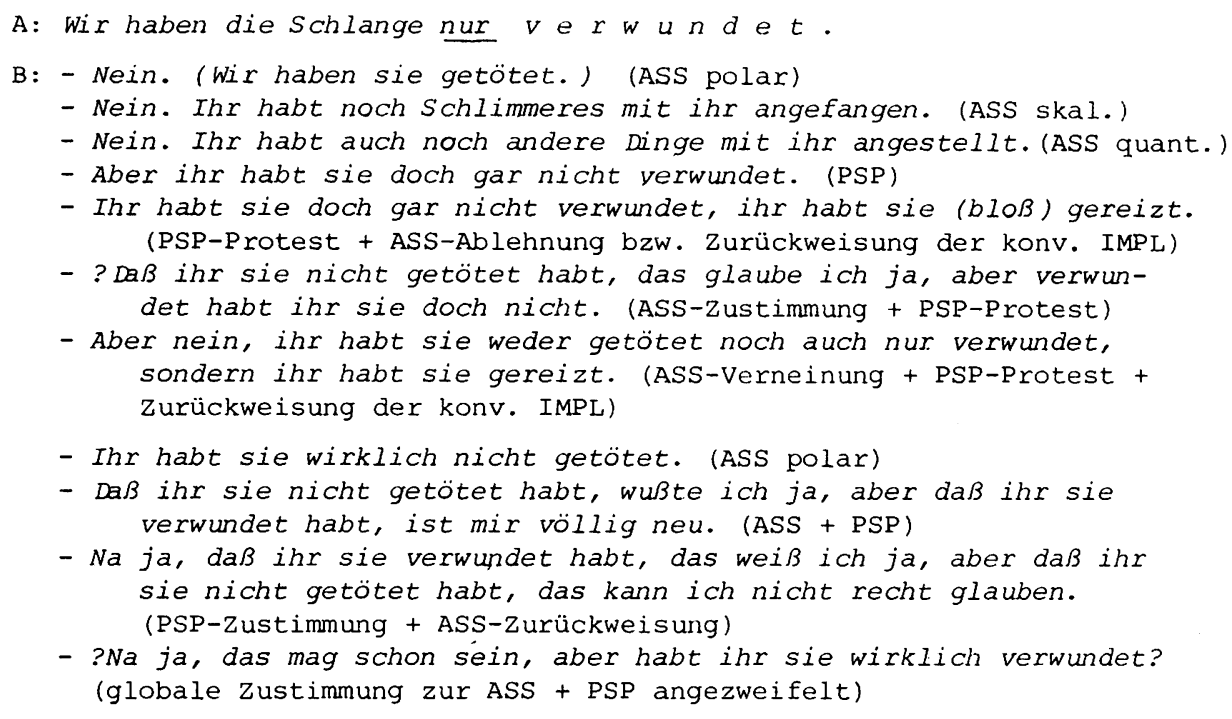

Das wichtigste Ergebnis ist die Inakzeptabilität der Verbindung von ASS-Zustimmung (global oder spezifisch) und PSP-Protest bzw. -Anzweifelung. Dies entspricht vollkommen der bekannten PSP-Gesetzmäßigkeit: laut Definition ist ja eine PSP Voraussetzung für einen Wahrheitswert der präsupponierenden Äußerung. Zu prüfen bleibt aber noch, ob diese definierende Eigenschaft für alle Skopus-Typen von nur zutrifft, und schließlich, ob auch - und sogar - Sätze ebenso reagieren.

Zusammenfassend muß man sagen, daß die semantische Analyse keine klare Entscheidung zwischen polarer, quantifizierender und skalierender Interpretation gebracht hat. Sie konnte nur zeigen, welche Voraussetzungen für jede dieser drei Interpretationsarten $z u$ erfüllen sind und welche Mechanismen den Disambiguierung zur Verfïgung stehen.

Polare Interpretation: sie setzt voraus, daß ein fest im Sprachgebrauch verankertes Paar von Verben mit entgegengesetzter Bedeutung, aber innerhalb der gleichen semantischen "Sphäre" vorhanden ist. Die Disambiguierung bei weiteren Möglichkeiten kann nicht ausschließlich durch eine spezifische GP erfolgen, sondern nur durch den Kontext. Meist wird dabei eine bestimmte "Richtung" zwischen den beiden Verben induziert.

Skalierende Interpretation: sie setzt das Vorhandensein einer "natürlichen" Skala von Verben voraus. Gleiche semantische "Dimension" ist dabei selbstverständliche Voraussetzung. Die Disambiguierung kann durch den jeweiligen Kontext, aber auch durch GPn erfolgen: ausschließlich "löscht" die skalierende Interpretation, lediglich gestattet vermutlich nur sie. 
Quantifizierende Interpretation: sie setzt voraus, daß zu einem Verbum eine Anzahl von weiteren, semantisch vergleichbaren Verben existiert, die mit diesem in einer bestimten Dimension kontrastieren können. Eine Disambiguierung erfolgt über den Kontext sowie mit Hilfe von ausschließlich, das nur quantifizierend möglich ist.

Schließlich kann man vielleicht noch eine temporale Interpretation anfügen, die wohl nur bei nur, ausschließlich und $b 2 O B$ (verdeutlicht durch die Hinzufügung von immer) möglich ist. Die PSP könnte dabei lauten: $x$ tut etwas fortwährend. Und die ASS: $x$ tut niemals etwas anderes. Beides kann auch auf einen bestimmten Zeitraum beschränkt werden.

\subsubsection{2. auch - Gruppe}

1. Synthetische Verbformen:

(1) Die Woge nagt am Renommee der international berühmten Sammlungen antiker Kunst, sie $t r \ddot{a} g t$ sie aber auch.

(2) Ich bearbeite sehr gerne meinen eigenen Garten, ich $I$ i e b e ihn auch sehr.

2. Analytische Verbformen einschließlich Passiv:

(3) Diese Volte wird nichts daran ändern, daß die Leute sich als Vertreter einer dritten Spezies, nämlich der leitenden Angestellten, fühlen und auch $v e r h$ a 1 t e $n$ werden.

(4) Gereist ist er schon in den vergangenen Jahren immer öfter, am liebsten $i m$ sportwagen. Auch $g e j a g t$ hat er in Niedermarsberg als Mitglied des örtlichen Hegeringes.

(5) Er hat mich nicht nur $g$ e $h o ̈ r t$, er hat mich auch $g$ e $s$ e $h$ e $n$.

(6) ...gab es imVerhältnis der BRD zur DDR viel Mißgunst und Gehässigkeit, die auch e $r$ wide $r$ wurde.

(7) Wie das Polizeipräsidium Nürnberg mitteilte, war es zwischen dem türkischen Ehepaar in der whoung zu einem $S$ treit gekommen, wobei die Frau von ihrem Manne auch $g$ e $s c^{\prime} l$ a $g$ e $n$ wurde.

3. Verb + Modalverb:

(8) Ich will die Sachen nicht immer nur anfangen, sondern auch $d u r c h-$ f üh $r$ e n.

(9) Ich soll nicht nur trinken, ich soll auch 1 i e b e n.

Zur Syntax:

Alle in die Untersuchung mit einbezogenen Elemente dieser Gruppe sind in den obigen Sätzen nicht einsetzbar, sie kommutieren bekanntlich nur mit der betonten Version von auch. Die übrigen GPn sind im Rahmen der bekannten semantischen Beschränkungen frei einsetzbar, ebenso fast alle Sorten von Adverbien, soweit sie in den hier vorkommenden Positionen möglich sind. 
Die Stellungsgesetze von auch entsprechen in diesem Falle völlig denen von nur (siehe S. 165). Die einzige Besonderheit bietet die kontrastive Betonbarkeit von auch in Distanzstellung nach topikalisierter Skopuskonstituente. Aber auch dies entspricht den schon in früheren Kapiteln behandelten Fällen:

(2a) Li e b e n tu ich ihn a uch sehr.

Satzarten: nur in Fragen tritt Funktionswechsel bzw. Ambiguität zwischen GPund MP-Funktion auf:

(2b) L $i$ e b s $t$ du ihn auch sehr?

(8a) her will es auch durch $\mathrm{füh} r$ e $n$ ?

(2c) Warum 1 i e b s $t$ du ihn auch?

Im Falle der Verwendung als rhetorische Frage kann auch $\mathbb{P}$ sein. - Fir die übrigen syntaktischen umformungen gilt dasselbe wie bei der nur-Gruppe (s.S. 167) . Negationsmöglichkeiten:

Typ 1: Hier wiederholen sich die für auch charakteristischen Einschränkungen:

(2d) "Ich 1 i e b e ihn nicht auch sehr.

Bei Fragesätzen ist die Kombination nicht auch möglich; jedoch ist auch in diesem Falle eine. MP und nicht keine Negation, sondern eine Fragepartikel.

Typ 2: wie iblich handelt es sich um die konjunktionale Verwendung von auch:

(2e) Ich 1 i e b e ihn auch nicht sehr.

(5a) Er hat mich auch nicht $g$ e s e he $n$.

Satz- und Satzglied-NEG zeigen die iblichen Beschränkungen, lediglich die NEG bei betontem auch ist noch erwähnenswert:

(2f) L i e b e $n$ tu ich ihn a uch nicht sehr.

(5b) Gesehen hat er mich a $\underline{a c h}$ nicht.

Skopus und Fokus

Die Probleme der Skopusabgrenzung, insbesondere ihrer formalen Indikatoren, treten bei diesem Skopustyp in unveränderter Form auf. Über die Anforderungen an die syntaktischen und semantischen Eigenschaften der Skopus-Verben gibt wieder der kontrastive Folgesatz die besten Auskünfte:

(2g) Ich 1 i e $b$ e meinen Garten auch sehr, ich mag/bearbeite ihn nicht nur.

(5c) Er hat mich auch $g$ e se hen, nicht nur ge hört.

(9a) L $i$ e b e $n$ soll ich $\underline{a u c h}$, nicht nur t $r i n k e n$.

Diese Kontrastsätze weisen auch in allen Fällen die Vollverben als GP-Skopus aus. Semantische Analyse

Auch bei diesem doch recht wichtigen Skopustys ird auch (bzw. engl. also/too) in der deutschen wie englischsprachigen litero. zum Thema vorwiegend als lästiges Anhängsel zu nur (engl. only) behandelt. Etwas genauere Angaben finden 
sich lediglich bei Horn (1972). Bartsch (1972: 192,194) erwähnt die Partikel lediglich und gibt eine verbale umschreibung der Bedeutung. Die Bemerkungen von Keenan (1971: 269f.) sind wohl kaum zutreffend in ihrer Bedeutung zu rekonstruieren, da bei ihnen jede Auseinandersetzung mit dem Skopusproblem fehlt.

Bei diesem Skopustyp möchte ich auch auf die Behauptung von Ebert (1973: 424,427) eingehen, wonach auch nur eine schwache pragmatische PSP aufweist: um den jeweiligen Äußerungen mit auch einen Wahrheitswert zuzuordnen, ma man die zugehörige PSP nicht kennen bzw. nicht fuir wahr halten; pragmatische PSPen gehen nicht als Argumente in die neue Aussagen ein, während logische PSPen als Konstanten fungieren. Fir nur hoffe ich das im letzten Abschnitt bereits widerlegt zu haben. - Verknüpft man die Feststellung von Ebert mit dem Hinweis von Bartsch (1972: 195 Anm. 11), daß Bedeutungselemente in der Art von "ErwartungsPSPen" nicht in die Wahrheitsbedingungen der betreffenden Äußerungen eingehen und damit auch nicht Gegenstand einer semantischen Analyse sowie darauf aufbauend der semantischen Repräsentation sein können, so wären diese Elemente in einem streng formalen Sinne gar nicht vorhanden, obwohl sie unzweifelhaft wichtige kommunikative Funktionen erfüllen. Dies macht deutlich, daß sich eine linguistische Beschreibung nicht mit der Erfassung der wahrheitswert-relevanten Eigenschaften lexikalischer Einheiten begnuigen kann.

Nach den bisherigen Vorschlägen, die auch (bzw. engl. also) nur im quantifizierenden Sinn interpretieren, käme man bei einigen der obigen Belegsätze zu folgenden semantischen Analysen:

(2h) ASS: Ich liebe meinen Garten sehr.

PSP: Ich tue noch anderes mit meinem Garten. (?)

(5d) ASS: Er hat mich gesehen.

PSP: Er hat mich auf andere heise wahrgenommen, nämlich gehört.

(7a) ASS: Die $F$ rau wurde von ihrem Mann geschlagen.

PSP: Der Mann hat mit der F rau etwas anderes getan, nämlich gestritten.

(9b) ASS: Ich soli lieben.

PSP: Ich soll noch andere $H$ andlungen durchführen, nämlich trinken.

Hierbei werden einige kritische Punkte sichtbar. Fir die ASS ist der Wortlaut sehr genau festgelegt: der jeweilige Satz ohne die GP auch (dies entspricht der PSP bei nur). Bei der Formulierung der PSP ist jedoch die Aufgabe, die jeweilige semantische Dimension, aus der die präsuppositionell gültigen Verben stammen sollen, zu charakterisieren, in einigen Fällen beinahe unlösbar.Skalierung, die spezifische Interpretation, die notwendigerweise mit sogar verbunden ist, wäre auch bei auch (zumindest in den hier zitierten Belegsätzen) möglich, wenn auch nicht zwingend: für diese skalierende Ordnung sind noch strengere Auswahlkriterien anzusetzen. - Es ist besonders erstaunlich, daß 
in vielen Fällen die PSP, oder doch ein in die generellere PSP-Formulierung eingeschlossener Tatbestand explizit ausgedrückt und mit der auch - Äußerung konjunktional verbunden wird. - Diese Themen sind bei den Tests zu beachten. Suspensionstest:

(2i) Ich $I$ i e $b$ e meinen Garten auch sehr,

- "und möglicherweise liebe $\overline{i c h}$ ihn nicht einmal. (ASS)

- "und möglicherweise bearbeite ich ihn nicht einmal. (PSP)

- und möglicherweise verehre ich ihn geradezu abgöttisch. (konv. IMPL)

(5e) Er hat mich nicht nur $g e h$ ö $r t$, er hat mich auch $g$ e $s$ e $h e n$,

- "und möglicherweise hat er mich nicht einmal gesehen. (ASS)

- "und möglicherweise hat er mich nicht einmal auf irgendeine andere heise wahrgenommen. (quant. PSP)

- und möglicherweise hat er mich sogar angefaßt. (konv. IMPL?)

- *und möglicherweise hat er mich nicht einmal gehört. (skal. PSP)

(9c) L i e b e n soll ich a uch,

- "und möglicherweise soll ich nicht einmal lieben. (ASS)

- "und möglicherweise soll ich sogar sonst gar nichts tun. (quant.PSP)

- "und möglicherweise soll ich nicht einmal trinken. (PSP quant. bzw. skal. PSP)

- und möglicherweise soll ich sogar überschwenglich schwärmen. (konv. IMPL)

Der Test bestätigt weitgehend die auch bei anderen Skopustypen bereits erarbeiteten Gesetzmäßigkeiten: dazu gehört, daß die ASS sich bei auch - Sätzen keinesfalls suspendieren läßt, weil dies zu logischen Widersprüchen führt. Es handelt sich ja auch nicht um eine bloße Grenzziehung, sondern um eine Bereichsangabe. Bei quant. PSP wird die Giltigkeit der (aller?) Verben der betreffenden semantischen "Dimension" vorausgesetzt: bei Satz (2) etwa die Verben, welche nögliche Relationen zwischen Barzel und seinem Gärtlein bezeichnen können (ev. nur positive); bei (5) die Verben, welche Wahmehmungsweisen zwischen Personen bezeichnen; bei (9) schließlich die Verben, welche mögliche Verhaltensweisen von Personen bezeichnen. Diese PSP darf nicht suspendiert werden, jedenfalls nicht global. - Bei skalierender Interpretation wird die Giltigkeit der "tieferen" Verben dieser Skala vorausgesetzt, die Giltigkeit einer bestimmten Stufe der Skala behauptet und die Ungültigkeit der höheren Stufen konversationell impliziert. Die Skala könnte etwa für (2) folgendemaßen lauten: mögen - gem haben - lieben - abgöttisch verehren; also eine Skala der (positiven) emotionalen Beziehung zu Gegenständen. Daß negative Werte in jedem Fall einer solchen Skala angehören, scheint mir nicht gesichert. - Hierbei sind PSP und ASS natürlich nicht suspendierbar, da die Suspensionsformel (sie enthält ja sogar bzw. nicht einmal!) nur eine Grenzüberschreitung in Richtung auf einen größeren Wert. erlaubt: damit ist die Suspension der konv. IMPL möglich. Man kann allerdings streiten, ob nicht lieben bzw. sehen jeweils als Endpunkte ihrer Skala gelten. 
Konjunktionstest:

(2j) Ich 1 i e b e meinen Garten auch sehr,

- “und ich liebe meinen Garten sehr. (ASS)

- "und ich tue anderes mit meinem Garten. (PSP quant.)

- und ich bearbeite meinen Garten. (spezifische quant. PSP)

- ?und ich mag meinen Garten. (skal. PSP)

- und ich verehre ihn nicht abgöttisch. (konv. IMPL)

Wie schon in 3.3.1.1. gezeigt, ist die Wiederholung der ASS nur bei stark divergierendem Wortlaut (wie im Falle von nur - Sätzen) voll akzeptabel, ist also für auch - Sätze nicht gut denkbar. - Bei der Formulierung der globalen quantifizierenden PSP stört einmal mehr die Unslcherheit des Wortlautes. Auffällig ist, daß eine spezifische quant. PSP ohne Schwierigkeiten angeschlossen werden kann; dies ist nur so zu deuten, daß eben durch diese Verbindung die quant. Interpretation bei (3) unterdrückt wird, andererseits aber die skalierende Interpretation keine solche PSP aufweist, es sich also nicht mehr um die Wiederholung einer PSP, sondern um eine unabhängige Behauptung handelt. - Die obige Formulienung der skal. PSP isoliert einen der möglichen vorausgesetzten Werte. Vielleicht verhindert dies eine klare Bewertung. Eine zusammenfassende Formulierung für alle "unteren Werte" ist aber recht prekär. - Der Anschluß der konv. IMPL geschähe besser mit aber; er ist jederzeit möglich, da dann eine keineswegs sichere Grenzmarkierung nachdrücklich behauptet wird.

- "Er hat mich gesehen, (ASS)

- ?Er hat mich auf andere heise (als Sehen) wahrgenommen, (quant.PSP)

- Er hat mich gehört, (spezifische quant. PSP)

- Er hatte einen weniger intensiven Kontakt mit mir als sehen (er hat mich gehört), (skal. PSP)

- :Er hat mich nicht berührt und nicht mit mir gesprochen, (konv. IMPL) und er hat mich auch $g$ e s e $h$ e $n$.

Irritierend ist, daß in diesem Falle die spezifische quant. PSP und die globale skal. PSP identisch sein können; vermutlich setzt sich aber in jedem Falle die skalierende Interpretation durch, da eine quant. PSP, die die Dimension der Wahrnehmung verläßt, nicht sehr sinnvoll erscheint.

Monologischer Widerspruchstest:

(2k) Ich 1 i e b e meinen Garten auch sehr,

- "aber ich liebe meinen Garten nicht. (ASS)

- ?aber ich tue nichts anderes mit meinem Garten. (quant. PSP)

- aber ich bearbeite meinen Garten nicht. (spezifische quant. PSP)

- "aber ich mag meinen Garten nicht. (skal. PSP)

- "aber ich verehre ihn abgöttisch. (konv. IMPL)

Der Anschluß der negierten Formen von ASS, skal. PSP und konv. IMPL mit aber führt zu den erwarteten Kontradiktionen. Beide Formulierungen von quantifizierenden PSPen führen jedoch zu Ergebnissen, die zwar erklärungsbediirftig, aber doch durchaus akzeptabel sind. Dies könnte man so deuten, daß die genaue Abgren- 
zung der präsupponierten Dimension nicht gelungen ist: vielleicht ist aber gerade diese Schwierigkeit Grund genug, um diese Interpretation von vornherein zu verhindern, es sei denn bei expliziter Aufzählung der sonst noch guiltigen Verben: das wäre auch eine Erklärung dafür, warum dieses auch so häufig als letztes Glied einer Aufzählung auftritt.

Dialogische Analyse:

(21) A: Ich bearbeite sehr gerne meinen eigenen Garten. Ich $I i$ e b e ihn auch sehr.

B: - Nein. (Du liebst ihn nicht.) (ASS)

- Aber du kümmerst dich doch nicht die spur darum. (quant. PSP)

- Aber du bearbeitest ihn ja gar nicht. (quant. PSP)

- Aber du magst ihn doch nicht einmal. (skal. PSP)

- Na ja, ich würde eher sagen, daß du ihn regelrecht vergötterst. (konv. IMPL)

- Ja. (Du liebst ihn). (ASS)

- Ja wirklich, du beschäftigst dich in jeder weise mit ihm. (quant.PSP

- Na ja, bearbeiten tust du ihn ja. (quant. PSP)

- Daß du ihn ganz gerne magst, würde ich schon zugestehen, aber lieben? (skal. PSP)

- Du hast schon das richtige Maß, vergöttern tust du ihn nicht.

- : Ja (du liebst ihn), aber daß du ihn magst, kann ich beim besten willen nicht glauben/aber magst du ihn denn? (ASS-Zustimmung + Protest gegen skal. PSP)

- Ja, du liebst ihn. Aber daß du ihn bearbeitest/aber daß du sonst irgendwas mit ihm zu schaffen hast, das bezweifle ich sehr/ ist nicht der Fall. (ASS-Zustimmung + Protest gegen quant. PSP)

Die Zurückweisung der quant. PSP wie die Zustimmung zu ihr haben nicht den Charakter von PSP-Protest bzw. -zustimung, sondern eher den einer Beschäftigung mit notwendigen/ibblichen Schlußfolgerungen bzw. Voraussetzungen. Ob das etwas mit dem Status dieser Bedeutungselemente zu tun hat, ist jedoch ungewiß. Damit ist aber auch die eingangs gestellte Frage, nämlich ob die PSP von Sätzen definitionsgemäß Voraussetzung für Wahrheit und/oder Falschheit des auch - Satzes ist, aufgrund der Analyseergebnisse dieses Skopustyps nicht eindeutig zu beantworten. Sie muß weiterhin im Auge behalten werden.

Zusammenfassend kann man feststellen, daß nur eine skalierende Interpretation zu einigermaßen akzeptablen Ergebnissen fïhrt. Andererseits glaube ich die quantifizierende Interpretation nicht ganz ausschließen zu können. Für die Festlegung des Status dieses quantifizierenden Bedeutungselementes ist die Verallgemeinerung des Negations-Tests, die dialogische ASS-Zuriickweisung, nicht präzise genug. - Abschließend ein sicher quant. zu interpretierender auch-Satz:

(10) Was muß der moderne Katholik alles tun? $Z$ um einen: Er betet, fastet, hält seinen Leib in strenger zucht, aber er $g$ ö $n n t$ sich auch etwas, geht tanzen oder $s$ ing $t$ aluch in froher Runde. 
3.3.1.3. sogar - Gruppe

1. Synthetische Verbformen:

(1) Paul $f 1$ i e $g t$ sogar.

(2) Böll: ... weil ich immer nach glaube und sogar we $i \beta$, dả es in der Sowjetunion zwei Gruppen gibt.

(3) ... daß er mich nicht einmal b e me rkte.

2. Analytische Verbformen einschließlich Passiv:

(4) Wir haben ihn nicht nur $g$ e $h o ̈ r t$, sondern sogar $g$ es e he $n$.

(5) Sogar $g$ e $j a g t$ hat er in Niedermarsberg.

(6) Das ist sogar teilweise schon ausdrücklich e $r k I \ddot{a} r t$ worden.

3. Verb + Modalverb:

(7) ... konnte jedermann - auch ein Sozi - eine Waffe kaufen, besitzen und sogar $b$ e $n$ u $t \boldsymbol{z}$ e $n$.

(8) Nicht einmal gr ü $\mathrm{B}$ e $\mathrm{n}$ wollen die jungen Leute.

(9) Davon wage ich nicht einmal zu trä umen.

zur Syntax:

In bezug auf die Klassenbildung ist erwähnenswert, daß die GP selbst bei diesem Skopustyp eine ausgesprochene Verteilungslïcke aufweist: in fast allen Fällen setzt sich die Verwendung als Reflexivum durch. Nur bei Topikalisierung des Skopusverbs könnte diese Regel vielleicht durchbrochen werden:

(5a) Selbst $g$ e jag $t$ hat er.

Diese Konstruktion dürfte aber i.d.R. gemieden werden. - Die Stellungsgesetze entsprechen ganz und gar den bereits bei der nur - Gruppe (Abschnitt 3.3.1.1. S. 165) dargestellten. Ein informatives Beispiel für die zwischen GP und Skopusverto möglichen Elemente stellt (6) dar. - Bei den Satzarten liegt Erwähnenswertes nur bei den wortfragen vor:

(5b) Was hat er sogar $g$ e $t$ a $n$ in Niedermarsberg?

(6a) was ist sogar teilweise schon ausdrücklich e $r k l \ddot{a} t$ worden? Fir alle Wortfragen dieser Art sind kaum Situationen denkbar, in denen sie angemessen geäußert werden könnten, es sei denn als Echofragen bei teilweiser Störung der Kommunikation, als Versuch zur Rekonstruktion von eben geäußerten sogar - Sätzen.

Semantische Analyse

Bisher wurde erst einmal beim Skopustyp Verbum eine semantische Analyse eines even - Satzes versucht, und zwar von Akmajian (1970a: 235f.):

(10) The Air Force even wan $s$ to bomb peasants. Akmajian zufolge könnten diesem Satz folgende "Implikationen" (im Sinne Austin's?) 
zugeordnet werden: The Air Force - has other 'attitudes'/not only $l i k e s$ to/has not only been o $r d e r e d$ to/not only mus $t /$ not only $c$ a $n$ bomb peasants. Dies zeige die Kontrastierbarkeit mit vielen Verben. Keines davon könne mit Sicherheit als di e Implikation (?) des Satzes (10) (115 bei Akmajian) identifiziert werden. Diese Feststellumg trifft sich teilweise mit. meinen Ausfuihrungen bei den übrigen Skopustypen von sogar sowie zu auch. Doch scheint mir der Ansatz Akmajians insgesamt zu pessimistisch. Deshalb noch einige Bemerkungen zu dem obigen Satz. Die Kontrastierung has other attitudes entspricht meinen Formulierungen bezüglich einer rein quantifizierenden Interpretation. Das scheint mir bei even/sogar jedoch nicht möglich; zumindest müßte die Kontrastierung lauten: Die Luftwaffe hat eine ganze Reihe von schlimmen "Haltungen", aber die Schlimmste davon ist doch, daß sie ... - Hingegen zielt die Kontrastierung likes - even $w$ a $n t s$ bereits sehr deutlich auf eine Skalierung von Verben zur Bezeichnung emotionaler Haltungen zu Handlungen. Im Deutschen ist allerdings eine vergleichbare Konstruktion nur schwer zu finden:

(11) Die Luftwaffe hat nicht nur ein gewisses $F$ aible für den Bombenabwurf

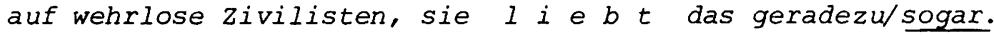

(11a) Die Luftwaffe wünscht, ja sie $s$ e $h \mathrm{n} t$ sich sogar danach, Bomben über wehrlosen zivilisten abzuladen.

Ich nehme an, daß furr diese Interpretation von (10) in jedem Falle ein disambiguierender Kontrastsatz nötig ist. - Die Kontrastierungen not only been ordered/ not only must/not only can (letzteres in der Bedeutung "Erlaubnis") möchte ich einer einzigen semantischen "Dimension" zurechnen: der Dimension 'Verhältnis zu einer bestimmten Aufgabe', ausdrückbar in dem polaren Paar nur gezwungenermaßen/auf Befehl - mit Begeisterung, wobei eine deutliche Abstufung herrscht. Die andere Bedeutung von can würde das polare Paar 'potentielle Fähigkeit' 'Absicht sie zu verwirklichen' definieren. - Damit reduzieren sich die Interpretationsmöglichkeiten beträchtlich, und zwischen den einzelnen Kontrastierungsmöglichkeiten sind sinnvolle Zusammenhänge herstellbar. Man kann generell feststellen, daß es für ein bestimmtes Verb eine sozusagen natürliche Skala oder ein übliches polares Paar gibt, in die es eingeordnet wird, wenn nicht ausdricklich durch den Kontext eine andere Zuordnung geleistet wird. In diesem Falle ist der Sprecher weitgehend frei in der Definition von völlig neuen, ja unerhörten Skalen, und sogar leistet hier die wichtige Aufgabe, die intendierte Richtung der Skala anzuzeigen. Unter Einbeziehung dieser überlegungen kann man dann furr einige Belegsätze folgende semantische Analysen formulieren:

(1a) ASS: Paul fliegt.

PSP: Paul hat noch andere, weniger gefährliche/aufwendige/teure ... Fortbewegungsmittel/Sportarten/Geldanlagen ... 
konv. IMPL: Es gibt noch gefährlichere/ aufwendigere/teuerere $F$ ortbebewegungsmittel/Sportarten/Geldanlagen ... denen sich Paul nicht widmet.

Man m fen kann; siehe etwa:

(12) Carla reitet, Tanja fährt Mòtorradrennen und Paul $f l i$ e $g$ sogar.

(4a) ASS: Wir haben ihn gesehen.

PSP: Wir haben ihn auf weniger eindeutige Art wahrgenommen/gehört.

konv. IMPL: Wir haben ihn nicht berührt/nicht mit ihm gesprochen.

(8a) ASS: Die jungen Leute wollen nicht grüßen.

PSP: Die jungen Leute wollen auch weniger zentrale Höflichkeitsforderungen nicht erfüllen.

konv. IMPL: ? Die jungen Leute erfüllen wichtigere Höflichkeitsforderungen als das Grüßen ...

Die Festlegung der Interpretation von (1) und (8) kann ohne klärenden Kontext (bzw. Ohne zusätzliche Information iber die jeweils thematisierten Bereiche) nicht gelingen. Satz (4) liefert den Beweis hierfür. Dies betrifft jedoch nur den genauen wortlaut der PSP bzw. konv. IMPL, nicht hingegen die Funktion von sogar/nicht einmal. Da mir die skalierende Interpretation für (1) nicht gesichert erscheint, biete ich für diesen Satz noch 3 Kontexte an:

(1b) Ich hätte paul nicht einmal eine anständige Bergtour zugetraut. Aber Paul $f 1$ i e $g$ sogar.

(1c) Paul ist sonst ein rechter Feigling. Aber er $f \mathrm{l}$ i e g t sogar.

(ld) Paul ist querschnittgelähmt. Aber er fli e gt sogar.

Alle drei Beispiele haben gemeinsam, daß sie relativ zu bestimmten Eigenschaften von Personen (Mut, Ausdauer, Kraft ... ) eine Skala von Leistungen definieren, wobei die mit sogar gekennzeichnete Stufe eine relativ hohe wenn nicht die höchste darstellt. Der in diesen Sätzen besonders deutliche Erwartungsaspekt geht in der Skalierung auf.

Suspensionstest:

(4b) Wir haben ihn nicht nur $g$ e hö $r t$, sondern sogar $g$ e $s$ e h e $n$,

- "und möglicherweise haben wir ihn nicht einmal gesehen. (ASS)

- "und möglicherweise haben wir ihn nicht einmal gehört. (skal.PSP)

- und möglicherweise haben wir sogar mit ihm gesprochen. (konv. IMPL)

(le) Paul $f l i$ e g $t$ sogar,

- “und möglicherweise fliegt Paul nicht einmal. (ASS)

- "und möglicherweise hat Paul nicht einmal weniger gefährliche Hobbys. (skal. PSP)

- und möglicherweise macht er sogar heltraumexpeditionen. (konv. IMPL)

Satz (le) zeigt, daß man genaugenommen erst einmal den Kontext festlegen muß, und sei es durch eine Suspensionsformel, um weiter mit einem sogar - Satz operieren zu können. Andererseits bestätigt (4b) sehr deutlich meine Annahmen. Festzuhalten ist, daß der disambiguierende Vorgängersatz die gleiche PSP auf- 
weist wie der sogar - Satz, und umgekehrt der sogar - Satz nur die ASS des nicht-nur - Satzes präzisiert.

Konjunktionstest:

(4c) Wir haben ihn nicht nur $g$ e $h \ddot{~} r t$, sondern sogar ge se he n,

- ?und wir haben ihn gesehen. (ASS)

- "und wir haben ihn gehört. (skal. PSP)

- und wir haben nicht mit ihm gesprochen. (konv. IMPL)

(4d) - ? Wir haben ihn gesehen, (ASS)

- Wir haben ihn gehört, (skal. PSP)

- "Wir haben nicht mit ihm gesprochen, (konv. IMPL)

und wir haben ihn nicht nur $g e h o ̈ r t$, sondern sogar $g$ e $s$ e $h e n$.

Dies erbringt also völlig reguläre Ergebnisse, besonders eindeutig bei der Vorweg-Behauptung der skal. PSP des sogar - Satzes.

Dialogische Analyse:

(4e) A: wir haben ihn nicht nur $g$ e $h \ddot{~} r t$, sondern sogar $g$ e $s$ e $h e n$.

B: - Nein. (Ihr habt ihn nicht gesehen.) (ASS)

- Na, gehört habt iht ihn vielleicht, aber gesehen? (ASS)

- Aber ihr habt ihn doch nicht einmal gehört. (skal. PSP)

- ?Ihr mögt ihn ja gesehen haben, aber gehört habt ihr ihn sicher nicht. (ASS-Zustimmung + PSP-Protest)

- Ja. (ihr habt ihn gesehen.) (ASS)

- Daß ihr ihn gehört habt, glaube ich auch. (PSP)

- ?Ja, (gesehen habt ihr ihn,) aber habt ihr ihn auch gehört?/aber gehört habt ihr ihn nicht. (ASS-Zustimmung + PSP-Protest)

Es zeigt sich also, daß die Kombination von ASS-Zustimmung (ev. global) und PSP-Protest nicht so kraß inakzeptabel ausfällt wie bei den ersten beiden Partikel-Gruppen. Dies mag damit zusammenhängen, daß die Abfolge hören - sehen berühren - sprechen mit offensichtlich keine so feste Skala ist, daß die Giltigkeit eines höheren Wertes jeweils die Gïltigkeit der niedrigeren Werte voraussetzt.

Dieser Abschnitt bringt also viele Probleme mit sich: offensichtlich sind die "semantischen Dimensionen" im Bereich der Verben nicht so klar festzulegen wie etwa im Bereich der NPn. Mit der Formulierung einer globalen "ErwartungsPSP" ist dem Dilemma auf keinen Fall beizukammen, man hätte vielmehr dle gleichen Schwierigkeiten zu erwarten. Zur Formulierung einer spezifischen Erwartungs-PSP sind die gleichen Informationen notwendig wie zur präzisen Formulierung einer skal. PSP, letztere aber hat einen größeren Giltigkeitsbereich. 
3.3.2. Exkurs: Skopus mit kontrastiv betonten Elementen des Auxiliar-Bereichs.

3.3.2.1./2./3.nur -, auch- und sogar-Gruppe

Passende Belege waren nur für einen Teilbereich bei auch aufzutreiben. Die meisten Beispiele sind also konstruiert, und so ist bei den Akzeptabilitätsurteilen doppelte Vorsicht geboten.

1. Passiv und passivnahe Konstruktionen:

(1) Ein Auto wi $r d$ nur in Bewegung gesetzt, es setzt sich nicht selbst in Bewegung (auch wenn es manchmal so aussehen mag).

(2) ?Ihm ge hö $r t$ nur/auch/ sogar gekündigt, er soll nicht selbst kündigen können.

(3) Hans kriegte nur/auch/sogar auf die schultern geklopft, er hat nicht (nur) selber jemandem auf die schultern geklopft.

(4) Ich $b$ e $k \circ \mathrm{m}$ e nur manchmal $\mathrm{F}$ ragen an mich herangetragen, ich äußere selber keine.

2. Tempus, Faktizität:

(5) Ich glaubte, er sei verreist, und er wa $r$ es auch/sogar.

(6) Die Kommandoaktion, die die sich damals abzeichenden Friedensinitiativen Ägyptens und Jordaniens stören sollte und auch/sogar gestört $h$ a $t, \ldots$

(7) Ich fühle mich krank, und ich $b i \mathrm{n}$ es wohl auch.

(8) Er wa $r$ bloß weggegangen, aber jetzt ist er da.

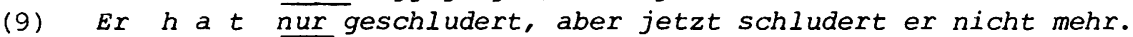

3. Modalverben:

(10) Ich wi 1 I nur immer, aber können tu ich nie.

(11) Er $d$ a $r f$ nur, er $m u B$ aber nicht.

(12) Er muß nicht einmal, er $d$ a $r f$ es (sogar)

(13) Er soll, und er w,i $r d$ sogar/auch.

(14) Wenn man das erst einmal nicht nur ändern $k$ a $n n$, sondern auch wi 11 , dann wird sich diesituation recht schnell ändern.

Hier will ich mich ausschließlich mit den Fragen der Kontrastierbarkeit bei diesen Skopuselementen beschäftigen. Die syntaktischen und semantischen Gesetzmäßigkeiten entsprechen natürlich den beim Skopustyp Verbum besprochenen.

Passiv: es scheint prinzipiell gut mit dem Aktiv kontrastiexbar zu sein, aber nicht im Sinne der Polarität oder der Skalierung. Ich vermute, daß der Eindruck der Skalierung erst durch die jeweiligen Vollverben induziert wird: kündigen, gekündigt werden. Vielleicht verursacht diese Divergenz zwischen semantisch kontrastierbaren Vollverben und formal kontrastierten Hilfsverben den eigenartigen Eindruck, den man von solchen Formulierungen hat. Bei den übrigen passivnahen Formulierungen würde ich einen etwas höheren Akzeptabilitätsgrad veranschlagen, doch kann man sie wohl noch nicht als standardsprachlich bezeichnen.

Tempus: synthetisch gebildete Tempora können nach meinen Beobachtungen keinesfalls kontrastiert werden. Ob dies bei analytischen Tempora möglich ist, wie Satz (8) und (9) vermuten lassen, kann nur dann entschieden werden, wenn man diese Form eindeutig von.einer sehr ähnlichen Form unterscheiden kann, die hauptsächlich (wenn nicht ausschlieBlich) mit auch und sogar auftritt: die Kontrastierung zwischen Intention, Annahme, Anschein einerseits und Faktizität andererseits: das Verhältnis zwischen diesen beiden Kontrastelementen kann das einer reinen Addition (vorwiegend auch) und auch das einer steigerung, Skalierung sein (sogar). - Eine interessante Frage bei den Tempora ist, ob sie als gleichberechtigte Einzelelemente (quantifizierend) oder als geordnete Auf- 
einanderfolge im Sinne einer Skala zu gelten haben: gibt es also, wenn überhaupt, eine vorgeschriebene "Richtung" der Kontrastierung? Dazu die folgende Satzreihe:

(15) Er ha $t$ nur geträumt, er wi $r d$ nicht mehr träumen.

(16) ?Er wir $d$ nur träumen, er $h$ a $t$ nicht geträumt/ex träumt jetzt nicht.

Die Anwendung von nur auf Vergangenheitstempora erbringt vermutlich günstigere Exgebnisse. Zur Kontrolle:

(17) Er träumte nur in der $V$ e $r g a n g e n h e i t$, in $z u k u n f t$ wird er nicht mehr träumen.

(18) ?Er träumt nur in der $z$ u $k$ un $f t$, in der $V$ e $r g a n g e n h e i t$ hat er nicht geträumt.

Dies würde für die "Richtung" Vergangenheit-Gegenwart-Zukunft sprechen. Hingegen liefern die Modalverben semantisch recht gut kontrastierbare Paare. Sehr fraglich ist jedoch, ob sie in eine vorgegebene feste Ordnung gebracht werden können, wie das naheliegende dürfen - sollen - müssen. Bei Testreihen zur ordnung dieser Elemente stören immer wieder temporale Aspekte bei nur (im Sinne von immer nur). Nach meinen Beobachtungen werden sogar und nur, ganz wie bei anderen Skopustypen auch, zur Errichtung von ad-hoc-Skalen verwendet, das quantifizierende auch hingegen zur Hinzufügung eines weiteren Aspektes.

Man kann nun noch versuchen, die bisherigen Feststellungen durch genauer ausgeführte Kontrastkonstruktionen (die zugleich die pragmatischen Faktoren einigermaßen festlegen) abzusichern. Ich wähle hierzu Vorgängersätze.

(19) A: Sie haben ja jetzt sicher finanzielle Schwierigkeiten, nachdem Ihr Mann gekündigt hat.

B: Mein Mann hat nicht selbst gekündigt, er w u r d e nur gekündigt.

Das nur ist in dieser Antwort ziemlich überflüssig: das deutet darauf hin, daß zwischen Aktiv und Passiv keine echten Abstufungsmöglichkeiten bestehen. Darauf verweist auch der folgende Satz:

(19a) B: Mein Mann hat nicht nur $s e l b s t$ gekündigt, ihm w u $r d e$ auch gekündigt.

Hingegen:

(3a) A: Hans hat Anja wieder mal ganz gönnerhaft auf die Schulter geklopft.

B: Nun, Hans h a $t$ ihr nicht (nur) auf die schulter geklopft, er $k r i e g t e$ nur (auch) auf die schulter geklopft.

Zum Tempus:

(20) A: Helmut arbeitet drüben im Labor.

B: Nein, er arbeitet jetzt $n i c h t$ drüben. Er hat nur drüben gearbeitet.

(20a) A: Helmut hat heute morgen im Labor gearbeitet.

B: ?Nein, er a $r$ b e i $t$ e $t$ nur im Labor.

(2Ob) A: Der Helmut wird morgen im Labor arbeiten.

B: ?Nein, er a $r$ b e i t e $t$ nur im Labor.

(20c) A: Der Helmut arbeitet (derzeit) drüben im Labor.

B: Nein, er wi $r d$ nur (in $z$ ukunft) drüben im Labor arbeiten.

(20d) A: Der Helmut hat drüben im Labor gearbeitet.

B: ?Nein, er $w$ i $r$ d nur im Labor arbeiten.

(20e) A: Helmut wird drüben im Labor arbeiten.

B: Nein, er $h$ a $t$ nur drüben gearbeitet/er a $r b e i t e t$ nur.

Aus dieser Folge von Äußerungen kann man den Schluß ziehen, daß offenbar nur das Perfekt - hat als Skopus von nur mit allen anderen Tempora kontrastiert werden kann, und möglicherweise nicht einmal dieses. 
3.3.3. Skopustyp prädikatives Adjektiv und Prädikatsnomen

3.3.3.1. nur - Gruppe

(1) Er ist nur $\circ h n m \ddot{a} c h t i g$, nicht tot.

(2) Es ist nur natür $n i c h, d a \beta \ldots$

(3) Nur s chade, daß er sich umsonst erhitzte.

(4) Alle Hoheit legte er ab und war nur $x$ uhig.

(5) Er ist nur $k 1$ e in.

(6) Er ist nur $k l$ e $i n e r$, nicht dümmer als seine Kameraden.

(7) Er ist nur der $K I$ e $i$ ns te,nicht der Dümste unter seinen Kameraden.

(8) Soll die erschütternde Geste vor den Kämpfern des Warschauer Ghettos nur $B$ e $x$ e $c h n$ u $g$ gewesen sein?

(9) Das ist ja nur eine $P r \ddot{u} f u n g$.

(10) Das ist nur der $A \cap f$ a $n g$, verriet ein japanischer Manager.

(11) So ist der beltsee nur eine $R$ a nderscheinung.

(12) Dort war er bloßein $A n g$ est e 1 l te $r$.

(13) Es war lediglich $B \circ r n i$ e $r t h$ e $i t$, nicht böser wille.

Vorweg ist darauf hinzuweisen, daß nicht alle obigen Belegsätze eindeutig dem genannten Skopustyp angehören, ebensowenig der untersuchten GP-Funktion. Sie sind aber zur genaueren Abgrenzung nötig.

Austauschbarkeit/Klassenbildung

Eine beschränkte Einsetzbarkeit weisen bei diesem Skopustyp nur einzig und allein auf, verursacht durch Funktionswechsel:

(14) Sie ist e inzig schön.

Wegen des deutlich verschiedenen Betonungsschemas besteht aber kaum Verwechslungsgefahr. Allein könnte in dieser Konstruktion im Sinne von als Einziger verwendet werden. Außerdem widersprechen sich die quantifizierende Interpretationsweise dieser Elemente und die vorwiegend skalierende Verwendung dieses Skopustyps. Aus dem gleichen Grunde weicht ausschließlich meist in eine temporale Interpretation aus.

Stellungsgesetze

TYP 1 (GP vor der Skopuskonstituente) ist hier ausnahmslos die Normalstellung. TYP 2 (GP unmittelbar nach der Skopuskonstituente) : diese GP-Position, i.d.R. am Satzschluß, ist nicht ohne weiteres akzeptabel, es sei denn mit starken stilistischen Wirkungen und in spezifischen Textsorten:

(la) Er ist ohnm àchtig nur, nicht tot.

Typ 4: GP in Distanzstellung vor oder nach der Skopuskonstituente. Hierfuir 
gibt es nicht sehr viele Möglichkeiten: zwischen GP und Skopuskonst1tuente können nur Formulierungen wie ein bißchen, ein wenig stehen, und natürlich können die Prädikatsnomina durch Attribute erweitert sein.

Typ 5 (Skopuselement topikalisiert): hier sind alle bereits beschriebenen Anordnungen möglich, also Satzanfangsposition der GP und Distanzstellung:

(5a) Nur $k 1$ e $i n$ ist er.

(9a) Eine $P$ r ü $f$ ung war das nur.

Möglichkeiten für Typ 6 (kontrastiv betonte GP) existieren nicht.

Syntaktische umformungen:

Fir die Form des Aussagesatzes sind alle Belegsätze Beispiele. Bei Wortfragen ebenso wie bei Imperativen kommt es wieder zu den bekannten Funktionswechseln bzw. -ambiguitäten GP - MP:

(1b) harum ist er nur ohnm ächtig ?

(15) Sei bloß höflich, aber nicht unterwürfig. (GP)

- sonst hast du nichts zu lachen! (MP)

Dies alles zeigt die große Nähe zum Skopustyp Verbum. Wie dort so sind auch hier die restlichen syntaktischen umformungen (Clefting usw.) nicht sinnvoll. Negationsmöglichkeiten:

Es existieren alle bereits von den anderen nur - Kapiteln her bekannten Typen:

(1c) Er ist nicht nur $\circ$ h $n m \ddot{a} c h t i g$, sondern tot.

(1d) Er ist nur nicht $\circ h n m \ddot{a} c h t i g \ldots$ (konjunktional)

(1e) Nur ist er nicht ohnmächtig... (konjunktional)

(1f) Nur $\circ h n m a ̈ c h t i g$ ist er nicht. (konjunktional bzw. GP)

(1g) Kein Typhuskranker ist nur $\circ \mathrm{h} \mathrm{nmächtig.}$

Skopus und Fokus

Die Festlegung der Skopusgrenzen würde hier in der Regel auch dann keine Schwierigkeiten bereiten, wenn man nur auf die Kriterien von GP-Stellung und Kontrastakzent angewiesen wäre. Dies läßt sich leicht aus den Stellungsgesetzen und aus der überwiegenden Eingliedrigkeit des Skopuselementes schließen. - Der Test der natürlichen Antwort begegnet den bekannten Schwierigkeiten:

(1h) was ist er (nur)? - Er ist (nur) ohnmächtig.

Aber auch die Anpassung an eine sinnvolle Verwendung von nur stößt bel diesem Skopustyp auf Schwierigkeiten, denn die entsprechende Frage erzwingt eigentlich eine quantifizierende Interpretation, die aber fir diesen speziellen Fall nicht sehr wahrscheinlich ist:

(1i) Was is $t$ er denn alles? - Er ist nur $\circ h n m a ̈ c h t i g$. Ein bislang noch vernachlässigtes Thema ist die Frage von koordinierten Konstituenten im Skopus: 
(16) Er ist nur $h \ddot{O} f I i c h$ und a ufgeschloss e $\mathrm{f}$...

(16a) Er ist nur $h o ̈ f l i c h$ und nur a ufgeschlos $c$ e $n$...

(16) Er ist nur $h$ öfI $i c h$ und er ist nur a ufgeschIoss en.

(16c) Er ist nur $h o ̈ f I i c h$, und (er) ist aufgeschlossen ...

In (16) bilden beide prädikativen Adjektiva zusammen den Skopus von nur, aber dieser Satz ist in seinen Wahrheitsbedingungen verschieden von (16a) und (16b), die wiederum bedeutungsgleich sind. (16c) weicht in der Bedeutung von allen anderen $\mathrm{ab}$.

Wichtige Hinweise sowohl fuir die Skopusabgrenzung als auch fuir die semantische Analyse kann man wieder den kontrastiven Folgesätzen entnehmen:

(1j) Er ist nur o hn $m \ddot{a} c h t i g$, nicht tot/nichts Schlimmeres.

- ?allein ohn $m \ddot{a} c h t i g$, nichts sonst.

- ?еinzig ohnm ä $c h t i g$, nichts sonst.

- bloß/lediglich o hn $m \ddot{a} c h t i g$, nicht tot/nichts Ennsteres

- ausschließlich $\circ h n m a ̈ c h t i g$, nichts sonst/ niemals etwas anderes.

Daran ist die Funktionsdifferenzierung deutlich ablesbar.

(2a) Es ist nur $n$ a $t \ddot{u} r I i c h$, nicht ungewöhnlich, daß...

Hier sind nur die skalierenden GPn möglich. Der Grund für die spürbare Abweichung vom Normaltyp ist damit jedoch noch nicht genannt. - Bei (3) liegt der Verdacht nahe, daß es sich nicht um die hier interessierende Verwendung von nur handelt, zumal auch die Abfolge schade nur ganz geläufig ist, und ein normaler Kontrastsatz kaum akzeptabel ist.

(3a) S ch a d e nur/bloß/lediglich/?allein/?einzig/"ausschließlich, daß..., ?nicht gottseidank ...

Trotz der "eindeutigen" Betonung liegen folgende Interpretationen näher:

(3b) Schade ist nur die Tatsache, daß er sich umsonst erhitzte.

(3c) Nur ist es schade, daß er sich umsonst erhitzte.

Weitere Kontrastsätze:

(4a) ... nur ruhig, nichts sonst.

(5b) $\ldots$ nur $k 1$ e $i n$, nicht mittelgroß.

(8a) ... nur $B$ e $r$ e chnung, nicht ehrliche Uberzeugung/nichts Besseres.

(9b) ... nur eine $P r \ddot{u} f$ ung, nichts wichtigeres/Schlimmeres.

(10a) ... nur ein $A n$ a $n g$, nicht die eigentliche Aktion.

(11a) ... nur eine $R$ and e $r s c h$ e i n ung, nichts wichtigeres.

(12a) ... bloß ein $A n g$ e $s$ e $l$ l t e $r$, kein "Leitender"/kein Beamter.

Die gegebenen Beispiele lassen eine bunte Mischung von skalierenden, quantifizierenden und polaren Interpretationen erwarten. 
Semantische Analyse

Fragestellung und Forschungslage sind im Prinzip dieselben wie S. 169 ff. beim Skopustyp Verbum bereits geschildert. Kernfrage bleibt also, ob eine skalierende Interpretation in eine nur quantifizierende Notation ibersetzt werden kann. - Selbst wenn man grundsätzlich der Ansicht ist, daß Skalen nicht in Lexikoneinträgen definiert werden, sondern vorrangig mit Hilfe von GPn, mu man doch zugeben, daß die Verwendung quasi-natürlicher Skalen bei diesen Untersuchungen gewisse Vorteile bietet: die Abfolge und Stufung der Werte ist. weitgehend starr; damit sind PSPen, ASSen und konv. IMPLen ziemlich genau bestimmbar; und die Anfangs- und Endpunkte der Skalen sind gesichert. In dieser Skalenfunktion treten die meisten Wörter nur im Prädikat voll in Erscheinung. Als Skalen bieten sich an: die Temperaturskala, Schönheitsadjektiva, Qualitätsadjektiva/Noten, Helligkeitsadjektiva; bei den prädikativen Nomina: Militär- und sonstige Grade (Berufe, Hierarchien), Altersstufen, kriminelle Vergehen, Ordensstufen usw. Die Skalen selber können dabei von der Untersuchung nicht ganz ausgeschlossen werden: Brüche, Inhomogenitäten, natürliche "Richtung" usw. Unter diesem Aspekt wurden die Analysesätze ausgewählt:

(1) ASS: Er ist nicht tot.

PSP: Er ist ohnmächtig.

konv. IMPL: Er schläft nicht.

Ich möchte dies als Beispiel für eine ad-hoc-Skala bezeichnen.

(4) ASS: Er ist nichts anderes/nie etwas anderes.

PSP: Er ist (stets) ruhig.

Dies ist ein Beispiel für quantifizierende Interpretation.

(7) PSP: $E r$ ist der Kleinste (aus einer bestimmten Menge).

ASS: Er steht sonst nie am untersten Ende einer Skala (bezüglich dieser bestimmten Menge).

Hier ist nicht einwandfrei zu entscheiden, ob quantifizierende oder skalierende Interpretation vorliegt.

(17) Der Gegenstand ist nur $w$ a $r \mathrm{~m}$.

PSP: Der Gegenstand ist warm.

ASS: Der Gegenstand ist nicht heiß.

konv. IMPL: Der Gegenstand ist nicht lauwarm.

Ein Beispiel furr eine lexikalisierte Skala.

(18) Eva ist nur b e f $r i$ e $d i g$ e $n d$ in Mathematik.

PSP : Eva ist befriedigend.

ASS: Eva ist nicht gut.

konv. IMPL: Eva ist nicht ausreichend.

Wiederum ein Beispiel für eine lexikalisierte Skala.

Das waren ausschließlich $A$ n $g$ e $t e l i t e$.

PSP: Das waren Angestellte.

ASS: Das waren keine Arbeiter/ BeamteN nternehmer. 
Gegen den ersten Anschein handelt es sich um ein cquantifizierendes Beispiel (bei ausschließlich ist ja auch nichts anderes möglich).

(20) Hans ist nur ein $A n g$ e $s$ e $l 1 t e r$.

PSP: Hans ist ein Angestellter.

ASS: Hans ist kein Beamter/kein'Jnternehmer ...

konv. IMPL: Hans ist kein Arbeiter.

Hier handelt es sich um ein Beispiel mit mehrfacher Skalierungsmöglichkeit.

Suspensionstest:

(1k) Er ist nur $O h n m \ddot{a} c h t i g$,

- "und möglicherweise ist er sogar tot. (ASS)

- und möglicherweise ist er nicht einmal ohnmächtig. (PSP)

- und möglicherweise schläft er sogar (?nur?). (konv. IMPL)

Also ein völlig nonmales Ergebnis für eine skalierende Interpretation.

(4b) Er war nur $r$ u hig,

- "und möglicherweise war er sogar noch etwa anderes. (ASS)

- ?und möglicherweise war er nicht einmal ruhig. (PSP)

Beim Festhalten an quantifizierender Interpretation muß die Suspension der PSP inakzeptabel werden: doch ist in solchen Situationen ein Ausweichen in eine ev. mögliche skalierende Interpretation das Normale.

(7a) Er ist nur der $K l e i n s t e$,

- " und möglicherweise ist er sogar der Dümmste. (A.SS)

- "und möglicherweise ist er nicht einmal der Kleinste. (PSP)

Dies deutet auf eine nur quantifizierende Interpretation.

(17a) Der Gegenstand ist nur w a $r \mathrm{~m}$,

- "und möglicherweise ist er sogar heiß. (ASS)

- und möglicherweise ist er nicht einmal warm. (PSP)

- und möglicherweise ist er sogar (?nur?) lauwarm. (konv. IMPL)

Auch hier wieder das reguläre Ergebnis fiur skalierende Interpretation.

(19a) Das waren ausschließlich $A n g$ e $s$ t $l_{1} t e$,

- "und möglicherweise waren es sogar Arbeiter/Beamte ... (skal. ASS)

- "und möglicherweise waren es nicht einmal Angestellte. (skal. PSP)

Durch ausschließlich wird ein Ausweichen auf eine skalierende Interpretation

wirksam verhindert.

(20a) Hans ist nur ein $A n g$ e $s$ e 1 l $t$ e $r$,

- "und $\overline{m o ̈ g l i c h e r w e i s e ~ i s t ~ H a n s ~ s o g a r ~ e i n ~ B e a m t e r / u n t e r n e h m e r . ~(A S S) ~}$

- und möglicherweise ist Hans nicht einmal ein Angestellter. (PSP)

- und möglicherweise ist Hans sogar (?nur?) ein Arbeiter. (konv. IMPL)

Fur dieses skalierende Beispiel gibt es eine, wenn auch vielleicht etwas vertrackte Interpretation. Man stelle sich vor, der folgende Satz (t die Suspensionen) werde von einem Vulgärmarxisten gesprochen:

(20b) Hans ist nur ein $A n g e s t e l l t e r$,

- "und möglicherweise sogar ein Arbeiter. (ASS)

- und möglicherweise nicht einmal ein Angestellter. (PSP)

- und möglicherweise sogar (nur) ein Beamter. (konv. IMPL)

Um die Grundlage zu verdeutlichen: für den Sprecher sind Arbeiter "die Krone 
der Schöpfung", Angestellte sind schon leise vom Kapitalismus angekränkelt, und Beamte schließlich sind geradezu der faschistische Abgrund. Dies zeigt wlederum, daß eine Skala nicht an sich und auch nicht in ihrer Richtung festliegt, sondern jeweils vom Sprecher zu definieren ist (falls er vom üblichen Sprachgebrauch/von den vorherrschenden Ansichten abweicht).

(20c) Hans ist nicht nur ein $A n g$ e s t e I l t e $r$,

- "und möglicherweise ist er nicht einmal ein Angestellter. (PSP?)

- "und möglicherweise ist er sogar ein Beamter. (?)

- "und möglicherweise ist er nicht einmal ein Beamter. (ASS)

Das ist ein ganz ungewöhnliches Ergebnis, das der Erklärung bedarf, zumal die mittlere Version auch nicht durch Abänderungen zu retten ist:

(20c) "Möglicherweise ist ex sogar ein Beamter.

Die folgende Version ist keine Suspension mehr, sondern die Verbindung der ASS mit dem zugehörigen GP-Satz:

(20d) Hans ist nicht nur ein $A n g$ e $t$ e 11 t e $r$, Hans ist ein Beamter. Die skalierende Interpretation von (20c) mïßte so aussehen:

(20C) PSP: Hans ist kein Angestellter ASS: Hans ist mehr als ein Angestellter, z. B. ein Beamter/ nternehmer. konv. IMPL: Hans ist nicht weniger als ein Angestellter.

Die Schwierigkeiten sind also zweifach begrindet: in dieser Berufsskala schlieBen sich die einzelnen Werte gegenseitig aus, also Angestellter $(x) \supset\urcorner^{B e c o n-}$ ter $(x)$. Dieser Sachverhalt ist auch die Ursache dafuir, daß bei (20c) gegenüber (20) die Negation nicht präsuppositionsneutral ist. Für dieses Dilemma gibt es derzeit wohl keine Lösung. - Die einzige "Grenzmarkierung", die in der Interpretation von (20c) auftritt, die konv. IMPL, liegt nicht "in Richtung" der Skala, ist damit auch nicht suspendierbar.- Möglicherweise hat (2OC) auch eine quantifizierende Interpretation:

(20e) Hans ist nicht nur (in seinem ersten Beruf) $A n g e s t e l l t e r$, sondern (in seinem 2. Beruf) auch Beamter.

In diesem Falle schließen sich die einzelnen Werte der Berufsbezelchnungen nicht gegenseitig aus.

Konjunktionstest:

(19b) Das waren ausschließlich $A n g$ e $t e l 1 t e$,

- und das waren keine anderen Menschen. (ASS)

- " und das waren Angestellte. (PSP)

(20f) Hans ist nur ein $A n g e s t e l l t e r$,

- und Hans ist kein Beamter/kein Unternehmer. (skal. ASS)

- "und Hans ist ein Angestellter. (PSP)

- "und Hans ist kein Arbeiter. (konv. IMPL)

(20g) Hans ist nicht nur ein $A n g$ e $t$ e 1 l te $r$,

- und Hans ist ein Beamter. (ASS)

- “und Hans ist kein Angestellter. (PSP?) 
Lediglich (20g) liefert also irreguläre Ergebnisse. Sie können aber erst nach Abschluß aller Tests beschrieben und gedeutet werden.

Monologischer Widerspruchstest

Er wird hier nur auf das nicht-nur - Beispiel angewendet:

(20h) Hans ist nicht nur ein $A \mathrm{n} g$ e $s$ t e $1 \mathrm{l} t$ e $r$,

- "aber Hans ist kein Angestellter. (PSP)

- ?aber Hans ist ein Angestellter. (PSP?)

- “aber Hans ist nichts Höheres/kein Beamter/Unternehmer. (ASS)

Auch dieser Versuch schafft also noch keine Klarheit über den Status der mit diesem Satz verbundenen Bedeutungselemente. Eigenartig ist, daß man die zweite Möglichkeit nicht als völlig inakzeptabel ausschließen kann, obwohl der Basissatz dieses Bedeutungselement eigentlich ausschließt: es scheint sich also um ein aufhebbares Bedeutungselement zu handeln.

Dialogische Analyse:

(20i) A: Hans ist nur ein $A n g e s t e l l$ t e $r$,

B: - Nein. (Hans ist doch ein Beamter.) (ASS)

- Aber er ist doch gar kein Angestellter! (PSP)

- Er ist sogar nur ein Arbeiter! (konv. IMPL)

- Aber nein, Hans ist nicht nur ein $A n g$ e $s$ t $l 1 t e r$, sondern ein Beamter/Unternehmer. (PSP-Protest + ASS-Verneinung)

- Ich find das gar nicht so übel. (Skala selbst)

- Ja. (Er ist kein Beamter/U nternehmer.) (ASS)

- Was, ein Angestellter ist Hans? Das wußte ich noch gar nicht. Na ja, immerhin ist er kein Arbeiter. (konv. IMPL)

- 'Ja, (Hans ist kein Beamter,) aber er ist doch kein Angestellter!

Dieser Test gestaltet sich beim vorliegenden Skopustyp besonders schwierig. Bei Befragungen von kompetenten Sprechern wird in der Regel die globale Zurickweisung der Behauptung $($ Nein) in der Form des PSP-Protestes expliziert. Auch der Einbau in "eindeutige" Kontexte veränderte das Ergebnis nicht wesentlich. Wie üblich fuihrt die Verknüpfung der ASS-Zustimung mit der PSP-Zurückweisung zu Inakzeptabilität. Unklar ist dabei, ob sie durch die Fortsetzung sonderm ein Arbeiter akzeptabel wird. - Eine mögliche Erklärung wäre, daß das globale ja nicht nur die ASS, sondern auch alle mit einer bestimmten Formulierung verbundenen PSPen usw. billigt: eine nachträgliche Zurückweisung der PSP würde damit notwendig zu einem Widerspruch fihren. Trifft dies $z u$, dann müßte die bloße explizite ASS-Zustimmung mit einem PSP-Protest kombinierbar sein:

(20k) A: Hans ist nur ein $A n g$ e $t$ e 1 l t e $r$.

B: Hans ist kein Beamter, aber er ist (auch) kein Angestellter, sondern ein Arbeiter. (ASS-Zustimmung + PSP-Protest + Verneinung der konv. IMPL)

Diese Antwort ist sicherlich nicht inkonsistent, aber doch zumindest sehr ungewöhnlich. Bei gleicher Sachlage wïrde die Antwort normalerweise lauten: 
(20k) B: - Du wirst es nicht glauben, aber er ist sogar nur ein A $r$ b e $i$ ter.

- Er ist nicht einmal ein $A n g$ e $s t e l l t e r$, sondern sogar nur ein $A r b$ e i $t$ e $r$.

Nun noch zu unserem nicht-nur - Beispiel:

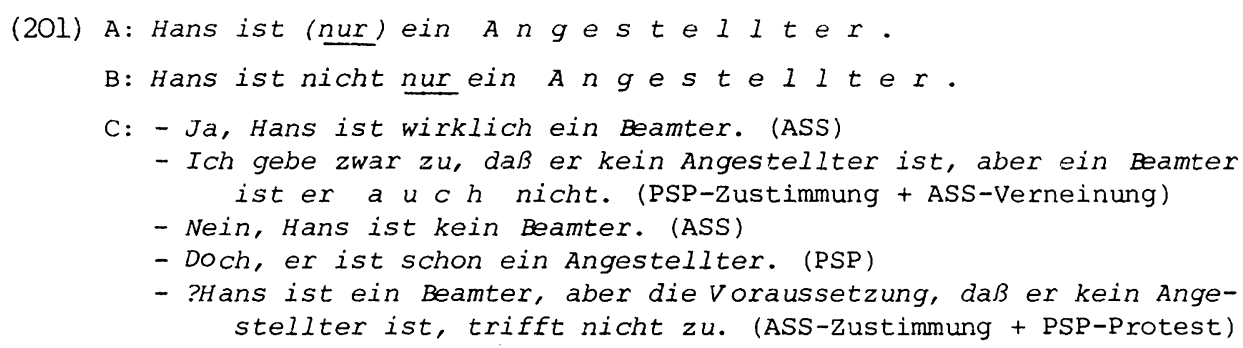

Dies wäre ein plausibles Ergebnis. Doch irritiert die Umkehrung des Konjunktionstests, die hier noch nachzutragen ist:

(2Om) - Hans ist ein Beamter, (ASS)

- ?Hans ist kein Angestellter, (PSP?)

und Hans ist nicht nur ein $A n g$ e $s t e l l t e r$.

Nun ist aber in aller Regel die Vorweg-Behauptung der PSP des folgenden Satzes ohne Einschränkung akzeptabel. Alle diese Schwierigkeiten treten ausschließIich mit der skalierenden Interpretation auf, nicht hingegen mit der quantifizierenden, die bei gleichem Wortlaut möglich ist:

(20n) Hans ist nicht nur ein $A n g$ e $s$ e $l$ l $t$ e $r$, sondern auch noch selbständigerUnternehmer.

PSP: Hans ist Angestellter.

Die quantifizierende Interpretation von nur, bloß, einzig, allein, ausschließZich entspricht also ganz und gar dem Utblichen, so, wie es bereits in 3.2.1.1. S. 106 durch ein Diagramm verdeutlicht wurde. Schwierigkeiten bereitet lediglich die Verneinungsform nur ... nicht. Beispiel (11) schließt zwar nicht völlig die GP-Interpretation aus, klingt dann aber sehr seltsam:

(11) Alles mögliche ist er, nur $\circ \mathrm{h} n \mathrm{~m} \ddot{a} c h t i g$ ist er nicht.

Die eigentlich hierfiir zuständige Satzform ist bezeichnenderweise inakzeptabel:

(1m) $\ddot{E r r}_{\text {Er }}$ nur $O h n m \ddot{a} c h t i g$ nicht.

Die eindeutig konjunktionale Form (1n)

(1n) Nur ist er nicht $\circ$ hn $m \ddot{a} c h t i g$, sondern er schläft.

ist höchstens im Status ihrer Bedeutungselemente von der GP-Interpretation von

(11) zu unterscheiden:

(1n) ASS: Er ist nicht ohnmächtig. (dialogische Analyse!)

PSP: Er befindet sich in einem anderen, ohnmachtsähnlichen zustand. (normale Interpretation für kontrastive Negation)

Die polare Interpretation tritt bei nur, bioß und lediglich auf. Fir diese Interpretationsform, die Eigenschaften der quantifizierenden wie der skalierenden 
Interpretation vereinigt, habe ich bislang keine adäquate Darstellur:g gefunden. Sie ist aber so einfach in ihren Eigenschaften, daß man darauf verzichten kann: bezogen ist sie immer auf zwei fest aufeinander zugeordnete Prädikate; bei nur wird das eine assertiv als ungültig, das andere präsuppositional als gültig bezeichnet; bei nicht nur wird das eine assertiv und das andere präsuppositional als gültig bezeichnet. Zwischen beiden Prädikaten herrscht in jedem Fall eine Richtungsbeziehung. Hinsichtlich der Negationsform nur $x \ldots$ nicht entstehen die oben bereits geschilderten Schwierigkeiten.

Bei der skalierenden Interpretation (nur, bloß, lediglich) muß man unterscheiden zwischen Skalen, deren Werte sich gegenseitig ausschließen, und Skalen, deren werte miteinander verträglich sind:

- nur: hier ist diese Skalenunterscheidung unerheblich.

- nicht nur: skalierende Interpretation ist hier sowohl mit einer Skala möglich, deren Werte sich nicht gegenseitig ausschließen (I) als auch mit dem Gegenteil(II). - nur $x$... nicht: hier überwiegt die konjunktionale Interpretation, wie oben bereits geschildert. Bei GP-Interpretation erlaubt nur eine Skala mit Werten, die sich nicht gegenseitig ausschließen, eine skalierende Interpretation; sehr viel wahrscheinlicher ist aber auch in diesem Falle eine quant. Interpretation.

nur nicht nur nur...nicht

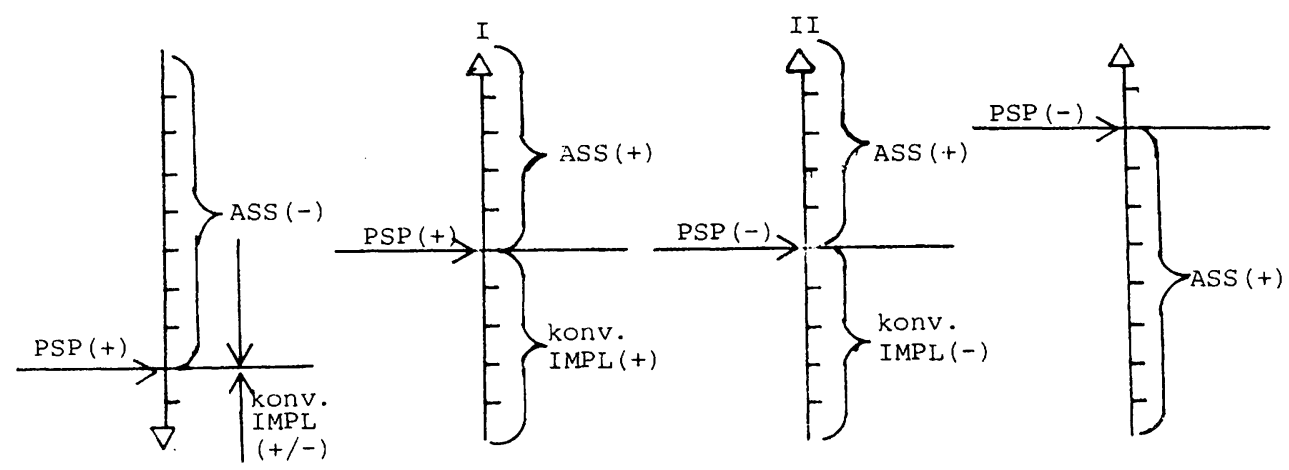

Zum Zweck der leichteren Orientierung werden Beispiele für alle Kombinationsmöglichkeiten bei skalierender Interpretation beigegeben:

(21) Sie ist nur $h$ ü b s ch (nicht schön oder gar einzigartig schön), mit Sicherheit aber einigermaßen attraktiv. (Skala, deren Werte sich nicht gegenseitig ausschließen)

(22) Er ist nur $A n g$ e $t$ e $l$ l t e $r$, nicht Beamter, aber zum Glück auch nicht Arbeiter. (Skala, deren Werte sich gegenseitig ausschließen)

(23) Sie ist nicht nur $h \ddot{u} b s c h$, sondern im wahrsten Sinne schön, von einigermaßen attraktiv gar nicht zu reden. (Skala, deren werte sich nicht gegenseitig ausschließen) 
(24) Er ist nicht nur $A n g$ e $t$ te 1 l t e $r$, sondern Beamter, von Arbeiter gar nicht zu reden. (Skala, deren werte sich gegenseitig ausschließen)

(25) 'Sie ist nur w un de $r s c h$ ö n nicht, aber sonst kann man getrost alle Prädikate der Schönheitsskala auf sie anwenden.

(Skala, deren Werte sich nicht gegenseitig ausschließen)

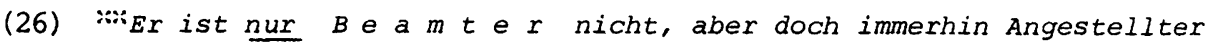
(jedoch nicht Afbeiter). (Skala, deren Werte sich gegenseitig ausschließen)

\subsubsection{2. auch - Gruppe}

(1) Dies ist auch $v$ e r s t änd 1 i ch.

(2) Von da $a b$ war er nicht nur sehr umgänglich, sondern er war auch $g e$ $s p r \ddot{a} c h i g$.

(3) Er ist auch $d i c k$, nicht nur langsam.

(4) Er ist nicht nur der Dïmste, sondern auch der $D i c k s t e$ in der Klasse.

(5) Es war auch $B \circ r n i$ e $r$ he it, nicht nur Unfähigkeit.

(6) In dieser Dienststelle war er der Portier und auch der $A m t s b \circ t e$

Zur Syntax:

Hier ist nicht viel Neues zu berichten: Austauschbarkeit und Stellungsgesetze entsprechen dem bereits Bekannten. - Bei Satzfragen und Wortfragen treten Funktionsambiguitäten zwischen GPn und MPn auf:

(7) Ist dies auch $v e r s t \ddot{n} d I i c h$ ?

(8) her ist auch $d i c k$ ?

Zur semantischen Analyse:

Vorweg wieder Kontrastsätze als Hilfsmittel firr die semantische Analyse:

(la) Dies ist auch $v e r s t a ̈ n d l i c h$, nicht nur richtig/verdammenswert/überraschend ...

(2a) Er war auch $g$ esp r ächig, nicht nur umgänglich/schweigsam (!)/ höflich ...

(3a) Er ist auch dick, nicht nur klein/groß/gemütlich/aufdringlich/ unsportich/lieb/unappetitlich ...

Diese wenigen Sätze zeigen bereits, daß nicht nur nahe verwandte wörter mit dem Skopuswort von auch kontrastieren können, sondern auch Antonyme usw. Im Einzelnen hängt der Bereich des Aufaddierbaren nur vom jeweiligen Sprecher bzw. von der jeweiligen Situation ab. - Wesentlich weniger Wahlmöglichkeiten bestehen bei Satz (6):

(6a) In dieser Dienststelle war er auch der $A m t s b \circ t e$, nicht nur der Portier/der Nachtwächter/“Sozialdemokrat/”der Beliebteste ... 
Nicht-Berufsbezeichnungen sind hierbei nicht grundsätzlich ausgeschlossen, werden aber doch in diese Richtung uminterpretiert:

(6b) In dieser Dienststelle war er auch der $A m t s b \circ t e$, nicht nur der Sozialdemokrat (von Beruf).

Nun ist noch das Verhalten von auch, gegenüber lexikalisierten Skalen zu prufen:

(9) Er ist nicht nur verletzt/"umnebelt, sondern auch $0 \mathrm{hnm} \ddot{\mathrm{a}} \mathrm{cht} i \mathrm{~g}$.

(10) Der Gegenstand ist nicht nur " 1 auwarm/weich, sondern auch $k a 1 /$ w a $r \mathrm{~m}$.

(11) 'Hans ist nicht nur Studienrat, sondern auch $O$ be rs t udi e n$r$ a $t$.

Dies zeigt sehr deutlich, daß skalierende Interpretation für auch bel diesem Skopustyp nicht in Frage kommt: ein uberraschendes Ergebnis, wenn man an das überwiegen der skalierenden Interpretation bei der nur - Gruppe fuir diesen Skopustyp denkt. - Skalen, deren einzelne Werte sich nicht gegenseitig ausschliessen, können natürlich auch quantifizierend interpretiert werden. Dies gilt auch fuir polare Paare:

(12) Es war nicht nur'Jnfähigkeit, sondern $\underline{\text { auch }} B \circ s h e i t$. Auch für diesen Skopustyp stellt sich das Problem der Formulierung der PSP:

(9a) Er ist auch o h n $\mathrm{a}$ a $c h t i g$,

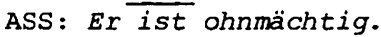

PSP: Er ist noch anderes/es treffen noch andere Prädikate auf ihn zu. (?)

(11a) Hans ist auch $O b$ e $r s t u d i$ e n $r$ a $t$.

ASS : Hans ist oberstudienrat.

PSP: Hans hat noch andere Berufe/Amter/Aufgaben.

Um eine nähere Charakterisierung des PSP-Berelches kormt man dabel nicht herum: die Schwierigkeit liegt darin, daß die pragmatischen Faktoren dabei iberwiegen. Keine Iösung sehe ich in den Formalisierungsansätzen bei Horn (1972) und. Bartsch (1972), die die Giltigkeit a 1 l e $x$ Prädikate für die jeweilige Argumentkombination zulassen.

Suspensionstest: treffen die Feststellungen zu, dann kann es in diesem Falle keine akzeptable Suspendierung von Bedeutungselementen geben.

(11b) Hans ist auch $S t u d i$ e $n$ a $t$,

- "und möglicherweise ist er nicht einmal studienrat. ("skal.ASS")

- "und möglicherweise ist er sogar oberstudienrat. ("konv. IMPL")

- "und möglicherweise ist er nur Assessor. ("skal. PSP")

- "und möglicherweise ist er nichts sonst. (quant. PSP)

Das Ergebnis ist eindeutig: nur die quant. Interpretation ist zulässig. Konjunktionstest: er zeigt eigenartig abweichende Ergebnisse.

(11c) Hans ist auch $S t$ u $d i$ e n $r$ a $t$,

- "und Hans ist Studienrat. (ASS)

- "und Hans ist sonst noch etwas/hat noch einen anderen Beruf. (PSP) 
(11d)

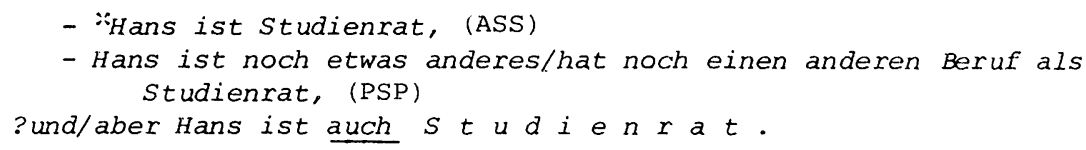

Hier muß man nun allerdings zwischen verschiedenen Arten der Inakzeptabilität unterscheiden. Durch die Wiederholung der ASS entstehen keinerlei logische Widersprüche, dies verstößt nur gegen die Grice'sche Maxime "be relevant". Erklärungsbediirftig ist hier nicht das Verhalten von auch - Sätzen, sondern von nur - Sätzen. Daß die Vorweg-Behauptung der PSP nicht sehr glücklich ist, liegt wohl mehr an der Formulierung; mit einer spezifischen PSP ist sie akzeptabel:

(1le) Hans ist Nebenerwerbslandwirt und Hobbyastronom, und ex ist auch $S t u d i$ e n r a $t$.

Beim Widerspruchstest ist nur eine Tatsache von Interesse: die ausdrückliche Verneinung eines ganz bestimmten Prädikats aus dem möglichen PSP-Bereich führt keineswegs zu Inakzeptabilität, wie etwa die globale PSP-Verneinung:

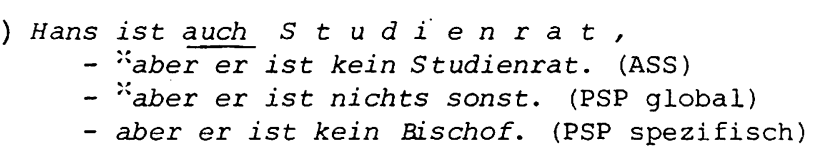

Dialogische Analyse:

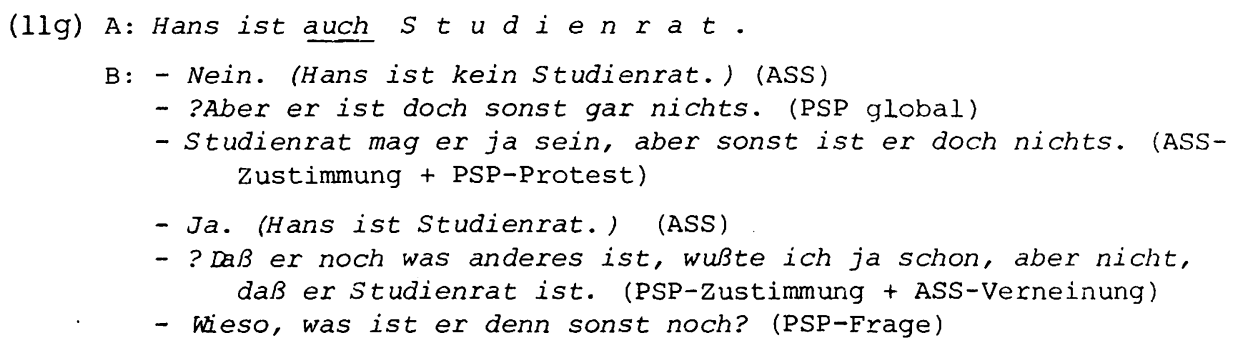

Die Versuche des PSP-Protestes und der PSP-Zustimmung sowie der PSP-Frage zeigen sehr deutlich, daß der "absolute" Gebrauch des quantifizierenden auch mit diesem Skopustyp nicht sehr sinnvoll ist, da die PSP für den Hörer nur in den seltensten Fällen (Erinnerung an frühere Gespräche usw.) rekonstruierbar ist. Es wird also wohl nur in expliziten Aufzählungen auftreten.

\subsubsection{3. sogar - Gruppe}

(1) Das ist sogar sehr a ns tändig von ihm.

(2) Von Deutschlands guten Seiten $z u$ reden ist heute in Deutschland ungewohnt, fast ein bißchen befremalich, sogar ein bißchen $p e i n l i c h$.

(3) ... oder der sympathische Dehler (der war sogar der a 1 l e r $s$ chli m $m s t e$ ). 
(4) Er ist nicht einmal sch ön, geschweige denn gewinnend.

(5) Selbst di $c k$ ist er, dabei durchaus beweglich.

(6) Hans ist sogar 0 b e r s t u d i en $r$ a $t$.

\section{Zur Syntax}

Hier sind nur zwei Stellungstypen von Interesse. Während wieder Typ 1 (GP unmittelbar vor der Skopuskonstituente) die Normalposition ist, ist Typ 2 bei diesem Skopustyp etwas akzeptabler für sogar:

(la) Das ist sehr a $n s t$ ändig ?sogar/ "selbst/" "nicht einmal von ihm. Stellungstyp 5 (topikalisierte Skopuskonstituente), und zwar in der Variante mit GP vor der topikalisierten Skopuskonstituente, ist die einzige Möglichkeit für selbst, das ansonsten bei diesem Skopustyp eine Verteilungslücke aufweist. Bei Distanzstellung der GP fällt selbst aus, nicht einmal ist wenig akzeptabel. Im übrigen können die bekannten Gesetzmäßigkeiten der sogar - Gruppe bzw. die Ergebnisse der Parallelabschnitte übertragen werden.

Semantische Analyse

Natürlich ist auch bei diesem Skopustyp nur skalierende Interpretation möglich; dies bestätigen auch die obigen Sätze. Das Interesse kann sich damit auf die Eigenschaften der Skalen richten, insbesondere auf die Möglichkeit, von dem bloßen sogar - Satz aus auf eine ganz bestimte Skala zu schließen. Deshalb zunächst eine Reihe von Kontrastierungsmöglichkeiten, aus denen man jeweils unschwer die Bezugsskala erschließen kann:

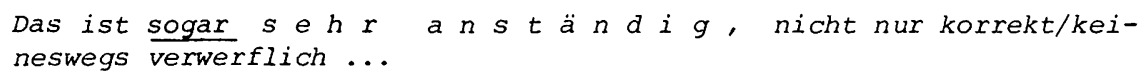

(4) Er ist nicht einmal schön, geschweige denn wunderschön/bezaubernd/ein Adonis // intelligent/geschickt ...

(5) Sogar $d i c k$ ist ex, nicht nur ein bißchen korpulent/vollschlank// Iangsam/dumm ...

(6) Hans ist sogar $O b$ e $r s t u d i$ e $n$ a $t$, nicht nur studienrat.Karl ist Prokurist, Detlev ist Aufsichtsratsmitglied, und Hans ist sogar $O b$ e r s t udienrat.

Folgende Typen kann man unterscheiden: a. Die Kombination aus sogar + Prädikatsnomen/-adjektiv läßt nur eine Skala zu, z.B. bei den Steigerungsformen von Adjektiven, i.d.R. wohl auch bei Berufen. - b. Die Kombination aus sogar + Prädikatsnomen/-adjektiv läßt eine Skala "in der Ebene des Skopuselementes" zu, aber auch quer dazu verlaufende Skalen: hübsch, schön, wunderschön, ein Adonis; schön, erfolgreich, intelligent, charaktervoll. - c. Das Skopuselement läßt über die bereits genannten Möglichkeiten hinaus beliebige ad-hoc-Skalen zu, 
siehe etwa Satz (6). - Eine Reihe von Beisplelen ließe sich auch als polares Paar interpretieren: schön - intelligent.

Die semantischen Analysen sehen demach folgendermaßen aus:

(2a) ASS: Es ist peinlich, von Deutschlands guten Seiten zu reden.

PSP : Es ist befremdlich/ungewohnt/minder peinlich ...

konv. IMPL: Es ist nicht unerträglich/schrecklich peinlich.

(4a) ASS: Er ist nicht schön.

PSP : Er ist nicht wunderschön/nicht intelligent/nichts Bedeutenderes. konv. IMPL: Weniger Bedeutendes/hichtiges trifft auf ihn $z u$.

(6a) ASS: Hans ist Oberstudienrat.

PSP: Hans ist nicht Studienrat/Andere haben weniger bedeutende Berufspositionen.

konv. IMPL: Hans ist nicht Studiendirektor.

(6b) Hans ist nicht einmal 0 b e $r s t u d i$ e $n$ r $t$.

ASS: Hans ist nicht Oberstudienrat/Studiendirektor ...

PSP: Hans ist nicht Studiendirektor.

konv. IMPL: Hans ist Studienrat.

Diese Beispiele zeigen schon sehr deutlich die Schwierigkeiten der Formulierung, wenn die jeweiligen Bezugsskalen nicht festliegen. Da diese Formulierungsschwächen die Tests erheblich stören, beschränke ich mich bei den Tests auf die Sätze (6a) und (6b), und dabei wiederum auf die Berufsskala; wichtig ist dabei der präsuppositionelle Ausschluß der unteren Werte im Falle von (6a). Das unterscheidet diesen Skopustyp offensichtlich von allen anderen.

Suspensionstest:

(6c) Hans ist sogar $O b$ e $r s t u d i$ e $n r a t$,

- "und möglicherweise ist er nicht einmal Oberstudienrat. (ASS)

- "und möglicherweise ist er sogar studienrat. (PSP)

- und möglicherweise ist er sogar studiendirektor. (konv. IMPL)

(6d) Hans ist nicht einmal ob e r s t udi en $r$ a $t$,

- "und möglicherweise ist er sogar oberstudienrat. (ASS)

- und möglicherweise ist ex nicht einmal Studienrat, (konv. IMPL) sondern sogar nur Assessor.

- " und möglicherweise ist er sogar studiendirektor. (PSP)

Trotz relativer Stabilität der Skala hat man große Schwierigkeiten, den Bereich von ASS, PSP und konv. IMPL festzulegen, und zwar auch bei sehr spezifischen Kontexten wie dem folgenden.

(6e) Ich dachte immer, Hans ist Studiendirektor. Und dabei ist er nicht einmal $O b$ e $r$ s $t$ u $i$ e $n r$ a $t$, sondern nur Studienrat, und (unzuverlässig wie er ist) möglicherweise sogar nur Assessor.

Konjunktionstest:

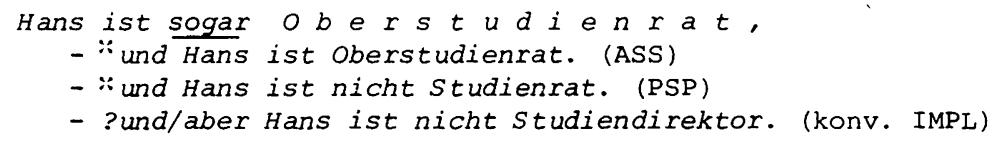


- Hans ist nicht Studienrat, (PSP)

- ?Hans ist nicht Studiendirektor, (konv. IMPL) und Hans ist sogar $O b$ e $r s t u d i$ e n $r a t$.

über die Inakzeptabilität der PSP-Wiederholung bin ich mir nicht völlig sicher, ebensowenig wie über die der Vorwegbehauptung der konv. IMPL: letzteres könnte deswegen inakzeptabel sein, weil dadurch verschiedene "Richtungen" derselben Skala aufeinandertreffen.

(6h) Hans ist nicht einmal $O b$ e $r s t u d i e n r a t$,

- "und Hans ist nicht Oberstudienrat. (ASS)

- und Hans ist Studienrat. (konv. IMPL)

- “Hans ist nicht Studiendirektor. (PSP)

- "Hans ist nicht Oberstudienrat, (ASS)

- Hans ist Studienrat, (konv. IMPL)

- Hans ist nicht Studiendirektor, (PSP)

und Hans ist nicht einmal $O b$ e r s $t$ u d $i$ e n $r$ a $t$.

Die Akzeptabilitätsurteile bestätigen sowohl die Bedeutungsanalyse wie auch die Statuszuweisungen. Erklärungsbedürftig bleibt nur das Verhalten der beiden Bedeutungselemente in (6i). Die Vorwegnahme der konv. IMPL, die sonst nicht voll akzeptabel ist, könnte hier deswegen akzeptabel sein, weil es sich um eine "schwache" konv. IMPL handelt. - Die Vorwegnahme der PSP Hans ist nicht Studiendirektor ist wie gewöhnlich akzeptabel.

Dialogische Analyse:

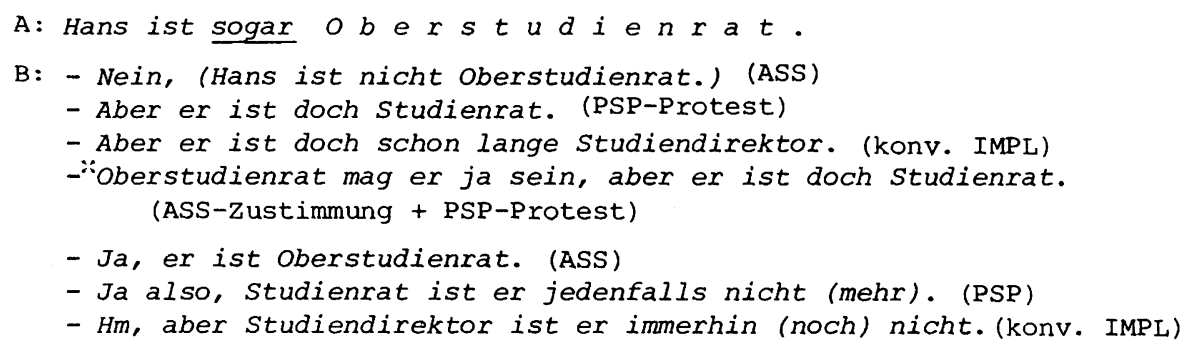

(6k) A: Hans ist nicht einmal $O b$ e $r s t u d i$ e n $r$ a $t$.

B: - Nein, oberstudienrat ist er nicht. (ASS)

- Aber er ist doch sogar Studiendirektor. (PSP-Protest)

- Das ginge ja noch. Aber er ist ja nicht einmal studienrat. (konv. IMPL)

- "Es mag ja sein, daß er nicht oberstudienrat ist, aber er ist doch Studiendirektor. (ASS-Zustimmung + PSP-Protest)

- Doch, er ist Oberstudienrat. (ASS)

- Na ja, daß er nicht Studiendirektor ist, kann ich mir ja denken. (PSP + "be relevant"-Maxime)

- Dafür ist er aber wenigstens Studienrat, und so wenig ist das ja nun auch wieder nicht. (konv. IMPL + Skala)

Dieser letzte Test bestätigt sehr zuverlässig die Statuszuweisung an die Bedeutungselemente von nicht-einmal - Sätzen. Damit ist die Symmetrie zwischen sogar und nicht einmal vollständig: während sogar in einer bestimmten Skala 
die Richtung von "unten" nach "oben" bestimmt, bedingt nicht einmal die ungekehrte Richtung: die Ungültigkeit des ersten wertes "in Richtung" wird präsupponiert, die Ungültigkeit des zweiten Wertes assertiert, und die Gültigkeit des dritten Wertes schwach konversationell impliziert.

\subsection{Skopustyp: Verbalphrase.}

3.4.1./2./3. nur -, auch -, sogar - Gruppe.

Das Belegmaterial wie die zentrale Fragestellung nach Kriterien für die Skopusabgrenzung lassen es geraten erscheinen, alle GP-Gruppen zusammenzufassen. Diskutiert werden fast ausschließlich nur-Beispiele, bei den beiden anderen Gruppen ergaben sich keine signifikanten Abweichungen hiervon bzw. von den bereits bekannten Gruppen-Regularitäten.

(1) Damit werden wiederum nur ... pragmatische Sachverhalte in den bestehenden syntaktisch-semant $\overline{i s c h e n ~ A p p a r a t ~ e i n g e g l i e d e r t . ~}$

(2) In diesem Falle könne es leicht dazu kommen, daß die CSU in München sogar/auch Stimmen verliert.

(3) Auch Adolf Hitler illustriert nur die Erkenntnis des britischen Historikers $A$. J. P. Taylor, daß die Staatsmänner eher ...

(4) Der Pakt bestätigt nur das jahrhundertealte Mißtrauen gegen die beiden Nachbarn.

(5) Er kümmerte sich lediglich um seine Angelegenheiten.

(6) Im wesentlichen bestätigte Nixon lediglich seine bisherige Verteidigungslinie.

(7) Sie können vom Marienplatz zum Stachus schlendern, wo es ihnen paßt stehenbleiben, ja sich sogar/auch vor einer großen Gaststätte an den Tischen im Freien niedersetzen, und in aller Ruhe ein Bier trinken oder eine Brotzeit zu sich nehmen.

Fokus und Skopus

In diesem Falle ist es vordringlich notwendig, zu klären, ob überhaupt ein Skopus VP vorliegt, bevor man an die syntaktischen und semantischen Eigenschaften sich heranwagen darf. Die Kernfrage ist hierbei, ob zusammengesetzte Ausdrücke (in einem oberflächenstrukturellen Sinn zunächst) wie Verb + Objekte, oder auch ein Satz, oder mehrgliedrige NPn Skopus einer GP sein können bzw. ob diese Gebilde in unterschiedlicher Weise "zusammengesetzt" und ev. dadurch auch unterschiedlich "skopusfähig" sind. - R. Bartsch (1972:196) nennt nur NPn, Adverbien, Nomina, Artikel und Quantoren als skopusfähige Elemente. Dies ist eine sowohl unvollständige (Prädikate, VP und Satz) als auch teilweise falsche (Artikel, Nomina) Liste, doch liegt dies wohl daran, daß sich die Verfasserin nicht ge- 
nauer mit den oberflächenstrukturellen Markierungspunkten des Skopus bei GPn auseinandersetzt. - Hingegen wird fast in der gesamten englischen Literatur zu diesem Thema sowohl ein Skopustyp VP als auch ein Skopustyp Satz als möglich angenommen; so bei Bruce Fraser (1971: 151) für das engl. even:

(8) The boys even destroyed the glass door. Die Fragen der Skopusabgrenzung, der syntaktischen und semantischen Eigenschaften dieses Skopustyps werden jedoch kaum angesprochen. Einen weiteren Beleg bietet L. Horn (1969: 225, Satz 23), der als Abgrenzungskriterium einen Kontrastsatz verwendet:

(9) Muriel only voted for Hubert, she didn't do the laundry. Wenn er aber von "Betonung der VP" spricht, dann ist dies ohne weitere Erläuterungen ein problematisches Kriterium. - Für das Englische wird ganz allgemein die Position der GP (bei normaler wortfolge!) zwischen Subjekt und VP als typisch für den Skopus VP bezeichnet. Dies hat im Deutschen bei Matrixsatzwortstellung keine Parallele. Lediglich bei der nur - Gruppe wäre sie als Nachstellung beim Skopus Subjekts-NP (mit starken stilistischen Wirkungen) möglich. Vielmehr steht beim Skopus Verb die GP nach dem Verbum (wenn dieses finit ist) bzw. vor diesem (infinit). Für den Skopus VP kormt im Matrixsatz nur die Stellung unmittelbar nach dem finiten Verb, wie sie alle Belegsätze mit Ausnahme von (2) zeigen, in Frage. Dabei ist allerdings ohne klärenden Kontext nicht sicher $z u$ entscheiden, ob das davorstehende finite verb kontrastiv betont und damit Skopus der GP ist (falls es sich um ein Vollverb handelt), oder ob das darauffolgende objekt oder Adverb kontrastiv betont und damit Skopus der GP ist; und schließlich, ob gegebenenfalls eine ganz normale Akzentverteilung vorliegt mit Betonung des letzten Hauptelementes im Satz. Die Problematik soll anhand von Satz (3) und seinen möglichen kontrastiven Folgesätzen erläutert werden:

(3a) Auch Adolf Hitler illustriert nur die Erkenntnis des britischen Historikers A.J.P. Taylor, daß die Staatsmänner ...

- er wi d e r l e g t sie nicht. Damit wird das Hauptverbum kontrastiert, im Basissatz muß also $i l$ u s $t r i$ e $r t$ betont werden.

- aber er illustriert nicht die These von Ranke, daß ... Damit wird das Akkusativobjekt kontrastiert, im Basissatz mu also $T$ a y $l$ o $r$ oder Erkenntnis des britischen Historikers A.J.P. TayZor (mit schwebendem Akzent) betont werden.

- aber er weckt in der Bevölkerung immer noch gewisse Illusionen. Damit ist die Kontrastierung der VP erreicht. Die Betonung miißte, wie oben bereits bemerkt, auf dem letzten "Hauptelement" der VP liegen.

Im Konstituentensatz mit. Inversion bleibt für diesen Skopustyp nur die Stellung der GP zwischen Subjekt und finitem Verb oder genauer zwischen Subjekt und Prä- 
dikat, also noch vor allen Objekten und Adverbien. Auch hier kann natürlich ohne klärenden Kontext nicht entschieden werden, ob nicht auch die Konstituenten unmittelbar vor oder nach der GP (also das Subjekt oder eines der objekte oder Adverbiale) kontrastiv betont und damit Skopus der GP sind. Aber im Al1gemeinen ist diese Position viel eindeutiger hinsichtlich des Skopus und wird deswegen wohl auch in aller Regel vorgezogen:

(3b) ... weil Adolf Hitler nur die Erkenntnis des britischen Historikers A.J.P. Taylor illustriert ... , aber nicht zu einem Schwinden der Illusionen in der Bevölkerung führt.

(4a) ... weil der Pakt nur das jahrhundertealte Mißtrauen gegen die beiden Nachbarn bestätigt, aber nicht freundschaftliche Beziehungen bewirkt.

(6a) ... weil Nixon lediglich seine bisherige verteidigungslinie bestätigt, aber nicht die Konsequenzen aus den Vorfällen zieht.

Bei beiden Stellungstypen ist also eine kontrastive Betonung nicht eindeutig erkennbar (als "abweichender" Satzakzent), sie ist auch nicht gut möglich, da nur der Normalakzent die gesamte VP als kontrastiert markieren kann. Möglich ist allein eine Bindung der Skopuskonstituente, die ja einen beträchtlichen Umfang erreichen kann, durch Phrasierung und Pausen am Anfang und Ende. Die Betonungsverteilung ist ansonsten ganz normal; die Kontrastierung wird ausschließlich durch den (meist verbalen) Kontext geleistet.

Zur Syntax

Die Klasseneigenschaften werden etwas verschleiert durch die an dieser GP-Stelle möglichen Funktionswechsel bzw. Funktionsambiguitäten (z.B. bei allein und einzig). Durch das Fehlen eines eindeutigen Kontrastakzentes wird dieses Problem noch verschärft, handelt es sich doch dabei um einen ziemlich zuverlässigen Indikator für GP-Funktion.

Die Stellungsgesetze wurden teilweise bereits oben dargestellt. Ergänzungen: Typ 1 (GP vor der Skopuskonstituente) ist auch bei diesem Skopustyp Normalstellung; sie ist allerdings nur im Konstituentensatz möglich.

TYP 2: GP nach der Skopuskonstituente. Sie wiirde in jedem Fall zu Skopusambiguitäten führen, da ja die letzte Konstituente jeweils betont ist und damit viel eher als ausschließliche Skopuskonstituente gelten kann.

Typ 3: GP in der Skopuskonstituente. Dies ist eine der wenigen Gelegenheiten, wo dieser Stellungstyp auftreten kann. Er führt zu nicht zu unterschätzenden Problemen bei der Skopusabgrenzung, zu Skopusambiguitäten, und wird deswegen gemieden; das zeigt seine Systemwidrigkeit bei deutschen Wortstellungsregeln. Typ 5: Skopuskonstituente topikalisiert. Alle derartigen Beispiele wirken einigermaßen gekuinstelt, sie setzen analytische Verbformen voraus: 
(5a) Lediglich um seine Angelegenheiten kümmern will er sich./Um seine Angelegenheiten kümmern will er sich lediglich.

In beiden Positionen können eigentlich keine Skopusambiguitäten aufkommen, da z.B. beim Skopus Präpositionalobjekt die Verbstellung inakzeptabel wäre.

Bei den syntaktischen Umformungen bieten Satz- wie Wortfragen ein schwer lösbares Problem durch die diskontinuierliche Anordnung der VP:

(3c) Illustriert Adolf Hitler nur die Erkenntnis des britischen Historikers A.J.P. Taylor ..., oder weckt er in der Bevölkerung immer noch gewisse Illusionen?

(4b) Bestätigt der Pakt nur das jahrhundertealte Mißtrauen gegen die beiden Nachbarn, oder bewirkt er freundschaftliche Beziehungen?

(5b) Kümmert er sich lediglich um seine Angelegenheiten, oder überarbeitet er den Katalog?

Man kann sich kaum vorstellen, daß hier jeweils ganze VPn miteinander kontrastieren; wenn es so wäre, dann würde es sich jedenfalls um eine ganz andere Art von Kontrastierung handeln als bei einer Kontrastierung Element um Element. So können etwa die Verben sich kümmerm und überarbeiten wohl schwerlich allein miteinander kontrastieren, ob num im quantifizierenden oder skalierenden Sinn.

Ähnlich verhält es sich beim Imperativ (der außerdem Funktionswechsel zur MP z.B. bei nur und bZoß zuläßt):

(5c) Kümmere dich nur um deine Angelegenheiten, überarbeite nicht den katalog! / Du sollst dich nur um deine Angelegenheiten kümmern und nicht den Katalog überarbeiten!

Das Thema der Skopusabgrenzung taucht auch wieder beim 'Clefting' auf:

(5d) Nur seine Angelegenheiten sind es, um die er sich kümmert, nicht die Uberarbeitung des Katalogs/ nicht der Katalog, den er überarbeitet.

(5e) ?Nur sich um seine Angelegenheiten kümmern ist es, was er tut, nicht den Katalog überarbeiten.

Es zeigt sich, daß ein NP-Skopus leicht abgespalten werden kann, aber offensichtlich nicht ein VP-Skopus. Lediglich Left Dislocation ist möglich, aber die Kontrastierung ist dann sehr schwierig:

(5f) Sich nur um seine Angelegenheiten kümmern, ja das mag er, aber den Katalog überarbeiten, dafür ist er nicht zu haben.

An Negationsmöglichkeiten existieren (außer der lokalen Negation) nur zwei Formen: nicht nur, das bei diesem Skopustyp mit der Satznegation identisch ist, und nur nicht, bei dem allerdings nur in der konjunktionalen Verwendung vorliegt (bzw. die Äquivalente, soweit vorhanden, bei den anderen GP-Gruppen).

Die Frage des Passivs stellt in diesem Falle eine ganz eigene Problematik dar: sie spielte eine wichtige Rolle bei der Diskussion um die Ebene innerhalb einer Transformationsgrammatik, auf der die GPn während der Ableitung ihrem jeweiligen Skopus zugeordnet werden sollen: in der Tiefenstruktur oder in der 
Oberflächenstruktur. Kann man zeigen, daß der Skopus einer GP von einer Transformation wie dem Passiv umberührt bleibt, so ist das ein Argument dafür, die Zuordnung auf einer früheren Stufe als die Transformation anzusetzen. Kann man jedoch zeigen, daß dadurch der Skopus verändert wird und daß damit auch eine Bedeutungsveränderung verbunden ist, so muß man, will man die Bedeutungsneutralität der Transformationen beibehalten, auf eine Zuordnung erst nach den Transformationen ausweichen. - Für diese Problematik hat nun (1) eine gewisse Bedeutung: dort umfaßt nämlich der Skopus der GP Subjekts-NP und Teile der VP eines passivischen Satzes. Das Gegenargument, es liege ein Skopus Satz vor, läßt sich mit dem Hinweis widerlegen, daß z.B. von Ross hinzugefügt werden kann und keinesfalls im Skopus liegt. Auch die Begrenzung des Skopus auf pragmatische Sachverhalte ist unhaltbar: in diesem Falle mïßten auch alle Beispiele mit dem Skopus VP im Aktiv aufgegeben werden, mit denen Satz (1) große Ähnlichkeit aufweist. - Dies würde (zumindest für diesen Fall) bedeuten, daß der tiefenstrukturelle Skopus VP durch die Passivtransformation nicht verändert wurde; also ein Argument für die Skopuszuweisung in der Tiefenstruktur. Doch ist für eine Entscheidung die empirische Basis natürlich zu schmal. Ein Gegenbeispiel, wo also der Skopus einer GP die ganze VP eines passivischen Satzes umfaßt, konnte ich nicht beibringen.

Semantische Analyse

Anhand der oben aufgeführten Kontrastsätze sind bereits einige Schlüsse möglich: In allen Fällen ist eine quantifizierende Interpretation denkbar. Dies zeigt schon die grumdsätzliche Verwendbarkeit von ausschließlich und einzig und alZein. - Eine skalierende Interpretation ist in keinem Fall mit Sicherheit auszuschließen. In beiden Fällen ist die PSP durch den wortlaut der Äußerung ohne nur fest gegeben. In beiden Fällen läßt sich die jeweilige ASS nicht mit Sicherheit (weder dem Sinne noch dem Wortlaut nach) bestimmen: die quantifizierende ASS kann aus einem weiten Bereich von auf ein Subjekt zutreffenden VPn gewählt werden; die skalierende ASS hat hier nicht den Vorzug von weitgehend lexikalisierten Skalen. - Dies erschwert jede eingehendere semantische Analyse beträchtlich. Deshalb seien die einzelnen Stationen nur an einem einfachen Beispiel durchgespielt:

(5g) ... weil er sich nur um die Signatur der Bücher kümmerte und nicht den Katalog überarbeitete.

PSP: Er kümmerte sich um die Signatur der Bücher.

ASS: Er tat nichts sonst/er erledigte keine andere bibliothekarische Aufgabe.

Er übernahm keine wichtigere bibliothekarische Aufgabe, z.B. den Katalog überarbeiten. 
konv. IMPL: Er erledigte keine von den weniger wichtigen bibliothekarischen Aufgaben, z.B. Bücher einsortieren.

Suspensionstest:

(5h) Er kümmerte sich lediglich um die Signatur der Bücher,

- und möglicherweise hat er nicht einmal das getan. (skal. PSP)

- “und möglicherweise übernahm er sogar wichtigere bibliothekarische Aufgaben. (skal. ASS)

- : und möglicherweise übernahm er sogar andere bibliothekarische Aufgaben. (quant. PSP)

- und möglicherweise erledigte er sogar noch weniger wichtige bibliothekarische Aufgaben. (konv. IMPL)

Konjunktionstest:

(5i) Er kümmerte sich lediglich um die Signatur der Bücher,

- :und er kümmerte sich um die Signatur der Bücher. (PSP)

- und er tat nichts sonst (quant. ASS)/und er erledigte keine anderen bibliothekarischen Aufgaben.

- und er übernahm keine wichtigere bibliothekarische Aufgabe/er überarbeitete nicht den Katalog. (skal. ASS)

- "und er erledigte keine weniger wichtige bibliothekarische Aufgabe. (konv. IMPL)

(5j) - Er kümmert sich um die signatur der Bücher, (PSP; GP betont)

- Er erledigt keine anderen bibliothekarischen Aufgaben, (quant.ASS)

- Er übernimmt keine wichtigere bibliothekarische Aufgabe/er überarbeitet nicht den Katalog, (skal.ASS)

- 'Er erledigt keine weniger wichtige bibliothekarische Aufgabe, (konv. IMPL)

und er kümmert sich lediglich um die Signatur der Bücher.

Dialogische Analyse:

(5k) A: Er kümmerte sich lediglich um die signatur der Bücher.

B: - Nein. (Er hat noch viele andere bibliothekarische Aufgaben erledigt.) (quant. ASS) / (er hat auch wichtigere bibliothekarische Aufgaben bearbeitet.) (skal. ASS)

- Aber er hat sich doch gar nicht um die Signatur der Bücher gekümmert. (Protest gegen quant. PSP)

- Du wirst es vielleicht nicht glauben, aber er hat sogar nur die Bücher einsortiert. (konv. IMPL)

- Aber er hat sich doch nicht einmal um die signatur der Bücher gekümmert. (skal. PSP)

- Na, ich find' das gar nicht so übel. (Skala selbst)

- "Um andere/wichtigere bibliothekarische Aufgaben mag er sich ja nicht gerissen haben (ASS-Zustimmung), aber er hat sich doch nicht nur um die Buchsignaturen gekümmert. (PSP-Protest)

- Nein also, er hat wirklich sonst gar nichts/nichts wichtigeres gemacht. (quant./skal. A.SS)

- Ja, find' ich auch ganz schrecklich primitiv. (Skala selbst)

Der Versuch zeigt, daß ein sehr eng gewählter pragmatischer Kontext die Testbewertungen wesentlich erleichtert und zu recht brauchbaren Ergebnissen führt. Allerdings darf man nicht ibersehen, daß die Abgrenzung des ASS-Bereiches im gesamten Beispiel keine Sache der Semantik, sondern allein der Pragmatik war. 
3.5. Skopustypen mit kontrastiv betonten Quantoren

3.5.1. Skopustypen mit kontrastiv betonten Zahlwörtern

\subsubsection{1. nur - Gruppe} ... bei den Landwirten nur $d r e i z e h n$ bis $d r e i \beta i g$ Prozent.

(2) Sibirien hat nur $f \ddot{u} n f u n d z$ wan $z i g$ Millionen Einwohner.

(3) In nur $s i$ e $b$ e $n$ Sekunden fallen die 56 Bomben aus den Schächten.

(4) Nur ein einzige $r$ (Traumbau) wurde verwirklicht.

(5) So ergeben die Recherchen, daß etwa nur jeder $v i$ e $r$ e Soziologe vermittelt werden konnte.

(6) Der Weg über die Expertenstellung wäre nur e i n e $r$ der möglichen Wege für die FDP.

(7) Aber das ist nur die e ine Ursache meines Nichtbeschäftigtseins. Zur Syntax

Bei der syntaktischen Untersuchung obiger Sätze hat man zu berücksichtigen, daß mit 'Quantor' nur semantisch ein einheitlicher Skopustyp vorliegt. Syntaktisch gehören die meisten obigen Beispiele zu anderen, schon behandelten Skopustypen. Insofern ist die Bildung eines eigenen Skopustyps nur von den semantischen Eigenschaften her gerechtfertigt. - Divergierende syntaktische Gesetzmäßigkeiten gibt es nur bei den Stellungsgesetzen. Um die anfallenden Aufgaben sinnvoll bewältigen zu können, werden die Stellungstypen geringfügig abgewandelt.

Typ 1 (GP unmittelbar vor der Skopuskonstituente, die das kontrastiv betonte Zahlwort enthält). Dies ist die Anordnung in allen obigen Sätzen außer (3). Typ 2: GP unmittelbar nach der Skopuskonstituente. Das ist grundsätzlich möglich, insbesondere wenn die GP dabei am Satzschluß zu stehen kommt: dadurch können nämlich keine Skopusambiguitäten mehr auftreten.

(4a) Verwirklicht wurde ein e inzige $r$ (Traumbau) nur.

(1a) ... bei den Landwirten 13 - 30 Prozent nur.

Bei (6) und (7) ist dies jedoch nicht möglich:

(7a) ?Aber das ist die e ine Ursache meines Nichtbeschäftigtseins nur. Auf die in diesem Fall deutlich abweichende Funktion von nur gehe ich bei der semantischen Analyse noch ein.

Typ 3: GP in derjenigen Konstituente, die das kontrastiv betonte Zahlwort enthält, und zwar unmittelbar vor letzterem. Dafür ist (3) ein gutes Beispiel. Diese Position ist nur möglich, wenn die NP einen Artikel oder eine Präposition 
enthält, und außerdem nur vor Kardinalzahlen:

(3a) In nur $s$ i e b e $n$ Sekunden ...

(8) In nur a cht Koffern ...

(9) In nur jeder $v i$ e $r$ te $n$ Stadt...

(10) "In nur der $v i$ e $r$ t e $n$ stadt...

(11) "In nur dem e $i n$ e $n$ Fall ...

Das gleiche Stellungsmuster ist also auch vor Ordinalzahlen, soweit sie mit jeder serialisiert sind, möglich. - Besondere Aufmerksamkeit verdient auch noch die Stellung des Artikels relativ zur GP, sowie von jeder.

(3b) In den nur $s i$ e $b$ e $n$ Sekunden seiner Abwesenheit ...

(3c) 'In nur den $s i$ e $b$ e $n$ Sekunden seiner Abwesenheit...

(9a) 'In jeder nur $v i$ e $r$ t e $n$ stadt...

Man beachte auch den semantischen Unterschied zwischen den Formulierungen in nur $s i e b$ e $n$ Sekunden und nur in $s i$ e $b$ e sekunden. Er läßt sich aber nicht in gleicher Weise paraphrasieren wie bei der so ähnlich erscheinenden Konstruktion mit kontrastiv betontem Adjektivattribut (3.2.4.1.).

TYp 4: GP in Distanzstellung vor und nach der Skopuskonstituente: dergleichen konnte ich bislang nicht beobachten.

Typ 5: Die Skopuskonstituente ist topikalisiert, die GP steht nahe dem Satzschluß. In den Fällen des Stellungstyps 3 scheint dies jedoch nicht möglich zu sein. Dies hängt wahrscheinlich mit der spezifischen semantischen Funktion dieser Stellung zusammen.

(1b) 13 - 30 Prozent sind es bei den Landwirten bloß.

(3d) "In $s i$ e $b$ e $n$ Sekunden fallen die 56 Bomben (nur) aus den Schächten (nur).

Es wiederholen sich also die Stellungsgesetze der jeweiligen Skopustypen, in denen die Zahlwörter enthalten sind.

Bei den Satzarten erwähne ich nur den Imperativ:

(4b) Verwirkliche nur einen e in $z$ i g e n Traumbau!

(5a) vermittle nur $v i$ e $r$ Soziologen!

Hier ist bei MP-Interpretation ein anderes Betontungsmuster notwendig: auf jeden Fall darf das Zahlwort keinen Kontrastakzent erhalten. - Bei der Spaltsatzbildung darf wiederum nur die gesamte Phrase, die das Zahlwort enthält, abgespalten werden (mit wechselnden Schwierigkeiten, je nach Art und Größe der abgespaltenen Konstituente). - Nun noch zu den Negationsmöglichkeiten:

Typ 1: NEG + GP. Offenbar ist diese Kombination bei der e $i n e$ im Skopus nicht möglich:

(2a) Sibirien hat nicht nur 25, sondern 30 Millionen Einwohner. 
(7b) "Das ist offenbar nicht nur die e in e Ursache, sondern auch die a $n d e r e$.

(4c) Nicht nur ein einzige r traumbau wurde verwirklicht, sondern viele.

(6a) 'Der Weg über die Expertenstellung wäre nicht nur e i n möglicher Weg, sondern... (?) / nicht nur de $r$ mögliche weg.

Typ 2: GP + NEG + Skopuskonstituente (wobei die GP, wenn möglich, in der konjunktionalen Verwendungsweise vorliegt) .

(2b) Sibirien hat nur nicht 25 Millionen Einwohner, sondern ... Nur hat Sibirien nicht 25 Millionen Einwohner, sondern ...

(3e) "In nur nicht si e b e n Sekunden ...

Inakzeptabel ist diese Form auch mit allen anderen Belegsätzen: ein weiteres Indiz für die von anderen Skopustypen abweichende semantische Grundstruktur. Typ 3: Satznegation. Sie ist in allen Fällen problemlos möglich.

(3f) In nur $s i$ e $b$ e $n$ Sekunden fallen die Bomben nicht aus den Schächten.

(5b) Nur jeder $v i$ e $r$ e Soziologe konnte nicht vermittelt werden.

Skopus und Fokus

Die Relation zwischen den Skopusgrenzen einerseits und der Kombination aus kontrastivem Akzent und den Stellungsmöglichkeiten der GP andererseits ist in diesem Fall außerordentlich unklar. Die reine Akzentpositionierung bietet dann keine Probleme, wenn auf die GP nur ein Zahlwort folgt. In diesem Fall ist die Bezugsmenge für die Zahl meist vorerwähnt oder durch den Kontext eindeutig festgelegt. Ebenso klar ist die Sachlage, wenn ein expliziter Kontrastsatz vorhanden ist: die kontrastierende Konstituente erhält den Kontrastakzent. - Schwieriger ist die Lage, wenn die Bezugsmenge explizit mit dem Zahlwort verbunden ist und kein Kontrastsatz vorhanden ist. Prinzipiell können dann natülich wieder alle Einzelelemente kontrastiv betont werden:

(2c) Sibirien hat nur $25 M i 11$ i o n e $n$ Einwohner, nicht 25 Mi 11 ia $r$ d e $n$.

(2d) Sibirien hat nur 25 Millionen $E$ in wohne $r$, nicht 25 Millionen $H \ddot{a} u s$ e $r$.

(2e) Sibirien hat nur $f \ddot{u} n f$ undzwanzig Millionen Einwohner, nicht $v i$ e $\overline{r z}$ ig Millionen Einwohner.

(2f) Stell dir vor, Sibirien hat nur 25 Millionen Einwohner. Ist das nicht sagenhaft wenig für dieses Riesenland?

Ich nehme an, daß zwischen den letzten beiden Versionen zumindest Gebrauchsunterschiede bestehen, und ich bin weiter der Ansicht, daß für die Version (2f) ein kontrastiver Akzent nur auf der Zahl nicht statthaft wäre. Andererseits liegen wohl auch nicht auf allen Elementen Kontrastakzente. Wahrscheinlicher ist wohl eine Bindung durch Phrasierung bei gleichbleibenden Akzentniveau. 
Zur semantischen Analyse

Zur Abgrenzung der semantischen Möglichkeiten und zur Trennung der Funktionen der einzelnen GPn werden zunächst wieder Kontrastsätze gebildet:

(2g) Sibirien hat nur/bloß/lediglich fünfundzwanzig Millionen Einwohner, nicht 30 Millionen Einwohner/das ist unglaublich wenig.

Sibirien hat ausschließlich/einzig und allein fünfundzwanzig Millionen Einwohner, sonst nichts.

Sibirien hat allein fünfundzwanzig Millionen Einwohner, anderes noch gar nicht gerechnet.

Man beachte, daß je nach Folgesatz im Basissatz ein anderes Akzentmuster vorliegt: für die kontrastive Betonung von fünfundzwanzig kommen nur nur, bloß und lediglich in Betracht, nur diese Konstellation ist hier unser Thema. Für die nur implizit kontrastive Interpretation im Sinne von nicht mehr/das ist unglaublich wenig kann man nur, bloß und lediglich anführen, also dieselben Elemente wie oben. Eine rein quantifizierende Interpretation, die man etwa mit sonst besitzt Sibirien gar nichts umschreiben könnte, bedingen wieder die drei Elemente allein, einzig, ausschließlich, sowie die Zusammensetzung einzig und allein. Dazu kommt noch die spezifische Verwendung von allein: einmal im Sinne der implizit kontrastiven Interpretation von nur etc.; die andere Verwendung (Sibirien ohne andere Gebiete) kann leicht ausgeschlossen werden. Es liegt also wieder die gewohnte Verteilung von skalierender und quantifizierender Interpretation vor. Nun ist dies noch für die Subtypen dieses Skopustyps zu überprüfen:

(3g) In nur/allein/"einzig/ausschließlich/einzig und allein s i e b e $n$ Sekunden fallen die 56 Bomben aus den Schächten.

Die hier mögliche Interpretation von allein läßt sich folgendermaßen verdeutlichen: gar nicht zu reden davon, wieviele Bomben in 60 sekunden herausfallen. Dies entspricht etwa der Semantik von schon in gleicher Position. - Die hier zutreffende Interpretation von ausschließlich ist: nie in einer anderen zeitspanne. Das dürfte auch für einzig und allein gelten; wir haben damit wohl wieder die quant. Interpretation vor uns. Diese Interpretation läßt sich auch für einzig bzw. allein nicht völlig ausschließen, aber ich wïrde sie doch für sehr ungewöhnlich halten. Die Möglichkeit zu einer quant. Interpretation, also:

(4d) $\frac{\text { Nur/allein/'einzig/ausschließlich/einzig und allein ein }}{g}$ e in $n$ zraumbau konnte verwirklicht werden. sollte man wohl allen Elementen nicht absprechen; bei ausschließlich weist sie wohl eine zusätzliche temporale Komponente auf.

(5c) Nur/allein/"einzig/ausschließlich/einzig und allein jeder $v i$ e $t e$ Soziologe konnte vermittelt werden.

Hier ist wieder dieselbe Gesetzmäßigkeit zu konstatieren, wenn auch mit gewis- 
sen Zweifeln hinsichtlich der Akzeptabilität solcher Formen:

(7) Das ist nur/?allein/“einzig/?ausschließlich/?einzig und allein die e in e Ursache meines Nichtbeschäftigtseins.

Eine nur quantifizierende Interpretation ist in diesem Fall von manifester polarer Interpretation für mich nicht recht denkbar.

In einem zweiten Abschnitt soll durch Kontrastsätze der jeweils mögliche Interpretationsbereich ermittelt werden.

(1c) Von den Beamtenkindern machen 27-44 Prozent das Abitur, bei den Bauern sind es nur 13-30 Prozent, nicht 25-35 Prozent.

(2h) Das europäische Rußland hat 226 Millionen Einwohner, Sibirien hat nur 25 Millionen Einwohner (nicht 35 Millionen Einwohner).

(3h) Nicht etwa in 10 Sekunden, nein, in nur $s i$ e $b$ e $n$ sekunden fallen die 56 Bomben aus den Schächten / " nur in $s i$ e $b$ e $n$...

(4e) Fünf Traumbauten waren geplant, aber nur ein e in $z i g$ e $r$ wurde verwirklicht.

(5d) In Hessen konnte jeder zweite Soziologe unterkommen, in Bayern hingegen konnte nur etwa jeder $v i$ e $r$ t e Soziologe vermittelt werden.

(6b) Der FDP bieten sich derzeit eine ganze Reihe von Chancen. Der weg über die Expertenstellung wäre nur e i $n$ möglicher weg ...

(7c) Du kennst ja meine Schläfrigkeit. Aber das ist nur die e i n e Ursache meines Nichtbeschäftigtseins. Die andere ist meine Freßlust.

Dies ist jedoch jeweils nur die skalierende sowie bei (6b) und (7c) die polare Interpretation, was sich in den meisten Fällen durch den Einsatz von sogar vor nur verdeutlichen läßt. Für die quantifizierende Interpretation lassen sich nur sehr mihevoll halbwegs einsehbare Kontexte finden:

(2i) Die BRD mag 58 Millionen Einwohner haben, Großbritannien 75, Japan 84; Sibirien hat ausschließlich/einzig und allein 25 Millionen Einwohner.

(3i) Die zeit, in der die Bomben aus den Schächten purzeln, ist nicht beliebig: in ausschließlich $s i$ e $b$ e $n$ sekunden fallen ...

Hierbei wird ein einziger Wert als der allein gültige bezeichnet. Nur diesen Gebrauch von nur + Quantor berücksichtigt Bartsch (1972: 197).

Analysevorschläge:

(2) ASS: Sibirien hat nicht mehr als 25 Millionen Einwohner.

PSP: Sibirien hat 25 Millionen Einwohner.

konv. IMPL: Sibirien hat nicht weniger als 25 Millionen Einwohner.

(3) ASS: In nicht mehr als sieben Sekunden fallen die 56 Bomben... PSP: In sieben Sekunden fallen die 56 Bomben aus den Schächten. konv. IMPL: In nicht weniger als 7 Sekunden fallen die 56 Bomben...

(5) ASS: Nicht mehr als jeder vierte Soziologe (also nicht jeder zweite Soziologe) konnte vermittelt werden.

PSP: Jeder vierte Soziologe konnte vermittelt werden.

konv. IMPL: Nicht weniger als jeder vierte Soziologe konnte vermittelt werden. 
Das ist nur die $e$ i n e Ursache meines Nichtbeschäftigtseins.

ASS: Das ist nicht die andere Ursache meines Nichtbeschäftigtseins. PSP: Das ist die eine Ursache meines Nichtbeschäftigtseins.

konv. IMPL: ---

Für die quantifizierenden Interpretationen gelten grundsätzlich dieselben Aussagen:

(2j) Sibirien hat ausschließlich/einzig und allein 25 Millionen Einwohner. PSP: Sibirien hat 25 Mill. Einwohner.

ASS: Sibirien hat keine andere Bevölkerungszahl.

(3j) Ausschließlich in $s i$ e $b$ e n sekunden fallen die 56 Bomben ... PSP: In sieben Sekunden fallen die Bomben aus den Schächten.

ASS: In keinem anderen Zeitraum fallen die 56 Bomben ...

(5e) Ausschließlich jeder $v i$ e $t$ e Soziologe konnte vermittelt werden. PSP: Jeder vierte Soziologe konnte vermittelt werden.

ASS: Keine andere Vermittlungsrate trifft $z u$.

Vergleicht man nun die Bedeutung von Nur in $s i$ e $b$ e $n$ Sekunden fallen... mit der quantifizierenden Interpretation von In ausschließlich $s i$ e $b$ en Sekunden fallen... , so sieht man, daß beide identisch sind. Bei nur und bloß werden also durch die Position der GP skalierende und quantifizierende Interpretation unterschieden. Das gleiche gilt für: in nur $z w e i$ Kofferm - nur in $z w e i$ Kofferm; auf nur $f \ddot{u} n f$ Schiffen-nur auf $f \ddot{u} n f$ Schiffen. Bei nur quantifizierend interpretierbaren GPn besteht kein Bedeutungsunterschied zwischen den beiden Konstruktionen.

Schwierig ist auch die Analyse der Negation nicht nur:

(2k) Sibirien hat nicht nur $z$ wa $n z i g$ Millionen Einwohner. ASS: Sibirien hat mehr als zwanzig Millionen Einwohner. PSP: ?Sibirien hat (mindestens?) 20 Millionen Einwohner. konv. IMPL: Sibirien hat nicht weniger als 20 Millionen Einwohner.

Die PSP-Formulierung kann hier nicht befriedigen: andererseits ist die aus der unnegierten Fassung bekannte PSP-Formulierung (die ja von der internen Negation unberührt bleiben mißßte) wohl nicht ganz zutreffend (genauer: zwar zutreffend, aber irreführend) .

Suspensionstests:

(21) Sibirien hat nur $z$ wa $n z i g$ Millionen Einwohner,

- “und möglicherweise hat es sogar 30 Mill. Einwohner. (skal. ASS)

- und möglicherweise hat es nicht einmal 20 Mill. Einwohner. (PSP)

- und möglicherweise hat es sogar nur 15 Mill. Einwohner. (konv. IMPL)

(2m) Sibirien hat ausschließlich $z$ wa $n z i g$ Millionen Einwohner,

- "und möglicherweise hat es nicht einmal 20 Mill. Einwohner. (PSP)

- "und möglicherweise treffen nicht einmal andere Einwohnerzahlen

$z u$. (quant. ASS)

(3k) In nur $s i$ e $b$ e n Sekunden fallen die 56 Bomben aus den Schächten, - "und möglicherweise sogar in 10 Sekunden. (skal. ASS)

- und möglicherweise in nicht einmal sieben Sekunden. (PSP)

- und möglicherweise sogar in nur 5 sekunden. (konv. IMPL) 
(31) In ausschließlich $s i$ e $b$ e $n$ sekunden fallen die Bomben ... - "und möglicherweise nicht einmal in 7 Sekunden. (quant. PSP)

- “und möglicherweise sogar noch in anderen Zeitintervallen. (ASS)

(5f) Nur jeder $v i$ e $r$ e Soziologe konnte vermittelt werden,

- "und möglicherweise sogar jeder dritte. (skal. ASS)

- und möglicherweise nicht einmal jeder vierte. (skal. ASS)

- und möglicherweise sogar nur jeder fünfte. (konv. IMPL)

(5g) Ausschließlich jeder $v i$ e $r$ e Soziologe konnte vermittelt werden,

- “und möglicherweise nicht einmal jeder vierte. (quant. PSP)

- : und möglicherweise treffen sogar noch andere Vermittlungsraten zu. (quant. ASS)

(7d) Das ist nur die e in e Ursache meines Nichtbeschäftigtseins,

- "und möglicherweise sogar die andere. (polare ASS)

- "und möglicherweise nicht einmal die eine. (polare PSP)

Die Inakzeptabilität von Suspensionen bei quantifizierender Interpretation bestätigt meine These, daß Suspendierung notwendig etwas mit gerichteten Skalen zu tun habe. Die Unmöglichkeit von Suspensionen bei polaren Interpretationen steht jedoch gegen meine These, daß diese Form ebenfalls gerichtet und damit der skalierenden Interpretation näher sei als der quantifizierenden.

Konjunktionstest:

(2n) Sibirien hat nur $z$ wa $n z$ ig Millionen Einwohner,

- und Sibirien hat nicht mehr als zwanzig Mill. Einwohner. (skal.ASS)

- "und Sibirien hat zwanzig Millionen Einwohner. (skal. PSP)

- "und Sibirien hat nicht weniger als 20 Mill. Einwohner. (konv. IMPL)

(20) Sibirien hat ausschließlich $z$ wa $n z$ ig Millionen Einwohner,

- und Sibirien hat keine andere Bevölkerungszahl. (quant. ASS)

- "und Sibirien hat zwanzig Millionen Einwohner. (quant. PSP)

(2p) - ?Sibirien hat nicht mehr als 20 Millionen Einwohner, (skal. ASS)

- Sibirien hat 20 Millionen Einwohner, (skal. PSP)

- Sibirien hat nicht weniger als 20 Millionen Einwohner, (konv. IMPL) und Sibirien hat nur $z$ w a $n z i g$ Millionen Einwohner.

(2q) - ?Sibirien hat keine andere Bevölkerungszahl, (quant. ASS)

- Sibirien hat zwanzig Millionen Einwohner, (quant. PSP)

und Sibirien hat ausschließlich $z w a n z i g$ Mill. Einwohner.

Das sind völlig reguläre Ergebnisse, wenn man davon absieht, daß im letzteren Fall wohl immer die GP kontrastiv betont werden mu; die übrigen Sätze weichen ebenfalls nicht davon ab. Hier interessiert nur noch (7) wegen seiner polaren Interpretation:

(7e) Das ist nur die e in e Ursache,

- und das ist nicht die a $n d$ e $r$ e. (polare ASS)

- "und das ist die eine Ursache. (PSP polar)

- "und das ist nicht keine Ursache. ("konv. IMPL")

- Das ist nicht die andere Ursache, (ASS)

- Das ist die eine Ursache, (PSP polar)

- "Das ist nicht keine Ursache, ("konv. IMPL")

und das ist nur die $e$ in e Ursache. 
Dies beweist, daß für die polare Interpretation dieses Satzes eine konversationelle IMPL nicht sinnvoll zu formulieren ist (im Sinne einer "reduzierten" skalierenden Interpretation). Die polare Interpretation stellt damit eine eigene Form neben Quantifizierung und Skalierung dar.

Dialogische Analyse:

(2r) A: Sibirien hat nur $z$ wa $n z i g$ Millionen Einwohner.

B: - Nein, Sibirien hat 30 Millionen Einwohner. (skal. ASS)

- Aber Sibirien hat doch gar keine 20 Mill. Einwohner. (PSP skal.)

- Du wirst es nicht glauben, aber es hat sogar noch viel weniger Einwohner. (konv. IMPL)

- "Daß es nicht mehr als 20 Millionen Einwohner hat, mag ja stimmen, aber $20 \mathrm{Mill}$. Einwohner hat Sibirien sicherlich nicht. (ASSZustimmung + PSP-Protest.

- Ja, Sibirien hat wirklich nicht mehr Einwohner. (skal. ASS)

- Was, zwanzig Millionen? Das wußte ich ja noch gar nicht. (PSP)

- Na ja, weniger hat es ja nun auch nicht.

- "Also, daß Sibirien zwanzig Millionen Einwohner hat, das nehme ich schon auch an, aber es hat sicherlich mehr Einwohner. (ASS-Verneinung, PSP-Zustimmung)

Die Ergebnisse sind regulär bis auf die Kombinationen: die Verknüpfung von ASSZustimmung und PSP-Protest führt, wie erwartet, zu totaler Inakzeptabilität; umgekehrt müßte die Verbindung ASS-Verneinung + PSP-Zustimmung akzeptabel sein, was mir hier nicht ganz gesichert scheint.

(3m) A: In ausschließlich $s i$ e $b$ e $n$ Sekunden fallen die Bomben aus den Schächten.

B: - Ja, die Bomben fallen tatsächlich in keiner anderen Anzahl von Sekunden aus ihren Schächten. (quant. ASS)

- Ich nehme schon auch an, daß sie in 7 Sekunden aus den Schächten fallen, (PSP-Zustimmung) aber andere Anzahlen von Sekunden dürften wohl auch zutreffen. (ASS-Verneinung)

- Nein also, die fallen schon auch in anderen Sekundenfristen raus. (quant. ASS)

- Na also, in 7 Sekunden fallen sie bestimmt nicht raus, (PSP-Protest) "aber in anderen Sekundenfristen fallen sie nicht heraus. (ASS-Zustimmung)

Hier erbringen also die Kombinationen wieder die regulären Ergebnisse. Bemerkenswert ist, daß in diesem Zusammenhang auch durchaus spezifische ASS-Verneinungen möglich sind. - Eine in gewisser Weise selbständige Variante bilden die folgenden Sätze:

(5h) Nur jeder $v i$ e $r$ e Soziologe wird vermittelt, nicht jeder dritte.

(5i) Hör mal, eben les' ich, daß nur jeder vierte Soziologe vermittelt werden kann. Ist das nicht schrecklich?

Bei (5i) ist kein eindeutiger Kontrastakzent auf vierte oder Soziologe zu erkennen. Vermutlich werden nur beide Elemente kontrastiert, etwa mit immerhin jeder zweite Germanist. Semantische Unterschiede folgen aber daraus nicht. 
3.5.1.2. auch - Gruppe

(1) Es ist auch nicht e i n e $r$ davongekommen.

(2) Bei der Tüchtigkeit unserer Landwirte werden auch d $r$ e. i $\beta$ i g Prozent von ihnen gewinnbringend wirtschaften können.

(3) Die $100 \mathrm{~m}$ spult er auch in e $1 \mathrm{f}$ Sekunden $a b$.

(4) Zwei sind ja ganz praktisch, aber auch ein e inzige $r$ würde in diesem Falle reichen.

(5) $\underline{\text { Auch }}$ ein $f \ddot{u} n f t$ e $r$ oder $s \in c h s$ t e $r$ start würde hier nichts $\overline{\text { mehr }}$ retten.

(6) Zwei wirklich liebe Kerle! Der eine versorgte die Rosen, aber auch der a $n d e r$ e war nie ganz unbeschäftigt. Baden würde mir auch $f \ddot{u} n f m$ a 1 (hintereinander) Spaß machen.

Zur Syntax

Die Tatsache, daß der Austausch mit Elementen der sogar - Gruppe auch durch keinerlei semantische Restriktionen behindert wird, ist ein Hinweis auf die Verwandtschaft in der Funktion. - Die Stellungsgesetze entsprechen ganz dem bisher Festgestellten, bis auf den Typ 5, also GP in Distanzstellung nach topikalisierter Skopuskonstituente. Während etwa Satz (1), (2) und (3) in dieser Form recht zweifelhaft in ihrer Akzeptabilität sind, sind die Sätze (4) bis (7) voll akzeptabel:

(1a) ?E $i n$ e $r$ ist $\underline{\text { a } u C h}$ nicht davongekommen.

(4a) Ein Einziger würde in diesem Fall a $u c h$ reichen.

(6a) $\ldots$ und der a $n d e r e$ war $a u c h$. nicht ganz unbeschäftigt.

(2a) ?D $r$ e $i$ B ig Prozent unserer Landwirte werden bei ihrer Tüchtigkeit $\underline{a} u \subset h$ gewinnbringend arbeiten können.

Die These, das unterschiedliche Verhalten hänge mit der Austauschbarkeit gegen sogar (und damit mit dem Unterschied quant. - skal. Interpretation) zusammen, muß erst noch durch weitere Argumente abgesichert werden. -

Bei Satzfragen tritt wieder der bekannte Funktionswechsel von auch zur MP ein. - Clefting ist auch dann, wenn nicht das kontrastiv betonte Zahlwort, sondern die gesamte NP, in der es enthalten ist, abgespalten wird, wegen der gegensätzlichen Semantik von auch und Spaltsatz inakzeptabel. - Im Zusammenhang mit dem Thema 'Negationsmöglichkeiten' möchte ich nur auf die in Satz (1), (5) und (6) enthaltenen Negationen kurz eingehen. In (5) und (6) liegt jeweils eine Satznegation vor, die die Semantik von auch nicht verändert. Anders bei (1):

(1b) ?Es ist auch e ine $r$ davongekommen.

(1c) ?Auch/ferner/außerdem .... ist nicht e in e $r$ davongekommen (sondern

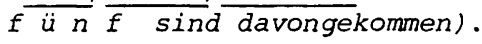


Die normale Interpretation von auch nicht in der Stellung unmittelbar vor einer kontrastiv betonten Konstituente ist aber: konjunktional verwendetes auch + lokale oder Kontrastnegation. Der Nachsatz in (1c) zeigt, daß dieser Satz nicht mit (1) bedeutungsgleich sein kann, behauptet doch dieser gerade, daß überhaupt keiner davongekommen ist:

(1d) ?Auch nicht e in e $r$ ist davongekommen.

(1d) hätte wohl die gleiche Bedeutung wie (1), doch ist er zumindest unüblich. Hingegen ist er mit nicht einmal akzeptabel (aber nicht mit sogar nicht):

(1e) Nicht einmal e ine $r$ ist davongekommen.

(1f) “Sogar nicht einer/ sogar e i n e r ist nicht davongekommen. Es kann sich also nur um eine idiomatische Wendung handeln, zumal auch die Hinzufügung eines Bestimmungsnomens nichts an der Lage ändert.

(8) Es sind auch nicht $f$ ün $f$ Rallyefahrer durchgekommen.

Zur semantischen Analyse

Hier sollen zunächst wieder Vorgänger- und Folgesätze den jeweiligen semantischen Bereich abstecken helfen.

(1g) Hans, Karl und Fritz hat es erwischt, auch nicht e in e $r$ ist davongekommen.

(3a) Die $100 \mathrm{~m}$ läuft er zwar jetzt nur in 12 Sekunden, aber die spult er auch in $e$ I f Sekunden ab / nicht nur in 11,5 Sekunden.

(6b) Der eine war ja immer mit den Rosen beschäftigt / nicht nur der eine hatte zu tun, auch der a $n d e r$ e war nie ganz unbeschäftigt / ?nicht nur der eine / von ersterem gar nicht zu reden.

Wie schon in 3.5.1.1. gezeigt, ist die Trennung von quantifizierender und skalierender Interpretation beim Skopustyp Zahlen schwieriger, da naturlich auch die quantifizierende Interpretation eine geordnete Skala verwenden mu, ohne sich allerdings auf diese Eigenschaft wirklich zu beziehen. Ein sehr sicheres Unterscheidungskriterium ist die Ersetzbarkeit von auch durch sogar bzw. nicht einmal, die hier mit Ausnahme vielleicht von (6) in allen Fällen gegeben ist. Die "natürliche" Skalenrichtung ist diejenige von kleineren zu größeren Zahlen, wie sie in (2), (5) und (7) vorliegt. - Viel mehr Schwierigkeiten bereiten die "verkehrten" Skalen, die bei auch (im Gegensatz zu nur) gar nicht so selten sind. Bei ihnen sind Kontrastsätze mit nicht nur nicht ganz akzeptabel, bzw. irreführend, weil man daraufhin gewöhnlich mit einer steigenden Skala rechnet. Als nicht eigentlich skalierend kann Satz (1) bezeichnet werden. In der Regel dürfte diese Konstruktion, übrigens auch bei Angabe einer höheren Anzahl, nur die Bedeutung überhaupt keiner haben:

(9) Der Knabe sitzt ja regelrecht auf seinem Geldsack. Auch nicht/nicht einmal $f \ddot{u} n f$ müde Märker hat er uns spendiert. 
Als Beispiele für quant. Interpretation werden folgende Sätze verwendet:

(10) Sie können das Magazin mit jeder beliebigen Anzahl von Patronen füllen, natürlich auch mit $f \ddot{u} n f z e h n$. Denn die Transportautomatik überspringt ja alle leeren Fächer.

- nicht nux mit 4, 6, 8 oder 10 Patronen, sondern auch mit fünfzehn.

(11) Die Zeitwahluhr kann auf jede beliebige Zeitspanne von 10 sekunden bis zu 2 Stunden eingestellt werden, auch auf 20 Minuten und 15 Sekunden, denn man ist nicht an gerade Minutenzahlen gebunden.

In beiden Fällen ist die Ersetzbarkeit durch sogar nicht auszuschließen, also auch nicht die skal. Interpretation. Wir besitzen aber ein syntaktisches Mittel zur Festlegung auf quant. Interpretation, nämlich Topikalisierung der Skopuskonstituente und kontrastive Betonung auf dem auch in Distanzstellung:

(10a) Mit $f \ddot{u} n f z$ e h $n$ Patronen können Sie das Magazin a $u c h$ laden. Dieser Sachverhalt erklärt auch die Inakzeptabilität dieser Konstruktion bei (1), (2) und (7): diese Sätze sind nämlich weitgehend auf skal. Interpretation festgelegt. Quant. Interpretation kann zwar durch nachgestelltes betontes auch erzwrungen werden, führt aber zu einem hohen Grad an Inakzeptabilität. -

Damit zu den Vorschlägen für eine semantische Analyse. Bei der skal. Interpretation ist die Formulierung einer konv. IMPL eine delikate Angelegenheit, da bei auch die feste obergrenze keinesfalls sicher aus der Formulierung folgt.

(3b) Die $100 \mathrm{~m}$ spult er auch in e $1 \mathrm{f}$ Sekunden $a b$.

ASS: Die $100 \mathrm{~m}$ spult er in elf Sekunden $a b$.

PSP: Die $100 \mathrm{~m}$ spult er in mehr Sekunden $a b$.

konv. IMPL: Die $100 \mathrm{~m}$ läuft er in nicht weniger als 11 Sekunden.

Nun noch ein Beispiel für quantifizierende Interpretation:

(10b) Mit $f \ddot{u} n f z$ e h $n$ Patronen können Sie das Magazin a uch laden. ASS: Mit 15 Patronen können Sie das Magazin laden.

PSP: Mit jeder anderen Anzahl Patronen können Sie das Magazin laden.

Und ein Beispiel fur polare Interpretation:

(6c) Auch der a $n$ de $r$ e war nicht ganz unbeschäftigt.

ASS: Der andere war nicht ganz unbeschäftigt.

PSP: Der eine/ein Zugehöriger war nicht ganz unbeschäftigt.

\section{Suspensionstests:}

(3c) Die $100 \mathrm{~m}$ spult er auch in e $1 \mathrm{f}$ Sekunden $a b$, - "und möglicherweise nicht einmal in 11 Sekunden. (skal. ASS)

- "und möglicherweise nicht einmal in 12 sekunden. (skal. PSP)

- und möglicherweise sogar in 10,5 Sekunden. (konv. IMPL)

(10c) Mit $f \ddot{u} n f z$ e h $n$ Patronen können Sie das Magazin a uch laden, - " und möglicherweise nicht einmal mit 15 patronen. (quant. ASS)

- "und möglicherweise mit keiner anderen Anzahl von Patronen. (quant. PSP)

- "und möglicherweise nicht einmal mit 14. (Imitation einer skal.PSP)

- "und möglicherweise sogar mit 16. (Imitation einer konv. IMPL) 
Die Ergebnisse bestätigen also voll die vorgeschlagene Analyse. Eine andere Akzeptabilitäts-Beurteilung wäre allerdings bei (10c) im Falle der Suspension einer "konv. IMPL" möglich. Akzeptabel wäre dies aber nur, wenn der Gültigkeitsbereich nicht definiert ist: von 2 bis 20 Patronen können Sie jede beliebige Anzahl Patronen in das Magazin einfüllen. Nach dieser Angabe des Gültigkeitsbereiches ist natürlich der Zusatz und möglicherweise sogar mit 16 indiskutabel. Aber auch im anderen Fall handelt es sich nicht um die Suspension einer konv. IMPL, sondern um die Bekundung der Unsicherheit über den Bereich:

(10d) Ich weiß nur, daß Sie das Magazin mit 5, 10 und 20 patronen füllen können. Ach ja, mit $f \ddot{u} n f z e h n$ können Sie es a $\mathrm{a}_{\mathrm{c} h}$ füllen, und möglicherweise sogar mit 16 oder 11, oder von mir aus auch mit einer einzigen. Das weiß ich einfach nicht so genau. Probieren sie halt mal!

Natürlich sind bei polarer Interpretation Suspensionen ausgeschlossen.

Die $100 \mathrm{~m}$ spult er auch in e I f Sekunden $a b$,

- ?und die $100 \mathrm{~m}$ spult er in elf Sekunden ab. (skal. ASS)

- "und die $100 \mathrm{~m}$ spult er in mehr Sekunden ab. (skal. PSP)

- "und die $100 \mathrm{~m}$ spult er nicht in weniger Sekunden ab. (konv. IMPL)

(3e) - ?Die $100 \mathrm{~m}$ spult er in elf Sekunden $a b$, (skal. ASS)

- Die $100 \mathrm{~m}$ spult er in mehr als elf Sekunden ab, (skal. PSP)

- "Die $100 \mathrm{~m}$ spult er in nicht weniger als 11 Sekunden $a b$, (konv.IMPL) und die $100 \mathrm{~m}$ spult er auch in e $1 \mathrm{f}$ Sekunden $a b$.

(10e) Mit $f \ddot{u} n f$ e h $n$ Patronen können Sie das Magazin a uch laden,

- ?und mit 15 Patronen können Sie das Magazin laden, (quant. ASS)

- "und mit jeder anderen Anzahl Patronen können Sie das Magazin laden. (quant. PSP)

(10f) - ?Mit 15 Patronen können Sie das Magazin laden, (quant. ASS)

- Mit jeder anderen Anzahl Patronen können Sie das Magazin laden, (quant. PSP)

und mit $f \ddot{u} n f z$ e $h$ Patronen können Sie das Magazin a $u c h$ laden.

Die Ergebnisse dieser Konjunktionstests entsprechen dem bei auch Üblichen; im Gegensatz zu nur ist also die Hinzufügung der ASS wenig oder gar nicht akzeptabel (wohl wegen der großen Ähnlichkeit der Formulierungen).

Dialogische Analyse:

(3f) A: Die $100 \mathrm{~m}$ spult Karl auch in e $1 \mathrm{f}$ Sekunden $a b$.

B: - Nein, die $100 \mathrm{~m}$ schafft Karl nie in dieser zeit. (skal. ASS)

- Ach wo, der schafft sie doch nicht einmal in 12 Sekunden, und dann redest du von elf. (skal. PSP)

- Irrtum, der schafft sie jetzt sogar in 10,5 sekunden. (konv. IMPL)

- 'Ich geb' ja gerne zu, daß er's in 11-Komma-Null schafft, aber. der läuft sie doch nicht in mehr Sekunden. (ASS-Zustimmung + PSP-Protest)

- Ja, in elf Sekunden schafft er es sicher noch. (skal. ASS)

- Na ja, mag ja sein, daß er es in mehr als 11 Sekunden runterkurbelt. (skal. PSP)

- Nein, in elf läuft er das nie, aber langsamer sicherlich. (ASS-Verneinung + PSP-Zustimmung) 
(10g) A: Mit $f \ddot{u} n f z$ e h n Patronen können Sie das Magazin a $u c h$ laden.

B: - Nein, diese Anzahl ist gerade ausgeschlossen. (quant. ASS)

- Aber das ist doch die einzig zugelassene Anzahl von Patronen! (quant. PSP)

- Mit fünfzehn schon, aber doch mit keiner anderen Anzahl!

(Zustimmung zur quant. ASS + Protest gegen quant. PSP)

- Mit fünfzehn nicht, und auch mit keiner anderen Anzahl. (ASS-Verneinung + PSP-Protest)

- Mit fünfzehn eben nicht, aber mit jeder anderen Anzahl. (ASS-Verneinung + PSP-Zustimmung)

- Ja, mit 15 kann man sie tatsächlich laden. (ASS quant.)

- Ja, und mit wieviel sonst noch? (PSP-Frage)

Dieser Test bestätigt einerseits meine Analyse, andererseits fügen sich seine Ergebnisse nahtlos in das bisher von auch Bekannte. Bemerkenswert nur, daß bei skalierender Interpretation die Kombination von ASS-Zustimmung und PSP-Verneinung zu Inakzeptabilität führt, bei quant. Interpretation hingegen nicht.

\subsubsection{3. sogar - Gruppe}

(1) Bis zum wirklichen Kriøg haben es die Deutschen denn doch nicht getrieben. Alle anderen geteilten Länder haben es getan: die Koreaner ebenso wie die Vietnamesen; die Inder sogar d $r$ e $i$ Mal.

(2) Hier ist die Landzunge nicht einmal $z$ we $i$ Kilometer breit.

(3) Bei den Abiturienten machen die Akademikerkinder sogar $z$ wa $n z i g$ Prozent aus.

(4) Unsere kleine Bundesrepublik hat jetzt sogar $s$ e $c h z i g$ Millionen Einwohner.

(5) Soweit ich weiß, kommt er sogar mit einer e in zigen Schreibhilfe aus.

(6) So ergeben die Recherchen, daß neuerdings nicht nur jeder vierte, sondern sogar jeder $d r i t t e$ Soziologe in eine feste Stelle vermittelt werden kann.

(7) Beim Tandemfahren trat der eine kräftig in die Pedale, und selbst der a $n d e r e$ mühte sich nach Kräften.

(8) Nicht einmal mit einer $d r i t t e n$ und $v i$ e $r$ e $n$ Schneekette kämen wir aus dem Schlamassel.

\section{Zur Syntax}

Hier gelten im Prinzip wieder die bereits bekannten Gesetze. Bekannt ist auch bereits, daß selbst wegen der möglichen Funktionswechsel praktisch nur satzeinleitend als GP möglich ist. - Die Position innerhalb einer NP, also nach dem Artikel bzw. der Präposition und vor dem kontrastiv betonten Zahlwort, ist für die Elemente der sogar - Gruppe nicht zulässig. Hingegen können sowohl sogar als auch nicht einmal nach ihrem Skopuszahlwort am Satzschluß stehen, wenn das Bezugsnomen fehlt: die Inder $d r e i m$ a $l$ sogar. 
Skopus und Fokus

Hier möchte ich nur auf 2 kleine Teilprobleme eingehen. Das eine steht im $\mathrm{Zu}-$ sammenhang mit dem Fragetest und betrifft die Erfragbarkeit von Sätzen, die sogar oder nicht einmal enthalten:

(4) Wieviele Einwohner hat die BRD denn? Sie hat 60 Millionen.

Als Antwort auf eine normale Wortfrage kommt also sogar nicht vor. Auch in der Antwort auf eine Echofrage mit sogar muß es nicht auftauchen. Sinnvoll ist es hingegen in der Antwort auf eine Satzfrage bestimmten Typs:

(4a) A: Hat die BRD eigentlich schon 50 Millionen Einwohner?

B: Sie hat sogar $s$ e $\mathrm{ch} z$ i g Millionen Einwohner.

Dies verdeutlicht sehr schön die skalierende Funktion von sogar, ist aber leider wertlos zur Ermittlung des Satzfokus.

Das zweite Problem ist die Akzentpositionierung in jenen Fällen, in denen mehrere Zahlwörter auf die GP folgen, wenn z.B. ein bestimter Bereich angegeben wird: sogar 13 - 30 Prozent. In diesem Ausdruck ist dreizehn zu betonen, wenn die zugehörige Kontrastphrase so lautet: nicht nur 10 - 30 Prozent. Die zweite Zahl ist kontrastiv zu betonen, wenn die Kontrastphrase nicht nur 13 - 20 Prozent lautet. Und schließlich sind beide Zahlen kontrastiv $z u$ betonen, wenn die Kontrastphrase nicht nur 10 - 12 Prozent oder aber auch nicht nur 18 - 25 Prozent lautet. Ob für die Kontrastierungsfähigkeit der Mittelwert oder die jeweiligen Einzelwerte oder die Spanne entscheidend sind, läßt sich nicht mit Sicherheit entscheiden. - Das gleiche Problem taucht auch bei zusammengesetzten Zahlwörtern und bei der Nennung der Bezugsmenge auf:

$$
\begin{aligned}
& \text {... sogar z wa } n z i g \text { Millionen, nicht nur } z \text { e h } n \text { Millionen. } \\
& \text {... } \\
& \text {... sogar zehn Millionen } E \text { in wo h } \mathrm{s} r \text {, nicht nur zehn Millionen } \\
& S \text { c } h \text { a f e. }
\end{aligned}
$$

Das Betonungsmuster bei zusammengesetzter Zahl hat keinen Einfluß auf die semantischen Eigenschaften und auf die Skopusabgrenzumg. Von einigem Interesse ist lediglich die Bereichsangabe, da sie eine andere Formalierung der ASS erzwingt; auf sie werde ich noch näher eingehen:

Zur semantischen Analyse

Als Einstieg dienen wieder einige Kontrastsätze:

(2a) Hier ist die Landzunge nicht nur nicht dre $i$, sondern nicht einmal $z$ we $i$ Kilometer breit.

(5a) Soweit ich weiß, kommt er nicht nur mit bloß $z w e i$, sondern sogar mit einer e inzigen Schreibkraft aus.

(7a) Nicht nur der e $i n e$, sondern sogar der a $n d e r e \ldots$ 
Alle Beispiele bis auf das polar-gerichtete (7) lassen natürlich nur eine skalierende Interpretation $\mathrm{zu}$, deren Gesetzmäßigkeiten bereits bekannt sind. Nur für einige nicht völlig klare Sätze wird noch ein Analysevorschlag geboten:

(2b) ASS: Die Landzunge ist hier nicht zwei $\mathrm{km}$ breit. PSP: Die Landzunge ist nicht mehr als zwei $\mathrm{km}$ breit.

konv. IMPL: Die Landzunge ist weniger als zwei Kilometer breit.

Die Formulierung der konv. IMPL kann nicht völlig befriedigen, wie der Suspensionstest zeigt:

(2c) Hier ist die Landzunge nicht einmal $z$ we $i$ Kilometer breit,

- "und möglicherweise ist sie hier sogar $2 \mathrm{~km}$ breit. (ASS)

- "und möglicherweise ist sie sogar breiter als $2 \mathrm{~km}$. (PSP)

- ?und möglicherweise ist sie nicht einmal 1,9 km breit.

- und möglicherweise ist sie sogar nur e i n e $n \quad k m$ breit.

Normalerweise besagt diese Formulierung, daß die Landzunge knapp unter $2 \mathrm{~km}$ breit ist. Eine genaue Grenze für die konv. IMPL läßt sich nicht angeben, doch muß eine vorhanden sein, weil sonst der folgende Satz normal sein miißte:

(2d) ... nicht einmal $z$ we $i \mathrm{~km}$ breit. Sie hat nämlich nur $100 \mathrm{~m}$ in der Breite.

Dies erfüllt den Tatbestand der Täuschung, wie er für die Verletzung einer konv. IMPL typisch ist. - Nun noch die Analyse für Satz (7):

(7b) ASS: Der andere mühte sich nach Kräften.

PSP: Der eine mühte sich nach Kräften.

konv. IMPL: --

Suspensionstest:

(7c) Selbst der a $n d$ e $r$ e mühte sich nach Kräften,

- *und möglicherweise nicht einmal der andere. (ASS)

- "und möglicherweise nicht einmal der eine. (PSP)

Dies zeigt dasselbe Bild wie beim Parallelbeispiel mit der GP auch, womit wohl bewiesen ist, daß die polare Interpretation relativ selbständig neben quant. und skal. Interpretation steht, denn selbst ist ungeeignet für quant. Gebrauch.

Es bedarf noch eines kurzen Hinweises auf die "Richtung" der Skalen. Für sogar und selbst gilt i.d.R. die normale Richtung der Zahlenreihe, für nicht einmal die umgekehrte Richtung. Während bei letzterem aber auch das Gegenteil möglich ist, stößt die Umkehrung der Skala bei sogar und selbst auf Schwierigkeiten, wie Satz (5) zeigt. In solchen Fällen wird zur Verdeutlichung gerne noch ein nur hinzugefügt:

(9) ?Es ist sogar e i n e $r$ davongekommen. Es ist sogar nur e i n e $r$ davongekommen.

Welchen Skopus dabei jede GP hat, kann vielleicht (9a) verdeutlichen:

(9a) Es ist sogar $s \circ$, daß nur e ine $r$ davongekommen ist. Die Problematik wird eingehend in Abschnitt 4.1.3. S. $264 \mathrm{ff}$. behandelt. 
3.5.2. Skopustypen mit den kontrastiv betonten Quantoren viel - wenig; viele - wenige - einige - alle.

3.5.2.1./2./3. nur -, auch -, sogar - Gruppe

Ich fasse alle GP-Gruppen zusammen, weil Belege fast nur für die nur - Gruppe zur Verfügung standen, und weil andererseits nach der Klärung der Grundstrukturen bei Quantoren im Skopus in 3.5.1. nur noch wenige Zusätze nötig sind.

(1) Der Gesetzesaufwand habe sich (auch dann) gelohnt, wenn nur we n i $g$ e $n$ das Leben gerettet werdis.

(2) Davon haben nur we nige Gebrauch gemacht.

(3) Auch/sogar/selbst we nige/vi e 1 e können davon Gebrauch machen, ohne daß wir dadurch aus dem Gleichgewicht geworfen werden.

(4) Von diesen Sprüchen möchte ich nur e in ig e wiedergeben.

(5) In diesem Falle können nur a 1 I e etwas erreichen.

(6) In diesem Superauto haben sogar/auch a 11 e platz.

(7) Nur/nicht einmal $v i$ e $l$ e können durch ihren Einsatz Verbesserungen bewirken.

(8) Zwei winter hindurch fiel in den Alpen nichts oder nur w e n $i g$ von diesem leichten lockeren Puderweiß.

(9) Über die Ursache des Absturzes ist nur we n ig bekannt.

(10) $\underline{\text { Auch/sogar }}$ we $n i g$ von dieser Medizin kann großen Schaden anrichten.

(11) Sogar/auch $v i$ e $I$ von dieser Suppe kannst du haben, wenn sie dir schmeckt.

Zur Syntax

Bei den Stellungsgesetzen gibt es eine fast vollständige Beschränkung auf den Stellungstyp 1, also GP umittelbar vor der Skopuskonstituente, die die oben genannten Elemente enthält. Typ 2, also Nachstellung der GP, sowie Typ 3, die Position der GP in der Konstituente, die die Quantoren enthält, verbunden mit einem Funktionswechsel bei diesen Skopustypen, ist ausschließlich bei der nur - Gruppe und auch da nur bei den Elementen nur und ev. bloß und ZedigZich möglich.

(12) Von nur/?bloß/? lediglich we nige $n$ Delegierten wurde der Entwurf des Vorstandes gutgeheißen.

Im Gegensatz zu allen anderen Skopustypen scheint auch Typ 5, also iP in Distanzstellung nach der topikalisierten Skopuskonstituente, nicht akzeptabel zu sein, oder zu nachhaltigen semantischen Veränderungen zu führen:

(2a) ?W e n i ge haben davon (nur) Gebrauch gemacht (nur).

(10a) ?W e $n$ i g von dieser Medizin kann (sogarl a uch) großen Schaden anrichten (sogar). 
Nicht ganz so scheint es bei nachgestelltem betontem auch zu sein:

(6a) A 11 e haben in diesem superauto a uch Platz.

Satz (6a) mag seltsam klingen, aber in bestimmten Situationen ist er ohne zweifel akzeptabel bzw. möglich:

(6b) A: Vier oder fünf können ohne weiteres in den wagen.

B: Wir sind aber sechs, und der Lothar fährt nicht.

A: $A I I$ e haben in diesem superauto a $u C h$ Platz.

Der Stellungs- und Betonungsumterschied bedingt einen deutlichen Funktionsunterschied.

Die verschiedenen Satztypen sind deswegen erwähnenswert, weil nur selten Funktionswechsel bzw. Funktionsambiguitäten auftreten; ibrigens gilt dies auch für selbst. Dies alles ist nur erklärbar mit einer besonders engen Verbindung zwi-schen diesem Skopustyp und der jeweiligen GP.

(4a) Gib nur e in $i g$ e von diesen Sprüchen wieder!

(13) Sag nur we nig!

(10b) Warum sollte auch we ni g von dieser Medizin großen Schaden machen?

(11) Du bekommst selbst $v i$ e 1 von dieser Suppe, wenn sie dir schmeckt.

Auch bei der Spaltsatzbildung sind gewisse Abweichungen von den üblichen Regeln zu konstatieren:

(1a) Es sind nur we nige, denen das Leben gerettet werden kann.

(4b) ?Es sind nur e i n $\mathrm{g} e$, die ich von diesen sprüchen wiedergeben will.

(4c) ?Es sind nur e in $i g$ e von diesen Sprüchen, die ich hier wiedergeben will.

(6c) Es sind sogar a $11 \mathrm{e}$, die in dem Superauto Platz haben.

Die gewisse Inakzeptabilität in (4b) kommt wohl nicht von der Abtrennung der Bezugsphrase, aber auch die Semantik von einige kann kaum die Ursache sein, da man das Gleiche bei wenig beobachten kann. Andererseits führt in Sätzen mit sogar (teilweise auch) die Spaltsatzbildung nicht zu jenem Grad an Inakzeptabilität, den man eigentlich erwarten würde.

Zur semantischen Analyse

Bei der Festlegung der Kontrastierungsbereiche sollen wieder Kontexte helfen:

(1b) Der Gesetzesaufwand habe sich (auch dann) gelohnt, wenn

- nur/bloß/lediglich $w \in n i g$ e $n$, nicht ?mehr/nicht vielen/ allen/"einigen das Leben gerettet werde.

- allein/einzig/ausschließlich/einzig und allein we $n$ i ge $n$, nicht keinem oder vielen oder allen das Leben gerettet werde.

Der Test erbringt wieder die Unterteilung in 2 GP-Gruppen und 2 Interpretationsweisen, die skalierende und die quantifizierende. Daß mit diesem Skopustyp skalierende bzw. polare Funktion möglich ist, dürfte nicht weiter umstritten 
sein. Das Problem bildet die quantifizierende Interpretation; in diesem Falle mïßte es ja eine definierbare Menge (mindestens 3) von Werten geben, die alternativ in derselben Situation möglich wären. Die Kontrastierung von einige und wenige ist inakzeptabel, auf der gleichen Ebene liegt mehrere. Die Kontrastierungsreihe lautet also: gar niemand/nichts - wenige/einige/mehrere - viele - alle. Die Versuche, daraus eine kontinuierliche Skala herzustellen (tür das Englische Horn:1972) halte ich aufgrund der Gebrauchseigenschaften dieser Elemente für verfehlt. - Es geht nun noch darum, das Verhalten der GPn gegenüber einem hohen sowie gegenüber den Extremwerten zu testen:

(2b) Davon haben nur/bloß/lediglich $v i$ e $l e$, nicht mehr/nicht wenige Gebrauch gemacht.

Davon haben allein/einzig/ausschließlich $v i$ e $l e$, nicht alle/ einige/gar niemand Gebrauch gemacht.

(2c) Davon haben nur/bloß/lediglich a 1 le, nicht weniger/nicht einige Gebrauch gemacht.

Davon haben allein/einzig/ausschließlich a $11 \mathrm{e}$, nicht viele/einige/ gar niemand Gebrauch gemacht.

(2d) "Davon hat nur/bloß/lediglich/allein/einzig/ausschließlich gar $k$ e i n e r Gebrauch gemacht.

Um die Art einer quantifizierenden Interpretation zu verdeutlichen bzw. wahrscheinlicher zu machen, konstruiere ich einen Dialog:

(14) A: Du weißt doch, daß man zwei wochen lang kostenlos die Stoßdämpfer prüfen lassen konnte. Da haben doch tatsächlich immer nur/ausschließlich an allen Tagen e $i \mathrm{n} i \mathrm{~g} e$ Gebrauch davon gemacht.

B: Du irrst dich wieder mal. Es waren ausschließlich/?immer nur $v i e-$ l e / gar ni e mand/" ${ }_{a} 1$ I e, die davon Gebrauch machten.

Die Version mit nur scheint hier zu mißverständlich, als daß sie üblich sein könnte. Die Version mit ausschließlich viele jedoch halte ich für voll akzeptabel. Das Beispiel zeigt weiter, daß bei dieser Quantifizierung die Kontrastierung mit alle/gar keiner von einige/wenige/mehrere aus wegfällt. Damit wird es wahrscheinlich, daß es sich um ein polares Paar handelt. - Zur Verdeutlichung sei ein weiteres Beispiel gegeben:

(15) (Situation: Lehrer mit Schulklasse vor einer Ausstellung. Ein Teil der Klasse will in die Ausstellung, ein anderer möchte lieber schwimmen gehen. Lehrer:)

Da können nur a $1 \mathrm{l}$ e (oder ga $r$ k $\mathrm{i} n$ e r) rein, ?nicht einige/ein paar...

Der Analysevorschlag für die quantifizierende Interpretation lautet also:

(15a) Da können nur a 11 e rein.

ASS: Da kann keine andere = geringere Anzahl rein.

PSP: Da können alle rein.

Und für die skalierende Interpretation lautet der Analysevorschlag folgendermaßen: 
(2e). Sie halten nur we nig e $n$.

PSP: Sie halfen wenigen.

ASS: Sie halfen nicht vielen/allen.

konv. IMPL: Sie halfen nicht gar niemandem.

Dies soll nun ummittelbar durch den Suspensionstest geprüft werden:

(15b) Da können nur a 11 e rein,

- "und möglicherweise können nicht einmal alle rein. (PSP quant.)

- "und möglicherweise können auch Teile der Gruppe rein. (quant. ASS)

(2f) Sie halfen nur we nige $n$,

- und möglicherweise halfen sie nicht einmal wenigen. (skal. PSP)

- "und möglicherweise halfen sie sogar vielen/allen. (skal. ASS)

- und möglicherweise halfen sie sogar überhaupt niemandem. (konv.IMPL)

Die skal. Interpretation wird also bestätigt. Allerdings dürfte sie bei nur auf wenige/einige beschränkt sein. Mehrere ist erstaunlicherweise in diesem Zusammenhang nicht möglich, es erzwingt offenbar eine andere "Richtung". Viele ist theoretisch möglich, kommt aber nur selten vor. Der oberste Wert ist von vornherein ausgeschlossen, für die Ausschließung des untersten Wertes weiß ich keine plausible Erklärung.

Dialogische Analyse:

A: Sie halfen nur we $n i g$ e $n$.

B: - Nein, das stimmt ja gar nicht. Sie haben vielen/allen geholfen. (skal. ASS)

- Aber sie haben doch gar nicht wenigen geholfen. (PSP)

- Da liegst du noch viel zu günstig mit deinen Vermutungen. Die haben nämlich gar niemandem geholfen. (konv. IMPL)

- Ja, ich gebe zu, sie haben tatsächlich nicht vielen/?allen geholfen. (ASS)

- Daß sie nicht gerade vielen geholfen hatten, konnte ich mir ja denken, aber nicht, daß sie immerhin einigen wenigen geholfen haben. (ASS-Zustimmung + PSP-Zustimmung)

- "Daß sie nicht gerade vielen geholfen haben, steht ja nun fest, aber es stimmt ganz einfach nicht, daß sie wenigen geholfen haben. (ASS-Zustimmung + PSP-Protest)

(15c) A: Da können nur a 1 l e rein.

B: - Aber nein, da kann doch auch ein reil reingehen. (ASS)

- Was redest du denn, da kann weder die Gesamtheit noch auch nur ein Teil rein: da darf überhaupt niemand rein. (ASS-Verneinung + PSP-Protest)

- Na ja, das mag schon sein, daß wir nicht in Schüben rein dürfen. (ASS)

- Ei freilich dürfen alle rein. (PSP)

- "Natürlich dürfen wir nicht in Teilen rein, aber alle dürfen wir auch nicht anmarschieren. (ASS-Zustimmung + PSP-Protest.

Die Tests bestätigen sowohl die skalierende als auch die quantifizierende Interpretation. - Die Elemente der beiden anderen GP-Gruppen weisen gegenüber dem bereits Bekannten keinerlei Besonderheiten auf. 
3.6. Skopustyp Adverbien

3.6.1. Skopustyp adverbiale Präpositionalphrase

3.6.1.1. nur - Gruppe

(1) Andere haben nur aus $V$ e $r s$ e h e $n$ mal etwas draufgedrückt.

(2) ... und so kennen viele Menschen den Kommunismus nur vom $H$ ö $r$ e $n$ $s$ a $g$ e $n$.

(3) Nur auf höchsten Lagen und Graten liegt noch der ersehnte Pulverschnee.

(4) Daß Legastheniker ins Kriminelle abgleiten, läßt sich nur durch rechtzeitiges Erkennen und eine pädagogische Behandlung vermeiden.

(5) Lediglich wegen dessen Mitgliedschaft in einer legalen Partei ... Die syntaktischen und semantischen Teilgruppen der adverbialen Präpositionalphrasen können in diesem Zusammenhang unberücksichtigt bleiben.

Zur Syntax

Die Stellungsgesetze entsprechen ganz dem bereits Bekannten. Beim überprüfen der Nachstellungsmöglichkeit für GPn stellt sich heraus, daß die Länge der Skopuskonstituente in direkter Verbindung mit der Akzeptabilität des Stellungstyps steht. Die Stellung der GP zwischen Präposition und NP ist nur dann möglich, wenn ein passendes kontrastiv betontes Adjektivattribut bzw. Numerale vorhanden ist. Die von Fraser (1971), Anderson (1972) und Schmerling (1972) für das Englische als häufig bezeichnete Stellung Subjekts-NP - GP - finites Verb, die dort zu vielfältigen Skopusambiguitäten führt, existiert im Deutschen nicht. - Beim Imperativ und bei der Wortfrage sind für nur und bloß wieder Funktionswechsel bzw. Funktionsambiguitäten zwischen GP und MP zu konstatieren; sie können jeweils durch Folgesätze verdeutlicht werden:

(5a) Entlasse ihn nur wegen seiner Mitgliedschaft in einer legalen Partei,

- nicht wegen irgendwelchen anderen Vergehen!

- du wirst schon sehen, wohin das führt!

- nicht wegen seiner $T \ddot{a} t i g k$ e $t$ in einer legalen Partei!

(5b) Warum entläßt du ihn nur wegen seiner Mitgliedschaft in einer legalen Partei,

- warum nicht auch aus anderen Gründen? (GP)

- das bleibt mir völlig unverständlich. (MP)

Je nach Funktion und Kontrastierung sind natürlich in den jeweiligen Basissätzen unterschiedliche Kontrastakzente notwendig. Bei der MP-Verwendung trifft der Satzakzent auf entlasse, bei der GP-Verwendung kann der Kontrastakzent auf Mitgliedschaft, legalen und Partei fallen. Trifft der Kontrastakzent auf das Adjektiv und wird die GP unmittelbar davor, also nach Präposition bzw. Artikel 
plaziert, so befindet sich naturlich nur das Adjektiv (paraphrasierbar als prädikatives Adjektiv eines restriktiven Relativsatzes) im Skopus der GP. Die Spaltsatzbildung erbringt erstaunlicherweise keine besseren Gebilde als bei Präpositionalobjekten:

(1a) Es ist nur (aus) $V$ e $r s$ e $h$ e $n$, woraus/weswegen/daß andere mal etwas draufgedrückt haben.

(1b) (Aus) $V$ e $r$ e $h$ e $n$ war es nur, weswegen andere mal etwas draufgedrückt haben.

Dies läßt sich nur so erklären, daß die Präposition in dieser Konstruktion stör Bei der Behandlung der Negationsmöglichkeiten müchte ich aus Anlaß eines passen den Beispiels auf die Verknüpfungen zwischen den verschiedenen Negationsformen hinweisen:

(6) Nur nicht aus Liebe weinen! (konjunktionale Verwendung?)

(6a) Nur aus Liebe nicht weinen!

(6a) kann als äquivalent mit (6) interpretiert werden, aber auch als GP mit dem Skopus Präpositionalphrase:

(6b) Aus jedem anderen Grunde, nur aus $L i$ e b e nicht weinen! Nur die GP-Version lassen jedoch die beiden folgenden, ebenfalls äquivalenten Sätze zu:

(6c) Nicht nur aus $L i$ e b e weinen! / Nicht aus $L i$ e b e nur weinen (es gibt noch so viele schöne Gründe für Tränen).

Und ich kenne noch eine weitere Version, die allerdings von der konjunktionalen Interpretation von nur sehr schwer zu trennen ist:

(6d) we ine nur nicht! / Nur nicht weinen! / Nur nicht aus Liebe weinen! In dieser Funktion ist nur durch doch, eben usw. ersetzbar, liegt also in der MP-Funktion vor.

Skopus und Fokus

Für die Folge GP - adverbiale Präpositionalphrase existieren sehr viel weniger Möglichkeiten der Akzent-Verteilung als bei Präpositionalobjekten. Da adverbiale Glieder relativ selten unmittelbar nach dem finiten Verb stehen, fällt dieses i.d.R. als Skopus der GP aus. Steht die GP aber zwischen zwei NPn, so wird sie gewöhnlich der folgenden NP zugeschlagen. - Die Kontrastierung ist bei allen adverbialen Präpositionalphrasen sehr viel einfacher als bei Präpositionalobjekten. Die Kontrastphrasen sind häufig so fest geworden, daß sie weggelassen werden können: nur aus Versehen - nicht absichtlich; nur vom Hörensagen nicht aus eigener Anschauung. - Eine Erwähnung verdient auch die Frage der Skopusabgrenzung bei durch und verbundenen Präpositionalphrasen:

(3a) Nur auf höchsten Lagen und Graten liegt noch ...

(3b) Nur auf höchsten Lagen und auf Graten liegt noch ... 
(3c) ?Nur auf höchsten Lagen und nur auf Graten liegt noch ...

(3d) "Nur auf höchsten Lagen und nur Graten liegt noch ...

(3a) ist nicht skopusambig, Kontrastierungsmöglichkeiten der einzelnen Elemente sind hierbei ohne Bedeutung; beide Präpositionalphrasen gehören trotz der beträchtlichen Verkürzung klar erkennbar zum Skopus von nur. Das ist bei (3b) nicht mehr der Fall: durch die bloße Wiederholung der Präposition wird die zweite Präpositionalphrase aus dem Skopus von nur ausgegliedert, ohne daß ich einen einleuchtenden Grund dafür angeben könnte. - Bei (3c) könnte man aus der Wiederholung des nur einen logischen widerspruch konstruieren: die eine Einzigkeitsbehauptung schließt die andere aus. Durch Zuordnung zu unterschiedlichen Mengen könnte dieser Widerspruch jedoch aufgelöst werden. - Wie (3d) zeigt, ist bei Wiederholung des nur jede Tilgung in der zweiten Präpositionalphrase ausgeschlossen. Dies könnte in zwei Richtungen gedeutet werden: einmal im Blick auf die "geschützte Umgebung" (protected environment), wie sie B. Fraser (1971) für den Skopus von GPn konstatiert; daciurch ist jede Tilgung, Extraposition usw. im Bereich des Skopus von GPn ausgeschlossen. Zum anderen durch den Hinweis darauf, daß offenkundig das nur eine unüberbrückbare Barriere für Aufzählungen darstellt. Da es jeweils eine Einheit aus einer bestimmten Menge ausgrenzt, können aus der gleichen Menge nicht noch weitere Einheiten genannt werden, sonst entstünde ein logischer widerspruch.

Zur semantischen Analyse

Die Mehrzahl der Sätze weist die normale quantifiziereıde oder skalierende Interpretation auf. Etwas anders verhält es sich mit den Sätzen (1) und (2), wie schon der Versuch, Kontrastsätze zu bilden, zeigt:

(1c) Andere haben nur aus $V$ e $r$ s e he $n$, nicht absichtlich, mal etwas draufgedrückt.

(2a) ... und so kennen viele Menschen den Kommunismus nur vom $H$ ö $r$ e ns a $g e n$, nicht wirklich/nicht aus eigener Anschauung.

Es ist also jeweils nur ein bestimmter Kontrastsatz möglich. Die Umkehrung des Tests mit nicht nur führt zum selben Ergebnis. Die Bedeutungsanalyse lautet:

(1) PSP: Andere haben aus Versehen mal etwas draufgedrückt. ASS: Andere haben nicht absichtlich mal etwas draufgedrückt.

(2) PSP: Viele Menschen kennen den Kommunismus vom Hörensagen. ASS: Viele Menschen kennen den Kommunismus nicht aus eigener Kenntnis. Suspensionstest:

(1d) Andere haben nur aus $V$ e $r s$ e h e $n$ mal etwas draufgedrückt, - "und möglicherweise nicht einmal aus diesem Grund. (PSP)

- "und möglicherweise sogar mit Absicht. (ASS) 
(2b) Viele Menschen kennen den Kommunismus nur vom $H$ ö $r$ e $n$ s a $g$ en, - ?und möglicherweise nicht einmal in dieser Art. (PSP)

- "und möglicherweise sogar aus eigener Anschauung. (ASS)

Das sind die üblichen Ergebnisse für polare Interpretation, abgesehen von der Suspension der PSP in (2b): sie führt nur deswegen nicht zu völliger Inakzeptabilität, weil als dritte Möglichkeit auch völlige Unkenntnis des Kommuismus nicht ausgeschlossen werden kann. -'Polar' nenne ich diese Interpretationsweise im Anschluß an Bartsch (1972: 196).

\subsubsection{2. auch - Gruppe}

(1) ... lebenslängliche Strafe ... Sie endet für die meisten Gefangenen auch ein Viertel jahrhundert nach Abschaffung der Todesstrafe noch immer tödlich.

(2) Und wie unter ihresgleichen erleben progressive Polizisten auch unter $B \ddot{u} r g$ e $r \mathrm{n}$ widerstand.

(3) Das alte Klassenkrankenhaus ... werde so in Rheinland-Pfalz auch für die $z$ u $k$ u $n \in t$ garantiert.

(4) Daß Jeannes Partner Paul heißt, das erfährt auch bei den unausweichlichen weiteren Begegnungen des Paares in der Leerwohnung nur das Publikum.

(5) ... im Juli 1970 seine Geheimdienste angewiesen, die angebliche Unterwanderung des Staates um jeden Preis zu stoppen - notfalls auch durch heimlichen Einstieg und Einbruch.

Die syntaktischen Eigenschaften von auch in diesem Skopustyp weichen nicht von dem bereits Bekannten ab. - Im Hinblick auf die Semantik ist Satz (5) ein selten schönes Beispiel dafür, daß auch nicht nur quantifizierend, sondern auch skalierend, und zwar synonym mit sogar verwendet werden kann. Dies zeigt der Suspensionstest recht deutlich:

(5a) ... im Juli 1970 seine Geheimdienste angewiesen, die angebliche Unterwanderung des Staates um jeden Preis zu stoppen - notfalls auch durch helmlichen Einstieg und Einbruch, und möglicherweise sogar durch Mord, wenn nicht sogar durch Vergewaltigung.

Der konstruierte Satz zeigt außerdem, falls er für völlig akzeptabel gehalten wird, daß die Stufen der Skala (hier nach Verwerflichkeit kriminellen Verhaltens) weitgehend frei mit Hilfe von sogar definiert werden können. - Der Vorzug von auch gegenuiber sogar besteht in den erweiterten Möglichkeiten der Interpretation; so ist auch bei (5) eine bloß quantifizierende Interpretation möglich. 
3.6.1.3. sogar - Gruppe

(1) ... die sich bereitgefunden hatten, sich in kollektive Schutzinstitutionen oder sogar $T$ a $r$ i f verträg e einzuordnen.

(2) ... statteten die Länder ihre Polizei mit "besonderen Waffen" aus, Handgranaten, Maschinengewehren und sogar Granatwe $r$ fer $n$.

(3) Selbst in $L$ a $t e i n$ a $e r i k$ a erreicht das Bruttosozialprodukt nur $1436 D M$.

(4) Es hat nicht etwa mit $H i t I$ e $r$ angefangen, nicht einmal mit Kaiser $w i l h e l \mathrm{~m}$, sondern sogar schon zur zeit $N$ a $p \circ 1$ e o n s.

Satz (1) und (2) zeigen, daß eine Verallgemeinerung des in 3.6.1.1. hinsichtlich der Tilgbarkeit von einzelnen Elementen im Skopus von nur Festgestellten auf den Gesamtbereich der GPn nicht möglich ist. In beiden Fällen fehlt die Präposition, ohne daß die Akzeptabilität der Sätze im Geringsten leiden würde. Das Faktum wird auch nicht durch Zweifel am Status der Skopusphrasen als adverbialen Präpositionalphrasen ausgeräumt. Eindeutige Umformungen zeigen keine anderen Eigenschaften. Es kann also nur an den einzelnen GPn liegen. - Alle Sätze sind übrigens gute Beispiele für die skalierende Funktion von sogar: und zwar dadurch, daß sie nacheinander mehrere Stufen der Skala aufzählen und die höchste jeweils vorhandene mit sogar kennzeichnen.

3.6.2. Exkurs zu Skopustypen mit kontrastiv betonten Präpositionen

Für diesen Skopustyp brauchbare Beispiele zu finden ist aus naheliegenden Gründen sehr schwer. Daher mußten überdurchschnittlich viele "konstruierte" Sätze eingefügt werden. Es besteht also Anlaß, alle Aussagen dieses Abschnitts mit Vorsicht zu betrachten. - Da bei den hier interessierenden Fragen keine Differenzen zwischen den drei GP-Gruppen auftreten, werden sie zusammengefaßt.

3.6.2.1./2./3. nur-, auch-, sogar-Gruppe

1. Präpositionen in adverbialen Präpositionalphrasen:

(1) Nur/auch na $c h$ unserer Scheidung rief ich meine Frau öfter an.

(2) Traudl schwimmt nur/sogar oh $n e$, nicht (nur) mit (Badeanzug).

(3) Nur üb e $r$, nicht un $t$ e $r$ Tag sind derartige Technologien derzeit verwendbar.

2. Präpositionen in einem Präpositionalobjekt:

(4) Er pocht bloß a uf sein Recht, nicht d a gege $n$.

(5) Er denkt nur a $n$ seine Freundin, er denkt nicht über sie $n a c h$.

3. Präpositionen in Funktionsverbgefügen:

Das Parlament setzt Gesetze nur in Kraft, nicht a u $\beta$ e $r$ Kraft. 
4. Trennbare Verbvorsilben:

(7) ?Die Strafe ist unverständlich, denn ich baute bloß a u $f$, aber nicht $a b$.

(8) Ex wollte sich nux $\ddot{u} b$ e $r$-, nicht un te $x$ ordnen.

(9) Die strafe ist unverständlich, da ich nur a uf -, nicht aber a $b$ bauen wollte.

5. Nicht trennbare Verbvorsilben:

'Sie wollte diesen text nur ü b e r setzen, nicht...

\section{Zur Syntax}

Bei den Stellungsgesetzen ist erwähnenswert, daß Typ 1, also GP unmittelbar vor dem Skopuselement, in den akzeptablen Beispielen ausschließlich erlaubt ist. Jede Form von Nachstellung ist verboten, insbesondere dann, wenn die GP dabei die Grenzen zwischen den Satzgliedern verläßt. Natürlich ist auch die Topikalisierung der Skopuskonstituenten (und die daraus folgende Distanzstellung der GP) unmöglich, abgesehen von der Topikalisierung eines Verbs mit betonter trennbarer Vorsilbe. - Das Clefting, obwohl sehr mühevoll, bringt einige wichtige Hinweise für die richtige Analyse der zur Debatte stehenden Fälle:

(1a) "Es war nur $n$ a $c h$, daß ich meine Frau unserer Scheidung öfter anrief.

(1b) Es war nur $n$ a $c h$ unserer Scheidung, daß ich meine Frau öfter anrief.

(2a) ?Es ist nur o h ne Badeanzug, wie Traudl schwimmt.

Abgesehen von der üblichen Ungebräuchlichkeit solcher Formen zeigt sich an den Sätzen doch einwandfrei, daß jeweils die gesamte adverbiale Präpositionalphrase im Skopus der GP liegt, nicht nur die Präposition. Die kontrastive Betonung der Präposition betrifft nur die innere Struktur der Präpositionalphrase. Noch deutlicher ist das bei den trennbaren verbvorsilben.

Fokus und skopus

Ich beschränke mich hier auf die Bedingungen der Kontrastierbarkeit von Präpositionen. Man muß davon ausgehen, daß in den meisten Beispielen dieser Art ein expliziter Kontrastsatz fehlt. Dies liegt einmal daran, daß alle Elemente des Kontrastsatzes bis auf die Präposition mit dem kontrastierten Satz identisch sind, zum anderen daran, daß es zu jeder Präposition, falls überhaupt, nur eine mögliche Kontrastpräposition, in der Art polarer Paare geordnet, gibt: vor nach; über - unter; in - auf; wegen - trotz usw. Gewisse Probleme kann es mit den Präpositionen-Paaren ohne - mit, gegen - mit geben: da sie jeweils unterschiedliche Kasusrektion aufweisen, dürfen bei expliziten Kontrastsätzen in den Satzpaaren keine Tilgungen vorgenommen werden.

Trotz der grundsätzlichen Möglichkeit zu solchem Gebrauch wirken alle derartigen Beispiele etwas gekünstelt; metasprachlicher Gebrauch läßt sich nicht immer ganz ausschließen bei diesem künstlich genug anmutenden Betonungsmuster. Damit besitzt man aber auch die Mittel für die Begründung der Inakzeptabilität bei den Untergruppen: 2. Präpositionalobjekt. 3. Funktionsverbgefüge. 5. Nicht trennbare Verbvorsilben. Da in allen diesen Fällen keinerlei semantische Differenz zwischen den einzelnen Präpositionen festzustellen ist (sie stellen ja reine Markierungszeichen für Kasus-, Argumentrelationen dar), sind auch keine semantisch fundierten Kontrastierungen möglich. Aus dieser Tatsache leitet sich auch die Unbetonbarkeit dieser Elemente her. Man besäße damit ein leicht merkbares Trennungskriterium, wenn nicht auch bei den "echten" Präpositionen die Kontrastierungsmöglichkeiten so beschränkt wären. - Die Möglichkeit einer rein formalen Kontrastierung wird damit aber keineswegs bestritten. 
3.6.3. Skopustyp Adjektiv-Adverbien und reine Adverbien

3.6.3.1. nur - Gruppe

(1) Nur $m \ddot{u} h s$ a $m$ konnte Christopher G. Janus seine Erregung verbergen.

(2) Mein Mann wurde von mir in dieser Funktion nur $r \circ u t i n e m a ̈ \beta i g$ bestätigt.

(3) Unterschiede sind nur $s c h w$ e $r$ feststellbar.

(4) ... in der Industrie, die bisher nur z nachwuchs einstellt.

(5) Die direkt abgeleiteten kommunalen Abwässer werden in der Regel nicht oder nur ma $\mathrm{n} g \mathrm{e} l \mathrm{~h}$ a $f t$ geklärt.

(6) Doch derlei Einbrüche geschehen lediglich sch ub we is e.

(7) Die Einsätze dauerten nur $s$ e 1 t e $n$ eine volle Stunde.

(8) Im Zentralhospital schaltet sich das Notaggregat ein. Nur $s$ o können die Ärzte eine Gehirnoperation beenden.

(9) Er war nur $h i$ e $r$ - und nicht $d \circ r t$ / seine Bücher hat er aber nicht mitgenommen.

Zur Syntax

Bei den Stellungsgesetzen ergeben sich geringfügige Abweichungen:

Typ 1, also die Voranstellung der GP, ist wieder die Normalstellung. Aber bei diesem Skopustyp ist auch Stellungstyp 2, die Nachstellung der GP, völlig normal; sie scheint hier außer der deutlichen Emphase und der Bevorzugung der Stellung am Satzanfang keine weitere stilistische Wirkung zu haben. Gerade derartige "Ausnahmen" dürften eine generelle Formulierung von Stellungsgesetzen für GPn äußerst schwierig machen. Natürlich könnte es sich bei den Beschränkungen der Nachstellung um reine Performanzerscheinungen handeln, da die Möglichkeit offenbar mit steigender Länge der Skopuskonstituente abnimmt. Das würde aber immer noch nicht erklären, warm Nachstellung bei nur (jedenfalls in einer Reihe von Fällen) möglich ist, bei den übrigen GPn aber kaum einmal. - Belege für Typ 4 (Distanzstellung) konnte ich nicht finden. Ausgesprochen kompliziert ist aber die Lage bei Typ 5 (Topikalisierung der Skopuskonstituente, GP in Distanzstellung danach). Während einige Beispiele dadurch völlig inakzeptabel werden, sind andere nur etwas ungewöhnlich, und wieder andere völlig normal:

(3a) ?S $c h$ w e $r$ sind Unterschiede nur feststellbar.

(1a) ?M üh 5 a $m$ konnte Chr. G. Janus seine Erregung nur verbergen.

(7a) is e $l$ t e $n$ dauerten die Einsätze (nur) eine volle Stunde (nur).

(8a) “s o können die Ärte eine Gehirnoperation (nur) beenden (nur). 
Natürlich können diese Sätze meist "gerettet" werden, wenn man dem nur einen anderen, nahegelegenen Skopus zuweist. - Auffällig ist auch, daß dieser Stellungstyp bei den eigentlichen reinen Adverbien offenbar immer (ohne Bedeutungsänderung gegenüber der Normalstellung) möglich ist, bei den Pronominaladverbien aber offenbar nicht:

(9a) $H$ i e $r$ war er nur.

(10) Nur d a mals war er glücklich. $/{ }^{\circ}$ a $m$ a $I s$ war er nur glücklich. Bei den Adjektivadverbien nützt auch die Zuordnung des Verbums nichts:

(1b) ?M üh $s$ a $m$ verbergen konnte Chr. G. Janus seine Erregung nur. Eine hallowegs einleuchtende Erklärung für dieses Verhalten kann ich nicht geben. Irgendwie scheint es aber mit dem Verbot der Abspaltung zusammenzuhängen, und dies wiederum deutet auf eine Kollision mit skalierender Interpretation:

(1c) "Es ist nur $m \ddot{u} h s$ a $m / m \ddot{u} h s$ a $m$ war es nur, daß Chr. G. Janus seine Erregung verbarg.

Dies wird auch dadurch nicht besser, daß man weitere Glieder dem Spaltsatz zuschlägt:

(1d) 'Es war nur mühsam seine Erregung verbergen; was Chr. G. Janus tat. Bei dieser Konstruktion muß natürlich ein anderes Akzentmuster verwendet werden. Dies zej.gt, daß die Schwierigkeiten jedenfalls nicht von einer falschen Skopusabgrenzung verursacht wurden.

Negationsmöglichkeiten:

Typ 1: NEG + GP. Erstaumlicherweise ist er in der Mehrzahl der Fälle nicht möglich:

(1e) “Nicht nur $m$ ühs a $m$, sondern schier überhaupt nicht konnte Chr. G. Janus seine Erregung verbergen.

(3b) ?Unterschiede sind nicht nur $s c h$ we $r$, sondern überhaupt nicht feststellbar.

(7b) "Die Einsätze dauerten nicht nur $s$ e $l$ t e $n$, sondern überhaupt niel sondern sehr oft eine volle stunde.

(9b) Er war nicht nur $h i e r$, sondern auch $d \circ r t$.

Daraus ist zu schließen, daß in den meisten Fällen eine echte Kontrastierungsmöglichkeit fehlt. - Typ 3, die Satznegation, ist natürlich möglich, wenn sie auch nicht in allen Fällen (insbesondere bei den Adjektivadverbien) zu akzeptablen Ergebnissen führt.

Skopus und Fokus

Von Akzent und Stellung der GP her ist die Abgrenzung des Skopus relativ klar. Überall dort, wo Kontrastsätze möglich sind, kann diese Abgrenzung auch semantisch untermauert werden. - Der Fragesatztest kann naturlich auch auf diesen Skopustyp nicht so abgestimmt werden, daß er zur Fokusabgrenzung etwas bei- 
tragen wïrde. Auf die Frage:

(5a) Wie werden denn die Abwässer geklärt?

ist nur mange lhaft eine mögliche, aber keinesfalls eine nötige Antwort. Notwendig ist nur bei Adjektivadverbien ausschließlich auf eine Frage wie:

(5b) Werden die Abwässer vollständig gereinigt? - Nein, sie werden nur ma $n g$ e $I$ ha $f t$ geklärt.

Bei den reinen Adverbien scheint es die Einzigkeits-PSP und die auf ihr beruhende Frage-Antwort-Kombination zu geben:

(9c) Wo war er denn überall? - Er war nur hi e $x$.

Zur semantischen Analyse

Sie wird wieder mit Kontrastsätzen eingeleitet:

(1f) - nur $m \ddot{u} h s$ a $m$, nicht mit Leichtigkeit. (?)

(2a) - nur $r \circ$ u $t$ i n e mäßig, nicht auf besondere Veranlassung.

(3c) - nur schwer, nicht leicht.

(4a) - nur $z$ ög e $r n d$, nicht zügig/rasch.

(5c) - nur mange 1 haf $t$, nicht gut/sehr gut.

(6a) - lediglich schub we ise, nicht kontinuierlich.

Wenn man die sehr begrenzten Möglichkeiten der Kontrastierung (auch der mit nicht nur, s.o.!) verknüpft mit der Feststellung, daß in praktisch keinem Fall die rein quantifizierenden GPn dieser Gruppe verwendbar sind, so muß man zu dem Schluß kommen, daß in den meisten Fällen polare Interpretation vorliegt, in (5) hingegen skalierende Interpretation. - Nun noch zu den Kontrastierungsmöglichkeiten für die reinen Adverbien:

(7c) - nur $s$ e 1 te $n$, nicht oft.

(8b) - nur $s o$, nicht anders.

(9d) - nur $h i$ e $r$, nirgendwo sonst.

Hier ist (7) offenbar polar zu interpretieren, (8) und (9) hingegen quantifizierend, wie die Einsetzbarkeit von ausschließlich, einzig und allein etc. nahelegt. - Die Analysevorschläge missen also folgendermaßen lauten:

Für polare Interpretation:

(3d) PSP: Unterschiede sind schwer feststellbar. ASS: Unterschiede sind nicht leicht feststellbar.

Für skalierende Interpretation:

(5d) Die kommunalen Abwässer werden nur $m$ a $n g e l$ h a $f t$ geklärt. PSP: Die kommunalen Abwässer werden mangelhaft geklärt. ASS: Die kommunalen Abwässer werden nicht besser als mangelhaft geklärt. konv. IMPL: Die kommunalen Abwässer werden nicht schlechter als mangelhaft geklärt. 
Für quantifizierende Interpretation:

(9e) PSP : Er war hier.

ASS: Er war nirgendwo sonst.

Suspensionstest:

(3e) Unterschiede sind nur $s c h w$ e $r$ feststellbar,

- "und möglicherweise sind sie nicht einmal schwer feststellbar. (polare PSP)

- "und möglicherweise sind sie sogar leicht feststellbar. (polare ASS)

- und möglicherweise sind sie überhaupt nicht feststellbar. (konv. IMPL, also auch skalierende Interpretation möglich).

(5e) Die kommunalen Abwässer werden nur $m a n g e l h$ a $f t$ geklärt,

- und möglicherweise werden sie nicht einmal mangelhaft geklärt. (skal. PSP)

- "und möglicherweise werden sie sogar befriedigend/gut/sehr gut geklärt. (skal. ASS)

- und möglicherweise werden sie sogar ganz ungenügend/überhaupt nicht geklärt. (konv. IMPL)

(9f) Er war nur $h$ i e $r$,

- "und möglicherweise war er nicht hier. (quant. PSP)

- 'und möglicherweise war er sogar irgendwo sonst. (quant. ASS)

Irregulär ist bei diesen Tests nur die inakzeptable PSP-Suspension von (3e). Insofern unterscheidet sich polare Interpretation deutlich von skal. Interpretation.

Konjunktionstest:

(3f) Unterschiede sind nur $s c h w$ e $r$ feststellbar,

- “und Unterschiede sind schwer feststellbar. (polare PSP)

- ?und Unterschiede sind nicht leicht feststellbar. (polare ASS)

- "und Unterschiede sind nicht überhaupt nicht feststellbar. (konv. IMPL)

(5f) Die kommunalen Abwässer werden nur $m a n g e l h$ a $f t$ geklärt,

- “und die kommunalen Abwässer werden mangelhaft geklärt. (skal.PSP)

- und die kommunalen Abwässer werden nicht gut geklärt. (skal. ASS)

- ‘und die kommunalen Abwässer werden nicht ungenügend geklärt.

(konv. IMPL, mit aber akzeptabel)

(9g) Er war nur hi e $r$,

- “und er war hier. (quant. PSP)

- und er war nicht dort/nirgendwo sonst. (quant. ASS)

Und die Umkehrung des Konjunktionstests:

- ?Unterschiede sind schwer feststellbar, (polare PSP)

- Unterschiede sind nicht leicht feststellbar, (polare ASS)

- ?Unterschiede sind nicht überhaupt nicht feststellbar, (konv. IMPL)

und Unterschiede sind nur $s c h w$ e $r$ feststellbar.

- ?Die kommunalen Abwässer werden mangelhaft geklärt, (skal. PSP)

- Die kommunalen Abwässer werden nicht gut geklärt, (skal. ASS)

- ?Die kommunalen Abwässer werden nicht ungenügend geklärt, (konv. IMPL)

und die kommunalen Abwässer werden nur $m a n g e l h$ a $f t$ geklärt. 
(9h) Er war hier (PSP), und er war $\frac{n u r}{r}$ hier.

Er war nicht dort (ASS), und er war nur $h i$ e $r$.

Das ist ein völlig reguläres Ergebnis. Es empfehlen sich aber noch einige Hinweise. - Die Möglichkeit, eine explizite Formulierung der ASS anzuschließen, ist eine Besonderheit von nur - Sätzen, ermöglicht durch die starken Unterschiede zwischen dem wortlaut des nur - Satzes und der expliziten Formulierung der ASS. Außerdem bedeutet eine explizite ASS-Formulierung meist gegenüber der ASS des nur - Satzes eine Präzisierung, Einschränkung. - Die Wiederholung der konv. IMPL ist nur dann akzeptabel, wenn sie mit aber angeschlossen wird. Dies liegt daran, daß das und den folgenden Ausdruck nicht als Abgrenzung gegen eine Fehlinterpretation charakterisiert, wie es das aber tut: als Abgrenzung gegen die Ansicht, das nur wïrde bereits die oberste Stufe der betreffenden Skala markieren, und als Abgrenzung gegen eine quantifizierende Interpretation des nur. - Die Voranstellung einer expliziten PSP-Formulierung ist bei skalierendem Gebrauch des nur deswegen unbefriedigend bzw. völlig inakzeptabel, weil die daraufhin allein mögliche kontrastive Betonung des nur eine quantifizierende Interpretation via Einzigkeitsbehauptung zur Folge hat. Die Voranstellung einer expliziten ASS-Formulierung ist voll akzeptabel, wiederum wegen der Verschiedenheit der beiden Formulierungen, und wegen der gegenseitigen Präzisierung der beiden Aussagen: nicht gut läßt nämlich die Möglichkeiten befriedigend, ausreichend, mangelhaft, ungenügend, überhaupt nicht offen, erst durch nur mangelhaft ist der Bereich klar abgegrenzt. Bei einer Bereichsformulierung hingegen ergeben sich Schwierigkeiten:

(5h) ?Die kommunalen Abwässer werden nicht besser als mangelhaft geklärt, und die kommunalen Abwässer werden nur $m a n g e l$ a $f t$ geklärt. Kontrastive Betonung des nur verbessert die Situation nicht, da hierdurch nur eine Einzigkeitsbehauptung entsteht, die im Widerspruch zur skalierenden Interpretation steht. - Die Voranstellung einer expliziten Formulierung der konv. IMPL ist deswegen unbefriedigend bzw. inakzeptabel, weil in dieser Form eine umgekehrte Skala induziert wird:

(5i) Die kommunalen Abwässer werden nicht ungenügend, sondern mangelhaft geklärt, und möglicherweise sogar ausreichend.

Hingegen wäre eine andere Formulierung durchaus akzeptabel:

(5k) Die kommunalen Abwässer werden zwar nicht ungenügend, aber doch nur ma $n g e l$ h a $f t$ geklärt.

Diese Fonmulierung stört nicht die durch das nur induzierte Skalenrichtung. All das gilt auch für die quantifizierende Interpretation von (9). Erschwerend komm in diesem Fall hinzu, daß die explizite ASS-Formulierung i.d.R. nicht einen Bereich deckt, sondern einen ganz bestimmten wert behauptet. 
Dialogische Analyse:

(51) A: Die kommunalen Abwässer werden nur $m a n g$ e $\mathrm{l}$ a $f t$ geklärt.

B: - Nein, die Abwässer werden ausreichend/befriedigend/gut .../werden besser als mangelhaft (?) geklärt. (ASS)

- Das stimmt doch gar nicht, daß die mangelhaft geklärt werden. (PSP-Protest)

- Sie werden sogar nur ungenügend/überhaupt nicht geklärt. (konv. IMPL)

- "Es stimmt, daß sie nicht besser als mangelhaft geklärt werden (nicht gut/befriedigend ...) (ASS-Zustimmung), aber sie werden doch nicht mangelhaft geklärt. (PSP-Protest)

- Ja, also, gut werden sie wirklich nicht geklärt. (ASS)

- Ich weiß, daß sie mangelhaft geklärt werden. (PSP)

- Na ja, wenigstens nicht schlechter als mangelhaft/immerhin werden sie geklärt, wenn auch schlecht. (konv. IMPL)

- "Die Voraussetzung, daß sie mangelhaft geklärt werden, (PSP), stimmt zwar, aber sie werden auch besser geklärt. (ASS-Verneinung)

- Ich finde das gar nicht so schlimm/das ist doch gar nicht so schlecht! (Skala selbst)

Erwähnenswert ist daran die Inakzeptabilität der Kombination von PSP-Zustimmung und ASS-Verneinung. Sie scheint spezifisch zu sein für skalierende Interpretation, allerdings nur bei Skalen, deren Werte sich gegenseitig ausschließen. Die Inakzeptabilität hat also nichts mit dem Status der kombinierten Äußerungen zu tum, sondern mit der Tatsache, daß durch die Verneinung der ASS ein oder mehrere Skalenwerte behauptet werden, und daß durch die Zustimmung zur PSP ein weiterer Skalenwert behauptet wird, wodurch ein logischer Widerspruch entsteht. Firr eine quantifizierende Interpretation ist diese Kombination durchaus akzeptabel, wie der nächste Abschnitt zeigen wird; hier handelt es sich lediglich um eine Ausweitung des Bereiches. Gegen diese Erklärung scheinen zunächst skalierende Bereichsabgrenzungen zu sprechen:

(5m) Die kommunalen Abwässer werden nur mangelhaft bis ungenügend geklärt, und möglicherweise sogar überhaupt nicht.

Die Suspensionsformel beweist durch ihre Akzeptabilität, daß es sich durchaus noch um eine skalierende Äußerung handelt. Der Versuch, die gültigen Skalenwerte zu vermehren, scheitert auch hier:

(5n) "Die kommunalen Abwässer werden nur mangelhaft bis ungenügend geklärt, aber sie werden auch befriedigend geklärt.

Eine kurze Bemerkung auch noch zur dialogischen Ablehnung der Skala; sie betrifft eigentlich nicht die Skala, sondern nicht notwendig mit dieser verbundene Sprecher-bzw. Hörereinstellungen. So kann A darauf antworten: Das habe ich ja auch nicht behauptet. Ich stelle lediglich fest, daß sie keinen höheren Wert erreicht. 
3.6.3.2. auch - Gruppe

(1) Er produziert seine Sachen nicht nur geschwind und sauber, ex produziert auch b $i$ l 1 i $g$.

(2) Die Kiste ist keinen roten Heller wert. Sie läuft unruhig, unsicher und auch $I$ a $u t$.

(3) ... Genau. Wobei man auch $h$ i e $r$ noch eines hinzufügen muß ...

(4) Diese Regierung wird sich auch we $i$ te $r h i n$ bemühen um verständnisvolle Partnerschaft mit den Kirchen.

(5) Wie schon in den beiden wintern zuvor, herrscht auch $h$ e u e $r$ allerorten Schnee- und Kältemangel.

\section{Zur Syntax}

Im Gegensatz zu den Verhältnissen bei der nur - Gruppe sind bei auch die Stellungsgesetze ganz regulär, auch beim Stellungstyp 5 (topikalisierte Skopuskonstituente mit GP in Distanzstellung):

(1a) B I I I g produziert er a $\underline{\mathrm{a} \mathrm{Ch}}$.

(5a) He ue $r$ herrscht a $\underline{a c h}$ allerorten Schnee- und Kältemangel.

Damit bietet sich für den Ausfall dieser Stellungsmöglichkeit bei der nur Gruppe (beim Skopustyp Adjektivadverb) eine Erklärungsmöglichkeit: er betrifft ausschließlich Fälle von skalierender Interpretation. Dies würde gleichzeitig die Unmöglichkeit des Spaltsatzes in diesen Fällen erklären: offenbar wird in beiden Fällen die Einzigkeit behauptet, und dies verträgt sich nicht mit der skalierenden Interpretation. -

Die einzige mögliche Negationsform ist auch nicht. Diese Kombination kann bei Topikalisierung der Skopuskonstituente auch getrennt werden:

(1b) $\ldots$ und er produziert auch nicht bi 11 i $g$.

(1c) $\ldots$ und auch bi 1 I ig produziert er nicht.

(1d) ... und bi I I g produziert ex $\underline{a c h}$ nicht.

Skopus und Fokus

Sätze mit auch sind die natürlichen Antworten auf Fragen, die nur enthalten:

(4a) A: Bemühte sich die Regierung nur b i s h e $r$ um vertrauensvolle Partnerschaft mit den Kirchen?

B: Nein, sie wird sich auch $z u k$ ü $n f t i g$ darum bemühen.

Die Semantik entspricht bei diesem Skopustyp völlig den üblichen quant. Analysen bei auch. Lediglich die Kombination von PSP-Protest und ASS-Zustimmung bei den dialogischen Tests interessiert:

(2a) Ja, er produziert billig, aber er produziert auf sonst keine weise/ aber er produziert nicht schnell und sauber.

Dies bestätigt die in 3.6.3.1. getroffenen Feststellungen. 
3.6.3.3. sogar - Gruppe

(1) Ich betreibe das schmutzige Geschäft sogar (sehr) a $n s t$ ä $n d i g$.

(2) Unseren Kindern bekommen unsere Schulen nicht so recht. Ja, sie bekommen ihnen sogar $s c h l e c h t e r$, als sie es ahnen.

(3) Er arbeitet langsam, und dabei nicht einmal s a u b e $r$.

(4) Die hochgiftigen Industrieabwässer werden in diesem werk sogar $g u t$ bis $s$ e hrg gut geklärt.

(5) Sogar hi e $r$ plagte uns die Sorge um unseren Arbeitsplatz.

(6) Sogar un $t$ e $n$, an der Basis, hat er ordnung geschaffen.

Zur Syntax

Bei diesem Skopustyp gibt es erstaunlicherweise eine akzeptable Konstruktion mit nachgestelltem sogar:

(2a) Die Schule bekommt den Kindern schlecht, schl e cht e $r$ sogar, als sie es ahnen.

Hierbei kann man allerdings eine Ausdehnung des Skopus auf den Vergleichssatz nicht von vornherein vermeinen. - Die Nachstellung von sogar in Distanz nach topikalisiertem Skopuselement ist ohne Einschränkung möglich, nicht jedoch die von selbst (wegen des Funktionswechsels zum Reflexivum), und wohl auch nicht die von nicht einmal. Bei letzterem könnte es semantische Gründe geben: die Topikalisierung eines Elementes bedingt offenbar seine nachdrückliche Behauptung (wenn auch vielleicht nicht, wie bei der Spaltsatzbildung, Einzigkeits-Behauptung), die natürlich in unauflöslichem Widerspruch zur Satznegation steht. Deshalb gilt dieses Verbot auch für nicht nur, auch nicht usw. Hingegen nicht für a $u c h$ nicht, da diesem in der Regel schon eine negative Behauptung vorangeht.

Zur semantischen Analyse

Kontrastsätze mit nicht nur sind bei den obigen Belegsätzen (abgesehen vielleicht von (4)) nur schwer zu bilden, da kaum einmal. eine quasilexikalisierte Skala vorliegt. Aber die skalierende Funktion dieser Gruppe steht außer Frage. Die Analyse wird deshalb auf Satz (4) beschränkt.

(4a) Die Industrieabwässer werden sogar gut geklärt.

ASS: Die Industrieabwässer werden gut geklärt.

PSP: Die Industrieabwässer werden nicht nur befriedigend geklärt. konv. IMPL: Die Industrieabwässer werden nicht sehr gut geklärt.

\section{Suspensionstest:}

(4b) Die Industrieabwässer werden befriedigend, ja sogar $g u t$ geklärt, und möglicherweise sogar sehr gut.

Wie bekannt, kann bei sogar nur die konversationelle Implikatur suspendiert werden. 
Konjunktionstest:

(4c) Die Industrieabwässer werden sogar gut geklärt,

- "und sie werden gut geklärt. (ASS)

- "und sie werden befriedigend gekiärt. (PSP)

- *und sie werden nicht sehr gut geklärt. (konv. IMPL)

$(4 \mathrm{~d})$

- "Die Industrieabwässer werden gut geklärt, (ASS)

- Die Industrieabwässer werden befriedigend geklärt, (PSP)

- "Die Industrieabwässer werden nicht sehr gut geklärt, (konv. IMPL) und die Industrieabwässer werden sogar $g u t$ geklärt.

Die zweite Version von (4d) widerlegt nur scheinbar das oben über Skalen, deren Werte sich gegenseitig ausschließen, Gesagte. Hier werden nicht eigentlich zwei Werte als gültig behauptet, sondern die Behauptung von gut hebt die Behauptung von befriedigend auf: ein ganz regulärer Fall von Suspension der konv. IMPL (allerdings ohne das geforderte konjunktivische Element in der Suspensionsformel).

Dialogische Analyse

(4e) A: Die Industrieabwässer werden sogar gu $g$ geklärt.

B: - Nein, sie werden nicht gut geklärt. (ASS)

- Aber sie werden doch nicht einmal befriedigend geklärt. (PSP)

- Ich möchte sogar annehmen, daß sie sehr gut geklärt werden. (konv. IMPL)

- 'Va, gut werden sie schon geklärt, aber sie werden doch nicht einmal befriedigend geklärt. (ASS-Zustimmung + PSP-Protest)

- Ja, sie werden wirklich gut geklärt. (ASS)

- Ich weiß nicht, werden die denn überhaupt befriedigend geklärt? (PSP)

- Ja, aber immerhin nicht sehr gut, und das reicht noch allemal. (konv. IMPL)

Damit haben wir also die normalen Gesetzmäßigkeiten der skalierenden Interpretation von sogar.

\subsubsection{Skopustyp Satzadverbien}

\subsubsection{1./2./3. nur -, auch -, sogar - Gruppe}

Zu diesem Skopustyp haben sich nur sehr wenige und meist auch noch in der genauen Skopusabgrenzung unsichere Beispiele finden lassen. Da ich also vorwiegend auf konstruierte Sätze angewiesen bin und zwischen den einzelnen Gruppen von GPn keine gravierenden Unterschiede bestehen, fasse ich alle Teilthemen in einem Abschnitt zusammen. - Die Einteilung der Satzadverbien in die Klassen $K_{0}$ bis $K_{6}$ folgt $R$. Bartsch (1972: 28 - 33). 
$\mathrm{K}_{\mathrm{O}}$ : Die Elemente dieser Gruppe lassen sich recht gut zu kontrastierbaren Paaren ordnen, so daß man positive Ergebnisse mit GPn glaubt erwarten zu duirfen: vermutlich - gewiB; wahrscheinlich - wirklich; hoffentlich - fraglos, zweifelZos; viezleicht - tatsächlich, bestimmt.

(1) A: Hans hat den zug sicher erreicht.

B: ?Oh nein, er hat ihn nur $V$ e $r m$ u $t$ l i $c h$ erreicht.

(2) A: Ich bin sicher, daß Hans den zug erreicht hat.

B: Oh nein, du $v$ e $r m u t$ e $t$ nur, daß Hans den zug erreicht hat.

(3) ?Hat Hans nur $v$ e $r m u t I i c h$ den $z u g$ erreicht?

(4) A: Bist du sicher, daß Hans den zug erreicht hat?

B: Nein, ich $v$ e r m u $t$ e nur, daß das zutrifft.

Die Gründe für die Divergenz in der Akzeptabilität zwischen Satzadverbien im GP-Skopus und ihren Paraphrasen werden bei $\mathrm{K}_{1}$ diskutiert. - Die Beispiele für metasprachliche Verwendung sind bei diesem Skopustyp besonders zahlreich:

(5) A: Bestimmt hat Hans den zug erreicht.

B: Nein, deine Annahme trifft nicht zu. Hans hat den $z u g$ nur $v i$ e 1 $1 \mathrm{e} i \mathrm{ch} t$ erreicht/du darfst statt "bestimmt" nur $\overline{v i}$ e $I$ -

$l e i c h t$ in diesem Satz verwenden, wenn er zutreffen soll.

(6) ?Hans hat den zug nicht $s i c h e r$, sondern nur $v i e l i e i c h t$ erreicht.

(7) ?Hans hat den zug nur $v i e l i e i c h t$, nicht aber $s i c h e r$ erreicht.

Nicht günstiger ist die Situation bei sogar:

(8) ?Das industrielle Wachstum wird sogar sich e $r$ das Leben unerträglich machen.

(9) ?Die katholischen Spitzen entschließen sich im Fall Denzler sogar wahrs cheinlich dazu, daß...

$\mathrm{K}_{1}$ : Auch diese Gruppe läßt sich wieder zu (isoliert betrachtet) kontrastierenden Paaren sortieren: bedauerlicherweise - begrüßenswerterweise, anerkennenswertemweise; enttäuschenderweise - dankenswerterweise; ärgerlicherweise erfreulicherweise; begreiflicherweise - unbegreiflicherweise, erstaunlicherweise; etc. - Num zum Versuch der Kontrastierung in einem Satz:

(10) "Nur b e d a u e r I i che rwe is e hat $K$. Lorenz den Kongreß verlassen, nicht anerkennenswerterweise/erfreulicherweise.

(11) Es e n $t \ddot{a} u s c h t$ mich nur/ich be d a u $r e$ nur, daß Konrad Lorenz den Kongreß verlassen hat; ich bin nicht todtraurig/ich freue mich nicht darüber.

Dies beruht darauf, daß die Satzadverbien in jedem Fall zum Iopic eines Satzes gehören, die Paraphrasen hingegen können auch zum Satzfokus gehören. Dies 
zeigt sich auch in folgenden zugegebenermaßen konstruierten Gebrauchsweisen: Beim Kondolieren am Grab ist folgender Satz wegen falscher Topic-Comment-Verteilung pragmatisch inakzeptabel:

$$
\text { "Bedauerlicherweise ist Ihr Mann gestorben. }
$$

Die Witwe könnte ja darauf nur antworten:

(13) Ja, das sehe ich/weiß ich.

Aber nicht:

(14) “oh nein, sie freuen sich ja darüber.

Das Gegenteil ergibt sich in folgender Situation: Ein Arzt teilt der eben zur Witwe Gewordenen mit:

(15) Bedauerlicherweise ist Ihr Mann eben gestorben.

Die folgende, aus Höflichkeitsgründen in dieser Situation oft gebrauchte Formulierung ist pragmatisch eigentlich unangemessen:

(16) ?Ich bedaure sehr (Ihnen mitteilen zu müsșen), daß Ihr Mann gestorben ist.

Als Antwort auf (15) ist möglich:

(17) Das stimmt nicht. Ich habe ihn ja eben noch springlebendig herumlaufen sehen.

Aber nicht:

(18) "Das stimmt ja gar nicht. Sie sind doch ganz froh, daß er gestorben ist. In Sätzen mit Satzadverbien wird jeweils ein Sprechakt des Behauptens ausgeführt: nur der Inhalt der Behauptung ist als Ganzes oder in Teilen fokusfähig, nicht das Satzadverb, das die persönliche Stellungnahme des Sprechers wiedergibt. Bei den Paraphrasen hingegen handelt es sich um Sprechakte des Bedauerns usw.: in diesem Fall bildet der Matrixsatz den Satzfokus = das Rhema der Aussage. Diese Verben sind in sprechaktbeschreibender Funktion (nicht in performativer Verwendung) kontrastierbar. - Dazu paßt auch, daß diese Satzadverbien weder erfragt noch verneint werden können, allerdings nicht aus pragmatischen, sondern aus semantischen Gründen: als Aussagen ïber den jeweiligen internen Zustand des Sprechers können sie nicht angezweifelt werden.

Im Skopus von auch und sogar scheinen diese Elemente nirgends möglich zu sein. Man darf sich hierbei nicht von Äußerungen täuschen lassen, in denen die GP zwar unmittelbar vor diesen Satzadverbien steht, aber einen anderen Skopus aufweist:

(19) Er hat sogar glücklicherweise das Es s e $n$ mitgebracht. Die Satzadverbien gehören nämlich zu jenen Elementen, die in quasiparenthetischer Verwendung zwischen GP und Skopuskonstituente stehen können: ein weiterer Hinweis, daß sie selber nicht skopusfähig sind. 
$\mathrm{K}_{2}$ : klugerweise - dummerweise; vorsichtigerweise - unvorsichtigerweise; ungerechterweise - gerechterweise; ausnahmsweise - üblicherweise.

(20) “Nur k l uge $r$ we ise / d u m me $r$ we i s e hat er das Schloß gut gesichert.

(21) Es war nur $k$ I ug von ihm, nichts sonst (quant.) / nicht genial (skal.), daß er das Schloß gut gesichert hat.

(22) Er hat nur $k 1$ uge $r$ we $i s e$, aber nicht ge rech t e r $w e i s e$ eine Lohnzulage gewährt.

Ganz das Gleiche gilt für die Gruppen $\mathrm{K}_{3}$ und $\mathrm{K}_{4}$ (wunschgemäß, unerwünscht, exwartungsgemäß, unemwartet; verabredungsgemäß; erlaubterweise, verbotenerweise, zulässigerweise, notwendigerweise etc.). Dabei sind offenbar ganz bestimmte Formulierungen durchaus möglich:

(23) Es ging alles nur w u n $s c h g e m a ̈ \beta /$ nach $w$ uns $c h$.

(24) Sogar verbot e n e $r$ we i s e ging er zu ihr.

$\mathrm{K}_{5}$ : angeblich, vorgeblich, den Berichten nach etc.

(25) $\underline{\text { Nur/bloß/lediqlich }}$ a $n g e b l i c h$ ist er jetzt H-4-Professor.

(26) Nur $s c h e$ i $n$ b a $r$ ging er auf das Angebot ein.

$\mathrm{K}_{6}$ : natürlich, bekanntlich, offenbar. Diese Gruppe verhält sich, trotz ihrer syntaktischen Ähnlichkeit mit $\mathrm{K}_{5}$, ganz anders als diese Gruppe:

(27) “ hat er vor, ans MIT zu gehen.

Es ist nur nat ür $\mathrm{I}$ ich/ of $f$ enbar/bekanntI i c h , daß er ans MIT geht. (Funktionswechsel bei natürlich)

Die Untersuchungen bestätigen weitgehend die von Bartsch (1972) beschriebentn Eigenschaften dieser Adverbgruppe, insbesondere ihre Affinität zum Topic. Die wenigen akzeptablen Beispiele für die Konstruktion GP + Skopustyp Satzadverb sind syntaktisch so starr, daß sie entweder als Idiame gelten miissen oder aber (wegen Funktionswechsel des Skopuselementes unter Kontrastakzent) nicht zu diesem Konstruktionstyp gehören. 
3.6.5. Skopustyp limitierende Adverbien

3.6.5.1./2./3. nur -, auch -, sogar - Gruppe

Wegen der Seltenheit der Beispiele mit diesem Skopustyp werden auch hier wieder alle Gruppen gemeinsam behandelt.

(1) Die Arbeit ist nur $\ddot{u} b e r s i c h t s m \ddot{~} B i g$ gut, aber methodisch schlecht.

(2) Er ist nur/auch/sogar wirtschaft 1 i $c h$ ein Ass, in sozi-. aler Hinsicht aber ein Versager.

(3) Dieser Termin paßt mir nur $z$ e $i t i c h$ nicht, vom ort her schon.

(4) Er fühlt sich derzeit nur $g$ e is $t i g$, aber nicht $k$ ör $p e r-$ 1 i $c h$ auf der Höhe.

(5) Diese Strafe ... leidet unter einem weiteren nicht unerheblichen Defekt: sie ist auch $r$ e cht $l i c h$ nicht $z u$ rechtfertigen.

Zur Syntax

In allen Positionen, in denen üblicherweise Funktionswechsel auftritt (z.B. allein in der adverbialen Stellung nach finitem Verb) ist bei diesem Skopustyp Funktionswechsel zwar nicht völlig auszuschließen, doch wird offenkumdig die GP-Interpretation deutlich vorgezogen, und zwar sogar bei selbst.

Auch bei den Stellungsgesetzen ergeben sich wieder gewisse Abweichungen. Die Normalstellung ist Typ 1, also GP ummittelbar vor dem limitierenden Adverb im Skopus. Die bei einigen Elementen der nur - Gruppe sonst nicht gerade seltene Möglichkeit der Stellung unmittelbar hinter der Skopuskonstituente fällt hier völlig aus, ebenso generell die Möglichkeit der Stellung in Distanz vor oder nach der Skopuskonstituente. - Nicht sicher bin ich iber die Akzeptabilität der Konstruktion topikalisierte Skopuskonstituente + GP in Distanzstellung:

(1a) ?Ubersichts mä $B i g$ ist sie nur gut, nicht methodisch.

(2a) ?wi tschaft $1 \mathrm{ich}$ ist er sogar ein Ass.

(3a) Und $z$ e $i t I$ i $c h$ paßt mir der termin a uch nicht.

(5a) Die Strafe ist unter humanitären Gesichtspunkten eine Katastrophe, aber $r e c h t l i c h$ ist sie $\underline{a} u c h$ nicht $z u$ rechtfertigen.

Einen Grund für dieses unterschiedliche Verhalten kann ich nicht angeben.

Spaltsatzbildung scheidet in allen Fällen, auch bei nur, aus. Wie bei allen vergleichbaren Fällen bei Adverbien, so ist auch hier wohl der spezifische Skopustyp an sich (auch ohne GP) dafür verantwortlich zu machen.

Skopus und Fokus

Ihre Abgrenzung bietet wegen der Eingliedrigkeit der Skopuskonstituente und wegen der stark eingeschränkten Stellungsmöglichkeiten der GPn keine Schwierig- 
keiten. Interessant ist vielleicht, daß die spezifische Form der Frage-Antwort-Paare für Sätze mit GPn auch hier adaptiert werden kann:

(2b) A: wo ist er denn überall gut? B: Er ist nur wirtschaft 1 i $c h$ ein Ass.

(5b) A: Ist die Strafe nur unter sozialen Gesichtspunkten zu hart?

B: Nein, sie ist auch $r e c h t I i c h$ nicht $z u$, rechtfertigen, von den formalen Fehlern ganz zu schweigen.

Wiederum ist dies natürlich nicht für die Abgrenzung des Satzfokus zu nützen. Semantische Analyse

Ich gehe wieder von kontrastiven Vorgänger- und Folgesätzen aus:

(3b) Nur/allein/?einzig/bloß zeitlich paßt es.

Abgesehen von der gewissen stilistischen Inakzeptabilität von einzig sind also alle übrigen Elemente der nur - Gruppe verwendbar. Dies zeigt, daß eine quant. Interpretation sicher ist, ob daneben auch noch skal. Interpretation möglich ist, muß erst noch durch eindeutige Beispiele geklärt werden.

(3c) Es paßt mir nur $z$ e it 1 i ch nicht so ganz, und möglicherweise behagt mit sogar nur das wetter nicht recht.

Dies zeigt, daß Möglichkeiten zur Skalierung nicht ganz einfach zu finden sind, daß sie aber, wegen der praktisch unbegrenzten Möglichkeit, beliebige Gegenstände, Sachverhalte, Handlungen usw. in eine Rangfolge zu bringen, nicht ganz ausgeschlossen werden kann, übrigens auch bei der GP auch (in der verbindung mit nicht, siehe Bsp. (5)) nicht:

(5c) Die Strafe ist von den für mich wichtigeren sozialen und humanitären Gesichtspunkten her eine reine Katastrophe, aber auch $r$ e cht $1 \mathrm{i} h \mathrm{~h}$ ist sie nicht zu rechtfertigen, ganz zu schweigen von den haarsträubenden formalen Fehlern.

Selbstverständlich ist die skalierende Interpretation für sogar/selbst/nicht einmal die einzig mögliche.

Analysevorschlag für ein quantifizierendes Beispiel:

(4) Er fühlt sich derzeit nur $g$ e is $t i g$ auf der Höhe.

PSP: Er fühlt sich derzeit geistig auf der Höhe.

ASS: Er fühlt sich derzeit in allen anderen Bereichen (seelisch, körperlich) nicht auf der Höhe.

Analysevorschlag für ein skalierendes Beispiel:

(5d) Die strafe ist auch $r$ e cht 1 i $c h$ nicht $z u$ rechtfertigen.

ASS: Die Strafe ist rechtlich nicht zu rechtfertigen.

PSP: a. Sie ist aus wichtigeren als rechtlichen Gründen nicht zu rechtfertigen.

b. Sie ist aus weniger elementaren Gründen als den rechtlichen nicht $z u$ rechtfertigen.

konv. IMPL: a. Sie ist aus weniger wichtigen Gründen als den rechtlichen $z u$ rechtfertigen. 
b. Sie ist aus elementareren Gründen als den rechtlichen nicht zu rechtfertigen.

Die doppelten Formulierungen für PSP und konv. IMPL bei Satz (5d) kennzeichnen nach meinem Dafürhalten zwei unterschiedliche Skalenrichtungen, die möglicherweise nur bei auch, nicht hingegen bei nicht einmal auftreten können. Suspensionstest:

(5e) b. Die strafe ist schon formal nicht $z$ u rechtfertigen, und sie ist auch $r$ e $c h t I i c h$ nicht $z u$ rechtfertigen, und möglicherweise ist sie sogar a'גs humanitären Gründen nicht haltbar.

b. "Die Strafe ist schon formal nicht $z$ u rechtfertigen, und sie ist nicht einmal $r e c h t I i c h$ zu rechtfertigen, und möglicherweise ist sie sogar aus humanitären Gründen nicht haltbar.

a. Die Strafe ist aus den wichtigeren humanitären und sozialen Gründen nicht zu rechtfertigen, sie ist auch aus $r$ e $c h t l i c h$ e $n$ Gründen nicht $z u$ rechtfertigen, und sie ist möglicherweise nicht einmal aus formalen Gründen haltbar.

Um dies aber zweifelsfrei behaupten zu können, ist das Beispiel wegen der recht unklaren Abfolge der Skalenwerte nicht ganz geeignet. Die These müßte also noch bei eindeutig skalierbaren prädikativen Adjektiven und Adjektivactverbien überprüft werden. Eine gewisse Überprüfung ist auch anhand des dialogischen Tests möglich:

(5f) A: Die Strafe ist auch $r e c h t I i c h$ nicht zu rechtfertigen.

B: - Nein, rechtlich ist sie wirklich nicht zu rechtfertigen. (ASS)

- Rechtlich vielleicht nicht. Aber ich möchte doch meinen, daß sie gerade von den auch für dich wichtigeren humanitären und sozialen Gesichtspunkten her $z u$ rechtfertigen ist. (ASS-Zustimmung + PSP-a-Protest)

- Rechtlich vielleicht nicht. Aber ich möchte doch glauben, daß sie zumindest von den vielleicht weniger wichtigen formalen Aspekten her $z u$ halten ist. (ASS-Zustimmung + PSP-b-Protest)

- Ja sicher, aber ich würde hinzufügen, daß sie auch aus den noch weniger wichtigen formalen Gründen nicht $z u$ rechtfertigen ist. (konv. IMPL a)

- Ja sicher, und ich würde sogar hinzufügen, daß sie sogar aus den noch viel wichtigeren sozialen und humanitären Gründen nicht $z u$ rechtfertigen ist. (konv. IMPL b)

- Rechtlich ist sie tatsächlich nicht zu rechtfertigen, aber doch aus den viel wichtigeren humanitären und sozialen Gründen. (ASS-Zustimmung, PSP-a-Protest)

- "Doch, aus den viel wichtigeren humanitären und sozialen Gründen ist sie zu halten, aber doch aus den rechtlichen nicht.

usw. Eine letzte Sicherheit kann man auch damit natürlich nicht gewinnen. ${ }^{16}$

16 Parenthetische Adverbien wie ubertrieben gesagt, genau betrachtet, offen gesagt usw. können, da sie weder kontrastierbar und (was daraus folgt) nicht fokusfähig sind, nicht den Skopus von GPn bilden. Sie werden daher nicht untersucht. 


\subsection{Skopustyp Satz}

Dieser Skopustyp hat in der englischen Literatur zu den GPn eine gewisse Rolle gespielt. Die Diskussion stützte sich jedoch ausschließlich ${ }^{17}$ auf ein einigenmaßen überzeugendes Beispiel von Bruce Fraser: ${ }^{18}$

(1) What happened in 1969 that was so exciting? Brady was finally located! Men landed on the moon! Tiny Tim got married! Why, Harvard even held a pep rally ...

und diskutierte nur die Stellungsgesetze der GPn in diesem Fall und einige spezielle Fragen der transformationellen Zuordnung von even. Die sonst häufig diskutierte Frage der Skopusabgrenzung, etwa durch die Stellung der GP und durch die Position des "heavy stress", also der kontrastiven Betonung, sowie der allgemeinen Bedingungen (semantisch, pragmatisch) dieses Skopustyps werden nicht beriihrt. Dies ist noch ausgeprägter und in Anbetracht des Themas ihres Aufsatzes noch unverständlicher bei S. Schmerling (1972: $245 \mathrm{ff.}$.). Sie bietet zwar eine Reihe von Sätzen, die aufgrund der typographischen Markierung nur als Beispiele für den Skopustyp Satz gemeint sein können, aber sie sagt nichts zu den in diesem Fall anstehenden Akzentfragen. Hier seien nur zur Orientierung ihre Beispiele zitiert:

(2) John also/even/only reads books on politics.

(3) John reads books on politics also/even.

Von allen zitierten Forschern werden 3 Positionen von even bei diesem Skopustyp angenormen: unmittelbar vor der VP, bzw. nach dem ersten Element der Auxiliar-Sequenz, und am Satzschluß. Die satzeinleitende Stellung wird von Fraser ausdrïkklich ausgeschlossen. Alle drei GP-Positionen sind nicht direkt auf das Deutsche ibertragbar. In Satzanfangstellung liegt, wie im Englischen, meist der Skopus Subjekts-NP vor (falls nicht irgendeine andere Konstituente topikalisiert wurde) bzw. konjunktionaler Gebrauch. Die Stellung vor der gesamten VP ist bei Matrixsatzwortstellung iberhaupt nicht mïglich. Lediglich bei nur könnte es ein Fall von Position nach dem topikalisierten Skopus sein. - Dieser Wortstellungsvariante des Englischen entspricht im Deutschen wohl die Stellung der GP unmittelbar nach dem finiten Verb, wobei allerdings pronominalisierte Konstituenten zwischen finites Verb und GP treten können. Skopus kann in die-

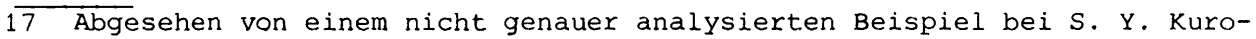
da (1969: 333):

(8) (a) The storm destroyed his house.

(b) The flood even devastated his farm.

18 B. Fraser (1971: 151 und 166). Wieder aufgenommen von S. R. Anderson (1972: 899). 
sem Falle sowohl das Vollverb (wenn es direkt folgt) als auch ein Objekt oder eine Adverbialphrase, aber auch die gesamte VP sein. Diese Ambiguität bezüglich der Skopusabgrenzung läßt den Schluß zu, daß bei dieser GP-Stellung auch ein Satzskopus möglich sein mïßte. - Die Position am Satzschluß ist im Deutschen nur bei einer synthetischen Verbform möglich (wenn auf das Verbum keine Ergänzungen folgen). Bei ihr ist gewöhnlich nur der Skopus Vollverb möglich (Matrixsatz!). Da dieser Stellungstyp insofern mit dem von oben identisch ist, sollte auch diese Position nicht ganz von den Untersuchungen ausgeschlossen werden.

Die Situation wird also durch folgende Fakten kompliziert: die Unklarheit über die formalen Eigenschaften des Satzskopus; die unsichere Abgrenzung gegenüber der konjunktionalen Verwendung, wie sie bei nur sicher und bei auch jedenfalls möglich ist; und schließlich: die unsichere Abgrenzung gegenüber der MPVerwendung bei auch und nur. - Wenn es so etwas wie einen Satzskopus gibt, dann muß er - dies folgt logisch aus der Abgrenzung gegenüber anderen Skopustypen folgende Eigenschaften aufweisen: Alle Glieder des Satzes müssen mit irgendeinem expliziten oder impliziten Vorgängersatz kontrastieren. Die Bedingung kann auch umgekehrt formuliert werden: befindet sich unter den (aufgrund der Partikelstellung) möglichen Skopi eine Konstituente, deren Kontrastierung mit einer Konstituente des vorhergehenden Satzes aufgrund der Identität anderer Konstituenten wahrscheinlich wird, so ist die Interpretation Satzskopus ausgeschlossen. - Da also die Gesamtheit des Satzes kontrastiert wird, ist jeder Kontrastakzent, der nicht mit dem normalen Akzent des Satzes übereinstimmt, von vornherein ausgeschlossen. Kontrastakzent und Partikelskopus bedingen sich ja gegenseitig. So ist es z.B. nicht möglich, einen Satz mit einem Kontrastakzent und einem davon getrennten Partikelskopus zu formulieren:

(4) $\ddot{H}_{H}$ a $\mathrm{s}$ hat nur $\ddot{\text { A }} p \mathrm{f}$ e 1 geklaut.

Um diese geforderte Verschiedenheit zwischen Vorgängersatz und GP-Satz sinnfällig zu demonstrieren, werden jeweils die Vorgängersätze hinzugefügt.

Aus dem oben Gesagten ergibt sich, daß bei sogar die geringsten, bei nur die größten Schwierigkeiten zu erwarten sind. Deshalb wähle ich in diesem Falle die umgekehrte Reihenfolge der drei Gruppen.

\subsection{1. sogar - Gruppe}

(1) ... ohne viel Dank dafür zu erwarten. Anja hat sogar das eine oder andere Mal die andere Backe hingehalten.

(2) Wehner preist die Marktwirtschaft und schmidt rühmt sogar die wirkung des Stabilitätsprogramms. 
(3) Der Außenhandel wächst ständig. Und die Sibirienofferte der sowjets läßt sogar ein phantastisches Bild auftauchen.

(4) Das Wirtschaftsembargo hat viele Löcher. Vor einigen wochen gelangten sogar drei Boeings aus dem Pleitenachlaß der Calair nach Salisbury.

(5) Überall bringen Sex-Filme die höchsten Einnahmen, und in ihrem Fischbecker Einfamilienhaus eröffnete sogar eine Dame mit einem vergnügten Unterleib mit dem Segen ihrer Familie ein Institut für Sexualinformation.

(6) Überall bläst den Gewerkschaften der wind ins Gesicht ... In einer rheinischen Metallfabrik bezeichnete sogar der Firmenrechtsanwalt die Kandidatur eines Lehrlings zum Betriebsrat als vertragswidrig.

\section{Zur Syntax}

Die Austauschgesetze sind ganz typisch für die GP-Position: nicht einmal ist überall einsetzbar, allerdings sind dann die Vorgängersätze ungeeignet. selbst wechselt in die Funktion des Reflexivrms. Modalpartikeln, soweit sie in Aussagesätzen möglich sind, können anstelle von sogar eingesetzt werden, aber sie können auch vor sogar hinzugefügt werden.

(3a)

$$
\begin{aligned}
& \text {... Und die Sibirienofferte der Sowjets läßt ja (sogar) ein phanta- } \\
& \text { stisches Bild auftauchen. }
\end{aligned}
$$

Doch ist dies kein Beweis für die Zugehörigkeit von sogar in diesen Fällen zur Funktionsgruppe der MPn; lediglich Stellung und Betonungsmuster sind identisch. Die Stellungsregeln sind denkbar einfach. Im Matrixsatz folgt sogar jeweils dem finiten Verb, pronominalisierte Konstituenten und Adverbiale können sich aber dazwischenschieben. Im Konstituentensatz geht sogar' dem finiten verb voraus, bzw. es folgt unmittelbar auf die Subjekts-NP. Die satzeinleitende Stellung verträgt sich bei sogar nicht mit diesem Skopustyp.

Eine einigermaßen überzeugende Umformung der zitierten Sätze in Wortfragen, Satzfragen und Imperative ohne gleichzeitige Verkleinerung des Skopus ist mir bisher nicht gelungen: gewöhnlich befindet sich dann nur noch die VP oder eine einzelne Konstituente im Skopus, wie die Akzeptabilität von Kontrastsätzen beweist.

(2a) Rühmt Schmidt sogar die wirkung des Stabilitätsprogramms? (oder mokiert er sich nur über die Inflationsraten?)

(1a) Warum hat Anja sogar das eine oder andere Mal die andere Backe hingehalten?

(2b) Rühme sogar die wirkung des Stabilitätsprogramms! (Mokiere dich nicht nur über die Inflationsraten!)

Auch bei den Negationen besteht eine enge Wechselwirkung mit der Skopusabgrenzung. Unmittelbar nach sogar kann keine Negation plaziert werden. Die Plazierung in größerem Abstand bewirkt, daß die dazwischenstehende VP zum Kontrastskopus von sogar wird. Ausschließlich nicht einmal konserviert alle Eigen- 
schaften des positiven Satzes:

(1b) :Anja hat sogar nicht das eine oder andere Mal die Backe hingehalten.

(1c) Anja hat sogar das eine oder andere Mal die $B$ a $c k e$ nicht hingehalten.

(1d) Anja hat nicht einmal das eine oder andere Mal die andere Backe hingehalten.

Skopus und Fokus

Die Position des Satzakzentes entspricht bei diesen Sätzen völlig der nichtkontrastiven Position des Satzakzentes. Daraus läßt sich aber kein verbindlicher Schlu3 auf Satzskopus ableiten, denn der gleiche Sachverhalt war bereits beim Skopus VP festzustellen. Der Unterschied zwischen beiden Skopustypen ist ein rein kontextueller. Beim Skopus VP ist die Voraussetzung, daß in einem ev. Vorgänger- oder Folgesatz das Subjekt mit dem des sogar - Satzes identisch ist (Referenzidentität natürlich!):

(1e) Anja ist ein Wunder an Sanftheit, und sie hat sogar das eine oder andere Mal die andere Backe hingehalten/und sie hat nicht nur die eine, sondern das eine oder andere Mal sogar die a $n d e r e$ Backe hingehalten.

Hingegen ist der sogar - Satz mit Satzskopus als ein Glied in eine Satzreihe einzuordnen, die immer höhere/wichtigere ... Werte markiert (in einer bestimmten Sphäre), und bei denen jeweils alle Glieder "neu" sind:

(2C) Alle Spitzenmänner der SPD führen sich wie tiefschwarze Unionschristen auf: Brandt predigt in einer evangelischen Kirche, wehner preist die Marktwirtschaft, und Schmidt rühmt sogar die Wirkung des Stabilitätsprogramms.

Die "Sphäre", in der die Skala angesiedelt ist, kann aus dem ersten Satz erschlossen werden: CDU-CSU-gemäßes Verhalten von SPD-Spitzenmännern. Jeder folgende Satz markiert darin einen (höheren) Wert. In gewisser Weise kann aber das jeweilige Subjekt durch die Angabe der Menge, der es zuzurechnen ist, als vorerwähnt gelten. - Eine semantische Analyse muß folgendermaßen aussehen:

(2d) ASS: Schmidt rühmt die wirkung des Stabilitätsprogramms.

PSP: Andere Leute der SPD legen ein Verhalten an den Tag, das auf der Skala des CDU-CSU-gemäßen Verhaltens tiefer rangiert.

konv. IMPL: Andere SPD-Leute als die genannten sind bezüglich des CDUCSU-gemäßen Verhaltens nicht höher einzustufen als 'Schmidt rühmt die wirkung des Stabilitätsprogramms'.

Natürlich ist die Formulierung sowohl der PSP wie auch der konv. IMPL völlig unzulänglich. Das Prinzip ist aber wohl klar: eine Reihe von Sätzen bzw. die dadurch ausgedrückten Sachverhalte werden in einer Skala geordnet, wobei der sogar - Satz als höchster gültiger Skalenwert (Sachverhalt) bezeichnet wird, die darunter liegenden Skalenwerte gelten präsuppositionell, die dariberliegenden werden durch konv. IMPL ausgeschlossen. Durch diese Anordnung sind die 
Möglichkeiten der Skalierung bei diesem Skopustyp praktisch unbegrenzt. - Man kann num versuchen, eine spezifische PSP bzw. konv. IMPL zu suspendieren:

(2e) ... und Schmidt rühmt sogar die wirkung des stabilitätsprogramms, - "und möglicherweise schwört kühn nicht einmal auf die Kräfte des freien Marktes.

- und möglicherweise wirbt Vogel sogar im nächsten wahlkampf mit Heiligenbildern.

Ebenso regulär läuft der dialogische Test ab:

(2f) A: ... und schmidt rühmt sogar die wirkung des stabilitätsprogramms.

B: - Nein, Schmidt rühmt nicht die wirkung des Stabilitätsprogramms. (ASS)

- Schmidt rühmi zwar die wirkung des Stabilitätsprogramms, aber die anderen SPD-Spitzenmänner verhalten sich doch nicht unionschristenmäßig. (ASS-Zustimmung + PSP-Protest)

- Ja, aber das Allerschlimmste in dieser Richtung ist, daß Vogel im nächsten Wahlkampf Heiligenbilder verteilen will. (konv. IMPL)

- Na ja, daß Brandt und wehner nach rechts schielen, das mag schon stimmen. Aber Schmidt rühmt doch nicht die wirkung des Stabilitätsprogramms. (PSP-Zustimmung, ASS-Verneinung)

- Ja also, das ist wirklich unglaublich, daß Schmidt jetzt die wirkung des stabilitätsprogramms rühmt. (ASS)

usw. Damit ist für den Satzskopus dieselbe skalierende Interpretation gesichert wie für die anderen Skopustypen von sogar.

\subsubsection{2. auch - Gruppe}

Der Versuch, auch anstelle von sogar bei allen Belegsätzen des letzten Abschnitts einzusetzen, zeigt, daß dies ohne Komplikationen möglich ist:

(1) ... ohne viel Dank dafür zu erwarten. Anja hat auch das eine oder andere Mal die andere Backe hingehalten.

(2) Wehner preist die Marktwirtschaft und Schmidt rühmt auch die wirkung des Stabilitätsprogrammes.

(3) Der Außenhandel wächst ständig. Und die Sibirienofferte der Sowjets läßt auch ein phantastisches Bild auftauchen.

(4) Das wirtschaftsembargo hat viele Löcher. Vor einigen wochen gelangten auch drei Boeings aus dem Pleitenachlaß der Calair nach Salisbury.

(5) Überall bringen Sex-Filme die höchsten Einnahmen, und in ihrem Fischbecker Einfamilienhaus eröffnete auch eine Dame mit einem vergnügten Unterleib mit dem Segen ihrer Familie ein Institut für Sexualinformation.

(6) Uberall bläst den Gewerkschaften der wind ins Gesicht ... In einer rheinischen Metallfabrik bezeichnete auch der Firmenrechtsanwalt die Kandidatur eines Lehrlings zum Betriebsrat als vert.ragswidrig.

Aber man muß feststellen, daß man doch i.d.R. bei dieser auch - Position eine Interpretation mit kleinerem Skopus vorziehen wïrde, also V, oder objekts-NP 
oder VP. In jedem Falle wirken die Texte merkwürdig unverbunden. - Aus diesem Grunde beziehe ich die gewöhnlich als konjunktionale Funktion interpretierte Satzanfangsposition von auch in die Untersuchung mit ein.

"Da gibt es", so Münchens SPD-Vorstandssprecher Christian Ude, "die abenteverlichsten Purzelbäume." Auch hat der früher auf Altgenossen gemünzte Protest gegen die Sitzfleischsozialisten nun eine gefährliche Uberspitzung erfahren.

$$
\text { Diesmal geht es nur um Geld und Abgeordnetenstimmen. Auch ist das Ka- }
$$
liber der Beteiligten geringer.

In beiden Fällen wird zu bereits als gültig bezeichneten Sachverhalten noch ein weiterer gültiger Sachverhalt hinzugefügt. Genau die gleiche Wirkung kann man mit diesem Stellungstyp bei den Sätzen (1) bis (6) erzielen:

(1a) ... ohne viel Dank dafür zu erwarten. Auch hat Anja das eine oder andere Mal die andere Backe hingehalten.

(2a) Auch rühmt Schmidt die wirkung des Stabilitätsprogramms.

(3a) Auch läßt die Sibirienofferte der Sowjets ein phantastisches Bild auftauchen.

In beiden Positionen besteht grundsätzlich Austauschbarkeit durch ferner, darüberhinaus, außerdem, zudem usw., obwohl auch für diese Elemente gilt, daß die satzeinleitende Postion deutlich bevorzugt wird. Zwischen diesen Positionen kann man, abgesehen von der Skopusambiguität in Mittelstellung, keine semantischen Unterschiede erkennen. Analysiert man Satz (1), so erhält man folgende semantische Bestandteile:

(1) ASS: Anja hat das eine oder andere Mal die andere Backe hingehalten. PSP: Andere Sachverhalte (die in irgendeiner weise mit dem assertierten Sachverhalt in Verbindung stehen) gelten/treffen $z u$.

Dies liegt ganz im Rahmen der GP-Funktion von auch. Fragen kann man sich allerdings, ob zu dieser quantifizierenden Interpretation noch ein gewisses "konzessives" Element hinzutritt. Wenn dies auch zuträfe, so ist es doch formal nicht faßbar. -

Gewisse Aufschliusse erbringt auch noch die Untersuchung der Möglichkeiten bei vorangehender Konjunktion (wamit gleichzeitig der Bereich der Konstituentensätze erfaßt wird) :

(1b) ..., weil auch An $j$ a das eine oder andere Mal ... ... , weil Anja auch das eine oder andere Mal ...

(2b) Wehner preist die Marktwirtschaft, und auch $S \mathrm{ch} \mathrm{m}$ id $t$ /und Schmidt rühmt auch die wirkung des Stabilitätsprogramms.

(5a) $\because .$. und auch eröffnete eine Dame mit einem vergnügten Unterleib ... ... und auch eine $D$ a $m$ e mit einem vergnügten Unterleib ...

Die Regeln können folgendermaßen formuliert werden: unmittelbar nach Konjunktionen, die Inversion bewirken, kann auch nur kontrastiven Subjekts-NP-Skopus 
haben. - auch nach dem Subjekt ist zumindest skopusambig: V und VP können Skopus sein, aber auch Satz-Skopus kann man nicht sicher ausschließen. Unmittelbar auf und, aber usw. folgendes auch kann nur Subjekts-NP-Skopus haben. auch zwischen Subjekt und finitem Verb ist nicht akzeptabel. - auch nach dem finiten Verb ist akzeptabel. Doch treten hier wieder die bereits bekannten Skopusambiguitäten auf. - Der Ausschluß der Position von auch mit Satzskopus nach einer Konjunktion ist ein gewichtiges Argument für die konjunktionale Funktion von auch in diesen Fällen. Doch bestünde dann ein nahtloser syntaktischer und semantischer übergang zwischen GP-inktion und konjunktionaler Funktion von auch.

\subsection{3 nur - Gruppe}

Gleiches gilt natürlich nicht von nur. Zunächst Sätze, in denen riur nach dem finiten Verb steht:

(1) Selbstverständlich muß die SPD reagieren. Diesen Konflikt gibt es nur nicht erst seit gestern.

(2) Ich hätte ihn ja ganz gerne mitgenommen. Das Auto war nur schon voll. Dieser Stellungstyp von nur ist, wie schon bei vielen Gelegenheiten bemerkt, nur konjunktional interpretierbar: typisch für dieses nur ist, daß es unmittelbar vor einer Negation oder nicht skopusfähigen Konstituente steht. Semantisch gleichwertig j.st nur in satzeinleitender Stellung:

(1a) Nur/bloß gibt es diesen Konflikt nicht erst seit gestern.

(2a) Nur/bloß war das Auto schon voll.

Austauschmöglichkeiten gibt es darüberhinaus in der nur - Gruppe nicht. Alzein verlangt eine andere Wortstellung:

(1b) Allein, diesen Konflikt gibt es nicht erst seit gestern. Alle diese Versionen haben semantisch nichts mit der GP-Funktion von nur zu tun (siehe auch den Abschnitt 4.2.1.1. S. 275 zur konjunktionalen Funktion von nur!). - Bei der weiteren Suche nach einem nur mit Satz-Skopus könnte nur noch die Rekonstruktion der Semantik helfen. Die Analyse von (2) müßte etwa lauten:

(2b) Nur war das Auto schon voll./Das Auto war nur schon voll. PSP: Das Auto war schon voll

ASS: ?Hinsichtlich meiner Bereitschaft, irgendjemand mitzunehmen, trifft kein anderer Sachverhalt zu.

Diese Interpretation trifft aber nicht die Semantik des vorliegenden (konjunktionalen) nur, und sie ist außerdem in sich selbst widersprüchlich bzw. zumindest völlig unwahrscheinlich. 


\subsection{Skopustyp Idiome}

3.8.1./2./3. nur -, auch -, sogar - Gruppe

(1) Du solltest jetzt nur die Kur $v$ e kratzen.

(2) Willst du nicht sogar $L$ e i n e ziehen?

(3) Von mir aus kann die Arbeit auch der $T$ e u f e 1 holen.

(4) Solche Dinge darfst du nicht einmal in $E r w \ddot{a} g u n g$ ziehen, von durchführen gar keine Rede.

Nicht ganz sicher bin ich mir, ob in jedem Falle das Idiom am Satzschluß stehen und unmittelbar und vollständig auf die GP folgen mu:

(2a) ?Nur die Kurve solltest du jetzt kratzen.

(4a) ? In E $r$ w ä $g u n g$ darfst du solche Dinge nicht einmal ziehen, von durchführen gar keine Rede.

(3a) ??Von mir aus kann der Teufel die Arbeit a uch holen/auch ho1 e $n$.

Im Falle (2a) ist der Satz nur dann einigermaßen akzeptabel, wenn man die Verbindung zwischen Kurve und kratzen nicht als absolut fest betrachtet.

Diese überlegungen führen unmittelbar zu der auch schon von Bruce Fraser ${ }^{19}$ gestellten Frage, ob Teile von idiomatischen Wendungen Skopus von GPn sein können. Seine Schlußfolgerung, daß dies keinesfalls möglich ist, kann ich nicht in dieser Schärfe teilen. Sicherlich ist es so, daß sehr feste Wortverbindungen nicht in dieser Weise trennbar sind (außer für Wortwitze u. dgl. m.). Gleichzeitig gibt es aber einen breiten übergangssaum, in dem dies mehr oder weniger gut denkbar ist. Ein Beispiel hierfür ist vielleicht Satz (3), wobei nicht ganz unwichtig sein mag, daß es sich hier um die Kombination SubjektsNP - Verbum handelt. Damit könnte dieses Konstruktionsmuster als Test für die jeweilige Festigkeit von Idiomen benutzt werden, also für das Maß, in welchem Einzelteile noch für sich kontrastiert werden können.

Die semantische Beschreibung erübrigt sich, da sie jeweils derjenigen entspricht, die man für eine einteilige Paraphrase zu geben hätte. Das Gleiche gilt übrigens für die syntaktische Beschreibung; also: nur die Kurve kratzen nur abhauen; sogar Leine ziehen - sogar verschwinden etc. Damit ist auch der Rahmen der Kontrastierbarkeit angedeutet.

19 Bruce Fraser (1971: 153 und 174 f.); siehe auch N. Chomsky (1969; 1972: 122 Anm. 9). 
4. ANALYSENTEIL II: EDNZELTHEMEN

4.1. Besondere Verwendungsweisen von Gradpartikeln

4.1.1. Betonte Gradpartikeln

4.1.1.1. nur - Gruppe

1. Bei Gliedsätzen: dieses Thema wurde bereits oben unter 3.2.6.1 S. 151 abgehandelt. Hier werden nur noch einige Beispiele gegeben.

(1) So reagiert nur, wer sich an einer besonders empfindlichen Stelle getroffen fühlt.

(2) Er weiß nur, daß etwas frisches wasser dazugehört.

(3) Eine Ursache kann begrifflich nu $\underline{r}$ /nur d a $n n$ gegeben sein, wenn eine Folge vorliegt.

(4) ?Meine Vorträge halte ich $\underline{n u r} / \underline{\text { nux }}$ dor $t$, wo ich auch Anerkennung finde.

Um eine kontrastive Betonung kann es sich keinesfalls handeln, da die GP in dieser Konstruktion nicht unmittelbar kontrastiert werden kann, abgesehen von der Kombination explizite PSP-Formulierung + nur - Satz:

(5) Der Präsident gab $z u$, was in Prozessen längst bekannt geworden war, und er gab $\underline{n u r} z u$, was in Prozessen längst bekannt geworden war. Dies dürfte auch tatsächlich die normale Verwendung des betonten nur sein. Ein weiterer Hinweis mag sein, daß die Betonung auf der GP in vielen Fällen bei völlig gleicher Konstruktion nicht beobachtet werden kann; wenn ich richtig beobachtet habe, dann wird in diesen Fällen die Satzfuge zwischen Konstituenten- und Matrixsatz ausgelöscht und es entsteht damit eine große Spannung auf das stark akzentuierte Verb am Schluß des Konstituentensatzes bzw. auf dessen letztes Namen hin. Diese Regelung dürte dann vorherrschen, wenn die GP nicht umittelbar vor dem Konstituentensatz stehen kann:

(5a) Nur was längst $b$ e $k$ a $n$ n war, gab der Präsident zu.

(6) Man antwortete mir, man werde mir nur zu trinken geben, wenn ich sch $r$ e i be. 
Dadurch kann man nicht mehr ausschließen, daß es sich nicht um eine wirkliche Betonung, sondern um einen starken Anstieg der Intonationskurve als Einleitung eines Konstituentensatzes handelt, der normalerweise auf den Platzhalter fällt; also eine Art "Ersatzbetonung".

2. nur im Fokus, kontrastiert gegen auch.

(1) Wir verkennen nicht, daß es in unserer Sammlung auch viele wörter gibt, die $n u r$ Adyerbien sind (nicht nur $\underline{a} u h$ Adverbien/und sonst gar nichts).

(2) A: wir verstehen unter "residence" die vertretung $n$ a $t$ ü $r$ I $c h e r$ und (auch) juristis che $r$ Personen.

B: Wir verstehen unter "residence" eben $n u r$ die vertretung natürlicher Personen (und nicht (nur) a u $\overline{C h}$ die vertretung juristischer Personen/und nichts sonst).

Die syntaktischen Eigenschaften entsprechen, bei aller Beschränkung, doch den bereits bekannten. Funktion und Semantik sind aus der Kontrastierung mit auch heraus, die offenbar in aller Regel explizit erfolgen mu, unmittelbar einsichtig: Einzigkeitsbehauptung mit Hilfe von nur gegen Nichteinzigkeits-PSP von auch.

3. Spezialfall mit bloß.

(1) Laß das $\underline{\text { b } 1 \circ \beta}$ sein!

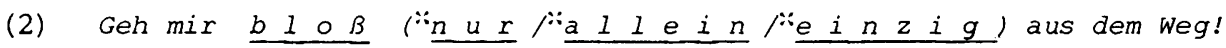
Um eine GP-Funktion kann es sich hierbei nicht handeln, da Kontrastsätze nicht möglich sind. Andererseits ist die Einordnung als MP nicht ganz unproblematisch: denn nur, Jas wie bloß als MP verwendet werden kann, darf in dieses Muster nicht eingesetzt werden:

$$
\text { Laß das n u } r \text { sein! }
$$

Außerdem sind MPn gerade durch die Eigenschaft definiert, nicht betonbar zu sein. Für die MP-Interpretation spricht allerdings die Stellung im Satz und die Bindung an den Imperativ. Eine plausible Erklärung wäre, daß es sich mit großer Wahrscheinlichkeit um eine (systemwidrige) regionale Variante handelt. 


\subsubsection{2. auch - Gruppe}

1. Bei Gliedsätzen: auch hier muß man auf Abschnitt 3.2.6.2. verweisen.

(1) Auch, wer die sehnsucht $n i c h t$ kennt, weiß, was ich leide.

(2) Daß Hans vorhin gelogen hat, stimmt, und es stimmt a uch, daß er überhaupt ein schlechter Mensch ist.

(3) Es kam nicht unerwartet, daß sich der letzte Schlag gegen eine arabische Botschaft richtete, und zwar a uch (auch d a nn), wenn $z u$ den Geiseln Amerikaner gehörten.

(4) Unsere Kollegen wissen das. Sie wissen a $\mathrm{uch}_{\mathrm{c}}$, wo die Verantwortlichen für jede Art Preistreiberei sitzen: im Unternehmerlager.

Auffällig ist, daß bei keinem dieser "betonten" auch ein Austausch gegen ebenfalls, ebenso, gleichfalls ... möglich ist. Dies deutet wiederum, zusammen mit der Möglichkeit, auch unbetont zu lassen und die Satzfuge zu beseitigen, darauf hin, daß es sich lediglich um ein bestimmtes Muster für den Anschluß von Gliedsätzen handelt. - Die syntaktischen und semantischen Eigenschaften dieses Konstruktionsmusters sind in dem oben genannten Abschnitt beschrieben.

2. auch im Fokus, kontrastiert mit nur.

Wir verkennen nicht, daß es in unserer sammlung viele wörter gibt, die nicht $\underline{n u r}$ Adverbien sind, sondern $\underline{a c h}$ Adverbien.

Diese Verwendung ist in allem das Spiegelbild des parallelen nur - Gebrauchs. Da sie äußerst selten ist, brauche ich mich wohl nicht mehr ausführlicher damit zu beschäftigen.

3. Spezialfall von betontem auch: in Distanzstellung nach dem topikalisierten Skopuselement. 1

(1) z we $r g$ e haben $\underline{a} u c h$ klein angefangen.

(2) Doch orde $r$, sich um einen Hering zu kümmern, falls er fälschlich im Milchladen ausliegt, haben sie a $u c h$.

(3) Auf die ohnmächtige öffentlichkeit im eigenen Land nahm Nixon a uch keine Rücksicht.

(4) ?Der kleinste Fehler darf a u $c h$ nicht passieren.

(5) ?Ein $l$ e $i d e n s c h$ a $t$ t $l$ i h e $r$ Jäger wird a uch nicht soviel für ein Revier anlegen wollen.

(6) Als er wieder gesund war, änderte er sein verhalten a $u c h$ nicht.

(7) Wenn wir ein Taxi nehmen, können wir den zug a $u c h$ nicht mehr erreichen.

1 Siehe zu diesem Thema auch Altmann (1976). 
(8) Dies schließt nicht aus, daß wir a uch sehr daran interessiert sind.

(9) De r kann uns a $u c h$ helfen.

(10) Ich habe an der Tombola einen "Golf" gewonnen, und irgendjemand (sonst) hat $\underline{a \mathrm{u} h}$ Glück gehabt.

(11) Üb e rordne $n$ wollte er sich a $\underline{\text { u ch }}$.

(12) ?Es ist jetzt immer Geld da, doch es $f$ e $h I t$ andererseits $a u c h$ immer Geld/ doch $f$ e $h l$ e $n$ tut das Geld a $u c h$ immer.

(13) Ich soll nicht nur t $r i n k$ en, li e be n soll ich a uch.

(14) "Ich glaubte, er sei verreist, und er $w$ a $r$ es $\underline{a c h}$.

(15) Ich will nicht nur, $k$ ön $n$ e $n$ tu ich a uch .

(16) Und verständlich ist es $\underline{a} u_{c h}$.

(17) Eine $P r \ddot{u} f u n g$ soll es ja a uch sein.

(18) Seine bisherige Verteidigungslinie bestätigen lassen wollte Nixon e $b$ enfalls.

(19) ?F ü $n f m a l$ würde mir das Baden $\underline{a} u c h$ Spaß machen.

(20) Der e in e versorgte die Rosen, aber der a $n d e r e$ war a $u c h$ nicht ganz unbeschäftigt.

(21) ?V i e 1 e können hier $\underline{a ~ u c h}$ nicht mehr helfen.

(22) ?Wie schon in den beiden Wintern zuvor, herrscht $h$ e ue $r$ a $u c h$ allerorten Schnee- und Kältemangel.

(23) Die Arbeit ist übersichtsmäßig gut, und $m e t h \circ d i s c h$ (ist sie) a $u \subset h$ ganz ansprechend.

\section{Zur Syntax}

Klassenbildung/Austauschbarkeit: in allen überprüften Fällen war der Austausch von auch gegen gleichfalls, ebenso, ebenfalls ... möglich. Natürlich kommen dadurch neue Bedeutungselemente, insbesondere bei ebenso (= in gleicher Weise) hinzu, ohne allerdings die Zuordnung nachhaltig zu stören.

Stellungsgesetze: das Skopuselement, auch wenn es mehrgliedrig ist, muß in jedem Falle topikalisiert sein. Diese Möglichkeit besteht in Reinform eigentlich nur im Aussagesatz. Die genannten GPn folgen meist in Distanzstellung, und zwar am Satzschluß bei synthetischen Verbformen, bei analytischen Verbformen in einer Position vor dem infiniten Verbteil. Probleme ergeben sich in jedem Fall dort, wo die GP durch Tilgungen bzw. durch Konstituentensatzwortstellung unmittelbar nach dem betonten Skopuselement zu stehen kammt: so etwa in (8), (22) und (23). Dies mag rein phonetische Grinde (2 schwere Betonungen unmittelbar nebeneinander) haben.

Satzarten: Fast alle Belegsätze sind Aussagesätze. Daneben kommt in (8) noch die Konstituentensatzwortfolge vor. Sie birgt gewisse Probleme durch die Stellung des finiten Verbums am Satzschluß: dadurch rücken Skopuselement und GP 
meist sehr eng aneinander, wie etwa in den folgenden Fragesätzen:

(1a) Haben $z$ werge $\underline{a u c h}$ klein angefangen.

(11a) ?wollte er sich üb e rord nen a $u c h$ ?

(8a) Wir sind a $\underline{\mathrm{a} C h}$ sehr daran interessiert.

(8b) Warum sind wi $\underline{a u c h}$ sehr daran interessiert?

(9a) Wann kann uns de $r$ (denn) $\underline{a} u c h$ helfen?

Alle Frageformen rücken Skopuswort und GP sehr eng aneinander (abgesehen von der Vergewisserungsfrage (8a)): i.d.R. wird dies wohl vermieden, um die "Zweigipfeligkeit" und die dazwischen automatisch entstehende Pause zu überwinden: dies kann auch durch "Füllwörter", meist MPn, dazwischen bzw. durch normale Aussagesatzwortstellung und Frageintonation erreicht werden. Imperativ:

(3a) Nimm auf die öffentlichkeit $\underline{a u c h}$ keine Rücksicht!

(13a) $T r i n k$ a $u c h$ !

Die Schwierigkeiten liegen hier darin, daß das betonte auch i.d.R. wohl auf das implizite Subjekt des Imperativsatzes bezogen wird (= sein Skopus) und nicht auf die meist ummittelbar davorstehende nominale oder verbale Konstituente (da Verben in diesem Falle nicht Skopuskonstituente sein können; hierzu miißte nachgestelltes auch unbetont bleiben).

Spaltsatzbildung erbringt bei diesem Konstruktionstyp keinerlei akzeptable Ergebnisse, schon wegen des Widerspruchs in der Semantik von betontem auch (das ja ausschließlich quantifizierende Funktion aufweist) und der Funktion des Spaltsatzes.

Negationsmöglichkeiten:

TYP 1 (NEG vor der GP) ist, wie allgemein bei auch und sogar, so auch bei betontem auch nicht möglich. Kein Gegenbeweis sind Kombinationen wie nicht a $u c h$ in Fragesätzen, die nur durch die Umstellung des auch entstehen:

(24) Warum hast du nicht a $\underline{a} c h$ gebrüllt?

(25) Das kann ich doch nicht a $\underline{a} \mathrm{ch}$ noch tun!

Typ 2: NEG unmittelbar nach betontem auch. Beispiele für diese normale Form der Negation in Sätzen mit betontem auch sind (3), (5), (6), (7) und (20). Es handelt sich um eine nicht PSP-neutrale Form der Negation. Die semantische Interpretation von solchen Sätzen entspricht völlig der von Sätzen mit unbetontem auch $+n i c h t$, nur entfällt die bei dieser Konstellation häufige Möglichkeit skalierender Interpretation.

Typ 3: Satznegation. Eine von Typ 2 unterschiedene Form der Negation konnte ich nirgends feststellen. Dieser Negationstyp 2 entspricht voll und ganz der normalen Satznegation bei unbetontem auch. 
Typ 4: Lokale Negation, Kontrastnegation. Offenbar kann die Kontrastnegation jeweils nur die Skopuskonstituente des betonten auch betreffen:

(1b) Nicht $z$ we $r g e$ haben $\underline{a u c h}$ klein angefangen, sondern ... Dies ist aber nicht der normale Fall der Kontrastnegation, sondern eine metasprachliche Form der Negation, die meist nur den Wortlaut betrifft.

Skopus und Fokus

In der wahl des Skopus ist betontes auch viel stärker eingeschränkt als normales auch. Nicht völlig ausgeschlossen sind zwar komplexe NPn, aber ihr Akzeptabilitätsgrad schwankt beträchtlich: siehe etwa Satz (2) und (3). Finite Verbformen scheiden völlig aus als Skopus; an ihre Stelle können aber infinite Ersatzformulierungen treten, siehe etwa (11), (12) in beiden Formen, (13) und (15). Etwas anders gelagert ist Satz (14), insofern es sich hier um die Kopula handelt, und damit nicht um eine bestimmte Verbsemantik, sondern um die Tatsächlichkeit des behaupteten Inhalts. - Schwierigkeiten bereiten auch Konstituentensätze im Skopus sowie nominale Skopuskonstituenten, die Konstituentensätze enthalten, offenbar wegen der dann in jedem Fall ungewöhnlichen Topikalisierung und wegen der in solchen Fällen auftretenden Schwierigkeiten bei der Akzentverteilung. Überhaupt gehen die meisten der Beschränkungen auf das Konto des Konstruktionstyps Topikalisierung.

Ausgeschieden werden auch alle Skopustypen, die nur skalierende Interpretation zulassen. Ein besonders deutliches Beispiel liegt in (4) vor, ferner bei (19) und (21). Dies ist der deutlichste Beweis, dais diese Konstruktion ausschließl1ch quantifizierende Interpretation zuläßt. - Daneben steht aber auch eine sehr erstaunliche Ausweitung der Möglichkeiten: Satz (10) würde, falls man ihn als akzeptabel einstufen kann, bedeuten, daß auch indefinite Pronomina Skopus sein können. Möglicherweise ist dies aber auf referentielle Indefinitpronomina beschränkt; doch auch für diese findet sich keine Parallele bei umbetontem auch. - Die Skopusgrenzen sind in dieser Konstruktion nur sehr schwer durch die Kombination von Stellung (der GP wie der Skopuskonstituente) und Akzentlage zu bestimmen. Vielleicht führt deshalb jede Abweichung von der absoluten Erststellung der Skopuskonstituente zu einem wechselnden Grad von Inakzeptabilität. In aller Regel wird aber das Problem durch explizite "Kontrast"-Vorgängersätze entschärft, die eine eindeutige Interpretation emöglichen.

Die semantische Analyse wurde bereits bei den einzelnen Skopustypen geleistet. Festzuhalten ist nur noch einmal, daß dieser Konstruktionstyp nur quantifizierende Interpretation zuläßt. 


\subsubsection{3. sogar - Gruppe}

Beim Skopustyp Gliedsatz finden sich zwar Beispiele, in denen der Platzhalter fehlt, die Betonung der GP ist aber in keinem Fall erkennbar vorgeschrieben, wahrscheinlich sogar nicht einmal möglich. Unter diesen Voraussetzungen werden die folgenden Beispielsätze nur als Entscheidungshilfe beigegeben.

(1) Sogar di e je nig e n, die bislang ständig SPD wählten, schwenkten angesichts wirtschaftlicher Schwierigkeiten ins konservative Lager.

(2) ?Sogar, die bislang SPD wählten, schwenkten ... ins konservative Lager.

(3) Sie weiß sogar, wer Einstein war.

(4) Ich sage nicht einmal, daß Israel ein ausgesprochen deutscher staat ist, obwohl es gute Gründe dafür gibt.

(5) Selbst, wenn Komarows Flug geglückt wäre, er hätte den gewaltigen Vorsprung der Amerikaner kaum verringern können.

(6) Sogar, als ich auf Mallorca war, hat es geregnet.

In allen diesen Beispielen ist eine expressive Hervorhebung der GPn das Übliche, eine wirkliche Betonung liegt aber nicht vor.

Mit den weiteren Typen von Kontrastbetonung in der nur - sowie auch - Gruppe vergleichbare Konstruktionen treten in der sogar - Gruppe nicht auf. Zu beachten ist, daß betontes selbst nur die Funktion des Reflexivums ausfüllen kann.

4.1.2. "Bindestrich-Zusammensetzungen" mit Gradpartikeln

\subsubsection{1. nur - Gruppe}

$$
\begin{aligned}
& \text {... kapselten sie sich von der Republik ab und flüchteten sich in eine } \\
& \text { Nur-Ideologie. }
\end{aligned}
$$

Bei diesen Bildungen mit nur handelt es sich um ein sehr produktives Muster. Erstaunlich ist, daß lediglich nur von dieser GP-Gruppe zugelassen ist, obwoinl die bei allen diesen Bildungen vorherrschende quantifizierende Interpretation von jedem Element der Gruppe erfüllt werden kann. Wörter wie Alzeinvertretungsanspruch gehören nicht diesem Typus an, da sie feste Verknüpfungen sind (die außerdem meist nicht eindeutig einer ganz bestimten Paraphrase zuzuordnen sind) und nicht ad-hoc-Bildungen, wie die Zusammensetzungen mit nur.

Das in diesem Zusammenhang allein interessierende Thema ist die semantische Analyse solcher Konstruktionen. Auf Anhieb kann man feststellen, daß sie nicht 
mit der Konstruktion nur + Skopus NP bedeutungsgleich sind, etwa bei Nur Wissenschaftzer:

Nur ein $w i s$ s e $n$ s $h$ a $f t l$ e $r$ kann dieses Problem sachgerecht lösen.

$$
\text { Ex ist nur ein } w i s s \text { n } s c h \text { a } f t l \text { e } r \text {, kein Millionär. }
$$

Er ist nur/stets/immer nur/ausschließlich/einzig und allein $W i s$ $s$ e n s chaft le $r$, nichts sonst/nie etwas anderes.

Lediglich Satz (4) könnte bedeutungsgleich mit Nur-Wissenschaftzer sein. Ein gewisser Unterschied besteht lediglich darin, daß (4) auch bzw. vorwiegend bewertungsneutral ist, während Nur-Wissenschaftler uberwiegend die negative Wertung 'Einseitigkeit' vermittelt. Die Interpretation entspricht also weitgehend dem quantifizierenden Gebrauch von nur mit dem Skopus prädikatives Nomen, die skalierende Interpretation kann mit Sicherheit ausgeschlossen werden. Dafür sprechen auch die geläufigen wendungen wie Nur-Hausfrau, Nur-Funktionär, Nur-Ehemann usw. Selbstverständlich können explizite PSP- und ASS-Formulierungen nur auf der Basis der oben in Satz (4) wahrscheinlich gemachten Paraphrase gebildet werden, entsprechen also den beim Abschnitt "Skopustyp prädikatives Nomen" vorgeschlagenen quantifizierenden Analysen (siehe 3.3.3.1. S. 187-196).

\subsubsection{2. auch - Gruppe}

Bildungen wie Auch-Dichter, Auch-Regisseur, Auch-Komponisten, Auch-Künstler, Auch-Maler, Auch-Sportler, Auch-Verbündete, Auch-Christen usw. sind wohl mindestens ebenso beliebt wie die entsprechenden Konstruktionen mit nur. Mit diesen teilen sie auch alle formalen Eigenschaften: es sind ad-hoc-Bildungen, und der Einsatz von bedeutungsgleichen Partikeln ist ausgeschlossen. Wie die nur Konstruktionen entsprechen sie auch mit dem Skopus prädikatives Nomen, und zwar in quantifizierender Interpretation. Für das Beispiel Auch-Maler:

(1) Charly ist ein widerlicher Auch-Maler.

(2) Charly ist auch ein $M a l$ e $r$ (und nicht $n$ u $r$ ein Maler).

\subsubsection{3. sogar - Gruppe}

Nach dem eben zur Semantik der Bindestrich-Zusammensetzungen mit nur und auch Gesagten kann es nicht ijberraschen, daß dergleichen bei den Elementen der sogar - Gruppe nicht möglich ist. Die Ursache hierfür ist die Festlegung der gesamten Gruppe auf skalierende Interpretation. 
4.1.3. Das inuftreten von mehreren Gradpartikeln in einem einzigen Satz

Die Frage, ob in einem Satz mehrere GPn auftreten können, hat seit Beginn der GP-Diskussion mit Kuroda (1969: 339) eine wichtige Rolle gespielt, ${ }^{2}$ wie ich glaube eine in vielen Fällen unglückliche wenn nicht verderbliche Rolle. Aus der Inakzeptabilität einiger konstruierter Beispiele hat Kuroda geschlossen, daß jeweils nur eine einzige GP pro Satz akzeptabel ist, und auf diese These baute er seine ganze formale Darstellung im Rahmen des Aspects-Modells auf, die sehr stark an die Behandlung der Negation im gleichen theoretischen Rahmen angenähert ist: das entscheidende Problem ist es ja, von Anfang an zu verhindern, daß mehr als eine GP in einem Satz erzeugt wird. Dies wird nicht dadurch erreicht, daß man vermittels Phrasenstrukturregeln GPn bei allen jenen Konstituenten erzeugt, die Skopus einer GP sein können, sonderm indem man generell eine Leerstelle für das GP-Symbol vorsieht, in der sie fakultativ erzeugt werden, und die GP dann transformationell der jeweiligen Skopuskonstituente zuordnet. Diese syntaktische Lösung führt zu vielen Ungereimtheiten und äußerst komplizierten Regelsystemen, von der Unzulänglichkeit der damit erzeugbaren Morphemketten gar nicht zu reden.

Die Annahme von Kuroda wurde erst von Anderson und Horn in Frage gestellt. Auf beide muß ich etwas näher eingehen. - Horn (1969; 1972: 224 f.) demonstriert anhand des auch für ihn nicht ganz akzeptablen Beispieles

(1) only $J \circ h n$ eats only $r i c e$.

daß derartige Sätze eine durchaus konsistente Interpretation ermöglichen (in beiden Fällen quantifizierende Interpretation!), daß sie aber bei Spaltsatztransformationen, die den obliquen Kasus betreffen, inakzeptabel werden; dies konnte er allerdings nicht erklären, wenn er auch die Verbindung zu Fragen der Topic-Comment-Gliederung des Satzes schon durchaus registrierte. - Ganz ähnlich argumentiert Anderson (1972: 893 und 902-905). Er zeigt, daß die Interpretation von

(2) Even $J \circ n$ e $s$ hates even Mi 11 a $r d \quad F i l l m o r e$. zu Widersprüchen führt, daß es aber bestinmte Situationen gibt, in denen dieser Satz eine durchaus konsistente Interpretation erhalten kann. Er schließt daraus, daß eine Lösung via Beschränkung der Vorkammen von GPn bereits in der Tiefenstruktur auf ein Exemplar pro Satz keineswegs das Problem aufklärt. Bezeich-

2 Vergleiche auch N. Chomsky (1969; 1972: 111); R. S. Jackendoff (1972: 250); L. Horn (1969; 1972: 224 f. und 228); B. Fraser (1971: 163 f.); S. R. Anderson (1972: 893 und 902-905). 
nend ist auch, daß sein Beispiel für die Verträglichkeit mehrerer GPn in einem Satz aus der Rekonstruktion einer Tilgung entsteht (Anderson 1972: 902):

(3) Jones beats even his wife, and she eyen encourages him to do so (so = to beat even his wife)

Hier stimmt aber schon die Grundannahme nicht: nämlich, daß in der getilgten Konstituente (bzw. in der pronominal vertretenen Konstituente) grundsätzlich alle Elemente vertreten sein müßten, die in der korrelierenden Einheit vorhanden sind. Dies müßte für die GPn erst einmal nachgewiesen werden.

(4) Stell dir vor, Hans prügelt sogar seine $F r a u$; und die verrückte Nudel e rmutigt ihn $\underline{\text { sogar }}$ noch dazu = sie/seine Frau ("sogar) zu prügeln.

Sogar stellt in diesem Falle eine von der reinen Information des Satzes zu trennende skalierende Stellungnahme des Sprechers dar; die Tilgung entspricht nur der Information, sie enthält nicht die Stellungnahme des Sprechers. Ganz ähnliche Fälle treten bei performativen Formeln auf; auch dies ist ein Argument dafür, daß die GP nicht Teil ihrer Skopuskonstituente wird.

B. Fraser (1971: 163 f.) führt das angebliche Verbot von mehreren GPn je Satz auf reine Ausgabe-Bedingungen zurück; er hält diese Sätze für schwer interpretierbar. Damit spielt er auf die von Chonsky für die Inakzeptabilität bestimmter Verzweigungstypen verantwortlich gemachte "begrenzte Kapazität" des menschlichen Gehirns/Gedächtnisses für bestimmte Operationen an. Andererseits erklärt er die Inakzeptabilität eines Satzes wie

(5) 'Even only Max will be there tonight.

mit semantischen Widersprüchen.

Gerade angesichts dieser Forschungssituation möchte ich noch einmal nachdrücklich auf meinen im theoretischen Vorspann dargelegten Prinzipien der Sprachanalyse insistieren: ich beschränke mich, um möglichst Fehlerquellen durch meine linguistisch verderbte Kompetenz auszuschließen, auf belegte Kambinationen von GPn und versuche, sie zu interpretieren. Aus diesem Grund muß ich für diesen Fall natürlich einige Lücken in Kauf nehmen. Unter den folgenden Beispielen sind auch einige, in denen eine GP in einer anderen Funktion vorliegt (z.B. MP). Sie werden zu Vergleichszwecken beigegeben.

(6) In keinem Fall konnte der Nachweis einer auch nur a $n g$ e de u t e$t e n$ Funktionswiederkehr festgestellt werden.

(7) Die Quote liegt sogar nur bei e in e m Prozent.

(8) Wer kann das auch nur wo 11 e $n$ ?

(9) $\underline{\text { Auch }}$ (!) wurde ja nur die $E$ h e geschieden.

(10) Und sogar $d i$ e's e Indizien machten im Fall des Arwed Imiela nur die Uberzeugung des Schwurgerichts tragfähig, kein anderer komme in Frage. 
(11) Er ist ja sehr nett. Nur (!) hat er in letzter zeit nur $P$ e $h$ gehabt.

(12) Grundsätzlich wird das elektronische Medium lediglich bei $k$ a $p$ i t $a l v e r b r e c h$ e $n$ und auch da $n$ a $\frac{n u r}{u n g e s c h a l t e t,}$ wenn der Tatverdächtige bereits ermittelt ist, und von ihm...

(13) Ich bin a $u c h$ nur ein $M$ e $n s c h$.

(14) Hans-Joachim Bäuchle hat auch d a $f \ddot{u} r$ nur ein $A c h s$ e 1 $z u c k$ e $n$.

(15) Fritz hat sogar nur e i n e $n$ Fehler gemacht.

(16) Die Gäste aßen sogar nicht einmal den $K u c h$ e $n$.

(17) ... das Druckhaus, dem nach der Auszehrung lediglich ein selbst für $R$ e g e $n$ s b u $r g$ e $r$ Verhältnisse veralteter Maschinenpark verblieben war.

Ich möchte nun so vorgehen, daß ich die Beispiele einzeln untersuche und erst zum Schluß einen Überblick gebe.

Satz (6) ist eines der Beispiele mit Kontaktstellung der beiden GPn. Bei ihnen ist die Abgrenzung des jeweiligen Skopus besonders schwer. Zunächst zur jeweiligen Funktion der beiden Partikeln: die Ersetzbarkeit von nur ausschließlich durch $b 20 \beta$ deutet darauf hin, daß skalierende Interpretation vorliegt. auch wiederum kann (bedeutungsneutral) nur durch sogar ersetzt werden. Es liegt also ebenfalls in der für dieses Element nicht gar zu häufigen skalierenden Funktion vor. nur dirfte stellungsstabil sein, auch kann ev. auch die Position vor einer, also vor dem Genitivattribut, einnehmen. Damit kann man die Skopi. folgendermaßen abgrenzen: der Skopus von auch umfaßt das gesamte Genitiv-Attribut, nur bezieht sich lediglich auf angedeuteten. Dies kann die folgende Paraphrase verdeutlichen:

(6a) In keinem Fall konnte der Nachweis auch/sogar einer Funktionswiederkehr, die nur a $n g$ e $d$ e u $t$ e $t$ ist, festgestellt werden.

Sind die beiden GPn getrennt, so kann nur auch quantifizierend interpretiert werden. Insofern wirkt also auch disambiguierend. Dariberhinaus ergänzen sich beide Partikeln in ihren semantischen Funktionen: was auch in skalierender Interpretation assertiert, das präsupponiert nur, und was skalierendes auch präsupponiert, das assertiert skalierendes nur: damit wird die Skalenrichtung und der (nicht zutreffende) allerunterste Skalenwert zweifelsfrei festgelegt.

Satz (7) und (8) entsprechen völlig dieser Konstellation, doch treten bei ihnen auch noch Sonderprobleme auf. In (7) kann sogar deshalb nicht durch auch ersetzt werden, weil letzteres in diesem Satz ohne Negation nicht in skalierender Funktion auftreten kann. Bei (8) kann man nicht restlos sicher sein, ob auch nicht in MP-Funktion vorliegt, übrigens wäre das auch für nur möglich: liegt jeweils nur eine GP vor, so ist der Satz ambig zwischen GP- und MP-Ver- 
wendung. Ein überzeugendes Entscheidungsverfahren ist mir leider nicht bekannt.

Bei Satz (9) zeigt die Austauschbarkeit von auch gegen fermer, außerdem usw., daß dieses Element in der konjunktionalen Funktion vorliegt.

Satz (10) ist das erste Beispiel, in dem zwei GPn deutlich getrennte Skopi haben. Wichtig ist hierbei, daß es sich um völlig verschiedene Skopustypen handelt und daß die Einheiten GP + Skopus durch mehrere Konstituenten getrennt sind, sowie, daß nur einmal ein deutlicher Kontrastakzent auftritt.

Satz (11) weist wieder ein nur in konjunktionaler Verwendung auf, sowie ein nur in GP-Funktion.

Satz (12) ist ein weiteres Beispiel für deutlich getrennte Skopi, wobei einmal Kontrastakzent auf dann und dann "Ersatzbetonung" auf nur vorliegt. Im Prinzip handelt es sich um den gleichen Fall wie bei Satz (6): auch wird skalierend verwendet, nur hingegen wird quantifizierend interpretiert, wie die Austauschklasse zeigt. Die Paraphrase könnte lauten:

(12a) ... und auch in $d i$ e s e $m$ Falle nux $d$ a $n n$ eingeschaltet... Noch schöner trennbar sind die jeweiligen Skopi bei Satz (13): der Skopus des nachgestellten betonten auch (quant.) ist ich, der von nur (in skalierender Funktion) ist ein Mensch.

Die Interpretation von (14) entspricht der von (12) bzw. (6), letzteres insofern, als sowohl auch als auch nur in skal. Funktion vorliegen.

Bei Satz (15) tritt wieder Amalgamierung der Partikelfunktionen bei Kontaktstellung mit identischen Skopus ein. Dabei bleibt die Funktion von sogar unbestimmt, da der gleiche Zweck auch von nur allein erfüllt werden kann. Bei nur allein könnte man allerdings auch dem Irrtum verfallen, die Skalenrichtung verliefe von "unten" nach "oben", mehr Fehler wären also besser. Diese Interpretation ist nach der Hinzufügung von sogar allerdings nicht mehr möglich.

Mysteriös ist Satz (16). Sogar und nicht einmal können hier nicht denselben Skopus haben, da sie sich sonst gegenseitig aufheben wïrden (da nicht einmal die nicht PSP-neutraie Negation von sogar ist). Man könnte folgern, daß sogar hier den Skopus Satz aufweist, nicht einmal hingegen den Skopus Kushen. Damit könnten zwei voneinander getrennte Skalen existieren.

(16a) Es ist sogar $s o$, daß die Gäste nicht einmal den $K$ u $c h$ e $n$ aßen. Satz (17) ist ein besonders schönes Beispiel für ineinandergeschachtelte Skopi. Während lediglich als Skopus ein für Regensburger Verhältnisse veralteter Maschinenpark hat, gehört zu selbst die Phrase ein für Regensburger Verhältnisse. Der Satz könnte damit folgendermaßen paraphrasiert werden:

(17a) ... dem lediglich ein Maschinenpark, der selbst für $R$ eg e n s b u r $g$ e $r$ verhältnisse veraltet war, verblieben war. 
Damit sind auch die Grundlinien erarbeitet:

1. Es existieren semantische Unverträglichkeiten zwischen einzelnen GPn, und zwar insbesondere dann, wenn sie quantifizierend gebraucht werden. Diese Widersprüchlichkeiten könnten wohl nur von Fall zu Fall aufgedeckt und eliminiert werden. Mit generellen Kombinationsbeschränkungen kann man inakzeptable Kombinationen nicht von vornherein ausschließen.

2. GPn können durchaus bei gleichem oder teilidentischem Skopus ergänzende Funktionen ibernehmen, allerdings offenbar nur, wenn zumindest eine GP in skalierender Funktion vorliegt. Nur mit derartigen semantischen Gründen hängt wohl auch die Regelung der Reihenfolge der GPn untereinander zusarmen.

3. GPn können im gleichen Satz verschiedene Skopi aufweisen, wenn diese deutlich typverschieden sind, und wenn nur einmal Kontrastakzent vorliegt; dabei könnte es sich sowohl um bloße Ausgabebedingungen als auch um tiefgreifende Regularitäten der Topic-Comment-Gliederung bzw. der Verwendung des kontrastiven Fokus handeln. In allen Konstellationen sind aber rein syntaktische Lösungen völlig unzureichend und unangemessen.

Die Auswertung der Belegsätze für GP-Kombinationen hat also einerseits zwei Ergebnisse der englischsprachigen GP-Forschung bestätigt: nämlich daß mehrere GPn mit typgleichem aber getrenntem Skopus in einem Satz inakzeptabel sind, und daß es semantische Unverträglichkeiten zwischen verschiedenen GPn gibt. Die Materialauswertung ermöglichte aber auch die Widerlegung einzelner Behauptungen bzw. die Formulierung neuer Regularitäten. So konnte festgestellt werden, daß alle GPn grundsätzlich miteinander kombinierbar sind, sei es nun mit identischem Skopus oder mit Skopusiuberlagerung oder mit getrennten typverschiedenen Skopi. Die Beschränkungen resultieren aus der Partikelsemantik und aus der Beschränkung des Kontrastfokus auf ein Exemplar pro Satz. Die Funktionsbeschreibung von Partikelkombinationen wurde schließlich erst durch die semantische Analyse der einzelnen GPn mit Hilfe der Begriffe 'quantifizierend' und 'skalierend' möglich. - Darauf aufbauend erscheint die Darstellungsproblematik in einem neuen Licht: die Beschränkung auf eine GP pro Satz ist nicht mehr notwendig. Die Lösung Kurodas (1969) mit Zuordnungstransformationen geht von falschen Voraussetzungen aus und beschreibt umgekehrt nicht alle tatsächlich vorhandenen Möglichkeiten, jedenfalls für das Deutsche. Der Ansatz Jackendoffs (1972: 250), die GP-Problematik mit der Frage der Fokuszuweisung zu verknüpfen, stimmt für einen weiten Bereich von Erscheinungen. Er scheitert aber an zwei Faktoren: an der Tatsache, daß mehrere GPn denselben Skopus haben künnen, und daß der Kontrastfokus einmal eine relativ selbständige Erscheinung (bezüglich des GP-Skopus) und andererseits vom Normalfokus deutlich zu trennen ist. - Insgesamt 
ist aber durch meine Beschreibungsansätze das Bild sehr viel komplexer geworden, und zwar durch die Hereinnahme der zahlreichen pragmatischen Faktoren, die im Einzelfall eine ganz bestimmte Interpretation sichern. Andererseits wïrde die Semantik der GPn allein nur einen sehr allgemeinen Rahmen abgeben, der nicht sehr viel über die spezifischen Eigenschaften natürlicher Sprachen aussagen wïrde.

\subsubsection{Kombinationen von Modalpartikeln mit Gradpartikeln}

Wie schon im vorigen Kapitel und bei vielen Anlässen in früheren Kapiteln erwähnt, bringt die Frage der sauberen Trennung zwischen der MP- und der GP-Funktion bei einigen der hier behandelten Partikeln einige Probleme mit sich. Um Anschauungsmaterial für die MP-Diskussion zu gewinnen, werden hier einige Beispiele für Kontaktstellung von MPn und GPn gebracht, darunter auch einige, die ambig bzw. unentscheidbar zu sein scheinen. Um größere Klarheit zu gewinnen, wurden auch Beispiele aufgenommen, die MPn enthalten, die nicht in GP-Funktion auftreten können.

(1) Denn Hammann war sicher, daß die Behörden in solchen Fällen doch nur $d e m$ e $n$ t i e $r$ e $n$.

(2) ... Aussagen, deren widersprüchlichkeit ex letztlich doch nur auf sich $b$ e $r$ u h e $n$ lassen kann.

(3) Wer kann das auch nur wo 11 e $n$ ?

(4) Ein lieber Kerl. Auch wurde ja nur die $E$ h e geschieden.

(5) Ge h doch nur! / $\underline{e}$ eh halt nur!

(6) Denn wir glauben ja auch an die Güte der deutschen Literatur trotz so mancher Dichterlinge.

(7) Sie kommt indessen nicht, sie ist ja auch mit ihrer Tochter Urte auf Reisen.

(8) Ließe er sich denn auch besser erklären, wenn man ihn unter die gleichsam "normalen" deutschen Kanzler einordnen würde?

(9) Dennoch wird die Titelgeschichte, die nur eben keine Titelgeschichte mehr ist, in diesem Heft veröffentlicht.

(10) Denn sie sind leicht zu verdauen, man schluckt sie nur eben/eben nur hinunter.

Dann geh'ich eben a uch zum Bäcker/eben auch zum B äck e r.

Auch hier möchte ich wieder zu den einzelnen Belegsätzen Stellung nehmen und dann eine Zusammenfassung der Regularitäten geben.

Satz (1) läßt kontrastive Vorgänger- und Folgesätze zu, die lediglich die Skopuskonstituente von nur kontrastieren bzw. variieren. Da doch in dieser Stel- 
lung nur als MP möglich ist, gibt es keine Interpretationsunsicherheiten. Außerdem kann nur in dieser Stellung regulär durch sowohl quantifizierende wie skalierende Elemente seiner Gruppe ersetzt werden.

Satz (2) entspricht Satz (1) weitgehend, nur sind Kontrastsätze nicht ganz so leicht zu formulieren, da der Skopusbereich von nur unterschiedlich groß sein kann und in jedem Falle nur global quantifizierende Assertionen (explizit ev. in Vorgängersätzen) formuliert werden können:

(2a) Gar nichts kann man tun, man kann es doch nur auf sich b e $r$ u h e $n$ lassen.

Satz (3) entspricht der Nummer (8) im vorhergehenden Abschnitt. Die Problematik ist dort bereits geschildert: beide Partikeln sind in dieser Satzform als MP und als GP möglich.

Satz (4) vereinigt in sich auch in konjunktionaler Funktion, $j a$ in MP-Funktion, und nur als GP mit den möglichen Skopi die Ehe (letzteres betont) bzw. die Ehe geschieden (ebenfalls Ehe betont), was durch entsprechende Folgesätze mit aber nicht demonstriert werden kann.

Ein Beispiel mit ambigem nur ist Satz (5). Doch kann zwar nur als MP auftreten, aber es sieht so aus, als ob auch 2 MPn pro Satz möglich wären:

(5a) Geh doch nur, ich halte dich nicht auf. (nur = MP)

(5b) Geh doch nur, fahr nie mehr mit dem Auto. (nur = quant. GP)

(5c) Geh doch nur, laufe/renne doch nicht immer. (nur = skal. GP)

Satz (6) besitzt mit ja eine zweifelsfreie MP. auch ist entweder GP, und zwar mit dem Skopus Güte, oder mit dem Skopus Güte der deutschen Literatur. Ferner möchte ich konjunktionale Verwendung nicht völlig ausschließen, wenn sie auch angesichts des cienn unwahrscheinlich sein muß. Die quant. GP-Funktion kann durch Topikalisierung der Skopuskonstituente sowie Distanzstellung und Betonung von auch nachgewiesen werden.

Ganz ähnlich ist Satz (7) gelagert. Hier ist allerdings die GP-Interpretation etwas unwahrscheinlicher, die konjunktionale Verwendung dagegen wesentlich wahrscheinlicher, sie kann in diesem Fall zudem durch Spitzenstellung und Ersatz durch darïberhinaus usw. plausibel gemacht werden.

In Satz (8) ist denn, wie üblicherweise in Satzfragen, MP (man könnte auch von einer Fragepartikel sprechen). Die Funktion von auch hingegen ist für mich nicht zweifelsfrei zu klären. Einwandfreie Kontrastformulierungen finde ich nicht, andererseits sind mir keine MP-Verwendungen von auch in derlei Satzformen geläufig, ohne daß ich andererseits den vorliegenden Satz als inakzeptabel einstufen könnte.

Satz (9) schließlich läßt nur folgende Interpretation zu: nur liegt, trotz 
Relativsatz, in konjunktionaler Verwendung vor, eben in MP-Funktion. Dazu stimmt auch die Form der Negation zwischen nur und der folgenden NP. Um die GP-Funktion von nur zu erzielen, müßte diese Partikel mit eben den Platz tauschen und die Negation beseitigt werden:

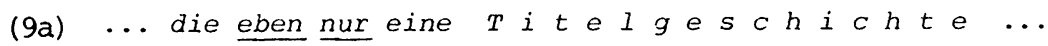

Bei Satz (10) halte ich die Stellungsvariante eben nur mit eben als $\mathbb{P}$ und nur als GP mit Skopus hinunterschlucken für wahrscheinlicher, ohne allerdings die Folge nur eben ausschließen zu können. In diesem Falle miißte nur wohl konjunktional interpretiert werden, da Kontrastsätze kaum denkbar sind.

Satz (11) endlich ist eine eindeutige Kombination von MP (eben) und GP (auch), allerdings je nach Akzentverteilung mit dem Skopus ich bzw. zum Bäcker. Damit kann man folgende Gesetzmäßigkeiten formulieren: Bei Kombinationen zwischen einer MP und einer GP darf nur diese Reihenfolge vorkommen. Das zweite Element ist also jeweils als GP zu interpretieren. Der jeweilige Skopus wird durch Stellung, Kontrastakzent bzw. Kontrastsätze bestimmt, und zwar ganz in der üblichen Art und Weise. - Bei Kombinationen zwischen GPn in konjunktionaler Verwendung und MPn muß wiederum diese Reihenfolge eingehalten werden. Natürlich kann die Konjunktion auch am Satzanfang stehen. - Daneben scheint es auch noch die Möglichkeit zur Kombination mehrerer MPn zu geben.

4.1.5. Feste Verbindungen von Gradpartikeln mit anderen Wörtern

4.1.5.1. nur - Gruppe

1. mit noch/schon:

(1) Die Industrie wird angewiesen, Radios nur noch o h n e UKW-Empfangsteile zu produzieren.

(2) Es war nur noch zu fragen, warum Arwed Imiela viex Frauen getötet hat.

(3) Wohl üben Polizeirekruten nur noch $s$ e $l$ te $n$ am $M G$, aber...

(4) Doch derlei Einbrüche geschehen lediglich $s c h$ u b w e i s e und $k \circ m p l i z i$ e $r$ e $n$ die ohnehin vertrackten Verhältnisse nur noch.

(5) Er kaufte sich eine Sepplhose, klebte sich einen Schnurrbart an und $j \circ d$ e $I$ t e nur noch.

(6) Daß eine Tariferhöhung allein schon aus te chnis $c h$ e $n$ Gründen frühestens am 25. Mai 1974 wirksam werden könnte, ...

Die Untersuchung der Austauschbarkeit fördert ein seltsames Ergebnis zu Tage: in den Fällen, in denen nur im Beispielsatz steht, können nur noch bloß und, mit einigen Bedenken, lediglich eingesetzt werden: 
(3a) Wohl üben Polizeirekruten nur/bloß/?lediglich/:allein/“einzig/“ausschließlich noch $s$ e 1 t en am MG.

Dieses Ergebnis könnte man, jedenfalls bei (3), auf die skal. Interpretation zurïckführen. Ein möglicherweise quantifizierendes Beispiel bringt aber keine völlige Klärung:

(2a) Es war nur/bloß/lediglich/?allein/:einzig/?ausschließlich/?einzig und allein noch $z u$ fragen, warum Arwed Imiela vier Frauen getötet hat.

Das legt den Schluß nahe, daß noch jeweils skal. Interpretation von nur erzwingt. Einen semantischen Widerspruch zwischen dem temporalen Aspekt von noch und dem möglichen temporalen Bedeutungselement bei ausschließlich kann man nur vermuten. Andererseits ist in dem Fall von allein + schon (Satz (6)) kein anderes Elernent dieser Partikel-Gruppe einsetzbar. Oder, um die Versuchsanordnung umzukehren, schon ist nicht nach nur und noch nicht nach alleinmöglich. Die GP-Funktion von allein in dieser Funktion steht allerdings nicht zweifelsfrei fest.

Die Normalposition für diese Verbindung ist die nach dem finiten Verb, aber auch alle anderen, für die jeweiligen Skopustypen geltenden Positionen von nur sind möglich. In jedem Fall muß die Reihenfolge nur + noch erhalten bleiben. Im Imperativ wird eine spezielle Funktion dieser Kombination offenbar: es ist nur noch die GP-Interpretation von nur, nicht mehr die MP-Interpretation möglich. Aus nicht weiter identifizierbaren Gründen scheidet die Negationsform nicht nur noch, obwohl nicht eigentlich falsch oder inakzeptabel, aus. Bei der Negationsform nur noch nicht wechselt nur, wie auch sonst bei dieser Negationsform, zur konjunktionalen Verwendung. - Alle diese formalen Eigenschaften (die semantischen entsprechen denen bei den jeweiligen Skopustypen von nur) lassen nur den Schluß zu, daß es sich um eine idiomatisierte Wendung handelt.

2. mit zu/alzzu:

(7) Er ist nur/“allein/“einzig/“oloß ... z u gerne bereit.

(8) Mit nur $z$ u großer Lust...

(9) ... so abstrakt, daß es Hitler nur a 1 I z u leicht fiel, es für seine zwecke ...

Wahrscheinlich handelt es sich bei dieser Partikelverbindung ebenfalls um ein Idiom; die Unmöglichkeit eines Austausches von nur spricht jedenfalls dafür. Bliebe noch zu fragen, ob nur irgendeine seiner üblichen Funktionen behält: die für die GP-Verwendung typischen Kontrastsätze mit nicht können nicht befriedigen, MP- und konjunktionale Funktion können ausgeschlossen werden. Möglich wäre höchstens noch die Erklärung als Form des indirekten Sprechens. - Der Versuch, die Idiomatizität von nur zu (oder auch nur noch) durch die Unmöglich- 
keit, eines der beiden Elemente wegzustreichen, zu erweisen, führt leider zu keinem eindeutigen Ergebnis:

(10) "Sein Verdacht erwies sich ols $\underline{z} \underline{u}$ begründet.

(9a) ... so abstrakt, daß es Hitler allzu leicht fiel ...

(5a) :Er kaufte sich eine Sepplhose und jodelte noch.

Damit beweist man offenkundig nur, daß die Einzelelemente deutlich andere Funktion haben als die Kombination.

3. immer nur:

(11) Bisher sind die alten Leute immer nur/"allein/"einzig/bloß/ausschließlich/'einzig und allein auf den $B$ e r g geklettert.

Auffällig ist hier die Unsicherheit bei ausschließlich, das auch allein die Bedeutung von immer nur haben kann. Platztausch zwischen nur und immer ist möglich, ebenso die Trennung, wenn auch meist mit gewissen semantischen Verschiebungen.

4.1.5.2. Feste Verbindungen mit auch

1. wenn auch:

ICh habe ... gesagt, es muß 1973 eine Steuererhöhung stattfinden. Das hat auch jeder - wenn $\underline{\text { auch }} z \ddot{a} h n$ e $k n i r s c h e n d$ - uns abgenommen.

(2) Die gesamte Nation schämt sich wegen Vietnam, wenn auch aus sehr un $t$ e $r s c h i$ e $l i c h$ e $n$ Gründen.

(3) Die finanzielle Lage bessert sich, wenn sie auch un üb e r sicht 1 i $c h$ bleibt.

(4) Wenn er auch dum $\mathrm{m}$ ist, das wird er doch wenigstens begreifen.

Man könnte glauben, daß es sich bei wenn auch um eine Verkürzung mit umstellung des auch handelt:

(4a) Auch (d a $n n$ ), wenn er dumm ist ...

Diese Umformung ist weitgehend bedeutungsneutral. Bei den mehr parenthetischen Formulierungen wie z.B. in (1) existiert eine derartige Möglichkeit jedoch nicht. Die Konjunktion wenn hat hier auch nicht die ubbliche konditionale Bedeutung, sondern eher einräumende/konzessive Bedeutung. Für eine idiomatische Betrachtung spricht auch, daß Kontrastsätze mit nicht nur in den meisten Fällen nicht möglich sind, und daß die Nachstellung von betontem auch bei topikalisierten Skopuskonstituenten nicht möglich ist. Andererseits können die beiden Partikeln durch eine NP getrennt werden. 
2. denn auch:

(5) Der Hitler der Jahre zwischen 1933 und 1939 ließe sich denn auch besser erklären, wenn ...

(6) Auf diese Formel brachten denn auch unlängst Psychiater ihren Protest.

Die formelhafte Verbindung ist nicht trennbar und steht meist unmittelbar hinter dem finiten Verb, kann aber durch einzelne Konstituenten auch dawon getrennt werden. In allen übrigen Eigenschaften ist sie wenn auch sehr ähnlich. GP-Funktion läßt sich für auch nicht (mehr) zweifelsfrei nachweisen.

3. auch noch:

(7) Der Freistaat Bayern leistet sich neben der staatlichen Landpolizei auch noch eine staatliche $G r$ e $n z$ polizei.

(8) ... massenhafte Absagen von Skiläufern, die nicht auch noch zum d $x$ i $t$ $t$ e $n$ m a $l$ die Gefoppten sein möchten.

(9) Das fehlte auch noch/ $D$ as fehlte a $u$ ch noch.

Die hier gültigen Gesetzmäßigkeiten entsprechen denen bei nur noch: die GPFunktion ist in allen Beispielen unbezweifelbar. Bemerkenswert ist das nicht vor unbetontem auch in Satz (8). Es ist der einzige Fall von PSP-neutraler Negation bei auch, den ich bisher finden konnte. Die PSP des Satzes mit sowie ohne nicht in dieser Position lautet gleichermaßen:

(8a) Die Skifahrer waren das exste- und zweitemal die Gefoppten.

Die Umkehrmg noch auch, bei nur inakzeptabel, gibt es, wenn auch selten:

(10) Weder mein Großvater noch auch mein $v$ a $t$ e $r \ldots$

Sie ist zu paraphrasieren als mein Großvater nicht und auch nicht mein Vater, und damit wohl auch vollständig erklärt.

4. auch nur (vgl. auch 4.1.3.):

(11) Ohne einen solchen Einbruch in die persönliche Sphäre auch nur im $g$ e $r$ i $n g s$ t e $n$ zu rechtfertigen, sollten wir...

(12) ... Ludwig-Drama, mit dem der Regisseur Nostalgie und Morbidezza seines "Tod in Venedig" weder zu übertrumpfen noch auch nur e inzu ho 1 e $n$ verstand.

(13) Würden auch nur $z$ e h $n$ Prozent der Zufuhren gedrosselt ...

(14) Uberdenkt man auch nur $k$ urz die Aussage dieses Satzes...

Die GP-Funktion von nur ist in allen diesen Fällen unbezweifelbar, weil durch Kontrastsätze belegbar. Die von auch bleibt für mich mysteriös. Denkbar wären höchstens (für einige Beispiele) folgende Paraphrasen:

(13a) $\underline{\text { Auch }}$ da $n \mathrm{n}$, wenn $\underline{\text { nur }} \boldsymbol{z}$ e $h \mathrm{n}$ Prozent der Zufuhren... 
(14a) $\underline{\text { Auch }}$ dan $n$, wenn man nur $k$ ur $z$ die Aussage dieses Satzes ... Dies würde bedeuten, daß es sich um ein auch mit Satz- oder Gliedsatzskopus handelt, während nur einen NP-Skopus aufweist.

\subsubsection{Feste Verbindungen mit sogar}

Feste Verbindungen wie bei auch und nur gibt es nach meinen Beobachtungen bei sogar, selbst und nicht einmal nicht, wenn auch einige Äquivalente zu Kombinationen bei nur und auch, allerdings in unfester Form. Dafür seien hier einige Beispiele angefuhrt:

(1) Am Fünften kletterte das Thermometer sogar noch einmal auf 29.5 Grad, so $d a \beta \ldots$

(2) Unter Wissenschaftlern besteht noch nicht einmal Einvernehmen, wie ...

(3) Und Lufthansa-Vorstand Werner Utter weiß, daß ungeachtet offizieller Durchhalteparolen aus dem Verkehrsministerium sogar schon über eine $P r i v a t i s i$ e $r$ u $g$ intensiv verhande $\overline{l t}$ wird.

In allen diesen Fällen steht die GP-Funktion von sogar und nicht einmal fest. Bemerkenswert ist nur noch die Stellung von noch vor nicht einmal, während es nicht vor sogar stehen kann.

\subsection{Nicht-Gradpartikel-Funktionen der hier behandelten Partikeln}

4.2.1. Konjunktionale Verwendung ${ }^{3}$

\subsubsection{1. nur - Gruppe}

Typ 1: mit Inversion.

(1) Sie ist sehr intelligent, nur/bloß müßte sie ein wenig hübscher sein,

(2) Böl1: "Meine Taktik hat sich nicht geändert, nur ist sie dem Zeitpunkt angemessen."

(3) Du sollst den Schmerz nicht nähren, nur such einmal mein Grab!

(4) Da hast du bare fünfig Taler; nur unterlasse den Gesang!

Zur Syntax

Die übrigen Elemente dieser Gruppe sind in dieser Konstruktion nicht verwend-

3 Es empfiehlt sich, den Abschnitt über den Skopustyp Satz 3.7.3. S. 254 wegen der Nähe der Problemstellung heranzuziehen: 
bar. Hingegen gibt es eine Reihe von weiteren Austauschrmöglichkeiten, die die Zugehörigkeit von nur und $b l O \beta$ in dieser Verwendungsweise zu den Konjunktionen dokumentieren: doch, jedoch, hingegen usw.

In allen oben angeführten Beispielen findet sich nur äie satzeinleitende Stellung. Doch gibt es noch eine andere Position, die aber möglicherweise ambig ist, wie die Austauschmöglichkeiten vermuten lassen:

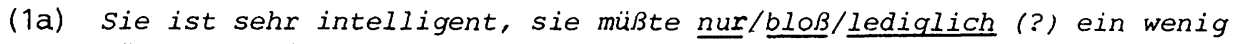
hübscher sein.

(2a) Meine Taktik hat sich nicht geändert, sie ist nur dem Zeitpunkt angemessen.

In diesem Falle muß also die konjunktional gebrauchte Partikel nach dem finiten Verb stehen. Auch in dieser Position besteht Austauschmöglichkeit mit den Konjunktionen jedoch, aber, hingegen usw. Daneben kann in dieser Position natürlich auch GP-Funktion auftreten, und zwar mit Verb-Skopus, wo dies die VerbSemantik zuläßt, oder mit einem nominalen oder adverbialen Skopus in der Position nach nur/bloß.

Von den Satzarten bleibt nur die Aussagesatzform ohne Komplikationen. Der Imperativ läßt wie die Fragesatzform nur Spitzenstellung der Konjunktion zu, wie Satz (3) zeigt. Im Falle von (3a):

(3a) Such nur einmal mein Grab!

kann sowohl MP-Gebrauch als auch GP-Funktion mit verschiedenen Skopusabgrenzungen vorliegen. Diese Ambiguität zwingt in der Regel zum Ausweichen auf andere Konstruktionen. - Nun noch zu den Fragesätzen:

(2b) Nur: ist sie dem Zeitpunkt angemessen?

(3b) Nur: suchst du einmal mein Grab?

(2c) Ist (" Die Negationsform nicht nur ist zwar satzeinleitend möglich, doch handelt es sich hierbei nicht um die Negation eines Satzes mit nur in konjunktionaler Verwendung. Diese besteht vielmehr im nachgestellten nicht: es befindet sich bei satzeinleitender Stellung von nur in der normalen Position der Satznegation bei Inversionsstellung (also nach dem nachgestellten Subjekt), bei Nachstellung von nur steht nicht entweder umittelbar oder in Distanz nach nur. Die Besonderheit ist hierbei, daß diese Negation disambiguierend in Richtung auf die konjunk.tionale Verwendungsweise wirkt.

Typ 2: Konjunktionale Verwendung ohne Inversion.

(5) Mernitzka: "Selbstverständlich ist dieser Konflikt unausweichlich. Nur: Diesen Konflikt gibt es nicht erst seit gestern ..." 
(6) Die Botschaft hör' ich wohl, allein mir fehlt der Glaube.

Diese Stellung ist die normale für das konjunktionale allein. Nur und bloß passen nur mit Einschränkungen in dieses Schema, wie der Satz (5) deutlich zeigt. Ansonsten gelten hier alle Regularitäten von Typ 1 sinngemäß, insbesondere auch die Austauschbarkeit von allein gegen aber, jedoch, hingegen usw.

3. Weitere satzeinleitende Verwendungsweisen bei Elementen der nur - Gruppe: ... dann wird es eben doch wieder dasselbe, nur/bloß u m ge $k$ e hr $t$. Gleiches liegt vor bei nur anders, nur schöner usw. In allen diesen Fällen spricht die Ersetzbarkeit durch aber, jedoch etc. für die Zuordnung zur konjunktionalen Verwendung. Auf der folgenden Konstituente liegt zwar ein kontrastiver Akzent, doch ist er nicht durch die GP bedingt, sondern durch die normale Bedeutung dieser Konstituente selbst sowie durch die Kontrastierung mit dem Inhalt des vorhergehenden Satzes. Kontrastive Vorgänger- und Folgesätze in der Art, wie sie sonst bei GP-Gebrauch von nur üblich sind, können hier nicht eingesetzt werden. Alle übrigen Eigenschaften entsprechen denen von Typ 1. Eínige Sätze jedoch, die diesem Typ äußerlich sehr ähnlich sind, zeigen deutlich andere Eigenschaften:

(8) Nur $s c h$ a $d e$, daß ich nicht von Anfang an dabei war.

Kennzeichnend ist, daß die Nachstellung von nur hier ohne Einschränkungen möglich ist. Im Gegensatz zum obigen Typ lassen sich aber und jedoch hier nicht einsetzen. Der Akzent auf schade kann nicht als kontrastiv gewertet werden: eine solche kontrastive Interpretation wäre völlig sinnlos. Für eine mögliche Paraphrase halte ich (bei quant. Interpretation von nur):

(8a) Schade ist nur die ratsache, daß ich nicht von Anfang an dabei war. Danacn müßte diese Verwendung von nur unter der Rubrik "Skopus: Subjektsatz" eingeordnet werden. Andererseits sind aber alle dort erwähnten Regularitäten in diesem Fall nicht aufzufinden, vielmehr teilweise die der konjunktionalen Verwendung. Bliebe nur noch die Erklärung als Idiom.

Zur Semantik der konjunktionalen Verwendung von nur, bloß, alzein: Gewöhnlich läuft diese Konjunktion unter dem Terminus "Einschränkung" (Duden Grammatik 1973: $319 \mathrm{Nr}$. 775) bzw. "konzessiv". Die Schwierigkeit, die konjumktionale Verwendung von der Semantik her (also nicht von ihren syntaktischen Gesetzmäßigkeiten, Austauschbarkeit, Stellungsgesetze etc., her) klar von der GP-Verwendung mit Satzskopus abzugrenzen, läßt den Schluß zu, daß wesentliche Teile der GP-Semantik erhalten geblieben sind. Eine der quant. Interpretation angenäherte Deutung müßte aber praktisch den gesamten Satz als Skopus betrach- 
ten, etwa in der Form:

(5a) ... ist dieser Konflikt unausweichlich. Es gilt nur: Diesen Konflik gibt es nicht erst seit gestern.

PSP: Diesen Konflikt gibt es nicht erst seit gestern.

ASS: Es gilt nichts anderes.

Diese quant. Interpretation entspricht aber sicherlich nicht der Bedeutung von (5), abgesehen davon, daß diese Interpretation, wie beim Skopustyp Satz (Abschnitt 3.7.) gezeigt, generell inakzeptabel ist. - Ebensowenig ist eine skal. Interpretation denkbar. Die Bedeutungsgleichheit mit aber und jedoch zeigt deutlich, daß mit einer tbertragung der GP-Semantik nichts gewonnen ist. Die Semantik von Konjunktionen ist aber nicht das Thema dieser Arbeit.

\subsubsection{2. auch - Gruppe}

Typ 1: Konjunktionale Verwendung von auch mit Inversion der Wortstellung:

(1) ... könnte der Staat assistieren - wobei ich mich nicht auf zinskonditionen festlegen will. Auch wird zu überlegen sein, mit welchen Mitteln der Staat sein Interesse zeigt, wenn ...

(2) Annemarie Schröder blieben 150 ooo Mark Bargeld, auch behielt sie die gemeinsam bewohnte Villa in Langen.

(3) Diesmal geht es nur um Geld und Abgeordnetenstimmen. Auch ist das Kaliber der Beteiligten geringer.

Weitere Stellungstypen gibt es bej. konjunktionalem auch nicht.

\section{Zur Syntax}

Wie schon bei den einzelnen Skopustypen mehrmals erwähnt, ist die Austauschbarkeit gegen fermer, außerdem, darüberhinaus usw., wobei die Bedeutung nur unwesentlich verändert wird, kennzeichnend für den konjunktionalen Gebrauch von auch. Von Partikeln mit anderer Semantik sind nur nur und bloß einsetzbar, ev. auch hingegen, jedoch, aber nicht allein und aber (da diese beiden keine Inversionswortstellung zulassen). - Außer der Satzanfangsstellung ist keine der versuchten Positionen für konjunktionales auch voll akzeptabel:

(3a) Es ist auch das, Kaliber der Beteiligten geringer.

(3b) Das Kaliber der Beteiligten ist a u ch /auch geringer.

In allen diesen Positionen kann auch einen ganz bestimmten Skopus, der keinesfalls den gesamten Satz umfaßt, haben. Bei konjunktionaler Verwendung ist jedoch kein bestimmter Skopus im Sinne dieser Erscheinung bei GPn auszumachen; von der Semantik her ist der Skopus Satz am wahrscheinlichsten (siehe 3.7.2.). Zur semantischen Analyse des konjunktionalen Gebrauchs von auch: Es ist unverkennbar, daß das hier beschriebene auch (wie fermer, darüberhinaus 
usw.) sozusagen den Inhalt des von ihm eingeleiteten Satzes zu den vorausgehenden Feststellungen hinzuaddiert:

(2a) Annemarie Schröder blieben 150 ooo Mark Bargeld ..., auch behielt sie die gemeinsam bewohnte Villa in Längen.

ASS: Sie behielt die gemeinsam bewohnte Villa in Langen.

PSP: Anderes trifft zu (z.B. Annemarie Schröder blieben 150 ooo Mark Bargeld ...)

Ich sehe keinen Grund, diese Interpretation abzulehnen. Sie würde aber bedeuten, daß wir hier keine konjunktionale Verwendung im eigentlichen Sinn vorliegen haben, sondern eine quantifizierend gebrauchte GP mit Satzskopus, die gewisse konjunktionale Funktionen erfüllt sowie formale Eigenschaften mit Konjunktionen teilt. - Als Aufgabe bliebe noch, den jeweiligen PSP-Bereich einigermaßen überzeugend festzulegen. Bei jedem anderen Skopustyp ist eine gewisse Festlegung durch die nicht im Skopus befindlichen Konstituenten des Satzes vorhanden; sie tauchen ja wieder unverändert in der PSP-Formulierung auf. Dies ist bei konjunktionaler Verwendung von auch nicht der Fall: daraus kann man aber nicht schließen, daß der PSP-Bereich universal sei, daß also jede beliebige Tatsache in Frage käme. So ist das folgende Paar pragmatisch nicht sehr plausibel, wenn auch nicht mit Sicherheit auszuschließen:

(2b) ASS: Annemarie Schröder behielt die gemeinsam bewohnte Villa in Langen. PSP: Morgen wird die Welt untergehen.

Was aber jeweils auszuschließen ist, dafür gibt es wohl Erfahrungsregeln, aber keine zuverlässigen sprachlichen Indikatoren.

Unberücksichtigt blieb bis jetzt, daß derartige Sätze, eine ganz nomale Akzentverteilung aufweisen, es gibt also keinen kontrastierenden Fokus, der mit einem erkennbaren Kontrastakzent ausgestattet wäre. Trotzdem läßt sich für keinen der obigen Sätze eine "passende Frage" angeben:

(1a) Was ist zu tun? - Es wird (?auch) zu überlegen sein, mit welchen ...

\subsubsection{3. sogar - Gruppe in konjunktionaler Verwendung}

Offenbar gibt es bei der sogar - Gruppe nichts, was dex konjunktionalen Verwendung bei der auch - und bei der nur -Gruppe in formaler Hinsicht entspräche:

(1) "Annemarie Schröder blieben 150 ooo Mark Bargeld, sogar behielt sie die gemeinsam bewohnte Villa in Langen.

(2) "Dann wird es eben doch wieder dasselbe, sogar umgekehrt.

Ein plausibler Grund für diese Lücke könnte die vorgeschriebene skal. Interpretation von sogar - Sätzen sein: um die jeweils gemeinte Skala rekonstruieren zu können, muß ein Minimum an Information über den PSP-Bereich vorhanden sein. Diese fehlt aber gerade bei dieser Konstruktion. 


\subsubsection{Modalpartikel-Funktion der untersuchten Elemente}

Der Funktionswechsel von GPn zu MPn wurde bei den einzelnen Skopustypen sehr häufig erwähnt. Vielleicht sind dabei auch schon die Grundlinien, die wichtigsten Eigenschaften dieser Funktionsgruppe einigermaßen klar geworden, so klar, wie dies eben in diesem Falle möglich ist. Denn trotz umfangreicher Arbeiten zu dieser Partikelgruppe, ${ }^{4}$ die natürlich außer den auch zur GP-Gruppe gehörigen Elementen auch noch einige andere Partikeln umfaßt, ist die genaue Abgrenzung, sind die sie definierenden Eigenschaften bis heute nicht ganz geklärt. Um nur einige wenige hier aufzuführen: die MPn sind unbetont und unbetonbar; sie können keine vollständige Äußerung bilden; sie sind nicht erfragbar (folgt aus der unbetonbarkeit); sie sind weglaßbar, ohne daß ein klar erkennbarer Informationsverlust eintreten wïrde; sie bilden kein eigenes Satzglied; jede MP ist meist mit einer bestimmten Satzart oder mit einer für sie typischen Kombination von Satzarten verbunden; die MPn sind keiner einzelnen Konstituente zugeordnet (etwa in der Art eines Operator-Skopus-Verhältnisses) ; ihre Position ist i.d.R. nach dem finiten Verb, doch können sich pronominalisierte NPn dazwischenschieben; etc. - Das Problem besteht nun in der möglichst einleuchtenden Trennung von der GP-Funktion derselben Elemente. Das ist deswegen nicht ganz einfach, weil in der gleichen Position, manchmal sogar mit demselben Akzentmuster, auch GP-Funktion des betreffenden Elements möglich ist. Um die verschiedenen Möglichkeiten klar zu machen, gehe ich nach GP-Gruppen und innerhalb derer nach Satztypen vor.

\subsubsection{1. nur - Gruppe}

\section{Imperativ:}

(1) Nur $M u t$ !

(2) Er soll nur $k \circ m m e n$ !

(3) Seht euch nur diese Gestalten an, die die Ehre dieser Stadt besudeln!

In entsprechenden Kontexten könnten alle diese Sätze mit kontrastiven Folgesätzen versehen werden, die auf GP-Funktion und Skopusgrenzen schließen lasseJ

(1a) - nicht Feigheit kann uns helfen.

(2a) - nicht weggehen!

4 Da ich nicht näher auf diese problematik eingehen kann, verweise ich nur aut die wichtigste Literatur dazu: Kriwonossow (1963; 1965; 1966); H. Weyc (1969); M. Schubiger (1965); W.Arndt (1960); G. Helbig (1970). 
(3a) - nicht jene reputierlichen Bürger, die unseren Ruhm über alle Grenzen tragen.

Natürlich können alle diese Sätze auch ohne expliziten Kontrastsatz im Sinne von GFn verwendet werden. In diesem Falle lautet der hinzuzudenkende Folgesatz (der der ASS entspricht): nichts anderes/nichts sonst. Diese nur implizit kontrastierende Interpretation ist nur noch sehr schwer von der üblichen MP-Interpretation zu trennen. Ob sich daraus ev. Anhaltspunkte für eine diachrone Herleitung dieser Verwendungsweise bzw. umgekehrt, ob sich aus der Untersuchung der Diachronie Anhaltspunkte für die innere Verwandtschaft ergeben, bedürte einer sehr eingehenden Prüfung.

\section{Zur Syntax}

Im Rahmen der üblichen semantischen Beschränkungen sind alle Mitglieder der nur - Gruppe wie auch alle sonstigen GPn einsetzbar. Bei Elementen wie ausschließlich, allein, einzig, lediglich tritt dann allerdings Disambiguierung in Richtung auf die GP-Funktion ein (abgesehen von anderen adverbialen Funktionen bei allein und einzig). Die Ambiguität bleibt erhalten, wenn nur und ev. auch bloß unmittelbar hinter dem imperativischen Verb verbleiben; nur pronominalisierte Objekte können sich zwischen beide schieben. - Erstaunlich ist, daß andere MPn nur $v \circ r$ der MP nur stehen können, wie Satz (2) zeigt. In diesem Falle ist allerdings die GP-Interpretation für nur wahrscheinlicher; sie ist die einzig mögliche, wenn ein Objekt oder ein Adverb darauf folgt. Dies zeigt sehr deutlich die Labilität der Situation.

Bei absoluten Verben sind nur diese betont, wie im Imperativ üblich. Ist das Verb Skopus der GP, so mu eine eindeutige Situation bzw. ein Kontrastsatz hinzukormen, um die Ambiguität zu beseitigen. Ganz offensichtlich existieren also nicht zwei unterschiedliche bzw. unterscheidbare Akzenttypen, die diese Disambiguierung leisten könnten.

Die MP-Interpretation wird durch alle in Frage kammenden Negationsformen verindert. So bewirkt nicht nur Disambiguierung in Richtung auf die GP-Funktion. ie Formen nur nicht bzw. nur ... nicht erzwingen die konjunktionale Interpreation von nur (siehe dazu Abschnitt 4.2.1.1.)

\section{- Ausruf:}

(4) Könnte ich ihm nur helfen!

(5) Wenn sie nur (bald) käme!

(6) Wenn du nur früher gekommen wärst, dann hättest du ihn noch getroffen.

ur wenige Mitglieder der nur - Gruppe sind in dieser Funktion möglich: 
(4a) Könnte ich ihm nur/bloß/?lediglich/?ausschließlich/"allein/"einzig/ "einzig und allein $h$ e f en!

Allein und einzig wären möglich mit dem Skopus ihm (mit kontrastiver Betonung!), nicht jedoch mit dem Skopus Verb. Lediglich und ausschließlich sind auch mit dem Skopus Verb akzeptabel. Doch sind sie nicht möglich mit der MP-Interpretation, wie sie ausschließlich nur und bloß zulassen, und wie sie vielleicht der Folgesatz ich witrde alles dafür opferm verdeutlicht.

Umstellungen sind in den Belegsätzen nicht möglich; das deutet auf sehr strenge Stellungsgesetze hin: bei synthetischen Verbformen steht die $\mathbb{P}$ am Satzschluß; bei analytischen Verbformen steht sie vor der infiniten Verbform, zwischen sie und die MP können sich Adverbien schieben; nach wenn und soferm steht die MP nach dem pronominalisierten Subjekt, aber $v \circ r$ der nicht pronominalisierten Subjekts-NP. Auf der Vollverbform wird in jedem Fall ein emphatischer Akzent plaziert.

Von den Negationsformen ist nicht nur bei allen Beispielen anwendbar. Es führt zu Disambiguierung in Richtung auf die GP-Interpretation. Nur nicht ist in allen Fällen verwendbar; sie führt zu der eigenartigen Erscheinung, daß man intuitiv nur als MP wertet und die Negation dem Hauptverb zuschlägt. Nur ... nicht führt zu einer Umgestaltung des Satzcharakters:

(4b) Nur könnte ich ihm nicht helfen.

(5a) Nur, wenn du nicht früher gekommen wärst, hätten ...

(6a) Nur, wenn sie nicht käme, wären wir aus dem Schneider.

In den Sätzen (5) und (6) liegt dann nicht konjunktionale Verwendung, sondern nur mit dem Skopus Konditionalsatz vor.

3. nur im indirekten Fragesatz:

$\because$ über den Preis aus dem Markt herauszuholen, was nur/?bloß/:allein/ "einzig/"lediglich/”ausschließlich herauszuholen ist.

(8) Sie erhält, was sie nur will.

(9) Man blockiert, mauert und verzögert dort, wo es nur irgendwie geht.

Die Akzeptabilitätsbewertungen bei den einzelnen Partikeln in Satz (7) lassen nicht erwarten, daß es sich hierbei um eine GP-Verwendung handeln könnte. Da nur in diesem Typ meist im Konstituentensatz auftritt, gestalten sich die Stellungsgesetze vergleichsweise einfach: nur steht unmittelbar vor dem Verbum, Adverbien können sich allerdings dazwischenschieben. - Das Betonumgsmuster entspricht demjenigen von nichtkontrastiven Sätzen.

Die Negationsform nicht nur kann in diesem Falle nicht eingesetzt werden. Aber auch nur nicht/nur... nicht können nicht verwendet werden. Andere Nega- 
tionsmöglichkeiten sind ebenfalls nicht erkennbar. Dies halte ich für ein starkes Argument für idiomatischen Gebrauch des nur.

\section{Fragen:}

(10) Der Pastor dachte: "Wie kriege ich nur/bloß die Kirche voll?" Wirde man die Kirche kontrastiv betonen, dann wären alle Elemente der Grupe in dieser Position möglich, da es auf quant. GP-Interpretation hinauslaufen würde. Ähnliche Beispiele sind:

(11) Wie nur? Warum nur?

Die syntaktischen Eigenschaften entsprechen weitgehend denen von Typ 3.

\section{Restgruppe:}

(12) Es krachte nur/bloß so.

(13) ... ein Luftraum, in dem es von sport- und Segelflugzeugen nur so wimmelt.

Die syntaktischen Eigenschaften entsprechen hier ebenfalls denen von Typ 3. Zur Semantik der MPn nur und bloß

Obwohl man die übergänge von der GP mit quant. Interpretation zu MPn auch von der Semantik her klar erkennen kann, ist doch eine Erklärung der MPn auf der Basis der GP-Semantik (die einen abgrenzbaren Skopus voraussetzt, im Hinblick auf welchen eine Quantifizierung vorgenommen werden kann) nicht sinnvoll. Wesentlich fruchtbarer sind Überlegungen zur pragmatischen Funktion dieser Partikeln, wie sie etwa Dieter Wunderlich mehrfach angestellt hat (im Sinne von Indikatoren der illokutionären Rolle einer bestimmten Äußerung).

\subsubsection{2. auch - Gruppe und MP-Funktion}

Typ 1: Aussagesatz.

(1) Darum ist es auch kein widerspruch, wenn ausgerechnet die konservative oberschicht um die letzten Flecken Natur bangt.

(2) Der Agrarbericht 1973 verrät ja auch die Gründe.

(3) ... eine Modifizierung des bestehenden Systems. Das soll sie auch sein. es soll nicht der Eindruck ...

(4) Eine spätere Eingemeindung in den hier vorgeschlagenen Grenzen käme dann ganz von selbst, und die wäre auch sehr zu wünschen.

(5) Vielleicht gab es auch nie einen Grund zu dieser Annahme.

(6) Man hat hierzulande freilich auch mehr für die Vertriebenen getan. 
(7) So sieht es auch aus.

(8) Er kann sich ziemlich viel leisten, er ist ja auch Beamter.

Typ 2: Ausruf.

(9) Du hältst aber auch $n$ i e den Mund.

(10) Das ist aber auch $z u$ ärgerlich.

Typ 3: Wortfrage.

(11) Wer auch schuld daran ist...

(12) Welche dieser drei Möglichkeiten ich auch wählte, immer geriete ich zwischen die Mühlsteine der beiden anderen.

(13) ... hinterfotziger Tritt gegen Brandts Entspannungspolitik entstanden: denn welcher auch sie sehnsüchtig wünschende Deutsche kann ernsthaft noch an die "Wiedervereinigung" glauben?

Typ 4: Ausruf.

(14) Teufel auch!

(15) Nein, was denkst du auch!

(16) Das fehlte auch noch!

Typ 5: Satzfrage.

(17) Ist das auch wahr?

(18) wird er auch kommen?

(19) Kann ich es auch glauben?

Man mag beim ersten Durchlesen der Belege der Ansicht sein, daß vielfach eine quant. GP-Interpretation vorliege oder aber doch zumindest möglich sei. Ich will die wichtigsten Möglichkeiten kurz auflisten, um eine bessere Abgrenzung des hier gemeinten Gebrauchs vorgängig zu erzielen:

In einer Reihe von Fällen kann auch kontrastiv betont werden. Dadurch wird in jedem Falle quant. Interpretation mit topikalisiertem Skopus erzwrngen:

(3a) Das soll sie $\underline{a c h}$ sein.

(9a) Du hältst aber $\underline{a u c h}$ nie den Mund!

(16a) Das fehlte a $u c h$ noch.

Die Beispiele zeigen, daß diese Form der Interpretation nur bei einem skopusfähigen Anfangselement des Satzes möglich bzw. akzeptabel ist, also z.B. nicht bei wortfragen, nur in seltenen Fällen bei Ausrufen und Satzfragen, jedoch fast immer bei Aussagesätzen.

In sehr vielen Fällen kann die folgende bzw. die vorausgehende Konstituente durch kontrastive Betonung zum Skopus von auch werden. Damit setzt sich die quant. GP-Interpretation durch: 
(2a) Der Agrarbericht 1973 verrät ja auch die $G$ r ün $d e$.

(8a) Er ist ja auch $B$ e a $m$ t e $r$ (und nicht nur Landtagsabgeordneter).

(18a) Wird er auch $k \circ \mathrm{m}$ e $n$ (und nicht nur schreiben)?

Aufgrumd der zuordnungsgesetze zwischen auch und seiner Skopuskonstituente können nur finite Verben als Skopus vor auch stehen (siehe Satz (16)), alle anderen Skopuskonstituenten müssen ihm folgen. - Diese beiden Möglichkeiten möchte ich ausdrücklich ausschließen. Trotzdem sind in einigen Fällen immer noch zumindest Ambiguitäten zu verzeichnen. Sie werden im weiteren Verlauf einzeln benannt

\section{Zur Syntax}

Die Klassenbildung stellt also das eigentliche Definitionsproblem dar. Im Gegensatz zur nur - Gruppe kann die Unterscheidung GP-Funktion - MP-Funktion nicht durch eine Anzahl von spezifischen Austauschmöglichkeiten plausibel oder doch wahrscheinlich gemacht werden: für die GP-Funktion gibt es kein bedeutungsgleiches Element in dieser Stellung und ohne kontrastive Betonung. Aber auch der Austausch gegen andere GPn bringt Probleme mit sich: nur ist in den meisten dieser Positionen ebenso ambig wie auch selber, andere Elemente dieser Gruppe sind wieder nicht in dieser Konstruktion verwendbar (sie scheinen eine kontrastiv betonte Konstituente zu fordern). Eine in der Bedeutung stark angenäherte Ersatzmöglichkeit bietet sogar. Es hat außerdem den Vorteil, keinesfalls in MP-Funktion verwendbar zu sein. Nur stellt es an die Skopuskonstituente wegen seiner ausschließlich skalierenden Interpretation andere Anforderungen als auch. Jedenfalls ergibt die Einsetzung, daß es in den Typen 2, 3, 4 und 5 nicht im Sinne von auch verwendbar ist. Es erzwingt entweder ein Akzentmuster, das eine GP-Interpretation zuläßt, oder der Satz wird inakzeptabel:

(10a) "Das ist aber sogar z u ärgerlich!

(11a) "Was sogar geschehen mag ...

(14a) "Teufel sogar!

(17a) "Ist das sogar wahr? / Ist das sogar wa hr? Hingegen sind bei Typ 1 einige Sätze durchaus korrekt:

(1a) Darum ist es sogar kein widerspruch, wenn ...

(5a) Vielleicht gab es sogar nie einen Grund dazu.

Es ist wohl nicht ganz abwegig, daraus den Schluß zu ziehen, daß es sich hier um GP mit Satzskopus handeln könnte. Andererseits ist die andere Konstruktion, die bei auch ev. Satzskopus bedeutet, nämlich die satzeinleitende Stellung von auch, bei diesen Sätzen nicht in jedem Fall akzeptabel bzw. bedeutungsgleich:

(1b) $\underline{\text { Auch }}$ ist es darum kein Widerspruch, wenn ...

(5b) $\underline{\text { Auch }}$ gab es vielleicht nie einen Grund dazu ... 
Eine größere Entscheidungssicherheit gäben hier wohl "Texte", die erkennen las-sen, ob diese Form des Anschlusses der Form und Bedeutung des nach dem finiten Verb stehenden auch entspricht:

(3b) ... eine Modifizierung des bestehenden Systems. "Auch soll sie das sein. Es soll nicht der Eindruck ...

(4a) Eine spätere Eingemeindung in den hier vorgeschlagenen Grenzen käme dann ganz von selbst, auch wäre die sehr zu wünschen.

(8b) Er kann sich ziemlich viel leisten. "Auch ist er ja Beamter. Dieses umeinheitliche Ergebnis läßt einerseits den Schluß zu, daß wohl nur in den wenigsten Fällen das "konjunktionale" auch bedeutungsgleich ist mit dem in Typ 1 verwendeten auch, und daß zweitens die GP-Funktion bei diesem Typ 1 zumindest sehr unsicher ist.

Der Versuch, die vorliegenden Beispiele mit der MP-Funktion zu vergleichen, indem man "sichere MPn" einsetzt, birgt nicht weniger Schwierigkeiten. Am einfachsten ist es noch bei Typ 5:

(17b) Ist es denn wahr?

(18b) Wird er denn kommen?

Typ 3 und Typ 4 sind jedoch so weit idiomatisiert, daß keine halbwegs akzeptable Ersatzmöglichkeit zur Verfügung steht. - Bei Typ 2 besitzen wir zum Glück eine hieb- und stichfeste MP-Ersatzmöglichkeit: doch.

(9b) Du hältst doch $n i$ e den Mund.

(10b) Das ist aber doch $z$ u ärgerlich.

Für eine Reihe von Sätzen des Typs 1 bringt die Einsetzung von doch ebenfalls akzeptable Ergebnisse:

(4b) ... und die wäre doch sehr zu wünschen.

(7a) So sieht es doch aus.

Für andere Sätze ist dies nicht sinnvoll möglich. Auch die Einsetzung von eben und $j a$, die in Aussagesätzen prinzipiell als MPn möglich sind, kann nur sehr selten befriedigen.

Die Stellungsregel für Typ 1 lautet wohl so, daß auch vor derjenigen Konstituente steht, die den Satzakzent trägt (einschließlich der ihr zugeordneten Wörter wie sehr in (4)). Zwischen auch und dem finiten Verbum sind aber wohl nur pronominalisierte Konstituenten möglich:

(5c) Vielleicht gab er ihm auch keinen Grund dazu.

(5d) Vielleicht gab er auch dem Fabrikanten keinen Grund dazu. Typ 2 ist als Idiom zu betrachten und dann auch so zu beschreiben. Typ 3, auch in der Wortfrage, ist eigentlich keine Frage und zeigt auch nicht deren Stellungseigenheiten. auch steht hier unmittelbar nach dem Fragepronomen soweit dieses nominativisch ist, bzw. nach dessen Ergänzungen, wie in (12), 
oder unmittelbar vor dem in Satzschlußstellung befindlichen Verbkomplex. Typisch ist für diese Form, daß man statt auch jeweils auch immer einsetzen kann. Die dem Typ 1 entsprechenden Frageformen (Wortfragen) lassen sich leicht anhand der Stellungseigenschaften und des Ausschlusses von auch immer unterscheiden:

(7b) Warum sieht es auch so aus?

(6a) Warum hat man hierzulande auch mehr für die Vertriebenen getan? Diese "Frage" hat eher den Charakter einer vorwurfsvollen Feststellung. Hingegen wirkt die Umformung für jene Sätze, für die ich die GP-Interpretation (mit Satzskopus) nicht ausschließen wollte, reichlich seltsam.

Auch Typ 4 ist nur als Idiom zu beschreiben. - In Typ 5, der Satzfrage, steht auch unmittelbar vor dem infiniten Verbteil, abgesehen von solchen Wörtern wie wirklich, tatsächlich usw., oder, falls eine synthetische Verbform vorliegt, am Satzschluß. Die dem Typ 1 entsprechenden Satzfragen sind wiederum nur bei jenen Sätzen voll akzeptabel, bei denen ich MP-Funktion von auch für wahrscheinlich erklärt habe, jedoch seltsam bei jenen Sätzen, bei denen GPFunktion nicht ganz auszuschließen ist.

Die bisherige Einordnung der einzelnen Typen wird bestätigt durch die jeweiligen Negationsmöglichkeiten. Beim Typ 1 entspricht die Normalnegation gänzlich der bei der konjunktionalen Verwendung von auch, nämlich auch nicht bzw. auch kein oder auch nie usw., siehe die Sätze (1) und (5). Steht die Negation erst nach der auf auch folgenden nominalen Konstituente, so wird diese zum kontrastiv betonten Skopus von auch:

(6b) Man hat hierzulande freilich auch für die $V$ e $r$ t $i$ e b e n e $n$ nichts getan.

Die restlichen Typen lassen Negationen nicht $z u$, oder doch nur bei gründlicher Änderung des jeweiligen Konstruktionstyps.

Zusammenfassend mu man also konstatieren, daß aus den formalen Eigenschaften von Typ 1 kein eindeutiger Schluß auf GP-Verwendung mit Satzskopus oder MP-Funktion von auch möglich ist. Für die übrigen Typen scheint mir jedoch die MP-Funktion gesichert. - Auch von den semantischen Eigenschaften her ist keine eindeutige Grenzziehung möglich. Klärende Kontrastsätze sind ausgeschlossen. Reihungen aber sind so wenig in ihren semantischen Eigenschaften festgelegt, daß daraus kein sicherer Schluß zu ziehen ist.

Da es kejnerlei Indizien für eine MP-Verwendung der Elemente der sogar Gruppe gibt, eribrigt sich die gesonderte Behandlung dieser Gruppe. 
4.3. Negationsmöglichkeiten bei Gradpartikeln

Dieser Abschnitt soll der Strukturierung der vielfältigen und eng miteinander verzahnten Negationsformen im Zusammenhang mit Gradpartikeln dienen. Eine rein syntaktische Behandlung dieses Themas, die nur die verschiedenen Negationsformen und ihr Zusammenwirken mit den GPn diskutiert, würde am Kern der Thematik vorbeigehen. In dieser Zusammenfassung soll vielmehr die Verknüpfung mit der Frage des kontrastiven Skopus und mit der Semantik (via Kontrollverfahren: Kontrastsätze) klar gemacht werden. Zu diesem Zweck müssen für die einzelnen Gruppen und die verschiedenen Skopustypen jeweils eine Anzahl von Beispielsätzen gebracht werden.

\subsection{1. nur - Gruppe}

1. Negation vor der GP:

Mit dieser Konstruktion besitzt man ein ideales Kontrollverfahren für die Analyse der Semantik des quantifizierenden nur, da sie semantisch die genaue umkehrung von nur bildet. Es handelt sich um die PSP-neutrale Negation. Über die genauen Eigenschaften bei skalierendem nur wurde bislang nur wenig gesagt; dieses Thema soll deshalb hier noch einmal zur Sprache kommen.

(1) Nicht nur A gui $r$ e übersteht die Fährnisse im Fieber, sondern auch Pizarro, Piedro... überleben.

(2) Nicht nur ich habe Glück gehabt, sondern auch $d u$.

(3) Bestraft wurde nicht nur, wer eine waffe besaß, sondern auch, wer...

(4) Dies $s$ a $g t$ er nichtallein, dies $t u t$ er auch.

(5) Weil der Pakt nicht nur das jahrhundertealte Mißtrauen bestätigt, sondern auch ...

(6) Ich wi l nicht nur anregen, ich $k$ a $n$ es auch/sogar.

(7) Nicht nur $m \ddot{u} h s$ a $m$, sondern auch $k \circ s$ s $p i$ e 1 i $g$.

(8) Nicht nur $s$ e 1 t e $n$, sondern auch/sogar o $t t$.

(9) Sie müßte nicht nur ein wenig $h \ddot{u} b s c h e r$, sondern auch ein wenig $i n t \overline{e l i g}$ e $n$ e $r$ sein.

Es überrascht einigermaßen, daß mit dieser Form von NEG nur bloß und nur uneirgeschränkt akzeptabel sind, allein, lediglich und ausschließlich sind nicht ganz inakzeptabel, einzig sowie einzig und allein hingegen können offenbar nicht mit dieser Negationsform kambiniert werden, ohne daß man bestimte semantische oder syntaktische Gründe angeben könnte.

Die Stellungsgesetze für die nicht nur - Gruppe sind etwas enger als die 
für nur allein. So ist die Nachstellung generell unmöglich (abgesehen von der allgemeinen Ausnahme beim Skopustyp finites Verb). Die Distanzstellung davor und danach scheint noch weniger normal zu sein als bei den einigermaßen akzeptablen nur - Beispielen. Nicht ganz klar ist die Akzeptabilität der Konstruktion topikalisierte Skopuskonstituente + nicht nur in Distanzstellung danach:

(1a) ?A guirre übersteht nicht nur die Fährnisse im Fieber ...

(2a) ?I c h habe nicht nur Glück gehabt, sondern auch...

Vermutlich ist hier dieselbe Ursache wirksam wie bei der gewissen Inakzeptabilität von Spaltsatzkonstruktionen mit nicht nur:

(1b) ?A guirre ist es nicht nur, der die Fährnisse ...

(2b) ?I $c h$ bin es nicht nur, der Glück gehabt hat, sondern ...

In beiden Fällen steht die Einzigkeitsfunktion der Topikalisierung bzw. Spaltsatzkonstruktion gegen die Nichteinzigkeitsbehauptung von nicht nur. Daraus wird wohl die Inakzeptabilität resultieren.

Besonders wichtig ist, daß die MP-Funktion von nur und bloß in allen dafur geeigneten Stellungen durch die Hinzufügung von nicht ausfällt. Es treten also keinerlei Funktionsambiguitäten mehr auf.

Zur Semantik von nicht nur

Wie schon einleitend festgestellt, besitzt man mit dieser Verbindung eine Kon-trollmöglichkeit für die Semantik sowohl von nur als auch von auch. Für auch Sätze sind nicht nur - Sätze Vorgängersätze, die eine der für den betreffenden Satz möglichen PSPen explizit ausformulieren. Damit sind sie auch wichtige Hinweise für eine allgemeinere Formulierung der PSP. - Für die Semantik von nur sind sie insofern interessant, als sie bei der Unterscheidung von quant. und skal. Interpretation von nur helfen: bei quant. nur darf im Kontrastsatz nur auch stehen, bei skal. Interpretation nur sogar (dessen Skopus dann eine "höhere" Stufe bezeichnet = die ASS von nicht nur). Umgekehrt kann in denjenigen Fällen, in denen sowohl quant. als auch skal. Interpretation möglich ist, (aufgrund der semantischen Eigenschaften des Skopuselementes), durch das auch bzw. sogar im Nachsatz die gewinschte Interpretation verdeutlicht werden. Die Skala muß ibrigens auch bei nicht nur keine bestimmte "objektive" Richtung" aufweisen, sie führt aber in jedem Falle von schwächeren zu stärkeren Skalenwerten.

Völlig selbstverständlich ist, daß bei konjunktionalem nur ebenso wie bei nur mit MP-Funktion dann, wenn für beide nicht gleichzeitig GP-Funktion möglich ist, das davorgestellte nicht zu Inakzeptabilität führt. 
2. Negation nach der Gradpartikel:

(10) Alle anderen überstehen die Fährnisse, nur nicht $A g$ u $r$ re.

(11) Bestraft wurde nur nicht, wer eine $w$ a $f f e$ mißbrauchte, sondern ... Bestraft wurde nurnicht, wer eine Waffe mißbrauchte, sondern ... Bestraft wurde $\underline{n u r}$, wer eine Waffe miBbrauchte, "nicht.

(12) Die Tür war nur nicht a $n g$ e $l$ e $h n t$, sondern... (:auch/?sogar).. "Die Türr war nur a $n g$ e e hn $t$ nicht.

Nur war die rür nicht a $n g$ e $l$ e h $\overline{n t}$, sondern...

Nur: a $n g$ e $l$ e hn $t$ war die Tür nicht, sondern...

(13) Er ist nur nicht $\circ h n m \ddot{a} c h t i g$, sondern... Nur ist er nicht oh $n m \ddot{a} c h t i g$, sondern... Nur: $\circ h n \overline{m a ̈} h t i g$ ist er nicht, sondern ...

(14) Er ist nur nicht $w i r t s c h$ a $f t l i c h$ ein Ass, sondern... Nur ist er nicht $w i r t s c h$ a $f t l i c h$ ein Ass, sondern ... Nur ist ex $\overline{w i} t s c h$ a $f t l i c h$ kein Ass. Er ist nur wi $r$ t $s$ c $h$ a $t l i c h$ kein Ass. Ein Ass ist er nur wi $r t s c h$ a $f t i \overline{i c h}$ nicht.

(15) Bei den Landwirten sind es nur nicht 13 - $30 \%$, sondern... Nur sind es bei den Landwirten nicht 13 - $30 \%$, sondern ... $\overline{{ }^{*} \mathrm{Be}} \mathrm{i}$ den Landwirten sind es nur $13-30 \%$ nicht.

Alle diese Beispiele umfassen 3 Negationsformen: ... nur nicht $x$, sonderm .../ Nur ... nicht $x$, sondern .../... nur $x$ nicht. Die beiden ersten Konstruktionen sind äquivalent; in beiden Fällen handelt es sich um das "konjunktionale" nur', verbunden mit einer Kontrastnegation. - Die dritte Negationsform ist davon völlig verschieden, was schon die klare Inakzeptabilität bei allen Skopi, die nur skalierende Interpretation zulassen, verrät. Im Hinblick auf ihre semantische Wirkung sind jedoch die drei Formen nicht ganz leicht zu unterscheiden:

(16) Die Leute lieben nur $A \cap j a$.

PSP: Die Leute lieben Anja.

ASS: Die Leute lieben niemanden sonst (aus einer Gruppe).

(16a) Nur lieben die Leute nicht $A n j a$, sondern ...

ASS: Die Leute lieben Anja nicht.

PSP: Die Leute lieben jemand anderen.

(16b) Nur: die Leute lieben Anja nicht.

ASS: Die Leute lieben Anja nicht.

PSP: ?

(16c) Die Leute lieben nicht nur Anja.

PSP: Die Leute lieben Anja.

ASS: Die Leute lieben auch andere Menschen als Anja.

(16d) Die Leute lieben nur nicht $A n j a$, sondern ...

ASS: Die Leute lieben Anja nicht.

PSP: Die Leute lieben jemand anderen.

(16e) Die Leute lieben nur An ja nicht.

PSP: Die Leute lieben Anja nicht.

ASS: Die Leute lieben alle anderen (aus einer Gruppe). 
Damit ist die letzte Form der Negation in ihrer Wirkung klar von allen anderen zu unterscheiden. Gleichzeitig ist aus der ASS-Formulierung alle anderen ersichtlich, daß dies auf eine skal. Interpretation nicht anwendbar ist: in diesem Falle miißten nämlich alle die sich gegenseitig ausschließenden Skalenwerte bis auf den mit nur gekennzeichneten gelten, außerdem ginge die Skalenrichtung verloren. - Das nur ... nicht bei quantifizierendem Skopus ist nicht die Negation von nur (das ist nicht nur): denn nicht nur die ASS wird negiert, sondern auch die PSP.

\subsection{2. auch - Gruppe}

1. Negation vor der GP:

Wie immer wieder festgestellt, ist diese Negationsform bei auch im Gegensatz zu nur nicht möglich:

(1) "Nun beklagt also nicht auch der $s p i$ e ge 1 den armen Steuerzahler. Auch Topikalisierung der Skopuskonstituente und nachgestelltes betontes auch ändert nichts daran:

(1a) :Der $S p i$ ege $I$ beklagt also nicht a $\underline{\mathrm{u} c h}$ den armen Steuerzahler. Hingegen in Fragesätzen, wobei nicht eher als Fragepartikel zu werten ist:

(1b) Warum beklagt nicht auch der $s$ p i e ge 1 den armen Steuerzahler?

(2) Kann uns nicht auch ein sol che r Mann helfen?

Damit existiert für auch keine PSP-neutrale Negation. Sie müßte folgende Analyse bewirken:

(1c) ASS: Der Spiegel beklagt den armen Steuerzahler nicht.

PSP: Andere beklagen den armen Steuerzahler.

Genau diese Bedeutungelemente, aber im jeweils umgekehrten Status besitzt der Satz:

(1d) Nun beklagt nur der $S p$ i e ge I nicht den armen Steuerzahler.

2. Negation nach der GP: auch nicht; a uch nicht; auch... nicht.

(3) :Auch nicht $z$ we $r g$ e haben klein angefangen.

(4) Auch $z$ we $r g e$ haben nicht klein angefangen.

(5) Zwerge haben a $\mathrm{uch}$ nicht klein angefangen.

(6) Das schließt nicht aus, daß “auch nicht wi $r$ / auch wi r nicht / wir a uch nicht daran interessiert sind.

(7) Auf niemanden nahm Nixon Rücksicht, auch nicht auf die ohnmächtige öffentlichkeit im eigenen Land. 
(8) Ich bearbeite nicht sehr gerne meinen eigenen Garten, ich $l i$ e b e ihn auch nicht sehr.

(9) Dies ist auch nicht $v$ e rständIich.

(10) Es war auch nicht $B$ ornierthe $i t$.

(11) Es ist auch nicht e ine $r$ davongekommen.

Aus den Belegsätzen kann folgendes geschlossen werden: die Negationsformen auch $x \ldots$ nicht und $x \ldots$ a $u c h$ nicht sind in allen Beispielen mit quant. Interpretation zulässig, in allen Beispielen mit skal. Interpretation, die gerade bei der Kombination von auch mit einer Negation häufig sind, sind sie jedoch inakzeptabel (dies entspricht der Inakzeptabilität von betontem auch bei skal. Interpretation).

Die Negationsform auch nicht bedarf eingehender Beobachtungen. Zunächst fällt eine rein formale Eigenheit auf: auch nicht darf nämlich nicht satzeinleitend erscheinen, siehe (3) und (6). Es gibt jedoch eine Ausnahme, die durch (7) exemplifiziert wird; die Bedingung ist ein negativer Vordersatz und ein durch Tilgung der identischen Konstituenten verkürzter auch nicht - Satz. In diesem Falle liegt skal. Interpretation vor. (7) ist insofern identisch mit:

(7a) Auf niemanden nahm Nixon Rücksicht, auch auf die ohnmächtige ö $f$ fent 1 ichkeit nicht.

Die Position nach dem finiten Verbum scheint jedoch die Normal-Position von auch nicht zu sein. Konjunktionale Verwendung neben GP-Funktion mit dem Skopus Verb bzw. VP von auch (in Anlehnung an die Verhältnisse bei nur) kann man hier zunächst nicht ganz ausschließen, zumal für (8) und (10) auch die satzeinleitende Stellung von auch ohne Bedeutungsveränderung möglich ist:

(8a) $\underline{\text { Auch }} 1 \mathrm{i}$ e $b$ e ich ihn nicht sehr.

(10a) Auch war es nicht $B$ or ni e $r$ the it.

Für (11) ist aber diese Paraphrase undenkbar. Hier ist vielmehr die umschreibung mit nicht einmal angebracht. Unmöglich ist jedoch folgende Paraphrase:

(11a) “Es ist auch e ine r nicht davongekommen.

Di.e Zusammenhänge bleiben aber umentwirrbar. - Abschließend seien noch einmal die Interpretationen der verschiedenen Negationsformen gegenübergestellt:

(3a) Auch $z$ we $r g$ e haben nicht klein angefangen. I $z$ we $r g$ e haben $\overline{a u c h}$ nicht klein angefangen.

ASS: Zwerge haben nicht klein angefangen.

PSP: Andere Leute haben nicht klein angefangen.

(7b) Auf niemand nahm Nixon Rücksicht, auch nicht / nicht einmal auf die ohnmächtige öffentlichkeit im eigenen Land.

ASS: Nixon nahm auf die ohnmächtige öffentlichkeit im eigenen Land keine Rücksicht.

PSP: Nixon nahm auf weniger wichtige Leute/Gruppen keine Rücksicht. 
konv. IMPL: Nixon nahm auf wichtigere Leute/Gruppen als die ohnmächtige öffentlichkeit im eigenen Land Rücksicht.

Normalerweise wird mit auch nicht/nicht einmal jedoch die absolut höchste Stufe bezeichnet, eine konv. IMPI entfälit dann natïrlich.

\subsection{3. sogar - Gruppe}

1. Negation vor der GP: "nicht selbst, "'nicht sogar

Diese Kombination ist bei sogar und selbst ebensowenig möglich wie bei auch. Horn $(1969$; 1972) hat für das engl. even bereits dasselbe festgestellt, konnte aber ebensowenig eine Erklärung für das Fehlen dieser Negationsmöglichkeit in der Oberflächenstruktur angeben, zumal sie durchaus denkbar wäre. In dieser "denkbaren Form" sind diejenigen Bedeutungselemente enthalten, die entsprechende Sätze mit nur nicht aufweisen, allerdings jeweils mit anderem Status. Nur einige Belege für die Inakzeptabilität dieser Form:

(1) "Nicht sogar / selbst Schweine nehmen bei Musikberieselung zu.

(2) "Nicht sogar $n a C h$ unserer Scheidung rief ich meine Frau öfter an. Die Kombination kann allerdings zufällig durch das zusarmentreffen mit der Fragepartikel nicht in Satzfragen entstehen:

(3) Sollte die erschütternde Geste vor den Kämpfern des Warschauer Ghettos nicht sogar $B$ e $r$ e chn ung gewesen sein?

2. Negation nach der GP: "sogar/selbst nicht, nicht einmal, sogar/selbst... nicht

(4) “Selbst nicht $S c h$ we ine nehmen bei ständiger Musikberieselung $z$ u.

(5) Selbst $S c h$ we ine nehmen nicht schneller zu.

(6) Nicht einmal $S c h$ we ine nehmen schneller zu.

(7) "Er hat sogar ein $s c h$ we i n nicht.

(8) Sogar ein $S c h w e i n$ hat er nicht.

(9) Er hat nicht einmal ein $S c h w e i n$.

Nach den Erfahrungen bei auch würde man erwarten, daß bei sogar eine form- und bedeutungsgleiche Parallele zu auch nicht vorhanden wäre. Dem ist aber nicht so. - Die äquivalenten Formen sogar ... nicht und nicht einmal sind keine PSP-neutralen Negationen, entsprechen also sowohl auch nicht wie auch auch ... nicht bzw. a u ch nicht. 
5. ZUSAMMENFASSUNG DER ANALYSEERGEBNISSE

Dieser abschließende Teil soll die wichtigsten Ergebnisse des im Vorausgehenden bewältigten Analyseprogramms in überschaubarer Weise zusammenfassen. Dabei muß ich mich auf die Herausarbeitung der Grundlinien beschränken; viele nicht unwichtige Details, insbesondere die zahlreichen Idiosynkrasien, miissen unerwähnt bleiben, so daß leicht der Eindruck überwiegender Regelmäßigkeit entsteht.

\subsection{Zur Syntax}

\subsubsection{Zur Frage der syntaktischen Klassenbildung}

Ich bin ausgegangen von der These, daß es sich bei der Gradpartikel-Funktion um eine klar abgrenzbare Funktion mit sicher festlegbaren Eigenschaften handle. Aufgrumd der Ergebnisse des syntaktischen Analyseprogramms halte ich es für erwiesen, daß es sich bei der Gruppe der Elemente, die diese Funktion erfüllen können, also bei den Gradpartikeln, um eine trotz zahlreicher Idiosynkrasien syntaktisch einheitliche Gruppe handelt. Dies ist einmal gewährleistet durch die grundsätzliche gegenseitige Austauschbarkeit (bei zahlreichen Interventionen durch die Semantik der jeweiligen Elemente bzw. Gruppen) sowie durch eine zumindest ähnliche Distribution, wie sie in Austauschbarkeit und Stellungsgesetzen zum Ausdruck kormt.

\subsubsection{Austauschbarkeit}

Die Untersuchung der Gradpartikeln wird stark behindert durch die zahlreichen Funktionsambiguitäten und Funktionswechsel einzelner Elemente der Gruppe, die zu Lïcken in der Distribution führen, aber auch durch die Festlegung einzelner Gradpartikeln auf bestimmte Interpretationweisen. Für diese Distributionslükken kann man jedoch erklärende Gesetzmäßigkeiten angeben. - Anders verhält es sich mit ummotiviert erscheinenden Verteilungslücken. Sie können einstweilen 
nur als Idiosynkrasien registriert werden.

5.1.2.1. Die verschiedenen Mitglieder der nur - Gruppe weisen untereinander wie mit den Elementen der übrigen Gruppen einen hohen Grad von Austauschbarkeit auf. Mit nur und $b$ LOß besitzen wir hier zwei Partikeln, die (auch semantisch!) eine absolut identische Distribution aufweisen (abgesehen von der Modalpartikel-Funktion in Imperativsätzen). Die freie Austauschbarkeit wird jedoch behindert durch folgende Faktoren:

1. nur, bloß und lediglich können sowohl quantifizierend (für lediglich ist das zweifelhaft) als auch skalierend und polar verwendet werden, hingegen einzig, einzig und allein, allein und ausschließlich nur quantifizierend. In eindeutig skalierenden Kontexten ist deshalb diese zweite Gruppe nicht verwendbar. 2. ausschließlich kann in bestimmten Kontexten ein temporales Element (im Sinne von immer nur) hinzugewinnen.

3. Daneben gibt es einige Fälle von Funktionsambiguitäten bzw. Funktionswechseln. Nur und bloß können in bestimmten Positionen in die Modalpartikel-Funktion überwechseln, meist kommt es aber nur zur Ambiguität zwischen GradpartikelFunktion und Modalpartikel-Funktion. - einzig und allein wechseln in einigen Positionen in andere Adverbialfunktionen uber, übrigens ohne daß das immer, wegen der Nähe der Bedeutungen, einwandfrei erkennbar wäre. Funktionsambiguität ist in diesen Fällen allerdings sehr selten. - Die Adjektiv-Interpretationen von $b$ Zoß und ausschließlich sind wegen der flexivischen Merkmale leicht von der Gradpartikel-Funktion zu unterscheiden. Die Funktion als Modaladverbiale ist bei bloß nicht üblich, bzw. wird durch Formulierungen wie nackt und bloß disambiguiert, die entsprechende Funktion von ausschließlich ist von der Gradpartikel-Funktion kaum zu unterscheiden.

5.1.2.2. Die auch - Gruppe bietet die größten Schwierigkeiten. Das wichtigste Element der Gruppe, auch selbst, tritt in zwei formal sehr unterschiedlichen Konstruktionsmustern auf: unbetont unmittelbar vor der jeweiligen Skopuskonstituente, betont in Distanzstellung nach der topikalisierten Skopuskonstituente. Nur in dieser letzten Fassung kann es durch gleichfalls, ebenfalls, ebenso ersetzt werden. - Problematisch ist auch die vielfach zu beobachtende Ambiguität zwischen Gradpartikel-Funktion und Modalpartikel-Funktion, wobei allerdings die eindeutigen Fälle von Modalpartikel-Verwendung meist so stark idiomatisiert sind, daß sie keine andere Verwendung mehr zulassen. Daneben besteht eine Gruppe von Konstruktionen, in der die beiden Funktionen nicht eindeutig zu trennen sind, sei es durch Ambiguität oder durch Regelunsicherheit (Diachronie!). 
5.1.2.3. Die sogar - Gruppe ist syntaktisch zweifellos die hamogenste Gruppe. Lediglich selbst wechselt in all den Positionen, wo dies möglich ist, in die Rolle des Reflexivums. Ambiguitäten entstehen dadurch kaum, denn der Vorrang des Reflexivums ist eindeutig.

5.1.2.4. Nach ihren distributionellen Eigenschaften lassen sich folgende Untergruppen benennen: a. eine außerordentlich schmale "Kerngruppe", die in praktisch allen Konstruktionsmustern möglich ist und dabei weder Funktionswechsel noch Funktionsambiguitäten aufweist: lediglich, einzig und allein, sogar, nicht einmal, ausschließlich. Die freie Austauschbarkeit wird hier nur durch semantische Eigenheiten beschränkt. - b. Eine Gruppe, die zwar in allen für die Gradpartikel-Funktion typischen Positionen auftreten kann, aber in gewissen Positionen Funktionsambiguitäten (Modalpartikel) zeigt: nur, bloß, auch. - c. Eine weitere Gruppe läßt auch Funktionswechsel zu: einzig, allein, selbst. Das heißt, daß bei diesen Elementen eine nicht-Gradpartikel-Funktion in Positionen, die bei beiden Funktionen möglich wäre, Vorrang vor der Gradpartikel-Funktion hat.

Die weiteren Ersetzungsmöglichkeiten betreffen vor allem andere Adverbialgruppen; sie beschränken sich aber i.d.R. auf die Positionen, die dem Skopus Verbalphrase oder Teile der Verbalphrase entsprechen. Keine dieser Ersetzungsmöglichkeiten weist jedoch die Summe aller für die Gradpartikel-Funktion typischen Positionen (beachte auch die Zuordnung des Kontrastakzentes!) auf, ganz abgesehen von der semantischen Funktion. Iediglich die Negation kann offenbar in allen Konstruktionsnustern die Gradqartikeln ersetzen. Dies kann bei Beachtung der Funktionsähnlichkeiten (Kontrastnegation, Satznegation) nicht überraschen. - Für die formale Darstellung erwachsen aus dieser Sachlage nicht unbedeutende Probleme. Einerseits muß man eine syntaktische (und semantische) Kategorie 'Gradpartikeln' (bzw. Satzoperatoren) einführen, andererseits müssen Funktionswechsel und Funktionsambiguitäten angemessen repräsentiert werden. Die Funktionsambiguität, wie sie etwa bei: Geh nur! (Gradpartikel und Modalpartikel) feststellbar ist, erfordert dieselben technischen Vorkehrungen wie bei anderen Fällen von Ambiguität: also einen Ambiguitätsconstraint, der die Zuordnung zu verschiedenen syntaktischen Tiefenstrukturen oder Semantischen Repräsentationen gewährleistet. - Wesentlich mehr Schwierigkeiten macht die (bislang offenbar noch nicht beschriebene) Erscheinung des Funktionsvorranges, wie er in dem Satz: Er ist selbst gefahren. zum Ausdruck kommt. Bezeichnend ist hierbei, daß sich die nicht-Gradpartikel-Funktion jeweils gegen die Gradpartikel-Funktion durchsetzt, allerdings wechselt dies je nach syntaktischer Umgebung. Die geschilderte Sachlage deutet auf eine allgemeinere Regularität, 
für die ich jedoch keine adäquate formale Darstellung kenne: Ableitungsbeschränkungen (Filterregeln) müßten praktisch für jede Gradpartikel formuliert werden, um die Erzeugung von Oberflächenstrukturen mit Gradpartikel-Funktionen in Positionen zu verhindern, in denen andere Funktionen Vorrang besitzen. Eine Steverung iber das Lexikon, das für jede einzelne Funktion, die ein bestimmtes Element erfüllen kann, alle und nur die möglichen Satzmuster verzeichnet, erscheint der Sachlage angemessener. Das typische Phänomen der "Verdrängung" einer bestimmten Funktion durch eine andere in einem bestimmten syntaktischen Muster ist aber damit nicht adäquat beschrieben.

\subsubsection{Stellungsgesetze}

Da Stellungsregeln einen wichtigen Teil einer grammatischen Beschreibung ausmachen, ist eine Klassifikation, die bei Stellungsregeln keine Generalisierungen zuläßt, nicht sehr nützlich. Im Laufe der Untersuchung haben sich erstaunlich konstante Grundmuster der Zuordnung zwischen Skopuselement(en) und Gradpartikeln herauskristallisiert, trotz zahlreicher Idiosynkrasien einzelner Gradpartikeln.

Typ 1, also die Plazierung der Gradpartikel unmittelbar vor der Skopuskonstituente bzw. vor der kontrastiv betonten Konstituente (wie im Falle der kontrastiv betonten Adjektivattribute), ist für alle Gradpartikeln die Normalstellung. Nur die Skopustypen Verbum, Verbalphrase sowie Satz machen hierin eine Ausnahme: in der Matrixsatz-Wortstellung, im Imperativ und in der Satzfrage folgt die Gradpartikel jeweils dem finiten Verbum. Hingegen nimmt sie bei analytischen Verbformen die Position vor dem infiniten Vollverb ein, im Konstituentensatz steht sie vor der gesamten Verbalphrase (bzw. vor dem Skopusverbum). Vielleicht ist dies ein Hinweis darauf, daß die Wortfolge Subjekt - Objekte - Verbum als elementarer zu gelten hat. Hierbei wird die diskontinuierliche Stellung von analytischen Verbformen vermieden. Ein wichtiger Hinweis darauf mag auch sein, daß gewöhnlich die Konstruktion finites Vollverb im Skopus - darauf folgende Gradpartikel zugunsten diskontinuierlicher Konstruktionen wie: Hans hat nur $g$ e $l \circ g$ e $n$ vermieden wird. - Noch eines bleibt anzumerken: führt die normale position von vorangestellter Gradpartikel und folgendem Skopus zu Ambiguitäten oder unerwiinschten Funktionswechseln, so wird auf die topikalisierte Stellung der Skopuskonstituente ausgewichen, wo dies möglich ist.

Typ 2: Er besteht in der Plazierung der Gradpartikel unmittelbar hinter der Skopuskonstituente. Außer der für alle Gradpartikeln verbindlichen Position 
hinter dem Skopustyp synthetische finite Vollverbform bei Matrixsatzwortstellung gibt es nur weitgehend unregelmäßig verteilte Möglichkeiten zur Nachstellung, konzentriert auf die Gradpartikeln nur und (in geringerem Maße) allein. Die Akzeptabilität der Nachstellung hängt bei diesen Elementen sowohl vam jeweiligen Skopustyp, von dessen Position im Satz und schließlich auch von der Länge der Skopuseinheit ab: die Akzeptabilität der Nachstellung nimmt mit der Länge der Skopuskonstituente ab. - Ferner muß man feststellen, daß alle akzeptablen Beispiele von Nachstellung der Gradpartikel stilistisch stark merkmalhaltig sind, bzw. bestimten Gebrauchssituationen oder Textsorten zugeordnet werden können. - Völlig inakzeptabel ist dieser Stellungstyp bei auch; bei den meisten anderen Elementen sind in einer Anzahl von Konstruktionsmustern keine eindeutigen Akzeptabilitätsurteile möglich. -

Auch diese Sachlage bereitet bei einer formalen Beschreibung sicherlich größte Schwierigkeiten. Kompliziert und vermutlich auch nicht erklärungsadäquat wäre sowohl die generelle Ermöglichung der Nachstellung der Gradpartikeln (die zahlreiche Filterregeln für das "Aussortieren" inakzeptabler Varianten erfordern wïrde) wie eine idiosynkratische Lösung (z.B. durch die Registrierung aller möglichen Formen von Nachstellung in den Lexikoneinträgen der jeweiligen Gradpartikeln). In beiden Lösungen wären nämlich die vielfältigen übergänge und Beurteilungsunsicherheiten, aber auch die stilistische Merkmalhaltigkeit nicht darstellbar. Nicht ausschließen möchte ich eine Erklärung der Konstruktion Skopuselement - nachgestellte Gradpartikel als eine Art "interner Topikalisierung", die in ihrer semantischen Funktion etwa der Topikalisierung der Skopuskonstituente mit Gradpartikel in Distanzstellung danach vergleichbar wäre: damit wäre nämlich der Weg frei, die zahlreichen Distributionlücken über semantische Unverträglichkeit zwischen der Funktion dieses Stellungstyps und der Gradpartikel-Interpretation zu erklären.

Typ 3: Hierbei befindet sich die Gradpartikel innerhalb derjenigen komplexen Konstituente, die das kontrastiv betonte Element enthält, und zwar unmittelbar vor diesem Element. Das betrifft vor allem Nominalphrasen und Präpositionalphrasen mit kontrastiv betontem Adjektivattribut oder Zahlwort, sowie analytische Verbformen in kontinuierlicher Stellung. Diese Konstruktion birgt ein doppeltes Problem: sie ist für eine Reihe von Gradpartikeln völlig normal (nur, bloß, lediglich, ausschließlich, nicht einmal), für eine weitere Gruppe ist sie nicht ausdrücklich inakzeptabel (sogar, selbst), für auch ist sie hingegen völlig unmöglich. Dazu kommt noch, daß diese umstellung aus der Position vor dem das Skopuselement enthaltenden Satzglied für einige Gradpartikeln eine 
einschneidende Interpretationsänderung zur Folge hat (nur), für andere hingegen nicht (sogar). Dies hängt sicher mit dem Wechsel des Skopus von Nominalphrase zu prädikativem Adjektiv und der damit verbundenen Interpretationsänderumg von quantifizierend zu skalierend zusammen, doch ist eine generelle Erklärung damit nicht möglich, da sich die Zahlwörter nicht in dieses Muster einordnen lassen.

Typ 4: Gradpartikel in Distanz vor und nach der Skopuskonstituente. Beispiele hierfür sind sehr selten und nur dann halbwegs akzeptabel, wenn durch besonders starken Kontrastakzent sowie durch bestimmte Phrasierungen die Zuordnung gesichert bleibt. Es bleibt in jedem Falle eine Performanzangelegenheit, die in der formalen Beschreibung wohl nicht berücksichtigt zu werden braucht.

Stellungstyp 5 ist demgegenüber eine sehr wichtige Möglichkeit. Bei ihm rückt die Skopuskonstituente an den Satzanfang (sie wird topikalisiert), die Gradpartikel folgt erst in größerem Abstand, und zwar nach dem finiten Verb, wobei sich zwischen finites Verb und Gradpartikel noch bestimmte Konstituenten einschieben können. Dieser Konstruktionstyp kann bei allen Gradpartikeln und Skopustypen auftreten (von wenigen "Lücken" abgesehen). Das Problem ist, daß auch in diesem Falle betont wird, und daß es nur die quantifizierende Interpretation erhalten kann. Ein ähnlicher Ausschluß der skalierenden Interpretation ist in der nur - und sogar - Gruppe nicht festzustellen. Vielmehr kann man bei der nur - Gruppe beobachten, daß die prinzipiell sowohl skalierend als auch quantifizierend möglichen Gradpartikeln nur und bloß (mit gewissen Einschränkungen auch lediglich) in dieser Konstruktion die skalierende Interpretation "wählen", während die nur in quantifizierender Interpretation möglichen Gradpartikeln einzig, einzig und allein, allein und ausschließlich entweder in eine andere Funktion ausweichen (z.B. allein und einzig), oder ein neues Bedeutungselement hinzugewinnen (wie ausschließlich im Sinne von immer nur) oder schlicht inakzeptabel sind. Eine mögliche Erklärung künnte lauten: die Funktion der Topikalisierung der Skopuskonstituente und die quantifizierende Interpretation von Gradpartikeln widersprechen sich. Die Akzeptabilität der Kombination von Topikalisierumg und quantifizierender Interpretation im Falle von auch widerspricht dem nicht: liegt doch durch die kontrastive Betonung von auch eine Abwandlung des Konstruktionsmusters vor.

Ein weiterer Konstruktionstyp, nämlich kontrastiv betonte Gradpartikel unmittelbar vor ihrer Skopuskonstituente, kormt nur in sehr starren Konstruktionen vor, etwa wenn die explizite Formulierung der Präsupposition oder Assertion eines Gradpartikel-Satzes (je nach Gradpartikel-Gruppe) mit diesem selbst ver- 
bunden wird. Grumdsätzlich scheint hierbei nur quantifizierende Interpretation möglich zu sein.

Damit ist der Weg für sehr allgemeine Stellungsgesetze sowie deren Verknüpfung mit bestimmten semantischen Funktionen gebahnt, trotz der Probleme im Bereich der Nachstellung der Gradpartikeln.

\subsubsection{Syntaktische Umformungen}

5.1.4.1. Die Beobachtung der Stellungsgesetzmäßigkeiten ergab, daß die Zuordnumg zwischen Gradpartikeln und Skopus in allen Satztypen in der gleichen Weise vor sich geht, mit geringfügigen Änderungen aufgrund der Änderung der Verbstellung, des Fehlens einzelner Konstituenten usw.

Die Erfassung von Funktionsambiguitäten bzw. Funktionswechseln war deswegen eine besondere Aufgabe dieses Abschnitts, weil etwa der Wechsel zur Modalpartikel-Funktion sehr eng mit der jeweiligen Satzart zusammenhängt. Die im folgenden vorgelegte Liste von Funktionswechseln und -ambiguitäten erfaßt der Übersichtlichkeit wegen auch diejenigen Fälle, die nicht unmittelbar mit einzelnen Satztypen zusammenhängen. Insofern versteht sich dieser Abschnitt als ein Nachtrag zur Distribution.

nur: diese Gradpartikel kann in Imperativen und Wortfragen sowohl in Gradpartikel-Funktion als auch in Modalpartikel-Funktion interpretiert werden; in manchen Fällen liegt al.lerdings auch "Vorrang" der Modalpartikel-Funktion vor. Dazu muß sich allerdings nur in Positionen befinden, die einem Skopus verb bzw. Verbalphrase entsprechen (wobei die kontrastiv betonte Konstituente keineswegs das Verbum oder die letzte Hauptkonstituente der Verbalphrase sein muß).

allein: die bei diesem Element zu verzeichnenden Funktionswechsel und -ambiguitäten hängen nicht mit den Satztypen zusammen. allein wechselt vielmehr in allen Positionen, in denen es auch als Modaladverb auftreten kann, in diese Funktion, also ein Fall von "Vorrang". Die Lücke betrifft also den Skopus Verb bzw. Verbalphrase, und die diesen Positionen entsprechenden Anordnungen bei anderen Skopustypen. Gleichzeitig wird dabei eine Änderung des Betonungsmusters erzwrungen: allein erhält den Satzakzent.

einzig: es wechselt ebenso wie allein und in genau denselben Positionen zur Funktion des Modaladverbs, unabhängig von der jeweiligen Satzart, und zieht dadurch den Satzakzent auf sich.

auch: dieses Element wechselt nur in sehr wenigen, halbidiamatischen Satzmu- 
stern die Funktion. Das betrifft nicht oder kaum die Aussagesatzform, aber die beiden Fragesatzformen und verkürzte Ausrufe, und zwar jeweils in der Position, die dem Skopus Verb bzw. Verbalphrase bzw. diesen äquivalente Stellungen entsprechen.

selbst: der bei dieser Partikel zu verzeichnende Vorrang der Reflexivum-Funktion ist unabhängig von allen Satztypen. Durch diesen Funktionsvorrang entsteht eine Distributionslücke in denjenigen Positionen, in denen auch das Reflexivum vorkammt, also wiederum bei der dem Skopustyp Verbum entsprechenden Stellung.

Stärker am Rand der Thematik liegt die Feststellung, daß starre Akzenttypen, wie sie etwa im Imperativ vorliegen, die Wahl bestimmter Skopustypen, etwa der Subjekts-Nominalphrase im Imperativ, verhindern oder doch stark einschränken.

Auf das Verhalten der Gradpartikeln gegenüber performativen Formeln wie Ich fordere dich hiermit auf ... bin ich im Analysenteil nicht näher eingegangen. Gegen die Feststellung von Fraser (1971:164), daß Gradpartikeln, die in ihrem Skopus die gesamte performative Formel oder Teile von ihr haben, die performative Kraft dieser Formeln zerstören, konnten keine Belege nachgewiesen werden. Der entsprechende Satz wird dadurch allerdings nicht ungrammatisch: Sogar $i c h$ fordere dich hiermit auf, daß ... Zur Erklärung dieser Erscheinung kann ich nur Vermutungen anführen: entweder duldet die Formel keine Kontrastierung, da dies automatisch zu einer Beschreibung oder Interpretation von Sprechakten aber nicht zu ihrer Durchführung zwingen wïrde; oder die Gradpartikeln enthalten selbst illokutionäre Elemente, die nicht zu anderen illokutionären Rollen passen. Dafür gibt es aber keinen Anhaltspunkt.

\subsubsection{Die Bildung von Spaltsätzen sollte bei der Lösung von zwei Aufgaben} helfen: bei der Abgrenzung des Skopus von Gradpartikeln (nach der Hypothese des "protected environment" von Fraser 1971) und bei der Aufdeckung der Semantik einzelner Gradpartikeln, ausgehend von der These Frasers (1971: 174), daß die Semantik von even mit der Funktion von Spaltsätzen unvereinbar sei. Hinderlich war dabei die im Laufe der Untersuchung bestätigte geringe Akzeptabilität dieser Konstruktion im Deutschen (siehe dazu auch R. Posner 1972: 68 ff.). Dazu kommt noch, daß keineswegs alle Skopustypen abgespalten werden können. Insbesondere Adverbien und finite Verben sind ausgesprochen spaltsatzunwillig. Darauf hat schon R. Jackendoff (1972: 233) in seiner Kritik an Akmajians "tiefenstruktureller" Lösung des focus-presupposition-Problems hingewiesen. Damit bleiben nur noch einfache Nominalphrasen, denn auch mit steigender Länge einer Nominalphrase wächst die Inakzeptabilität dieser Spaltsatzkonstruktion. 
Schließlich ergab sich noch der wichtigste Einwand: wenn der Skopus einer Gradpartikel kleiner ist als die Nominalphrase, dann muß dennoch die gesamte Nominalphrase abgespalten werden:

$$
\begin{aligned}
& \text { Nur eine quantifizierende Interpretation kann den Tatbestand erklären. } \\
& \text { Nur eine quantifizierende Interpretation ist es, die ... erklären kann. } \\
& \text { Eine nur quantifizierende Interpretation kann den Tatbestand nicht } \\
& \text { erklären. } \\
& \text { Eine nur quantifizierende Interpretation ist es, die den Tatbestand } \\
& \quad \text { nicht erklären kann. } \\
& \text { Eine nur quantifizierende ist es, die Interpretation den Tatbestand } \\
& \text { nicht erklären kann. }
\end{aligned}
$$

Dies zeigt, daß man mit dem Spaltsatztest nur Daten über Nominalphrasen, aber nicht über den Skopus von Gradpartikeln erhält.

Noch schwieriger gestalten sich die Verhältnisse bei der Beobachtung des Zusammenwirkens der Spaltsatzfunktion mit der Semantik einzelner Gradpartikeln. Die Unschärfen von Akzeptabilitätsurteilen sind in diesem Falle beträchtlich. Zusammenfassend läßt sich feststellen: die Funktion des Spaltsatzes ist die nachdrückliche Identifizierung verbunden mit einer Einzigkeitsbehauptung. Gradpartikeln sind offenbar nur in ihrer skalierenden Interpretation damit vereinbar, soweit dabei nicht Nichteinzigkeit behauptet (nicht nur) oder präsupponiert (sogar) wird. Nur und $b$ LOB sind also in ihrer skalierenden Interpretation möglich, ebenso sogar, soweit es in einer Version ohne NichteinzigkeitsPräsupposition vorliegt, also bei einer Skala, deren Werte sich gegenseitig ausschließen. Auch hingegen fällt wegen seiner konstanten NichteinzigkeitsPräsupposition aus. Zur Absicherung dieser Aussagen wären aber gründliche Untersuchungen über die Funktion von Wortstellungstypen wie Spalten, Topikalisieren, Extraponieren und Left Dislocation notwendig.

Im Zusammenhang mit der Frage nach dem Verhalten des Skopus von Gradpartikeln gegenuber der Spaltsatzbildung kann auch die Frage des Verhaltens von Idiamen gegenüber Gradpartikeln behandelt werden. Dies ist kein spezifisches Problem für Gradpartikeln, denn die Unteilbarkeit von Idiamen bei der Spaltsatzbildung, bei Umstellungen und z.T. bei der Umwandlung von Sätzen vom Aktiv ins Passiv usw. gilt auch ohne Gradpartikeln. Die Tatsache, daß die Gradpartikel nicht einen Teil eines Idiams als Skopus abtrennen kann, erklärt sich aus dieser allgemeinen Gesetzmäßigkeit, aber auch aus der Tatsache, daß der Skopus eine primär semantische Erscheinung ist.

Ein wesentlich wichtigerer Punkt ist mit der Frage angesprochen, wie sich der Skopus von Gradpartikeln gegeniber anderen transformationellen Veränderungen verhält, z.B. gegenüber der Passivtransformation. Die möglichen Konstellationen sind hierbei: man macht bestimmte Annahmen ïber die möglichen Skopus- 
typen hinsichtlich ihrer formalen Eigenschaften: so etwa, daß nur vollständige Konstituenten (mit allen Teilästen) Skopus einer Gradpartikel sein können, und daß die Verknüpfung einer Gradpartikel mit einer Konstituente des Typs a wieder eine Konstituente des Typs a ergibt. Die Schwierigkeiten dieser Hypothese liegen auf der Hand: man muß entweder die Strukturbeschreibung von Sätzen einer natürlichen Sprache so gestalten, daß nur solche Einheiten als Konstituenten auftreten können, die tatsächlich den Skopus von Gradpartikeln bilden können. Danach könnten Artikel usw. nicht mehr als Konstituenten auftreten. Unberücksichtigt ist dabei noch die Unterscheidung zwischen Konstituenten der Oberflächenstruktur und solchen der Tiefenstruktur. - Oder aber man mu eine Anzahl von Zuordnungsrestriktionen formulieren, um unzulässige Kombinationen zu vermeiden. Konkreter: von den in der Oberflächenstruktur erscheinenden Konstituenten können Nominalphrasen in allen denkbaren Formen, Verbalphrasen, Verben, prädikative Adjektiva und prädikative Nomina sowie Adverbien, Pronomina, Gliedsätze, Attribute, Konjunktionen, Präpositionen ... Skopus von Gradpartikeln sein. Tiefenstrukturell lassen sich diese Möglichkeiten vielleicht reduzieren auf folgende Skopustypen (die semantischen Restriktionen mïssen hier natïlich unberücksichtigt bleiben):

a. Nominalphrasen bzw. Argumente; die Tatsache, daß bei komplexen Nominalphrasen einzelne Glieder kontrastiv hervorgehoben werden können, verändert nicht im eigentlichen Sinn den Skopus (die Skopusgrenzen), sondern nur dessen innere Struktur. Insofern gehören auch kontrastiv betonte Attribute und Gliedsätze, soweit sie einzelnen Argumenten zugeordnet sind oder sie vertreten, in diese Gruppe, und zwar unabhängig von der jeweils in ihnen herrschenden kontrastiven Betonung. Ebenso selbstverständlich gehören alle Formen von Pronomina in diese Gruppe.

b. Prädikate im logischen Sinn: also Verben mit ihren Untergruppen, sowie prädikative Adjektiva und Nomina. Dazu gehören auch attributive Adjektive, soweit sie kontrastiv betont sind und die Gradpartikel unmittelbar vor ihnen steht. c. Adverbiale in ihren sämtlichen Untergruppen. Kontrastiv betonte Einzelteile etwa von adverbialen Präpositionalphrasen (z.B. Präpositionen) sind nicht selbst Skopus, sondern jeweils die vollständigen Adverbialphrasen.

Doch bleibt es nicht bei dieser plausiblen Unterteilung in die Hauptkonstituenten der logischen Stıuktur. Es können nämlich auch, wie aus der Untersuchung der Oberflächenstruktur einwandfrei hervorgeht, sehr kamplexe Oberflächenstrukturen) im Skopus von Gradpartikeln liegen: so z.B. eine vollständige Verbalphrase, oder ein Satz, oder, wie ich glaube gezeigt zu haben, sogar die Verbindung Subjekt + Hauptverb, ev. sogar die Kambination von verschiedenen Ob- 
jekten ohne das Verb und das Subjekt.

Daraus ergeben sich fundamentale Schwierigkeiten für jede Form der Darstellung. Sowohl in einer Tiefenstruktur in der Art der generativen Syntax wie in einer Semantischen Repräsentation, die sich an der Prädikatenlogik orientiert, ist das Operator - Skopus - Verhältnis bei einfachen Konstituenten als Skopus ohne größere Schwierigkeiten darstellbar, sei es nun durch die Erzeugung der Gradpartikeln mit Hilfe von Phrasenstrukturregeln unmittelbar bei ihren Skopuskonstituenten oder durch die transformationelle zuordnung noch vor anderen transformationellen Veränderungen. In beiden Fällen müssen semantische und formale Filter die inakzeptablen Kombinationen blockieren. Ein Unterschied besteht hier lediglich insofern, als das System der Konstituenz für die Darstellung des Operator - Skopus - Verhältnisses wenig geeignet ist.

Man kann allerdings auch die Argumentation umdrehen und davon ausgehen, daß die Oberflächenstruktur jeweils genügend formale Hinweise auf die Zuordnung einzelner Einheiten $z u$ bestimmten anderen Einheiten enthalten muß. Mittel dieser Zuordnumg können Stellung, Kontrastakzent sowie verschiedene Formen der Übereinstimmung in zugeordneten grammatischen Kategorien usw. sein. Nun ließ sich aber in einer ganzen Reihe von Fällen nachweisen, daß der Skopus von Gradpartikeln bzw. genauer die Skopusgrenzen allein aufgrund formaler oberflächenstruktureller Eigenschaften nicht festzulegen waren, daß vielmehr Informationen aus dem jeweiligen verbalen oder situationellen Kontext dazu nötig waren. Dies zeigt den semantischen bzw. pragmatischen Kern des Problems. Eine nur syntaktische Zuordnung kann diese Aufgabe keinesfalls bewältigen, da sie ja nicht nur ein Einbahnstraßen-Definitionssystem sein soll, das die Zuordnung bestimmter Tiefenstrukturen zu bestimmten Oberflächenstrukturen festlegt, sondern auch die Zuordnung bestimmter Oberflächenstrukturen zu bestimmten Tiefenstrukturen. Dabei genügt nicht die Feststellung der Ambiguität, vielmehr muß der Vorgang der Disambiguierung noch beschreibbar sein, und dieser ist nicht allein von formalen Merkmalen bestimmt. - Die Diskussion sowohl um das Verhalten der Einheit Gradpartikel + Skopus gegenüber der Spaltsatzbildung als auch gegenuber der Passivtransformation muß unter diesem Aspekt gesehen werden. In allen Fällen kann man die unverletzliankeit des Skopus konstatieren, auch im Falle des Passiv, wie der folgende Satz sehr schön zeigt: Damit werden von Ross wiederum nur pragmatische Sachverhalte in den bestehenden syntaktisch-semantischen Apparat eingegliedert. Im zugehörigen Aktivsatz umfaßt der Skopus die gesamte Verbalphrase, in dem zitierten Passivsatz umfaßt er die gleichen Elemente, nun aber aufgeteilt auf Subjekts-Nominalphrase und einen Teil der Verbalphrase. Aber selbst wenn im Falle des Passiv Skopusformen auftreten würden, die eine 
Ableitung vom parallelen Aktivsatz unmöglich machen würden, wäre dies kein ausschließliches Argument für eine Skopuszuweisung erst in der Oberflächenstruktur, sondern ebensogut für eine andere Behandlung passivischer Sätze; deren Selbständigkeit in pragmatischer und vielleicht sogar semantischer Hinsicht wird ohnehin immer mehr zugestanden. Das heißt, sie miißten eine vom parallelen Aktivsatz verschiedene Tiefenstruktur bzw. Semantische Repräsentation erhalten. Allerdings bestünde dann die Notwendigkeit, eine eigene Ebene für die Darstellung rein formaler Beziehungen zwischen Sätzen unterschiedlicher Bedeutung zu definieren.

5.1.4.3. Bei der Untersuchumg der Negationsmöglichkeiten in Gradpartikel-Sätzen waren drei Teilaufgaben zu bewältigen: die rein formale Frage nach den jeweiligen Negationsmöglichkeiten in einem Gradpartikel-Satz, die Suche nach der präsuppositionsneutralen Negationsform für Gradpartikel-Sätze, und die Frage der überlagerung von Negationsskopus und Gradpartikel-Skopus. Dabei ergaben sich folgende Grundkonstellationen:

1. Negation + Gradpartikel + Skopuskonstituente: in dieser Kombination behält die Gradpartikel ihren Skopus, der eng verknüpft ist mit dem Kontrastfokus des Satzes. Der Skopus der Negation ist dieser Kontrastfokus. Fokusnegation kann aber als die Normalform der Satznegation (starken Negation) gelten. Dies deckt sich mit der Tatsache, daß es sich bei dieser Form, soweit sie auftritt, um die präsuppositionsneutrale Negation von Gradpartikelsätzen handelt. Im einzelnen stellt sich die Sachlage folgendermaßen dar: nicht nur ist ohne Komplikationen bei allen Skopustypen möglich. "nicht auch, "nicht sogar: beide Fonmen sind in aller Regel inakzeptabel, obwohl im Gedankenexperiment zusammen mit den jeweiligen Bedeutungselementen rekonstruierbar. Man kann nur vermuten, daß dies damit zusammenhängt, daß bei diesen Gradpartikeln die explizite Formulierung der Assertion weitgehend dem Wortlaut des Satzes selbst entspricht.

2. Gradpartikel + Negation + Skopuskonstituente. In dieser Konstruktion handelt es sich um die Kontrastnegation, die die Gradpartikel aus ihrem Skopus, der mit dem Kontrastfokus des Satzes eng verknüpft ist, verdrängt. Dadurch ist die Partikel nur in konjunktionaler Funktion bzw. in Gradpartikel-Funktion mit Satzskopus möglich, falls die jeweilige Partikel in einer von diesen beiden Funktionen überhaupt vorkommen kann. Im Falle von nur nicht gibt es keinerlei Komplikationen, da die konjunktionale Funktion von nur semantisch gut von der Gradpartikel-Funktion zu trennen ist. Bei auch nicht herrscht jedoch große Verwirrung, da diese Kombination bei einigen Skopustypen auch im Sinne der Gradpartikel nicht einmal möglich ist (in skalierender Funktion also). Von sogar 
nicht kenne ich keine akzeptablen Beispiele. Dies mag mit der fehlenden konjunktionalen Funktion von sogar zusammenhängen. - In dieses Erklärungsschema paßt allerdings nicht die folgende Konstruktion: Alle haben gelogen, nur nicht $H a n s$. Die zutreffende Paraphrase hierfur lautet: Alle haben gelogen, nur Han $s$ hat nicht gelogen. Der einzige Ausweg bestiunde darin, das obige Beispiel als Fall von fehlerhafter (wenn auch häufiger) Stellung der Negation zu erklären. Die richtige müßte demach Lauten: Alle haben gelogen, nur $H$ a $n s$ nicht. Überraschen muß dann aber, daß die obige Konstruktion für auch nicht akzeptabel, für sogar nicht jedoch deutlich inakzeptabel ist.

3. Gradpartikel + Skopuskonstituente + Negation. Folgt der Skopuskonstituente das finite Verb, so muß die Negation nach diesem stehen, folgt jedoch die Einheit Gradpartikel + Skopuskonstituente dem finiten Verb, so kann die Negation umittelbar hinter der Skopuskonstituente stehen. Es handelt sich also von der Stellung her um die gewöhnliche Satznegation (starke Negation), die aber hier auf keinen Fall präsuppositionsneutral ist. Dies liegt wohl daran, daß ihr Skopus nicht den Kontrastfokus umfaßt. Andererseits widerlegt diese Negationsmöglichkeit die Ansicht, daß der Negationsskopus grundsätzlich auf den (oder einen) Satzfokus beschränkt ist. - Die geschilderte Konstruktion ist ausnahmslos ohne Schwierigkeiten verwendbar, außer wenn sich das finite Verbum im Skopus der. Gradpartikel befindet. In diesem Fall ist die normale Satznegations-Position nicht mehr gut möglich.

4. Eine Kontrastnegation (lokale Negation), die neben dem mit dem GradpartikelSkopus meist verknüpften Kontrastfokus einen weiteren Kontrastfokus bedingen wïrde, führt, wie jede Vermehrung der Anzahl von Fokus-Konstituenten, zu wachsender Inakzeptabilität.

5. Die schwache Negation hat sich als für linguistische Untersuchungen irrelevant erwiesen.

\subsection{Skopus und Fokus}

Die grundsätzlich für alle Gradpartikeln gültige Liste der möglichen Skopuskonstituenten wurde bereits im vorausgehenden Abschnitt (S. 303) gegeben. Die gelegentlichen Lücken bei einzelnen Gradpartikeln stellen nicht das syntaktische Grundmuster der Distribution in Frage.

Die semantischen Anforderungen an die jeweiligen Skopuselemente beschränken sich bei allen drei Gradpartikel-Gruppen auf die quantifizierbarkeit bzw. Skalierbarkeit, bzw. auf die mögliche Anordnung in polare Paare. Die Quant:fi- 
zierbarkeit ist naturgemäß bei den Argument-Konstituenten höher als bei den verschiedenen Prädikatstypen, umgekehrt sind letztere eher für die Skalierung geeignet.

Die Relationen zwischen den Stellungsmöglichkeiten der Gradpartikeln einerseits und den Skopusgrenzen andererseits wurden bereits in den Grundrissen skizziert (S. 297). Entscheidend ist die Feststellung, daß nur in relativ wenigen Fällen die Stellung der Gradpartikel eine einwandfreie Identifizierung des Skopusbereiches zuläßt, und auch da eigentlich nur durch das Zusammenwirken der verschiedenen möglichen Stellungstypen.

Auch die Hinzunahme des kontrastiven Akzentes führt nicht in jedem Falle zu einer einwandfreien Identifizierung des Skopus, und zwar aus folgenden Gründen: nicht nur die kontrastiv betonte Konstituente befindet sich jeweils im Skopus, sondern meist die gesamte Konstituente, in der sie enthalten ist (das Satzglied also). Der Kontrastakzent ist zumeist nur ein wichtiges Mittel zur internen Strukturierung der Skopuskonstituente. Dazu kommt, daß in einer Reihe von Fällen der Kontrastakzent auch auf einem Element plaziert sein kann, das auch einen ganz normalen Satzakzent tragen kann. Ist dann nicht ein expliziter Kontrastsatz vorhanden, so ist ein solches Gebilde formal nicht von einem normalen Satz zu unterscheiden. Betroffen sind davon die Skopustypen aus dem Bereich der Verbalphrase. - Andererseits ist die Verknüpfung des GradpartikelSkopus mit dem Satzfokus so fest, daß Elemente, die nicht fokusfähig sind, wie etwa die Satzadverbien, auch nicht den Skopus von Gradpartikeln bilden können.

Das Hauptproblem ist, daß bei nur geschriebenen Äußerungen die Zuweisung des Kontrastakzentes nur in den allerseltensten Fällen absolut sicher festliegt. Aber auch bei festliegendem Kontrastakzent sind in den meisten Fällen mehrere Möglichkeiten der Skopusabgrenzung gegeben, wiederum vor allem im Bereich der Verbalphrase, wenn sich der Kontrastakzent mit dem nomalen Satzakzent deckt: nämlich die Konstituente mit dem Kontrastakzent selber, oder die größeren Konstituenten, in denen sie enthalten ist, z.B. Objekts-Nominalphrase, Verbalphrase, Satz.

'Kontrast' ist ebensowenig wie 'Skopus' primär eine syntaktische Angelegenheit, sondem eine semantische, obwohl es natürlich auch formale Kontrastierung gibt. Diese stellt aber eine Randerscheinung dar. - Das adäquate Mittel zur Erforschung von beidem sind deshalb auch Kontrastsätze, die strengen formalen Anforderungen genügen müssen: sie mïssen bis auf die jeweils kontrastierte Konstituente bedeutungsgleich (also keineswegs formgleich) sein. Es stellte sich heraus, daß jede Gradpartikel bzw. jede Gradpartikelgruppe für jede ihrer semantischen Funktionen ganz bestimmte Kontrastierumgsschablonen aufweist: 
a. für nur:

nicht..., sonderm nur... (skalierend und quantifizierend) nur..., aber nicht (auch) ... (iberwiegend quantifizierend)

nicht nur..., sonderm auch... (uberwiegend quantifizierend)

... , nicht nur... (skalierend und quantifizierend)

nicht nur..., sonderm sogar... (nur skalierend)

nur..., nicht einmal... (nur skalierend)

nur... nicht..., aber doch... (nur quantifizierend)

b. für auch:

nicht nur..., sonderm auch ... (skalierend und quantifizierend)

auch ..., nicht nur... (überwiegend quantifizierend)

nicht..., und auch nicht... (nur quantifizierend)

$\ldots$, und ... a u ch... (nur quantifizierend)

... nicht, and ... a u ch nicht... (nur quantifizierend)

c. für sogar:

nicht nur..., sondern sogar... (skalierend)

sogar..., nicht nur... (skalierend)

nicht einmal..., ganz zu schweigen von ... (skalierend)

... nicht..., und nicht einmal... (skalierend)

Diese kontrastierenden Formuliermgen zeigen die enge Funktionsverknüpfung zwischen den drei Gradpartikel-Gruppen. - Diese Kontrastsätze haben eine mehrfache Funktion: wir können mit ihrer Hilfe feststellen, welches Element jeweils kontrastiert wird. Damit können wir auch feststellen, wie weit der Skopus der Gradpartikel reichen kann. Es sei ausdruicklich darauf hingewiesen, daß die Skopuskonstituente nicht mit dem kontrastierten Element identisch ist, sondern bis auf wenige Fälle die gesamte Phrase umfaßt, in der das kontrastierte Element enthalten ist. Um ein Beispiel zu bringen: wenn nur das Adjektivattribut kontrastiert wird, so ist im Normalfall die gesamte Nominalphrase, in der das kontrastierte Adjektivattribut enthalten ist, der Skopus. Er reicht in diesem Falle aber auch nicht über die Nominalphrase hinaus. Dies wäre erst der Fall, wenn nicht nur alle Elemente der Nominalphrase, sondern z.B. auch noch das Hauptverbum des Satzes kontrastiert werden. Natuirlich ist es auch möglich, ein absolutes Verb mit einer vollständigen Verbalphrase zu kontrastieren, die außer dem Verbum noch Objekte und Adverbiale enthält. In dieser Richtung mu also die bisherige Rede vom kontrastiven Skopus von Gradpartikeln korrigiert werden. Gleichzeitig zeigt uns die Kontrastierungsformulierung bzw. die Kontrastierungsschablone, welche Möglichkeiten der Gradpartikelfunktion in Betracht kammen. Allerdings ist nur in einigen wenigen Fällen eine sichere Entscheidung zwischen 
skalierender und quantifizierender Interpretation aufgrund dieser Formeln möglich. - Außerdem liefert uns der Inhalt der einzelnen Kontrastsätze Hinweise auf die Sphäre, auf den Bereich, aus dem bei nur - Sätzen die Assertion, bei auch - und sogar - Sätzen die Präsupposition stammen kann. Und schließlich stellen die Kontrastsätze spezifische Fälle von Assertionen bei nur - Sätzen und von Präsuppositionen bei auch - und sogar - Sätzen dar. Damit besitzen wir außer verwertbaren Hinweisen auf die Skopusabgrenzung auch noch wertvolle Anhaltspunkte für eine adäquate semantische Analyse sowie für eine einigermaßen befriedigende explizite Formulierung der einzelnen Bedeutungsfaktoren, die insbesondere bei den zahlreichen Fällen der impliziten Kontrastierung sehr schwer fällt.

Hinzuweisen bleibt nun noch im Rahmen dieses Teilthemas auf zwei Einzelaspekte, und zwar zunächst einmal auf das Verhalten des Gradpartikel-Skopus zum Skopus anderer Operatoren wie etwa der Negation. Aussagen dazu finden sich auch im Abschnitt über die Negationsmöglichkeiten in Gradpartikel-Sätzen (S. 305). Folgendes läßt sich sagen: steht die Negation nicht unmittelbar vor der Gradpartikel (dies ist nur bei nur möglich), so umfaßt der Skopus von nicht Gradpartikel und Gradpartikelskopus. Der Form nach handelt es sich also um eine lokale Negation oder Kontrastnegation, der Wirkung nach um eine Fokusnegation. Das Eigenartige ist hierbei, daß dies die einzige präsuppositionsneutrale Negierungsmöglichkeit bei Gradpartikeln ist. Sie verkehrt lediglich die Assertion in ihr Gegenteil. Steht die Negation unmittelbar nach der "Gradpartikel", so handelt es sich wiederum um eine kontrastive Negation, die allerdings diesmal die Gradpartikel aus ihrem Skopus verdrängt und zum Wechsel in den konjunktionalen Gebrauch zwingt, wo dies möglich ist (nämlich bei nur ; nicht möglich ist es bei sogar; bei auch gibt es beide Möglichkeiten, je nach syntaktischer Ungebung). - Schließlich gibt es noch die Möglichkeit, wo Stellung und Anzahl der Satzglieder dies zulassen, daß eine normale Satznegation (starke Negation) eingefügt wird, und zwar i.d.R. unmittelbar nach dem finiten Verb, wenn dieses nach dem Gradpartikelskopus steht. Wiederum ist das Erstaunlichste an dieser Negationsform, die in allen anderen bekannten Fällen von Präsuppositionen als präsuppositionsneutral gilt, daß sie im Falle der Gradpartikel-Sätze die Präsupposition betrifft, ebenso wie die Assertion (also nicht nur, wie die externe = schwache Negation, im Hinblick auf die Präsupposition ambig ist). Die Erklärung für dieses vam üblichen Negationsverhalten abweichende Ergebnis liegt in der jeweiligen Relation der Negation zum kontrastiven Satzfokus. Dies liefert auch die Begründung für die Notwendigkeit der Unterscheidung von Assertion und 
Präsupposition bei einer Paraphrase etwa für nur. Die früher üblichen Parphrasen und ihre Formalisierungen führen zu fehlerhaften Ergebnissen bei lem jeweiligen Negationen:

$$
\begin{aligned}
\text { nur } x & \equiv x \text { und nicht nicht-x } \\
\text { nicht nur } x & \equiv \text { nicht }(x \text { und nicht nicht-x) } \\
& \equiv \text { nicht } x \text { und nicht } x \\
\text { nur } x & \equiv(\mathrm{x}) \wedge \neg(\neg \mathrm{x}) \\
\text { nicht nur } x & \equiv \neg(\mathrm{x}) \wedge \neg(\neg \mathrm{x}) \\
& \equiv \neg \mathrm{x} \wedge \neg \mathrm{x}
\end{aligned}
$$

Daß dieser Notierungsvorschlag in semantischer Hinsicht unzulänglich ist, hat bereits Bartsch (1972: 195) deutlich gemacht.

Ein weiterer Aspekt ist die Problematik des Fragetests oder besser ds Tests der natürlichen Antwort im Zusammenhang mit Gradpartikel-Sätzen. Di:ser Test ist für nichtkontrastive Sätze ein geeignetes Mittel, um die Fokusgnnzen für sie zu ermitteln. Für kontrastive Sätze jedoch, also nicht nur fü: Gradpartikelsätze, ist er - für diesen Zweck - nicht geeignet. Andererseis lassen Frage-Antwort-Paare bei Gradpartikel-Sätzen gewisse Schlüsse auf de Partikelsemantik zu. Etwa bei dem folgenden Satzpaar: Wer alles war denn 'a? Nur $H a n s$ war da. Die Frage enthält eine Nichteinzigkeits-Präsuppositon, die in der Antwort durch die Einzigkeits-Assertion von nur zurückgewiesen wird. Für auch sieht eine vergleichbare Konstellation folgendermaßen aus: war nx $K a \operatorname{rr} l a$ ? - Nein, auch $H$ an $s$ war da. Hier wird die Entscheidungsfr.ge um Einzigkeit - Nichteinzigkeit durch Präsupposition wie Assertion des auth Satzes beantwortet. Und schließlich für sogar: Ist er etwa/nur/nicht einmi $b$ e f $r i$ e $d i g$ end ? - Nein, er ist sogar gut.

Wie die formale Beschreibung des Kontrastfokus auszusehen hätte, dariier kann ich keine konkreten Angaben machen. Keinesfalls jedoch ist der sonst verschiedentlich gemachte Vorschlag, daß ein normaler Satzfokus als höchste 'roposition in einer Baum-Struktur dargestellt wird, ohne tiefgreifende Modi:ikationen auf die Beschreibung eines Kontrastfokus übertragbar (abgesehen vol den Problemen, die dieser Iösungsvorschlag bereits bei der Darstellung eines lormalen Satzfokus bewirkt). Eine erklärungsadäquate Darstellung mïßte entwecer die Satzgrenzen überschreiten und die allfälligen kontrastierten Sätze in die Beschreibung einbeziehen, oder aber, er müßte die mit einem Kontrastfokus jeweils verbundenen spezifischen Präsuppositionen in die Beschreibung einbalen. Für die Darstellung von Präsuppositionen dieses Typs ist aber bislang keir befriedigendes Verfahren bekannt. 
5.3. Zur Semantik und Pragmatik der Gradpartikeln

Ich behandle die einzelnen Gruppen getrennt, gehe dabei auf die Besonderheiten der jeweiligen Einzelelemente ein und ziehe innerhalb der einzelnen Kapitel Querverbindungen zu den anderen Gruppen.

\subsection{1. nur - Gruppe}

Diese Gruppe zeigt in semantischer Hinsicht eine deutliche Zweiteilung: nur, bloß und lediglich können sowohl quantifizierend als auch skalierend sowie polar gebraucht werden; allein, einzig, einzig und allein und ausschließlich können nur quantifizierend gebraucht werden. Daneben weist allein noch eine partikelähnliche Funktion im Sinne von schon auf, und ausschließlich kann in gewissen Texten auch die Funktion von immer nur erhalten. Für beide Interpretationsarten (wenn man die polare als "Mischform" zunächst ignoriert) steht in diesem Falle (abgesehen von lediglich und einzig sowie einzig und allein, wo diese Negationsform nicht üblich ist) eine präsuppositionsneutrale Negation zur Verfügung: nicht nur. Sie erleichtert die Analyse nach Assertion und Präsupposition sowie konversationeller Implikatur sehr.

Die quantifizierende Interpretation stellt bei der nur - Gruppe an die jeweilige Skopuskonstituente bzw. an das darin enthaltene kontrastierte Element keine andere Anforderung als Kontrastierbarkeit. Die präsuppositionsneutrale Negation erweist dieFormulierung des jeweiligen Satzes abzüglich der Gradpartikel als die explizite Präsuppositionsformulierung des Satzes. Die Assertion des Gradpartikel-Satzes beinhaltet, daß für keine andere Skopusformulierung als die im Gradpartikel-Satz vorliegende der betreffende Satzzusammenhang eine zutreffende Äußerung ergibt. Oder in einer Paraphrase: nur $x=x$ und nicht $n i c h t-x$. , wobei $\mathrm{x}$ fïr den Skopusinhalt unabhängig vom jeweiligen Skopustyp stehen soll. Diese trmulierung, gleichgültig ob sie nun nur als informelle Paraphrase oder aber als prädikatenlogische Notation geboten wird, weist bei allen Vorteilen auch zwei entscheidende Nachteile auf: a. Sie löst den GradpartikelSatz in zwei durch Konjunktion verbundene Assertionen auf. Damit wird ein nicht geringer Teil der sprachlichen Gesetzmäßigkeiten, die für diesen Satztyp gelter, von jeder Beschreibung ausgenommen. Will man sie berücksichtigen, so muß man das erste Glied der Adjunktion als Präsupposition deutlich vom zweiten Glied als der eigentlichen Assertion des Gradpartikel-Satzes abtrennen.b. Als weiterer Mangel muß betrachtet werden, daß sie den Bereich der möglichen Assertion nicht definiert. Sieht man einmal davon $a b$, daß man diesen Bereich formal auf 
typgleiche Elemente (gemeint bezüglich der Skopuskonstituente, im linguistischen oder prädikatenlogischen Sinn) beschränken könnte, so fehlt nicht nur in dieser Paraphrase, sondern generell die Möglichkeit, diesen Assertionsbereich $\mathrm{zu}$ definieren. Er ist je nach Skopuskonstituente unterschiedlich groß, unterschiedlich genau festgelegt und damit auch unterschiedlich genau rekonstruierbar. Trotzdem ist ex keineswegs beliebig: kompetente Sprecher des Deutschen können mit ziemlicher Sicherheit und weitgehend übereinstimmend sowohl die Assertion eines solchen Gradpartikel-Satzes paraphrasieren als auch beurteilen, ob eine bestimmte, dem Satz hinzugefügte explizite Assertions-Formulierung in dem betreffenden Falle möglich ist. Wenn auch in vielen Fällen dabei das "Wissen" der Befragten die entscheidende Rolle spielt, so zeigten die Einzelnalysen doch, daß es vielfältige sprachliche Indikatoren gibt, die der linguistischen Untersuchung durchaus zugänglich sind. Für die Analyse selbst ist die Feststellung, ob es sich jeweils noch um die Untersuchung sprachlicher Gesetzmäßigkeiten handelt, zunächst wenig relevant. Das Herausfinden der Grenze macht zunächst einmal die Grenzïberschreitung nötig.

Auch die Formalisierung von Bartsch (1972: 197) bewältigt nicht die Aufgabe der Abgrenzung des möglichen Assertionsbereiches. Hier sei als Beispiel nur die Formel für die Auswahl aus Argumenten zitiert:

"F (nur a)" $\leftrightarrow$ "F(a). (x) $(x \in M \backslash\{a\} \rightarrow-F(x)) "$

oder: $F(a) .(x)(F(x) . x \in M \rightarrow x=a) "$

Diese Notation hat im Prinzip dieselben Mängel wie die oben angesprochene. Sie macht keinen Unterschied zwischen Präsupposition und Assertion, und sie kann keine überzeugende Abgrenzung der negativen Assertion geben. Die Definition für die Menge $M$ der in das Satzmuster passenden Argumente, von denen a eines ist, bleibt, jedenfalls aus pragmatischer Sicht, unzureichend, oder genauer, sie ist nicht vorhanden und auch nicht Aufgabe einer prädikatenlogischen Notation von diesem Allgemeinheitsanspruch. Für die Beschreibung der pragmatischen Akzeptabilität bestimmter Sätze ist sie jedoch unabdingbar.

Die zur Überprüfung der Analyse jeweils eingesetzten Tests hatten im Fall der quantifizierenden Interpretation bei der nur - Guppe folgendes Ergebnis: Vorgänger- wie Folgesätze bringen spezifische Assertionsformulierungen, d.h. sie definieren sehr genau die oben erwähnte Menge $M$ der möglichen, aber auszuschließenden Skopuselemente. Im normalen Sprachgebrauch ist diese Definition fast immer vorhanden, ob nun explizit in Vorgänger- oder Folgesätzen, bzw. in Sätzen, die weiter vorausliegen, oder implizit durch die Situation.

Mit dem Suspensionstest ließ sich nachweisen, daß bei nur - Sätzen sowohl die Assertion als auch die Präsupposition bei diesem Interpretationstyp nicht 
suspendierbar sind. Trotzdem angesetzte Suspensionsformeln, die im Falle der Assertion und möglicherweise sogar nicht-x, im Falle der Präsupposition und möglicherweise nicht einmal $x$ lauten müßten, führen zur Inakzeptabilität des dadurch entstehenden Satzgebildes. Diese Tatsache wird beim Gebrauch von nur und $b Z O \beta$ durch die Ambiguität dieser Gradpartikeln zwischen quantifizierender und skalierender Interpretation verdeckt und fiuhrt leicht zu dem Fehlschluß, beide Bedeutungselemente wären, im Gegensatz zu der Lage bei auch, suspendierbar. Tatsächlich ist es aber so, daß die Suspensionsformel durch die projektive Kraft des in ihr enthaltenen sogar bzw. nicht einmal eine skalierende Interpretation (die fast bei allen Skopustypen möglich ist) erzwingt.

Der Konjunktionstest mit und führt im Falle einer expliziten Präsuppositionsformulierung als Folgesatz zu einem inakzeptablen Ergebnis. Die explizite Präsuppositions-Formulierung als Vorgängersatz ist jedoch, wie zu erwarten, voll akzeptabel. - Ebenso ist die explizite globale oder spezifische Assertionsformulierung als Folgesatz voll akzeptabel; durch sie wird ja erst die genaue Abgrenzung des Assertionsbereiches möglich. Als Vorgängersatz ist die spezifische Assertionsformulierung ohne weiteres akzeptabel, die globale Formulierung verneint hier jedoch die gesamte Menge der möglichen Elemente eirschließlich der durch den Partikelsatz als zutreffend bezeichneten Einheit; trotz dieses logischen Widerspruchs wird diese Kombination häufig verwendet und gilt als akzeptabel. - Ein Spezifikum der Präsuppositions-Formulierung als Vorgängersatz ist es, daß er (wohl wegen seiner vollständigen Formulierungsgleichheit mit dem Gradpartikel-Satz) nur dann akzeptabel ist, wenn nur kontrastiv betont wird -wobei quantifizierende Interpretation in jedem Fall erzwungen wird und wenn die identischen Konstituenten getilgt werden.

Der monologische Widerspruchstest mit aber bringt für alle Kombinationen die erwarteten inakzeptablen Ergebnisse. Er zeigt dadurch, daß sowohl Präsupposition als auch Assertion in den vorgeschlagenen Formulierungen wichtige Aspekte der Bedeutung eines Gradpartikel-Satzes sind, und daß ihre explizite Verneinung durch denselben Sprecher zu logischen Widersprüchen führt.

Der dialogische Test schließlich bestätigt den präsumptiven Status der angesetzten Bedeutungselemente, und zwar dadurch, daß die globale Zustimmung wie auch Verneinung die als Assertion gekennzeichnete Formulierung betrifft. Präsuppositionszustimmung wie Präsuppositions-Protest sind, wie üblich, schwer formulierbar, und meist nur in Verbindung mit einer Assertions-Zustimmung bzw. -Verneinung voll akzeptabel. Erstaunlicherweise führt die Verknüpfung der Assertionszustimmmg mit dèm Präsuppositions-Protest nicht zur Inakzeptabilität, was nur den Schluß zuläßt, daß diese Art der Präsupposition keine $n \circ t$ w e n - 
d i g e Voraussetzung für die Wahrheit oder Falschheit des Gradpartikel-Satzes ist, obwohl die Intuition dagegen spricht.

Die skalierende Interpretation ist bei dieser Gruppe nur bei den Gradpartikeln nur, $B Z O B$ und Zediglich möglich. Sie stellt an das jeweilige Skopuselement nicht nur die Bedingung der Kontrastierbarkeit, sondern auch die der skalaren Lokalisierbarkeit. Das Skopuselement muß also auf irgendwelchen Skalen als ein wert möglich sein. Wie ich an vielen Stellen zu zeigen versuchte, ist die Eigenschaft der Skalierbarkeit eines bestimmten Elementes nicht abhängig von bestimmten objektiven Gegebenheiten, und auch nur in geringem Maße abhängig von lexikalisierten Skalen, sondern in der Regel von der Skalierungsabsicht des Sprechers. Allerdings gibt es übliche Skalen, Ansätze zu lexikalisierten Skalen mit einer bestimmten Richtung. Doch ist der Sprecher prinzipiell frei in der Wahl sowohl der Skalierungsebene als auch der Skalierungsstufen sowie der Skalierungsrichtung. Das größte Problem für die Beschreibung ist hierbei, daß weder durch eine explizite Formulierung noch durch Kontextmerkmale diese drei "Kennumgen"einer Skala angegeben werden müssen; der Hörer wird also im Unklaren gelassen über Skalierungsebene, Skalierungsstufen, und, mit Einschränkungen, Skalierungsrichtung. Die relative Richtung kennt er in jedem Falle durch das nur; dies zeigt die projektive Kraft dieser Gradpartikel. Sie markiert ihr Skopuselement als entweder relativ tief auf der jeweiligen Skala angeordnet, oder als niedriger im Vergleich zu bestimmten anderen Elementen. Das unterste Skalenglied kann ebenso mit nur markiert werden, nicht jedoch das höchste. Man lasse sich hierbei nicht davon täuschen, daß auch "objektiv" höchste Skalenstufen mit nur gekennzeichnet werden können. Sie werden in diesem Falle (in einer Umkehrung der üblichen Skalenrichtung durch den Sprecher) als tiefe oder tiefste Stufen der "persönlichen Skala" bezeichnet; natürlich kann der Sprecher auch bloß die ihm bekannte Skalenordnung seines jeweiligen Hörers antizipieren. Für die Analyse gelten also folgende Grundregeln: die Präsupposition entspricht der Formulierung des Gradpartikel-Satzes abzüglich der Gradpartikel; durch die Assertion wird festgelegt, daß an der Stelle des Skopuselementes keine "höhere" Skalenstufe möglich ist; durch die konversationelle Implikatur wird festgelegt, daß an der Stelle des Skopuselementes keine "niedrigere" Skalenstufe möglich ist. Dabei bezieht sich das "höher" und "tiefer" jeweils auf die im Skopus genannte Stufe der Bezugsskala. - Schon daraus ist ersichtlich, wie schwer in einzelnen Fällen eine präzise Formulierung von Assertion und konversationeller Implikatur fällt. - Eine formale Darstellung dieses Interpretationstyps, den man als bloße pragmatische Variante, als eine "stärkere" Ver- 
sion der quantifizierenden Interpretation verstehen kann, mïßte auf skalar geordnete Mengen von Skopuselementen Bezug nehmen können. Eine linguistische Beschreibung miißte darüberhinaus die in Abhängigkeit von einem bestimmten Skopuselement definierbaren Skalen festlegen können, oder wenigstens für eine Anzahl von lexikalisierten Skalen dies leisten. Es gibt aber sehr starke Gründe für die Annahme, daß dies nur in sehr begrenztem Umfang möglich sein dürfte.

Bei diesem Interpretationstyp verhält sich die Negation nicht nur erstaunlicherweise nicht in jedem Falle präsuppositionsneutral. Dies ist abhängig von der jeweiligen Skala: schließen sich die Stufen der Skala gegenseitig aus, so wird bei skalarer Interpretation die Präsupposition ins Gegenteil verkehrt. In der Regel wird aber in diesem Falle eine quantifizierende Interpretation vorgezogen, bei der natürlich mehrere Werte derselben Skala gleichzeitig gelten können. Bei Skalen, deren Werte sich nicht gegenseitig ausschließen, wie etwa die Zahlenskala, bleibt die Präsupposition unverändert. Die Skalenrichtung hingegen wird in ihr Gegenteil verkehrt. Damit ändert sich auch die konversationelle Implikatur: sie betrifft die "tieferen" Werte, die bei Skalen mit sich gegenseitig ausschließenden Skalenstufen als ungültig, bei Skalen mit Stufen, die sich nicht gegenseitig ausschließen, als giultig bezeichnet werden. Die Assertion betrifft nach wie vor die "höheren" Werte. Aber sie werden nicht insgesamt als gültig bezeichnet, es wird nur behauptet, daß mindestens eine Stufe, die höher einzuordnen ist als die im Skopus genannte, gültig ist. - Die geschilderte Karplikation muß keine weiteren Konsequenzen für die semantische Analyse haben. Welchem Typ eine ganz bestimmte Skala angehört, ist eine Angelegenheit unseres Wissens, unserer Erfahrung. Sie bewirkt bei Skalen mit Werten, die sich gegenseitig ausschließen, eine Modifikation der skalierenden Interpretation, also eine weitere Einschränkung des quantifizierenden Grundmusters.

Vorgänger- und Folgesätze sind sehr nützlich bei der jeweiligen Definition der Skala selbst wie der Skalenstufen, sie liefern außerdem explizite spezifische Assertionsformulierungen wie auch Formulierungen für die konversationelle Implikatur, sie definieren also wiederum die skalar geordnete Menge der möglichen Skopuselemente. Im normalen Sprachgebrauch sind aber derartige Vorgänger- und Folgesätze nicht allzu häufig.

Im Falle von nur, $b$ ZOB und lediglich führt der Versuch, die Assertion zu suspendieren, in jedem Falle zu inakzeptablen Ergebnissen. Hingegen ist sowohl die Suspension der Präsupposition (mit der Formel und möglicherweise nicht einma $(x)$ wie der konversationellen Implikatur (mit der Formel und möglicherweise sogar nur y) möglich. Im Falle von nicht nur, nicht bloß führen hingegen alle Suspensionsversuche zur Inakzeptabilität. Hier existiert ja auch keine 
scha:-fe Grenze, die in Richtung auf einen "stärkeren" Wert überschritten wer den könnte.

Beim Konjunktionstest führt die Nachstellung der expliziten Präsuppositionsformulierung in jedem Falle zur Inakzeptabilität, die Voranstellung hingegen ist akzeptabel, wenn die Gradpartikel betont wird; damit erfolgt dann allerdings ein Wechsel zur quantifizierenden Interpretation. - Eine explizite Assertions-Formulierung ist aus denselben Gründen wie bei der quantifizierenden Interpretation sowohl als Vorgänger- wie als Folgesatz akzeptabel. - Komplizierter ist die Lage bei der konversationellen Implikatur. Ihre explizite Formulierung ist als Folgesatz mit der Konjunktion aber akzeptabel, als Vorgängersatz jedoch nicht; hier wïrde sie nämlich die falsche Skalenrichtung induzieren.

Der monologische Widerspruchstest mit aber führt bei allen drei Bedeutungselementen zu logischen Widersprüchen, die Inakzeptabilität verursachen; er bestätigt aber damit global die Relevanz dieser Bedeutungselemente für die Interpretation des betreffenden Gradpartikel-Satzes.

Die dialogische Bedeutungsanalyse wiederm bestätigt, daß bei direkter Verneinung bzw. Bejahung jeweils die als Assertion bezeichnete Formulierung betroffen ist. Die Tatsache, daß diese Form der Zustimmung bzw. Ablehnung von vielen Informanten bei isolierten Gradpartikel-Sätzen eher auf die Präsupposition bezogen wird, muß nicht umbedingt gegen die vorgelegte Analyse sprechen. Die Präsupposition kann wiederum meist nur im Zusammenhang mit einer AssertionsBejahung oder -Ablehnumg vermünftig angenommen oder zurückgewiesen werden. Demgegenüber ist die konversationelle Implikatur ohne weiteres zustimmung- und ablehnungsfähig. Zustimmung zur Assertion und gleichzeitige Ablehnung der Präsupposition führt bei Skalen, deren Stufen sich gegenseitig ausschließen, zur Inakzeptabilität. Ebenso führt die Verknüpfung von Assertions-Zustimmung und Verneinung der konversationellen Implikatur zur Inakzeptabilität.

Sehr kurz kann und muß die Besprechung der polaren Interpretation bei dieser Partikelgruppe ausfallen. Soweit ich sehe, können nur die skalierenden Elemente dieser Gruppe auch diese Interpretation erfüllen. Sie stellt außerdem an die Skopuselemente sehr strenge Anforderungen: meist sind hier nur lexikalische Antonyme zugelassen. Da diese aber zum einen nicht selten in Skalen eingebaut werden können und andererseits natürlich auch quantifizierende Interpretation zulassen, ergeben sich so viele tberlagerungen, daß kaum einmal die Gesetzmäßigkeiten störungsfrei untersucht werden können. - Die semantische Analyse von Gradpartikel-Sätzen mit polarer Interpretation ist im Grunde sehr einfach: 
In der Präsupposition steht der niedrigere der beiden Werte. Die Assertion verneint den höheren Wert. Eine konversationelle Implikatur tritt naturlich nicht auf, kann aber meist durch Übergang zur skalierenden Interpretation ohne weiteres plausibel gemacht werden.

Die Negationsform nicht nur sprengt automatisch die polare Interpretation und führt entweder zu quantifizierender Interpretation (die wahrscheinlichste Lösung) oder zu skalierender Interpretation. - Vorgänger- wie Folgesätze formulieren gewöhnlich nur die Assertion explizit aus; sie werden als redundant empfunden. - Der Suspensionstest hat in diesem Falle seine Tücken. Seine Anwendung auf die Assertion führt natürlich zu Inakzeptabilität, seine Anwendung auf die Präsupposition bzw. auf eine hinzuerfundene konversationelle Implikatur hingegen ist vollkormen akzeptabel, erzwingt sie doch den ja jederzeit möglichen Utbergang zur skalierenden Interpretation; derartige Utbergänge sind auch in täglichen Gesprächen nicht ganz selten.

Diese Analyse wird in jeder Hinsicht vom dialogischen Test bestätigt. Die Kombination Assertions-Bejahung und Präsuppositions-Protest scheint zwar regulär zur Inakzeptabilität zu führen, läßt aber Lücken für eine skalierende Interpretation. - So zeigt diese polare Interpretation alle Eigenschaften einer auf zwei Glieder verkürzten Skala, die durch diese Verkürzung jedoch manchmal gewisse züge der quantifizierenden Interpretation annimmt.

\subsection{2. auch - Gruppe}

Auch diese Gruppe ist deutlich zweigeteilt. auch selbst kann sowohl quantifizierend als auch skalierend interpretiert werden, letzteres allerdings vergleichsweise selten und meist nur in negativen Kontexten. In diesem Gebrauch entspricht es völlig nicht einmal, kann daher als bei der sogar - Gruppe abgehandelt gelten. Im normalen umbetonten sebrauch mit der üblichen Position vor dem Skopuselement ist auch sowohl quantifizierend als auch skalierend möglich, in der betonten Version nach topikalisierter Skopuskonstituente hingegen kann es nur quantifizierend interpretiert werden. In dieser Position und damit auch Funktion gibt es bedeutungsgleiche Partikeln: gleichfalls, ebenfalls, ebenso usw. - Nun also zur quantifizierenden Interpretation.

Auch und die übrigen Elemente dieser Gruppe stellen an die jeweilige Skopus-Konstituente bzw. bei komplexer Skopuskonstituente an das darin enthaltene kontrastierte Element keine andere Anforderung als Kontrastierbarkeit; diese sichert nämlich die Aufaddierbarkeit. - Eine präsuppositionsneutrale Negation gibt es bei auch nicht; damit ist man bei der Absicherung des Status der 
vorgeschlagenen Bedeutungselemente allein auf die übrigen Tests und auf den Vergleich mit anderen Partikels angewiesen. Man erhält dann folgende Analyse: Der Assertion entsprichic die Formulierung des Satzes abzüglich der Gradpart:kel; die Präsupposition beinhaltet, daß andere Skopuselemente zutreffen. Um dies wieder in einer den Status der jeweiligen Bedeutungselemente vernachlässigenden Paraphrase zu verdeutlichen:

auch $x \equiv x \cdot$ und nicht-x

wobei wiederum x für den Skopusinhalt stehen soll, gleichgültig, welcher oberflächenstruktureller Skopustyp vorliegt. In einer Übertragung der Bartsch'schen Formulierung (1972: 195) würde dies für den Skopustyp Argument so aussehen:

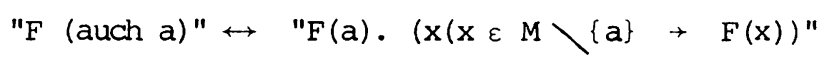

Auch hier gelten die bereits oben gemachten Einwände, daß Assertion und Präsupposition in dieser Formalisierung nicht unterschieden werden, und daß die durch das Skopuselement (und durch den Kontext) definierte Auswahlmenge für die Präsupposition nicht hinreichend gekennzeichnet wird. Dagegen macht die Notation sehr gut klar, daß mindestens ein Skopuselement existieren muß, das ebenfalls gilt. Dabei ist durchaus nicht ausgeschlossen, daß alle Elemente der definierten Menge zutreffen.

Vorgänger- und Folgesätze, meist mit nicht nur eingeleitet, bringen spezifische Präsuppositions-Formulierungen und definieren darüber hinaus sehr gut die Menge der möglichen Skopuselemente. Im nomalen Sprachgebrauch ist diese Abgrenzung praktisch immer, implizit oder explizit, vorhanden. - Für die Anwendung des Suspensionstests besitzt man in diesem Falle ein sehr gutes Mittel, um das Ausweichen eines Gradpartikel-Satzes unter dem Einfluß der Suspensionsformel in die skalierende Interpretation zu verhindern: man benützt nur das betonte, nachgestellte auch. Dann ist die Inakzeptabilität einer Suspendierung sowohl der Assertion wie auch der Präsupposition unverrückbar.

Der Konjunktionstest mit und bringt ein völlig anderes Ergebnis als bei der nur - Gruppe. So ist die explizite Assertionsformulierung sowohl als Vor gänger- wie als Folgesatz völlig inakzeptabel, auch bei betontem auch, offenkundig wegen der weitgehenden Formulierungsgleichheit. Die Präsupposition kann, wie üblich, nicht als Folgesatz verwendet werden, hingegen ist sie als Vorgängersatz akzeptabel. - Der monologische Widerspruchstest mit aber bringt für alle Kombinationen die erwarteten inakzeptablen Ergebnisse. Er zeigt dadurch, daß sowohl die jeweiligen Präsuppositionsformulierungen als auch Assertionen wichtige Aspekte der Bedeutung von Gradpartikel-Sätzen sind, und daß ihre explizite Verneinung durch denselben Sprecher zu logischen Widersprüchen führt.

Der dialogische Test. erweist die als Assertion bezeichnete Formulierung als 
richtig in ihrem Status bestimmt. Globale Zustimmung wie Ablehnung betreffen immer nur sie, nie die als Präsupposition bezeichnete Formulierung. Wie üblich wird zweckmäßigerweise die Zustimmung zur Präsupposition oder ihre Ablehnung mit der Zustimmung oder Ablehnung der Assertion verbunden. Die Kombination von Assertions-Zustimmung und Präsuppositions-Protest führt hier nicht zur Inakzeptabilität.

\subsection{3. sogar - Gruppe}

Diese Gruppe läßt in allen drei Elementen nur die skalierende Interpretation zu. Wie die skalierende Interpretation von nur stellen sogar, selbst und nicht einmal an das jeweilige Skopuselement nicht nur die Bedingung der Kontrastierbarkeit, sondern die der skalaren Lokalisierbarkeit. Hinsichtlich der Einrichtung derartiger Skalen gilt wiederum, daß hierfür die projektive Kraft der Gradpartikel viel wichtiger ist als irgendwelche semantischen Eigenschaften der Skopuselemente. Während aber nur jeweils die Richtung auf "schwächere" Stufen der bezeichneten Skala angibt, fordert sogar die Richtung auf "stärkere" Stufen. Die Umkehrung der Richtung ist möglich durch ciie Kombination sogar nur. Hingegen ist nicht einmal hinsichtlich der Skalenrichtung ambig. Auch hier braucht natïrlich die "persönliche Skalenrichtung" durchaus nicht mit einer objektiven Skalenrichtung übereinzustimmen (abgesehen vielleicht von der Zahlenskala). Für sogar und selbst gilt also folgende Analyse: die Assertion entspricht der Formulierung des Gradpartikelsatzes abzüglich der Gradpartikel. Die explizite Präsuppositionsformulierung beinhaltet entweder, daß kein tieferer Wert gilt (bei Skalen, deren Werte sich gegenseitig ausschließen) oder, daß alle tieferen Werte gelten (bei Skalen, deren Werte sich nicht gegenseitig ausschließen). Durch die konversationelle Implikatur ist festgelegt, daß kein "höherer" Wert gilt als der in der Assertion genannte. - Da in vielen Fällen die gemeinte Bezugsskala nicht identifiziert wird, ist eine genauere Formulierung sowohl der Präsupposition als auch der konversationellen Implikatur nicht oder nur sehr schwer möglich.

Im Hinblick auf die Formulierung der skalierenden Interpretation halte ich die Fixierung einer bestimmten "Erwartungspräsupposition" für sogar (wie für die skalierende Interpretation von nur) für überflüssig. Erwartungshaltungen von Kormunikationsteilnehmem sind eine Angelegenheit, die sich nicht auf Gradpartikeln beschränken läßt; sie sind außerdem sehr viel mehr von der jeweiligen Situation als von bestimmten Formulierungen abhängig, also kaum ein Beschreibungsgegenstand der Linguistik. Sie mu ja, um generalisieren zu können, auf 
konstante Gebrauchseigenschaften sprachlicher Einheiten abzielen. Diese Konstanz scheint mir aber für "Erwartungspräsuppositionen" nicht gegeben. Außerdem zeigen die Tests, daß es sich nicht um festlegbare und explizierbare Bedeutungskomponenten von Gradpartikelsätzen handelt.

Nun noch die Analyse für nicht einmal: diese Gradpartikel stellt eine nicht präsuppositionsneutrale Negation des sogar - Satzes dar. Dabei besagt die Assertion, daß der durch das Skopuselement bezeichnete Wert nicht zutrifft; die explizite Formulierung der Präsupposition verneint die Gültigkeit "stärkerer" Werte; und die konversationelle Implikatur besagt, daß "schwächere" als der Skopuswert zutreffen. Es ist allerdings unsicher, ob die konversationelle Implikatur tatsächlich so vorliegt. In den meisten Fällen diirfte nicht einmal die Ungültigkeit des tiefsten wertes einer bestimmten Skala assertieren.

Vorgänger- und Folgesätze liefern bei dieser Gradpartikelgruppe jeweils explizite Präsuppositions-Formulierungen und definieren gleichzeitig die Skala. Auch explizite Formulierungen der konversationellen Implikatur kommen, wenn auch selten, vor. - Eine Suspension von Assertion oder Präsupposition ist bei sogar - Sätzen nicht möglich, ebensowenig bei nicht einmal - Sätzen. Eine Suspension der konversationellen Implikatur mit Hilfe der Formel und möglicherm weise sogar/nicht einmal $y$ ist nicht gerade inakzeptabel, aber doch etwas ungewöhnlich. Im Falle von sogar ist die Grenze zu scharf, im Falle von nicht einmal zu schwach markiert, als daß die "Grenzibberschreitung" jeweils ohne Komplikationen erfolgen könnte.

Im Konjunktionstest mit und ist die explizite Assertions-Formulierung weder als Vorgänger- noch als Folgesatz möglich. Die explizite PräsuppositionsFormulierung ist als Folgesatz wohl nicht akzeptabel, als Vorgängersatz bedarf es einiger Formulierungstricks, um zu einwandfrei akzeptablen Ergebnissen zu kommen. Der Anschluß der konversationellen Implikatur als Folgesatz ist nur mit aber möglich, als Vorgängersatz ist sie inakzeptabel, da sie dann die "falsche Richtung" induzieren wïrde. - Der monologische Widerspruchstest mit aber führt bei den Formulierungen für alle drei Bedeutungselementen zu logischen Widersprüchen, die Inakzeptabilität verursachen. - Auch die dialogische Bedeutungsanalyse bestätigt die vorgeschlagenen Interpretationsweisen. Bei globaler Verneinung oder Bejahung ist immer nur die als Assertion bezeichnete Teilbehauptung betroffen. Zweckmäßigerweise wird die Verneinung oder Bejahumg der Präsupposition jeweils mit der Verneinung oder Bejahung der Assertion verbunden. Auffällig ist hierbei, daß die Verknüpfung von Assertions-Bejahung und Präsuppositions-Protest zu unauflöslichen Widersprichen führt. - Hingegen muß die Bejahung oder zurückweisung der konversationellen Implikatur keineswegs 
mit Zustimmung zur Assertion oder ihrer Ablehnung verbunden werden. Verknüpfungen mit Assertions-Vermeinung führen nicht automatisch zur Inakzeptabilität.

Die Kombinationstests innerhalb der dialogischen Analyse wurden hauptsächlich mit der Erwartung inszeniert, auf diese Weise ein verläßliches Kriterium für die Wahrheitswertfunktionalität der als Präsuppositionen eingestuften Bedeutungselemente von Gradpartikel-Sätzen zu erhalten. In der Utberschau zeigt sich, daß diese Erwartung nicht ganz begründet war, und zwar aus folgenden Gründen: Die Akzeptabilitätsurteile werden auch von ganz anderen Faktoren beeinflußt als von der Bedingung für die Verwendung/den Wahrheitswert von Äußerungen, etwa von den Eigenheiten von Skalen, deren Werte sich gegenseitig ausschließen, also von vorwiegend pragmatischen Faktoren. Außerdem könnte man aus diesem Test höchstens auf die Bedingungen für die Verwendung, keineswegs auf eine Bedingung für den Wahrheitswert deklarativer Äußerungen schließen. Ein weiterer Störfaktor ist, daß jede globale Zustimmmg oder Ablehnung einer deklarativen Äußerung unweigerlich zur Annahme der damit verbundenen Präsuppositionen führen muß, so daß die anschließende Ablehnung der Präsupposition schon aus diesem Grund zu logischen Widerspruichen führen mu. - Auch bei Beachtung dieser Faktoren erhält man Ergebnisse, die je nach Skopustyp und Interpretationsart sowie Gradpartikel variieren; eine Erklärung dieser Variation gestaltet sich äußerst schwierig wegen der Vielzahl der in Betracht kommenden Faktoren

Die aufgrund der Analyseergebnisse wie der Materialsammlung möglichen Aussagen bestätigen die Richtigkeit eines sehr viel stärker materialorientierten Vorgehens als derzeit in der linguistischen Diskussion üblich. Diese gegenüber allen vorausgehenden relevanten Äußerungen zu den Gradpartikeln wesentlich verbreiterte empirische Basis erlaubte begründete Aussagen uber die Kategorienbildung, iber die Stellungstypen von Gradpartikeln, iber die Skopuseigenschaften, die Frage des Kontrastfokus und die semantische Analyse einzelner Gradpartikeln wie der von ihnen bedingten Interpretationsarten. Gleichzeitig schützte die intensive Materialarbeit durch die Erfassung der zahlreichen Idiosynkrasien und Regelunsicherheiten vor vorschnellen Generalisierungen. 


\section{LITERATUR}

\section{Wörterbïicher}

Agricola, Erhard (Hg.). 1965. Wörter und Wendungen. Wörterbuch zum deutschen Sprachgebrauch. Leipzig, VEB Bibl. Institut.

(allein 21, auch 45, ausschließlich 66, bloß 110, eben 146, ebenfalls 146, nicht einmal 155, einzig 161, ferner 192, lediglich 358, nur 429, selbst 541 f., sogar 552).

Dornseiff, Franz. 1959. Der deutsche Wortschatz nach Sachgruppen. 5. Aufl. mit alphabet. Generalregister. Berlin.

(zu Gradpartikeln unter anderem S. 179, 182 ff., 191, 196 f., 200, 373 f., 380).

Grimm, Jakob, Wilhelm Grimm. 1854 - 1960. Deutsches Wörterbuch. Leipzig, Hirzel.

(allein Bd. I, Sp. 216 f.; auch Bd. I, Sp. 598 - 601; ausschließlich Bd. I, Sp. 956; bloß Bd. II, Sp. 144 - 150, vor allem 149 f.; nicht einmal Bd. III, Sp. 234; einzig Bd. III, Sp. $356-358$; lediglich $\mathrm{Bd}$. VI, Sp. 506; nur Bd. VIIa, Sp. 998 - 1005; selbst Bd. X,2,3, Sp. 448 - 453; sogar Bd. X,1, Sp. 1406).

Klappenbach, Ruth, Wolfgang Steinitz. 1961 ff. Wörterbuch der deutschen Gegenwartssprache. Bislang 4 Bde. Berlin, Akademie-Verlag. (allein 106, auch $230 \mathrm{f}$. , ausschließlich 362, bloß 635, ebenso 908, nicht einmal 966, einzig 1009, ferner 1257, lediglich 2332).

Mackensen, Lutz. 1952. Neues Deutsches Wörterbuch. Laupheim. (allein 30; auch 68; ausschließlich 88; bloß 147; ebenfalls, ebenso 241; nicht einmal 254; einzig 261; ferner 213; nur 660).

Ullstein Lexikon der deutschen Sprache. 1969. Hrsg, und bearb. von R. Köster unter Mitarbeit von Harald Hahmann, H. Hartmann, F. Mehling. Berlin, Ullstein.

(allein 37; auch 72; ausschließlich $97 \mathrm{f} . ;$ bloß 162; ebenso 245; ebenfalls 245; nicht einmal. 257; einzig 265; ferner 315; lediglich 552 f.; nur 645 ; selbst 810 ; sogar 822 ).

Wehrle, Hugo, Hans Eggers. 1961. Deutscher Wortschatz. Ein Wegweiser zum treffenden Ausdruck. 12. Aufl. Stuttgart, Klett.

(zu Gradpartikeln unter anderem S. 7 - 11). 
Abraham, Werner. 1970. Kompetenz und Mittelwertgrammatik. Lingua 2\% 339-357. , R. J. Binnick (Hgg.). 1972. Generative Semantik. Linguistische Forschungen Bd. 11. Frankfurt (Main), Athenäum.

1973. Über suggerierte Schiußfolgerungen. Referat auf dem VIII. Ling. Koll. Löwen 19.-22. Sept. 1973.

Admoni, Wladimir. 1960. Der deutsche Sprachbau. Theoretische Grammatik der deutschen Sprache. Leningrad. - 3. Aufl. München, Hueber 1970.

(zu Partikeln S. 206; zu Konjunktionen S. 208).

Akmajian, Adrian. 1970a. Aspects of the grammar of focus in English. (Masch.) Phil. Diss. MIT.

1970b. On deriving cleft sentences from pseudo cleft sentences. Linguistic Inquiry 1,2. 149-168.

, R. S. Jackendoff. 1970. Coreferentiality and stress. Linguistic Inquiry 1. 124-126.

1972. Getting tough. Linguistic Inquiry 3,3. 373-377.

(zu too, enough und just).

Althaus, Hans Peter, Helmut Henne. 1971. Sozialkompetenz und Sozialperformanz. ZDL 38. 1-15.

- Herbert Ernst Wiegand (Hgg.). 1973. Lexikon der Germanistischen Linguistik. Tübingen, Niemeyer.

Altmann, Hans. 1976. Gradpartikeln und Topikalisierung. Braunmüller, Kurt, Wilfried Kürschner (Hgg.). Akten des X. Ling. Koll. 1975 Tübingen. Bd. II: Grammatik. Tübingen, Niemeyer.

Anderson, Stephen R. 1972. How to get even. Language 48,4. 893-906.

1973. Universal quantifiers. Lingua 31. 125-176.

Arndt, W. 1960. Modal particles in Russian and German. Word 16. 323-336.

Austin, John L. 1962. How to do things with words. Oxford. - Übersetzung: Zur Theorie der Sprechakte (How to do things with words). Deutsche Bearb. v. Eike v. Savigny. Stuttgart, Reclam 1972.

Ayer, Alfred Jules. 1960. Language, truth and logic. London, Gollancz. - Ubersetzung: Sprache, Wahrheit und Logik. Aus dem Engl. übersetzt und hg. von H. Herring. Stuttgart, Reclam 1970.

Bach, Emmon, R. T. Harms (eds.). 1968. Universals in linguistic theory. New York, Holt.

1968. Nouns and noun phrases. Bach/Harms (eds.). 1968. 91-122.

Ballmer, Thomas. 1972. Einführing und Kontrolle von Diskurswelten. Wunderlich, (Hg.) . 1972. 183-206.

Bartsch, Renate. 1970. Die logische Analyse von Modaladverbien. Ling. Ber. 10. 27-34.

1972. Adverbialsemantik. Linguistische Forschungen Bd. 6. Frankfurt (Main), Athenäum. - Rez. Steinitz, Renate, Ewald Lang, Ms. Berlin 1975.

, Theo Vennemann. 1972. Semantic structures. A study in the relation between semantics and syntax. Athenäum-Skripten Linguistik Bd. 9.

Frankfurt (Main), Athenäum.

1973. Negative Transportation gibt es nicht. Ling. Ber. 27. 1-7. 
Bechert, Johannes. 1973. Sind Transformationen referenzerhaltend? Papiere zur Linguistik 5. 32-43.

Beeh, Volker. 1973. Ansätze zu einer wahrheitswertfunktionalen Semantik, dargestellt am Deutschen. Linguistische Reihe 11. München, Hueber.

Behaghel, Otto. 1923-1932. Deutsche Syntax. Bd. 1-4. Heidelberg.

(selbst, sogar I, 336; nur II, 4; III, 232; auch IV, 162; III, 172; allein III, 62; III, 75; III, 76).

Bellert, Irena. 1969. Arguments and predicates in the logico-semantic structure of utterances. Kiefer, Ferenc (Hg.), Studies in syntax and semantics. Dordrecht, Reidel. 34-54.

1971. On the use of linguistic quantifying operators in the logicosemantic structure representation of utterances. Poetics 2. 71-86. Wieder abgedruckt in Petöfi/Franck (Hgg.). 1973. 221-239.

Bierwisch, Manfred. 1966. Regeln für die Intonation deutscher Sätze. Studia Grammatica VII. Berlin, Akademie Verlag. 99-201.

Binnick, R. I. 1972. Zur Entwicklung der generativen Semantik. Abraham/Binnick (Hgg.). 1972. 1-50.

Black, M. 1958. Presuppositions and implication. Seizi Uyeda (ed.). A way to the philosophy of science. Waseda University Press. - Wieder abgedruckt in Petöfi/Franck (Hgg.). 1973. 55-70.

Blau, Ulrich. 1971/72. Die Logik der adverbialen Konstruktionen. Teil I. Münchner Papiere zur Ling. 2. 8-61. Teil II. MPzL 3. 1-29.

- 1973. Zur 3-wertigen Logik der natürlichen Sprache. Papiere zur Linguistik 4. 20-96.

- 1973/74. Dreiwertige Sprachanalyse und Logik. Manuskript. München.

Blumenthal, Peter. 1974. Eine kontrastive Präsuppositionsanalyse: nur/erst seulement. Linguistik und Didaktik 18. 50-58.

Bolinger, D. L. 1965. Forms of English: accent, morpheme, order. Ed. by Isamu Abe and Tetsuya Kanekiyo. Cambridge (Mass.), Harvard Univ. Press.

1968. Judgements of grammaticality. Lingua 21. 34-40.

1972. Degree words. Janua linguarum series maior 53. The Hague, Mouton.

Brekle, Herbert Ernst. 1970a. Generative Satzsemantik und transformationelle Syntax im System der englischen Nominalkomposition. München, Fink.

- 1970b. Generative Satzsemantik versus generative Syntax als Komponenten eines Grammatikmodells. Linguistik und Didaktik 2. 123-136.

1972. Semantik. Eine Einführung in die sprachwissenschaftliche Bedeutungslehre. UTB 102. München, Fink.

Bresnan, Joan W. 1972. Sentence stress and syntactic transformations. Language 47. $257-281$.

Brinkmann, Hennig. 1962. Die deutsche Sprache, Gestalt und Leistung. Düsseldorf. (zu auch und allein S. 492 f.; zu den Konjunktionen auch, allein, nicht nur - sondern auch S. 585 ff.)

Chafe, Wallace L. 1970. Meaning and the structure of language. Chicago, Univ. of Chicago Press. 
Chomsky, Noam. 1957. Syntactic structures. - 8th printing. The Hague, Mouton 1969 .

1965. Aspects of the theory of syntax. - Ubbersetzung: Aspekte der Syntaxtheorie. Frankfurt, Suhrkamp. 1969.

- Morris Halle. 1968. The sound pattern of English. New York, Harper and Row.

1969. Deep structure, surface structure, and semantic interpretation. Paper. - Abgedruckt in Kawamoto/Jacobson (eds.), Studies in general and oriental linguistics. Tokyo, T.E.C. Corporation 1970. 52-91. Ferner in: Steinberg/Jakobovits (eds.). 1971. 163-216. - Deutsch auszugsweise in F. Kiefer (Hg.). 1972. 101-124.

1972. Some empirical issues in the theory of transformational grammar. St. Peters (ed.), Goals of linguistic theory. Englewood Cliffs (N.J.), 63-130.

Clément, Danièle, Wolf Thümmel. 1975. Gründzüge einer Syntax der deutschen Standardsprache. Fischer Athenäum Taschenbücher Sprachwissenschaft 2057. Frankfurt (Main), Athenäum-Fischer.

(zu Gradpartikeln = "Rangierpartikeln" siehe die Kapitel 15, 18, 25 und 28; zu "Tonbruch" siehe Kapitel 31).

Cohen, Daniel. 1971. On the linguistic representation of presuppositions. University of Texas dissertation.

1973. On the mis-representation of presuppositions. glossa 7,1. 21-38.

Dahl, Östen: 1973. Presuppositions and propositions. Petöfi/Franck (eds.). 1973. 409-420.

DaneS, Frantisek. 1960. Sentence intonation from a functional point of view. Word 16. 34-54.

1967. Order of elements and sentence intonation. To honor Roman Jakobson. I. The Hague, Mouton. 499-512.

1968. Some thoughts on the semantic structure of the sentence. Lingua 21. 55-69.

, J. Firbas (eds.). 1971. Papers on functional sentence perspective. Proceedings from the symposium on FSP. Marianske Lázne.

Davidson, Donald, G. Harman (eds.). 1972. Semantics of natural language. Dordrecht, Reidel.

Dik, S. C. 1968. Coordination: its implications for the theory of general linguistics. Amsterdam.

Donnellan, K. 1966. Reference and definite descriptions. Philosophical Review LXXXV. 281-304. - Auch in Petöfi/Franck (eds.). 1973. 71-96.

Dressler, w. 1969. Theorie und Empirie in der Sprachwissenschaft. Ling. Ber. 4. $70-75$.

Ducrot, O. 1969. Présupposés et Sous-Entendus. Langue Francaise. Décembre 1969: "La Sémantique". 30-43. - Auch in Petöfi/Franck (eds.). 1973. 241-260.

Duden.1966. Grammatik der deutschen Gegenwartssprache. Bearb. von P. Grebe u.a. Mannheim, Bibliograph. Institut. - 3., neu bearb. und erw. Auflage. 1973.

(zu Partikeln ab Nr. 729, S. 303; Gradpartikeln = "Modaladverbien" Nr. 
744 f., S. 308 f.; konjunktionale verwendung. Nx. 775, S. 319 und Nr. 769 f. S. 317. allein Konj. 775, Modaladv. 746; auch 744, 746, 748, 770; ausschließlich nur 761; nur Adv. 244, 745 f., 748; selbst Adv. 158, 691, 746; sogar 746; nicht nur - sondern auch 776, 1451).

Ebert, Karen H. 1973. Präsuppositionen im Sprechakt. Petöfi/Franck (eds.). 1973. 421-440.

Egli, Urs. 1971. Zweiwertigkeit und Präsupposition. Ling. Ber. 13. 74-78.

Ehrich, Veronika, Günter Saile. 1972. Über nicht-direkte Sprechakte. Wunderlich, Dieter (Hg.). 1972. 255-287.

Exben, Johannes. 1967. Abriß der deutschen Grammatik. 10. Auflage. Berlin, Akademie-Verlag.

(u.a. S. 154 zur Gradpartikel-Funktion von nur, allein; S. 167-171 zur konjunktionalen verwendung von auch, nicht nur - sondern auch, ja sogar, allein, nur; S. 249 zur Gradpartikel-Funktion von allein; S. 265 zur Gradpartikel-Funktion von nur; S. 161 zur Polyfunktionalität von bloß. Zahlreiche kleinere Hinweise. Siehe hierzu das Wortregister!).

Fillmore, Charles. 1965. Entailment rules in semantic theory. Project on Linguistic Analysis (POLA) Report 10. Columbus (Ohio). - Wieder abgedruckt in Rosenberg/Travis (eds.). 1971. 533-548.

- , D. Terence Langendoen (eds.). 1971. Studies in linguistic semantics. New York, Holt.

Firbas, J. 1964. On defining the theme in functional sentence analysis. TCLP 1. 267-280.

1965. A note on transition proper in functional sentence analysis. Philologica Pragensia 8. 170-176.

1966. Non-thematic subjects in contemporary English. TCLP 2. 239-256.

1973. Some aspects of the czechoslovak approach to problems of functional sentence perspective. Danes, F., J. Firbas (eds.). Papers on functional sentence perspective. Prague.

Fischer, Susan. 1968. On cleft sentences and contrastive stress. Ms. MIT.

van Fraassen, B. C. 1969. Presuppositions, supervaluations and free logic. Lambert (ed.). The logical way of doing things. New Haven, London. 67-91.

Franck, Dorothea. 1973. Zur Problematik dex Präsuppositionsdiskussion. Petöfi/ Franck (eds.). 1973. 11-41.

Fraser, Bruce. 1969. An analysis of concessive conditionals. Papers from the fifth regional meeting Chicago Linguistic Society. 66-75.

1970. Idioms within a transformational grammar. Found. of Lang. 6,1. $22-42$.

1971. An analysis of even in English. Fillmore/Langendoen (eds.). $150-178$.

Frege, Gottlob. 1892. Über Sinn und Bedeutung. ZS für Phil. und Kritik No. 100. 25-50. - Wieder abgedruckt in Funktion, Begriff, Bedeutung. Fünf 10gische Studien, hg. und eingel. von G. Patzig. Göttingen, Vandenhoeck. 1969. 40-65.

Gabriel, Gottfried. 1971. Kennzeichnung und Präsupposition. Ling. Ber. 15.27-31.

Garner, R. 1971. "Presupposition" in philosophy and linguistics. Fillmore/ Langendoen (eds.). 23-42. 
Geis, M. L., A. M. Zwicky. 1971. On invited inferences. Linguistic Inquiry 2. 561-566. - Ubersetzung: Über suggerierte Schlußfolgerungen. Abraham/Binnick (eds.). 1972. 236-241.

Gleitman, Lila. 1965. Coordinating conjunctions in English. Language 41. 260-293.

Glinz, Hans. 1947. Geschichte und Kritik der Lehre von den Satzgliedern in der deutschen Grammatik. Phil. Diss. Zürich.

1961. Die innere Form des Deutschen. 2. Aufl. Bern, München, Francke. (zu Gradpartikeln S. 220-223 und S. 231).

1971. Deutsche Grammatik II. Studienbücher zur Linguistik und Literaturwissenschaft 3. Frankfurt (Main), Athenäum.

(zu Partikeln S. 219-234 und 243-248).

Gordon, D., G. Lakoff. 1971. Conversational postulates. Papers from the 7 th regional meeting Chicago Linguistic Society. 63-85.

Green, Georgia M. 1968. On too and either, and not just too and either, either. Papers from the third regional meeting Chicago Linguistic Society.22-39.

Greenberg, R. 1971. A note on Strawson's theories of presuppositions. Mind 80. 258-261.

Grewendorf, Günther. 1972. Sprache ohne Kontext. Zur Kritik der performativen Analyse. Wunderlich, Dieter (Hg.). 1972. 144-182.

Grice, Paul. 1968. Logic and conversation. The William James Lectures. Unveröff. Manuskript.

Grosu, Alexander. 1973. A note on implicatures, invited inferences and syntactic form. Die Sprache. ZS für Sprachwissenschaft. XIX, 1. 57-62.

Gutknecht, Ch.1971. Präsuppositionen als ein Kriterium für die Grammatikalität von Sätzen. Ling. Ber. 15. 32-39.

Habermas, Jürgen. 1972. Vorbereitende Bemerkungen zu einer Theorie der kommunikativen Kompetenz. Habermas, Jürgen, Niklas Luhmann. Theorie der Gesellschaft oder Sozialtechnologie. Frankfurt (Main), Suhrkamp. 101-141.

Hajicová, E. 1973. Negation and topic vs. comment. Philologica Pragensia 16. $81-93$.

Hall-Partee, Barbara. 1970. Negation, conjunction and quantifiers. Syntax vs. semantics. Found. of Lang. 6,2.153-165.

Halliday, M. A. K. 1967. Notes on transitivity and theme in English. Journal of Ling. 3. 1967. 37-81 und 199-244; 4. 1968. 179-215.

Hartman, E. G. 1966. Emphasizing and connecting particles in the thirteen principal Upanishads. Annal. Acad. Scient. Fennicae, Ser. B. 143, 2. Helsinki.

Harweg, Roland. 1972. Funktion und Textologie der sowohl-als auch-Koordination. ZPSK 24. 360-372.

Hausser, Roland. 1974. Quantification in an extended Montague grammar. Phil. D. Dissertation. Austin.

1975. On the formal implementation of presuppositions. Manuskript. München.

Heidolph, K. E. 1970. Zur Bedeutung negativer Sätze. Bierwisch, Manfred, K. E. Heidolph. (eds.). Progress in linguistics. 86-101. 
Helbig, Gerhard. 1970. Sind Negationswörter, Modalwörter und Partikeln im Deutschen besondere Wortklassen? Deutsch als Fremdsprache 6. 393-401.

Henn, Beate. 1973. Die wissenschaftstheoretischen Voraussetzungen formaler Grammatiken und deren Kritik von seiten hermeneutischer und dialektischer Wissenschaftstheorie. deutsche sprache 3. 27-44.

Heringer, H. J. 1972. Deutsche Syntax. 2., völlig neubearb. Aufl. Sammlung Göschen Bd. 5246. Berlin, de Gruyter.

1973. Praktische Semantik. Stuttgart, Klett.

Hill, L. A. 1968. Prepositions and adverbial particles. An interim classification. Semantic, structural and graded. London, Oxford Univ. Press.

Horn, Laurence Robert. 1969. A presuppositional analysis of only and even. Papers from the fifth regional meeting Chicago Linguistic Society. 98-107. - Übersetzung: Die präsuppositionelle Analyse von only und even. Abraham/Binnick (eds.). 1972. 221-235.

1970. Ain't it hard (anymore). Papers from the sixth regional meeting Chicago Linguistic Society. 318-327.

1972. On the semantic properties of logical operators in English. Masch. Phil. Diss. Los Angeles.

1973. Greek Grice: a brief survey of proto-conversational rules in the history of logic. Papers from the ninth regional meeting Chicago Linguistic Society. 205-214.

Hornby, P.A. 1974.Surface structure and presupposition. Journal of Verbal Learning and Verbal Behavior 13. 530-538.

Hunger, Gert. 1972. Untersuchungen zu den modalen Partikeln in der deutschen Gegenwartssprache. Syntax und kommunikative Funktion der Partikeln doch, ja, denn. Masch. Phil. Diss. Leipzig.

Hungerland, I. 1960. Contextual implication. Inquiry 4, 211-258.

Immler, Manfred. 1973. Versuch einer integrierten Theorie von Bedeutung, Referenz, Kompetenz und Performenz. Papiere zur Linguistik 5. 89-115.

1974a. Generative Syntax - Generative Semantik. UTB 207. München, Fink.

1974b. Grundlinien einer Kritik der gegenwärtigen Linguistik (vorläufige Fassung). Manuskript. Stuttgart.

Isacenko, A. V., H. J. Schädlich. 1971. Untersuchungen über die deutsche Satzintonation. Studia Grammatica VII. 2. Aufl. 7-68.

Jackendoff, Ray S. 1969a. An interpretative theory of negation. Found. of Lang. 5. 218-241.

1969b. Some rules of semantic interpretation for English. Masch. Phil. Diss. MIT.

1971. On some questionable arguments about quantifiers and negation. Language $47.282-297$.

1972. Semantic interpretation in generative grammar. Cambridge (Mass.), MIT Press.

Jacobson, Sven. 1964. Adverbial positions in English. Uppsala Diss. Stockholm, AB Studentbok. - Rez. S. J. Keyser. Language 44. 1966. 357-373. 
Juilland, A., H. H. Lieb. 1968. Klasse und Klassifikation in der Sprachwissenschaft. Jan. ling. ser. min. 74. The Hague, Mouton.

Jфrgensen, E. 1974. Only with a temporal value. English studies Vol 55, Nr. 3. 247-260.

Kaneko, Tohru. 1971. Zum Problem der Topikalisierung. Dieter Wunderlich (Hg.). Probleme und Fortschritte der Transformationsgrammtik. Referate des 4. Ling. Koll. Berlin 1969. München, Hueber. 1971. 127-147.

Kanngießer, Siegfried. 1972a. Untersuchungen zur Kompetenztheorie und zum sprachlichen Handeln. LiLi 7. 11-43.

1972b. Ansätze zu einer Theorie von Synchronie und Diachronie. Linguistics. 5-71.

Karttunen, Lauri. 1971a. Counterfactual conditionals. Ling. Inquiry 2,4. 566-569. 1971b. Implicative verbs. Language 47,2. 340-358. - Wieder abgedruckt in Petöfi/Franck (eds.). 1973. 285-314.

1972. The logic of English predicate complement constructions. IULC. Übersetzung: Die Logik der englischen Prädikatkomplementkonstruktionen. Abraham/Binnick (Hgg.). 1972. 243-275.

1973. Presuppositions of compound sentences. Ling. Inquiry 4. 169-193.

Katz, J.J. 1970. Interpretative semantics. vs. generative semantics. Found. of Lang. 6,2. 220-259.

Keenan, E. L. 1969. A logical base for transformational grammar of English. Phil. Diss. Univ. of Pennsylvania. (Masch)

1971. Quantifier structures in English. Found. of Lang. 7,2. 255-284. 1972. On semantically based grammar. Ling. Inquiry 3,4. 413-462.

- Robert D. Hull. 1973. The logical presuppositions of questions and answers. Petöfi/Franck (eds.) . 1973. 441-466.

Kiparsky, Paul. 1966. Über den deutschen Akzent. Studia Grammatica VII. 69-98.

Klima, Edward S. 1964. Negation in English. Katz/Fodor (eds.), The structure of language. 246-323.

Klüber, J. 1971. Operationalismus. Kritik und Geschichte einer Philosophie der exakten Wissenschaften. Braunschweig, Vieweg.

Kolde, Gottfried. 1970. Zur Funktion der sogenannten Modaladverbien in der deutschen Sprache der Gegenwart. Wirk. Wort 20. $116 \mathrm{ff}$.

Kriwonossow, Alexej. 1963. Die modalen Partikeln in der deutschen Gegenwartssprache. (Masch) phil. Diss. Berlin.

1965a. Die Rolle der modalen Partikeln in der kommunikativen Gliederung der Sätze in bezug auf die Nebensatzglieder. ZS für Phon. XVIII. 487-503.

1965b. Die Wechselbeziehungen zwischen den modalen Partikeln und der Satzintonation im Deutschen. ZS für Phon. XVIII. 573-589.

1966. Die Rolle der modalen Partikeln in der kommunikativen Gliederung der Aussagesätze, der Fragesätze, der Befehlssätze und der Nebensätze in Bezug auf die Hauptsatzglieder II. ZS für Phon. XIX. 131-140.

Kummer, Werner. 1971. Quantifikation und Identität in Texten. A.v.Stechow (Hg.). Beiträge zur generativen Grammatik. Braunschweig, Vieweg. 122-141. 
Kummer, Werner. 1973. Pragmatic implication. Petöfi/Rieser (eds.). Studies in text grammar. Dordrecht, Reidel.

Kuroda, Sige-Yuki. 1965. Generative grammatical studies in the Japanese language. (Masch.) Phil. Diss. MIT.

1969. Attachment transformations. Reibel, D., S. Schane (eds.). Modern Studies in English. Englewood Cliffs (N.J.), Prentice Hall. 331-351.

Kutschera, F. von. 1971. Sprachphilosophie. München, Fink.- 2., erw. Aufl. 1974.

Lakoff, George. 1968. Instrumental adverbs and the concept of deep structure. Found. of Lang. 4. 4-29.

, P. Railton. 1970. Some types of presupposition and entailment in natural language. mimeo.

1970. Linguistics and natural logic. Synthese 22. 151-271. - Übersetzung: Linguistik und natürliche Logik, hg. v. Werner Abraham. Frankfurt (Main), Athenäum. 1971.

1971. Presupposition and relative well-formedness. Steinberg/Jakobovits (eds.). 1971. 329-340.

Lakoff, Robin. 1971. If's, and's, and but's about conjunction. Fillmore/ Langendoen (eds.). 114-149.

1972. Language in context. Language 48. 907-928.

1973. The logic of politeness; or, minding your P's and $Q^{\prime} s$. Papers of the ninth regional meeting Chicago Linguistic Society. 292-305.

Larkin, Don, Michael O'Malley. 1973. Declarative sentences and the rule-ofconversation hypothesis. Papers from the ninth regional meeting Chicago Linguistic Society. 306-319.

Leech, G. N. 1970. On the theory and practice of semantic testing. Lingua 24. 343-364.

Lehmann, Dolly, Ursula Spranger. 1966. Modalwörter in der deutschen Sprache der Gegenwart. ZS für Phon. XIX. 241-259.

Leys, Odo. 1973. Nicht-referentielle Nominalphrasen. deutsche sprache 1. 1-15.

Lieb, H. H. 1966. Das Sprachstadium: Entwicklungsabschnitt und System? Lingua 16. $352-363$.

Lindqvist, Axel. 1961. Satzwörter. Eine vergleichende syntaktische Studie. Göteborg.

Lipka, Leonhard. 1971. Grammatical categories, lexical items and word-formation. Found. of Lang. 7,2. 211-238.

Longacre, R. E. 1964. Grammar discovery procedures. A field manual. Jan. ling. ser. min. 33. The Hague, Mouton.

Lyons, John. 1966. Towards a notional theory of the 'parts of speech'. Journal of Ling. 209-236.

Maas, Utz, Dieter Wunderlich. 1972. Pragmatik und sprachliches Handeln. Mit einer Kritik am Funkkolleg Sprache. Frankfurt (Main), Athenäum.

1972. Ein Problem der Fragelogik: Sind zurückgewiesene Präsuppositionen Antworten? Ling. Ber. 19, 69-73.

1972a. Subjekt, Präsuppositionen, mein Freund Falk und das Ungeheuer von Loch Ness. Linguistics 93. 44-69. 
Mathesius, V. 1929. Zur Satzperspektive im modernen Englisch. Archiv für das Studium der neueren Sprachen und Literaturen. 155. 202-210.

McCawley, James D. 1971. Where do noun phrases come from? Steinberg/Jakobovits (eds.) . 1971. 217-231. 1975. If and only if. Ling. Inquiry $5.632 \mathrm{ff}$.

Miller, J. 1973. A note on so-called "discovery procedures". Found. of Lang. 10. $123-139$.

Miller, Rachel. 1971. Adverbs: some syntactic rules and semantic notions. Bachelor's thesis. Harvard University.

Morgan, Jerry. 1969. On the treatment of presuppositions in transformational grammar. Papers from the fifth regional meeting Chicago Linguistic Society. 167-177. Wieder abgedruckt in Petöfi/Franck (eds.). 1973. 373-388.

Muraki, Masa: 1970. Presupposition and pseudo-clefting. Papers from the sixth regional meeting Chicago Linguistic Society. 390-400.

Murphy, J. e.a. (eds.). 1975. Proceedings of the Texas Conference on performatives, presuppositions and conversational implicatures. Washington D.C.

Nerlich, G. 1965. Presupposition and entailment. American Philosophical Quarterly 2. 33-42. - Wieder abgedruckt in Petöfi/Franck (eds.). 1973. 137-158.

Nolan, Rita. 1970. Foundations for an adequate criterion of paraphrase. Jan. ling. ser. min. 84. The Hague, Mouton.

Palacas, A. L. 1971. The higher predicate status of modals and implications for the lexicon. glossa 5,1. 31-47.

Paul, Hermann. 1916-1920. Deutsche Grammatik. 4 Bde. - Repr. der 1. Aufl. Halle. Tübingen, Niemeyer. 1968.

(allein IV, 66 u. A.1; auch IV, 66, 469; bloß IV, 243; nur IV, 394 A.; nur daß 447; sogar IV, 66 A.2. - Die Stellen zu Lautform und Schreibung, überwiegend in Bd. I und II, können dem Register entnommen werden)

Perlmutter, David. 1968. Deep and surface structure constraints in syntax. Diss. MIT. - New York, Holt. 1971.

Petöfi, Janos, Dorothea Franck (eds.). 1973. Präsuppositionen in Philosophie und Linguistik. Presuppositions in philosophy and linguistics. Linguistische Forschungen Bd. 7. Frankfurt (Main), Athenäum.

Posner, Roland. 1972. Theorie des Kommentierens. Linguistische Forschungen Bd. 9. Frankfurt (Main), Athenäum.

Quine, W. V. O. 1943. Notes on existence and necessity. The Journal of Philosophy 40. - Deutsche Übersetzung in Sinnreich (Hg.). Zur Philosophie der idealen Sprache. München. 1972. 34-52.

Quirk, R., J. Svartvik. 1966. Invesigigating linguistic acceptability. The Hague, Mouton.

Reichenbach, H. 1948. Elements of symbolic logic. New York, The free Press. Paperback 1966 .

Reis, Marga. 1974. Rez. zu Petöfi/Franck (eds.). 1973. deutsche sprache 4. 287-304.

1974a. Assertion, Negation und "Fact". Anmerkungen zu P. \& C. Kiparsky. 1971. Kapitel 2. Papiere zur Linguistik 6. 73 - 89. 
Reis, Marga. 1975. Präsuppositionen und Syntax. Eine Vorstudie.(Masch.) Habil.-Schrift. München.

Rohrer, C. 1971. Die Beziehung zwischen Disjunktion und Quantifizierung mit Existenzzeichen. A. v. Stechow (Hg.), Beiträge zur generativen Grammatik. 228-237.

1970.zur Theorie der Fragesätze. D.Wunderlich (Hg.), Probleme und Fortschritte der Transformationsgrammatik. 109-126.

Rosenberg, J. F., Ch. Travis (eds.). 1971. Readings in the philosophy of language. Englewood Cliffs (N.J.), Prentice-Hall.

Ross, John Robert. 1967. Constraints on variables in syntax. (Masch.) Diss. MIT. Russell, Bertrand. 1905. Theory of descriptions.

1957. Mr. Strawson on referring. Mind 66. 385-389. - Wieder abgedruckt in Olshewsky (ed.). Problems in the philosophy of language. New York 1969. 333-338.

1905. On Denoting. Mind 30. 479-493. - Wieder abgedruckt in Olshewsky (ed.). Problems in the philosophy of language. New York,1969. 300-311.

Schank, Gerd. 1973. Zur Korpusfrage in der Linguistik. deutsche sprache 3.16-27.

Schmerling, Susan F. 1972. Presupposition and the notion of normal stress. Papers from the seventh regional meeting Chicago Linguistic Society 242-254.

1974. A re-examination of 'normal stress'. Language 50,1. 66-73.

Schmid, Wolfgang.P. 1970. Skizze einer allgemeinen Theorie der Wortarten. Mainzer Akad. der Wiss. u. d. Lit. Abhdlgn. der Geistes- und Sozialwiss. Klasse. Jg. $1970 \mathrm{Nr} .5$.

Schmidt, Wilhelm. 1964. Grundfragen der deutschen Grammatik. Berlin. (zu Partikeln siehe S. 204-207.).

1967. Lexikalische und aktuelle Bedeutung. Ein Beitrag zur Theorie der Wortbedeutung. Berlin, Akademie Verlag.

Schnelle, Helmut. 1970. Theorie und Empirie in der Sprachwissenschaft. H. Pilch, H. Richter (Hgg.). Theorie und Empirie in der Sprachforschung. Basel, München. 51-65.

1973. Sprachphilosophie und Linguistik. Prinzipien der Sprachanalyse a priori und a posteriori. rororo studium 30. Reinbek, Rowohlt.

Schnitzer, M. L. 1971. Presupposition, entailment and Russell's theory of description. Found. of.Lang. 7,2. 279-299.

Schnurr, Dagmar. 1973. Wozu überhaupt "überhaupt"? Ling. Ber. 26. 25-34.

Schubiger, Maria. 1965. English intonation and german modal particles. A comparative study. Phonetica XII. 65-84.

Searle, John R. 1968. Speech acts. An essay in the philosophy of language. Cambridge. Paperback 1970. - Übersetzung: Sprechakte. Aus dem Englischen von R. und R. Wiggershaus. Frankfurt, Suhrkamp. 1971.

Settekorn, W. 1971. Akzeptabilität, Kontext, Konsituation. Muttersprache. 209-223.

Seuren, Pieter A. M. 1969. Operators and nucleus. A contribution to the theory of grammar. Cambridge, Univ. Press. 
Sgall, Petr. 1972. Fillmore's mysteries and topic vs. comment. Journal of Linguistics 8. 201-207.

, Hajicová Eva, Eva Benesová. 1973. Topic, focus and generative semantics. Forsch. Ling. u. Komm.wiss. 1. Kronberg, Scriptor.

Sinnreich, Johannes.(Hg.). 1972. Zur Philosophie der idealen Sprache.München,dtv.

Sitta, H., W. Boettcher. 1972. Deutsche Grammatik III. Zusammengesetzter Satz und äquivalente Strukturen. Frankfurt, Athenäum.

Spencer, N.J. 1973. Differences between linguistics and nonlinguistics in intuitions of grammaticality - acceptability. Journal of psycholinguistic research, Vol. 12, No. 2. 83-98.

Spranger, Ursula. 1969. Modalwörter der Potentialität in der deutschen Gegenwartssprache. Masch. phil. Diss. Halle.

Stalnaker, R. C. 1972. Pragmatics. Davidson/Harman (eds.). Semantics of natural languages. North Holland. 380-397. - Auch in Petöfi/Franck (eds.). 1973. 389-408.

Stechow, A. v. 1971. Zur Theorie der Präsupposition. Münchner Papiere zur Linguistik 1. 1-18.

Steinberg, D. D., L. A. Jakobovits (eds.). 1971. Semantics. An interdisciplinary reader in philosophy, linguistics and psychology. Cambridge.

Steinitz, Renate. 1969. Adverbial-Syntax. Studia Grammatica Bd. X. Berlin, Akademie Verlag.

, Schädlich. 1972. Probleme der adverbialen Subkategorisierung. Actes Ling. 10,2. 977-984.

, Ewald Lang. 1975. Rez. zu R. Bartsch. 1972. Manuskript Berlin.

Steube, Anita. 1968. Gradation der Grammatikalität. Ruzicka (ed.), Probleme der strukturellen Grammatik und Semantik. 87-113.

Stickel, Gerhard. 1970. Untersuchungen zur Negation im heutigen Deutsch. Schriften zur Linguistik 1. Braunschweig, Vieweg.

1972. Ja und Nein als Kontroll- und Korrektursignale. Ling. Ber. 17. 12-17.

Strawson, P. F. 1950. On Referring. Mind 59. 330 ff.

1952. Introduction to logical theory. London. - Reprint London, Methuen. 1971 .

1954. Reply to Mr. Sellars. Philosophical Review 63. 216-231.

1970. Meaning and truth. Oxford.

Tarski, Alfred. 1944. The semantic conception of truth and the foundations of semantics. Philosophy and phenomenological research 4. - Übersetzung: Die semantische Konzeption der Wahrheit und die Grundlagen der Semantik. Johannes Sinnreich (Hg.). 1972. 53-100.

Vater, Heinz. 1967. Zur Tiefenstruktur deutscher Nominalphrasen. Beiträge zur Linguistik und Informationsverarbeitung 11. 53-71.

Vennemann, Theo. 1973. Topics, sentence accent, ellipsis: a proposal for their formal treatment. - Paper presented at the seminar on formal semantics. cambridge. 
Welte, Werner. 1971. Bemerkungen zur Problematik der Adverbialsyntax im Lichte verschiedener Modelle. LB-Papier Nr. 17.

Werner, Jürgen. 1960. Zu Gradadyerbien negativen Bedeutungsgehaltes. Forschungen und Fortschritte 34. 244.

Weydt, Harald. 1969. Abtönungspartikeln. Die deutschen Modalwörter und ihre französischen Entsprechungen. Bad Homburg v.d.H., Gehlen. 1969.

Wimmer, Rainer. 1972. Zur Theorie der Eigennamen. Ling. Ber. $17.70-75$.

Wittgenstein, Ludwig. 1967. Philosophische Untersuchungen. Frankfurt, Suhrkamp.

Wunderlich, Dieter (Hg.).1972a. Linguistische Pragmatik. Schwerpunkte Linguistik und Kommunikationswiss. 12. Frankfurt (Main), Athenäum.

1972b. Zur Konventionalität von Sprechhandlungen. Wunderlich (Hg.). 1972a. 11-58.

1973. Präsuppositionen in der Linguistik. Petöfi/Franck (eds.). 1973. 467-484.

- 1974. Grundlagen der Linguistik. rororo studium 17. Reinbek, Rowohlt.

Yamanashi, Masa-aki. 1972. Lexical decomposition and implied proposition. Papers from the eighth regional meeting Chicago Linguistic Society 388-401.

Zaefferer, Dietmar. 1974. Untersuchungen zu einer Theorie der sprachlichen Mißverständnisse. Vortrag auf dem 9. Ling. Koll. Bielefeld.

Zemb, Jean Marie. 1972. Satz, Wort, Rede. Semantische Strukturen des deutschen Satzes. Freiburg, Herder. 